Discovery of prognostic biomarkers from the prostate cancer membrane proteome using bioorthogonal chemistry.

Lynn Asante-Asare

St John's College

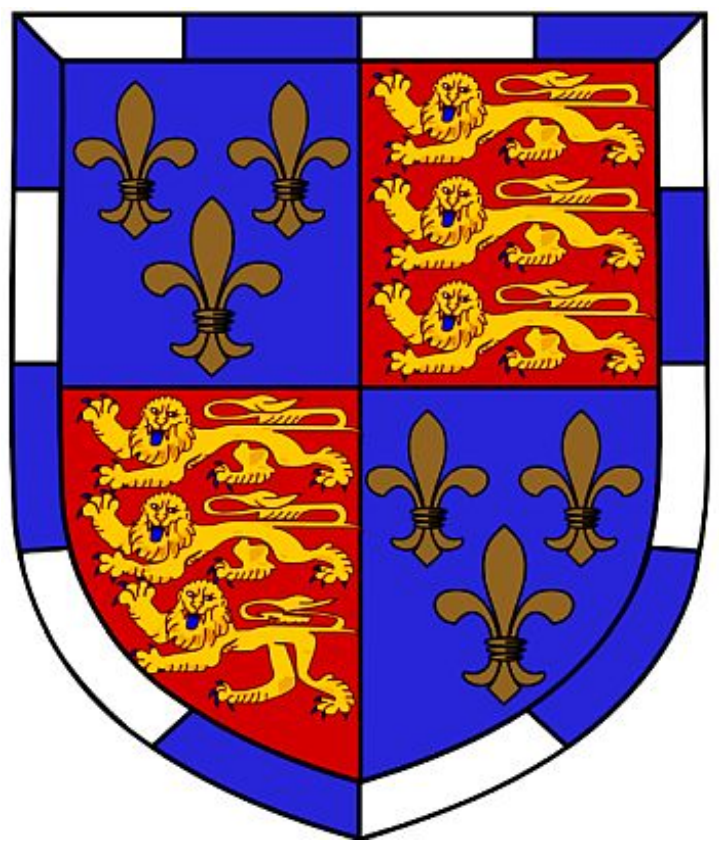

This thesis is submitted for the degree of Doctor of Philosophy, University of Cambridge September 2019 


\section{$\underline{\text { Declaration }}$}

This thesis is the result of my own work and includes nothing which is the outcome of work done in collaboration except as declared in the Preface and specified in the text. It is not substantially the same as any that I have submitted, or, is being concurrently submitted for a degree or diploma or other qualification at the University of Cambridge or any other University or similar institution except as declared in the Preface and specified in the text. I further state that no substantial part of my thesis has already been submitted, or, is being concurrently submitted for any such degree, diploma or other qualification at the University of Cambridge or any other University or similar institution except as declared in the Preface and specified in the text. It does not exceed the prescribed word limit for the relevant Degree Committee 


\title{
Discovery of prognostic biomarkers from the prostate cancer membrane proteome using bioorthogonal chemistry by Lynn Asante-Asare
}

\begin{abstract}
The link between aberrant glycosylation and cancer progression has formed the basis for detection of cell surface glycans and glycoproteins in the clinical management of cancer. However, the utility of biomarkers has been challenged in recent years due to low cancer specificity and high false-positive rates. The aim of this study was to use synthetic precursors of sialic acid and $\mathrm{N}$-acetylgalactosamine that had been labelled with an azide group ( $\mathrm{N}$ azidoacetylmannosamine ( $\mathrm{Ac}_{4} \mathrm{ManNAz}$ ) and N-azidoacetylgalactosamine ( $\left.\mathrm{Ac}_{4} \mathrm{GalNAz}\right)$ ), and which are incorporated biosynthetically into cell surface glycans, to monitor changes in cell surface glycosylation in models of prostate and breast cancer progression, in order to identify novel biomarkers.
\end{abstract}

A panel of human prostate, breast and pancreatic cancer cell lines was used, which represented the stages of cancer progression. The metastatic potential of the established prostate and breast cancer cell lines used in the study was confirmed in the scratch wound and Boyden chamber assays and by demonstrating a correlation with increased expression of vimentin, loss of PTEN expression, and decreased E-cadherin expression. Collectively, the data validated the panels as suitable in vitro models of prostate and breast cancer progression.

Global azidosugar labelling of glycoproteins and glycolipids was detected by NeutrAvidin ${ }^{\mathrm{TM}}$ DyLight $^{\mathrm{TM}} 650$ (NA650) fluorescence using flow cytometry. Azidosugar labelling of glycoproteins was detected by Alkyne-Alexa Fluor 488 fluorescence using 1D gel electrophoresis, and Streptavidin 800 fluorescence using 2D gel electrophoresis. An increase 
in the overall NA650 fluorescence was seen across the breast cancer cell lines using flow cytometry. An increase in the overall Alexa-488 fluorescence on 1D gels and Streptavidin800 fluorescence on 2D gels was seen across the prostate, breast, and pancreatic cancer cell lines. A general increase in azidosugar-specific sialylation ( $\left.\mathrm{Ac}_{4} \mathrm{ManNAz}\right)$ and $\mathrm{N}$ acetylgalactosamine ( $\left.\mathrm{Ac}_{4} \mathrm{GalNAz}\right)$ glycosylation was associated with an increase in metastatic potential. Proteomic analysis of the prostate cancer cell lines revealed that Basigin (CD147) glycosylation, which showed a strong increase in $\mathrm{Ac}_{4} \mathrm{ManNAz}$ and $\mathrm{Ac}_{4} \mathrm{GalNAz}$ labelling, correlated with an increase in metastatic potential, despite its total expression decreasing across the panel of prostate cancer cell lines.

In summary, we have demonstrated the use of bioorthogonal chemistry for detecting differential sialyation and $\mathrm{N}$-acetylgalactosamine glycosylation of proteins from human cell lines that represent the progression of cancer. Our data suggest that differential glycosylation may be a more sensitive and specific marker for monitoring prostate and breast cancer progression, than increases in total protein expression alone. 


\section{Acknowledgements}

It has been a privilege to work on this project with Professor Kevin M. Brindle. We set out to image tumour glycosylation using bioorthogonal chemistry but soon took a detour, opting to use the technique to address an evident clinical need to find new and improved prognostic biomarkers. I am very grateful to Kevin for supporting this change in project direction, allowing me to explore my scientific interests, remaining positive during the times of uncertainty, and always helping me troubleshoot my data. I would like to thank Dr. André Neves who would always send me newly published papers relevant to my project, which helped me stay on track with recent developments in glycobiology and biomarker discovery fields. I am very grateful for André's input when developing experimental approaches and always discussing them with me in the context of current advancements in the literature. I would also like to thank the students and postdocs that have previously worked on this project, providing me with invaluable chemicals, and techniques that formed the foundation of this project. I give a special mention to Henning Stöckmann, Yelena Wainwan, André Neves, Flaviu Bulat, Joe Kuo, De-en Hu, and Tom Whittaker.

My project would have been impossible without the CRUK CI core facilities. I imagine I spent equal amounts of time running gels in Research Instrumentation as I did on my lab bench. I am grateful to have had access to such a well organised, knowledgeable and friendly facility. I would also like to thank the flow cytometry, histopathology, proteomics, and microscopy cores for their priceless technical support, and sound advice. Other members have also played important roles in helping me complete my $\mathrm{PhD}$. In my 4 years at the $\mathrm{CRUK} \mathrm{Cl}$, I have never been served a coffee from Abdul in the café without a smile. I would like to thank all the evening security guards who made for great company during post-12am-experiments. I would also like to thank Joshua Bello, Building Services technician at the CRUK Cl, Gen Loaker from Mosaic Lives Ministries, Marion Karniely, Research Administer at the $\mathrm{CRUK} \mathrm{Cl}$, and Professor Jim Metcalfe and Dr Heide Kirschenlohr, from the Department of Biochemistry, for providing me with moral support and encouraging conversations at timely moments during my PhD. 
I would like to thank Sarah McGuire, Maria Fala, Dr. Friederike Hesse, and Dr. Anastasia Tsyben, for being such a funny, witty, and compassionate colleagues, and for memorable engaging conversations about every topic under the sun. I would also like to thank Drs. Richard Mair, Richard Hesketh, Susana Ros, Linda Julian, Stefanie Reichelt, and Aarthi Gopinathan, for being my secret postdoc role models. I have always admired their work ethic, integrity, and passion for science. I am especially happy to thank Dr Ann Kaminski. I have sought an audience with Ann several times to discuss things ranging from my PhD to my British Sign Language exams. I cannot thank Ann enough for her listening ear, invaluable advice, and caring nature.

I would like to thank my best friends Yvette Agyako, Donjeta Miftari, Alicia Neophytou, and Dr. Jennifer Murray, for their moral support, and continual encouragement. My brothers Michael Mensah and William Tagoe, nephew Karell Tagoe, and niece Kiana-Mae Tagoe have been invaluable in supporting my career aspirations and keeping my spirits high. Finally, I would like to give a precious thank you to my mum Veronica Hibbert for everything and more, but especially for keeping me filled with all the best food Ghana has to offer, throughout my PhD. 


\section{Abbreviations}

$\mathrm{Ac}_{4} \mathrm{GalNAz} \quad$ Peracetylated $\mathrm{N}$-azidoacetylgalactosamine

$\mathrm{Ac}_{4} \mathrm{ManNAz}$ Peracetylated $\mathrm{N}$-azidoacetylmannosamine

AFP Alpha fetoprotein

AFP-L3 Abberantly fucosylated alpha fetoprotein

Asn Asparagine

CA15-3 Cancer antigen 15-3

CEA Carcinoembryonic antigen

CT Computerised tomography

$\mathrm{EBL} \quad$ Elderberry bark lectin

EGFR Epidermal growth factor receptor

ELISA Enzyme-linked immunosorbent assay

FBS Foetal bovine serum

Fuc Fucose

Gal Galactose

GalNAc N-acetyl-galactosamine

Glc Glucose

GlcA Glucuronic acid

GlcNAc N-acetyl-glucosamine

H\&E Hematoxylin and eosin

HER 2 Human epidermal growth factor receptor 2

HPA Helix pomatia agglutinin

HRP Horseradish peroxidase

KC p48Cre; LSL-KrasG12D

LCA Lens culinaris agglutinin lectin

LCMS Liquid chromatography-tandem mass spectrometry 


\begin{tabular}{|c|c|}
\hline MAL I & Maackia amurensis lectin \\
\hline Man & Mannose \\
\hline MRI & Magnetic resonance imaging \\
\hline MUC1 & Mucin 1, cell surface associated \\
\hline NA650 & NeutrAvidin ${ }^{\mathrm{TM}}$ DyLight $^{\mathrm{TM}} 650$ \\
\hline NeuAc & $\mathrm{N}$-acetylneuraminic acid \\
\hline NSAF & Normalised Spectral Abundance Factor \\
\hline PanIN & Intraepithelial neoplasia \\
\hline PBS & Phosphate Buffered Saline \\
\hline PDA & Pancreatic ductal adenocarcinoma \\
\hline PET & Positron-emission tomography \\
\hline PNGase F & Peptide N-glycosidase F \\
\hline PSA & Prostate specific antigen \\
\hline PSMA & Prostate-specific membrane antigen \\
\hline PTEN & Phosphatase and tensin homolog \\
\hline Ser & Serine \\
\hline$S L e^{A}$ & Sialyl Lewis A antigen \\
\hline$S L e^{x}$ & Sialyl Lewis $\mathrm{X}$ antigen \\
\hline SNA & Sambucus Nigra Lectin \\
\hline SPECT & Single-photon emission imaging \\
\hline STI & Soybean trypsin inhibitor \\
\hline $\mathrm{T}$ & Thomsen-Friedenreich antigen \\
\hline Thr & Threonine \\
\hline TMDIBO & Tetramethoxydibenzocyclooctyne \\
\hline $\mathrm{Tn}$ & Thomsen-nouveau antigen \\
\hline TRIS-HCL & Tris hydrochloride \\
\hline WGA & Wheat germ agglutinin lectin \\
\hline Xyl & Xylose \\
\hline
\end{tabular}




\section{$\underline{\text { Table of contents }}$}

Declaration . i

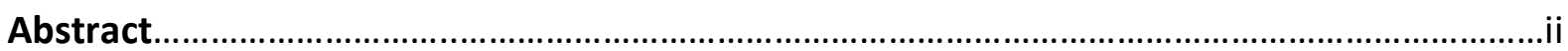

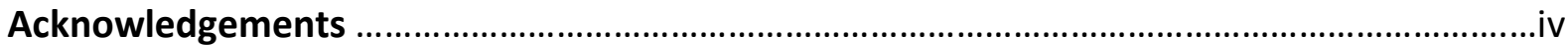

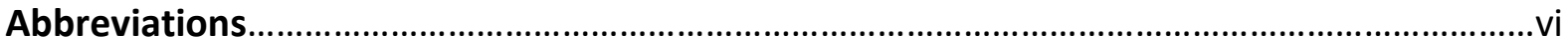

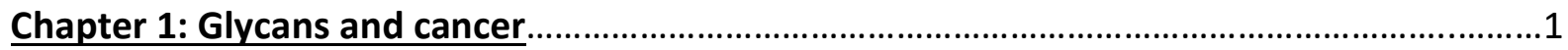

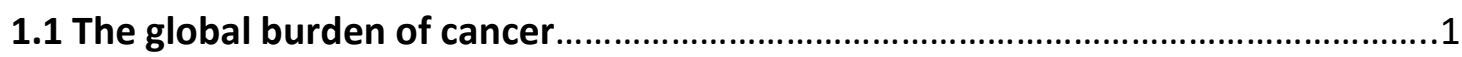

1.1.1 The global burden of cancer........................................................................

1.1.2 Reducing the burden of cancer with precision oncology and glycomics...3

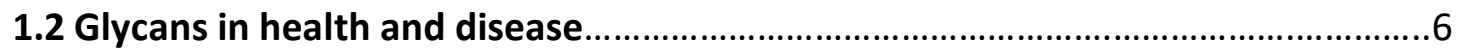

1.2.1 Glycan structure, diversity and biosynthesis.............................................6

1.2.2 Glycan function.........................................................................................

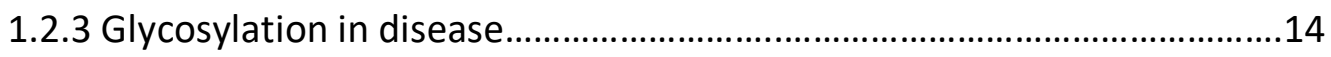

1.2.4 Aberrant glycosylation in cancer................................................................15

1.2.5 The functional role of aberrant glycosylation in cancer progression......19

1.2.6 The discovery of tumour-specific markers..............................................21

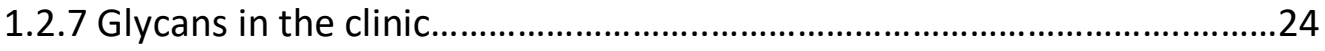

1.3 Exploiting the cancer glycome in biomarker discovery .........................................28

1.3.1 The challenges associated with using glycans as cancer biomarkers......28

1.3.2 Overcoming the challenges associated with using glycans as cancer

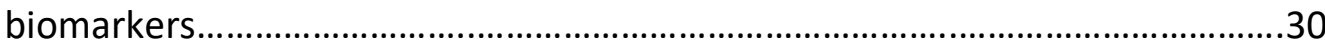

1.3.3 Detecting glycosylation using lectins......................................................33

1.3.4 Detecting glycosylation using antibodies...................................................37

1.3.5 Detecting glycosylation using bioorthogonal chemical reporters............39

1.3.6 Additional metabolic labelling strategies.................................................45

1.4 Project aims, objectives and experimental approaches..........................................47

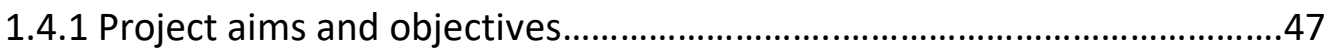


Chapter 2: Validation of in vitro panels of cancer progression...............................................55

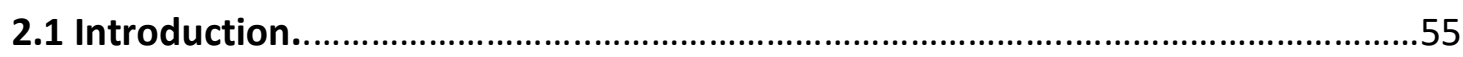

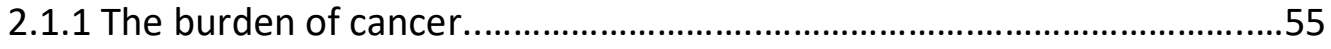

2.1.2 Histological and molecular progression of cancer....................................55

2.1.3 Pre-clinical models of cancer progression..............................................57

2.1.4 in vitro models of cancer progression.....................................................58

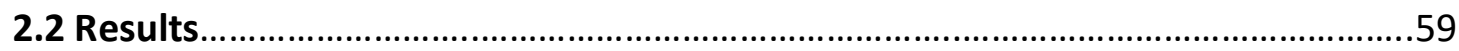

2.2.1 Validation of in vitro panels of prostate cancer progression....................59

2.2.2 Validation of in vitro panels of breast cancer progression.........................69

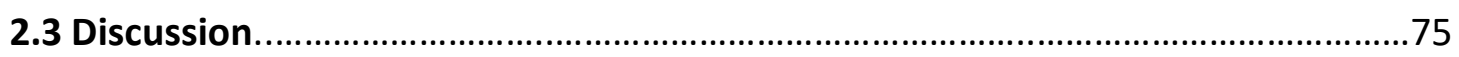

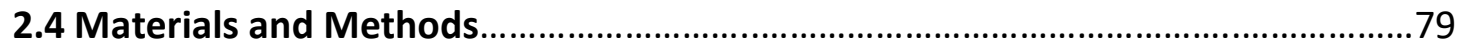

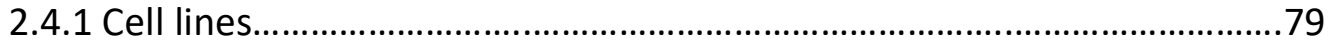

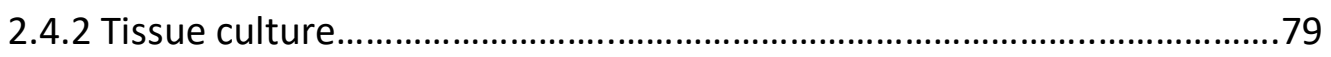

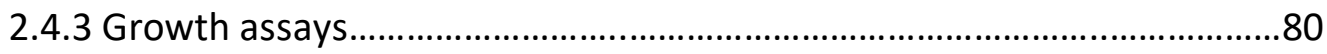

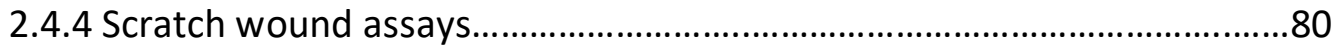

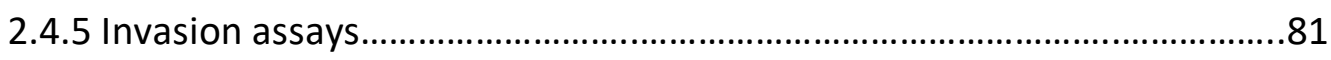

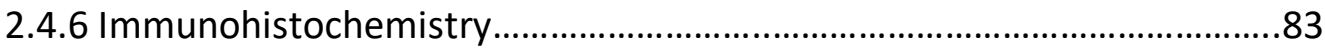

2.4.7 Preparation of whole-cell lysates................................................................. 83

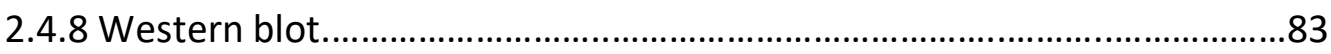

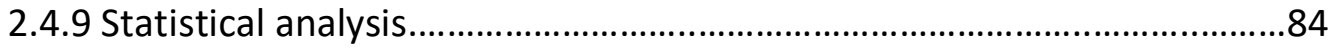

Chapter 3: Detection of differential glycosylation using bioorthogonal chemistry in in vitro models of cancer progression with the aim of imaging of tumour glycosylation in vivo.......85

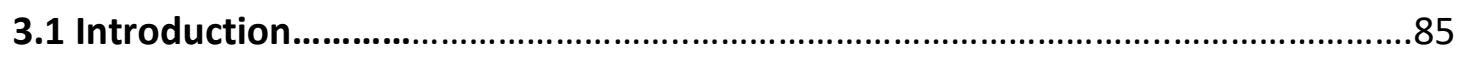

3.1.1 Aberrant sialyation and $\mathrm{N}$-acetylgalactosamine glycosylation in cancer 85

3.1.2 Imaging aberrant tumour glycosylation using bioorthogonal chemistry 86 
3.2.1 Flow cytometric analysis of metabolically labelled breast cancer cell lines.

3.2.2 Metabolic labelling of the p48Cre; LSL-KrasG12D (KC) mouse model of pancreatic cancer progression

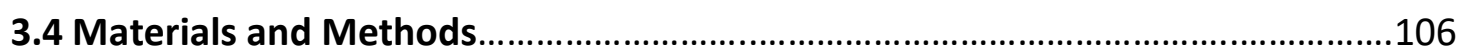

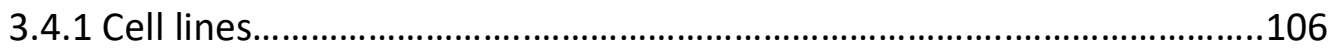

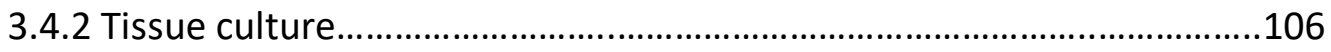

3.4.3 Bioorthogonal metabolic labelling in vitro for flow cytometry..............107

3.4.4 Pancreatic tumour organoid cultures.....................................................108

3.4.5 Bioorthogonal metabolic labelling of pancreatic tumour organoids for confocal microscopy

3.4.6 Bioorthogonal metabolic labelling of p48Cre; LSL-KrasG12D (KC) mice in vivo and chemoselective ligation of the metabolically labelled membrane proteins ex vivo.

3.4.7 1D gel electrophoresis and in-gel imaging of azidosugar labelled glycoproteins.

3.4.8 Statistical analysis.

Chapter 4: Investigation of differential glycosylation using bioorthogonal chemistry and gel electrophoresis in in vitro and in vivo models of cancer progression.

4.1 Introduction..

4.1.1 Cell surface protein glycosylation in cancer.

4.1.2 Detection of aberrantly glycosylated cell surface glycoproteins using bioorthogonal chemistry

4.2 Results.

4.2.1 Enrichment of the membrane proteome.

4.2.2 Azidosugar labelling of the membrane proteome of prostate cancer cell Lines.

4.2.3 Azidosugar labelling of the membrane proteome of breast cancer cell 
lines.

4.2.4 Azidosugar labelling of the membrane proteome of pancreatic cancer cell lines

4.2.5 Specific azidosugar labelling of $\mathrm{N}$-linked glycoproteins probed using deglycosylation enzymes.

4.2.6 Azidosugar labelling of the membrane proteome analysed using 2D gel Electrophoresis.

4.3 Discussion

4.4 Materials and Methods.

4.4.1 Cell lines.

4.4.2 Tissue culture. 148

4.4.3 Extraction and purification of the membrane protein fraction 148

4.4.4 Western blot .149

4.4.5 LC-MS/MS 150

4.4.6 Database searching. 150

4.4.7 Bioorthogonal metabolic labelling of cell lines in vitro for gel electrophoresis.

4.4.8 1D gel electrophoresis and in-gel imaging of azidosugar labelled glycoproteins

4.4.9 Deglycosylation of metabolically labelled glycoproteins using PNGase F

4.4.10 2D western blot analysis of azidosugar labelled glycoproteins. 153

4.4.11 Statistical analysis. .155

Chapter 5: Identification of bioorthogonally labelled proteins using liquid chromatographytandem mass spectrometry. 156

5.1 Introduction. 156

5.1.1 Glycan's as biomarkers. 156

5.1.2 Identification of new glycosylated biomarkers using bioorthogonal Chemistry. 157

5.2 Results 159 
5.2.1 Preparation of azidosugar-labelled proteins for mass spectrometry analysis. 160

5.2.2 Identification of azidosugar-labelled proteins using liquid chromatography-tandem mass spectrometry.......

5.2.3 Glycoprofiling of membrane bound and secreted basigin using lectin blotting

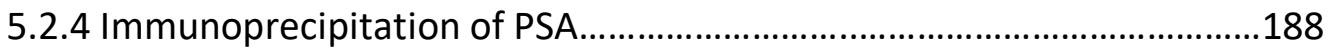

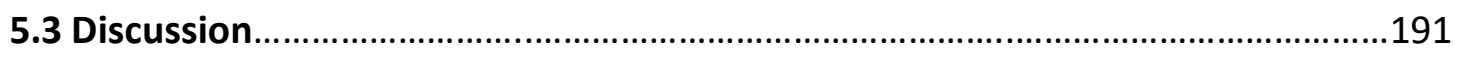

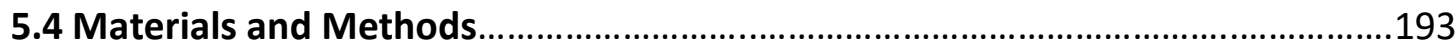

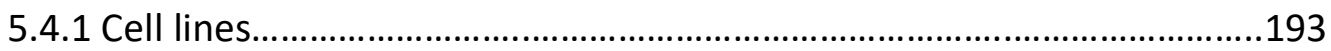

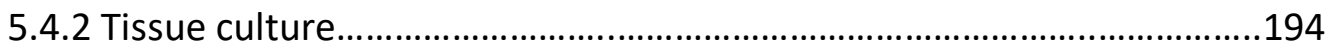

5.4.3 Bioorthogonal metabolic labelling of cell lines in vitro for mass spectrometry.

5.4.4 Enrichment of metabolically labelled glycoproteins using Streptavidin

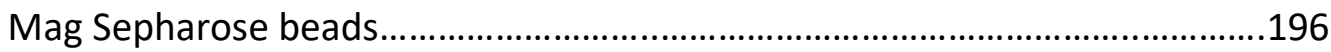

5.4.5 Sample preparation for LCMS/MS ........................................................197

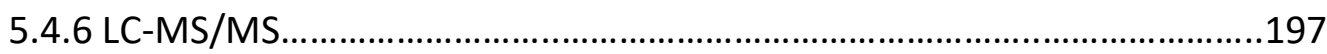

5.4.7 Database searching................................................................................198

5.4.8 Quantitative analysis of protein levels.................................................198

5.4.9 Preparation of whole-cell lysates and conditioned media.....................199

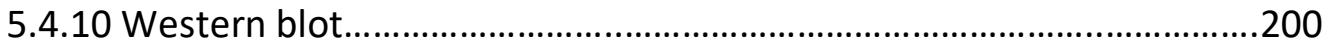

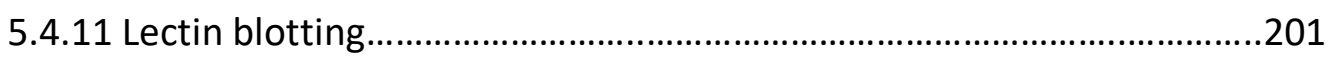

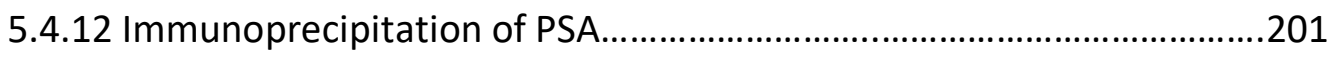

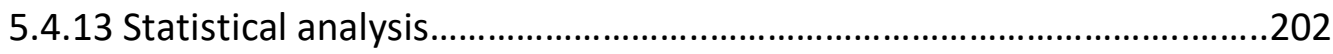




\section{Chapter 1: Glycans and cancer}

\subsection{The global burden of cancer}

\subsubsection{The global burden of cancer}

Trends in cancer-related morbidity (Fitzmaurice et. al. 2015) and mortality worldwide, highlight cancer as a threat to public health. Neoplasms were the third leading cause of deaths globally in 1990, after cardiovascular disorders, infections, and parasitic diseases (Murray et. al. 1997). Neoplasms accounted for 5.7 million deaths in 1990, 8.2 million deaths in 2013, and 9.9 million deaths in 2016 (Naghavi et. al. 2015; Foreman et. al. 2018). The number of global cancer deaths is forecasted to rise to 14.9 million by 2040 (Foreman et. al. 2018). A surge in the incidence of age-related cancers, such as prostate and breast cancers, is also anticipated due to increasing life expectancy, an aging population, and the association between cancer risk and age (Cook et. al 1969). For example, the global incidence of prostate cancer increased by $217 \%$ between 1990-2013 (Fitzmaurice et. al. 2015; DrudgeCoates et. al. 2018), although this trend has been associated with increases in the use of prostate specific antigen (PSA) testing and the incidental detection of tumours in patients undergoing a transurethral prostatectomy for the treatment of benign prostatic hyperplasia (Potosky et. al. 1990, Zhou et. al. 2016). In the UK alone, prostate and breast cancers account for the greatest proportion of cancer cases in males and females, respectively (Smittenaar et. al. 2016) (Figure 1.1). 

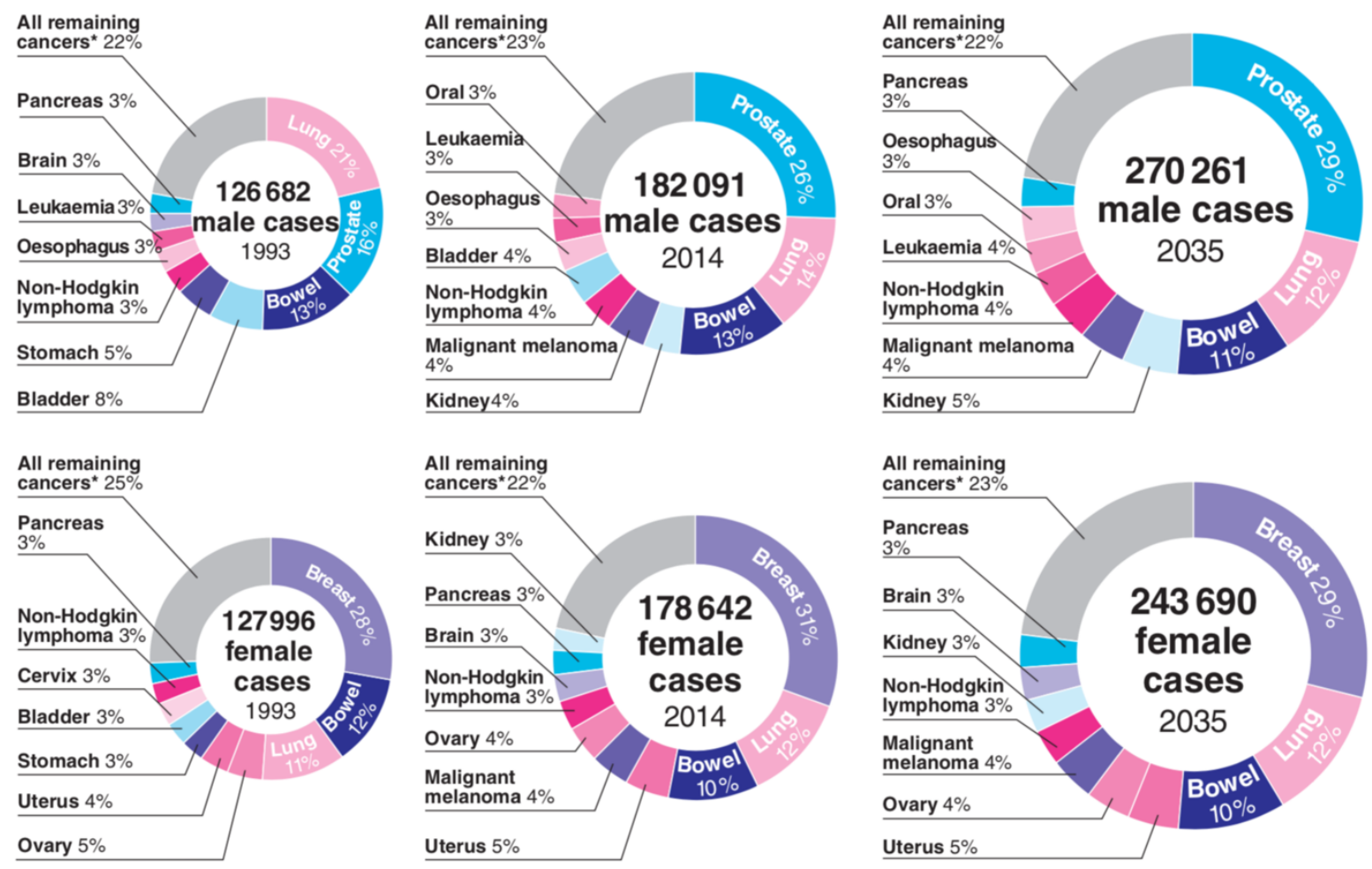

Figure 1.1: Total number of cancer cases recorded in the UK in 1993, 2014 and forecasted

for 2035, divided by organ site, and gender. (Figure taken from Smittenaar et. al. 2016).

Several epidemiology studies have identified a link between unhealthy dietary patterns and cancer risk (Grosso et. al. 2017). Therefore, the projected increases in global cancer incidence and mortality, have been attributed to the prevalence of cancer-associated risk factors. Without intervention, high blood pressure is forecast to be the leading risk factor contributing to poor health outcomes in 2040 , followed by a high body mass index, high fasting plasma glucose, tobacco, alcohol use, ambient particulate matter, and high total cholesterol (Foreman et. al. 2018). In response to the rising incidence of cancer in Europe (Quinn et. al. 2003; Naghavi et. al. 2015), the European Code Against Cancer was created to influence dietary habits across the EU and minimise population exposure to avoidable risk factors (Boyle et. al. 2003) (Figure 1.2). In the UK alone, tobacco smoking and obesity have been identified as the most preventable causes of cancer (Brown et. al. 2018). 
1) Do not smoke. Do not use any form of tobacco.

2) Make your home smoke free. Support smoke-free policies in your workplace.

3) Take action to be a healthy body weight.

4) Be physically active in everyday life. Limit the time you spend sitting.

5) Have a healthy diet: Eat plenty of whole grains, pulses, vegetables and fruits. Limit high-calorie foods (foods high in sugar or fat) and avoid sugary drinks. Avoid processed meat; limit red meat and foods high in salt.

6) If you drink alcohol of any type, limit your intake. Not drinking alcohol is better for cancer prevention.

7) Avoid too much sun, especially for children. Use sun protection. Do not use sunbeds.

8) In the workplace, protect yourself against cancer-causing substances by following health and safety instructions.

9) Find out if you are exposed to radiation from naturally high radon levels in your home. Take action to reduce high radon levels.

10) For women: Breastfeeding reduces the mother's cancer risk. If you can, breastfeed your baby. Hormone replacement therapy (HRT) increases the risk of certain cancers. Limit use of HRT.

11) Ensure your children take part in vaccination programmes for: Hepatitis B (for newborns) Human papillomavirus (HPV) (for girls).

12) Take part in organised cancer screening programmes for: Bowel cancer (men and women) Breast cancer (women) Cervical cancer (women).

Figure 1.2: The European code against cancer. (Figure taken from The World Health Organization, 2019, and Boyle et. al. 2003).

Overall, cancer incidence and mortality continue to increase worldwide, a trend accelerated by rising life expectancy, an ageing population, and exposure to cancer-associated risk factors (Smittenaar et. al. 2016).

\subsubsection{Reducing the burden of cancer with precision oncology and glycomics}

Screening in disease prevention remains a priority for policymakers, to improve the early detection of cancer, and offset the grave increases in cancer incidence observed worldwide (Shieh et. al. 2016; Schröder et. al. 2009; Foreman et. al. 2018) (Figure 1.3). Furthermore, non-imaging-based screening programmes have reduced the incidence of colorectal cancer, such as the faecal occult blood test, which screens healthy individuals aged over 50 for blood in the faeces (Duffy 2012). Prevention with the Human Papilloma Virus vaccine has also been instrumental in cervical cancer prevention (Penny and Wallace 2015). 


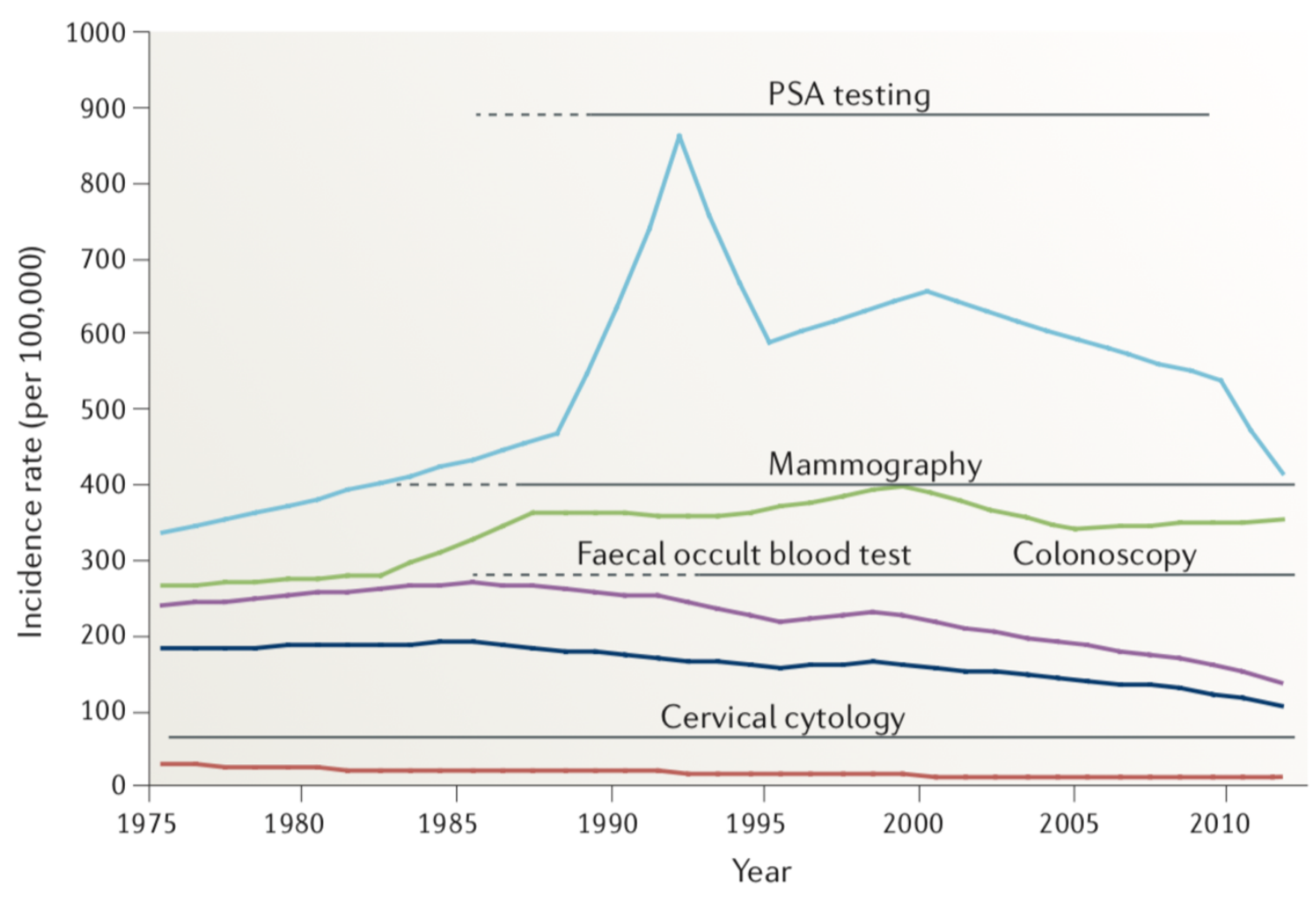

— Colorectal (females) - Cervical and uterine (females) - Breast (females)

- Colorectal (males) — Prostate (males)

Figure 1.3. The impact of population-based screening on cancer incidence in the USA.

Annual incidence rates of selected cancers in individuals aged over 50, between 1975-2012, were collated from the Surveillance, Epidemiology, and End Results registry. The implementation of routine screening programmes associated with each cancer type is represented (black lines). A reduction in the incidence of cervical and colorectal cancers are associated with the implementation of cervical cytology and the faecal occult blood test, respectively (Figure taken from Shieh et. al. 2016).

Whilst public health interventions have improved cancer detection and prevention, advancements in precision molecular oncology are furthering improvements in cancer outcomes. Molecular medicine is "a branch of medicine that develops ways to diagnose and treat disease by understanding the way genes, proteins, and other cellular molecules work" (National Cancer Institute Dictionary of Cancer Terms, 2019). Precision oncology, involves 
using patients biological, genetic and clinical information, to individualise clinical interventions, and treatment regimens (Biankin 2017). The rise of the "omics" revolution has expanded the field of precision oncology by exploiting our understanding of the role of biomolecules in cancer such as DNA (genomics), gene expression (transcriptomics), epigenetic mutations (epigenomics), protein expression (proteomics), and metabolism (metabolomics) (Epstein and Lin 2017).

The impact of "omics" research on precision oncology and disease outcomes can be illustrated through the development of immunotherapy, which specifically targets molecules unique to cancer cells to block or slow their proliferation and spread (Lee et. al. 2018). Trastuzumab is a monoclonal antibody, which targets human epidermal growth factor receptor 2 (HER2), a protein receptor overexpressed in breast cancers (Lee et. al. 2018). Breast cancer patients receiving anti-HER2 trastuzumab, are first tested for the presence of HER2 overexpression or HER2 gene amplification using a Food and Drug Administration (FDA)-approved multiple gene expression diagnostic assay called Oncotype DX (Schmidt et. al. 2016). Administering trastuzumab specifically in HER2 positive patients has been shown to provide optimal treatment response, reduce time to disease progression, and improve survival (Schmidt et. al. 2016). Next-generation sequencing has also been used to improve drug prescription guidelines by identifying gene mutations associated with drug toxicity. For example, leukaemia patients being treated with thiopurine based chemotherapies, receive up to a $90 \%$ dose reduction if they express a mutant thiopurine-S-methyl transferase, which has reduced thiopurine metabolism activity (Bainkin 2017).

Glycomics is the study of glycans, carbohydrates covalently attached to proteins or lipids, within cells and tissues, and their variation during biological events such as development and disease (Bertozzi and Sasisekharan 2009; Bennun et. al. 2016). Cancer glycomics involves the use of technologies such as mass spectrometry, glycan-binding lectin and antibody arrays, carbohydrate stains, enzymatic cleavage, metabolic labelling techniques, and genetic mutation models, to study the structure, distribution, abundance, and function of glycans in cancer (Springer and Gagneux 2016). This field of research has contributed towards a growing body of evidence implicating glycosylation as a vital driver in tumourigenesis (Christiansen et. al. 2014). Collaborative organisations such as the Consortium for Functional 
Glycomics (CFG), and the National Cancer Institute's (NCl) Alliance of Glycobiologists, have been created to advance our understanding of glycan biology to improve precision oncology (Hart and Copeland 2010).

This chapter will review the role of glycans in cancer, and their utility in promoting precision oncology through biomarker discovery, and molecular imaging.

\subsection{Glycans in health and disease}

\subsubsection{Glycan structure, diversity and biosynthesis}

Glycans are the complex carbohydrate structures formed when monosaccharides link together via glycosidic bonds. These monosaccharides are glucose (Glc), N-acetylglucosamine (GlcNAc), Galactose (Gal), N-acetyl-galactosamine (GalNAc), Mannose (Man), Xylose (Xyl), Glucuronic acid (GlcA), Fucose (Fuc) and N-acetylneuraminic acid (NeuAc), also known as sialic acid (Prestegard and Widmalm, 2017). As with proteins and nucleic acids, diversity in carbohydrate structures is conferred by the composition of monosaccharide units and their sequences. However, monosaccharides, unlike amino acids and nucleotides, are unique due to their ability to bond at different carbon positions, and in linear or branched forms (Seeberger 2017; Prestegard and Widmalm, 2017). D-sugars can link via $\alpha$ glycosidic bonds where the $\mathrm{OH}$ group on the anomeric carbon is below the plane of the monosaccharide ring, or via $\beta$-glycosidic bonds where the $\mathrm{OH}$ group on the anomeric carbon is above the plane of the monosaccharide ring (Seeberger 2017; Prestegard and Widmalm, 2017). Additional layers of complexity exist by the covalent attachment of chemical groups to glycan structures such as sulphates, phosphates, and acetyl groups (Seeberger 2017). Furthermore, monosaccharides can conjugate to acyl (non-sugar) groups within proteins or lipids that are embedded in the cell membrane or secreted. This gives rise to the different classes of glycoconjugates, which are the glycoproteins, glycolipids, glycosphingolipids, glycosaminoglycans, and proteoglycans (Pinho and Reis 2015) (Figure 1.4). 


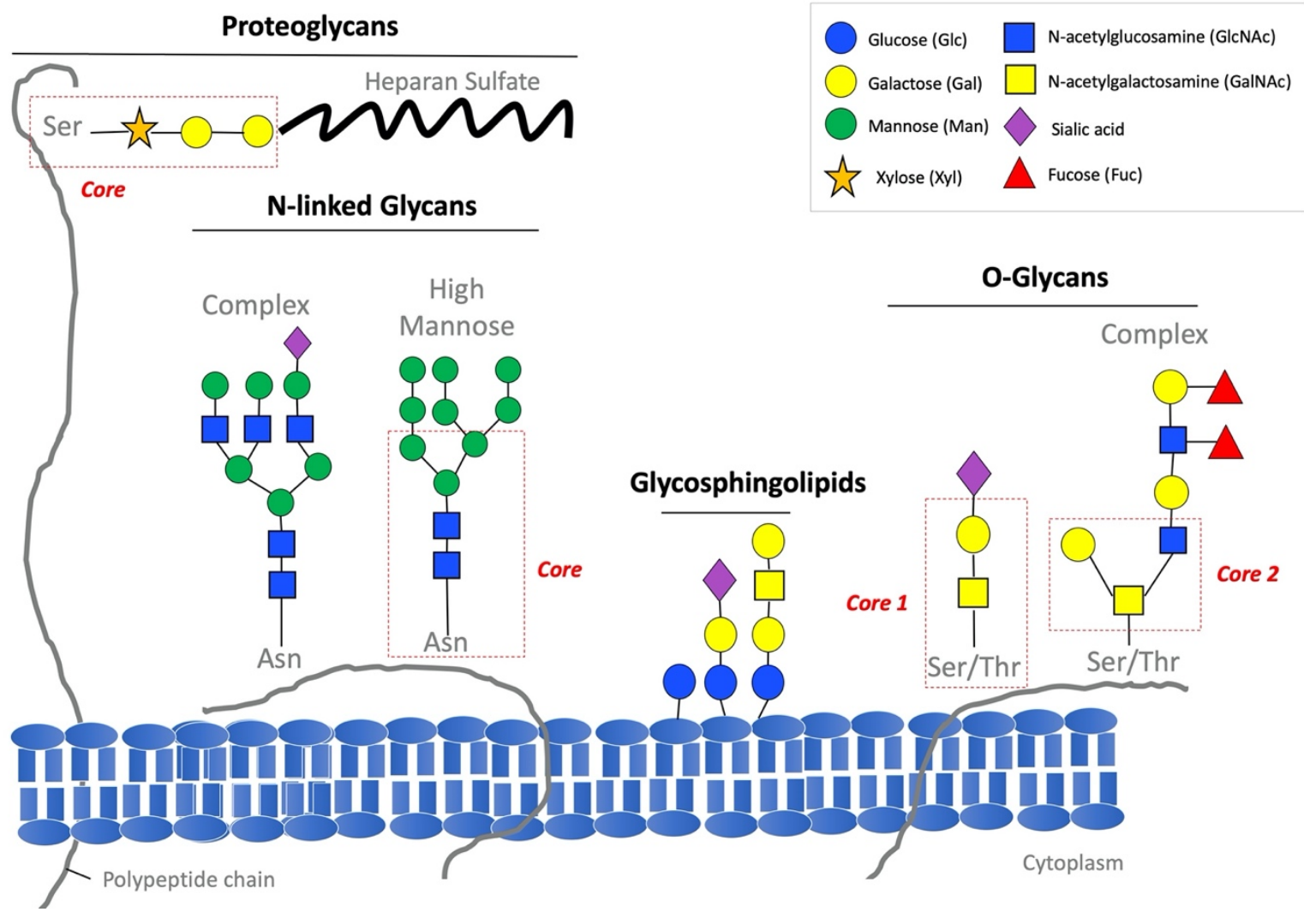

Figure 1.4: Common classes of glycoconjugates in mammalian cells. Schematic

representations of the structures of proteoglycans, N-glycans, O-glycans, glycosphingolipids, and proteoglycans. Within the glycoproteins, subclasses are defined by the amino acid upon which the glycan structures are installed. The synthesis of the N-glycans involves the binding of $\mathrm{N}$-acetylglucosamine to asparagine residues within the target polypeptide chain via an amide side chain. The synthesis of the O-glycans involves the covalent addition of carbon 1 in $\mathrm{N}$-acetylglucosamine to serine or threonine residues via a hydroxyl group. Core structures conserved within each class of glycoconjugates are highlighted (red perforated box). Abbreviations: serine (Ser), asparagine (Asn), and threonine (Thr). (Author diagram).

The majority of mammalian glycoconjugates are synthesised de novo by assembly through the Endoplasmic reticulum (ER)-Golgi secretory pathway. The enzymes responsible for processing the glycosidic linkages between monosaccharides and target conjugates include the glycosyltransferases and glycosidases (Ghazarian et. al. 2011). By segregating the 
location of glycoenzymes in different cell compartments, glycosylation occurs in a sequential, and non-templated fashion (St. Amand et. al. 2014).

The synthesis of the N-glycan's (Stanley et. al. 2017) is initiated by the ER-resident enzyme UDP-GIcNAc dolichol phosphate N-acetylglucosamine-1-phosphate transferase, which transfers $\mathrm{N}$-acetylglucosamine-phosphate (GlcNAc-1-P) to dolichol phosphate (Dol-P), to generate dolichol pyrophosphate $\mathrm{N}$-acetylglucosamine (Dol-P-P-GIcNAc). Thirteen monosaccharides are transferred sequentially to Dol-P-P-GICNAc by glycosyltransferases, to generate a mature 14-sugar $\left(\mathrm{Glc}_{3} \mathrm{Man}_{9} \mathrm{GlcNAc}_{2}\right)$ dolichol pyrophosphate precursor (Stanley et. al. 2017; Lowe and Marth 2003) (Figure 1.5a). The oligosaccharyltransferase complex embedded within the ER membrane facilitates the transfer of $\mathrm{Glc}_{3} \mathrm{Man}_{9} \mathrm{GICNAc}_{2}$ from the dolichol pyrophosphate precursor to select asparagine (Asn) residues within growing polypeptide chains (Schedin-Weiss et. al. 2014). Glucose is trimmed from Glc ${ }_{3} M_{a n}{ }_{9} G_{c} N_{A c}-$ Asn by ER-resident glucosidases, generating the high mannose $\mathrm{N}$-glycans (class I) (Nagae and Yamaguchi, 2012) (Figure 1.5b). Sequential trimming and monosaccharide extension events throughout the medial and trans Golgi, generate the hybrid (class II) and complex multiantenna N-glycan's (class III) (Nagae and Yamaguchi, 2012) (Figure 1.5b). The trimming and re-addition processing events are vital for protein folding in the ER and quality control in the Golgi (Helenius 2001). 


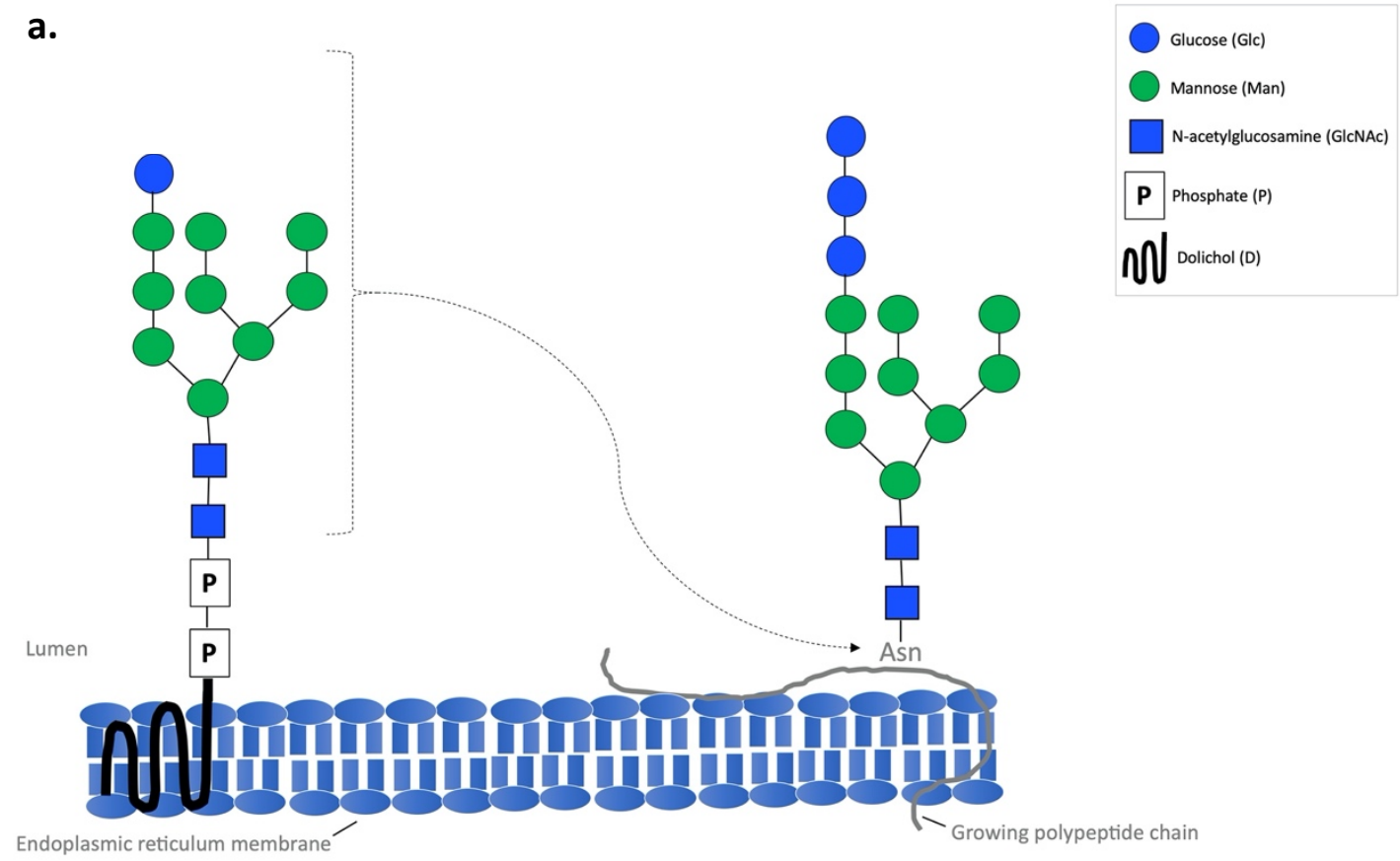

b.

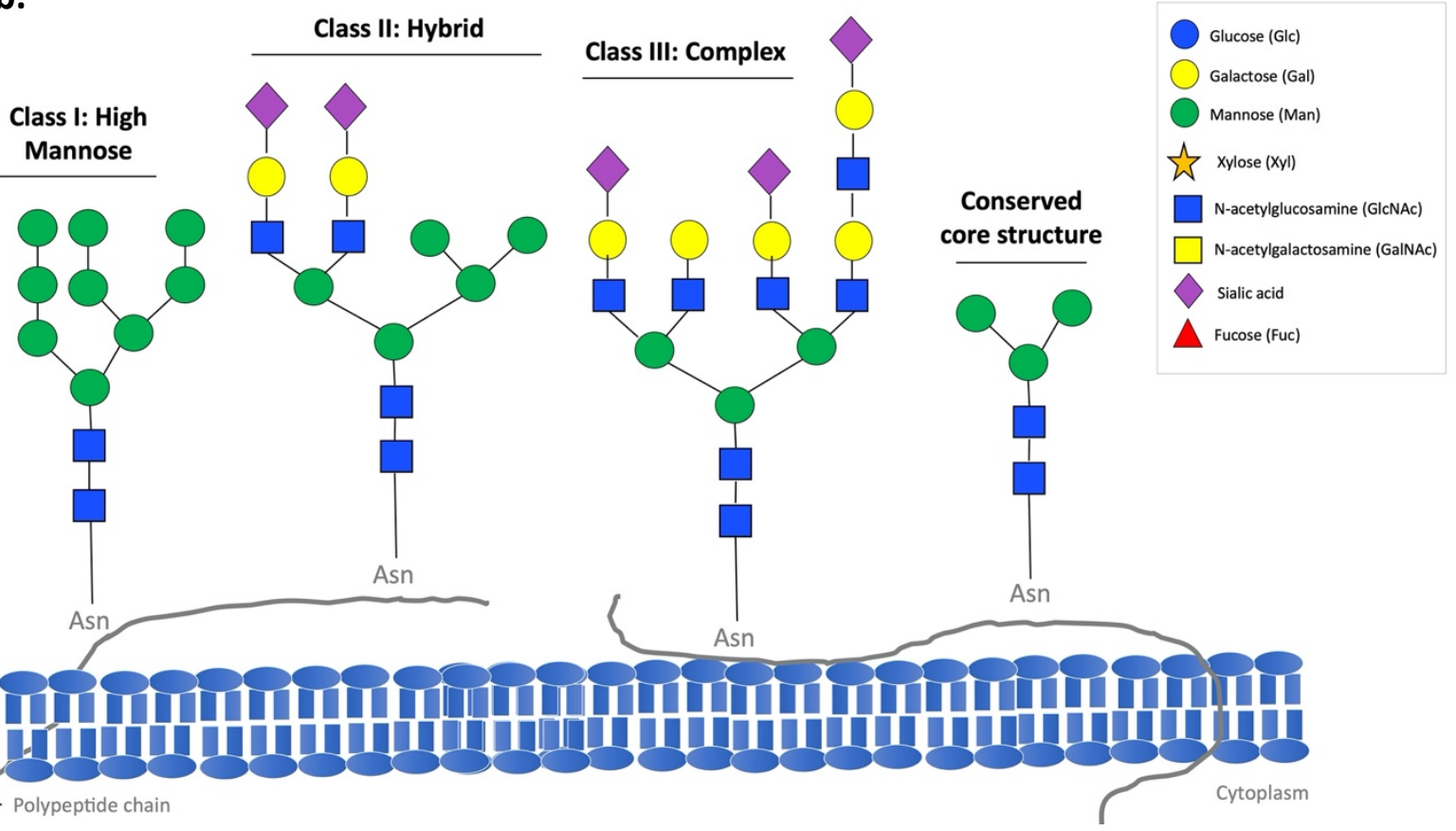

Figure 1.5. The synthesis of the $\mathbf{N}$-glycans. Schematic representations of key structures generated during N-glycan synthesis. (a) Glc $c_{3}$ an $_{9} \mathrm{GICNAC}_{2}$ from the mature 14-sugar dolichol pyrophosphate precursor is transferred (dotted lines) to select asparagine residue within growing polypeptide chains translocating into the ER lumen from the cytoplasm. This transfer is facilitated by the oligosaccharyltransferase complex embedded within the ER transmembrane (not shown). (b) Trimming and extension events throughout the ER-Golgi 
pathway generate the three types of $\mathrm{N}$-glycan. A core structure, composed of two $\mathrm{N}$ acetylglucosamine and three mannose residues, is conserved across all N-glycans. Abbreviations: Ser $=$ Serine, Asn $=$ Asparagine,$T h r=$ Threonine.$($ Author diagram $)$.

The synthesis of the O-glycan's (Brockhausen and Stanley, 2017) can occur through multiple pathways, which can be initiated by modification of serine and threonine residues with $\mathrm{N}$ acetylgalactosamine, fucose, glucose or mannose. The pathway responsible for generating the most highly abundant subset of O-glycan's called the Core 2 O-glycan's (Figure 1.4, red perforated box), involves the installation of $\mathrm{N}$-acetylgalactosamine onto specific serine and threonine residues, by one of several polypeptide $\mathrm{N}$-acetylgalactosamine transferases (Lowe and Marth 2003). In the Golgi, the polypeptide chains are extensively modified by branchspecific glycosyltransferases and sulfotransferases, which is distinct from the glycosidemediated modifications that occur with the N-glycans (Lowe and Marth 2003). The synthesis of the mucin-type-O-glycans is predominantly localized to the Golgi, which enables their synthesis without the need for glycosidase-dependent trimming events (Haltiwanger and Lowe 2004).

The synthesis of the glycolipids involves the addition of glucose or galactose to ceramide embedded in the plasma membrane, which is mediated by glucosylceramide synthase or galactosylceramide synthase, respectively (Lowe and Marth 2003). In contrast to the microheterogeneity of glycoproteins, the glycosphingolipids carry only one single glycan chain (Kopitz 2017). Glucosylceramide or galactosylceramide synthesised in the cytosol are then elongated by glycosyltransferases throughout the Golgi.

The terminal ends of the glycoconjugates are further modified in the trans Golgi with the addition of other monosaccharides such as sialic acid by sialyltransferases, and fucose by fucosidases (Schedin-Weiss et. al. 2014). After release from the Golgi, glycoconjugates are embedded within the cell membrane, packaged into lysosomes or packaged into secretory granules for secretion (Colley et. al. 2017). Such diversity in localisation and structure is incredible given that this co-translational and post-translational modification is not genome encoded and based on only ten monosaccharides (Ghazarian et. al. 2011). For example, 
within a single polypeptide, multiple asparagine, serine, and threonine residues are available for glycan installation. This can occur at different positions, across different polypeptides, at any given time, illustrating the complexity of glycoproteins. The glycome remains the most diverse universal characteristic of all living cells (Varki et. al. 2017).

\subsubsection{Glycan function}

Glycan structures are widely distributed at the cell surface and within the extracellular matrix, making them abundant contact sites for inter and intraspecies cellular recognition and communication (Varki et. al. 2017).

Intrinsically, glycan structures can be recognised by glycan-binding proteins called galectins (Cummings et. al. 2017). These interactions are vital for mammalian carbohydratedependent regulation of intracellular signalling, glycoprotein endocytosis, quality control in the ER, and targeting of glycoconjugates to intracellular compartments through the secretory pathway (Moremen et. al. 2012). For example, galectin 4-dependent recognition of $\mathrm{N}$-glycosylated neural adhesion molecule L1, causes its sorting to and clustering at the axon membrane, a process vital for polarisation, elongation, and branching of mammalian neurons (Higuero et. al. 2017) (Figure 1.6). 
a.
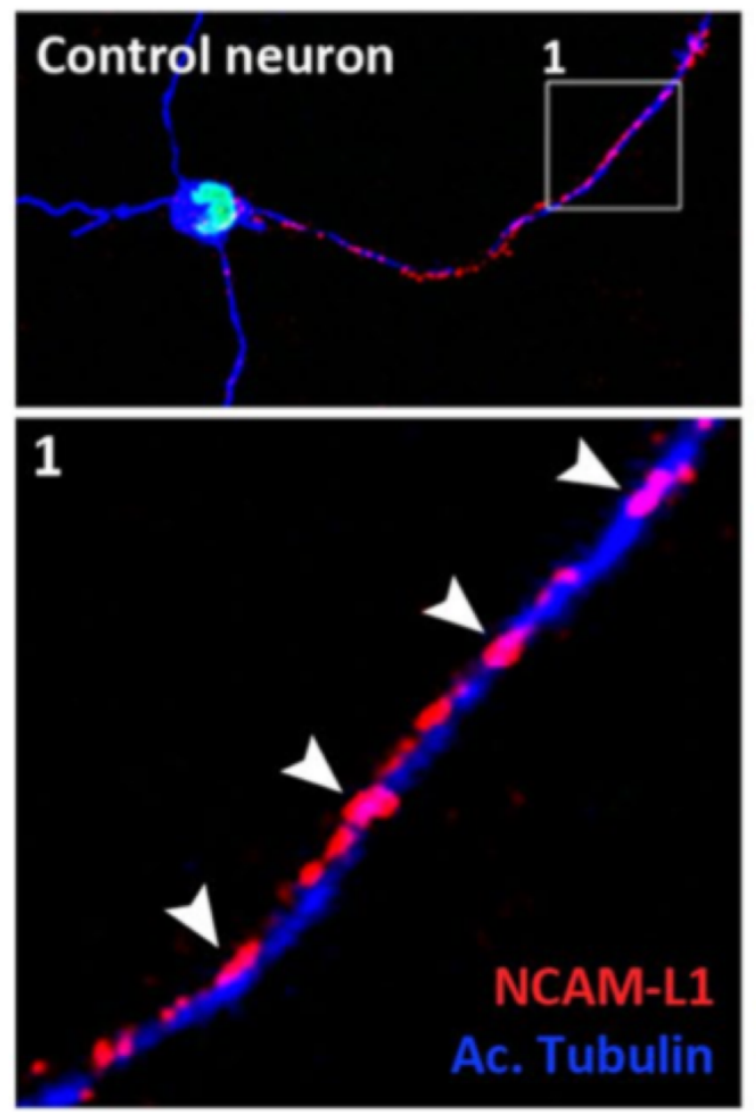

b.
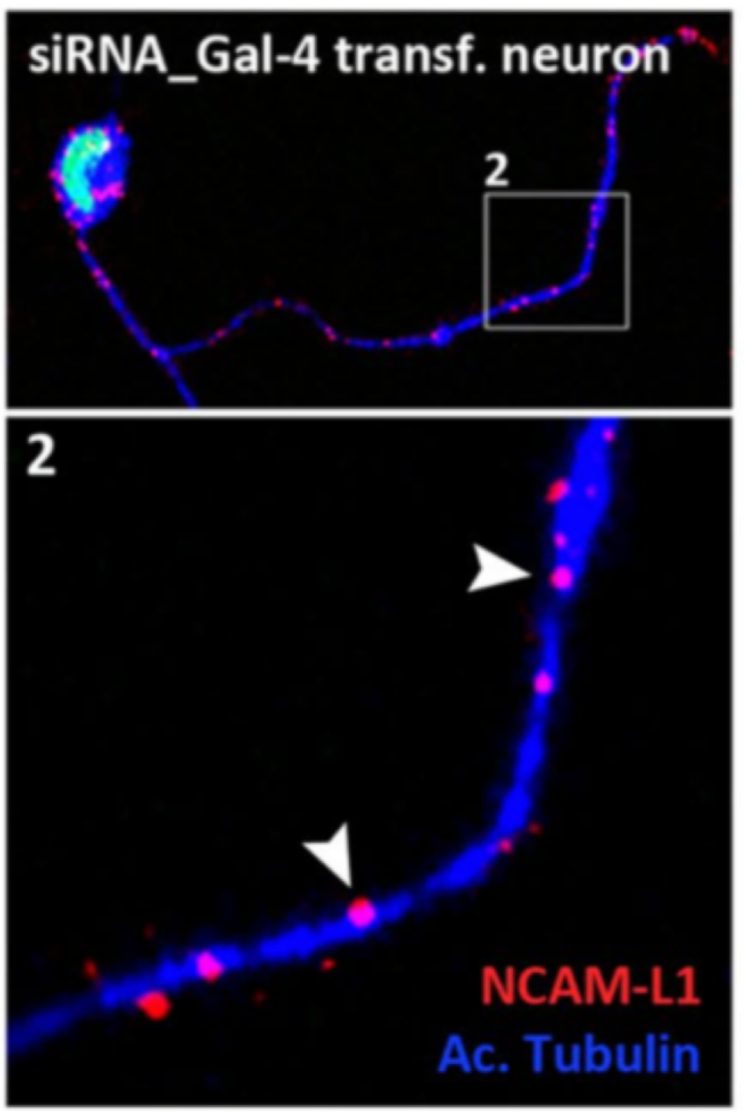

Figure 1.6: $N$-glycosylation of neural adhesion molecule $L 1$ is required for galectin-4 mediated targeting to the axon membrane. In the Golgi, galectin 4 binds the N-glycosylated C-terminus of NCAM-L1. Galectin-4 then binds to glycosylated sphingolipids in the axon membrane, facilitating NCAM-L1 axon delivery. (a) Mammalian neurons expressing galectin 4 exhibited NCAM-L1 (red) clustering throughout the axon membrane (blue). (b) siRNA knockdown of galectin-4 severally impaired NCAM-L1 sorting to the axon membrane. Abbreviations: neural adhesion molecule L1 (NCAM-L1, in red), and acetylated tubulin (Ac. tubulin, in blue) (Figure taken from Higuero et. al. 2017).

Differential glycosylation of glycoconjugates at any given time is required for regulating cell differentiation and development (Moremen et. al. 2012). For example, glycan-dependent variation amongst the $A B O$ blood groups and stage-specific embryonic antigens (SSEAs) has been identified and extensively characterised using immunohistochemistry (Haltiwanger and Lowe 2004). Differentiation of plasmalemmal glycoconjugates has also been correlated with 
the development of epithelial cell types in the embryonic rat pancreas (Maylié-Pfenninger et. al. 1980). Furthermore, it is believed that extensive $\alpha 1,2$-fucosylation of pluripotent stem cells is the principal feature distinguishing them from differentiated cells (Berger et. al. 2016).

Sialic acid has been shown to have a specific role in the survival and regulation of hormones in the circulation. Enzymatically de-sialylated gonadotrophic hormones injected into mice were rapidly removed from circulation and recovered in the liver (Morell et. al. 1971). Subsequent studies attributed this to exposure of galactose residues following neuraminidase mediated de-sialyation, specific recognition of the exposed galactose residues by asialoglycoprotein receptors in the hepatocyte cell membranes, and endocytosis of the hormones for lysosomal degradation in the liver (Ashwell and Harford 1982). Desialylated hormones were also removed from the circulation by secretion into the bile (Ashwell and Harford 1982).

Glycosphingolipids can regulate signal transduction by spontaneously forming lipid rafts, which complex with galectins and facilitate the topological organisation of proteins within the plasma membrane (Kopitz 2017) (Figure 1.6). Furthermore, studies have suggested that sialylated glycosphingolipids such as ganglioside 3, regulate epidermal growth factor receptor (EGFR)-tyrosine kinase signalling pathways by binding to $\mathrm{N}$-acetylglucosamine resides decorating the extracellular face of EGFR (Todeschini and Hakomori 2008).

Glycolipids and O-linked proteins at the surface of leukocytes, glycosylated with fucose and sialic acid, interact with glycan-binding selectins on the vascular endothelium, facilitating the migration of immune cells to sites of inflammation (Schnaar 2015). These interactions are exploited by some pathogens, notably viruses, through "molecular mimicry", which enables pathogens to highjack mobile immune cells for the dissemination of infection, and immune evasion (Krasnova and Wong 2016; Varki et. al. 2017).

Glycans also facilitate extrinsic interactions with chemical compounds, which has been studied extensively using clones of Chinese hamster ovary cell lines exhibiting defects in the pathways governing protein glycosylation. The $15 \mathrm{~B}$ clone was isolated based its acquired 
resistance to ricin, a cytotoxic galactose-binding lectin, and showed reduced plasma membrane glycosylation, and plant-lectin binding capacity (Gottlieb et. al. 1974). This phenotype was attributed to the absence of $\mathrm{N}$-acetylglucosaminyltransferase, and its associated glycan structures, resulting in less of the galactose epitopes usually responsible for ricin sensitivity (Stanley et. al. 1975).

In all, glycans act as cellular decorations with roles in intracellular signalling, hormone regulation, cell migration, plasma membrane structuring, immunity, host-pathogen recognition, and drug sensitivity (Pinho and Reis 2015; Varki 2017).

\subsubsection{Glycosylation in disease}

The synthesis of glycans and their associated functions are dependent on the dynamic interactions between glycoenzymes within a specific cell type. These dynamic interactions are sensitive to physiological and pathological changes (Reily et.al. 2019; Krasnova and Wong 2016; Mariño et. al. 2010). Abnormal changes in the expression and degradation of glycoconjugates have been implicated in a variety of human diseases (Ohtsubo and Marth 2006; Varki et. al. 2017).

Aberrant $\mathrm{N}$-glycosylation of Tau protein has been shown to increase its susceptibility to phosphorylation, generating the hyperphosphorylated variant responsible for the neurofibrillary tangles observed in Alzheimer pathology (Schedin-Weiss et. al. 2014). Decreased glycosylation of the anti-apoptotic protein $\alpha_{1}$ antitrypsin (AAT), which is secreted by hepatocytes and immune cells, is known to reduce its half-life, and anti-inflammatory properties in the circulation, leading to some chronic inflammatory conditions (McCarthy et. al. 2014). Altered N-glycosylation of scavenger receptor class BI (SR-BI) decreases its insertion within the plasma membrane of hepatocytes, subsequently reducing SR-BI mediated uptake of high-density lipoprotein cholesterol from the circulation (van den Boogert et. al. 2017). Therefore, aberrant glycosylation has been associated with an increased risk of cardiovascular disease. A rare paediatric Lysosomal Storage disease called Hurler syndrome is caused by a deficiency in $\alpha$-L-iduronidase, leading to accumulation of 
glycosaminoglycans in the lysosomes (Bie et. al. 2013). Osteoarthritis can result from degradation of aggrecan, a cartilage proteoglycan normally responsible for resisting compressive pressure (Huang and Wu 2008). Deficiencies in particular glycoenzymes have also been associated with the human congenital disorders of glycosylation (Freeze et. al. 2015; Marques-da-Silva et. al. 2017).

\subsubsection{Aberrant glycosylation in cancer}

Aberrant glycosylation has been identified in several cancers (Stowell et. al. 2015). Changes in glycan expression can occur as a result of cancer-associated alterations in the expression of glycoenzymes and their location within the Golgi compartments, alterations to the tertiary conformation of peptide backbones and nascent glycan chains, as well as fluctuations in the abundance of acceptor substrates, sugar nucleotide donors and cofactors (Pinho and Reis 2015; Adamczyk et. al. 2012). Aberrant glycosylation can also be caused by incomplete synthesis of glycan structures, and neo-synthesis of glycan structures due to changes in the gene expression of carbohydrate determinants (Pinho and Reis 2015). The overall consequence is the generation of tumour-associated glycan patterns such as hypersialylation, aberrant fucosylation, glycan truncation, hyperbranching, and overexpression of glycosphingolipids (Pinho and Reis 2015) (Figure 1.7). 


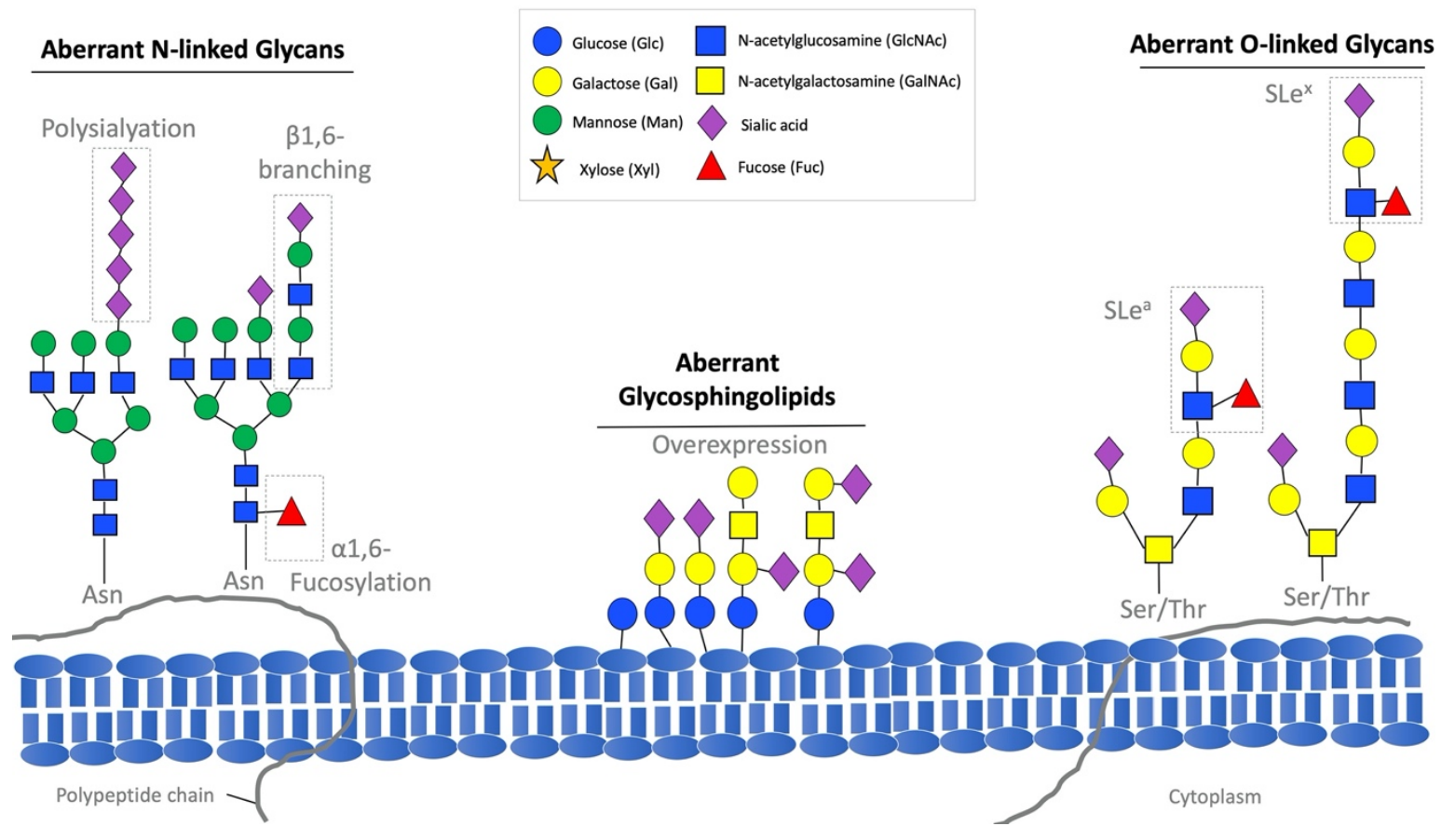

Figure 1.7: Cancer-associated glycans. Schematic representation of a subset of glycans found on tumour cells. Tumour-associated glycan patterns include hypersialylation, aberrant fucosylation, glycan truncation, hyperbranching, and overexpression of glycosphingolipids. Tumour-specific modifications within the glycoproteins are highlighted (grey perforated box). Abbreviations: serine (Ser), asparagine (Asn), and threonine (Thr) (Author diagram).

Sialyl Lewis X (SLe ${ }^{X}$ ) and sialyl Lewis A (SLe $\left.{ }^{A}\right)$ are a set of Lewis type blood group antigens, which are overexpressed in cancer (Natoni et. al. 2016; Pinho and Reis 2015) (Figure 1.8a). Under normal conditions, glycoconjugates containing SLe ${ }^{\mathrm{X}}$ and SLe ${ }^{\mathrm{A}}$ act as ligands for calcium-dependent receptors called selectins, which are present on the cell surface of endothelial cells, red blood cells and leukocytes. The interaction of selectins with the Lewis antigens facilitates adhesion and migration of activated platelets to physiologically perturbed sites in inflammation (Pinho and Reis 2015). Expression de novo of SLe ${ }^{\mathrm{X}}$ and SLe ${ }^{\mathrm{A}}$ on mucins and glycosphingolipids at the tumour cell surface enhances the ability of tumour cells to adhere to the vasculature (Figure 1.8b). This process is vital for tumour dissemination through the bloodstream, formation of emboli at distant endothelial sites and metastasis (Fuster and Esko 2005). 
a.

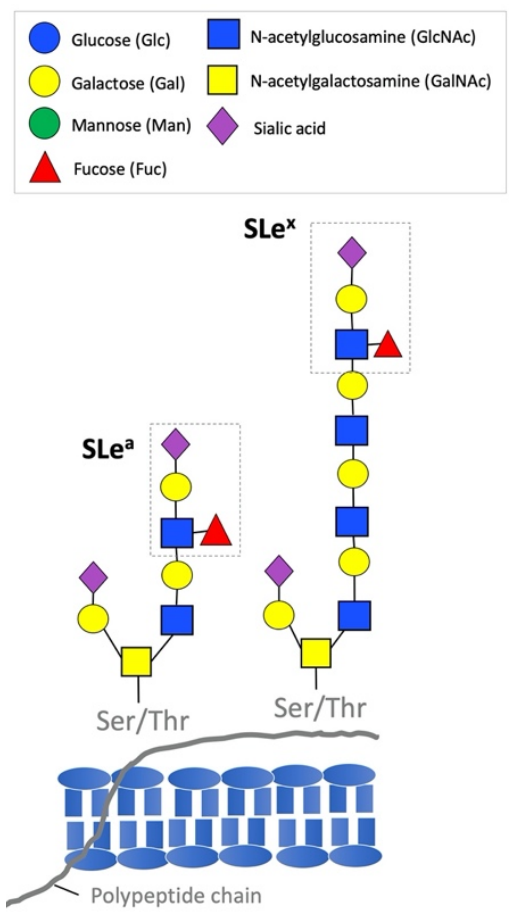

b.

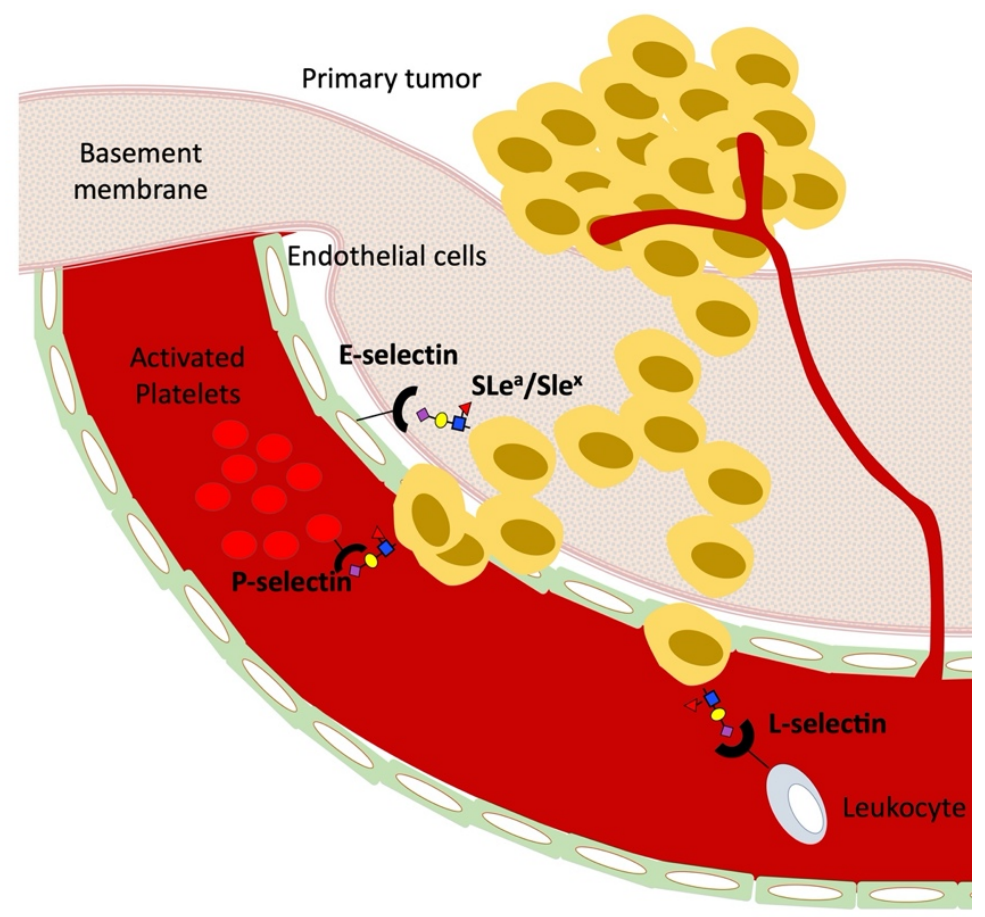

Figure 1.8: The Sialyl Lewis antigens promote tumour progression. (a) Schematic representation of the Sialyl Lewis X (SLe $\left.e^{X}\right)$ and sialyl Lewis $A\left(S L e^{A}\right)$ antigens. (b) Selectins expressed on endothelial cells (E-selectin), activated platelets (P-selectin) and host leukocytes (L-selectin) specifically bind glycan receptors on the surface of tumour cells containing SLe ${ }^{X}$ and SLe ${ }^{A}$. These glycan specific interactions facilitate dissemination of the tumour cells through the bloodstream. Tumour-specific modifications are highlighted (grey perforated box). Abbreviations: serine (Ser), and threonine (Thr) (Author diagram).

Cancer-associated mutations in the Golgi enzyme T synthase or Cosmc, the molecular chaperone responsible for correct folding of T synthase, leads to the abnormal generation of the Thomsen-nouveau (Tn) antigen (Ju et. al. 2011) (Figure 1.9). The overexpression of Tn antigen, a mucin-type O-glycan, has been correlated with poor prognosis in several cancers (Ju et. al. 2011). The interaction between Tn antigen on colorectal cancer cells, and macrophage galactose type lectins (MGLs) expressed on macrophages and dendritic cells, are believed to facilitate immunosuppression and immunoevasion (Ju et. al. 2011). Aberrant 
sialyation of the Fas receptor-Fas ligand has been shown to block the induction of apoptosis in cancer cells, promoting their resistance to cell death (Vajaria and Patel et. al. 2017). Overexpression of glycosphingolipid GM1 causes ganglioside-dependent heterodimerisation and activation of the receptor tyrosine kinases ERBB2-ERBB3, within lipid rafts (Fuster and Esko 2005). More recently, differential glycosylation of cell surface markers present on cancer stem cells has been implicated in tumour initiation, and metastasis (Barkeer et. al. 2018).
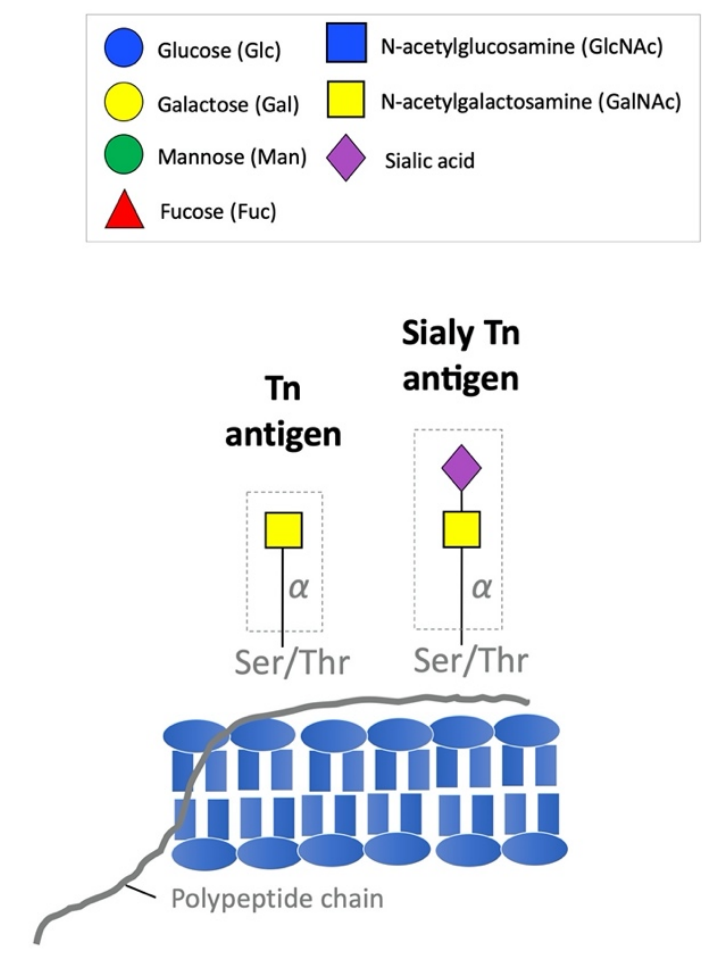

Figure 1.9: The Thomsen-nouveau (Tn) antigen. Schematic representation of the the Thomsen-nouveau (Tn) antigen. Tumour-specific modifications are highlighted (grey perforated box). Abbreviations: serine (Ser), and threonine (Thr) (Author diagram).

Overall, aberrant glycosylation can affect growth factor signalling, cell proliferation, adhesion, and migration, and promote tumour progression through enhanced cell-matrix interactions, invasion, angiogenesis, and metastasis (Moremen et. al. 2012). 


\subsubsection{The functional role of aberrant glycosylation in cancer progression}

Observing changes in glycosylation during physiological and pathological processes does not necessarily provide evidence for its function in normal development and cancer progression. Therefore, probing the function of sialyltransferases using gene editing has provided an avenue to begin to understand the biological mechanisms of glycosylation and cancer progression.

Murugaesu and colleagues identified the sialyltransferase ST6GaINAc2 as a suppressor of late breast cancer metastasis. Knockdown of ST6GalNAc2 in mouse mammary 4T1 tumour cells resulted in increased tumour burden in the lungs and the liver, and seeding of tumour cells at secondary sites (Murugaesu et. al. 2014). This phenotype was correlated with increases in the binding of recombinant galectin 3 due to enhanced presentation of its ligand, unmodified core 1 O-glycan called the Thomsen-Friedenreich $(T)$ antigen (Figure 1.10). In a complementary study, Reticker-Flynn and colleagues showed that knockdown of ST6GalNAc4 could drive lung cancer metastasis, which correlated with disrupted branching and elongation of the T-antigen, increased presentation of T-antigen on the surface of metastatic cells and an increase in galectin-3 binding to leukocytes (Reticker-Flynn and Bhatia 2015). Collectively, these studies provided a mechanism where patients with low expression of particular sialyltransferases would have increased levels of T antigen at the tumour cell surface, enhancing galectin 3 binding. Since galectin 3 is a known carbohydratebinding protein, which facilitates tumour cell interactions with endothelial cell and the cellmatrix, this could promote metastasis. 

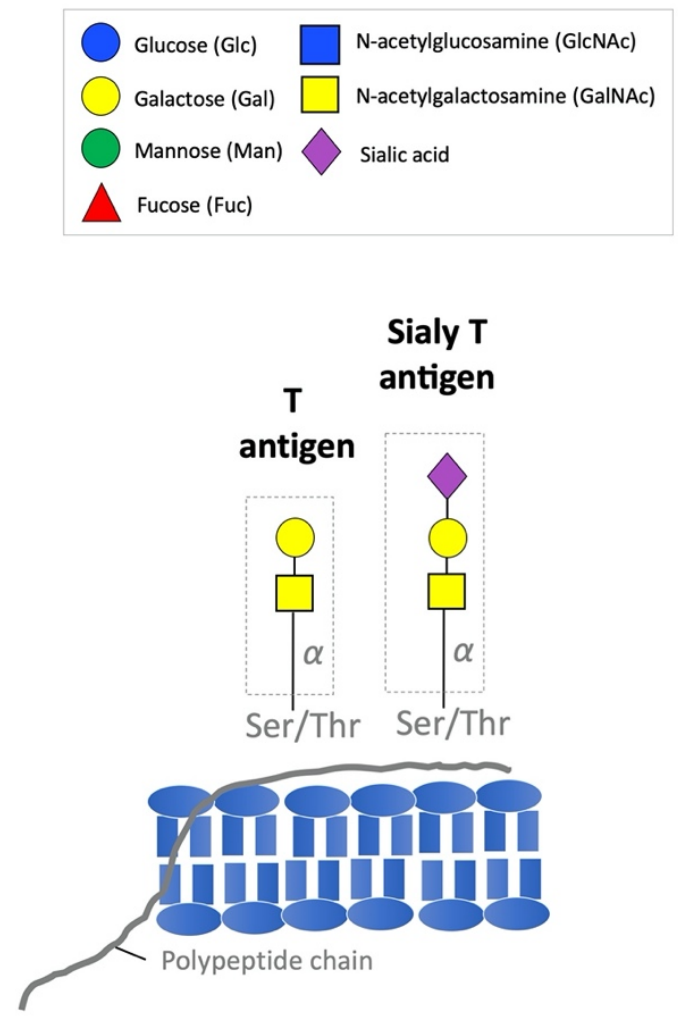

Figure 1.10: The Thomsen-Friedenreich (T) antigen. Schematic representation of the the Thomsen-Friedenreich (T) antigen. Tumour-specific modifications are highlighted (grey perforated box). Abbreviations: serine (Ser), and threonine (Thr) (Author diagram).

Increased expression of the Golgi $\beta 1,6 \mathrm{~N}$-acetylglucosaminyltransferase V (MGAT5) has been shown to result in increased $\beta 1,6$ GlcNAc-branching of $\mathrm{N}$-linked integrins, metastasis and poor prognosis in breast cancer (Dennis et. al. 1999). Granovsky and colleagues genetically engineered MGAT5 deficient mice to probe a functional link between MGAT5 expression, abnormal $\beta 1,6$ GlcNAc-branching, and breast cancer progression in vivo. The absence of $\beta 1,6$ GIcNAc-branching within MGAT5 deficient tissue lysates was confirmed by lectin blotting, with minimal Phytohaemagglutinin binding (Granovsky et. al. 2000). Both wild type and MGAT5 deficient mice formed microscopic tumour foci within the mammary fat pads by 14 weeks, indicating that MGAT5 was not important for cancer initiation (Granovsky et. al. 2000). However, by 20 weeks, the wild type mice were devoid of any normal ductal epithelium (0\% tumour-free), while the MGAT5 deficient mice exhibited a close to $500 \%$ decrease in tumour burden, with only a $5 \%$ incidence of lung metastases and a lesser proportion of proliferating cells within their tumours. Tumour cells extracted from MGAT5 
deficient mice also exhibited less membrane ruffling and formation of focal adhesion points. This phenotype was attributed to the absence of $\beta 1,6$ GIcNAc-branching within MGAT5 deficient tissue, which usually triggers phosphoinositide 3 (PI3) kinase-mediated membrane reorganisation (Granovsky et. al. 2000). Collectively, the data highlighted the importance of glycosylation in promoting cancer progression through plasma membrane plasticity.

These are but a few studies where the functional role of aberrant glycosylation in cancer progression has been probed, and causally associated with the hallmarks of cancer (Munkley and Elliott 2016; Hanahan and Weinberg 2011). It should be noted that since glycosylation is not directly gene-encoded, manipulating the activity of glycoenzymes only provides an indirect analysis of how changes in glycan expression result in cancer-associated phenotypes (Cheng et. al. 2016). Furthermore, differential glycosylation may not always be driven by tumour pathology as the upregulation of glycosylation is important for normal cellular processes. For example, endothelial cells express the SLe ${ }^{\mathrm{x}}$ antigen (Figure 1.8a) in response to chronic inflammation to facilitate leukocyte infiltration (Renkonen et. al. 2002; Peracaula et. al. 2008).

\subsubsection{The discovery of tumour-specific markers}

In the 1840s, Bence-Jones and colleagues discovered fragments of immunoglobulin light chains, now known as Bence-Jones Proteins, in the acidified urine of patients with multiple myeloma (Beetham 2000). Their data underpinned the idea that detecting a cancer-specific protein in a readily available clinical sample such as blood or urine, could be used to confirm a cancer diagnosis, indicate recurrence, monitor treatment response or treatment failure (Virji et. al. 1988; Hounsell 1997). Based on the role of glycosylation in cancer progression, several studies have gone on to characterise glycome differentiation between healthy individuals and cancer patients, and identify a repertoire of tumour-specific glycoconjugates.

Abelev and colleagues observed a globulin-like protein present in mouse embryos, whose expression disappeared in adult mice, but reappeared when mice were transplanted with mouse hepatocellular carcinoma cells (Abelev et. al. 1963) (Figure 1.11). This protein was 
later identified to be alpha fetoprotein (AFP), a glycosylated oncofoetal antigen normally produced in the yolk sac and liver during foetal development, but which is re-expressed by hepatocellular carcinoma cells and secreted into the blood (Pinho and Reis 2015). Elevated levels of AFP detected in serum using an immunological assay is a marker for hepatocellular carcinoma (Meany and Chan 2011).

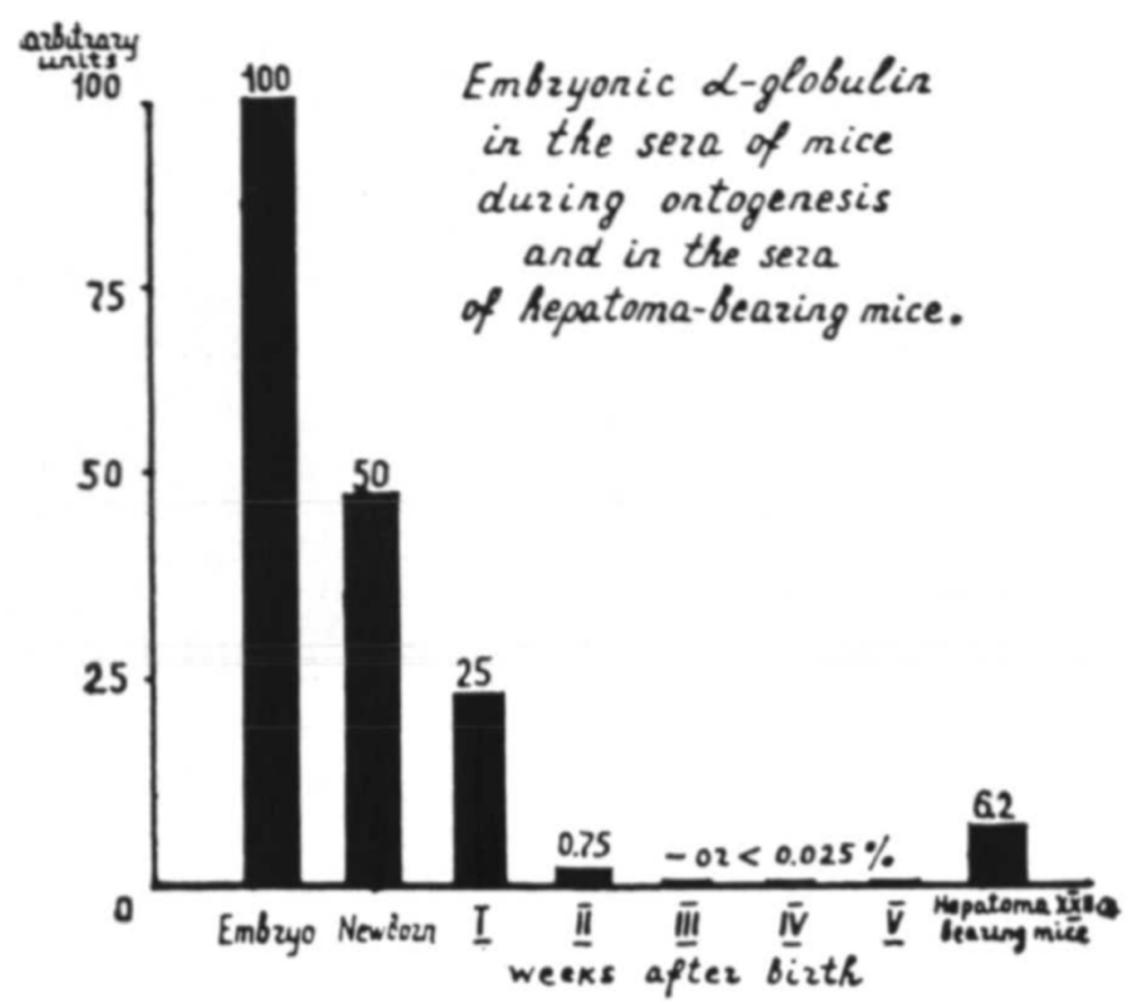

Figure 1.11: Detection of an $\alpha$-globulin like protein in the sera of mice during development and disease. Age-dependent decreases in the concentration of an $\alpha$-globulin like protein were observed during mouse development. Detection of the antigen disappeared from sera in adult mice but reaccumulated when mice were implanted with mouse hepatocellular carcinoma cells (Figure taken from Abelev et. al. 1963).

The first breast tumour-associated antigen, the T antigen (Figure 1.10), was later observed by Springer and colleagues, exclusively in membrane extracts and blood samples obtained from patients with malignant infiltrative breast adenocarcinoma (Springer et. al. 1975). The detection of anti-T antigen autoantibodies in the sera of patients post-mastectomy was 
increased by over $20 \%$ when patients were injected with T-antigen intradermally (Springer et. al. 1975). Their data suggested that the T-antigen was capable of triggering the hosts' immune response against the tumour (Springer et. al. 1975).

Chan and colleagues used a xenograft mouse model of lymphosarcoma, and Wheat germ agglutinin (WGA) lectin, which binds preferentially to $\mathrm{N}$-acetylglucosamine residues (Cummings and Etzler 2009), to compare the composition of glycoproteins extracted from primary tumours and liver metastases. Extracts obtained from liver metastases exhibited less WGA binding than lymphosarcoma tissue extracts, showing an association between decreases in glycosylation and increases in tumour aggressiveness (Chan et. al. 1984). When lymphosarcoma cells, exhibiting the highest level of WGA binding, were re-implanted into regions of the liver, which previously harboured a metastasis deposit, the resulting tumour exhibited less WGA binding (Chan et. al. 1984). Their data suggested that a tumours immediate environment contributed towards driving differential glycosylation during the development of a metastatic phenotype. The cross-talk between the tumour microenvironment and glycosylation has been confirmed in subsequent studies (Peixoto et. al. 2019). For example, Higai and colleagues treated hepatocellular carcinoma cells with interleukin-1 $\beta$ in vitro, to mimic the secretion of proinflammatory cytokines from cells within a tumour's growth environment (Higai et. al. 2006). This induced the expression of sialyltransferase ST3Gal IV and the fucosyltransferase FUT VI, resulting in the expression of Sle ${ }^{\mathrm{x}}$ antigens (Figure 1.8a) at the cell surface (Higai et. al. 2006).

Turner and colleagues measured the concentration of serum fucose in a cohort of patients with early disease (primary cervical cancer only), clinical disease (various tumour sites) and advanced disease (various tumour sites) (Turner et. al. 1985). Raised concentrations of fucose, detected by the fucose dehydrogenase method, correlated with an increase in advanced disease, which they hypothesised was being spontaneously released into the circulation as the tumour grew or by cell death resulting from the hosts' immune response (Turner et. al. 1985).

$\mathrm{Ng}$ and colleges used WGA lectin autoradiography to show differential glycan content within whole-cell extracts obtained from breast biopsies from healthy breast tissue and patients 
with invasive breast carcinoma. Tumour extracts exhibited less WGA binding, with lectin western blot, than extracts from healthy breast tissue (Ng et. al. 1987). This inverse correlation between WGA binding and disease aggressiveness was also seen in models of lymphosarcoma discussed previously (Chan et. al. 1984) and years later, during the progression of pre-invasive Barrett's oesophagus to adenocarcinoma (Bird-Lieberman et. al. 2012).

Collectively, these early studies supported the notion that differential glycosylation, and the unique presence of glycoconjugates in clinical samples, may have diagnostic and prognostic value.

\subsubsection{Glycans in the clinic}

Targeting glycosylation to improve cancer diagnosis is now embedded in clinical practice and involves detecting aberrantly glycosylated glycoproteins or oligosaccharide signatures in tissue or serum (Nimse et. al. 2016). These tumour markers, also known as biomarkers, are defined as "a measurable phenotypic parameter that characterises an organism's state of health or disease, or a response to a particular therapeutic intervention" (Negm et. al. 2002). Biomarkers are generally classed as genomic markers (RNA or DNA), protein markers or molecular imaging markers (Peracaula et. al. 2008).

Several of the clinical biomarkers used routinely for cancer diagnosis, therapeutic monitoring, detecting disease recurrence and examining tumour burden, are in fact glycoproteins or oligosaccharides (Peracaula et. al. 2003) (Figure 1.12). Current biomarkers approved by the FDA include AFP in hepatocellular carcinoma, prostate-specific antigen (PSA) in prostate cancer, and carcinoembryonic antigen (CEA) in colorectal, pancreatic, gastric and breast carcinomas (Pinho and Reis 2015). Biomarkers can be detected with monoclonal antibodies in liquified clinical samples using enzyme-linked immunosorbent assays (ELISA) or in tissue biopsies using immunohistochemistry (IHC) (Sharma 2009). For example, the CA19-9 assay detects the SLe ${ }^{a}$ tetrasaccharide (Figure 1.8a) and is used to monitor treatment response in biliary, colorectal, gastric and pancreatic cancers (Pinho and 
Reis 2015). The measurement of these markers is non-invasive and does not interfere with standard diagnostic methods such as biopsy, histology, and computerised tomography (CT) imaging.

\begin{tabular}{llll}
$\begin{array}{l}\text { Serological } \\
\text { marker }\end{array}$ & $\begin{array}{l}\text { Glycoprotein or } \\
\text { glycoform }\end{array}$ & Cancers & Application in the clinic \\
\hline AFP & $\begin{array}{l}\text { AFP'core' fucosylation } \\
\text { (AFP-L3) }\end{array}$ & $\begin{array}{l}\text { Hepatocellular } \\
\text { carcinoma }\end{array}$ & Early diagnosis and monitoring \\
\hline CA19-9 & SLe & $\begin{array}{l}\text { Biliary, colorectal, } \\
\text { gastric and pancreatic }\end{array}$ & $\begin{array}{l}\text { Therapeutic monitoring, } \\
\text { recurrence and tumour burden }\end{array}$ \\
\hline CA72-4 & STn & Gastric & Monitoring \\
\hline CA15-3 & MUC1 & Breast & Monitoring \\
\hline CA125 & MUC16 & Ovarian & Monitoring and recurrence \\
\hline CEA & CEA & Colorectal & Monitoring and recurrence \\
\hline PSA & PSA & Prostate & $\begin{array}{l}\text { Diagnosis, monitoring and } \\
\text { recurrence }\end{array}$ \\
\hline B-hCG & B-hCG & Gynaecological & Monitoring \\
\hline
\end{tabular}

Figure 1.12: Serological glycobiomarkers approved by the U.S. Food and Drug

Administration for use in the clinic. Abbreviations: alpha-fetoprotein (AFP), Sialyl Lewis A $\left(S L e^{a}\right)$, sialylated Thomsen-nouveau (STn), mucin 1, cell surface associated (MUC1), mucin 16, cell surface associated (MUC16), carcinoembryonic antigen (CEA), prostate specific antigen (PSA), and human chorionic gonadotropin (hCG) (Figure taken from Pinho and Reis 2015).

Targeting glycosylation has also been achieved through the development of glycotherapeutics. Early forms of glycotherapeutics involved treating Carbohydrate Deficient Glycoprotein Syndromes (Varki et. al. 2017) and Congenital Disorders of Glycosylation with mannose replacement therapy (Rush et. al. 2000), or Leukocyte Adhesion Deficiency Type II with oral fucose (Marquardt et. al. 1999). Now, glycotherapeutics has been used to treat cancer (Hudak and Bertozzi 2014) by inhibiting glycosyltransferases (Granovsky et. al. 2000), blocking oligosaccharide-mediated cell-cell interactions with inhibitors (Nangia-Makker et. al. 2002), or triggering an anti-tumour immune response using carbohydrate-based vaccines (Krasnova and Wong 2016). 
Heparin is an anticoagulant used clinically, which inhibits binding of Sle ${ }^{\mathrm{X}}$ (Figure 1.8a) to the selectins known to promote invasion (Fuster and Esko 2005; Magnani 2004). Other therapeutics in clinical trials aim to block the interactions between glycans and glycanbinding proteins such as the galectin-3 inhibitor, GSC-100, for pancreatic cancer and conjugated ganglioside GM2 vaccine for melanoma (Fuster and Esko 2005). Glycoprotein targeting immunotherapies used in standard clinical practice include the anti-CD20 monoclonal antibody, rituximab, for non-Hodgkin lymphoma, the anti-tyrosine kinase monoclonal antibody, imatinib, for leukaemia, anti-HER2 antibody, trastuzumab, for breast cancer, and the anti-EGFR antibody, cetuximab, for colorectal cancer (Weiner et. al. 2012; Madden 2018).

The Fc region and antigen-binding fragment $(\mathrm{Fab})$ of immunoglobulins contain several $\mathrm{N}$ - and O-glycosylation sites, which facilitate their binding to Fc receptors, and complement during the adaptive immune response (Seeling et. al. 2017; Arnold et. al. 2007) (Figure 1.13). Fcglyco-engineering involves site-directed mutagenesis of the Fc regions within existing immunotherapies, to generate glycosylated or deglycosylated variants, which improves their effector functions, specificities and clinical utility (Reusch and Tejeda 2015; Yu et. al. 2017). Fc-glyco-engineering was used to generate mogamulizumab, an afucosylated anti-CCchemokine receptor 4 antibody approved to treat T-cell lymphomas in Japan, and obinutuzumab, a low fucose anti-CD20 antibody approved to treat follicular lymphoma (Yu et. al. 2017). 


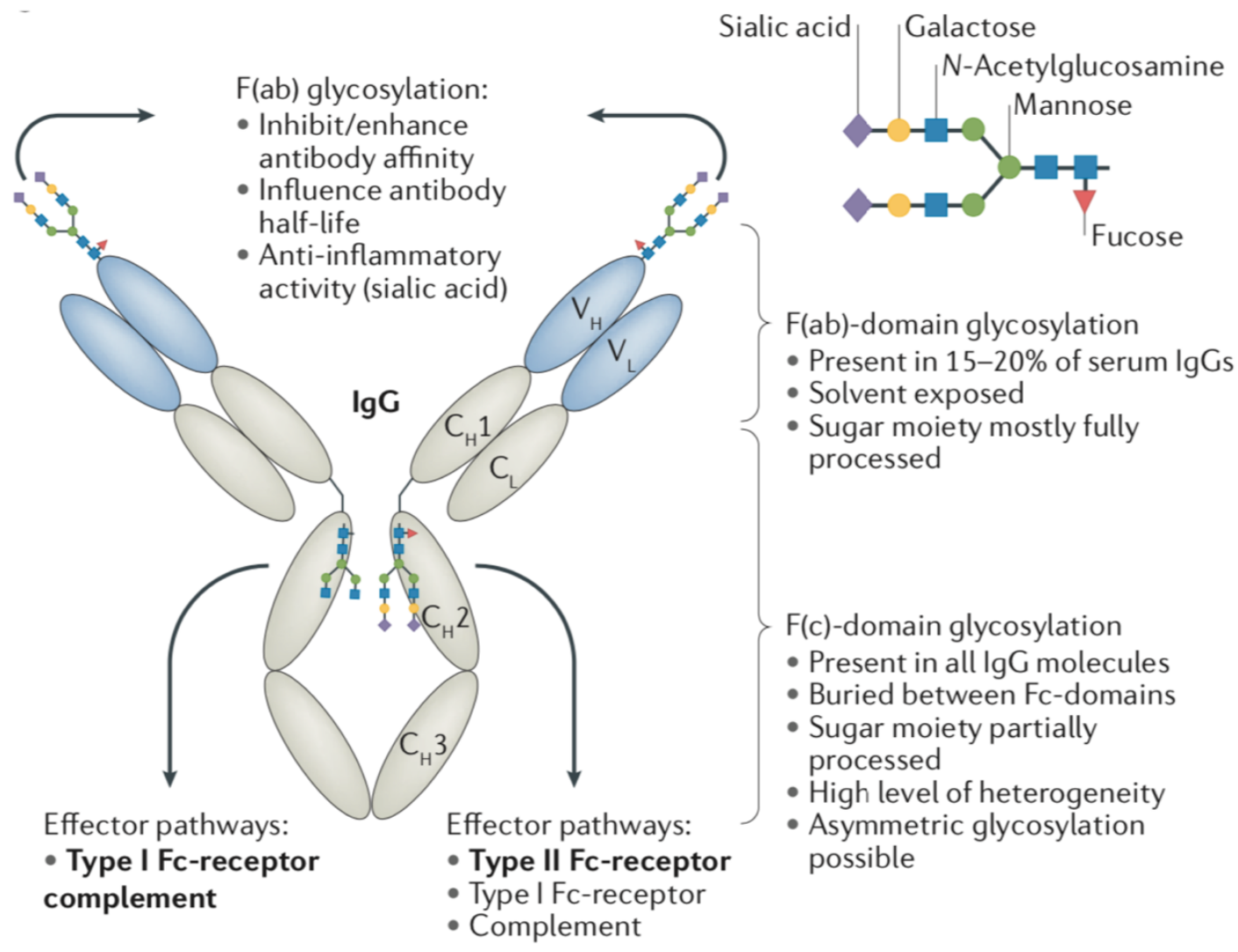

Figure 1.13: The effect of glycosylation on immunoglobulin function. Glycosylation of an immunoglobulins (Ig) fragment Fc region or antigen-binding fragment (Fab), can inhibit or enhance antigen binding, effector functions, or plasma half-life (Figure taken from Seeling et. al. 2017).

The presence of circulating immune biomarkers is based on the knowledge that autoantibodies are released in response to contact with tumour-associated antigens, which act as a "fingerprint" indicating the presence of a tumour (Chapoval et. al. 2015). Immune biomarkers that recognise tumour antigens have been identified by culturing antibodysecreting cells such as B cells, extracted from the lymph nodes of patients with cancer (Meeusen et. al. 2017). Immunodiagnostic assays to measure antibodies against glycanbased tumour antigens are currently in development (Bogen and Sompuram 2004). 
Understanding the role of glycosylation in cancer has immense clinical value and may provide novel ways to tackle one of the biggest causes of mortality worldwide (Foreman et. al. 2018).

\subsection{Exploiting the cancer glycome in biomarker discovery}

\subsubsection{The challenges associated with using glycans as cancer biomarkers}

The link between aberrant glycosylation and cancer progression has formed the basis for using glycans and glycoproteins in the clinical management of cancer (Munkley and Elliott 2016; Oliveira-Ferrer et. al. 2017). However, the utility of biomarkers has been challenged in recent years due to low cancer specificity and high false-positive rates (Mayeux 2004).

An ideal biomarker should correctly diagnose people with cancer (be sensitive), identify healthy people without cancer (be specific), correlate with tumour size, and be capable of monitoring disease recurrence or treatment response (Sharma 2009). Unfortunately, current clinical biomarkers fail to meet the ideal characteristics of a tumour biomarker. Although increased levels of AFP are associated with an increase in tumour size, elevated AFP is also observed in chronic hepatitis and cirrhosis, making it difficult to distinguish hepatocellular carcinoma from benign liver diseases (Adamczyk et. al. 2012). PSA is a serine protease whose serum levels, alongside Gleason scoring and clinical staging, is used to monitor tumour progression in prostate cancer (Meany and Chan 2011). Serum levels of PSA are elevated in prostate cancer but also in other benign conditions such as benign prostatic hyperplasia, prostatitis, prostatic intraepithelial neoplasia, and obesity (Adamczyk et. al. 2012). Therefore, levels of PSA alone are insufficient in distinguishing prostate cancer from other benign prostate diseases (Meany and Chan 2011). Multiple biomarkers measured simultaneously within the same patient can often show conflicting results. For example, Ishikawa and colleagues describe a breast cancer case report where levels of cancer antigen 15-3 (CA15-3) dropped significantly in response to a weekly dose of paclitaxel indicating successful response to therapy, whilst in contrast, carcinoembryonic antigen (CEA) levels 
continued to rise indicating disease progression within the same time window (Ishikawa et. al. 2012) (Figure 1.14).

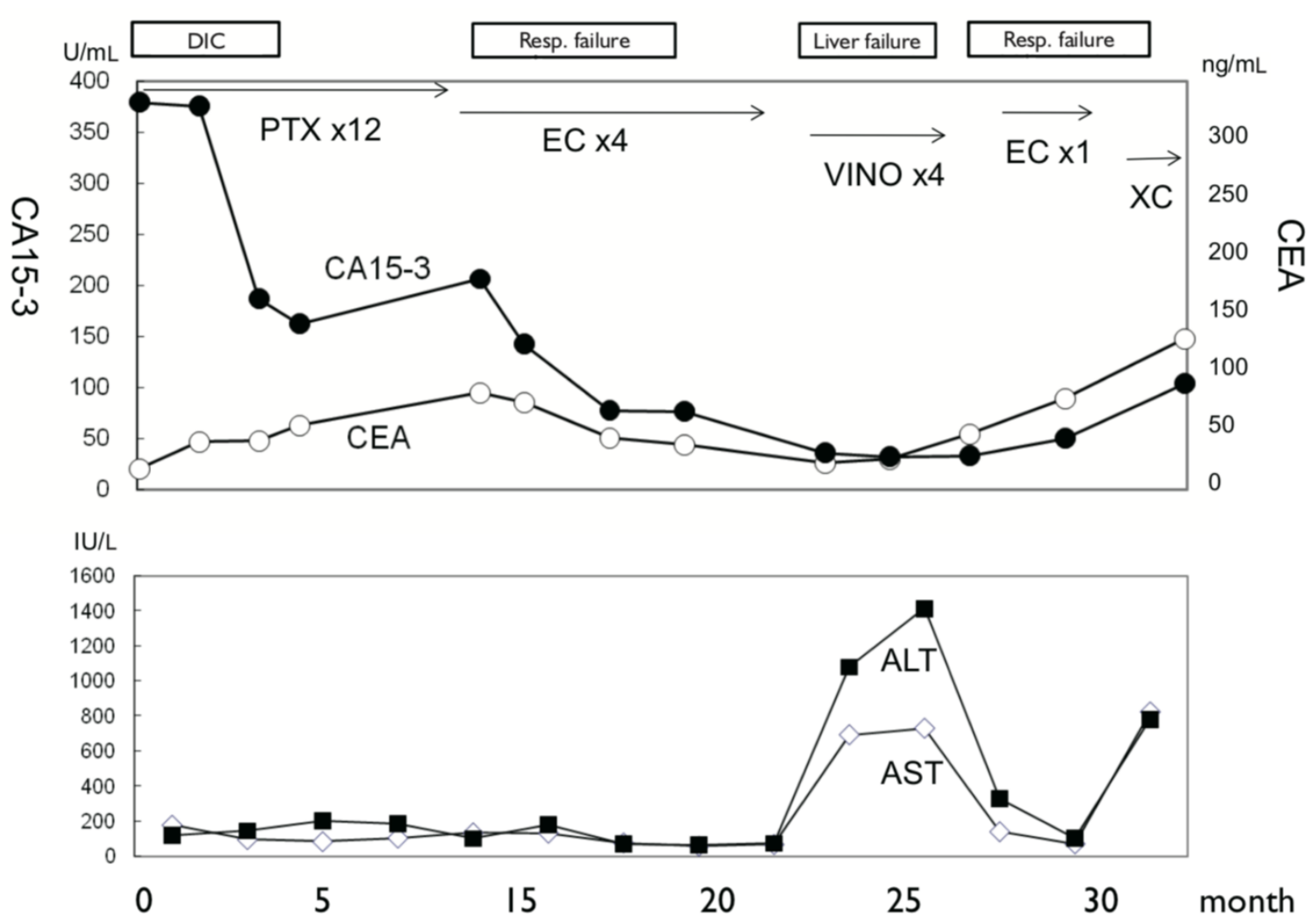

Figure 1.14: Changes in the concentration of CA15-3, CEA and liver function markers during the disease course of a 35-year-old female patient with breast cancer. CA15-3 is used to measure treatment response in breast cancer. Elevated CA15-3 was reduced in response to PTX treatment, but masked disease progression occurring between 0-5 months. CEA is used to monitor metastatic breast cancer and recurrence. Elevated levels of CEA were indicative of disease progression, which later correlated with elevated liver function markers, ALT and AST. Abbreviations: cancer antigen 15-3 (CA15-3), carcinoembryonic antigen (CEA), paclitaxel (PTX), epirubicin (EC) cyclophosphamide (VINO), vinorelbine (XC) and disseminated intravascular coagulation (DIC), alanine aminotransferase (ALT) and aspartate aminotransferase (AST) (Figure taken from Ishikawa et. al. 2012). 
Screening biomarkers in asymptomatic populations has reduced the incidence of several cancers (Shieh et. al. 2016) (Figure 1.3). Unfortunately, this has come at the cost of overdiagnosis and overtreatment (Shieh et. al. 2016; Bhatt and Klotz 2016). Overdiagnosis can result in multiple unnecessary biopsies being taken from patients with benign conditions and has been implicated in breast (Wallis 2018) and thyroid (Pandeya et. al. 2016) cancers.

In the European Randomized Study of Screening for Prostate Cancer in men aged 50-74, 82,000 men were randomly enrolled for prostate cancer screening once every 4 years, and 99,000 men were assigned to the no-screening control group (Schröder et. al. 2009). Elevated PSA was detected in $16.2 \%$ of all screening tests taken, and resulted in a reduction of 0.7 prostate-cancer deaths per 1000 men. However, $75.9 \%$ of the tests positive for elevated PSA, were confirmed to be false positives following biopsy (Schröder et. al. 2009). Furthermore, the UK's biggest trial for prostate cancer screening, the Prostate Testing for Cancer and Treatment (ProtecT) trial, showed no significant improvement in preventing prostate cancer mortality using PSA monitoring (Hamdy et. al. 2016). Therefore, the effectiveness of current clinical biomarkers for screening, diagnostic or prognostic purposes remains unclear.

\subsubsection{Overcoming the challenges associated with using glycans as cancer biomarkers}

Personalised risk-based screening models analyse biomarker levels in the context of other clinical variables to improve the clinical utility of biomarkers. The Stockholm-3 (STHLM-3) model analyses a set of predefined parameters such as plasma proteins (including free and bound PSA), genetic polymorphisms, previous prostate biopsies, age, and family history (Grönberg et. al. 2015). The detection of high-grade prostate cancers with a Gleason score of $\geq 7$, in a population cohort of 47,000 asymptomatic men aged $50-69$ years, was significantly improved using the STHLM-3 model, in comparison to PSA screening alone (Grönberg et. al. 2015). Of the 603 high-risk prostate cancers detected by the STHLM-3 model, only 124 of those patients had elevated PSA levels $\geq 3 \mathrm{ng} / \mathrm{ml}$ usually needed to warrant biopsy referral (Grönberg et. al. 2015). A two-step prostate cancer screening protocol has now been proposed in Europe, which involves a PSA elevation screen to identify high-risk patients, 
immediately followed by personalised prediction of cancer risk using the Stockholm model. This structured screening programme has been shown to reduce unnecessary multiple biopsies in patients with elevated PSA levels induced by benign conditions (Ström et. al. 2018). However, the STHLM-3 prediction risk-based screening programming has yet to be integrated into routine clinical practice across Europe.

New tissue and body fluid-based biomarkers are notoriously difficult to get into the clinic due to the need for large scale studies to test the sensitivity of the proposed biomarkers to individual variation, as well as the development of robust assays for their detection that are clinically feasible to conduct without the need for specialist interpretation (Adamczyk et. al., 2012; Bird-Lieberman et. al. 2012). Molecular imaging, defined as the "visualisation, characterisation, and measurement of biological processes at the molecular and cellular levels in humans [using] radiotracer imaging, magnetic resonance imaging (MRI) and optical imaging" (Mankoff 2007), provides an alternative approach to tumour characterisation that is independent of traditional tissue or serum-based biomarkers (Peracaula et. al. 2008). For example, treatment response has been assessed using positron-emission tomography (PET) by monitoring the uptake of zirconium-89 radiolabelled anti-HER2 trastuzumab $\left({ }^{89} \mathrm{Zr}\right.$ trastuzumab) within breast cancer metastases (Dijkers et. al. 2010) (Figure 1.15a). Uptake of indium-111 radiolabelled trastuzumab ( ${ }^{111}$ In-trastuzumab) has also been used to detect new HER2 positive metastatic breast cancer lesions in the liver using fused CT and single-photon emission (SPECT) imaging (Perik et. al. 2006) (Figure 1.15b). These are examples where molecular imaging is enabling a personalised approach to monitoring treatment response and diagnosing metastases, independent of biopsy-based tissue sampling (Mankoff et. al. 2017). 
a.

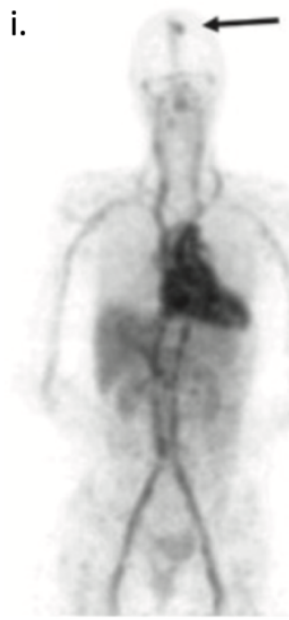

1 day p.i.

b.

Fused

CT

SPECT
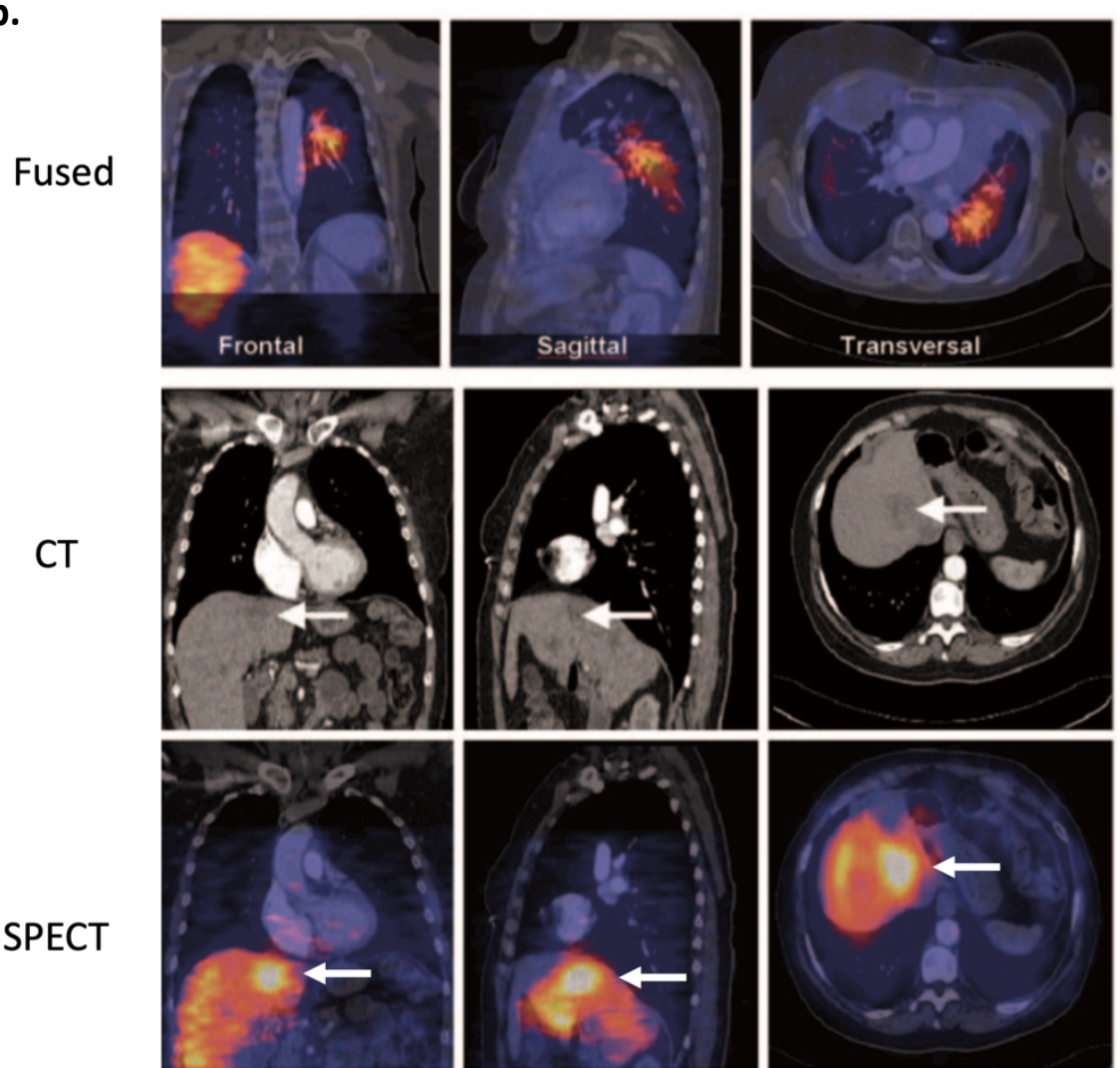

Frontal ii.
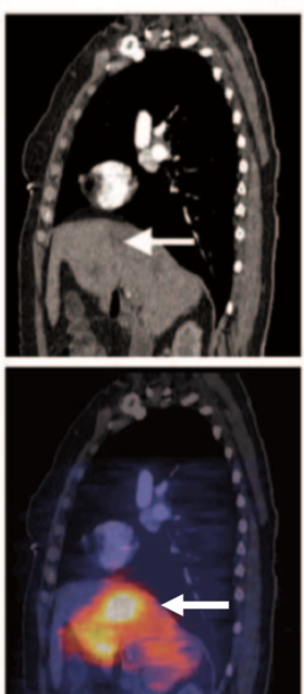

Sagittal iii.
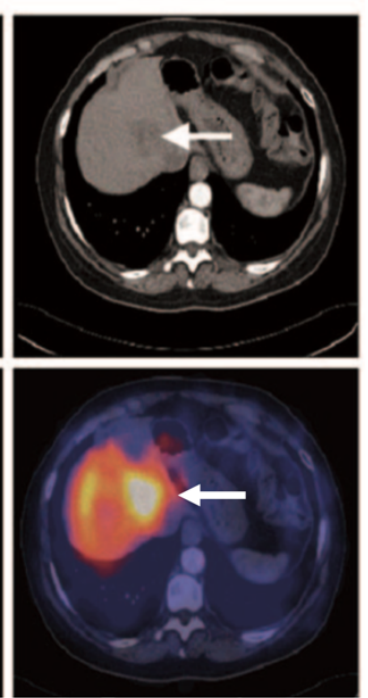

Transversal

Figure 1.15: Molecular imaging of breast cancer metastases using radiolabeled

trastuzumab. (a) Positron-emission tomography (PET) imaging showed increased uptake of radiotracer within a single brain metastasis (black arrow), 1 (i), 2 (ii) and 5 (iii) days post injection with ${ }^{89} \mathrm{Zr}$-trastuzumab. The study proposed 4-5 days post administration as the best time to assess tumour immunotherapy uptake (Modified from Dijkers et. al. 2010). (b) Fused 
single-photon emission-computed tomography (SPECT-CT) shows uptake of radiotracer within liver metastases (white arrow) 96 hours post-injection with ${ }^{111}$ In-trastuzumab (Modified from Perik et. al. 2006).

Molecular imaging is low-throughput in comparison to tissue-based methods, where multiple biomarkers can be assessed in a single clinical sample (Mankoff et. al. 2017), and also requires specialist interpretation. Therefore, clinical translation of molecular imaging may still require traditional tissue or serum-based biomarkers to determine the ideal timing of imaging or treatment administration, monitor potential toxicity and side effects to novel targeted therapies, and stratify patients into high-risk groups (Fuster and Esko 2005).

\subsubsection{Detecting glycosylation using lectins}

Several methods have been adopted to study the distribution, composition, and function of glycans in cancer to search for new and improved cancer biomarkers (Springer and Gagneux 2016). These techniques include mass spectrometry, generation of carbohydrate-specific monoclonal antibodies, editing of glycoenzymes, and lectin affinity assays, to name but a few (Adamczyk et. al. 2012; Biskup et. al. 2013; Block et. al. 2005; Saldova et. al. 2011; Hizal et. al. 2014; Tao et. al. 2008). Affinity reagents such as lectins and antibodies are the most extensively used because their specificities enable them to discriminate among a variety of glycan structures, with high-affinity binding due to their bivalency (Cummings and Etzler 2009). These affinity reagents can be used in combination with basic carbohydrate stains such as Periodic acid-Schiff for visualising glycoproteins and glycolipids, alcian blue for mucins and hyaluronic acid, which allows visualisation of the glucosaminoglycan hyaluronan within cartilage, and ruthenium red for the glycocalyx (Corfield 2017).

Lectins are naturally occurring carbohydrate-binding proteins conserved in plants, viruses, bacteria, protozoa, fungi, and animals (Sharon and Lis 1989). Generally, they facilitate adhesion by promoting intercellular bridging between soluble glycoconjugates on the surface of apposing cells, and within the extracellular matrix (Sharon and Lis 1989). Most 
commercially available lectins are purified from plants or animals. Their binding specificities are well characterised, they are abundant in nature, inexpensive to synthesise, stable under a range of experimental conditions, and bind a variety of human tissues (van Kooyk 2008; Bird-Lieberman et. al. 2012). Examples include Wheat germ agglutinin (WGA) from Triticum vulgaris, which binds $\mathrm{N}$-acetyl-D-glucosamine and sialic acid, and Helix pomatia agglutinin (HPA) from the edible snail, which binds to $\alpha$-N-acetylgalactosamine (Cummings and Etzler 2009).

Combining lectin affinity assays with glycoproteomic approaches have allowed deeper analysis of glycoforms to improve specificity and early diagnosis. For example, in 2006, the FDA approved the use of AFP-L3 for detecting hepatocellular carcinoma. AFP-L3 is an aberrantly fucosylated version of AFP, generated due to the overexpression of fucosyltransferase FUT 8 (Meany and Chan 2011). AFP-L3 levels are significantly elevated in hepatocellular carcinoma patients in comparison to benign liver diseases such as cirrhosis, providing a marker for the onset of hepatocellular carcinoma (Block et. al. 2005; Meany and Chan 2011; Adamczyk et. al. 2012; Pinho and Reis 2015). The AFP-LC3 capture assay developed by FUJIFILM Wako Diagnostics U.S.A uses the Lens culinaris agglutinin (LCA) lectin to capture aberrantly fucosylated AFP (Figure 1.16). Patients with chronic liver disease and elevated AFP-L3 were observed to have a 10.6-fold increased risk of developing hepatocellular carcinoma within 21 months (http://www.wakodiagnostics.com/afpl3test.html, 2019). AFP-L3 is an example of how the identification of aberrantly glycosylated isoforms of an existing biomarker has improved the early diagnosis of cancer and enabled identification of high-risk groups (Meany and Chan 2011). 


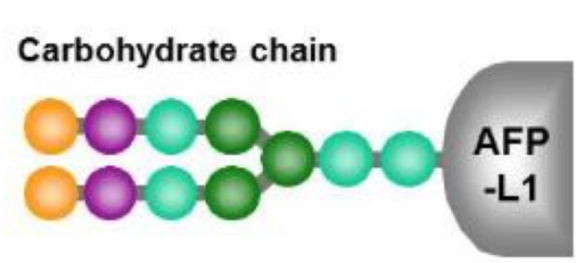

Sia

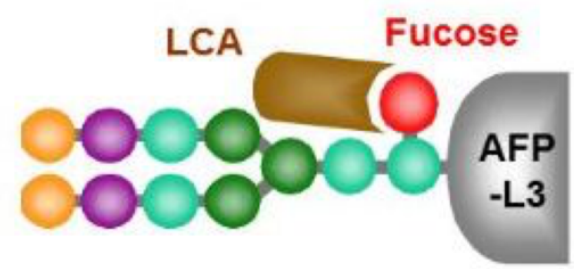

GIcNac
Man

Figure 1.16: The Fuji AFP-L3 diagnostic assay. Lens culinaris agglutinin (LCA) captures aberrantly fucosylated AFP. The ratio of AFP-L3 (abnormal) over AFP-L1 (unmodified) is highly specific for hepatocellular carcinoma compared to AFP-L1 alone. (Figure taken from http://www.wakodiagnostics.com/afp/3test.html, 2019).

Radiolabelled lectins have been used to successfully image glycans in tumour-bearing mice using radionuclide imaging (Kojima and Jay 1986). However, lectins can exhibit low tissue penetration and can cause concentration-dependent toxicity (Laughlina et. al. 2009). Furthermore, lectins have multiple carbohydrate-binding sites, leading to the formation of oligomeric structures. These can be difficult to clear and can reduce contrast in vivo (Berg et. al. 2002).

Despite the disadvantages associated with lectin imaging, their low tissue penetration can be beneficial for superficial imaging of tissue sections such as at the gastrointestinal surface. Various glycan targets can be observed at the mucosal surface, providing qualitative evidence for the different binding specificities of lectins (Kuo et. al. 2016) (Figure 1.17a). A decrease in the binding of a variety of fluorescently labelled lectins to the colorectal luminal surface epithelium have been observed during progression from normal epithelium to lowgrade dysplasia, high-grade dysplasia and carcinoma (Kuo et. al. 2016) (Figure 1.17b). The progression of oesophageal cancer has also been detected using clinical fluorescence endoscopy simply by topical application of fluorescein-labelled WGA (Bird-Lieberman et. al. 2012). Decreased binding of WGA was seen during the progression of pre-invasive Barrett's oesophagus to adenocarcinoma (Bird-Lieberman et. al. 2012) (Figure 1.17c). 
a.
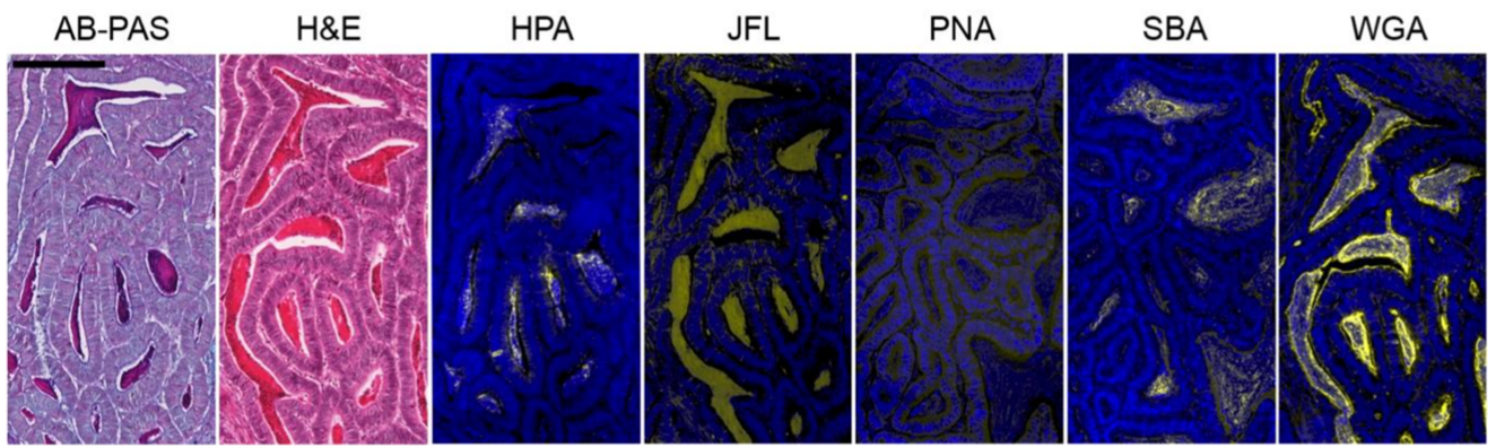

b.
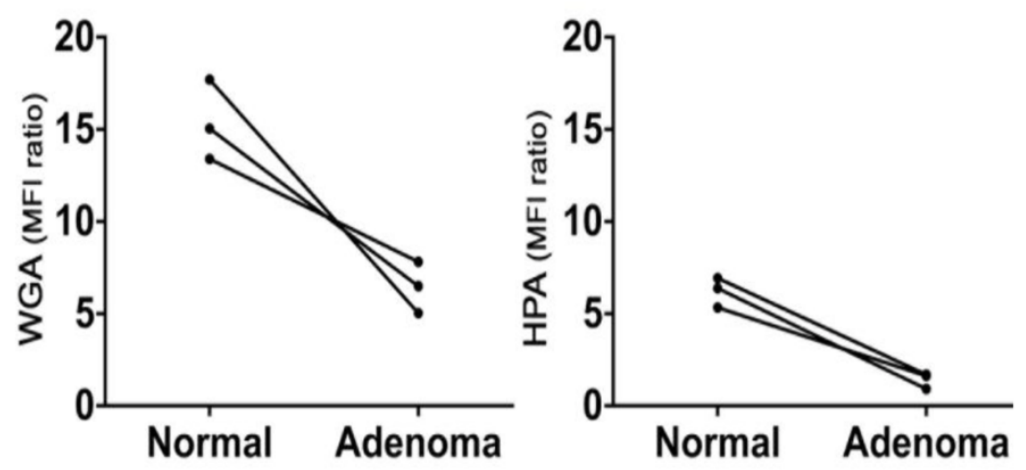

c.

\begin{tabular}{|c|c|c|}
\hline & OES1 & OES2 \\
\hline Indication & Intramucosal $\mathrm{Ca}+\mathrm{HGD}$ & Poor diff EAC \\
\hline Cancer stage (EUS) & T1NOMX & T3NOMO \\
\hline Treatment & EMR; esophagectomy & $\begin{array}{l}\text { Chemotherapy; } \\
\text { esophagectomy }\end{array}$ \\
\hline Pathological outcome & T1NOMX & ypT1NOMX \\
\hline Background fluorescence ${ }^{a}$ & $1.2(1.0-1.4)$ & $1.3(1.0-1.6)$ \\
\hline Signal-to-background ratio ${ }^{a}$ & $10.0(5.9-14.1)$ & $6.7(5.9-7.5)$ \\
\hline Signal-to-noise ratio ${ }^{a}$ & $46.5(44.9-48.1)$ & $38.0(37.4-38.6)$ \\
\hline Squamous esophagus ${ }^{a}$ & $56.9(44.8-69.7)$ & $48.7(45.3-52.1)$ \\
\hline Barrett's esophagus ${ }^{a}$ & $19.7(18.3-21.1)$ & $19.8(18.6-21.0)$ \\
\hline Dysplasia $^{a}$ & $N / A$ & $7.4(6.8-8.0)$ \\
\hline Intramucosal carcinoma ${ }^{a}$ & $6.1(5.4-7.8)$ & N/A \\
\hline $\begin{array}{l}\text { Esophageal } \\
\text { adenocarcinoma }^{\text {a }}\end{array}$ & N/A & $6.1(5.2-7.0)$ \\
\hline Scar tissue ${ }^{a}$ & $11.7(9.1-13.3)$ & N/A \\
\hline
\end{tabular}

Figure 1.17: Differential lectin staining of mucosal surfaces. (a) Differential lectin staining of deep luminal mucus deposits within carcinoma tissue (Figure taken from Kuo et. al. 2016). (b) Quantification of fluorescence imaging showed decreased binding of WGA, and HPA, associated with progression from normal tissue to adenoma in the colon of $A p c^{\text {min }}$ mice 
(Figure taken from Kuo et. al. 2016). (b) Esophageal lumen tissue was resected from two patients (OES1 and OES2) and labelled with WGA lectin and Alexa Fluor 488 ex vivo.

Decreased binding of WGA was associated with progression from squamous esophageal to esophageal adenocarcinoma. (Figure taken from Bird-Lieberman et. al. 2012). Abbreviations: Alcian Blue (AB), periodic acid Schiff (PAS), hematoxylin and eosin (H\&E), Helix pomatia agglutinin (HPA), jackfruit lectin (JFL), peanut agglutinin (PNA), soybean agglutinin (SBA), and wheat germ agglutinin (WGA). Scale bar represents $500 \mu \mathrm{m}$.

Issues of sensitivity are being addressed through enhanced multivalency, which has been generated using "glycan-decorated biomaterials" such as glyconanoparticles, and glycoarrays (Bojarová et. al. 2016; Wang et. al., 2016). Furthermore, the affinity of avidin for lectins expressed endogenously by cancer cells has been harnessed to specifically deliver avidin-fluorophore conjugates to tumours for optical imaging of tumour-bearing mice (Hama et. al. 2006; Hama et. al. 2006). This technique has been used to improve contrast during fluorescence endoscopy and surgical procedures required for the detection of peritoneal metastases (Hama et. al. 2006).

\subsubsection{Detecting glycosylation using antibodies}

The use of antibodies in the context of glycosylation usually involves detecting glycoproteins through epitopes within their polypeptide backbone. Common glycoprotein targeting immunotherapies used in standard clinical practice include the anti-CD20 monoclonal antibody, rituximab, for non-Hodgkin lymphoma, the anti-tyrosine kinase monoclonal antibody, imatinib, for leukaemia, the anti-HER2 monoclonal antibody, trastuzumab, for breast cancer, and the anti-EGFR monoclonal antibody, cetuximab, for colorectal cancer (Weiner et. al. 2012; Madden 2018). However, there are antibodies which recognise tumour cell-associated carbohydrate epitopes (Haji-Ghassemi et. al. 2015; Soliman et. al. 2017). For example, dinutuxmab, a glycolipid inhibitor, which targets the overexpressed ganglioside GD2, has been approved for the treatment of high-risk cases of neuroblastoma and is the first carbohydrate targeting antibody to be approved for use in clinical practice (Dhillon 2015). Antibodies against the Tn antigen (Figure 1.9) are also being developed to detect Tn 
antigen on circulating glycoproteins to improve the early detection of cancer (Kveton et. al. 2017; Ju et. al. 2011).

Hybridoma screening is a technique used to purify monoclonal antibodies against tumour antigens from mice immunised with cancer cells or glycoconjugates (Cummings and Etzler 2009). Antibodies targeting carbohydrates have varying specificities and cross-react with multiple epitopes non-specifically, due to the ubiquitous nature of glycosylation. Monoclonal antibodies against the $\mathrm{N}$-acetylgalactosamine (GalNac) component of the $\mathrm{Tn}$ antigen (Figure 1.9) have been shown to cross-react with GalNac residues within the blood group A antigen (Ju et. al. 2011). Monoclonal antibodies against MUC1, a mucin glycoprotein overexpressed in several cancers, has also been shown to lack carbohydrate specificity due to their affinity for several O-glycan's decorating the cell surface of MUC1 (Naito et. al 2017) (Figure 1.18).

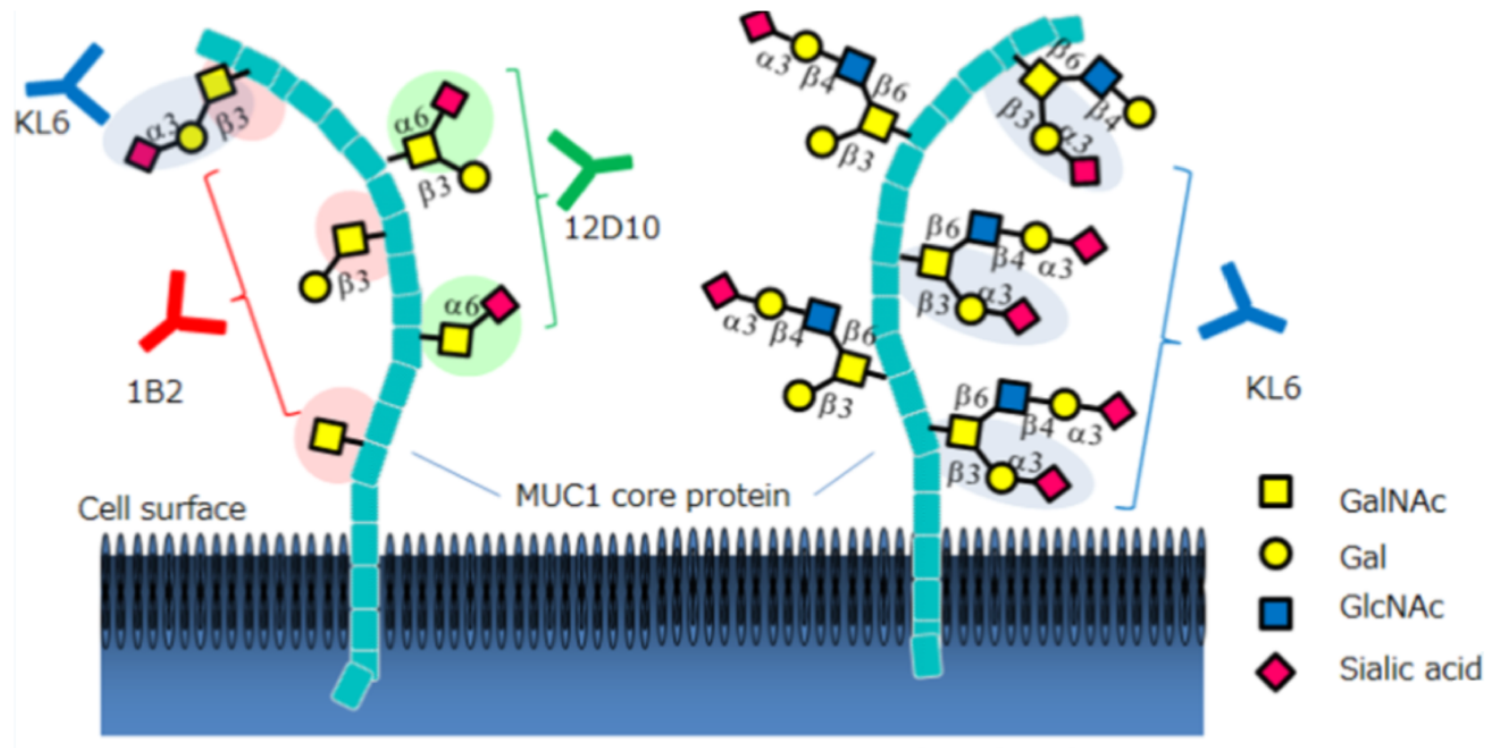

Figure 1.18: The low specificity of anti-MUC1 antibodies. MUC1 antibodies display varying specificities. Antibody KL6 (blue) recognises multiple oligosaccharide structures (Figure taken from Naito et. al 2017). 
Other commercially available antibodies against common tumour antigens such as heparan and SLe ${ }^{x}$ (Figure 1.8a) have been conjugated to radionuclides and used for tumour detection using PET and SPECT imaging, as well as MRI, optical, and ultrasound imaging (Barbet et. al. 2012; Warram et. al. 2014). The disadvantage of using antibody-based imaging is their immunogenicity at high concentrations, slow clearance from the blood, which reduces contrast, and accumulation in organs responsible for clearance, such as the liver (Kojima and Jay 1986). However, tumour background contrast can be improved by using antibody fragments, which clear more rapidly from the circulation, or by increasing the time between administration and imaging (Warram et. al. 2014).

\subsubsection{Detecting glycosylation using bioorthogonal chemical reporters}

Another strategy for detecting glycosylation involves the use of chemical reporters. Here, cells are treated with a monosaccharide analogue containing a functional group or "chemical reporter" not normally present in the cell, such as an azide- or alkyne- group (Laughlin et. al. 2009). For example, precursors of sialic acid and $\mathrm{N}$-acetylgalactosamine have been labelled with an azide group ( $\mathrm{N}$-azidoacetylmannosamine ( $\mathrm{Cc}_{4} \mathrm{ManNAz}$ ) and $\mathrm{N}$ azidoacetylgalactosamine ( $\left.\mathrm{Ac}_{4} \mathrm{GalNAz}\right)$ (Figure 1.19). 


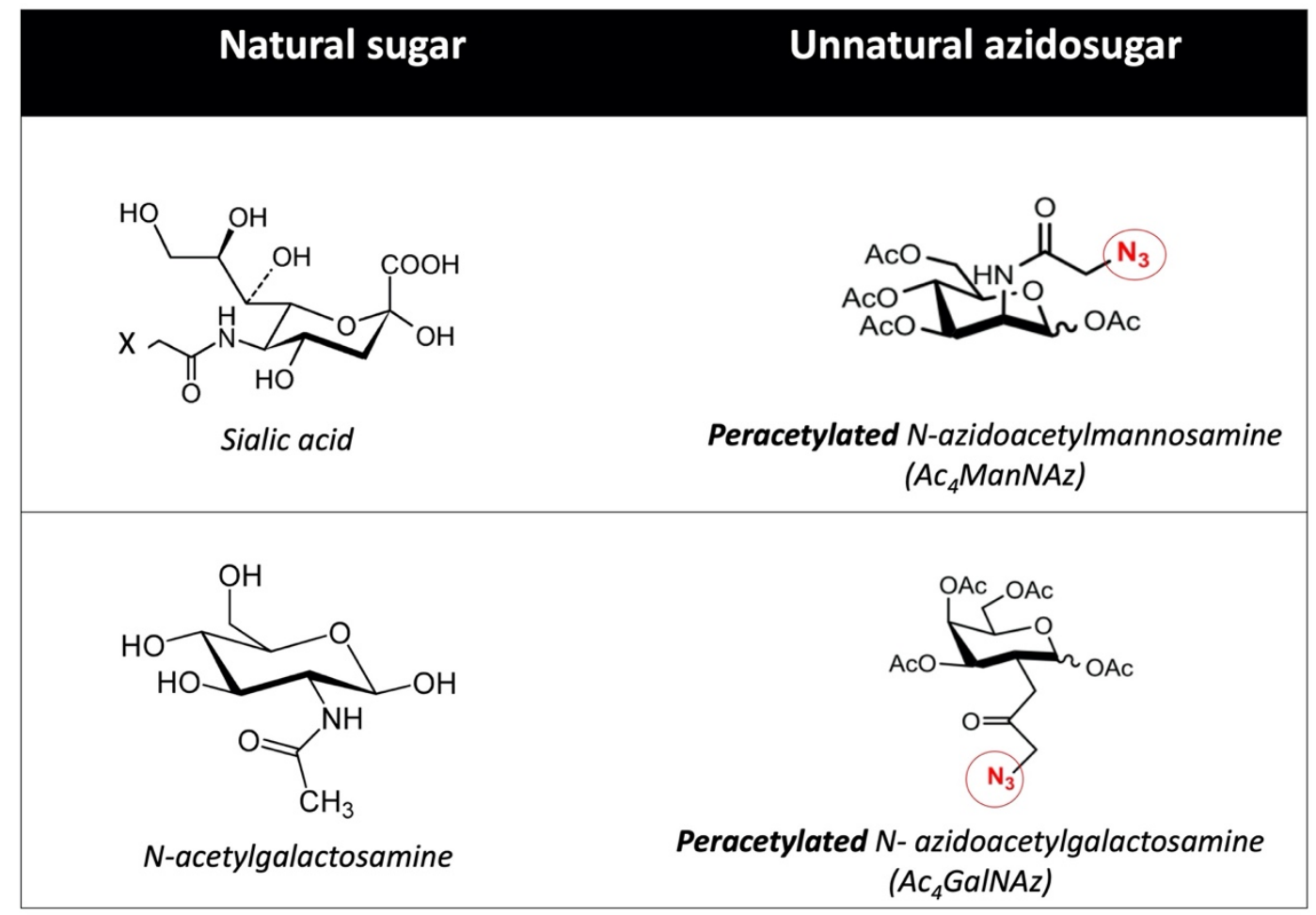

Figure 1.19: Bioorthogonal chemical reporters. Chemical structures of synthetic precursors of sialic acid and $\mathrm{N}$-acetylgalactosamine labelled with an azide group (red) (Nazidoacetylmannosamine (Ac4ManNAz) and N-azidoacetylgalactosamine (Ac $\left.\left.{ }_{4} G a l N A z\right)\right)$. Multiple acetylation helps the azidosugar to cross the cell membrane but these groups are later lost (Author diagram).

The azidosugars are incorporated metabolically into cellular glycan structures (Figure 1.20a). These modified glycans can then be detected by covalent ligation of imaging probes or affinity reagents that rapidly react with the azide or alkyne group, by Staudinger ligation (Saxon and Bertozzi 2000; Saxon et. al. 2002), or cycloaddition (Laughlin et. al. 2006) (Figure $1.20 \mathrm{~b})$. Hence, this technique is termed "bioorthogonal" and applies to dynamic imaging in live cells using a variety of imaging modalities (Borrmann and van Hest 2014) (Figure 1.20b). 
a.
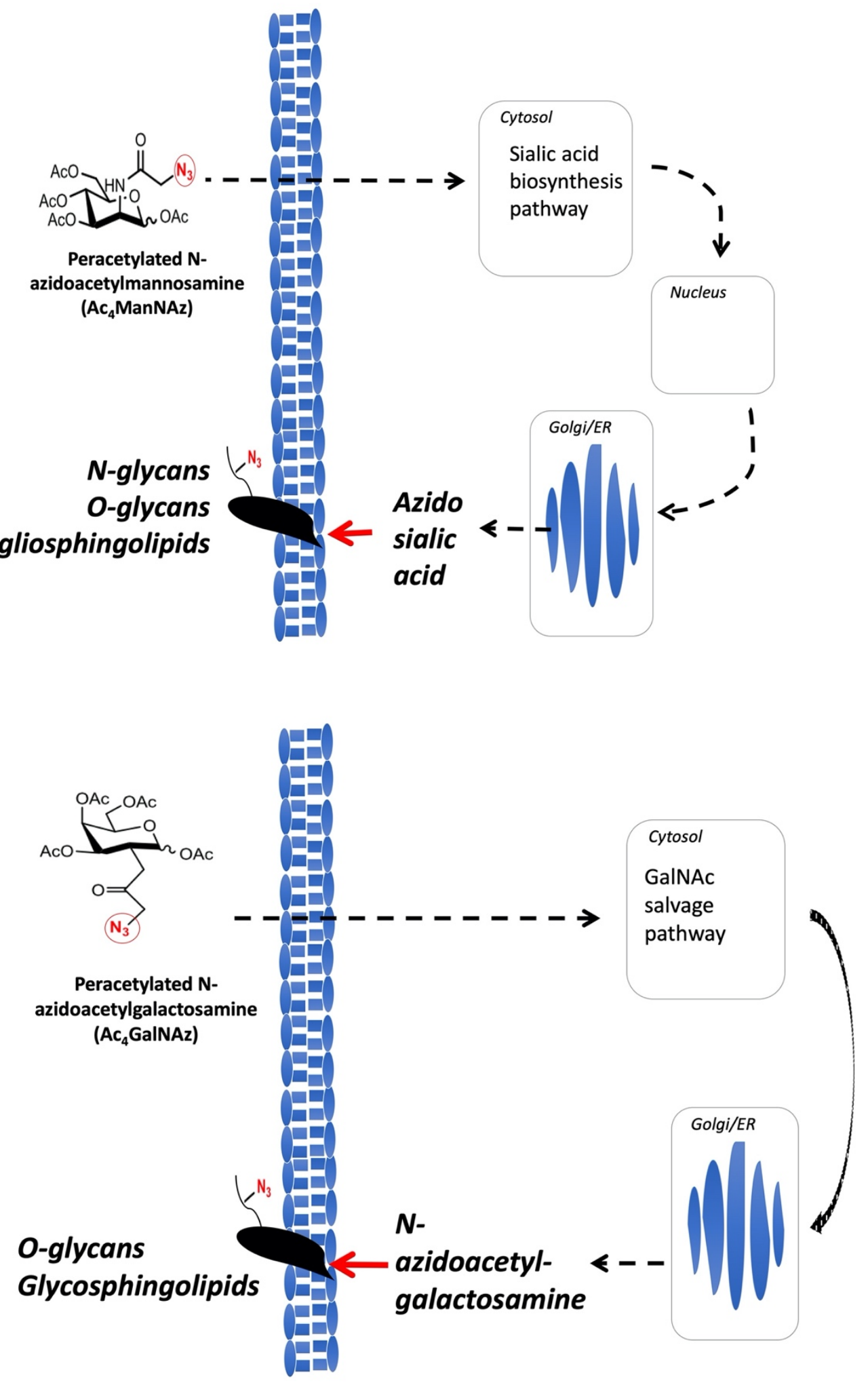


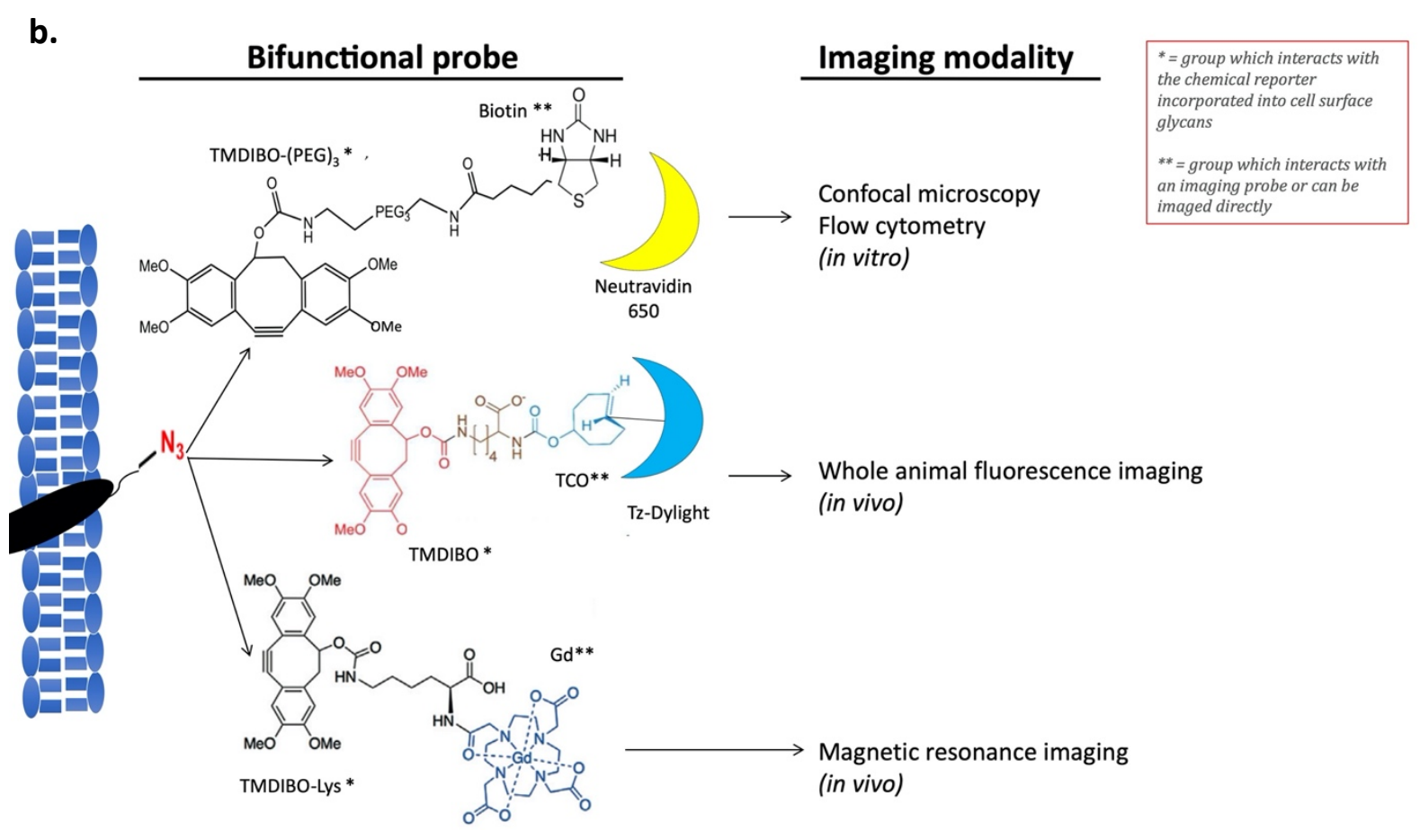

Figure 1.20: Metabolic labelling of glycan's using bioorthogonal chemistry. (a) The azidosugars $\mathrm{N}$-azidoacetylmannosamine (Ac4ManNAz) and $\mathrm{N}$-azidoacetylgalactosamine (Ac $\left.\left.{ }_{4} G a l N A z\right)\right)$ are incorporated biosynthetically into cell surface glycans through the sialic acid biosynthesis pathway and N-acetylgalactosamine salvage pathway, respectively. (b) Bifunctional probes are composed of one group (*), which covalently reacts with the azido $\left(N_{3}\right.$, red) sugar incorporated into cell surface glycans. The second group $\left(^{* *}\right)$ can be detected by high affinity binding imaging probes (Neutravidin 650), covalent ligation to fluorescence molecules (Tz-Dylight), or direct imaging (TMDIBO-Gd). Imaging probes can be fluorescent, conjugated to a radionuclide or conjugated to a contrast agent, which determines the imaging modality used to detect azidosugar labelling. Abbreviations: tetramethoxydibenzocyclooctyne (TMDIBO), trans-cyclooctene (TCO), phosphine (PHOS), Iysine (Lys), tetrazine (Tz), and gadolinium (Gd). (Author diagram; TMDIBO-TCO and PHOSTCO chemical structures from Neves et. al. 2013. TMDIBO-Lys-Gd chemical structure taken from Neves et. al. 2015).

Detecting metabolically labelled glycans using chemical reporters has been used to study glycosylation during the development of Zebrafish embryos (Laughlin et. al. 2008), image glycoprotein sialyation in live Zebrafish (Agarwal et. al. 2015), locate sialylated proteins 
within the membrane of Trypanosoma cruzi, investigate the role of lipooligosaccharide sialyation in Haemophilus ducreyi virulence, and visualise glycosylation in Caenorhabditis elegans (Goon et. al. 2003; Laughlin et. al. 2009).

Bioorthogonal chemistry has also been applied in human cells (Saxon and Bertozzi 2000; Dube and Bertozzi 2003). For example, Chang and colleagues used chemical reporters to simultaneously quantify cell surface sialyation, and $\mathrm{N}$-acetylgalactosamine glycosylation, within the same human cells, using flow cytometry, and fluorescent microscopy. Jurkat cells were subjected to dual treatments with peracetylated N-levulinoylmannosamine, a ketonelinked precursor of sialic acid, and $\mathrm{Ac}_{4} \mathrm{GalNAz}$, an azide-linked analogue of Nacetylgalactosamine (Chang et. al. 2007). Peracetylated N-levulinoylmannosamine was detected with hydrazide-biotin, which reacted covalently with the precursors ketone group, followed by biotin visualisation using FITC-avidin. $\mathrm{Ac}_{4} \mathrm{GaINAz}$ was detected with a fluorescein-phosphine probe, which reacted covalently with the analogue's azide group. The chemoselectivity of covalent reactions between complementary chemical groups, such as between ketones and hydrazides, or azides and phosphines, enables highly specific detection by this bioorthogonal metabolic labelling.

The most extensively used chemical reporters in cancer research are variations of sialic acid, $\mathrm{N}$-acetylgalactosamine or fucose, due to their associations with cancer progression (Fuster and Esko 2005; Laughlin et. al. 2009; Cheng et. al. 2016). Confocal microscopy (Figure 1.21a), and flow cytometry (Figure 1.21b) have been used to visualise and quantify azidosugar labelling in cancer cell lines (Stöckmann et. al. 2011; Kuo 2015, PhD thesis, unpublished). Azidosugar labelling has also been visualised in tumour-bearing mice using whole animal fluorescence (Figure 1.21c and d), MRI (Figure 1.21e) and PET imaging (Neves et. al. 2011; Neves et. al. 2013; Neves et. al. 2015). Peracetylated alkynyl N-acetylmannosamine, a precursor of sialic acid, has been used to study sialyation in oral cancer (Chen et. al. 2015). 
a.

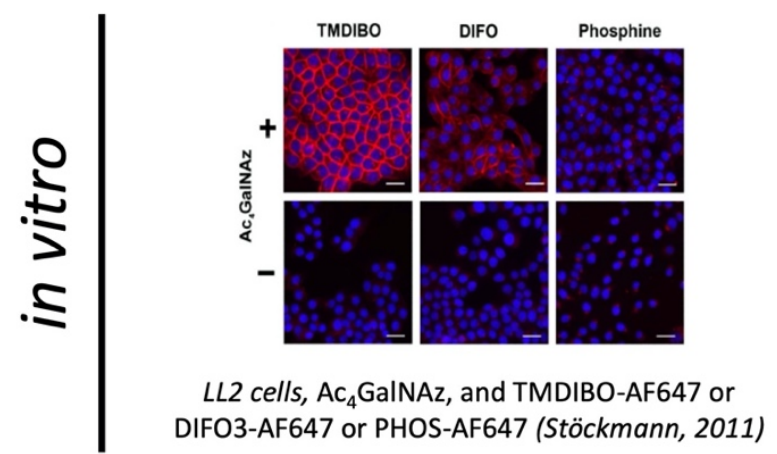

b.

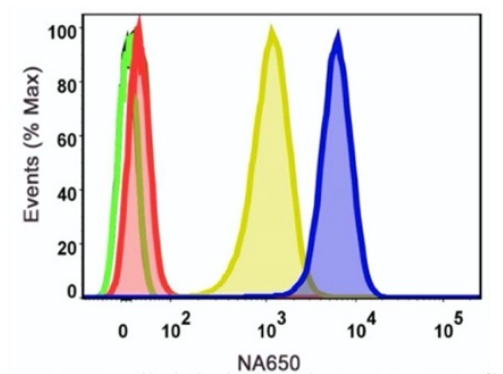

MDA MB 231 cells labeled with $A c_{4}$ ManNAz (blue) or $\mathrm{Ac}_{4}$ GaINAz (yellow), TMDIBO-Biotin, and NA650 (Kuo 2015, PhD thesis, unpublished ) c.

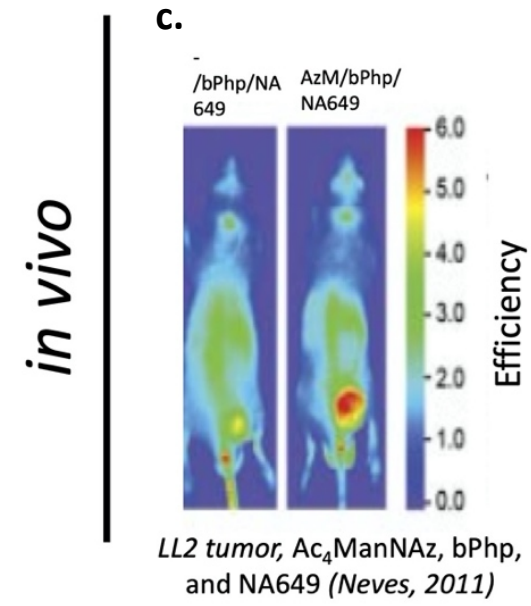

d.

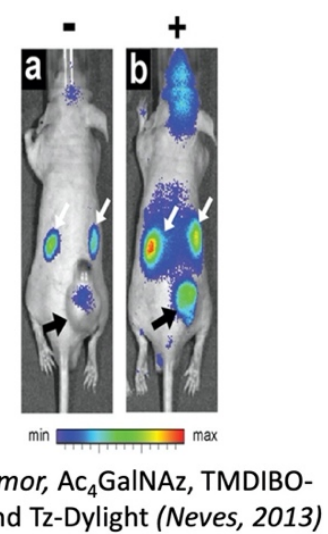

e.

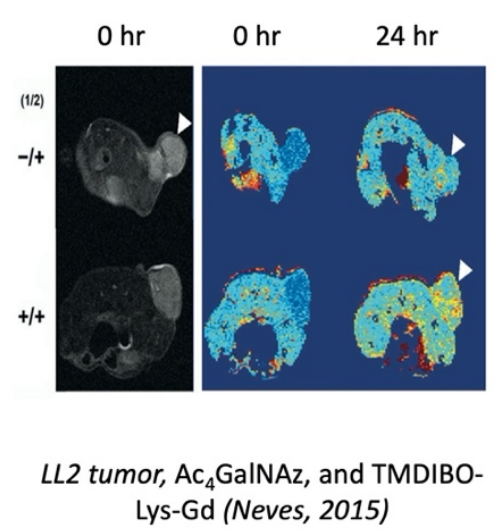

Figure 1.21: Molecular imaging of cell surface glycosylation of cancer cells in vitro and in vivo. (a) Lewis lung carcinoma cells labelled with combinations of Ac 4 GalNAz, TMDIBO-AF647 (red) and DAPI (blue) visualised using fluorescence microscopy. (b) Flow cytometric analysis of human breast cancer cells (MDA MB 231) labelled with combinations of $A c_{4} M a n N A z$ (blue) or Ac ${ }_{4} G a I N A z$ (yellow), TMDIBO-Biotin, and NeutrAvidin labelled with a fluorescent dye (NA650). (c) Fluorescence images of Lewis lung carcinoma (LL2) tumour-bearing BALB/c male mice treated with combinations of $A c_{4} M a n N A z$, biotin-phosphine probe (bPhp) and NeutrAvidin 649 (NA649) at 24 h after injection with NA649. (d) Fluorescence images of murine Lewis lung carcinoma (LL2) tumour-bearing BALB/c female mice treated with combinations of $A c_{4} G a I N A z, T M D I B O-T C O$ and Tz-DyLight at $6 \mathrm{~h}$ after injection with TzDyLight. The tumour (black arrow) and kidneys (white arrows) are highlighted. (e) Noninvasive MRI detection of $\mathrm{Ac}_{4}$ GalNAz-dependent metabolic labelling of tumours in vivo (white arrow head). BALB/C male mice were treated with combinations of $A c_{4} G a I N A z$ and TMDIBOLys-Gd at 0 and $24 \mathrm{~h}$ after injection of TMDIBO-Lys-Gd. Abbreviations: peracetylated $\mathrm{N}$ azidoacetylmannosamine (Ac4ManNAz), peracetylated $N$-azidoacetylgalactosamine (Ac4GalNAz), NeutAvidin ${ }^{T M}$ Dylight $^{T M} 650 / 649$ (NA650/649), tetrazine (Tz), 
tetramethoxydibenzocyclooctyne (TMDIBO), trans-cyclooctene (TCO), phosphine (PHOS), and lysine (Lys) (Author diagram created from figures adapted from Stöckmann et. al., 2011; Kuo 2015, PhD thesis, unpublished; Neves et. al., 2011; Neves et. al., 2013; Neves et. al., 2015).

The use of chemical reporters exhibits further superiority over affinity-based reagents such as lectins and antibodies due to the use of small molecules requiring much smaller amounts of material. However, the ubiquitous nature of glycosylation can generate non-tumour labelling, for example, in the organs responsible for clearance and excretion of the probes (Neves et. al., 2013) (Figure 1.21d, white arrows). Efforts to improve contrast and the specificity of glycan imaging have involved packaging of azidosugars into functionalised liposomes to encourage tumour-specific uptake of the labelling reagents (Xie et. al. 2012; Xie et. al. 2014).

\subsubsection{Additional metabolic labelling strategies}

Molecular imaging can be conducted by combining affinity reagents and chemical tools. Imaging glycosylation using both lectins and bioorthogonal chemical reporters has been used to image cell surface fucosylation (Figure 1.22a) and sialylation (Figure 1.22b) in the human hepatocellular carcinoma Hep3B cell line (Sawa et. al. 2006; Hsu et. al. 2007). 
a.

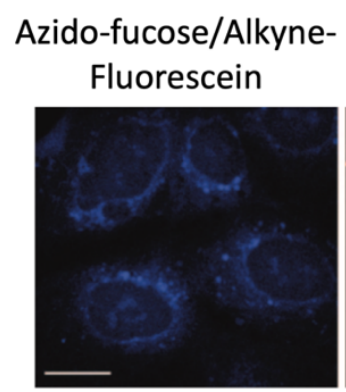

WGA-Alexa

594
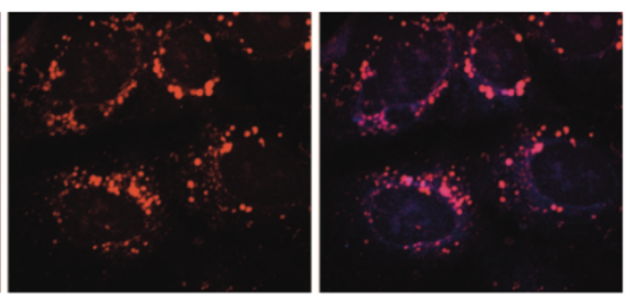

b.

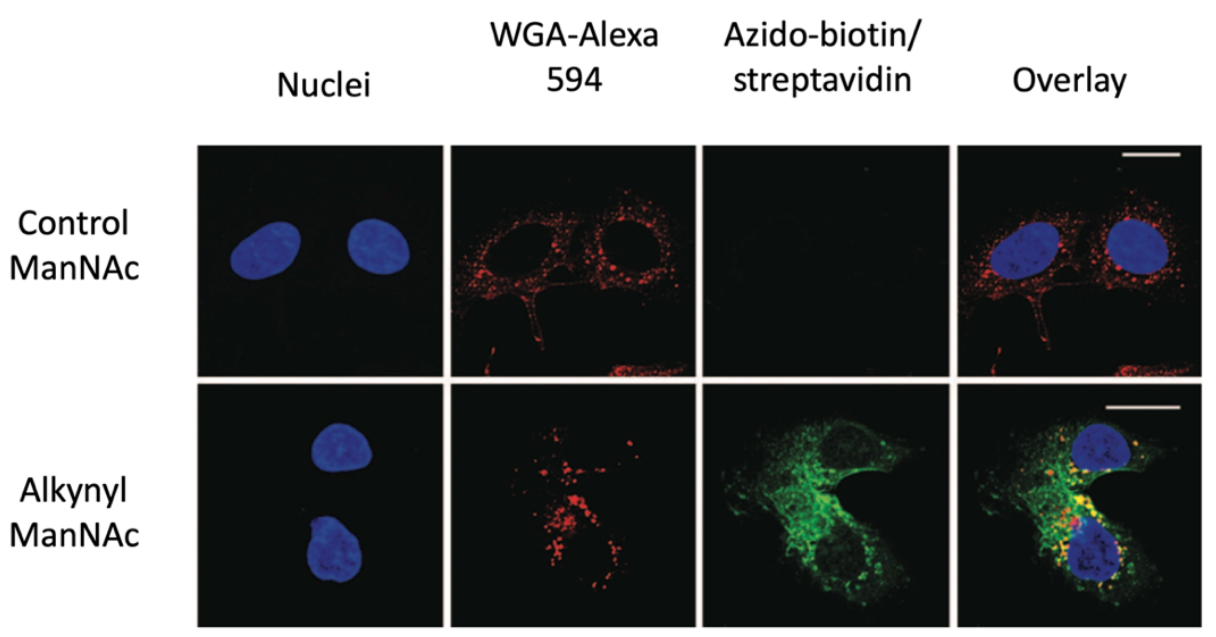

Figure 1.22: Dual confocal imaging of glycosylation in a hepatocellular carcinoma cell line using lectins and bioorthogonal chemical reporters. (a) Human hepatocellular carcinoma cells (Hep3B) were grown in the presence of synthetic azido-fucose, a synthetic analogue of fucose, which labels cell surface glycans. After 3 days, azido bearing glycans were covalently reacted with a fluorescein-conjugated alkyne probe (blue), and subsequently stained with Alexa Fluor 594-conjugated wheat germ agglutinin (WGA) lectin (red) (Figure adapted from Sawa et. al. 2006). (b) Hep3B cells were grown in the presence of exogenous natural $N$ acetylmannosamine (control ManNAc) or synthetic N-4-pentynoy/mannosamine (Alkynyl ManNAc), which both label cell surface glycans. After 3 days, alkyne-bearing glycans were covalently reacted with a biotinylated azido probe (biotin coupled to 1-azido-3aminopropane), and the cells stained subsequently with fluorescein-conjugated streptavidin (green), Alexa Fluor 594 conjugated WGA lectin (red), and Hoechst (blue). Scale bars represent $20 \mu \mathrm{m}$ (Figure adapted from Hsu et. al. 2007). 
A recently developed technique for the metabolic labelling of glycans uses monosaccharideimprinted fluorescent nanoparticles, which are more stable than lectins and antibodies (Wang et. al. 2016; Shinde et. al. 2015). Here, monosaccharide polymers are synthesised in the presence of a target monosaccharide, such as sialic acid, which is subsequently removed. The biopolymers are left with sialic acid-deficient cavities, also known as a molecularly imprinted polymer (MIP) or nanoparticle. These particles exhibit a pre-engineered specificity and affinity for sialic acid when added to cells, which can be detected by conjugating the MIP to a fluorophore. This represents a novel technique, which uses chemical engineering to enhance the specificity of fluorescence-based glycan imaging.

\subsection{Project aims, objectives and experimental approaches}

\subsubsection{Project aims and objectives}

Glycan's play an important role in cancer (Figure 1.7 and 1.8), and several studies have demonstrated the utility of targeting glycosylation for precision oncology through biomarker discovery (Figure 1.16), or the molecular imaging of glycans in vivo using lectins (Figure 1.17) and bioorthogonal azidosugars (Figure 1.21c-e). However, the clinical relevance of imaging glycosylation is not simply in the identification of tumours, for which many techniques already exist, but in the ability to visualise changes in glycosylation that we know occur during disease progression (Stowell et. al. 2015; Pinho and Reis 2015; Fuster and Esko 2005; Ju et. al. 2011; Vajaria and Patel 2017).

Lectin-based fluorescence imaging has revealed a link between glycosylation and cancer progression within mucosal surfaces, as discussed previously (Bird-Lieberman et. al. 2012;

Kuo et. al. 2016) (Figure 1.17). The association between glycosylation and cancer progression has also been observed using lectins and chemical reporters (Figure 1.19) using panels of human cell lines constructed to represent the different stages of cancer progression (Figure 1.23). For example, an increase in Maackia amurensis lectin binding, which binds sialic acid, was observed in a panel of breast cancer cell lines (Kuo 2015, Ph.D. thesis, unpublished) 
(Figure 1.23a). An increase in $\mathrm{Ac}_{4} \mathrm{ManNAz}$ labelling, a precursor of sialic acid, has also been associated with an increase in the metastatic potential of panels of breast (Figure 1.23b), prostate (Figure 1.23c) and melanoma (Figure 1.23d) cell lines (Kuo 2015, Ph.D. thesis, unpublished; Whittaker, 2015, Part III Dissertation, unpublished). These data confirm that as the cancer progresses, glycan expression is altered. Therefore, the correlation between increases in glycosylation and metastatic potential suggest that detecting changes in glycosylation could be useful for monitoring the propensity of a tumour to metastasise in vivo i.e. for assessing the aggressiveness of the disease. The ability to visualise non-invasively tumour glycosylation in vivo, using bioorthogonal glycan labelling, would provide qualitative insights into glycan dynamics and turnover (Colley et. al. 2017) in real-time. Furthermore, the benefits of basic cancer imaging are also realised, such as measuring tumour responses to therapy earlier and reducing health care costs by reducing overdiagnosis (Neves et. al. 2014). Glycan imaging may also be beneficial in clinical trials, where visualising tumour regression or stabilisation at a molecular level could reveal the effectiveness of drugs earlier (Amur et. al. 2005).

a.

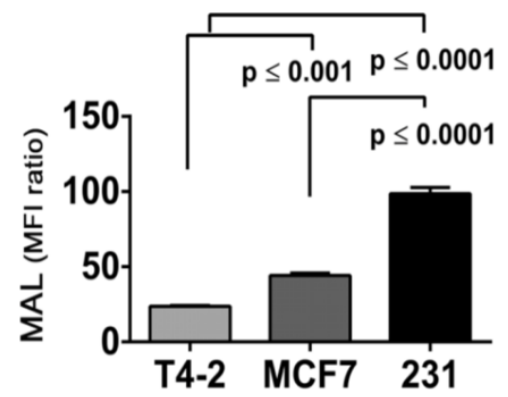

c.

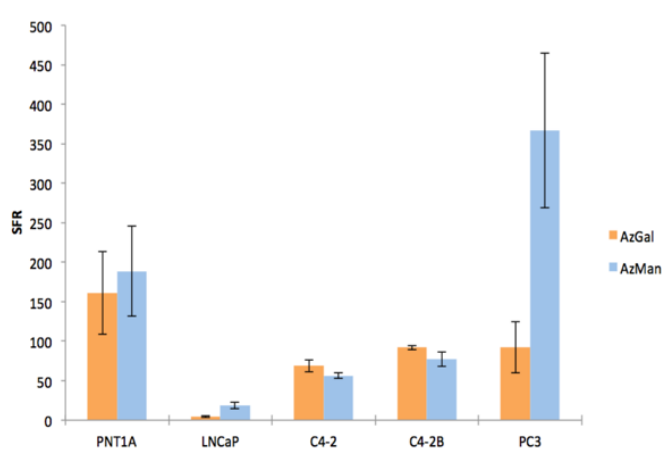

b.

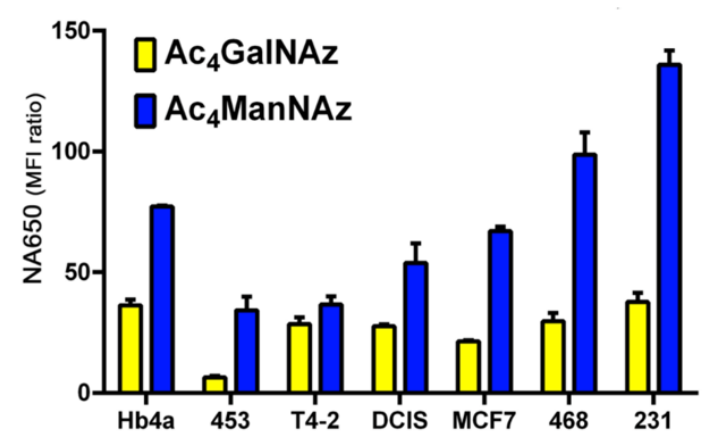

d.

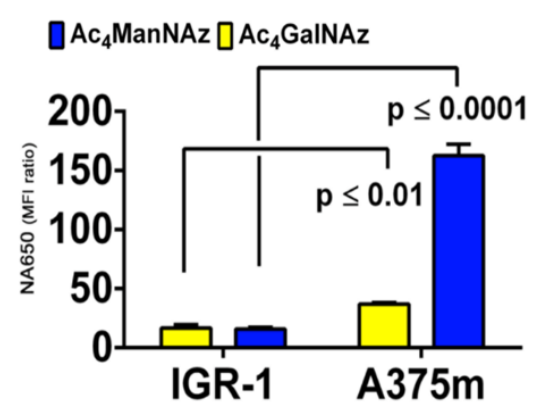

Figure 1.23: Detection of lectin binding and azidosugar incorporation in panels of human cell lines with increasing metastatic potential. Human breast, prostate and melanoma 
human cell lines were selected from the literature, and ordered based on their increasing tumourigenicity and metastatic potential following orthotopic implantation into nude mice. (a) An increase in Maackia amurensis lectin binding, which preferentially binds sialic acid, was observed in a panel of breast cell lines. (b) An increase in Ac ${ }_{4}$ ManNAz labelling (dark blue), a precursor of sialic acid, was observed a panel of breast cell lines. (c) An increase in Ac ${ }_{4}$ ManNAz labelling (light blue), a precursor of sialic acid, was observed in a panel of prostate cell lines. (d) An increase in Ac 4 ManNAz labelling (dark blue), a precursor of sialic acid, was observed in a limited panel of melanoma cell lines. The darker shades in the arrows' greyscales represent an increase in metastatic potential. ( $a, b$ and $d$ taken from Kuo 2015, Ph.D. thesis, unpublished; c taken from Whittaker, 2015, Part III Dissertation, unpublished).

What is yet to be answered is whether bioorthogonal metabolic labelling (Figure 1.21 and 1.23b-d) detects global increases in sialyation or increased turnover (Colley et. al. 2017) of cell surface glycan's. It is unknown whether azidosugars are preferentially incorporated into glycan structures via specific glycosidic linkages, and if this occurs at multiple epitopes within a glycoconjugate in a site-specific or tissue-specific manner. This would be influenced by changes in the expression or activity of sialyltransferases in the more metastatic cell lines, which also has not been investigated. It is possible that the glycans being differentially detected during progression using fluorescence-lectin imaging (Figure 1.17 and 1.23a) or azidosugar metabolic labelling (Figure 1.23b-d), may be part of a larger array of 'metastatic codes' associated with a tumour's glycan profile at any given time point (Fuster and Esko 2005). In other words, differential azidosugar labelling implies the presence of differentially labelled glycoconjugates, which if identified using mass spectrometry, may be predictive biomarkers of cancer progression.

The goal of this study was to use bioorthogonal chemistry, to monitor dynamic changes in cell surface glycosylation in models of cancer progression, to identify novel biomarkers, and to investigate if changes in tumour glycosylation could be imaged using metabolic labelling in vivo in advanced models of cancer progression. 


\subsubsection{Experimental approach}

The efficiency of glycan labelling using the affinity reagents (e.g. lectins, antibodies) or the bioorthogonal chemical reporters (e.g. azido-sugars) can differ between cancer types, often requiring extensive optimisation of appropriate reagent concentrations and incubation times, which can be time-consuming. Therefore, cell lines were used to optimise protocols and preliminarily investigate the utility of targeting glycosylation for biomarker discovery and glycan imaging.

Panels of human cell lines representing the progression of cancer have been selected previously from the literature, based on their increasing tumourigenicity and metastatic potential following orthotopic implantation into nude mice (Kuo 2015, Ph.D. thesis, unpublished, and Whittaker, 2015, Part III Dissertation, unpublished). To validate these panels as suitable models of cancer progression, the migration and invasion characteristics of each cell line were investigated using in vitro assays and immunohistochemistry to compare the relative expression of known invasion biomarkers.

Flow cytometry and gel electrophoresis were used to visualise how $\mathrm{Ac}_{4} \mathrm{ManNAz}$ and $\mathrm{Ac}_{4} \mathrm{GaINAz}$ (Figure 1.24) differentially label glycoconjugates across panels of cell lines, representing the stages of cancer progression in vitro. The most widely available electrophoresis techniques focus on resolving proteins. Therefore, this projected focused on glycoprotein dynamics, despite metabolic labelling also being affected by glycolipid turnover (Hakomori 1985; Colley et. al. 2017). Differentially labelled glycoconjugates were identified using proteomics. Cell lines were treated with an azidosugar, metabolically labelled glycoproteins extracted and covalently tagged with a bifunctional-biotin probe such as alkyne-biotin, and the resulting biotin-tagged azidosugar-labelled proteins enriched using streptavidin-coupled beads (Figure 1.24). Liquid chromatography-tandem mass spectrometry was used to identify proteins differentially azidosugar labelled across the in vitro models of cancer progression, which it was anticipated might lead to the discovery of new prognostic biomarkers. 
a.
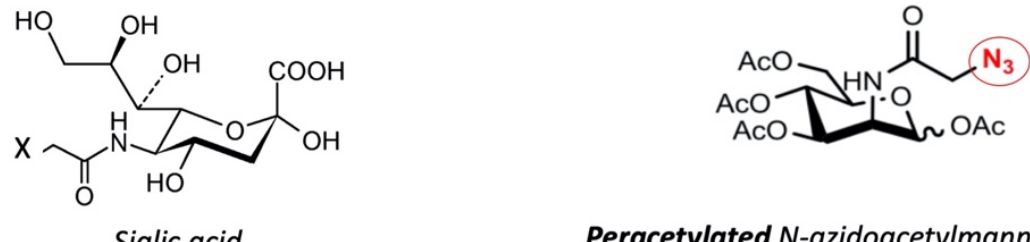

Sialic acid

Peracetylated $\mathrm{N}$-azidoacetylmannosamine ( $\left.\mathrm{Ac}_{4} \mathrm{ManNAz}\right)$

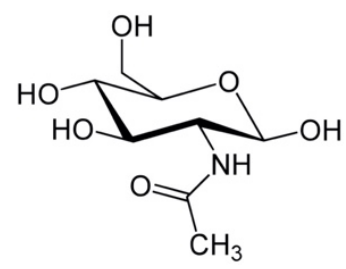

$\mathrm{N}$-acetylgalactosamine

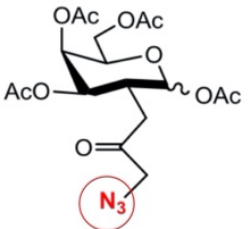

Peracetylated $\mathrm{N}$ - azidoacetylgalactosamine ( $A c_{4}$ GalNAz)

b.

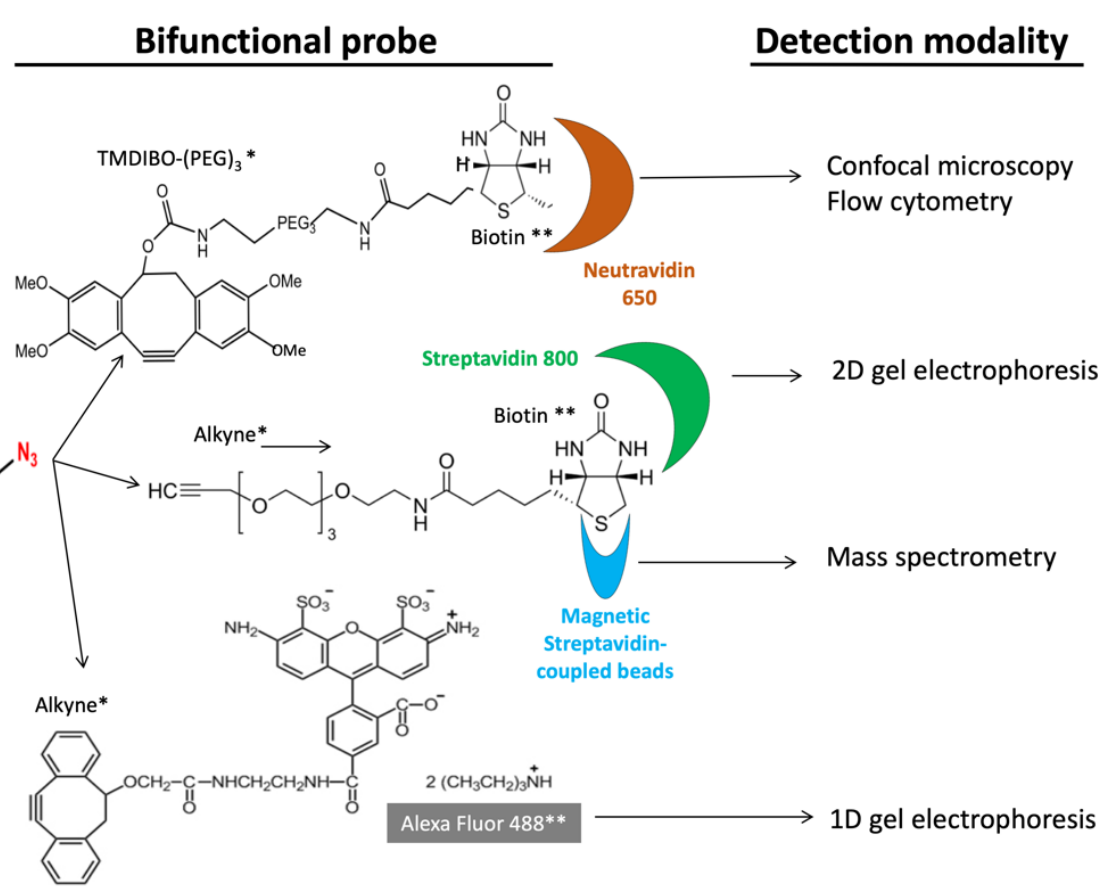

Figure 1.24: Summary of bioorthogonal chemical tools used in this project. Human breast and prostate cell lines have been selected from the literature previously and ordered based on their increasing tumourigenicity and metastatic potential following orthotopic implantation into nude mice. To validate these panels as suitable models of cancer progression, the migration and invasion characteristics of each cell line were investigated 
using in vitro assays. (a) Synthetic precursors of sialic acid and N-acetylgalactosamine labelled with an azide group ( $\mathrm{N}$-azidoacetylmannosamine (Ac4ManNAz) and $\mathrm{N}$ azidoacetylgalactosamine (Ac $\left.{ }_{4} G a I N A z\right)$, were used to study sialyation and $\mathrm{N}$ acetylgalactosamine glycosylation respectively. (b) For 1D gel electrophoresis analysis, azidosugar-labelled glycoproteins were covalently reacted with Alkyne-Alexa Fluor 488 and detected in-gel using Alexa-488 fluorescence. For 2D electrophoresis analysis, azidosugarlabelled glycoproteins were covalently reacted with Alkyne-biotin, resolved by 2D western blot and detected by fluorescence imaging using Streptavidin conjugated to Alexa Fluor 800. For mass spectrometry analysis, azidosugar-labelled glycoproteins were covalently ligated to alkyne-biotin, enriched using streptavidin-coupled beads, and identified by mass spectrometry (Author diagram).

The use of panels of cell lines is limited due to the loss of cell-cell interactions that exist in tissues, and within the tumour microenvironment (Burdall et. al. 2003). Therefore, the question exists whether the correlation between azidosugar labelling and progression in vitro (Figure 1.23) can be validated in advanced mouse models. The evolution of pancreatic ductal adenocarcinoma (PDAC) from intraepithelial neoplasia (PanIN) precursor lesions to tumours, has been replicated in p48Cre; LSL-KrasG12D (KC) mice (Hingorani et. al. 2003; Westphalen and Olive 2012) (Figure 1.25a and b). Samples from human and mouse pancreases have been successfully extracted and grown in matrigel, whilst still maintaining the ability to recapitulate the morphology and disease characteristics observed in humans in vivo (Boj et. al. 2015; Baker et. al. 2016) (Figure 1.25c). Therefore, azidosugar labelling protocols were initially optimised in 3D organoid models of PDAC. Metabolic labelling of pancreatic organoids generated from KC mice ex vivo provided preliminary evidence for whether increases in $\mathrm{Ac}_{4} \mathrm{ManNAz}$ between normal tissue, PanIN lesions, and tumours, would be observed in vivo. Metabolic labelling studies in $\mathrm{KC}$ mice in vivo were then conducted, which could support the future use of glycan imaging for monitoring tumour progression using clinically relevant imaging modalities. 
a.

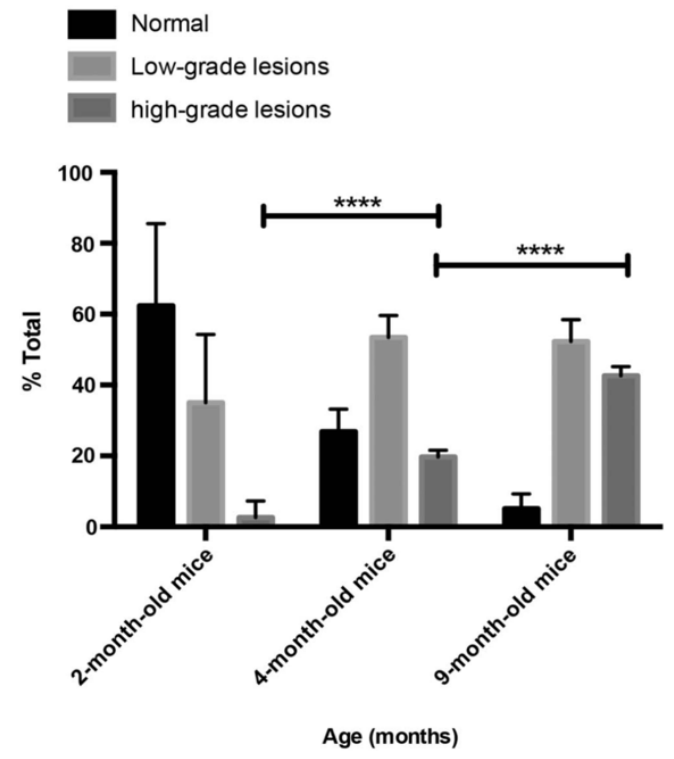

b.

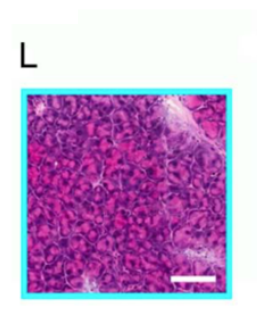

M

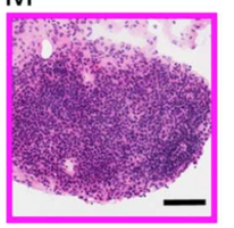

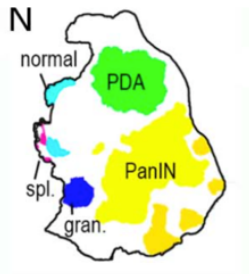

0

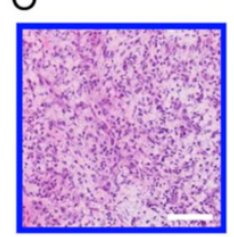

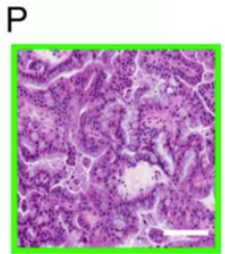

Q

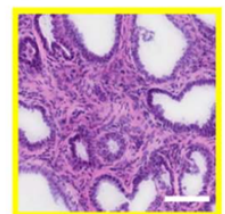

c.

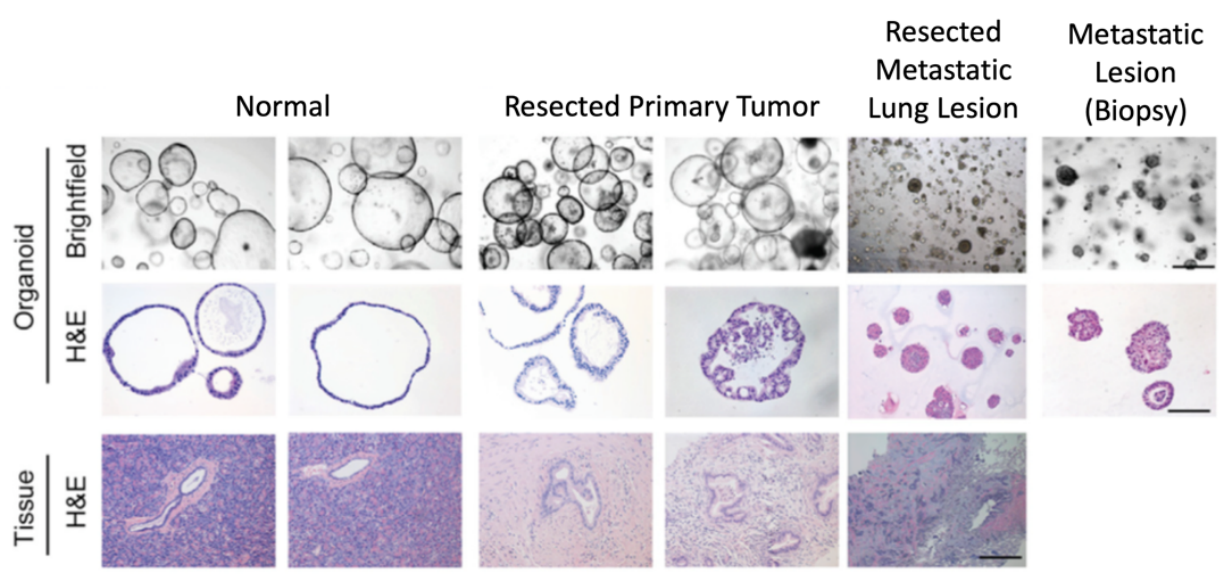

Figure 1.25: Advanced mouse model of pancreatic ductal adenocarcinoma resulting from

expression of mutant Kras. (a) Histological progression of intraepithelial neoplasia precursor lesions in p48Cre; LSL-KrasG12D (KC) mice over 9 months (Figure taken from Serrao et. al. 2015). (b) Confirmation of the spectrum of lesions observed in KC mice by H\&E staining of normal pancreatic tissue (L), splenic lymphoid tissue $(M)$, granuloma tissue $(O)$, pancreatic ductal adenocarcinoma $(P)$ and mouse pancreatic intraepithelial neoplasia $(Q)$ (Figure taken from Serrao et. al. 2015). (c) Organoid models of ductal pancreatic cancer from human tissues. Image shows organoid cultures generated from human tissue extracted from normal pancreas, resected primary tumours, resected metastatic lung lesion. Pancreatic organoids 
can be passaged, cryopreserved and orthotopically implanted into mice. Scale bars: $500 \mu \mathrm{m}$ (Brightfield image), 250 m (H\&E image) (Figure taken from Boj et. al. 2015).

The link between aberrant glycosylation and cancer progression has formed the basis for using glycoproteins and specific polysaccharide structures in the diagnosis, monitoring, and treatment of cancer (Pinho and Reis 2015). However, the utility of cancer biomarkers has been challenged in recent years due to low cancer specificity and high false-positive rates (Mayeux 2004). Lectins, antibodies, and bioorthogonal monosaccharides have facilitated visualisation of glycan distribution, and composition in the context of cancer (Figure 1.17, 1.21, and 1.22), and are useful tools for discovering novel or improved protein or imaging biomarkers (Springer and Gagneux 2016). Overall, targeting glycosylation to advance precision oncology and improve cancer outcomes will likely need a combination of detecting an aberrantly glycosylated biomarker in tissue and serum, and then monitoring of tumour glycosylation using dynamic molecular imaging (Nimse et. al. 2016). 


\section{Chapter 2: Validation of in vitro panels cancer progression}

\subsection{Introduction}

\subsubsection{The burden of cancer}

Trends in cancer-related morbidity (Fitzmaurice et. al. 2015) and mortality worldwide, highlight cancer as a threat to public health. A surge in the incidence of age-related cancers, such as prostate and breast cancers, is also anticipated due to increasing life expectancy, an aging population, and the association between cancer risk and age (Cook et. al 1969). The global incidence of prostate cancer increased by 217\% between 1990-2013 (Fitzmaurice et. al. 2015; Drudge-Coates et. al. 2017), which has been attributed to increases in early detection through PSA screening, digital rectal examinations and biopsies (Smith-Palmer et. al. 2019). Furthermore, in 2006, prostate cancer treatment costs totaled an estimated $\$ 9$ billion in the US alone, highlighting the economic burden of prostate cancer (Roehrborn and Black 2011). Although breast cancer mortality has declined in Europe (Carioli et. al. 2017), the Americas and Australasia (Carioli et. al. 2018), incidence continues to rise, and in the UK alone accounts for the greatest proportion of cancer cases in females (Smittenaar et. al. 2016) (Chapter 1: Figure 1.1). Pre-clinical models are vital to aid our understanding of the molecular events driving cancer progression, metastatic risk, and to test novel therapeutic interventions.

\subsubsection{Histological and molecular progression of cancer}

Prostate cancer tumourigenesis starts with the development of proliferative inflammatory atrophy (PIA) thought to be initiated by inflammation-induced dysplasia as a result of exposure to carcinogens, infectious agents (Taichman et. al. 2007), and unhealthy dietary lifestyle patterns (Grönberg 2003), or a familial predisposition due to the inheritance of germline mutations (Eeles et. al. 2014). PIA can develop into pre-cancerous prostatic 
intraepithelial neoplasia (PIN), which can progress to prostate cancer (Taichman et. al. 2007). The histological progression of prostate cancer is dependent on androgen, a hormone vital for proliferation and inhibition of apoptosis in prostate cancer cells (Debes and Tindall 2002), and is often accompanied by genetic alterations (Yokota 2000). Molecular classification markers of prostate cancer progression (Logothetis et. al. 2013) include phosphatase and tensin homolog (PTEN) loss, TP53 inactivation, E-cadherin loss, B-cell lymphoma 2 (BCL-2) overexpression and androgen receptor amplification (Quigley et. al. 2018; Dong 2006).

Metastatic prostate cancer, commonly to the bone (Jin et. al. 2011), remains a public health burden (Kelly et.al. 2018) and has been observed in 90\% of autopsies in men who died from prostate cancer (Bubendorf et. al. 2000). In advanced stages of prostate cancer, cells can become androgen-independent, which is accompanied by resistance to castration and androgen deprivation therapies (Taichman et. al. 2007), leading to the development of androgen-independent prostate cancer (Dutt and Gao 2009).

Breast cancers originate from different mammary cell types, and follow a traditional multistep tumourigenesis model, from flat epithelial atypia, to atypical ductal hyperplasia, to ductal carcinoma in situ (DCIS), to breast cancer (Bombonati and Sgroi 2011), which is divided into luminal, receptor tyrosine kinase erbB-2 (HER2) positive, triple-negative or basal-like, claudin-low, ERBB2-positive, and normal-like molecular subtypes (Dai et. al. 2015). Up to $40 \%$ of pre-malignant DCIS lesions progress to metastatic disease (Cowell et. al. 2013), a process influenced by the interaction of breast cancer cells with immune cells, endothelial cells and stroma within the breast tumour microenvironment (Soysal et. al. 2015). However, a "parallel progression model" (Klein 2009) has also been proposed for breast cancer where metastases are believed to develop early in tumourigenesis, with disseminating tumour cells being released from small-sized primary tumours before they are detectable with standard mammographic techniques (Autier 2018).

$10-15 \%$ of breast cancer patients present with metastatic disease, with invasive ductal carcinoma making up 50-80\% of histological metastatic breast cancer subtypes (Weigelt et. al. 2005). The principal sites of metastases can depend on the breast cancer subtype. For example, luminal ( $A$ and $B$ ) breast cancers have a high propensity to metastasise to the 
brain, which occurs less frequently in triple-negative breast cancers, whilst HER2+ breast cancers have been shown to have a high propensity to metastasise to the lungs in comparison to other breast cancer subtypes (Kimbung et. al. 2015). Therefore, primary breast cancers and metastases are very heterogeneous and present different histological, molecular, prognostic and treatment response features (Byler et. al. 2014; Zardavas et. al. 2015).

\subsubsection{Pre-clinical models of cancer progression}

Pre-clinical models of prostate (Arriaga and Abate-Shen 2019; Cunningham and You 2015; Ittmann et. al. 2013; Parisotto and Metzger 2013; Wu et. al. 2013) and breast (Whittle et. al. 2015; Fantozzi and Christofori 2006; Park et. al. 2018; Sakamoto et. al. 2015; Holen et. al. 2017) cancer progression exist in the form of cell lines, spontaneous mouse models and xenograft models, which replicate the spectrum of cancer progression states. The PTEN knockout transgenic mouse model exhibits a spectrum of pre-cancerous PIN and adenocarcinoma lesions and is a suitable model of early prostate cancer progression. The transgenic adenocarcinoma of the mouse prostate (TRAMP)-derived orthotopic prostate syngeneic (TOPS) model, exhibits primary tumours, as well as lung and lymph node metastases, and is a suitable model of advanced metastatic disease (Lardizabal et. al. 2018). Transgenic models maintain the immune system and stroma, generating a tumour growth environment more representative of that in humans. 3D organoid cultures, which grow faster than transgenic models, have been established from transgenic mice, human tissues, and circulating tumour cells (Huang et. al. 2016; Drost et. al. 2016; Liu et. al. 2012). However, 3D organoid models generally exhibit low proliferation and viability rates, due to the absence of nutrient supply from the vasculature, which is often counteracted with the use of growth factor-rich media preparations (Lancaster and Knoblich 2014).

Transgenic mouse models of breast cancer have been developed based on genetic engineering or induced models using a virus or ionising radiation (Sakamoto et. al. 2015; Fantozzi and Christofori 2006). However, the most extensively used pre-clinical models of breast cancer are xenograft models (Whittle et. al. 2015). Primary human tumour cells or 
tissue are implanted into mice, leading to the development of tumours, which maintain the histological, growth and metastatic features of the original tumour or tissue (Park et. al. 2018). However, xenograft models require access to fresh patient samples, exhibit low engraftment rates in less aggressive breast cancer subtypes, and are often developed in immunocompromised mice, omitting the immune system's role in tumorigenesis and making them unsuitable for testing immunotherapies (Holen et. al. 2017). Patient-derived xenograft models have also failed to recapitulate the 3D architecture observed in de novo prostate tumours (Huang et. al. 2016).

Overall, there remains a lack of available mouse models, which accurately recapitulate the progression of cancer from early disease to late-stage aggressive disease, that maintain tumour-host interactions and that are accessible to multiple research institutes (Pienta et. al. 2008; Holen et. al. 2017).

\subsection{4 in vitro models of cancer progression}

To date, there exists no single murine, or xenograft model, which replicates the progression of prostate or breast cancer from normal epithelium, to pre-cancerous lesions, to primary cancer, to advanced metastatic or drug-resistant cancer. However, there exists an extensive array of human prostate (Russell and Kingsley 2003) and breast (Dai et. al. 2017; Holliday and Speirs 2011) cancer cell lines, which are accessible, easy to grow, and enable high throughput studies. We constructed a panel of prostate cancer cell lines, which represented a progression model from normal prostate epithelium to metastatic prostate cancer, and a panel of breast cancer cell lines, which represented the complexity and heterogeneity of breast cancer molecular classifications and advanced metastatic disease states. Panels of cell lines, which collectively represent the general epithelial-mesenchymal transition (Chen et. al. 2017) of apical-basal polarised, tightly adhered, epithelial cells, to highly migratory and invasive cancer cells (Northcott et. al. 2018; Lamouille et. al. 2014), were assembled to provide a flexible in vitro system to study the role of glycosylation in the progression (Arvelo et. al. 2016) of prostate and breast cancers. 


\section{$\underline{2.2 \text { Results }}$}

Six human prostate cancer cell lines (Table 2.1a) and four human breast cancer cell lines (Table 2.1b) were selected from the literature, based on their increasing tumourigenicity and metastatic potential following orthotopic implantation into nude mice.

To validate these progression models, the characteristics of each cell line were investigated in vitro using scratch wound assays to compare migration, Boyden chambers to compare invasion and immunohistochemistry to compare the relative expression of known invasion biomarkers (Pijuan et. al. 2019; Justus et. al. 2014).

\subsubsection{Validation of in vitro panels of prostate cancer progression}

The growth properties of each prostate cancer cell line were characterised. An inverse relationship between the cell line growth rates and metastatic potential was observed (Figure 2.1). The most metastatic cell line, PC3, grew the slowest over a 72-hour period, as measured by the Incucyte's "Confluence (\%)" analysis metric (Figure 2.1a). The LNCAP cell line, the least metastatic and non-tumourigenic cell line, had the highest confluence $(83 \%)$ at 72 hours and therefore grew the fastest (Figure 2.1b). The doubling times calculated for each cell line were generally consistent with reports in the literature (Table 2.1a). As expected, the non-malignant PNT1A cell line, representing normal prostate epithelium, exhibited the slowest doubling rate, with cell numbers doubling, on average, every 45 hours (Figure 2.1c). Doubling time and confluence were strongly correlated $\left(r^{2}=0.8087\right)$ (Figure 2.1d). Therefore, migration, assessed by scratch wound assays, may be influenced by cell proliferation. 


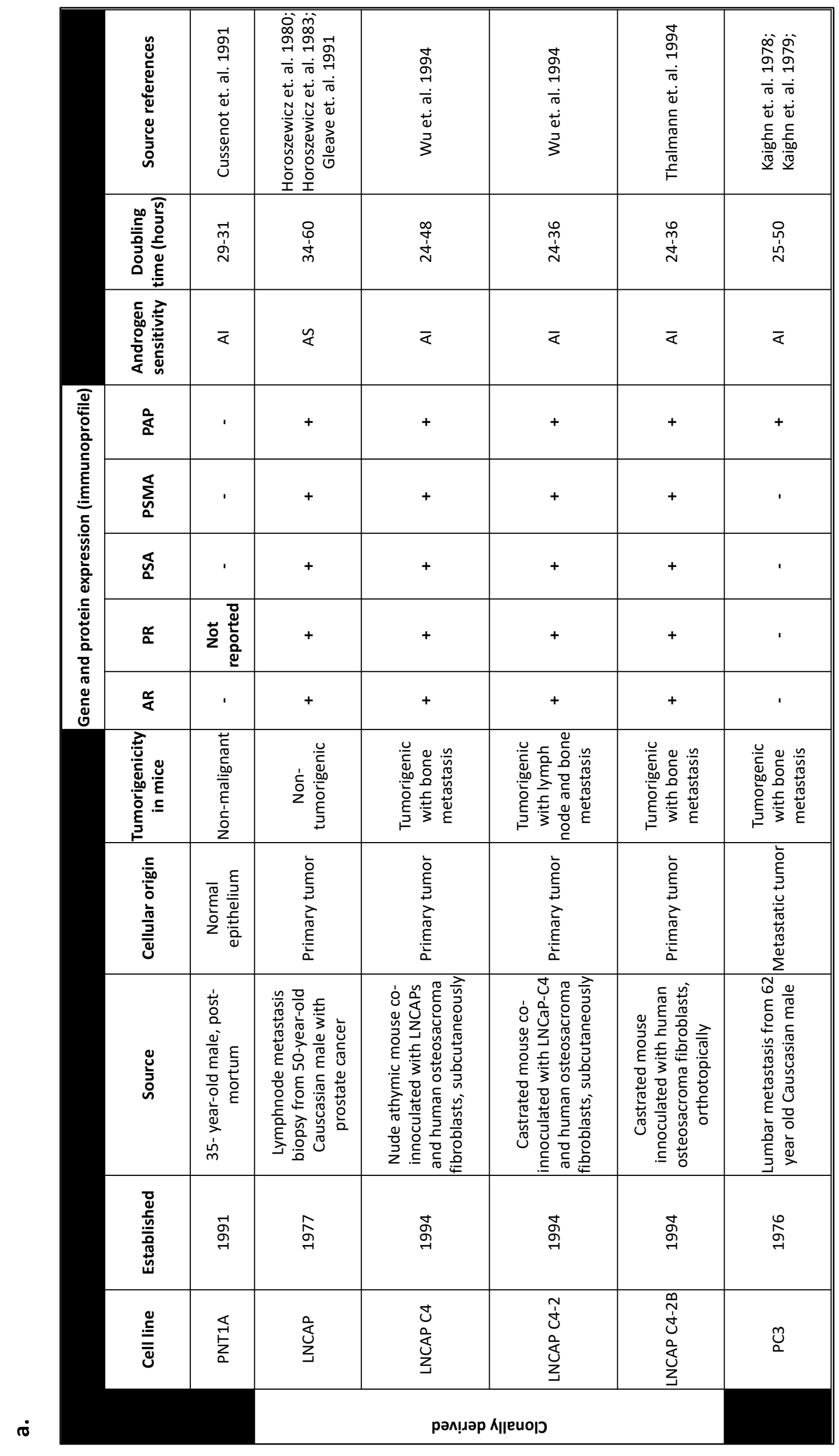




\begin{tabular}{|c|c|c|c|c|c|}
\hline & 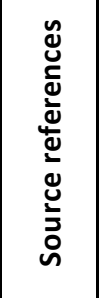 & 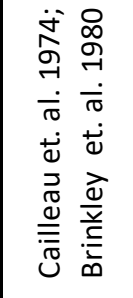 & 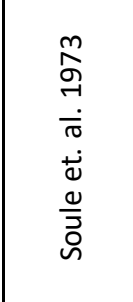 & 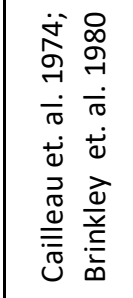 & 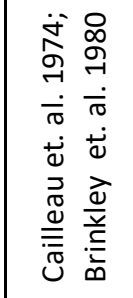 \\
\hline & 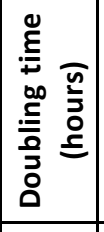 & $\begin{array}{l}\text { o } \\
\text { D } \\
\text { m. }\end{array}$ & $\begin{array}{l}\infty \\
\dot{1} \\
\stackrel{N}{N}\end{array}$ & $\begin{array}{l}\text { oे } \\
\text { ò }\end{array}$ & $\begin{array}{l}\text { ₹ } \\
\stackrel{\text { N }}{ }\end{array}$ \\
\hline \multirow{4}{*}{ 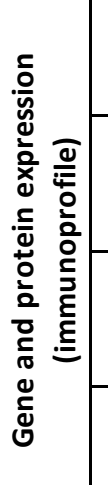 } & 岳 & ' & & + & + \\
\hline & $\begin{array}{l}\text { N } \\
\underset{w}{\underline{I}}\end{array}$ & + & & ' & \\
\hline & $\stackrel{x}{a}$ & ' & + & ' & . \\
\hline & 䍃 & ' & + & ' & ' \\
\hline & 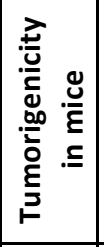 & 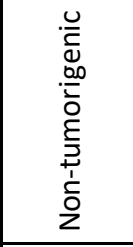 & 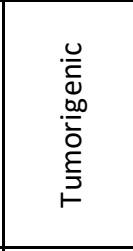 & 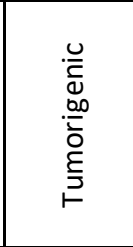 & 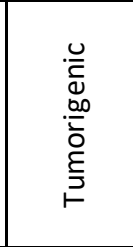 \\
\hline & $\begin{array}{l}\stackrel{0}{0} \\
\sum \\
\vdots \\
\xi \\
\xi\end{array}$ & 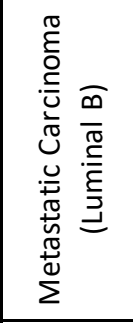 & 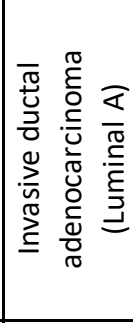 & 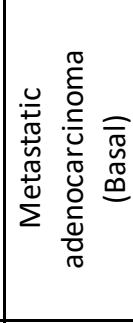 & 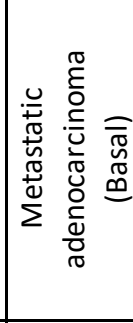 \\
\hline & 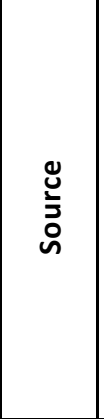 & 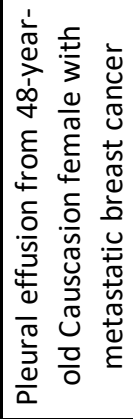 & 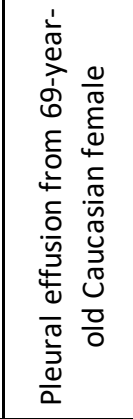 & 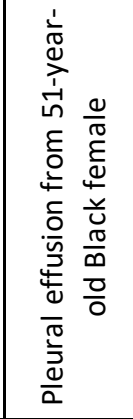 & 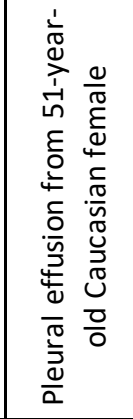 \\
\hline & 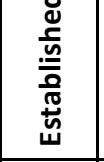 & $\begin{array}{l}\stackrel{0}{\sigma} \\
\stackrel{\sigma}{\sigma}\end{array}$ & 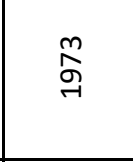 & 令 & $\underset{\stackrel{n}{\sigma}}{\stackrel{n}{\sigma}}$ \\
\hline & $\begin{array}{l}\stackrel{\mathscr{\Xi}}{\Xi} \\
\overline{\bar{\Xi}}\end{array}$ & 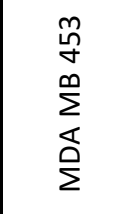 & $\frac{\vec{U}}{\Sigma}$ & 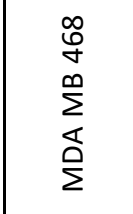 & $\begin{array}{l}\vec{N} \\
\underset{N}{\infty} \\
\sum^{\infty} \\
\stackrel{\alpha}{\Sigma}\end{array}$ \\
\hline
\end{tabular}


Table 2.1: in vitro models of prostate and breast cancer progression. (a) Summary of the characteristics of the prostate cancer cell lines used in this study (Ford et. al. 1985; van Steenbruggen et. al. 1989; Thalmann et. al. 1994; Russell et. al. 2003; Mitchell et. al. 2000; Avancès et. al. 2001; Burton et. al. 2018). (b) Summary of the characteristics of the breast cancer cell lines used in this study (Brinkley et. al. 1980; Cailleau et. al. 1974, Soule et. al. 1973, Rhodes et. al. 2002; Longueville et. al. 2005; Neve et. al. 2006; Grigoriadis et. al. 2012). Abbreviations: androgen receptor (AR), progesterone receptor (PR), prostate-specific antigen (PSA), prostate-specific membrane antigen (PSMA), prostatic acid phosphatase (PAP), androgen sensitive (AS), androgen insensitive (AI), estrogen receptor (ER), receptor tyrosineprotein kinase erbB-2 (HER 2), epidermal growth factor (EGFR). (Author diagram).

A scratch wound assay was conducted to assess the migratory properties of each prostate cancer cell line (Figure 2.2). Given the strong correlation between doubling time and confluence (Figure 2.1d), we anticipated that the slowest growing cell lines, PNT1A and PC3 (Figure 2.1a) would exhibit the lowest rates of wound closure. Interestingly, the slowest growing cell line, PC3, exhibited one of the fastest rates of wound closure over a 72-hour period (Figure 2.2a). Therefore, as expected, the most metastatic cell line, PC3, was the most migratory. Unexpectedly, the least metastatic cell lines in the panel, PNT1A and LNCAP, predicted to be the least motile, showed close to $100 \%$ wound closure at 72 -hours (Figure $2.2 b)$. Since there was no correlation between doubling time (Figure 2.2c) and relative wound density $\left(r^{2}=0.2380\right)$ (Figure $\left.2.2 d\right)$, the rapid wound closure seen with these cell lines was not caused by proliferation. This was especially true for the PNT1A cell line, which was the slowest growing cell line in the panel (Figure 2.1a, and c). The data suggested that the "normal" PNT1A cell line, was, in fact, highly migratory and thus exhibited a metastatic characteristic. The LNCAP cell line was the fastest growing cell line in the prostate cancer panel. Given the strong correlation between doubling time and confluence $\left(r^{2}=0.8087\right)$ (Figure 2.1d), its high relative wound density at 72 hours (Figure 2.2b) may have been influenced by proliferation. Generally, the data validated the panel of cell lines as a suitable in vitro model of prostate cancer progression. 
a.

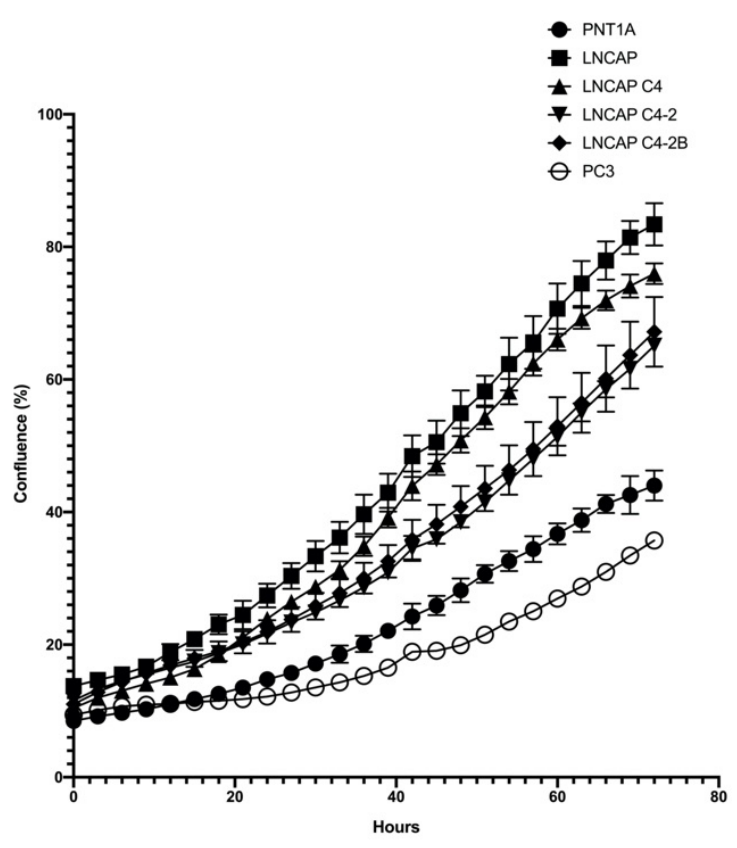

c.

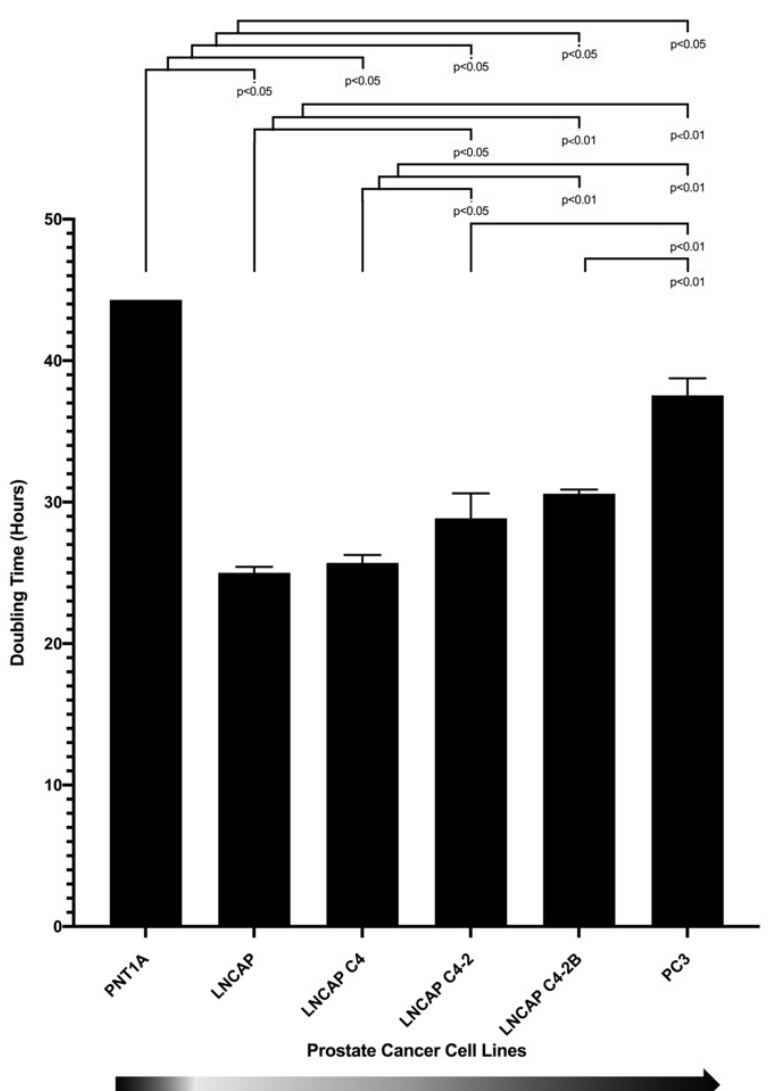

b.

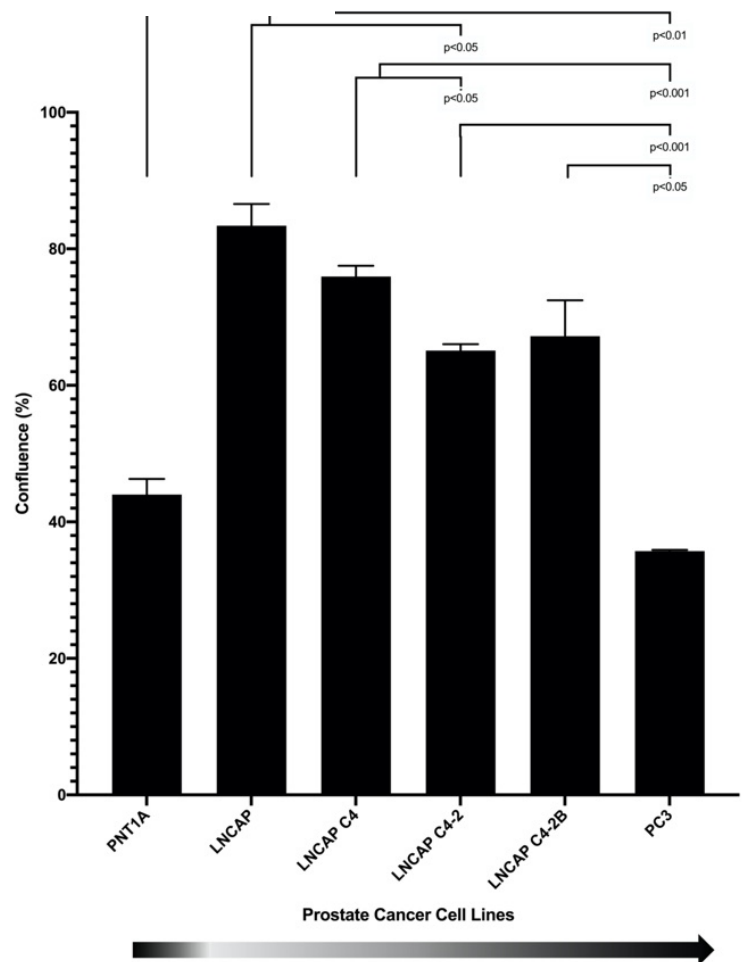

d.

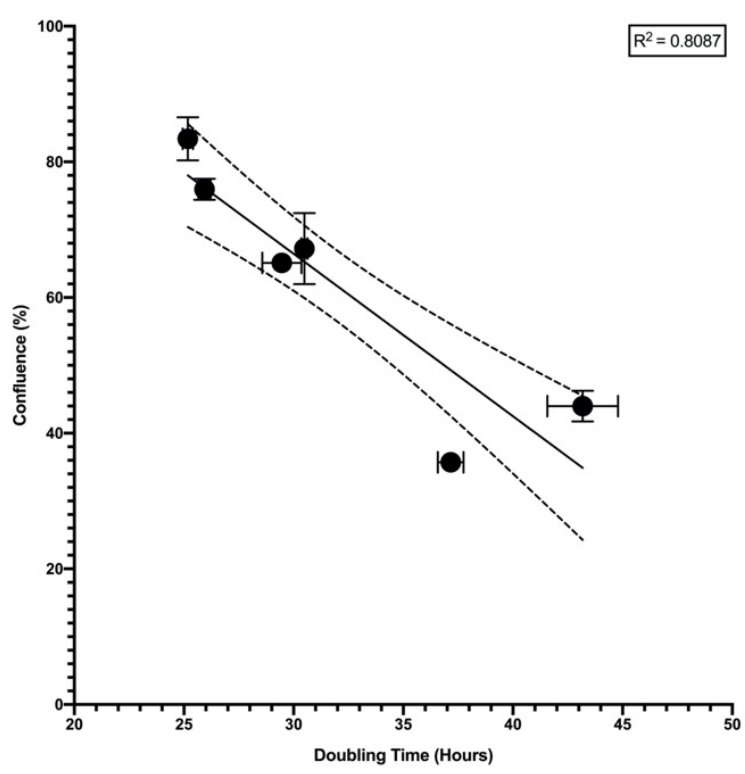


Figure 2.1: Growth analysis of a panel of prostate cancer cell lines with increasing metastatic potential. (a) Cells were imaged using the Incucyte, every 3 hours, over a 72-hour period. At each time point, the percentage of the area that was occupied by cells was calculated using the Incucyte's inbuilt "Confluence (\%)" metric. (b) Confluence (\%) at 72 hours for each prostate cancer cell line. (c) Whole cell counts were obtained every 24 hours, over a 72-hour period using the LUNA ${ }^{T M}$ Automated Cell Counter, and used to plot a growth curve. The gradient of the natural log of the regression line of each curve was used to calculate the doubling time (hours) for each prostate cancer cell line. (d) Linear regression of confluence (\%) at 72 hours as a function of doubling time. All data represent the average \pm S.D. of three independent experiments, each with 3 replicates. $p$ values represent significance by one-way ANOVA. The darker shades in the arrow's greyscale ( $b$ and $c$ ) represent an increase in the metastatic potential of the panel of prostate cancer cell lines.

The scratch wound assay does not sufficiently reflect the migration of cell lines through 3D tissue. Therefore, the invasive properties of each prostate cancer cell line were investigated using the Boyden chamber assay (Figure 2.3). Fluorescence imaging of the Boyden chambers, after a 24-hour incubation period, showed a correlation between the presence of invading cells and the metastatic potential of the cell lines (Figure 2.3a). An increase in Relative Fluorescence Units, which is directly proportional to cell numbers, correlated with an increase in the metastatic potential of the cell lines (Figure 2.3b). The high rate of invasion through the basement membrane, seen with the PNT1A cell line, confirmed the highly migratory phenotype shown in the scratch wound assay (Figure 2.2). Furthermore, high expression of vimentin, a marker of epithelial-mesenchymal transition (EMT) (Chen et. al. 2017), was observed in the highly metastatic PC3 cell line, as expected, but also in the PNT1A cell line (Figure 2.3c). Again, the data suggested that the PNT1A cell line exhibited metastatic characteristics and was not a suitable model for normal prostate epithelium. 
a.

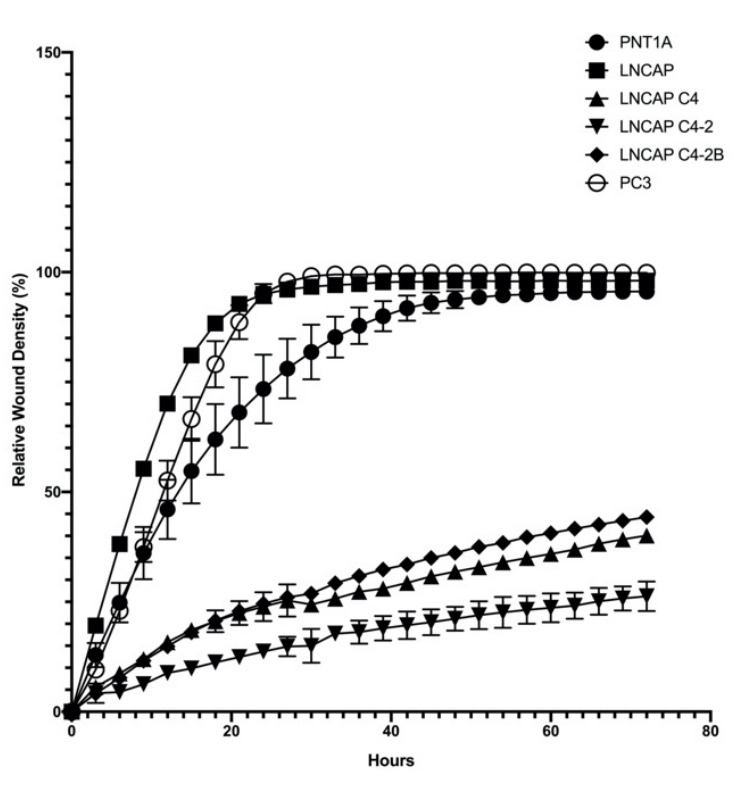

c.

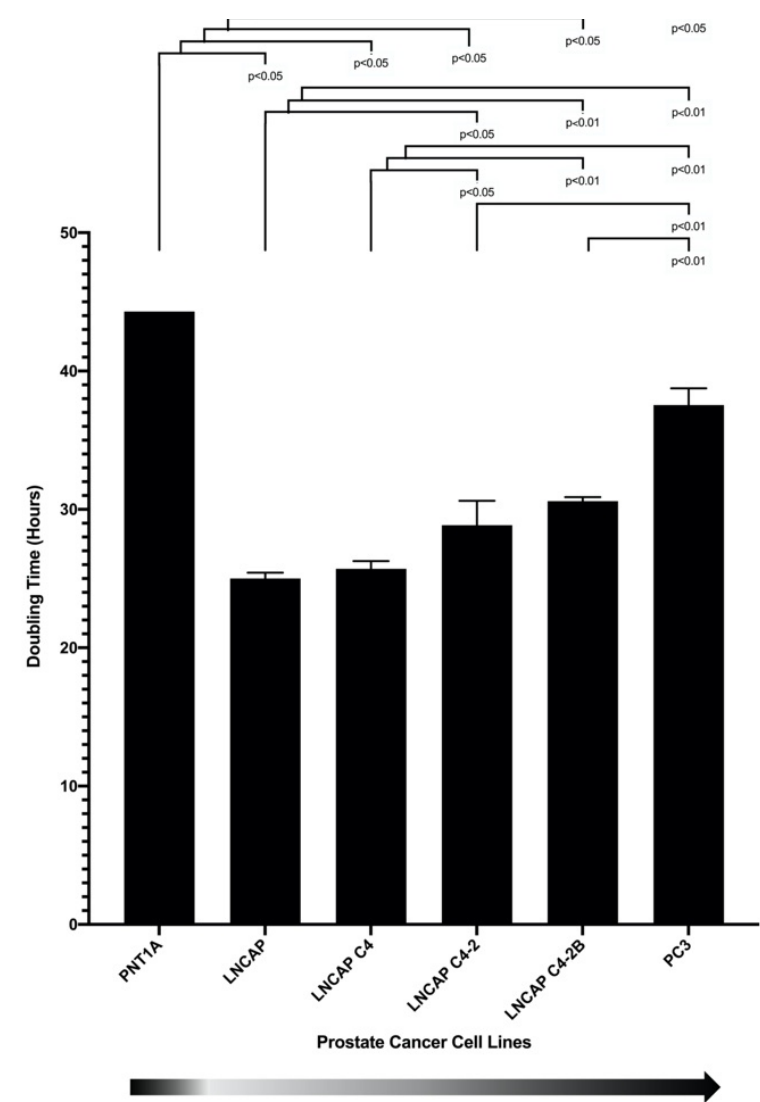

b.

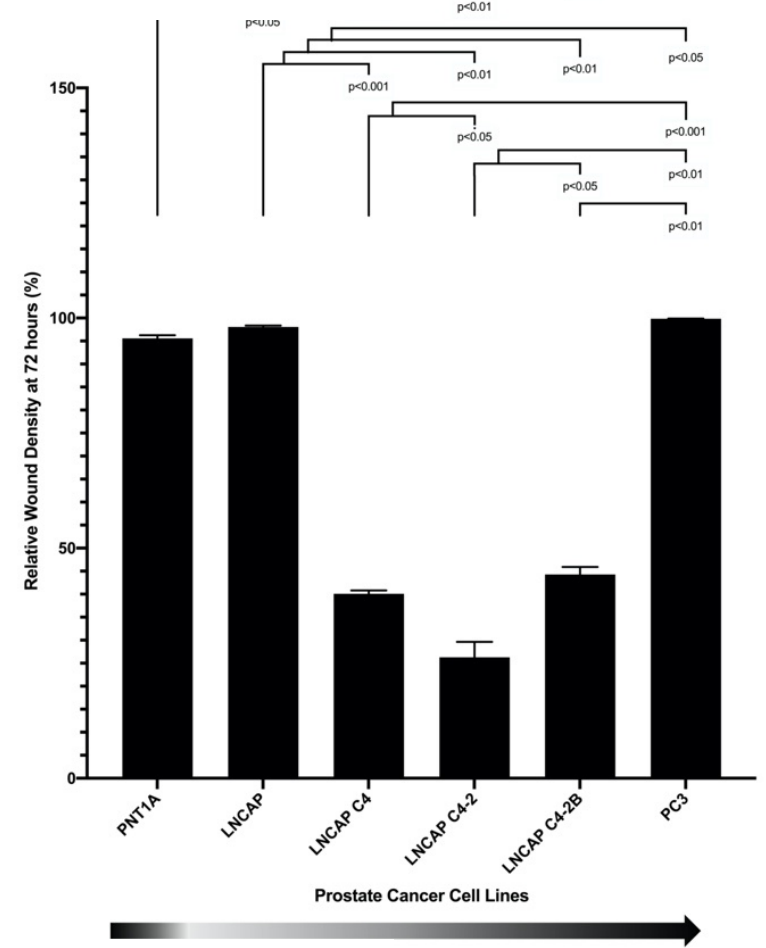

d.

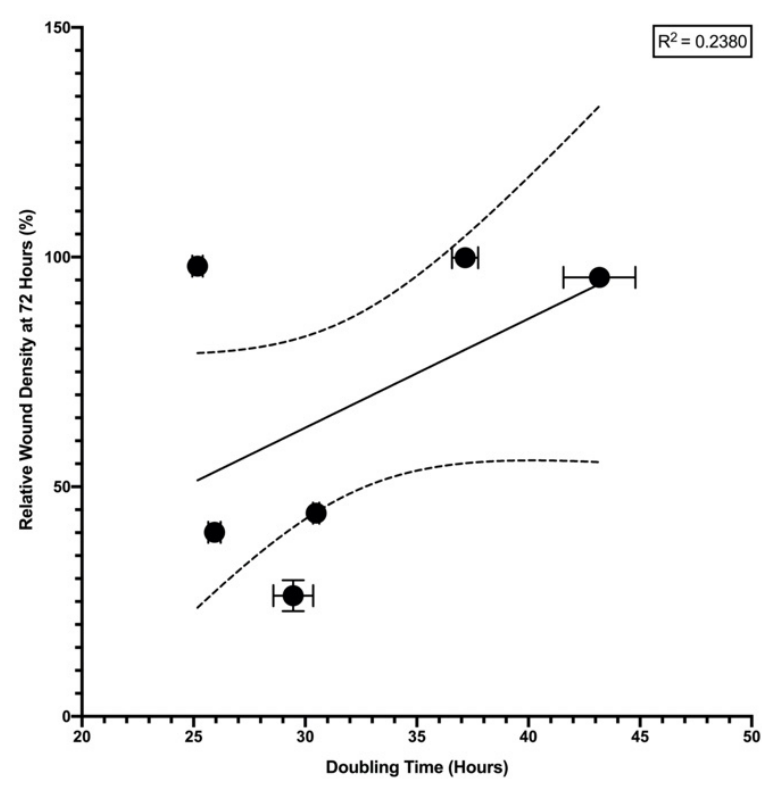

Figure 2.2: Migration analysis of a panel of prostate cancer cell lines with increasing metastatic potential. (a) Cells were grown to 95\% confluence and a scratch introduced using 
the 96-pin IncuCyte ${ }^{\circledR}$ WoundMaker (Essen Bioscience). The scratch area was imaged using the Incucyte, every 3 hours, over a 72-hour period. At each time point, the percentage cell density in the wound area relative to the cell density outside of the wound area was calculated using Incucyte's inbuilt "Relative Wound Density (\%)" metric. (b) Relative Wound Density (\%) at 72 hours for each prostate cancer cell line. (c) Whole cell counts were obtained every 24 hours, over a 72-hour period using the LUNA ${ }^{T M}$ Automated Cell Counter, and used to plot a growth curve. The gradient of the natural log regression line for each curve was used to calculate the doubling time (hours) for each prostate cancer cell line. (d) Linear regression of Relative Wound Density (\%) at 72 hours as a function of doubling time. All data represent the average \pm S.D. of three independent experiments, each with 3 replicates. $p$ values represent significance by one-way ANOVA. The darker shades in the arrow's greyscale (b and c) represent an increase in the metastatic potential of the panel of prostate cancer cell lines.

The prostate cancer cell lines, which exhibited a significant $(p<0.05,0.05$ or 0.001$)$ difference in invasion (Figure 2.3b), were analysed by immunohistochemistry to compare the relative expression of known invasion biomarkers and validate the PC3 cell line as a suitable model for metastatic prostate cancer (Figure 2.3d). The PC3 cell line stained negative for PSA (column 2) and PSMA (column 3), as documented in the literature (Table 2.1a). PSMA exists in a soluble form within normal prostate epithelial cells but during the progression of cancer, switches to an insoluble membrane-bound form (Laidler et. al. 2005), which is overexpressed in high-grade prostate cancers in comparison to benign tissues (Chang et. al. 2004). It is also negatively regulated by androgen and subsequently overexpressed in androgen-insensitive prostate cancers (Denmeade et al. 2003). Therefore, the expression of PSMA has been associated with invasive disease, despite the lack of evidence supporting a functional role in promoting prostate cancer progression. In contrast, the anti-invasive role of PSMA has been investigated. For example, PSMA-expressing cell lines (LNCAP and CWR22Rv1), had reduced invasive capacity through Matrigel, in comparison to cell lines which did not express PSMA (DU145 and PC3) (Ghosh et. al. 2005). This is consistent with the minor reductions in PSMA expression, which correlated with increases in the metastatic potential of the prostate cancer cell lines used in this study (Figure 2.3c, column 3). Furthermore, loss of PSMA expression has been observed during the switch from androgen-sensitive to androgen- 
insensitive in highly metastatic PC3 and Du 145 cell lines (Laidler et. al. 2005), consistent with our IHC data (Figure $2.3 \mathrm{c}$, column 3). The metastatic PC3 cell line exhibited the highest expression of vimentin (column 4), whose overexpression has been implicated in prostate cancer progression and metastasis (Satelli and Li 2011; Wei et. al. 2008). A reduction in the expression of E-cadherin was observed in the PC3 cell line compared to the LNCAP cell lines (column 6), consistent with E-cadherin loss associated with EMT in tumour cells (Petrova et. al. 2016). Therefore, the IHC data validated the PC3 cell line as a suitable representative of the advanced stages of prostate cancer.

Collectively, the migratory (Figure 2.2), and invasive (Figure 2.3) phenotypes of the prostate cancer cell lines validated the panel as a suitable in vitro model of prostate cancer progression.

a.

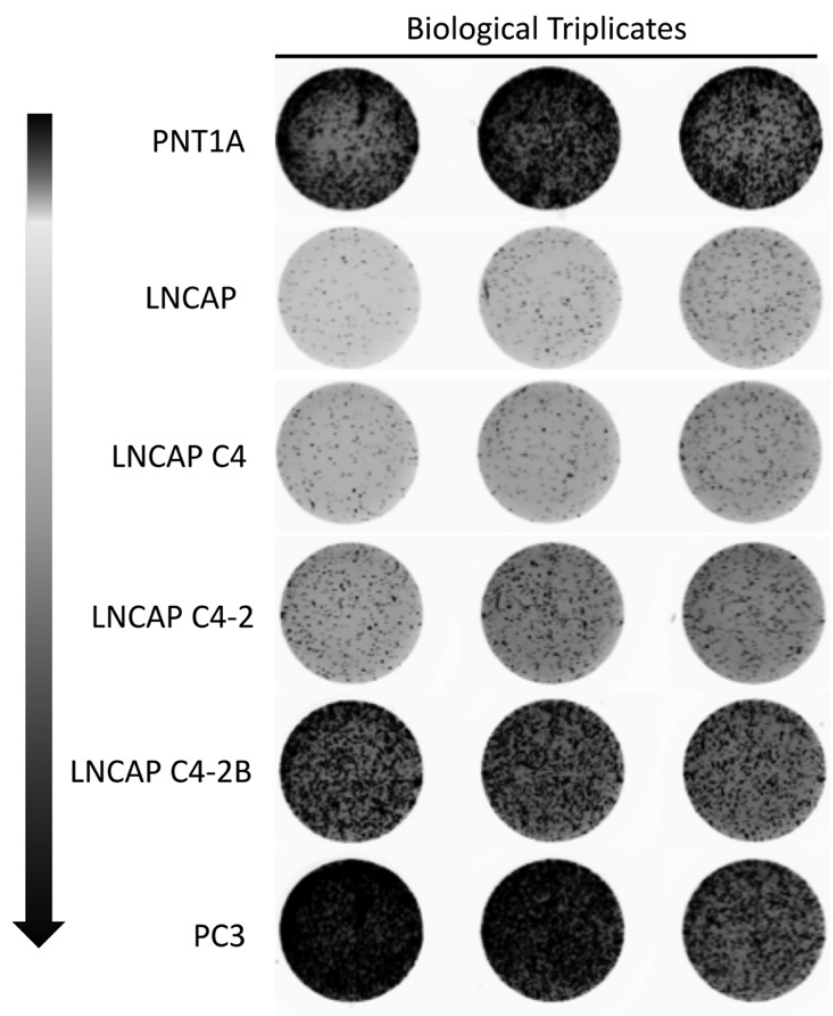

b.

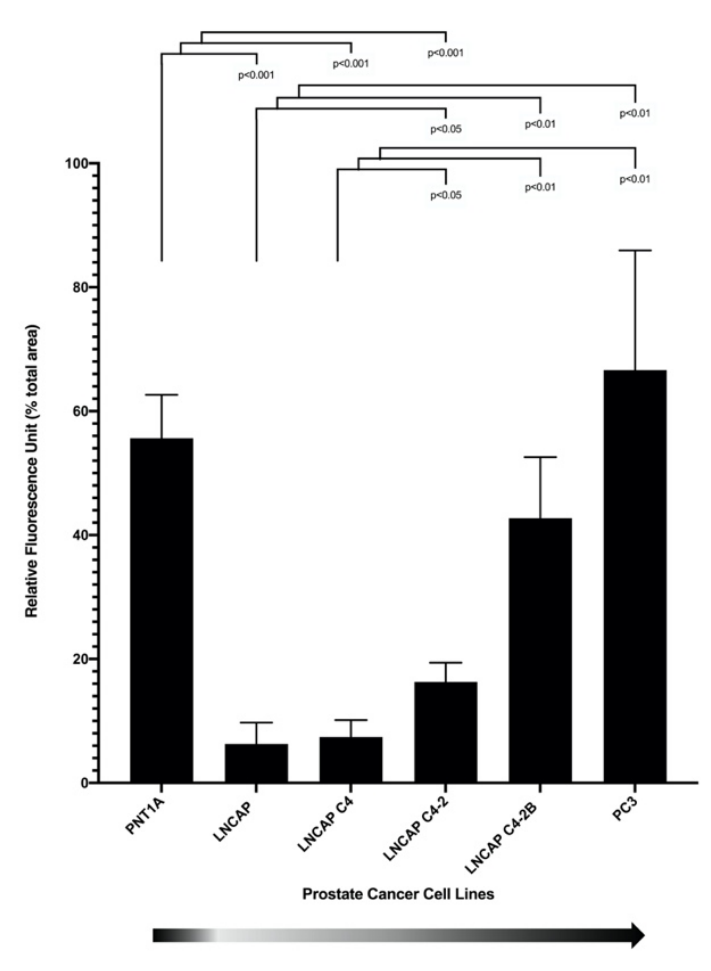


c. Whole cell lysate

\begin{tabular}{cccccc}
\hline PNT1A & LNCAP & LNCAP & LNCAP & LNCAP & PC3 \\
& & C4 & C4-2 & C4-2B &
\end{tabular}
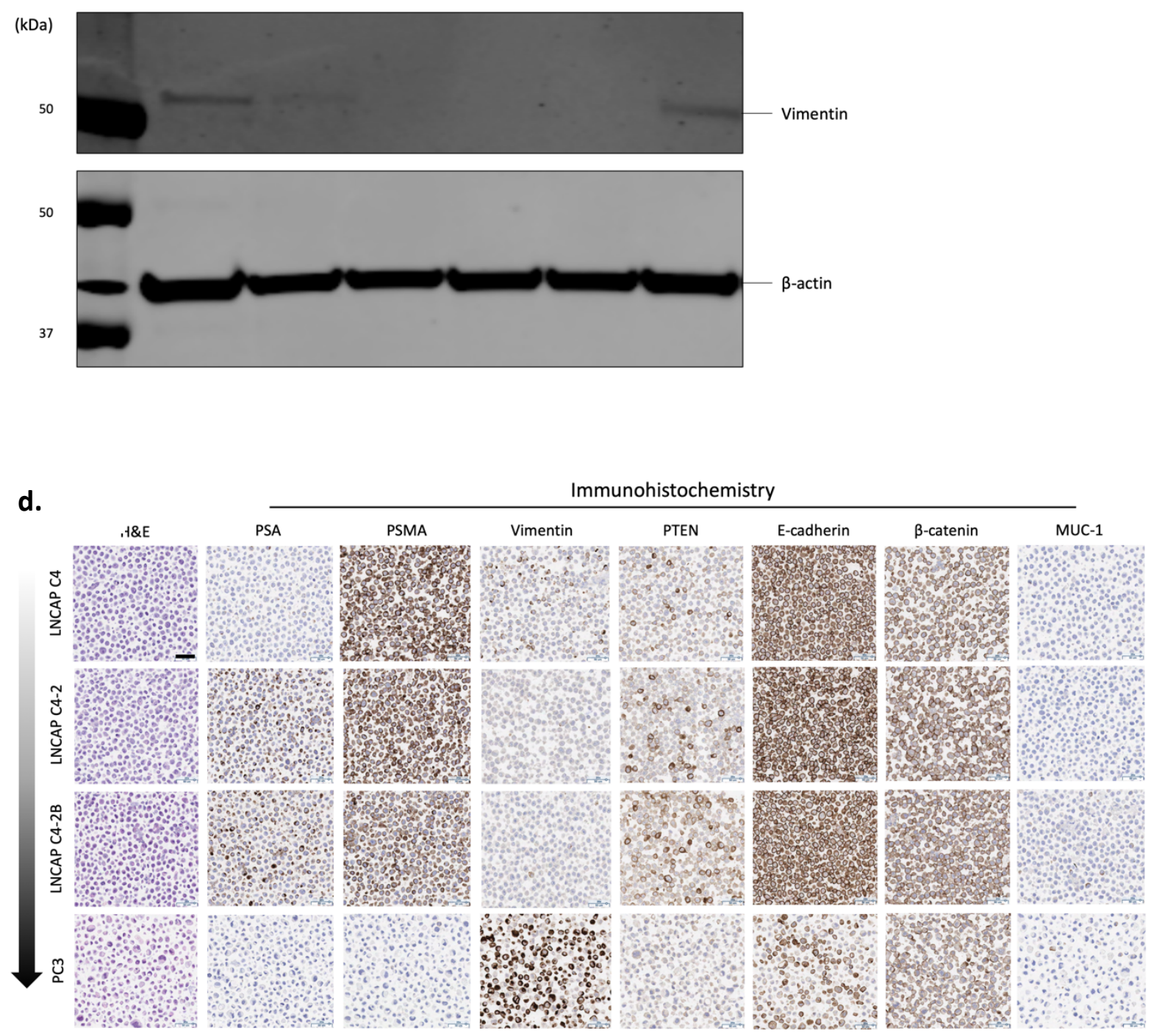

Figure 2.3: Invasion analysis of a panel of prostate cancer cell lines with increasing metastatic potential. (a) Cells were subjected to Boyden chamber analysis to measure migration across the basement membrane. Representative images of invading cells at the bottom of the polycarbonate membrane insert, 24 hours after seeding in the upper chamber, are shown. (b) Invading cells were detached from the bottom of the polycarbonate membrane insert, lysed and stained with $C y Q u a n t^{\circledR}$ GR dye. Fluorometric quantification of invading cells is represented for each prostate cancer cell line. (c) Representative western blots showing the relative abundance of vimentin in $30 \mu \mathrm{g}$ of extract from the prostate cancer cell lines. (d) Immunohistochemistry staining (in brown), was used to measure relative 
protein expression of known invasion markers: prostate-specific antigen (PSA; column 2), prostate-specific membrane antigen (PSMA; column 3), vimentin (column 4), phosphatase and tensin homolog (PTEN; column 5), E-cadherin (column 6), 6-catenin (column 7), and mucin 1 cell surface associated (MUC1; column 8). Sections were also stained with haematoxylin and eosin (H\&E, column 1). All data represent the average \pm S.D. of two independent experiments, each with 3 replicates. $p$ values represent significance by one-way ANOVA. Scale bars in histology represent 50 MM, magnification 20x. The darker shades in the arrow's greyscale represent an increase in the metastatic potential of the panel of prostate cancer cell lines.

\subsubsection{Validation of in vitro panels of breast cancer progression}

The growth properties of each breast cancer cell line were characterised. All growth rates were similar over a 72-hour period (Figure 2.4a). Only the least metastatic cell line, MDA MB 453 , and most metastatic cell line, MDA MB 231, showed significant differences $(p<0.05)$ in their degree of confluence at 72 hours (Figure 2.4b). All doubling times were similar (Figure 2.4c), and did not correlate with degree of confluence $\left(r^{2}=0.01787\right)$ (Figure 2.4d). Therefore, migration of the breast cancer cell lines, assessed by scratch wound assays, should not be influenced by cell proliferation, allowing assessment of real migratory phenotypes.

As expected, the least metastatic cell line, MDA MB 453, exhibited the slowest wound closure over a 72-hour period (Figure 2.5a). The most metastatic cell lines in the panel, MDA MB 468 and MDA MB 231, showed the highest relative wound density at 72 hours (Figure 2.5b). As there was no correlation between doubling time (Figure 2.5c) and relative wound density $\left(r^{2}=0.01199\right)$ (Figure $\left.2.5 d\right)$, the general increase in relative wound density at 72 hours (Figure 2.5b) was caused by an increase in the motility of the cell lines. This correlated with an increase in the metastatic potential of the breast cancer cell lines. 
a.

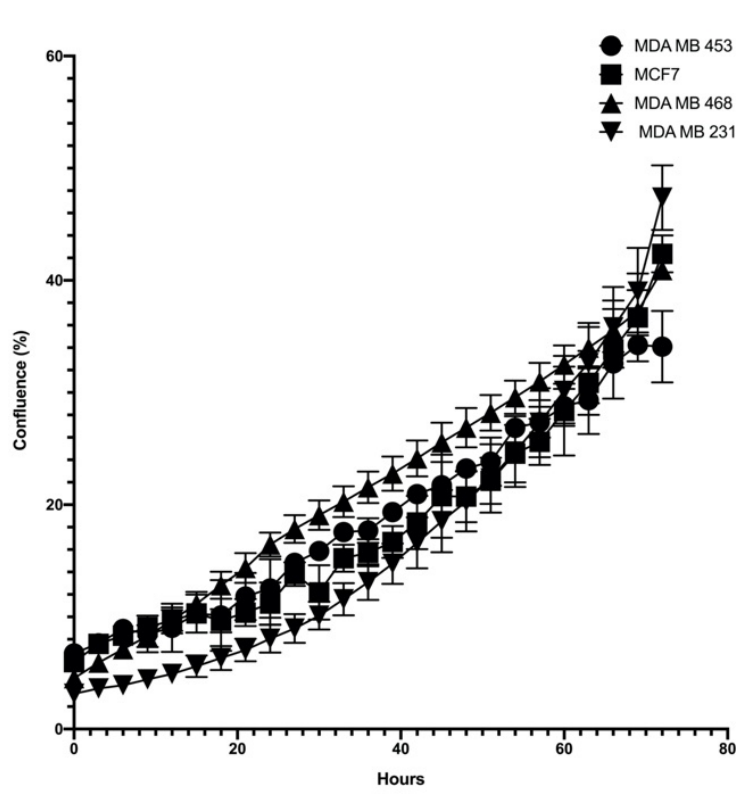

c.

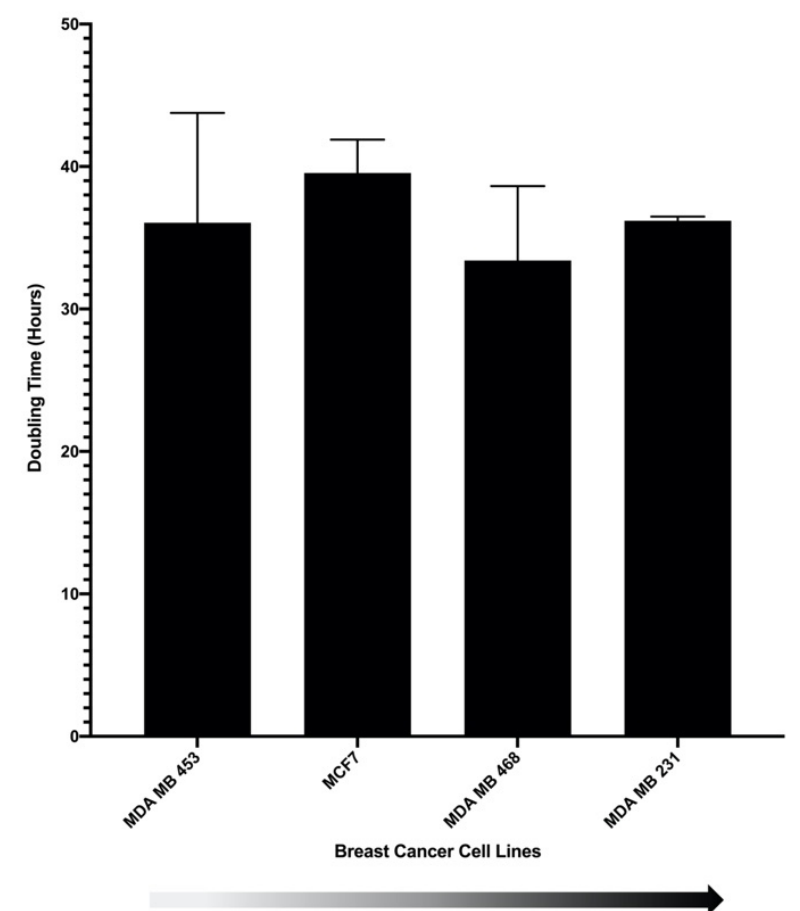

b.

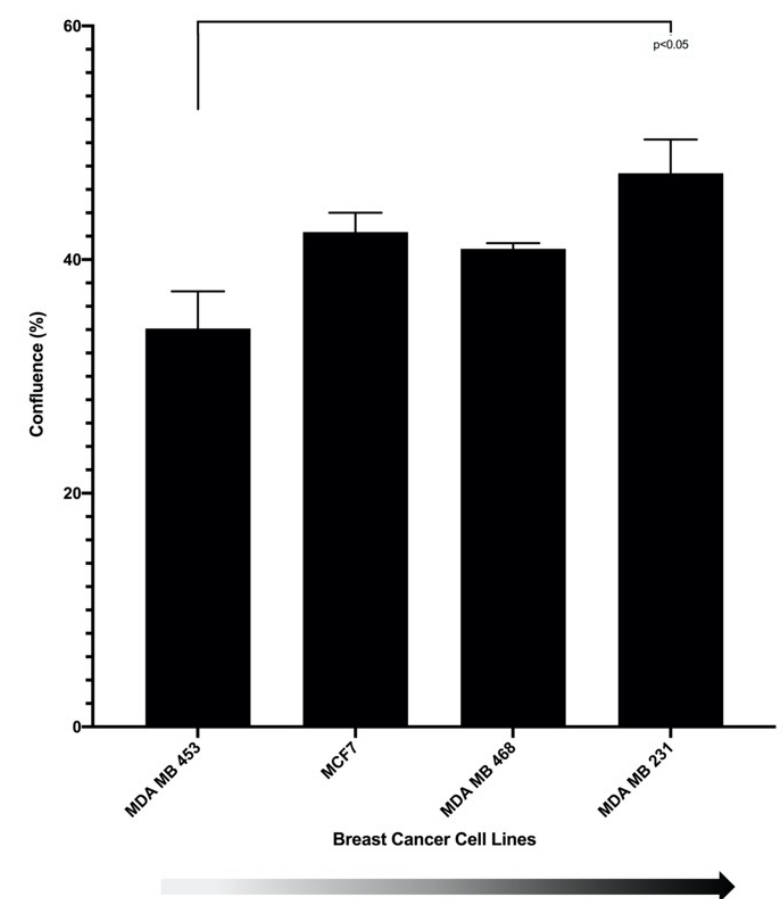

d.

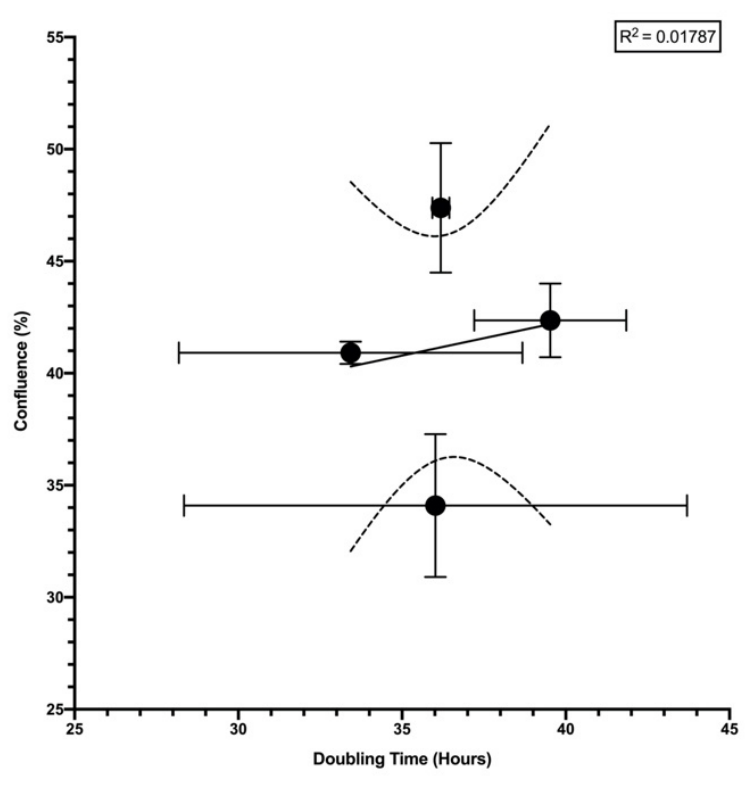

Figure 2.4: Growth analysis of a panel of breast cancer cell lines with increasing metastatic potential. (a) Cells were imaged using Incucyte, every 3 hours, over a 72-hour period. At each time point, the percentage of the image area occupied by cells was calculated using the Incucyte's inbuilt "Confluence (\%)" metric. (b) Confluence (\%) at 72 hours for breast cancer cell line. (c) Whole cell counts were obtained every 24 hours, over a 72-hour period, using the 
LUNA ${ }^{T M}$ Automated Cell Counter, and used to plot a growth curve. The gradient of the natural log regression line of each curve was used to calculate the doubling time (hours) for each breast cancer cell line. (d) Linear regression of the degree of confluence (\%) at 72 hours as a function of doubling time. All data represent the average \pm S.D. of three independent experiments, each with 3 replicates. $p$ values represent significance by one-way ANOVA. The darker shades in the arrow's greyscale ( $b$ and $c$ ) represent an increase in the metastatic potential of the panel of breast cancer cell lines.

The invasive properties of each breast cancer cell line were investigated using the Boyden chamber assay (Figure 2.6). There was a correlation between the presence of invading breast cancer cells and the metastatic potential of the cell lines (Figure 2.6a). An increase in Relative Fluorescence Units, also correlated with an increase in the metastatic potential of the cell lines (Figure 2.6b). The breast cancer cell lines were analysed by immunohistochemistry to compare the relative expression of known invasion biomarkers (Figure 2.6c). The MCF 7 cell line was the only cell line to stain positive (in brown) for estrogen receptor (ER; column 2) and progesterone receptor (PR; column 3), consistent with the immunoprofile documented in the literature (Table 2.1b). A decrease in the expression of PTEN (column 5) correlated with an increase in the metastatic potential of the cell lines. However, the literature provides conflicting information about the inactivation, reduced expression and loss of PTEN during the progression of breast cancer, with reduced PTEN expression being observed in anywhere between 4 to $63 \%$ of breast cancers ( $L i$ et. al. 2017; Álvarez-Garcia et. al. 2019). A clear increase in expression of E-cadherin (column 6) correlated with an increase in the metastatic potential of the cell lines, contrary to Ecadherin loss frequently associated with EMT in tumour cells (Petrova et. al. 2016). 
a.

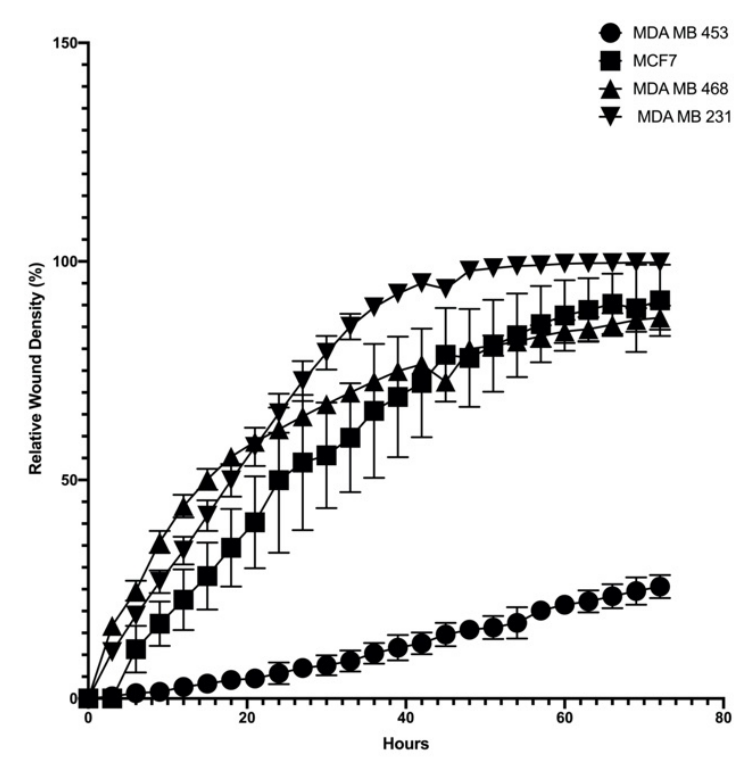

C.

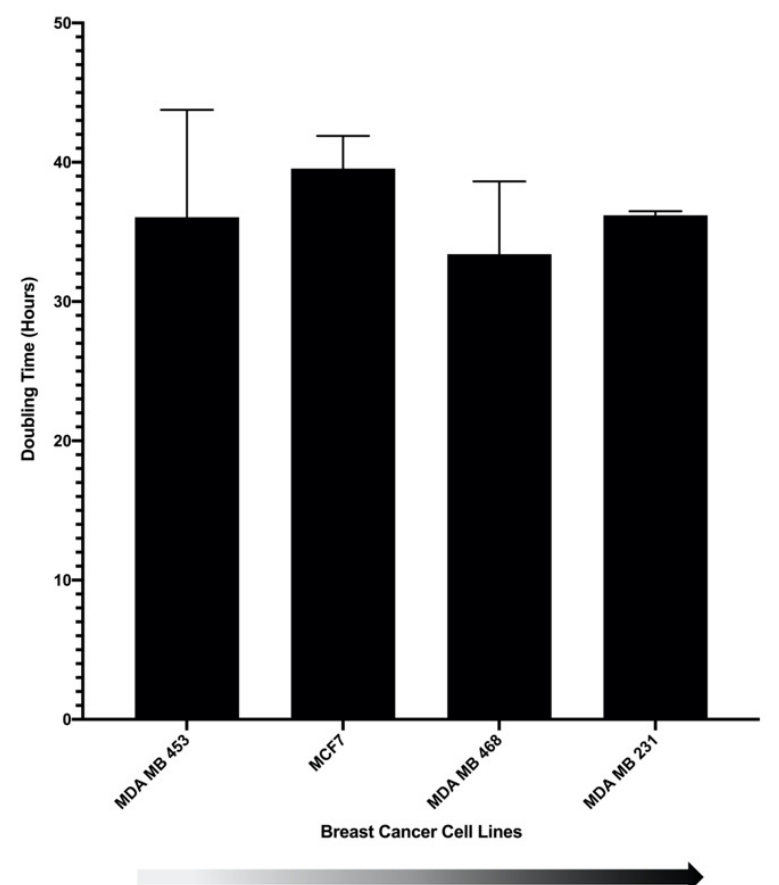

b.

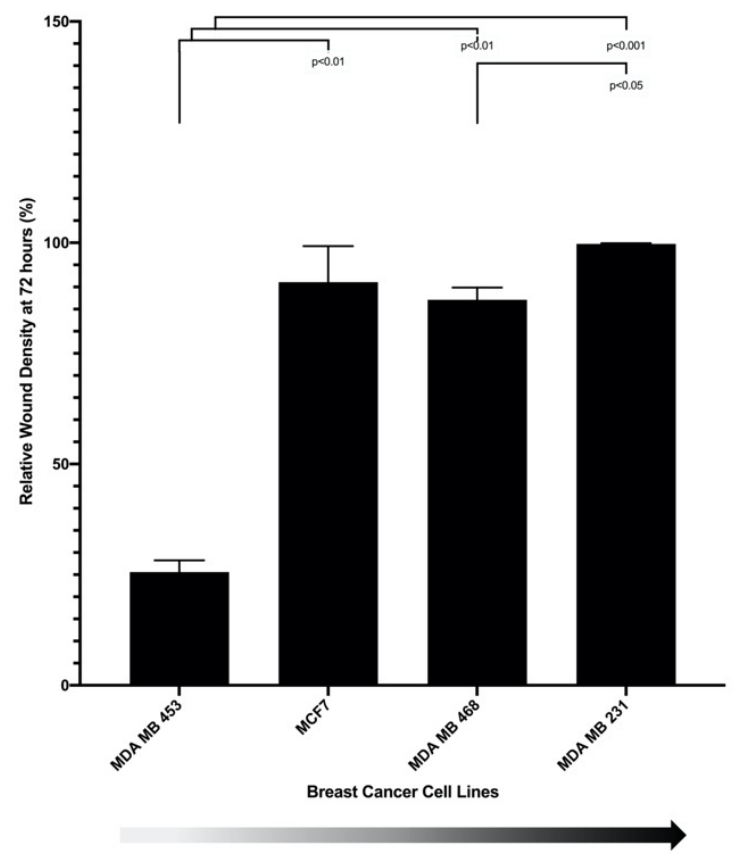

d.

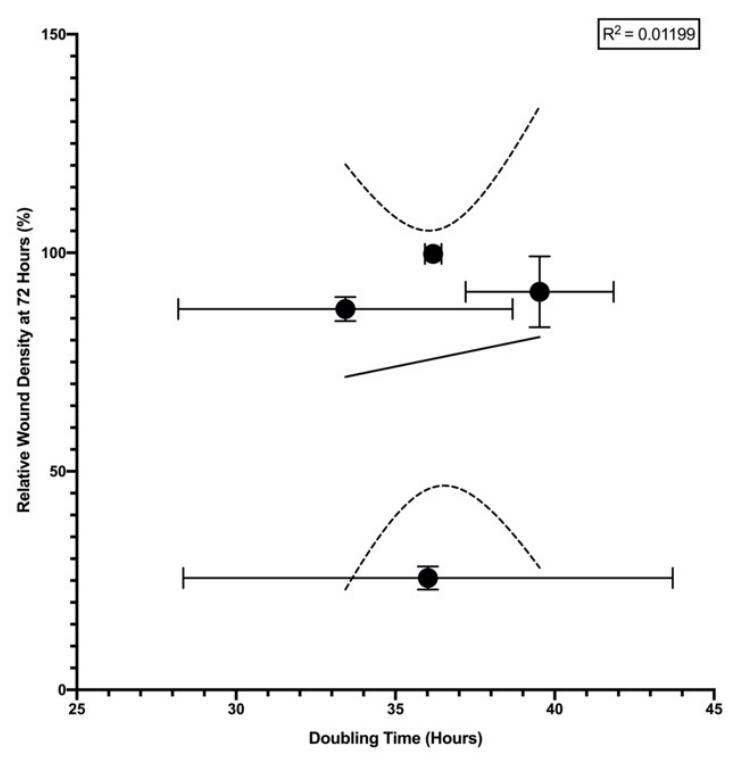

Figure 2.5: Migration analysis of a panel of breast cancer cell lines with increasing

metastatic potential. (a) Cells were grown to 95\% confluence and a scratch introduced using the 96-pin IncuCyte ${ }^{\circledR}$ WoundMaker (Essen Bioscience). The scratch area was imaged using Incucyte, every 3 hours, over a 72-hour period. At each time point, the percentage cell density in the wound area relative to the cell density outside of the wound area was calculated using 
Incucyte's inbuilt "Relative Wound Density (\%)" metric. (b) Relative Wound Density (\%) at 72 hours for each breast cancer cell line. (c) Whole cell counts were obtained every 24 hours, over a 72-hour period, using the LUNA ${ }^{T M}$ Automated Cell Counter, and used to plot a growth curve. The gradient of the natural log regression line of each curve was used to calculate the doubling time (hours) for each breast cancer cell line. (d) Linear regression of Relative Wound Density (\%) at 72 hours as a function of doubling time. All data represent the average \pm S.D. of three independent experiments, each with 3 replicates. $p$ values represent significance by one-way ANOVA. The darker shades in the arrow's greyscale ( $b$ and $c$ ) represent an increase in the metastatic potential of the panel of breast cancer cell lines.

Collectively, the migratory (Figure 2.5), and invasive (Figure 2.6) phenotypes of the breast cancer cell lines validated the panel as a suitable in vitro model of breast cancer progression.

a.

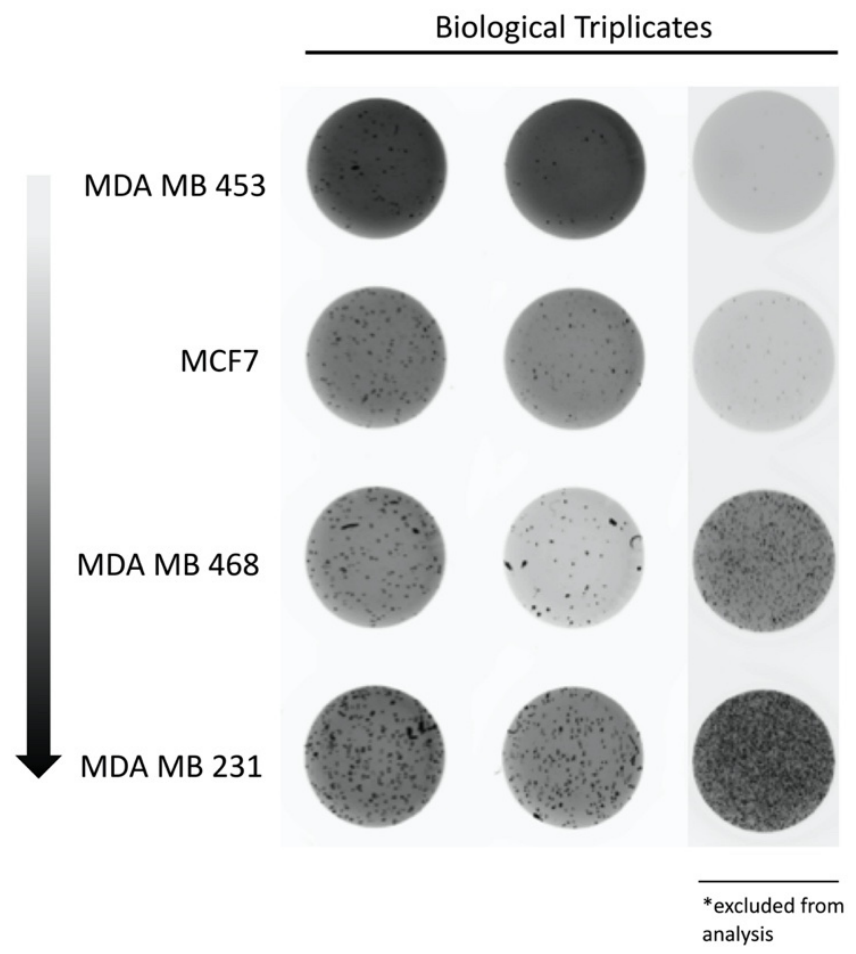

b.

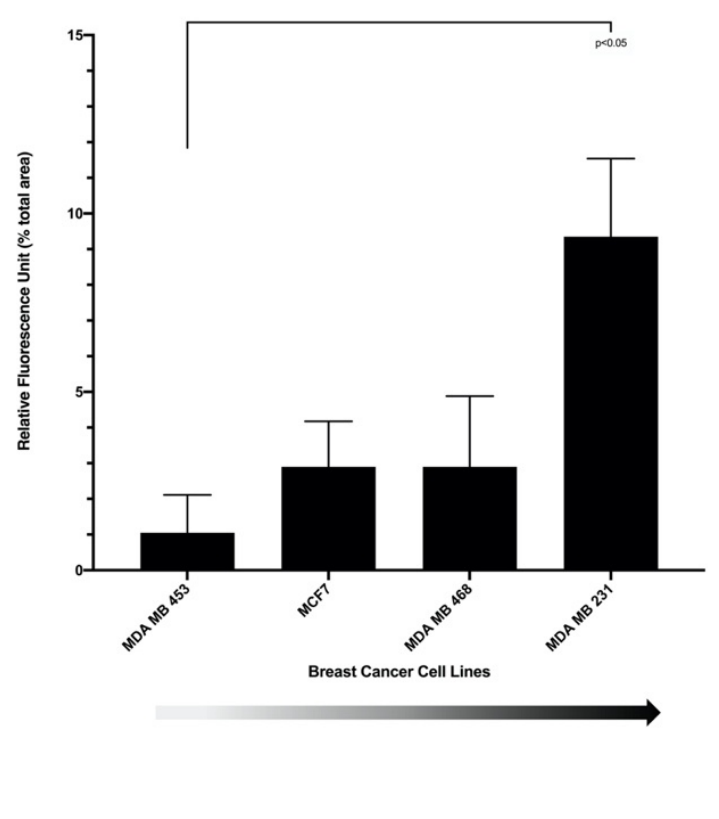


c. Immunohistochemistry

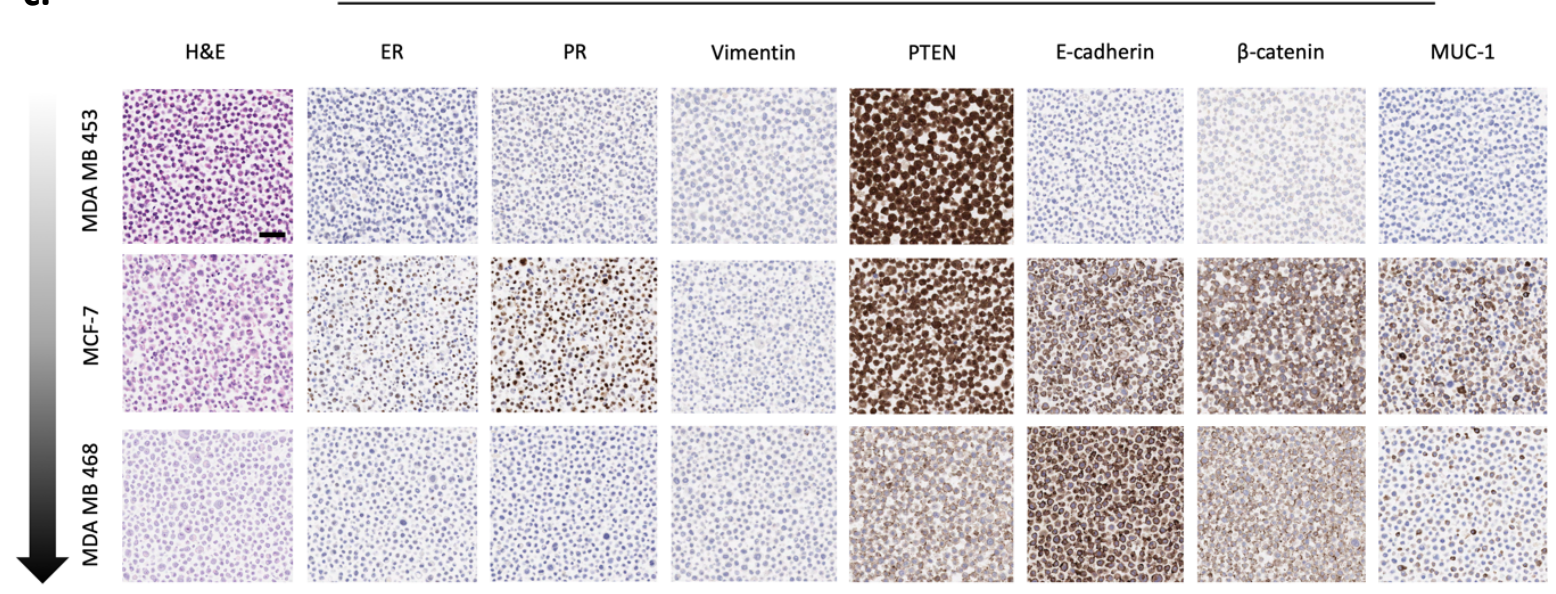

Figure 2.6: Invasion analysis of a panel of breast cancer cell lines with increasing metastatic potential. (a) Cells were subjected to Boyden chamber analysis to measure migration across the basement membrane. Representative images of invading cells at the bottom of the polycarbonate membrane insert, 24 hours after seeding in the upper chamber, are shown. (b) Invading cells were detached from the bottom of the polycarbonate membrane insert, lysed and stained with CyQuant ${ }^{\circledR}$ GR dye. Fluorometric quantification of invading cells is shown for each breast cancer cell line. (c) Immunohistochemistry staining (brown), was used to measure relative protein expression of known invasion markers: estrogen receptor (ER; column 2), progesterone receptor (PR; column 3), vimentin (column 4), phosphatase and tensin homolog (PTEN; column 5), E-cadherin (column 6), 6-catenin (column 7), and mucin 1 cell surface associated (MUC-1; column 8). Sections were also stained with haematoxylin and eosin (H\&E; column 1). All data represent the average \pm S.D. of three independent experiments, each with 3 replicates. $p$ values represent significance by one-way ANOVA. Scale bars in histology represent $50 \mu M$, magnification 20x. The darker shades in the arrow's greyscale ( $b$ and $c$ ) represent an increase in the metastatic potential of the panel of breast cancer cell lines. 


\subsection{Discussion}

There remains a lack of available preclinical models, which accurately recapitulate the progression of cancer from early disease to the late-stage aggressive disease observed in patients (Pienta et. al. 2008; Holen et. al. 2017). However, there exists an extensive array of human prostate (Russell and Kingsley 2003) and breast (Dai et. al. 2017; Holliday and Speirs 2011) cancer cell lines, derived from different disease states, which are easy to culture for high throughput studies. In this study, we constructed a panel of prostate (Table 2.1a) and breast cancer cell lines (Table 2.1b), which represented a progression model from normal epithelium to metastatic cancer, based on their cellular origin's and their characteristics when orthotopically implanted into mice in vivo.

During the metastatic process, tumour cells with enhanced plasticity migrate from the primary tumour, and express de novo glycans such as Sialyl Lewis ${ }^{\mathrm{X}}$ and Sialyl Lewis ${ }^{\mathrm{A}}$ on mucins and glycosphingolipids. These interact with glycan-binding selectins present on endothelial cells, activated platelets and leukocytes, and secreted extracellular matrix proteins, facilitating adherence and transversion through the vasculature, and promoting tumour dissemination via the bloodstream, and formation of emboli at distant endothelial sites (Bogenrieder and Herly 2003; Fuster and Esko 2005). Therefore, we expected cell lines derived from metastases to be highly migratory, complemented by reduced expression of cell-adhesion promoting proteins such as E-cadherin (Petrova et. al. 2016), and highly invasive, confirmed by high expression of proteins associated with EMT, such as vimentin (Chen et. al. 2017). The tumourigenic PC3 prostate cancer cell line, derived from a lumbar metastasis, exhibited one of the fastest rates of wound closure over a 72-hour period (Figure 2.2a and $b$ ), the highest number of invasive cells traversing the basement membrane (Figure 2.3a and b), the highest expression of vimentin (Figure 2.3d, column 4), and the lowest expression of E-cadherin (Figure 2.3d, column 6), consistent with molecular characteristics commonly observed during the metastatic process. The non-tumourigenic LNCAP cell line was the least invasive cell line in the prostate cancer panel (Figure 2.3a and b). Therefore, the LNCAP and PC3 cell lines represented less aggressive and aggressive disease, respectively. 
A unique characteristic of prostate cancer progression observed in humans is the switch from androgen dependence to androgen independence (Dutt and Gao 2009), which has detrimental effects on treatment interventions due to the accompanied resistance to castration and androgen deprivation therapies (Taichman et. al. 2007). Our panel of prostate cancer cell lines collectively represent a progression from androgen sensitivity in the AR expressing LNCAP cell line, to androgen insensitivity in the LNCAP C4, LNCAP C4-2 and LNCAP C4-2B cell lines, to an eventual loss of AR expression and androgen insensitivity in the PC3 cell lines (Table 2.1a). However, the highly migratory and invasive PC3 cell line, although of metastatic origin, is negative for PSA (Table 2.1a; Figure 2.3, column 2), and is not representative of the high expression of PSA observed in patients with aggressive prostate cancer. Several studies have reported loss of AR expression between the LNCAP and PC3 cell lines, and the absence of PSA expression in a PC3 cell line (Mitchell et. al. 2000; Tai et. al. 2011), which are consistent with our IHC data, and the immunoprofiles reported in the literature (Table 2.1a). Therefore, the PC3 cell line is limited in its ability to model the key clinical characteristics of prostate cancer progression observed in patients. This is the benefit of conducting studies in panels of multiple cell lines, rather than in a single cell line.

The MDA MB 453, MCF 7, MDA MB 468 and MDA MB 231 breast cancer cell lines, selected from the literature based on their increasing tumourigenicity, exhibited an increase in relative wound density at 72 hours (Figure $2.5 \mathrm{~b}$ ), which correlated with an increase in the metastatic potential of these breast cancer cell lines, which was illustrated by the increasing presence of invasive cells traversing the basement membrane during Boyden chamber analysis (Figure 2.6a). PTEN, a tumour suppressor protein, is frequently lost in cancers, causing unregulated activation of the phosphatidylinositol 3 kinase (PI3K)/protein kinase $B$ (AKT) pathway and its downstream effector functions such as cell cycle progression and stimulation of angiogenesis (Milella et. al. 2015). Consistent with these reports, a decrease in the expression of PTEN correlated with an increase in the metastatic potential of the breast cancer cell lines used in this study (Figure 2.6c, column 5). However, the literature provides conflicting information about the inactivation, reduced expression and loss of PTEN during the progression of breast cancer, with reduced PTEN expression being observed in anywhere between 4 to $63 \%$ of breast cancers (Li et. al. 2017; Álvarez-Garcia et. al. 2019). A clear 
increase in the expression of E-cadherin (Figure 2.6c, column 6) correlated with an increase in the metastatic potential of the breast cancer cell lines and was contrary to loss of Ecadherin frequently associated with EMT in tumour cells and aggressive disease (Petrova et. al. 2016). E-cadherin loss has been strongly correlated with invasive lobular breast carcinoma (Horne et. al. 2018; Singhai et. al. 2011), and proposed as a prognostic marker for this breast cancer subtype. However, few studies have correlated E-cadherin expression in other breast cancer histological subtypes. Furthermore, re-expression of E-cadherin has been reported in breast cancer metastases and overexpression plays an oncogenic role in the MDA MB 468 cell line (Hugo et. al. 2017), consistent with increases in E-cadherin expression observed across our panel of breast cancer cell lines.

The panel of breast cancer cell lines used in this study collectively represent the heterogeneous nature of breast cancer molecular subtypes (Dai et. al. 2015) such as luminal A (MCF 7), luminal B (MDA MB 453), receptor tyrosine kinase erbB-2 (HER2) positive (MDA MB 453), and triple-negative or basal-like (MDA MB 468 and MDA MB 231) (Dai et. al. 2015) (Table 2.1b). However, their varied cellular origins do not accurately represent the clonal evolution of cancer, which begins in a single cell type (Greaves et. al. 2012). In this respect, the metastatic end of the breast cancer panel could be improved based on lentivirus barcoding techniques used by Wagenblast and colleagues to analyse drivers of breast cancer metastasis (Wagenblast et. al. 2015). In this study, 4T1 cell lines were infected with lentivirus barcode libraries. Individual clones were isolated using FACS, and implanted into mammary fat pads, followed by the formation of tumours and metastases. Samples from the tumours and metastases to the brain, lung, lymph nodes, liver, and blood were extracted and sequenced. Clones of $4 \mathrm{~T} 1$ cells responsible for metastases to specific regions of the body could then be traced back to the original cell lines cultured in vitro based on their unique lentivirus barcodes. These cell lines may be available at the $\mathrm{CRUK} \mathrm{CI}$ (Hannon group) and would provide a panel of metastatic breast cancer cell lines derived from the same primary tumour.

Collectively, the migratory and invasive phenotypes of the cell lines, were consistent with molecular characteristics commonly observed during the metastatic process, validating the 
panels as suitable in vitro models of prostate (Table 2.1a) and breast (Table 2.1b) cancer progression.

The panels of cell lines used in this study were limited by the availability of cell lines representing normal prostate or breast tissue epithelium. The PNT1A cell line is the most extensively used model of normal prostate cells. However, despite being the slowest growing cell line in the panel (Figure 2.1a, and c), the PNT1A cell line was highly motile in scratch wound closure analysis (Figure 2.2) and invasive in Boyden chamber analysis (Figure 2.3a-c), and subsequently deemed an unsuitable model for normal prostate epithelium.

The Hb4a cell line (Stamps et. al. 1994), derived from normal breast tissue, exhibits highly rearranged chromosomes, which limited its use as a model for normal breast epithelial cells (Davidson et. al. 2000). The MCF10A cell line, derived from non-malignant breast tissue (Chavez et. al. 2010), has been shown not to stain for markers usually expressed in normal breast tissue, such as $\beta$-casein and $\alpha$-lactalbumin, and also expresses stem-like, luminal and basal cell markers when cultured in 2D or 3D culture (Qu et. al. 2015). Due to its instability under different culturing conditions, its utility as a model of normal breast epithelium remains unclear. Abnormal changes in morphology have been observed when adapting normal primary Human Mammary Epithelial Cells (HMECs) to HuMEC (Invitrogen ${ }^{\text {TM}}$ ) or MEGM (LONZA) media (Kuo 2015, PhD thesis, unpublished). There remains a need to find a common media preparation that will allow the growth of "normal" breast cell lines.

In this study, we validate a flexible in vitro system to study the role of glycosylation in prostate and breast cancer progression (Arvelo et. al. 2016). 


\subsection{Materials and Methods}

\subsubsection{Cell lines}

The PNT1A, LNCAP, and LNCAP C4-2B human prostate cell lines were obtained from the Cancer Research UK Cambridge Institute Biorepository, based on stocks made available by Joe Kuo. The LNCAP C4, LNCAP C4-2, and PC3 human prostate cancer cell lines were purchased from ATCC. All prostate cell lines were cultured in RPMI with L-glutamine (Gibco ${ }^{\circledR}$, Invitrogen ${ }^{\mathrm{TM}}$ ) and 10\% Foetal bovine serum (FBS Gold, PAA Laboratories).

The MDA MB 453, MCF 7, MDA-MB-468, and MDA MB 231 human breast cancer cell lines were obtained from the Cancer Research UK Cambridge Institute Biorepository, based on stocks made available by Joe Kuo. All breast cell lines were cultured in DMEM with Lglutamine (Gibco ${ }^{\circledR}$, Invitrogen ${ }^{\mathrm{TM}}$ ) and 10\% Foetal bovine serum (FBS Gold, PAA Laboratories).

Cell lines were genotyped and authenticated by Short Tandem Repeat (STR) DNA profiling and comparison with available databases from ATCC or DSMZ. All cell lines tested negative for Mycoplasma using the MycoProbe ${ }^{\mathrm{TM}}$ Kit (R\&D Systems).

\subsubsection{Tissue culture}

Cell lines were maintained in Nunc ${ }^{\mathrm{TM}} 175 \mathrm{~cm}^{2}$ flasks (Thermo Fisher Scientific ${ }^{\mathrm{TM}}$ ) at $5 \% \mathrm{CO}_{2}$, in $37^{\circ} \mathrm{C}$ humidified incubators, and cultured to $80 \%$ confluence. Briefly, cells were washed once with warm Phosphate Buffered Saline (PBS; 20 ml/flask; $8 \mathrm{~g} \mathrm{NaCl}, 0.2 \mathrm{~g} \mathrm{KCl}, 1.44 \mathrm{~g}$ $\mathrm{Na}_{2} \mathrm{HPO}_{4}, 0.24 \mathrm{~g} \mathrm{KH}_{2} \mathrm{PO}_{4}$ per litre, $\mathrm{pH} 7.4$ ), and trypsinised. Prostate cancer cell lines were trypsinised for 4 minutes at $37^{\circ} \mathrm{C}$, apart from the PC 3 cell line, which was trypsinised for 7 minutes. Breast cancer cell lines were trypsinsed for 7 minutes at $37^{\circ} \mathrm{C}$. Trypsinised cells were added to equal volumes of media, centrifuged at $700 \times \mathrm{g}$ for 4 minutes $\left(4^{\circ} \mathrm{C}\right)$, and resuspended in fresh media. Viable cell counts were obtained by Trypan blue dye exclusion 
using a LUNA ${ }^{\mathrm{TM}}$ Automated Cell Counter (Logos Biosystems), and cells re-seeded at $3 \times 10^{4}$ cells $/ \mathrm{cm}^{2}$.

\subsubsection{Growth assays}

Cell lines were seeded at $3 \times 10^{4}$ cells $/ \mathrm{cm}^{2}$ in Nunc ${ }^{\text {TM }} 24$ well plates (Thermo Scientific ${ }^{\text {TM}}$ ) with $0.5 \mathrm{ml}$ media and placed in a 20x FLR Incucyte (Essen Bioscience) for 72 hours. Each well was imaged in 9 positions, every 3 hours for 72 hours. At each time point, the percentage of the area that was occupied by cells was calculated using the Incucyte's inbuilt "Confluence (\%)" metric. The average confluency from the 9 images, across the 3 wells was calculated and used to plot a growth curve.

In parallel, cell lines were seeded at $3 \times 10^{4}$ cells $/ \mathrm{cm}^{2}$ in Nunc ${ }^{\mathrm{TM}} 24$ well plates (Thermo Fisher Scientific ${ }^{\mathrm{TM}}$ ) with $0.5 \mathrm{ml}$ media. Whole-cell counts (including dead cells) were obtained every 24 hours, over 72 hours using the LUNA ${ }^{\mathrm{TM}}$ Automated Cell Counter (Logos Biosystems) and used to plot a growth curve. The gradient of the natural log of the regression line of each curve was used to calculate the doubling time (hours) for each cell line.

\subsubsection{Scratch wound assays}

Cells were seeded at $3 \times 10^{4}$ cells $/ \mathrm{cm}^{2}$ in 96 well Imagelock (Essen Bioscience) plates with $100 \mu \mathrm{l}$ media. At 90\% confluence, wells were scratched manually using the 96-pin IncuCyte ${ }^{\circledR}$ WoundMaker (Essen Bioscience), the debris washed from the wounds with $100 \mu$ l warm PBS, and each well topped up with $100 \mu \mathrm{l}$ fresh media. Plates were immediately placed in a 20x FLR Incucyte (Essen Bioscience), and each scratch imaged at 1 central position every 3 hours, over 72 hours. At each time point, the percentage cell density in the wound area relative to the cell density outside of the wound area was calculated using Incucyte's inbuilt "Relative Wound Density (\%)" metric. 


\subsubsection{Invasion assays}

The invasive properties of the cell lines were assayed using the CytoSelect ${ }^{\mathrm{TM}}$ Cell Invasion Assay Kit (Cell Biolabs), according to the manufacturer's protocol (Figure 2.7).

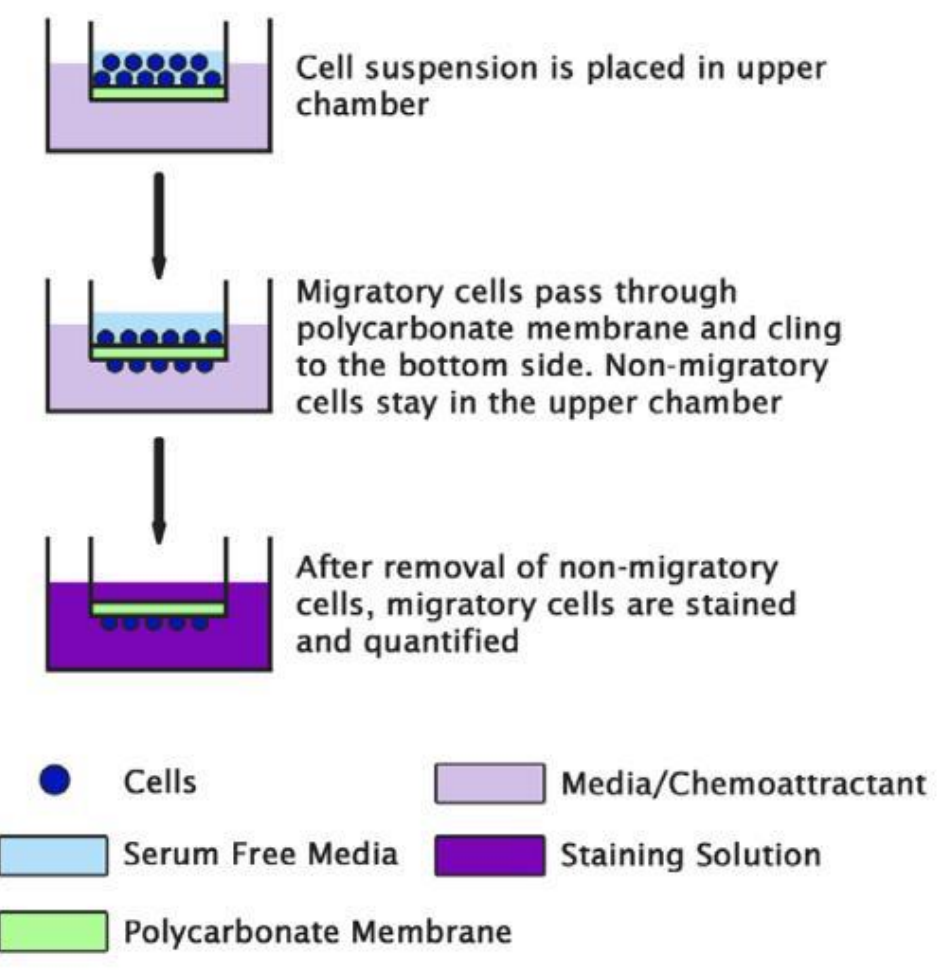

Figure 2.7: Schematic representation of the Cytoselect ${ }^{\circledR}$ Boyden Chamber assay. (Figure taken from Cambridge Bioscience, https://www.bioscience.co.uk/cpl/132954/, 2019).

Briefly, $100 \mu \mathrm{l}$ of serum-free media was added to the upper chambers to rehydrate the basement membrane lining the polycarbonate insert. After a 1-hour incubation at room temperature, the rehydration medium was removed. $150 \mu \mathrm{l}$ of phenol red-free media (10\% FBS) was added to the feeder tray beneath the polycarbonate insert. The upper chamber was seeded with $100 \mu \mathrm{l}$ of cell suspension containing $2 \times 10^{6}$ cells $/ \mathrm{ml}$ in phenol red-free media (10\% FBS). After a 48 -hour incubation at $5 \% \mathrm{CO}_{2}$, in $37{ }^{\circ} \mathrm{C}$ incubators, invaded cells were dislodged from the underside of the polycarbonate insert using $150 \mu$ l Cell Detachment Solution, dyed with CyQuant ${ }^{\circledast}$ GR Dye (diluted 1:75 in the kits $4 x$ lysis buffer), and 
transferred into a fresh clear bottom, black sided, 96-well microtiter plate (Costar). Cy2 fluorescence (489 $\mathrm{nm}$ maximum excitation wavelength, $506 \mathrm{~nm}$ maximum emission wavelength) was imaged using a Typhoon scanner (GE Healthcare) to obtain a plate image, and subsequently quantified with a PHERAstar FS plate reader (BMG Labtech) at $480 \mathrm{~nm} / 520$ $\mathrm{nm}$, and the Fiji analysis software.

\subsubsection{Immunohistochemistry}

$5.5 \times 10^{7}-1 \times 10^{8}$ cells were washed with $1.5 \mathrm{ml} \mathrm{PBS}$ and pelleted at $12,000 \times \mathrm{g}$ for 1 minute. The supernatant was removed, and the cell pellets fixed in $1 \mathrm{ml} \mathrm{10 \%} \mathrm{neutral} \mathrm{buffered}$ formalin at room temperature for 24-hours. Fixed pellets were harvested by centrifugation $(12,000 \times$ g for 1 minute), resuspended in $0.5 \mathrm{ml}$ of $70 \%$ ethanol solution, and incubated for 30 minutes at room temperature. Cells were pelleted at $12,000 \times \mathrm{g}$ for 1 minute, the supernatant removed, and air-dried to remove residual ethanol. Dried cell pellets were resuspended in $0.5 \mathrm{ml}$ of $2 \%$ agar ( $20 \mathrm{mg} / \mathrm{ml}$ in Milli-Q $18.2 \Omega$ water) and left to solidify at room temperature for approximately 1 hour.

Cells embedded in agar were submitted to the Histopathology core at the Cancer Research UK Cambridge Institute, where they were sliced into $3 \mu \mathrm{m}$ sections and stained using the Bond ${ }^{\mathrm{TM}}$ Polymer Refine Detection protocol on the Bond-III Automated Platform (Leica Biosystems Ltd). Briefly, sections were de-waxed and de-hydrated using the automated Leica ST5020 (Leica Biosystems Ltd), and incubated in hydrogen peroxide (HRP; 3-4\% v/v) to quench endogenous peroxidase activity. Sections obtained from the prostate cancer cell pellets were stained with the following primary antibodies: rabbit anti-PSA (1:2500; Dako), rabbit anti-PSMA (1:50; Novocastra), mouse anti-vimentin (1:400; Novocastra), mouse antiPTEN (1:200; Dako), mouse anti-E-cadherin (1:25; Dako), mouse anti- $\beta$-catenin (1:5000; BD Biosciences), and mouse anti-MUC1 (1:2500; Abcam). Sections obtained from the breast cancer cell pellets were stained with the following primary antibodies: mouse anti-ER (1.071 $\mu \mathrm{l} / \mathrm{ml}$ Novocastra), mouse anti-PR (1:50; Dako), mouse anti-vimentin (1:400; Novocastra), mouse anti-PTEN (1:200; Dako), mouse anti-E-cadherin (1:25; Dako), mouse anti- $\beta$-catenin (1:5000; BD Biosciences), and mouse anti-MUC1 (1:2500; Abcam). Mouse or rabbit primaries 
were localised using a Post-Primary IgG linker reagent or Poly-HRP IgG, respectively, and the complexes visualised by addition of chromogen 3,3'-Diaminobenzidine tetrahydrochloride hydrate (DAB). DAB Enhancer was used for all antibodies (Leica, AR9432), except for the anti-ER and anti-PR assays. Slides were counterstained using the Automated Schedule for Haematoxylin and Eosin Stain on a Leica ST5020 (Leica Biosystems). Slides were mounted and scanned on a Leica AT2 at 20x magnification at a resolution of $0.5 \mu \mathrm{m}$ per pixel.

\subsubsection{Preparation of whole-cell lysates}

Prostate cancer cell lines were cultured and counted as described previously, seeded at $3 \mathrm{x}$ $10^{4}$ cells $/ \mathrm{cm}^{2}$ in $100 \mathrm{~mm}$ cell culture dishes (15 ml media/dish) (Corning), and incubated in 5 $\% \mathrm{CO}_{2}$, at $37^{\circ} \mathrm{C}$ for 24 hours. After 24 hours, dishes were washed twice with warmed PBS and replaced with fresh media ( $15 \mathrm{ml}$ media /dish). After 24 hours, cells were harvested by trypsinisation and centrifugation, as described previously. Cell viability was measured by Trypan blue dye exclusion using a LUNA ${ }^{\mathrm{TM}}$ Automated Cell Counter (Logos Biosystems). Cells were incubated with $1 \mathrm{ml}$ RIPA buffer (Thermo Fisher Scientific ${ }^{\top M}$ ) supplemented with $1 \mathrm{x}$ Halt ${ }^{\mathrm{TM}}$ Protease and Phosphatase Inhibitor Cocktail (Thermo Fisher Scientific ${ }^{\mathrm{TM}}$ ) and $1 \times 0.5 \mathrm{M}$ EDTA solution (Thermo Fisher Scientific ${ }^{\mathrm{TM}}$ ), for 30 minutes at $4^{\circ} \mathrm{C}$ on an end-over-end shaker (30 rpm). Lysates were centrifuged at maximum speed $(21,000 \times \mathrm{g})$ for 20 minutes at $4^{\circ} \mathrm{C}$ and the supernatant transferred into a fresh Nostick ${ }^{\circledR}$ hydrophobic microcentrifuge tubes (Scientific Specialties, Inc.).

The total protein concentrations were determined using the DC ${ }^{\mathrm{TM}}$ Protein Assay (BIO-RAD), using BSA as a standard. Whole-cell lysates and conditioned media fractions were stored at $80^{\circ} \mathrm{C}$

\subsubsection{Western blot}

Whole-cell lysates from each prostate cancer cell line were resolved on precast $4-12 \% 1 \mathrm{~mm}$ Bis-Tris gels (Thermo Fisher Scientific ${ }^{T M}$ ) in 1x NuPAGE MOPS buffer (Invitrogen) (30 $\mu \mathrm{g} /$ /ane, 
$30 \min 60 \mathrm{~V}, 120 \mathrm{~min} 120 \mathrm{~V})$, and 1D gels transferred onto nitrocellulose membranes using an iBlot 2 (Life). Blots were incubated in 1x Odyssey ${ }^{\circledR}$ PBS block buffer (LI-COR) for 1 hour, followed by an overnight incubation at $4^{\circ} \mathrm{C}$ with the following primary antibodies in PBS blocking buffer ( $0.1 \%$ tween, $0.1 \%$ SDS): rabbit monoclonal anti-vimentin (1:1000; Cell Signalling Technologies), and rabbit monoclonal anti- $\beta$-actin (1:1000; Cell Signalling Technologies). After overnight incubation, primary antibodies were removed, and the blots washed 3 times (PBS + Tween (0.1\%), 5 minutes). Blots were incubated for 1 hour at room temperature with a goat anti-rabbit IRDye 680 RD secondary antibody $(1: 15,000$; LI-COR) in PBS blocking buffer (0.1\% tween, $0.1 \%$ SDS). Blots were washed 3 times in PBS ( $0.1 \%$ Tween) for 5 minutes, once in PBS for 5 minutes, and imaged on an Odyssey CLx Infrared Imaging System (LI-COR Biosciences).

\subsubsection{Statistical analysis}

All p-values were calculated using a one-way ANOVA test. 


\section{Chapter 3: Detection of differential glycosylation using}

bioorthogonal chemistry in in vitro models of cancer progression with the aim of imaging of tumour glycosylation in vivo

\subsection{Introduction}

\subsubsection{Aberrant sialyation and $\mathrm{N}$-acetylgalactosamine glycosylation in cancer}

Glycans are the complex carbohydrate structures formed when monosaccharides, namely glucose (Glc), N-acetyl-glucosamine (GlcNAc), Galactose (Gal), N-acetyl-galactosamine (GalNAc), Mannose (Man), Xylose (Xyl), Glucuronic acid (GlcA), Fucose (Fuc) and Nacetylneuraminic acid (NeuAc), also known as sialic acid, link together via glycosidic bonds (Prestegard and Widmalm, 2017). Glycans act as cellular decorations with roles in intracellular signalling, hormone regulation, cell migration, plasma membrane structuring, immunity, host-pathogen recognition, and drug sensitivity (Pinho and Reis 2015; Varki 2017). Their synthesis is dependent on the dynamic interactions between glycoenzymes within a specific cell type. These dynamic interactions are sensitive to physiological and pathological changes (Reily et.al. 2019; Krasnova and Wong 2016; Mariño et. al. 2010). In cancer, alterations in the expression of glycoenzymes and their location within the Golgi compartments, alterations to the tertiary conformation of peptide backbones and nascent glycan chains, as well as fluctuations in the abundance of acceptor substrates, sugar nucleotide donors and cofactors (Pinho and Reis 2015; Adamczyk et. al. 2012), change the repertoire of cell surface glycan structures. The overall consequence is the generation of tumour-associated glycan patterns such as hypersialylation, aberrant fucosylation, glycan truncation, hyperbranching, and overexpression of glycosphingolipids (Pinho and Reis 2015) (Chapter 1: Figure 1.7). 
Increases in sialyation (Büll et. al. 2014) of cell surface glycoproteins (Schultz et. al. 2012) and glycolipids (Daniotti et al. 2013) has been observed in prostate (Munkley et. al. 2016), breast (Kölbl et. al. 2016), and pancreatic cancers (Munkley 2019), and is associated with increased disease progression and metastasis (Stowell et. al. 2015). This has been attributed to overexpression of sialyltransferases (Bhide and Colley 2017), secretion of active sialyltransferases into the circulation (Zhang et. al. 2018) and downregulation of sialic acid cleaving sialidases (Uemura et. al. 2009), all of which contribute towards the generation of tumour-associated antigens such as the Sialyl Lewis antigens (Natoni et. al. 2016; Pinho and Reis 2015) (Chapter 1: Figure 1.8a).

Increases in cell surface $\mathrm{N}$-acetylgalactosamine during cancer progression has also been observed, for example, elevated levels of unmodified or sialylated Thomsen-nouveau (Ju et. al. 2011) (Chapter 1: Figure 1.9) and Thomsen-Friedenreich (Springer et. al. 1975) (Chapter 1: Figure 1.10) antigens, which are composed of an $\mathrm{N}$-acetylgalactosamine residue connected to serine or threonine residues by an $\alpha$-glycosidic bond. As disease progresses, glycosylation is altered.

\subsubsection{Imaging aberrant tumour glycosylation using bioorthogonal chemistry}

Traditionally, global increases in cell surface glycosylation associated with cancer progression have been quantified using glycan-binding lectins with various monosaccharide specificities (van Kooyk 2008; Kuo et. al. 2016) (Chapter 1: Figure 1.17a). For example, decreased binding of topically applied fluorescein-labelled wheat germ agglutinin lectin was detected during the progression of pre-invasive Barrett's oesophagus to adenocarcinoma (Bird-Lieberman et. al. 2012) (Chapter 1: Figure 1.17c). This correlation between increases in glycosylation and metastatic potential suggest that detecting changes in glycosylation could be useful for monitoring the propensity of a tumour to metastasise in vivo i.e. for assessing the aggressiveness of the disease.

Novel ways of detecting aberrant glycosylation include the use of bioorthogonal monosaccharide analogues containing a functional azide group or "chemical reporter" not 
normally present in the cell (Laughlin et. al. 2009), which are incorporated metabolically into cellular glycan structures, and subsequently detected by covalent ligation of imaging probes or affinity reagents that react rapidly with the azide group (Saxon and Bertozzi 2000; Saxon et. al. 2002) (Chapter 1: Figure 1.20). Bioorthogonal azide-based chemical reporters, generally called azidosugars, have been used to study glycosylation in zebrafish, Trypanosoma cruzi, Haemophilus ducreyi, Caenorhabditis elegans and human cell lines (Laughlin et. al. 2008; Lantos et. al. 2016; Agarwal et. al. 2015; Goon et. al. 2003; Laughlin et. al. 2009; Saxon and Bertozzi 2000; Dube and Bertozzi 2003). The most extensively used chemical reporters in cancer research are variants of sialic acid, $\mathrm{N}$-acetylgalactosamine and fucose, due to their association with cancer progression (Fuster and Esko 2005; Laughlin et. al. 2009; Cheng et. al. 2016), such as precursors of sialic acid and N-acetylgalactosamine, which have been labelled with an azide group ( $\mathrm{N}$-azidoacetylmannosamine ( $\mathrm{Ac}_{4} \mathrm{ManNAz}$ ) and $\mathrm{N}$-azidoacetylgalactosamine ( $\left.\left.\mathrm{Ac}_{4} \mathrm{GalNAz}\right)\right)$. We previously validated a panel of prostate (Chapter 2: Table 2.1a) and breast (Chapter 2: Table 2.1b) cancer cell lines as suitable in vitro models of cancer progression using scratch wound assays to compare migration, Boyden chambers to compare invasion and immunohistochemistry to compare the relative expression of known invasion biomarkers (Pijuan et. al. 2019; Justus et. al. 2014). Here, we applied bioorthogonal labelling techniques, which have been used previously to visualise cell surface glycosylation in cancer cell lines in vitro (Stöckmann et. al., 2011; Kuo 2015, PhD thesis, unpublished) (Chapter 1: Figure 1.21a and b) and in tumour bearing mice in vivo (Neves et. al. 2011; Neves et. al. 2013; Neves et. al. 2016) (Chapter 1:21c-e). These bioorthogonal labelling techniques were used in our in vitro model of breast cancer progression (Chapter 2: Table 2.1b), and the p48Cre; LSL-KrasG12D (KC) mouse model (Hingorani et. al. 2003; Westphalen and Olive 2012) of pancreatic ductal carcinoma (Chapter 1: Figure 1.25).

The clinical relevance of imaging glycosylation in models of cancer progression is not simply in the identification of tumours, for which many techniques already exist, but in the ability to visualise changes in glycosylation that we know occur during disease progression (Stowell et. al. 2015; Pinho and Reis 2015; Fuster and Esko 2005; Ju et. al. 2011; Vajaria and Patel 2017). The ability to visualise non-invasively tumour glycosylation in vivo, using bioorthogonal precursors of sialic acid ( $\mathrm{Ac}_{4} \mathrm{ManNAz}$ ) and $\mathrm{N}$-acetylgalactosamine ( $\mathrm{Ac}_{4} \mathrm{GalNAz}$ ), and clinically 
relevant imaging modalities, would provide qualitative insights into glycan dynamics and turnover (Colley et. al. 2017) in real-time. Glycan imaging may also support cancer grading in order to distinguish between benign lesions and those with the potential to become aggressive and metastasise, which is a diagnostic problem in prostate cancer (Meany and Chan 2011).

\subsection{Results}

Human breast cancer cell lines were selected from the literature, based on their increasing tumourigenicity and metastatic potential following orthotopic implantation in the mammary fat pads of nude mice (Chapter 2: Table 2.1b). Previous work in the lab showed a correlation between increases in $\mathrm{N}$-azidoacetylmannosamine labelling, a precursor of sialic acid, and increases in the metastatic potential of these breast cancer cell lines (Kuo 2015, Ph.D. thesis, unpublished) (Chapter 1: Figure 1.23a, b, and d). In this study, the migratory (Chapter 2: Figure 2.5), and invasive (Chapter 2: Figure 2.6) phenotypes of these breast cancer cell lines were used to validate the panel as a suitable in vitro model of breast cancer progression. The, dynamic increases in sialylation in these cell lines, observed using bioorthogonal chemistry, suggested that glycosylation was altered during cancer progression and that changes in tumour glycosylation could be imaged using metabolic labelling in vivo in advanced models of cancer progression.

Metabolic labelling of the breast cancer cell lines was conducted in Quantum 286 media (PAA Laboratories), which was eventually discontinued. Therefore, the cell lines were adapted to new DMEM media preparations and the metabolic labelling protocol optimised to ensure that the correlation between azidosugar labelling and metastatic potential could be replicated. 


\subsubsection{Flow cytometric analysis of metabolically labelled breast cancer cell lines}

Breast cancer cells were incubated with precursors of sialic acid and $\mathrm{N}$-acetylgalactosamine that had been labelled with an azide group ( $\mathrm{N}$-azidoacetylmannosamine ( $\mathrm{Cc}_{4} \mathrm{ManNAz}$ ) and $\mathrm{N}$ azidoacetylgalactosamine ( $\mathrm{Cc}_{4} \mathrm{GalNAz}$ ) respectively), and which are incorporated biosynthetically into cell surface glycans. Cell line viability was similar when treated with vehicle (0.1\% DMSO), Ac 4 ManNAz $(50 \mu \mathrm{M})$ or $\mathrm{Ac}_{4}$ GalNAz $(50 \mu \mathrm{M})$. Therefore, azidosugarinduced cytotoxicity was undetectable during the incubation period required for metabolic labelling (24 hours). The incorporated azide groups were reacted sequentially with TMDIBO(PEG) ${ }_{3}$-biotin and NeutrAvidin ${ }^{\mathrm{TM}}$ DyLight $^{\mathrm{TM}} 650$ (NA650), followed by fluorescence detection using flow cytometry (Figure 3.1).

Debris (Figure 3.1a, i), cell aggregates (Figure 3.1a, ii) and dying cells (Figure 3.1a, iii) were excluded from the analysis. NA650 fluorescence on viable cells was quantified (Figure 3.1a, iv). The Mean Fluorescence Intensity ratio (MFI ratio) for a single cell line was calculated (Figure 3.1b) by normalising the average fluorescence intensity from $\mathrm{Ac}_{4} \mathrm{ManNAz-labelled} \mathrm{(in}$ red) or $\mathrm{Ac}_{4}$ GalNAz-labelled (in green) cells, against background fluorescence. Minimal background was observed in the absence of the azido-sugars, in cells treated with TMDIBO(PEG) ${ }_{3}$-biotin/NA650/Sytox ${ }^{\circledR}$ Green (in brown), NA650/Sytox ${ }^{\circledR}$ Green (in black), Sytox ${ }^{\circledR}$ Green only (in dark grey) or vehicle only (in light grey). 
a.

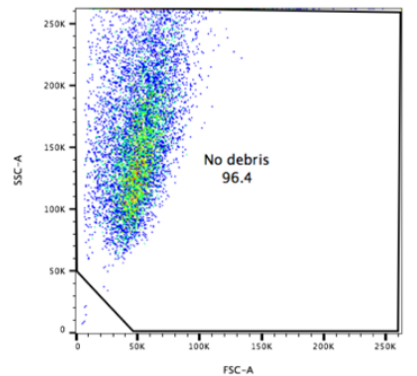

i. Exclude debris

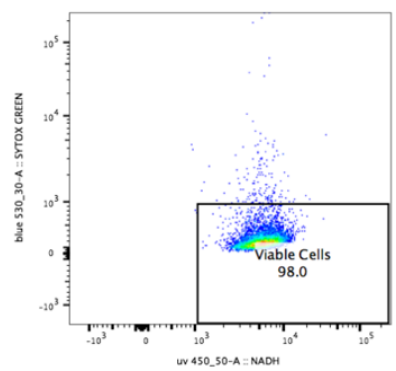

iii. Select viable cells

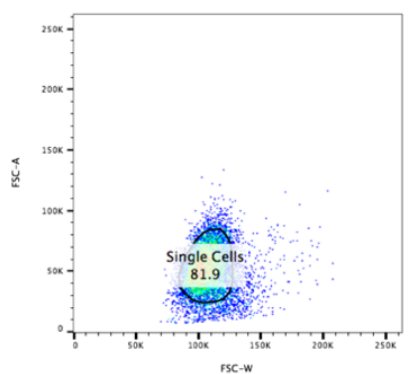

ii. Select single cells

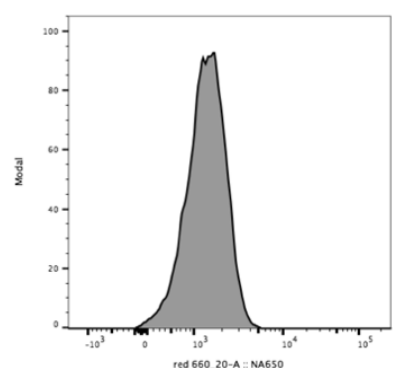

iv. Quantify NA650 signal b.

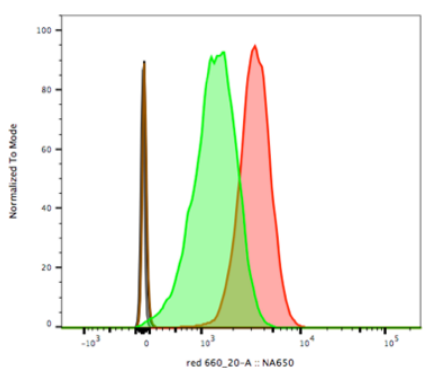

\begin{tabular}{|c|c|c|c|}
\hline Azidosugar & TMDIBO-biotin & NA650 & Sytox Green \\
\hline $\mathrm{Ac}_{4} \mathrm{ManAz}$ & $(+)$ & $(+)$ & $(+)$ \\
\hline $\mathrm{Ac}_{4} \mathrm{GalAz}$ & $(+)$ & $(+)$ & $(+)$ \\
\hline$(-)$ & $(+)$ & $(+)$ & $(+)$ \\
\hline$(-)$ & $(-)$ & $(+)$ & $(-)$ \\
\hline$(-)$ & $(-)$ & $(-)$ & $(+)$ \\
\hline$(-)$ & $(-)$ & $(-)$ & $(-)$ \\
\hline
\end{tabular}

Figure 3.1: Flow cytometric analysis of metabolically labelled MDA MB 231 breast cancer cells. (a) Gating parameters, based on forward scatter cell area analysis plots, were implemented to exclude debris (i), and aggregated cells (ii). Non-viable cells were excluded based on high Sytox ${ }^{\circledR}$ Green fluorescence and a low intracellular NADH concentration determined by measuring UV fluorescence. (iii) NA650 fluorescence was quantified on viable cells only (iv). (b) Representative histograms showing NA650 fluorescence from MDA MB 231 breast cancer cells metabolically labelled with $N$-azidoacetylmannosamine (Ac4ManNAz, red) or $N$ - azidoacetylgalactosamine (Ac4GalNAz, green). Background controls were included to detect non-specific fluorescence (brown, black, dark grey, and light grey).

The mean fluorescence intensity (MFI) ratios from each of the metabolically labelled breast cancer cell lines were compared (Figure 3.2). An increase in $A c_{4} M a n N A z$ and $A c_{4} G a l N A z$ labelling correlated with an increase in the metastatic potential of the cell lines (Figure 3.2a). The most metastatic cell line, MDA MB 231, showed the highest labelling with $\mathrm{Ac}_{4} \mathrm{ManAz}$ and $\mathrm{Ac}_{4} \mathrm{Gal} A z$. In each cell line, there was at least a 2 -fold increase in labelling with $\mathrm{Ac}_{4} \mathrm{ManNAz}$ when compared with $\mathrm{Ac}_{4} \mathrm{GalNAz}$ (Figure 3.2b). 
a.

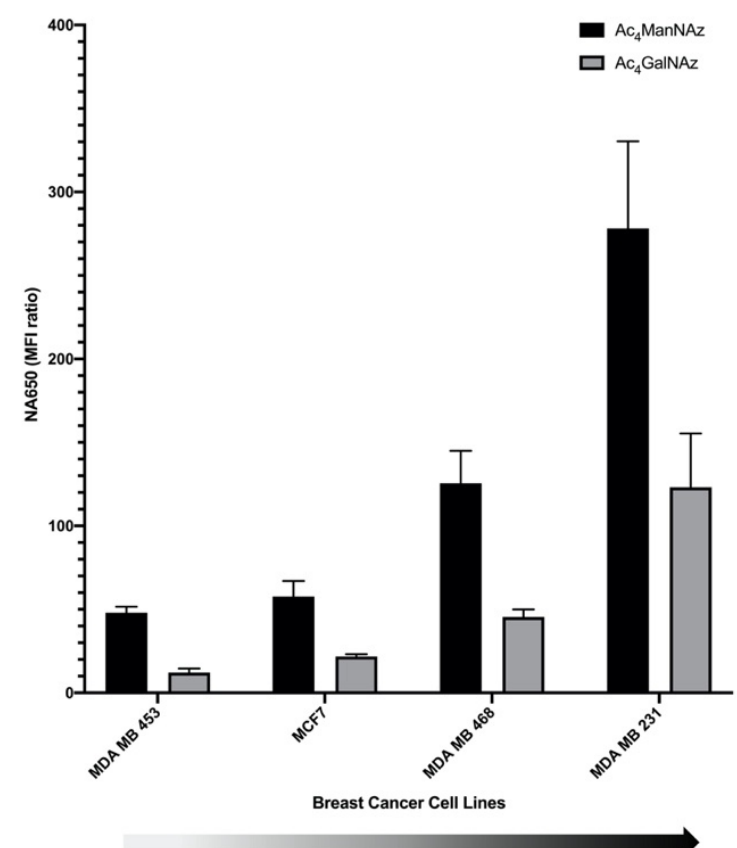

b.

Breast cancer $\quad \mathrm{Ac}_{4}$ ManNAz:Ac $\mathrm{GalNAz}_{4}$ cell line (MFI ratios)

\begin{tabular}{|cc|}
\hline MDA MB 453 & 4.0 \\
\hline MCF-7 & 2.7 \\
\hline MDA MB 468 & 2.8 \\
\hline MDA MB 231 & 2.5 \\
\hline
\end{tabular}

Figure 3.2: Metabolic labelling of a panel of breast cancer cell lines with increasing metastatic potential. Breast cancer cells were labelled sequentially with $\mathrm{N}$ azidoacetylmannosamine (Ac4ManNAz) or $\mathrm{N}$ - azidoacetylgalactosamine (Ac4 GalNAz), and then TMDIBO-(PEG)3-biotin and NeutrAvidin ${ }^{T M}$ DyLight ${ }^{T M} 650$ (NA650). Fluorescence detection of NA650 using flow cytometric analysis followed. The Mean Fluorescence Intensity ratio (MFI ratio) for each cell line was calculated by normalising the average fluorescence intensity from azidosugar-labelled cells against background fluorescence. (a) MFI ratios in breast cancer cell lines metabolically labelled with Ac 4 ManNAz (black) or Ac GalNAz (grey). (b) The fold difference in $A_{4} M a n N A z$ labelling versus $A c_{4} G a I N A z$ labelling for each cell line. All data represent the average \pm S.D. of three independent experiments, each with 3 replicates. $p$ values represent significance assessed by one-way ANOVA. The darker shades in the arrow's greyscale $(a, b)$ represent an increase in the metastatic potential of the panel of breast cancer cell lines.

There was no correlation between doubling time (Figure 3.3a) and $\mathrm{Ac}_{4} \mathrm{ManAz}$ (Figure 3.3b) or $\mathrm{Ac}_{4} \mathrm{GalNAz}$ (Figure 3.3c) incorporation into cell surface glycans. 
a.
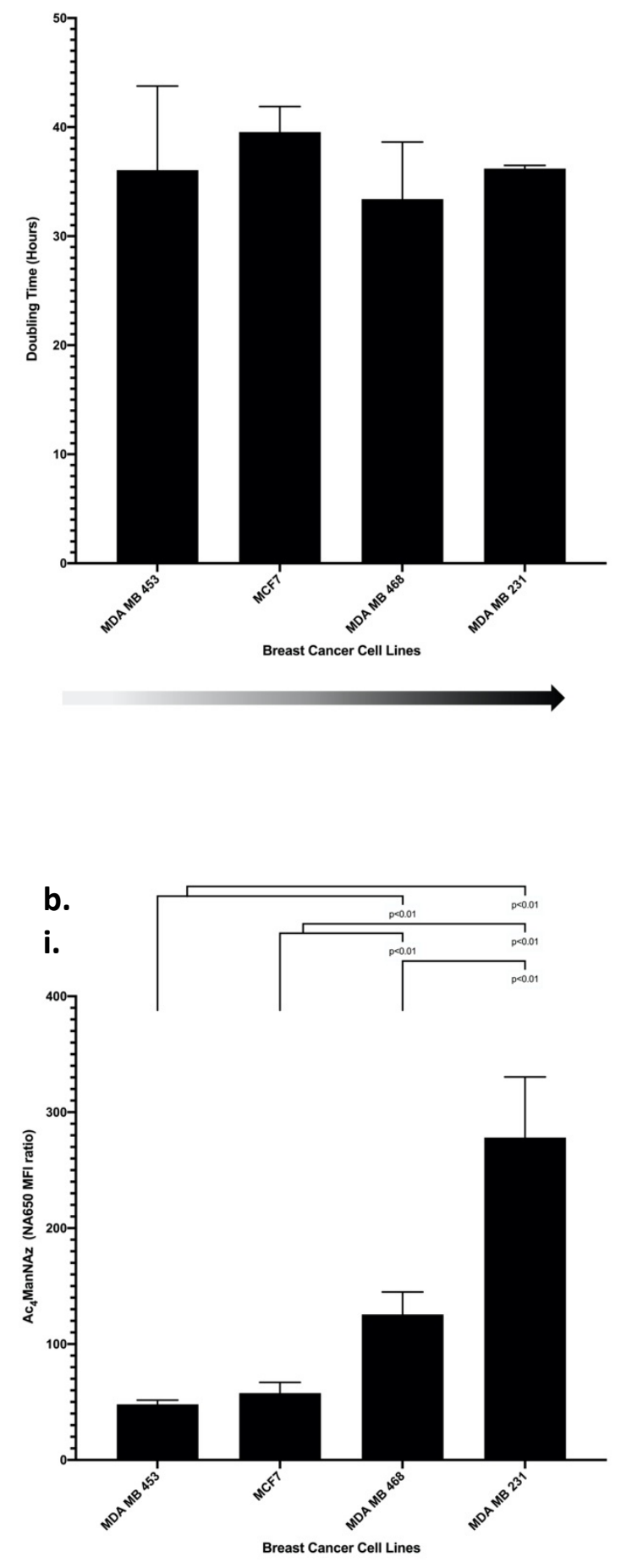

ii.

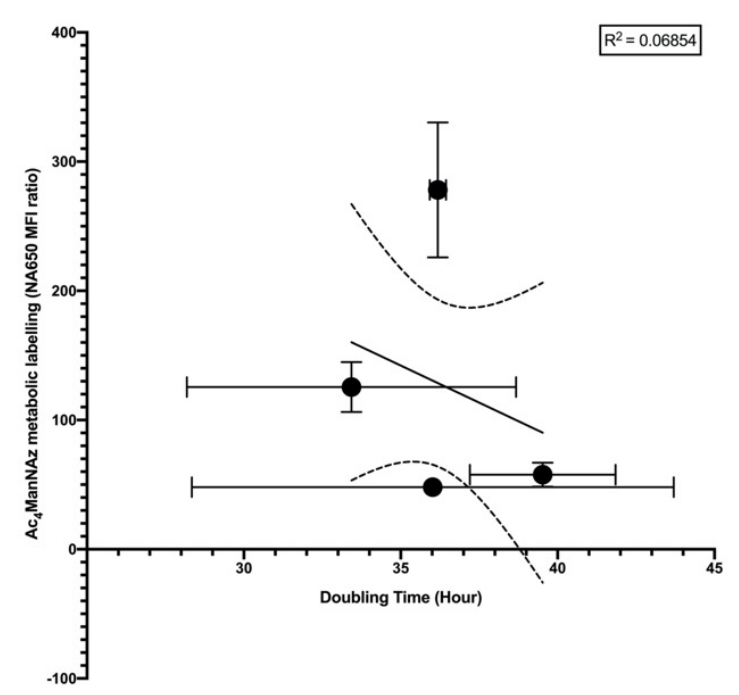




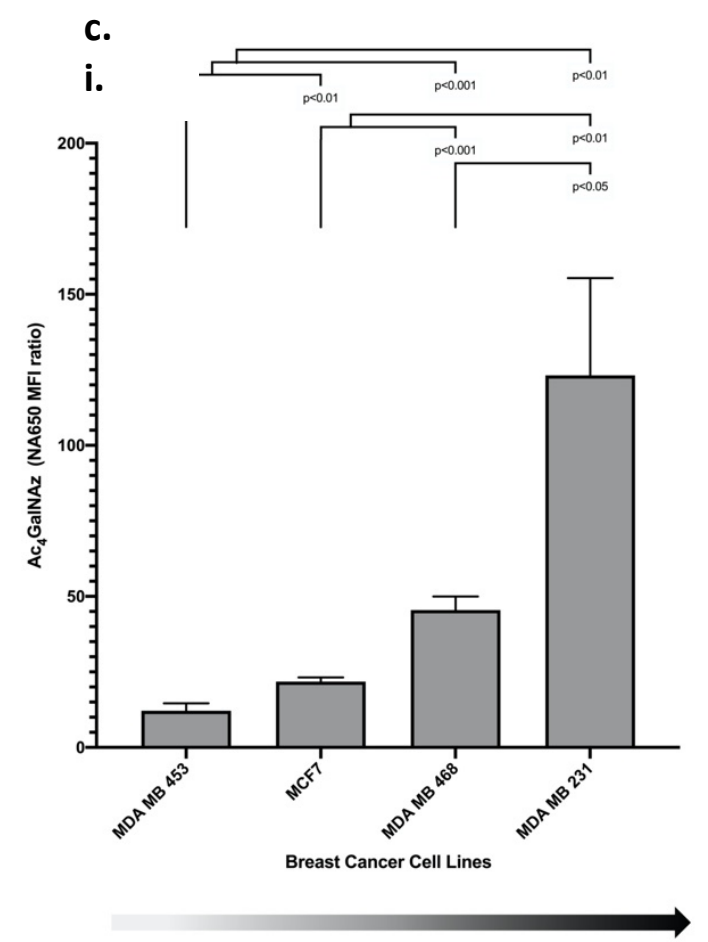

ii.

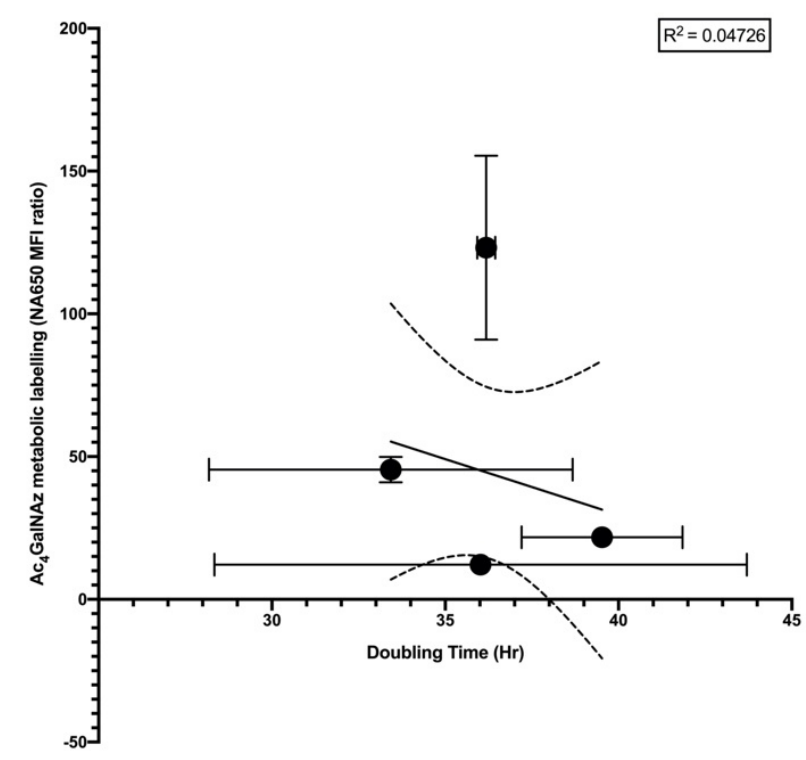

Figure 3.3: Investigating the effect of doubling time on metabolic labelling using linear regression correlation analysis. Breast cancer cells were labelled sequentially with $\mathrm{N}$ azidoacetylmannosamine ( $\mathrm{C}_{4} \mathrm{ManNAz}$ ) or $\mathrm{N}$ - azidoacetylgalactosamine (Ac4 GalNAz), and then TMDIBO-(PEG)-biotin and NeutrAvidin ${ }^{T M}$ DyLight $^{T M} 650$ (NA650). Fluorescence detection of NA650 using flow cytometric analysis followed. The Mean Fluorescence Intensity ratio (MFI ratio) for each cell line was calculated by normalising the average fluorescence intensity from azidosugar-labelled cells against background fluorescence. (a) Whole cell counts were obtained every 24 hours, over a 72-hour period using the LUNA ${ }^{\text {TM }}$ Automated Cell Counter, and used to plot a growth curve. The gradient of the natural log regression line of each curve was used to calculate the doubling time (hours) for each cell line. (b) MFI ratios for each cell line treated with Ac4ManNAz (i) plotted as a function of doubling time (ii). (c) MFI ratios for each cell line treated with Ac ${ }_{4} G a I N A z$ (i) plotted as a function of doubling time (ii). All data represent the average \pm S.D. of three independent experiments, each with 3 replicates. $p$ values represent significance by one-way ANOVA. The darker shades in the arrow's greyscale represent an increase in the metastatic potential of the panel of breast cancer cell lines. 
Previous work in the lab (Whittaker 2015, Part III dissertation, unpublished data) showed an increase in $\mathrm{Ac}_{4}$ ManNAz labelling in a panel of prostate cancer cell lines, which this study has validated to be a suitable in vitro model of prostate cancer progression (Chapter 2: Figure 2.2 and Figure 2.3). Collectively, these data have demonstrated the use of bioorthogonal chemistry for detecting differential $\mathrm{Ac}_{4} \mathrm{ManAz-specific} \mathrm{sialyation} \mathrm{and} \mathrm{Ac}_{4}$ GalNAz-specific Nacetylgalactosamine glycosylation of plasma membrane glycoconjugates in human tumour cell line models of breast and prostate cancer progression. Differential glycosylation may be a sensitive and specific marker for monitoring cancer progression.

\subsubsection{Metabolic labelling of the p48Cre; LSL-KrasG12D (KC) mouse model of pancreatic cancer progression}

The correlation between increases in glycosylation and metastatic potential (Chapter 1: Figure 1.23; Figure 3.2) could be useful for monitoring tumour glycosylation in vivo. Towards imaging of differential glycosylation, and in the absence of available in vivo models of breast cancer progression (Holen et. al. 2017), biorthogonal labelling techniques were tested in advanced mouse models of pancreatic cancer progression that have been studied previously in our lab (Chapter 1: Figure 1.25).

3D pancreatic tumour organoids were generated from p48Cre; LSL-KrasG12D (KC) mice (Hingorani et. al. 2003; Westphalen and Olive 2012) and labelled with Ac 4 ManNAz or $\mathrm{Ac}_{4}$ GalNAz, TMDIBO-(PEG) -biotin, and NeutrAvidin $^{\mathrm{TM}}$ DyLight $^{\mathrm{TM}} 650$ (NA650). Metabolically labelled organoids were fixed in formalin, and NA650 fluorescence visualised using a Leica Tandem confocal microscope (Figure 3.4). A slice through the z-stack generated from a region of interest (Figure 3.4a and b, black boxes) showed a hollow center devoid of cells, typical of organoids of ductal origin, and concentration of NA650 fluorescence at the cell surface. These data, demonstrated, what we believe to be the first instance of bioorthogonal metabolic labelling of cell surface glycans in 3D pancreatic organoids. 
a.

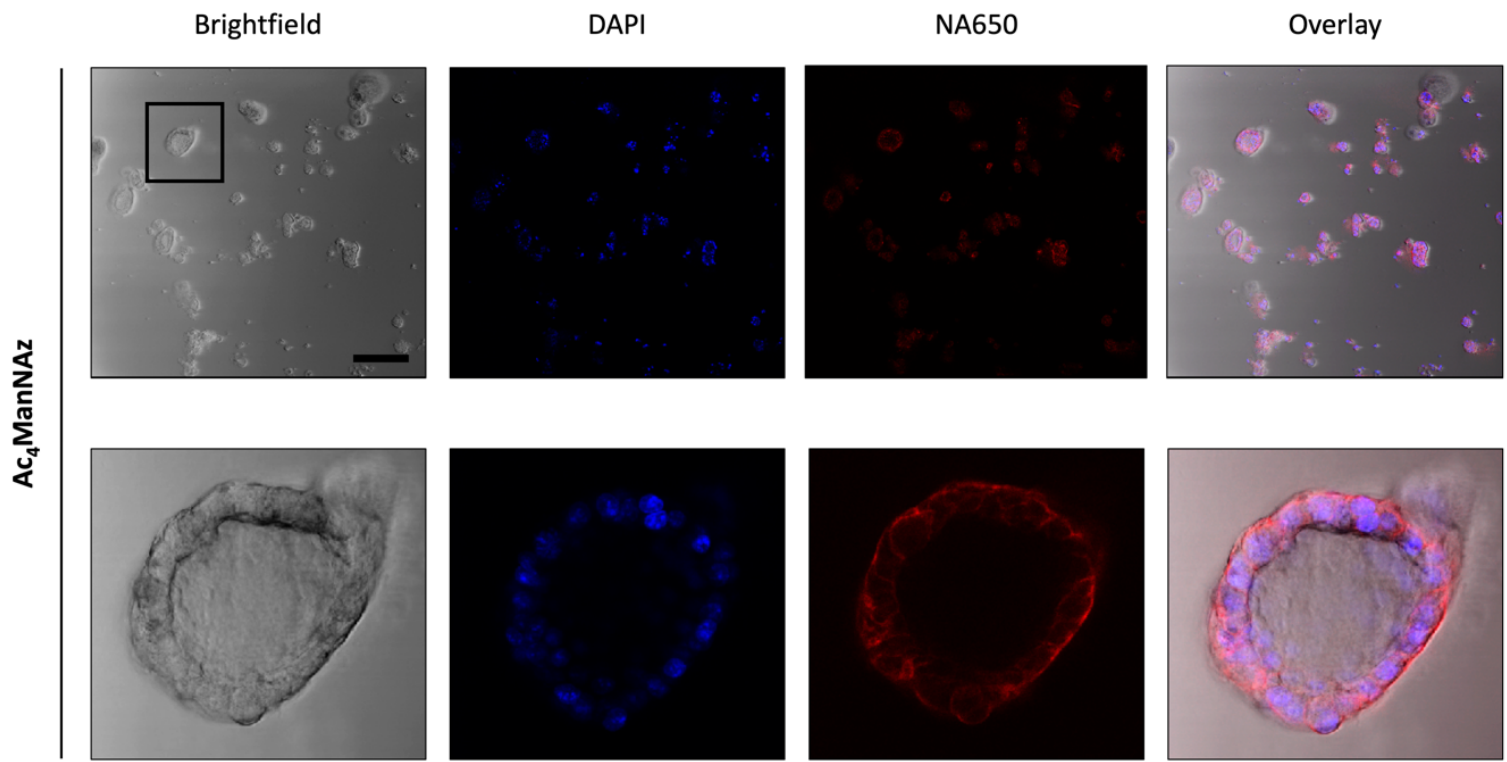

b.

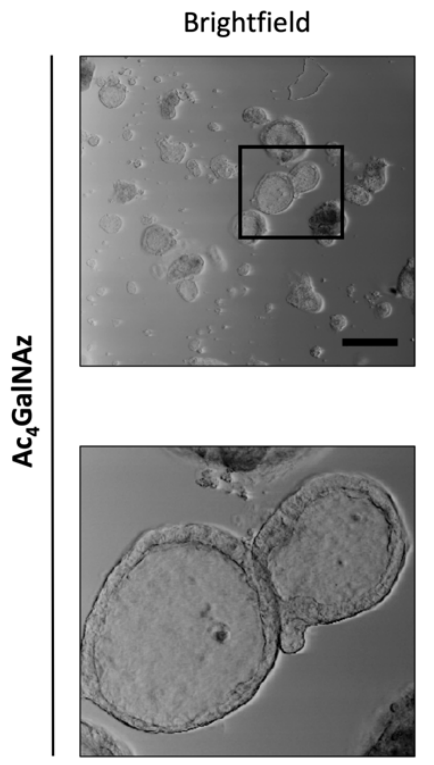

DAPI
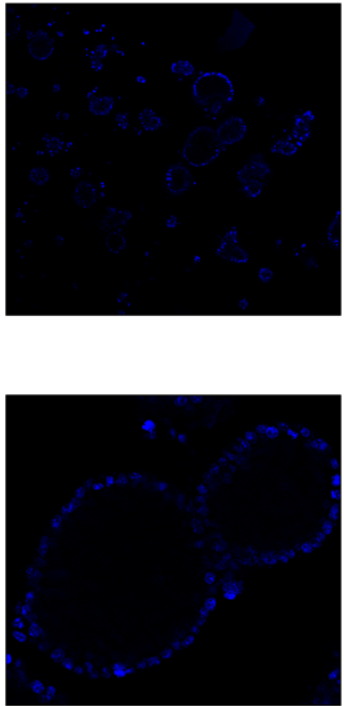

NA650
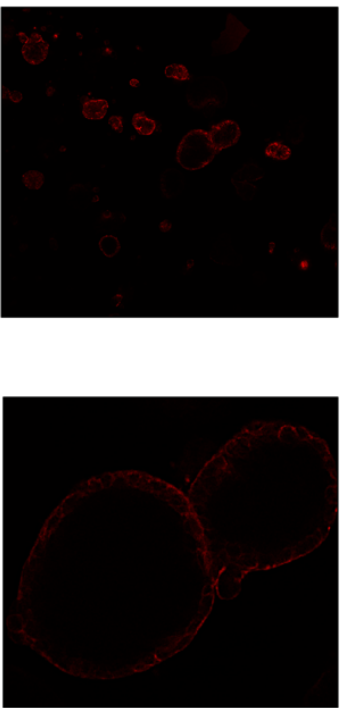

Overlay
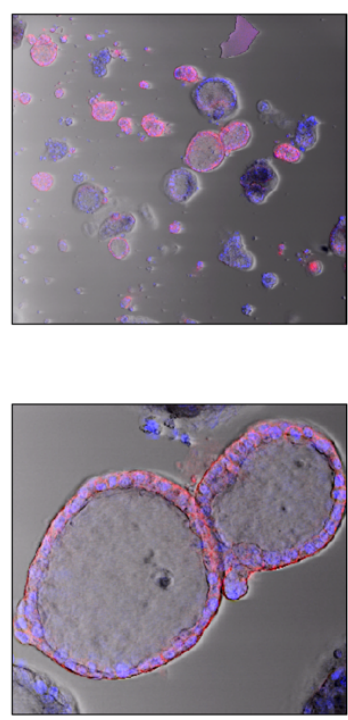

Figure 3.4: Fluorescence confocal microscopy of metabolically labelled 3D pancreatic tumour organoids. Azidosugar-specific-NA650 fluorescence (red) detected in organoids labelled with Ac 4 ManNAz (a) or Ac ${ }_{4} G a l N A z(b)$. A slice through the z-stack generated from a region of interest (black boxes in a and b). DAPI (blue), Bright field (grey). Scale bars represent $100 \mu \mathrm{m}$. 
Following successful azidosugar labelling of pancreatic tumour organoids (Figure 3.4), metabolic labelling was tested in vivo, using a mouse model of pancreatic cancer progression. Generally, control mice (with a normal pancreas), and KC mice (with a diseased pancreas), aged 3, 6 and 12 months, were treated with vehicle or varying dosing regimens of $\mathrm{N}$-azidoacetylmannosamine $\left(\mathrm{Ac}_{4} \mathrm{ManAz}\right)$ via oral gavage or i.p. injection. Pancreatic tissue was collected and the membrane proteome isolated by mechanical homogenisation and detergent-based fractionation. The incorporated azide groups were covalently ligated to alkyne-Alexa Fluor 488, ex vivo, using copper-catalysed chemistry. Labelled extracts were resolved by gel electrophoresis, and subjected to western blot analysis or in-gel fluorescence imaging.

Enrichment of the membrane proteome was confirmed by western blot analysis (Figure 3.5). Abundant proteins from the plasma membrane (E-cadherin) and the mitochondria (mitochondrial import inner membrane translocase subunit Tim23), were detected (Figure 3.5a). As expected, detection of proteins abundant in the cytosol ( $\beta$-actin) was minimal (Figure 3.5a).

Next, metabolic labelling was compared between 3 month and 12-month-old KC mice treated with three doses of $\mathrm{Ac}_{4} \mathrm{ManNAz}$ by oral gavage. Minimal protein was detected by Coomassie stain in extracts obtained from the 3-month-old KC mouse (Figure 3.5b). To confirm if this was due to poor protein extraction from the tissue, pancreatic tissue from the 3-month-old KC mice was subjected to mechanical homogenisation and detergent-based fractionation three times. For comparison, this was also conducted in pancreatic tissue from a 12-month-old KC mouse. The protein concentration of the cytosolic and membrane fractions was measured using the Biorad assay after each round of extraction and showed that the majority of protein was extracted from the tissues in the first round of processing (Figure 3.5c). Although $\mathrm{Ac}_{4}$ ManNAz-specific glycosylation was detected in the extracts from the 3-month-old KC mouse using Alexa-488 fluorescence, degradation of protein in the sample was inferred by band smearing on the silver stained gel (Figure 3.5d). All buffers used in the fractionation process contained protease inhibitors. Therefore, protein degradation may have been caused by delays in the time between tissue extraction and freezing the tissue in liquid nitrogen. 
a.

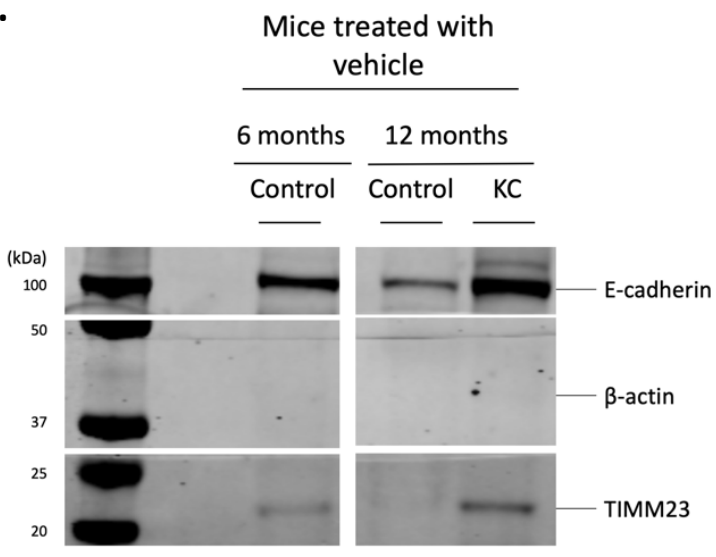

b.

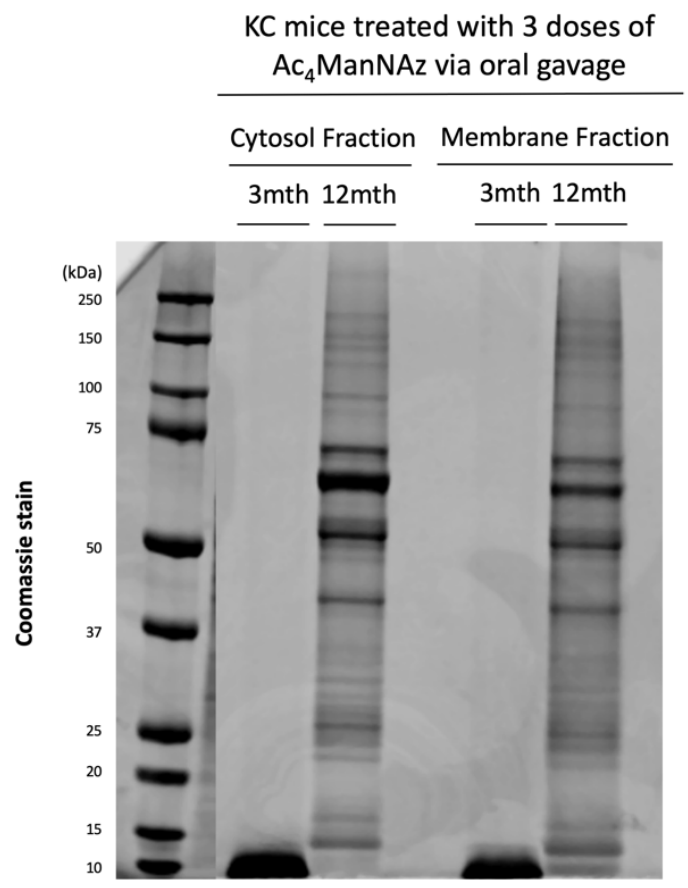

d.

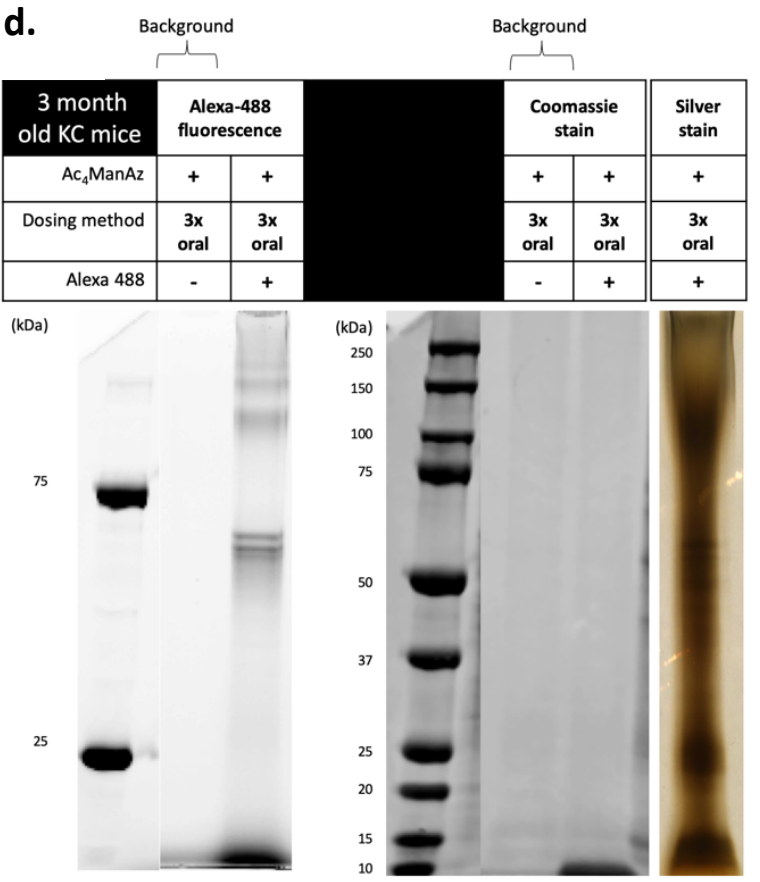

c.

\begin{tabular}{|c|c|c|c|c|c|c|c|}
\hline & & & Tot & $\mu \mathrm{g}$ & f prot & & \\
\hline & & & $\begin{array}{l}\text { toso } \\
\text { actio }\end{array}$ & & & $\begin{array}{l}\mathrm{mbr} \\
\text { actic }\end{array}$ & \\
\hline & $\begin{array}{l}\text { Extraction } \\
\text { Round }\end{array}$ & $1^{*}$ & 2 & 3 & $1^{*}$ & 2 & 3 \\
\hline 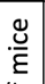 & 3 months & 103 & 46 & 29 & 170 & 37 & 75 \\
\hline 品 & 12 months & 249 & 61 & 7 & 313 & 35 & 10 \\
\hline
\end{tabular}

*Majority of protein retrieved in $1^{\text {st }}$ round of extraction

Figure 3.5: Enrichment of the membrane proteome from mouse pancreatic tissue

metabolically labelled in vivo. Alkyne-Alexa Fluor 488-labelled membrane extracts were obtained from control mice and $\mathrm{KC}$ mice, aged 3 and 12 months, that were treated with vehicle or varying dosing regimens of $\mathrm{N}$-azidoacetylmannosamine (Ac $\left.{ }_{4} M a n A z\right)$ by oral gavage or i.p. injection. $10 \mu \mathrm{g}$ of the extracts were resolved by gel electrophoresis and subjected to 
western blot analysis or in-gel fluorescence imaging. (a) Representative western blots showing the relative abundance of fraction controls in $10 \mu \mathrm{g}$ of extract from pancreatic tissue treated with vehicle: E-Cadherin (plasma membrane protein, 97 kDa), 6-actin (cytoskeletal protein, 42 kDa), mitochondrial import inner membrane translocase subunit Tim23 (TIMM 23, mitochondrial membrane protein, $22 \mathrm{kDa}$ ). (b) Coomassie stains of cytosolic and membrane protein extracts from 3 month and 12-month-old mice treated with three doses of Ac4ManNAz by oral gavage. (c) Pancreatic tissue extracted from 3 month and 12-month-old KC mice was subjected to mechanical homogenisation and detergent-based fractionation three times. Protein concentrations of the cytosolic and membrane fractions are shown. (d) In-gel Alexa-488 fluorescence detection of azidosugars incorporated into the membrane proteome of pancreatic tissue extracted from a 3-month-old $\mathrm{KC}$ mouse, treated with three doses of $A c_{4} M a n A z$ by oral gavage. Total protein was visualised using Coomassie and silver staining. Background controls were included to detect non-specific fluorescence. $10 \mu \mathrm{g}$ was loaded per lane.

Metabolic labelling, using Alexa-488 fluorescence, was compared between 6 month and 12month-old $\mathrm{KC}$ mice treated with vehicle or varying dosing regimens of $\mathrm{Ac}_{4} \mathrm{ManAz}$ by oral gavage or i.p. injection (Figure 3.6). Stronger metabolic labelling was detected in the 6month-old mice treated with three doses of $\mathrm{Ac}_{4} \mathrm{ManAz}$ by oral gavage, in comparison to treatment using i.p. injection (Figure 3.6a). Administering the azidosugar via i.p. injection may have caused its dilution in the circulation, resulting in a lower concentration reaching the pancreas, and reducing glycosylation-specific Alexa-488 fluorescence.

Coomassie stains of samples extracted from the 12-month-old control mice showed consistent extraction of tissue from similar regions of the pancreas (Figure 3.6b). We expected the pancreas of the control mice, irrespective of age, to be homogeneous. However, different banding patterns were observed in samples extracted from the KC mice. This was likely due to heterogeneity in disease burden, and thus cell types present in the pancreas of KC mice. 
The extent of metabolic labelling was compared between 6 month and 12-month-old mice, based on the reasoning that older KC mice, exhibiting greater tumour burden, would show higher azidosugar labelling (Figure 3.6c). We could not confidently investigate agedependent metabolic labelling due to high levels of background fluorescence, which was generally higher in the older, 12-month-old KC mouse (Figure 3.6b). This is likely to reduce image contrast and hinder our ability to detect tumour glycosylation in vivo during the late, more aggressive stages of the disease.

a.

\begin{tabular}{|c|c|c|c|c|c|c|c|c|}
\hline & & & & & \multicolumn{2}{|c|}{ Background } & & \\
\hline 6 month old mice & \multicolumn{4}{|c|}{ Control mice } & \multicolumn{4}{|c|}{ KC mice } \\
\hline $\mathrm{Ac}_{4} \mathrm{ManAz}$ & + & + & + & + & - & - & + & + \\
\hline Dosing method & $\begin{array}{c}1 \mathbf{x} \\
\text { oral }\end{array}$ & $\begin{array}{c}1 \mathrm{x} \\
\text { oral }\end{array}$ & $\begin{array}{l}3 \mathbf{3 x} \\
\text { IP }\end{array}$ & $\begin{array}{l}3 x \\
\text { IP }\end{array}$ & Vehicle & vehicle & $\begin{array}{l}3 x \\
\text { oral }\end{array}$ & $\begin{array}{l}3 x \\
\text { IP }\end{array}$ \\
\hline Alexa 488 & - & + & - & + & - & + & + & + \\
\hline
\end{tabular}

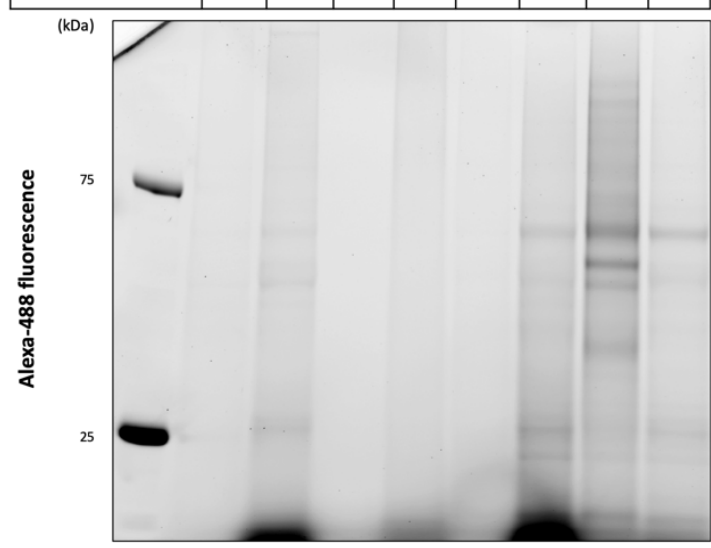

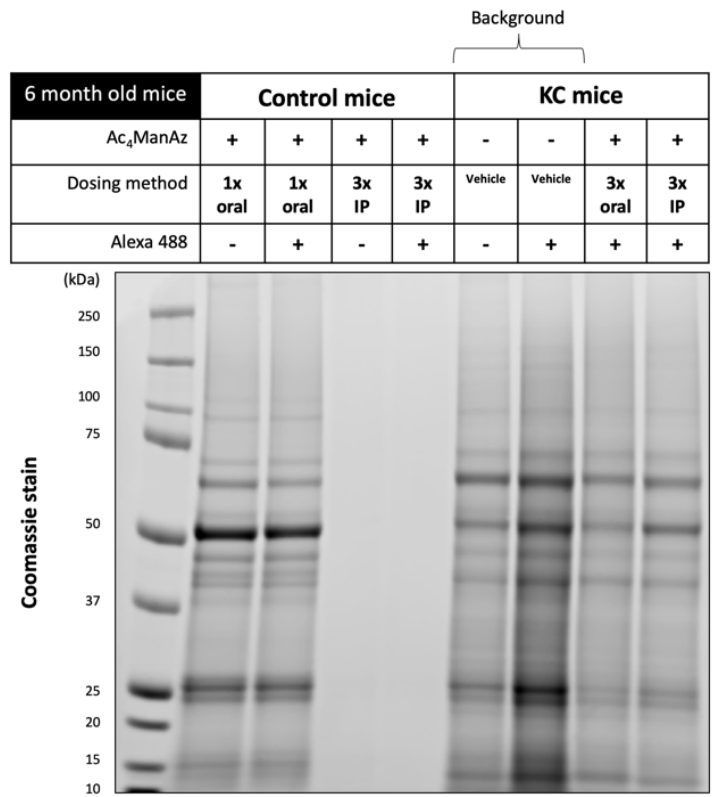


b.

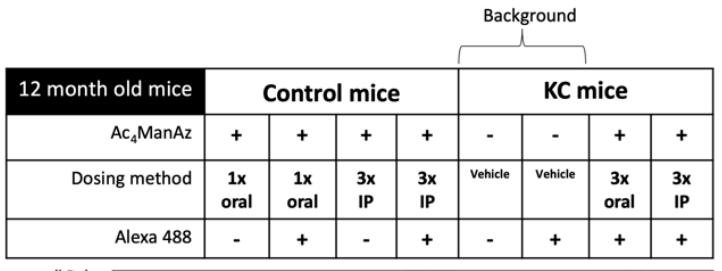

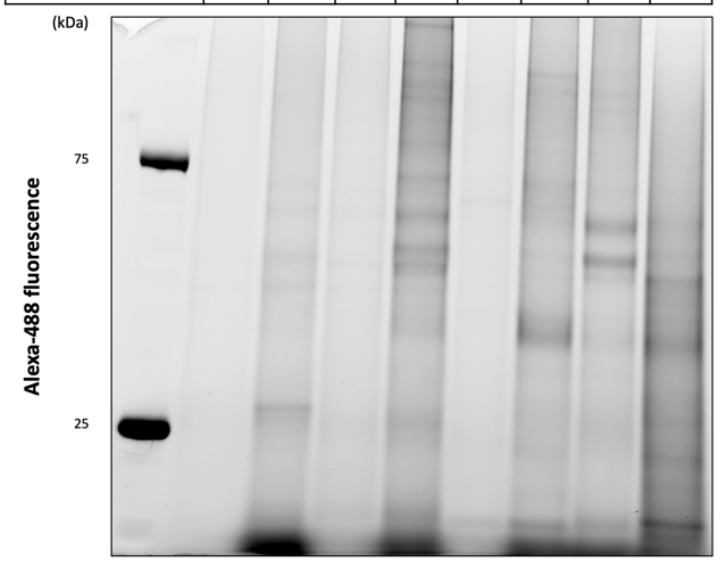

c.
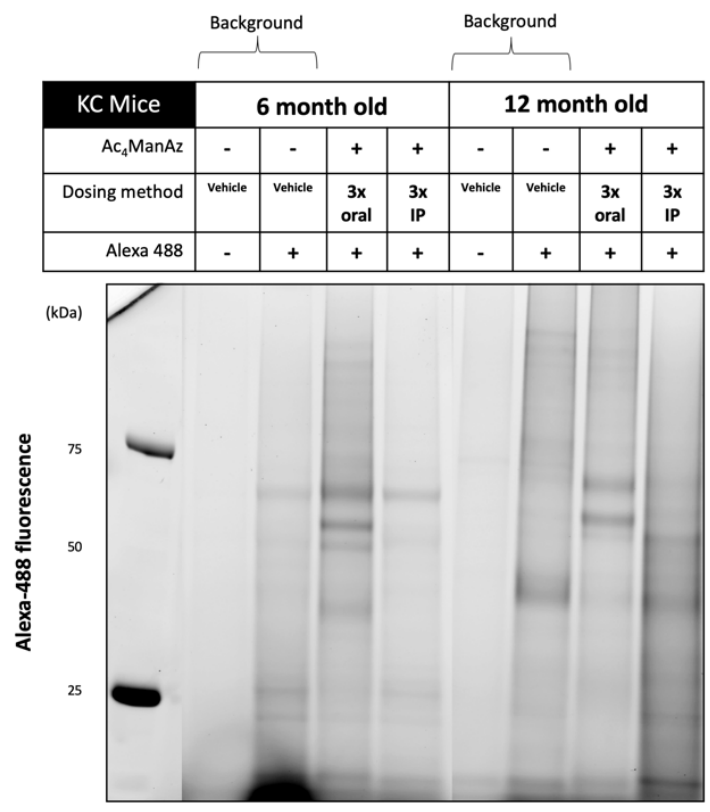
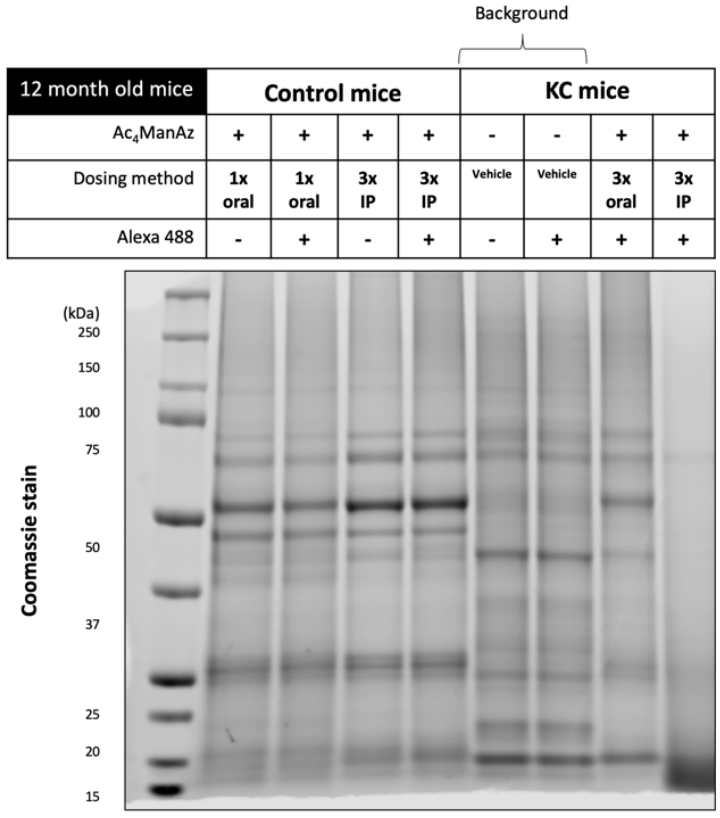

Figure 3.6: Age-dependent metabolic labelling of KC mice in vivo. Alkyne-Alexa 488 labelled membrane extracts were obtained from control mice and KC mice, aged 6 and 12 months, that were treated with vehicle or varying dosing regimens of $\mathrm{N}$-azidoacety/mannosamine (Ac4ManAz) via oral gavage or i.p. injection. $10 \mu \mathrm{g}$ of the extracts were resolved by gel electrophoresis and subjected to in-gel fluorescence imaging. Total protein per lane was visualised using Coomassie staining. (a) In-gel Alexa-488 fluorescence detection of azidosugars incorporated into the membrane proteome of pancreatic tissue extracted from a 6-month-old KC mouse, treated with three doses of Ac4ManAz by oral gavage or i.p. injection. 
Less than $10 \mu g$ of protein was loaded for samples $+A c_{4} M a n A z / 3 x$ i.p. and $+A c_{4} M a n A z / 3 x$ i.p./+Alexa-488, due to negligible protein extraction from the pancreatic tissue. The total protein amount in the same gel was visualised using Coomassie staining. (b) In-gel Alexa-488 fluorescence detection of azidosugars incorporated into the membrane proteome of pancreatic tissue extracted from a 12-month-old KC mouse, treated with three doses of Ac4ManAz by oral gavage or i.p. injection. Degradation of protein in the sample $+A c_{4} M a n A z / 3 x$ i.p./+Alexa-488 was inferred by band smearing on the Coomassie stained gel. (c) Comparison of Alexa-488 fluorescence in Ac 4 ManAz labelled 6 month and 12-month-old KC mice. Background controls were included to detect non-specific fluorescence.

Twelve-month-old control and $\mathrm{KC}$ mice were metabolically labelled with $\mathrm{Ac}_{4} \mathrm{ManAz}$ using varying dosage regimens: one, two, or three doses of $A c_{4} M a n A z$ by oral gavage or three doses of $A c_{4} M a n A z$ by i.p. injection (Figure 3.7). The effect of dosing regimen on metabolic labelling was compared. No significance difference in Alexa-488 fluorescence was observed in mice treated with one, two or three doses of $\mathrm{Ac}_{4} \mathrm{ManAz}$ by oral gavage.
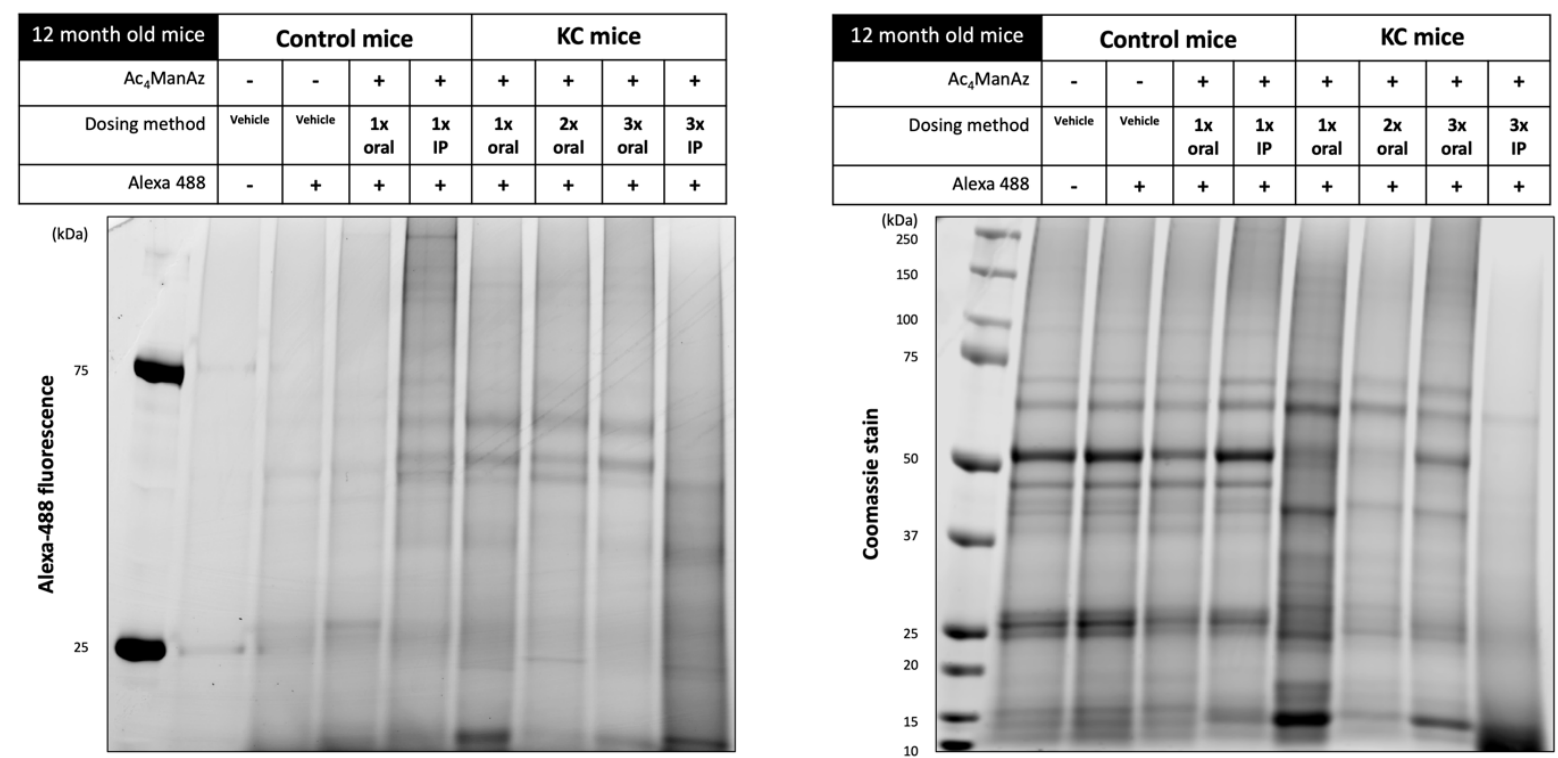

Figure 3.7: Dose regimen-dependent metabolic labelling of 12-month-old KC mice in vivo.

Alkyne-Alexa 488 labelled membrane extracts were obtained from 12-month-old control mice and $K C$ mice, that had been treated with vehicle or varying dosing regimens of $N$ - 
azidoacetylmannosamine (Ac4ManAz). Regimens were one, two, or three doses of $A c_{4} M a n A z$ by oral gavage or three doses of $A c_{4} M a n A z$ by i.p. injection. $10 \mu g$ of the extracts were resolved by gel electrophoresis and subjected to in-gel fluorescence imaging. Total protein per lane was visualised using Coomassie staining. Degradation of protein in the sample +Ac4ManAz/3x i.p./+Alexa-488 was inferred by band smearing on the Coomassie stained gel. Background controls were included to detect non-specific fluorescence.

Overall, the data suggested that monitoring differential tumour glycosylation using in vivo models of pancreatic cancer progression may pose challenges such as reduced contrast caused by non-specific background. Maintaining tissue integrity after extraction may need to be optimised, although this occurred randomly and in a minority of samples.

\subsection{Discussion}

Bioorthogonal azide-based chemical reporters (Laughlin et. al. 2009), generally called azidosugars, have been used to visualise glycosylation in tumour-bearing mice using whole animal fluorescence (Figure 1.21c and d), MRI (Figure 1.21e) and PET imaging (Neves et. al. 2011; Neves et. al. 2013; Neves et. al. 2015). However, these protocols have yet to be translated into preclinical models of cancer progression. In this study, we quantified azidosugar labelling in a previously validated in vitro model of breast cancer progression (Chapter 2) and an advanced mouse model of pancreatic cancer, with the ultimate aim of imaging tumour glycosylation in vivo. Imaging tumour glycosylation using a clinically relevant imaging modality to measure the propensity of a tumour to metastasise, and may enable us to distinguish lowly glycosylated benign lesions from highly glycosylated aggressive lesions to reduce unnecessary biopsies, and misdiagnosis.

An increase in $\mathrm{Ac}_{4} \mathrm{ManNAz}$ and $\mathrm{Ac}_{4}$ GalNAz labelling correlated with an increase in the metastatic potential of the breast cancer cell lines (Figure 3.2a). Zhou and colleagues used lectin arrays to study differential glycosylation in the metastatic MDA MB 231 cell lines, and 
5 additional cell lines with increasing metastatic potential, derived from the tumourigenic MDA MB 231 cell line. An increase in Ricinus communis agglutinin binding, which recognises lactose and galactose, an increase in Sambucus nigra lectin binding (also called elderberry bark lectin), which recognises sialic acid (Zhou et. al. 2015), and a decrease in wheat germ agglutinin lectin binding, which recognises $\mathrm{N}$-acetylglucosamine, was correlated with an increase in the metastatic potential of the MDA MB 231 derived cell lines. This is consistent with the increases in cell surface $\mathrm{Ac}_{4} \mathrm{ManNAz}$ observed here (Figure 3.3b), and increases in sialylation associated with an increase in the metastatic potential of several cancers (Natoni et. al. 2016). Levels of Helix pomatia agglutinin (HPA) binding, which recognises Nacetylgalactosamine, has been previously quantified using flow cytometry in breast cancer cell lines. Increased HPA binding was detected in the HMT 3522 cell line, representing normal breast epithelium (Briand et. al. 1987), and tumourigenic MDA MB 453, MCF 7 and MDA MB 468 cell lines (Chapter 2: Table 2.1b), illustrating a general increase in Nacetylgalactosamine incorporation into cell surface glycoconjugates (Brooks et. al. 2001). Increased levels of HPA binding in primary breast cancer paraffin-embedded tissue sections have also been correlated with tumour size and the presence of axillary lymph node metastases (Brooks and Leathem 1991). Furthermore, glycoproteomic analysis of N-glycan's released from human breast tissue extracts and serum proteins, using high-performance liquid chromatography, revealed significant increases in the levels of $\mathrm{N}$-acetylgalactosaminecontaining Sialyl Lewis X in breast cancer patients, relative to healthy individuals, and even higher levels in breast cancer patients with circulating tumour cells (Saldova et. al. 2011). These studies are consistent with an increase in $\mathrm{Ac}_{4} \mathrm{GalNAz}$ labelling, which correlated with an increase in the metastatic potential of the breast cancer cell lines observed in this study (Figure 3.3c).

Chan and colleagues used a xenograft mouse model of lymphosarcoma, and WGA, to compare the composition of glycoproteins extracted from primary tumours and liver metastases. When lymphosarcoma cells, exhibiting the highest level of WGA binding, were re-implanted into regions of the liver, which previously harboured a metastatic deposit, the resulting tumour exhibited less WGA binding (Chan et. al. 1984). Their data suggested that a tumours immediate environment contributed towards driving differential glycosylation during the development of a metastatic phenotype, a now well-reported concept (Peixoto 
et. al. 2019). Therefore, we investigated if the differential glycosylation observed in cell lines with increasing metastatic potential could be replicated in advanced mouse models, which were more representative of a tumours 3D architecture, and the host-tumour microenvironment. In the absence of available in vivo models of breast cancer progression (Holen et. al. 2017), biorthogonal labelling techniques were tested in the p48Cre; LSLKrasG12D (KC) mouse model (Hingorani et. al. 2003; Westphalen and Olive 2012), where the evolution of pancreatic ductal adenocarcinoma (PDAC) from intraepithelial neoplasia (PanIN) precursor lesions to tumours, has been replicated (Hingorani et. al. 2003; Westphalen and Olive 2012) (Chapter 1: Figure 1.25a and b).

We demonstrated, what we believe to be the first instance of bioorthogonal metabolic labelling and visualisation of cell surface glycans in 3D pancreatic tumour organoids (Figure 3.4). $A c_{4} M a n N A z$ and $A c_{4} G a l N A z$ labelling were localised to the plasma membrane, as observed previously by confocal microscopy in metabolically labelled Lewis lung carcinoma (Stöckmann et. al. 2011), and MDA MB 231 breast cancer (Kuo 2015, PhD thesis, unpublished) cell lines.

We cultured and cryopreserved pancreatic organoids obtained from normal pancreatic tissue, and PanIN precursor lesions, to quantify azidosugar labelling in this 3D organoid model of pancreatic cancer progression. Organoids derived from less aggressive phenotypes required complicated and expensive growth factor-rich media preparations. Furthermore, organoids exhibited low proliferation rates, which hindered the high throughput culturing needed to obtain enough cell numbers for quantitative flow cytometry. Studies using the pancreatic organoids was consequently abandoned.

Next, we adopted an alternative approach by conducting metabolic labelling of KC mice in vivo, following by quantification of azidosugar labelling ex vivo. Control mice (with a normal pancreas), and KC mice (with a diseased pancreas), aged 3, 6 and 12 months, were treated with vehicle or varying dosing regimens of $A c_{4} M a n A z$ via oral gavage or i.p. injection. Pancreatic tissue was collected and the membrane proteome isolated based on the observation of extensive azidosugar labelling within the plasma membrane of KC pancreatic tumour organoids (Figure 3.4). The incorporated azide groups were covalently ligated to 
alkyne-Alexa Fluor 488, ex vivo, using copper-catalysed chemistry. Labelled extracts were resolved by gel electrophoresis, and subjected to western blot analysis and in-gel fluorescence imaging.

Increases in sialyation (Büll et. al. 2014) of cell surface glycoproteins (Schultz et. al. 2012) has been observed in pancreatic cancers (Munkley 2019) and associated with increased disease progression and metastasis (Stowell et. al. 2015). Therefore, the extent of Ac 4 ManNAzsialylation was compared between 6 month and 12-month-old mice, based on the reasoning that older KC mice, exhibiting greater tumour burden, would show higher levels of azidosugar labelling. We could not confidently investigate age-dependent metabolic labelling due to high levels of background fluorescence, which was generally higher in the older, 12month-old KC mouse (Figure 3.6b). Improving delivery of the azidosugars to pancreatic tumours may reduce background labelling, and could be achieved using liposomes, nasal administration or osmotic pumps (Xie et. al., 2012; Xie et. al., 2014). This would be beneficial for fluorescence imaging, which despite being highly sensitive and high throughput, is limited by light absorption and scattering, which prevents deep imaging in opaque organisms such as mice (Ntziachristos 2006). Overall, the correlation between mouse age and nonspecific background suggest that imaging of tumour glycosylation in vivo may be more suitable in monitoring the early stages of the disease.

Our data have yet to support the notion that bioorthogonal glycan imaging could be useful for monitoring the propensity of a tumour to metastasise in vivo i.e. for assessing the aggressiveness of the disease. Translating this concept would require biorthogonal labelling of live tissue by administering azidosugars directly to patients in vivo. A more realistic scenario would be to conduct metabolic labelling of patient tissue biopsies to quantify azidosugar labelling ex vivo. Spiciarich and colleagues successfully metabolically labelled prostate tissue slices derived from radical prostatectomy specimens and observed $\mathrm{Ac}_{4} \mathrm{ManNAz}$ specific labelling of cell surface glycoconjugates using confocal microscopy (Spiciarich et. al. 2017). However, this ex vivo bioorthogonal metabolic labelling technique has not been translated into clinical practice. Our flow cytometry data, although limited, suggest that the glycoprotein fraction may be principally responsible for generating the increases in sialyation and $\mathrm{N}$-acetylgalactosamine glycosylation observed with an increase in 
the metastatic potential of the breast cancer cell lines (Figure 3.3). Assessing the dynamics of azidosugar incorporation into cell surface glycoproteins using gel electrophoresis may confirm the presence of differentially glycosylated glycoproteins, which if identified using mass spectrometry, may be predictive biomarkers of cancer progression.

\subsection{Materials and Methods}

\subsubsection{Cell lines}

The MDA MB 453, MCF 7, MDA-MB-468, and MDA MB 231 human breast cancer cell lines were obtained from the Cancer Research UK Cambridge Institute Biorepository, based on stocks made available by Joe Kuo. All breast cell lines were cultured in DMEM with Lglutamine (Gibco ${ }^{\circledR}$, Invitrogen ${ }^{\mathrm{TM}}$ ) and 10\% Foetal bovine serum (FBS Gold, PAA Laboratories).

Cell lines were genotyped and authenticated by Short Tandem Repeat (STR) DNA profiling and comparison with available databases from ATCC or DSMZ. All cell lines tested negative for Mycoplasma using the MycoProbe ${ }^{\mathrm{TM}}$ Kit (R\&D Systems).

\subsubsection{Tissue culture}

Cell lines were maintained in Nunc ${ }^{\mathrm{TM}} 175 \mathrm{~cm}^{2}$ flasks (Thermo Fisher Scientific ${ }^{\mathrm{TM}}$ ) at $5 \% \mathrm{CO}_{2}$, in $37^{\circ} \mathrm{C}$ humidified incubators, and cultured to $80 \%$ confluence. Briefly, cells were washed once with warm Phosphate Buffered Saline (20 ml/flask; $8 \mathrm{~g} \mathrm{NaCl}, 0.2 \mathrm{~g} \mathrm{KCl}, 1.44 \mathrm{~g} \mathrm{Na}_{2} \mathrm{HPO}_{4}$, $0.24 \mathrm{~g} \mathrm{KH}_{2} \mathrm{PO}_{4}$ per litre, $\mathrm{pH} 7.4$ ), and trypsinised for 7 minutes at $37^{\circ} \mathrm{C}$. Trypsinised cells were added to equal volumes of media, centrifuged at $700 \times \mathrm{g}$ for 4 minutes $\left(4^{\circ} \mathrm{C}\right)$, and resuspended in fresh media. Viable cell counts were obtained by Trypan blue dye exclusion using a LUNA ${ }^{\mathrm{TM}}$ Automated Cell Counter (Logos Biosystems), and cells seeded at $3 \times 10^{4}$ cells $/ \mathrm{cm}^{2}$. 


\subsubsection{Bioorthogonal metabolic labelling in vitro for flow cytometry}

$\mathrm{N}$-azidoacetylmannosamine ( $\left.\mathrm{Ac}_{4} \mathrm{ManNAz}\right), \mathrm{N}$-azidoacetylgalactosamine ( $\mathrm{Ac} \mathrm{C}_{4} \mathrm{GalNAz}$ ) and TMDIBO-PEG 3 -biotin were synthesised by Henning Stöckmann, Department of Chemistry.

Cells were seeded at $1.75 \times 10^{4}$ cells $/ \mathrm{cm}^{2}$ in Nunc ${ }^{T M} 175 \mathrm{~cm}^{2}$ flasks (Thermo Fisher Scientific ${ }^{\mathrm{TM}}$ ) and maintained at $5 \% \mathrm{CO}_{2}$, in $37^{\circ} \mathrm{C}$ humidified incubators for 3 days before metabolic labelling experiments. Flasks were then washed once with $20 \mathrm{ml}$ warm Phosphate Buffered Saline (PBS; $8 \mathrm{~g} \mathrm{NaCl}, 0.2 \mathrm{~g} \mathrm{KCl}, 1.44 \mathrm{~g} \mathrm{Na}_{2} \mathrm{HPO}_{4}, 0.24 \mathrm{~g} \mathrm{KH_{2 }} \mathrm{PO}_{4}$ per litre, $\mathrm{pH} 7.4$ ), and cells trypsinised for 7 minutes at $37^{\circ} \mathrm{C}$. Trypsinised cells were added to equal volumes of media, centrifuged at $700 \times \mathrm{g}$ for 4 minutes $\left(4^{\circ} \mathrm{C}\right)$ and resuspended in fresh media. Viable cell counts were obtained by Trypan blue dye exclusion using a LUNA ${ }^{\text {TM }}$ Automated Cell Counter (Logos Biosystems), and cells seeded at $3 \times 10^{4}$ cells $/ \mathrm{cm}^{2}$ in Nunc ${ }^{\mathrm{TM}} 6$ well plates (Thermo Fisher Scientific ${ }^{\mathrm{TM}}$ ) with $3 \mathrm{ml}$ media/well. Plates were incubated in $5 \% \mathrm{CO}_{2}, 37^{\circ} \mathrm{C}$ for 24 hours. After 24 hours, the media was replaced with fresh media only (0.1\% DMSO, vehicle

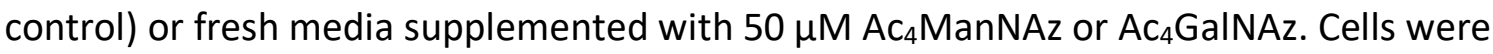
incubated in $5 \% \mathrm{CO}_{2}$ at $37^{\circ} \mathrm{C}$ for 24 hours. After 24 hours, cells were harvested by trypsinisation and counted as described previously.

$4 \times 10^{5}$ cells from each treatment group were transferred into separate Nostick ${ }^{\circledR}$ hydrophobic microcentrifuge tubes (Scientific Specialties, Inc.), pelleted by centrifugation (700 x g for 4 minutes at $4^{\circ} \mathrm{C}$ ), and washed with $1 \mathrm{ml}$ ice-cold Flow Cytometry Staining (FACS) buffer (PBS with 1\% FBS). From here onwards, all samples and dyes were kept on ice. The washed pellets were resuspended in $100 \mu \mathrm{l}$ FACS buffer or $100 \mu \mathrm{l}$ FACS buffer supplemented with $20 \mu \mathrm{M}$ TMDIBO-PEG 3 -biotin, and incubated in a heating block for 25 minutes at $37^{\circ} \mathrm{C}$ with agitation (400 rpm). Reactions were quenched with $700 \mu$ FACS buffer, cells harvested, and the pellets washed 3 times with FACS buffer as before. After washing, cells were resuspended in $100 \mu$ I FACS buffer, or $100 \mu$ I FACS buffer supplemented with $0.835 \mu \mathrm{M}$ NeutAvidin ${ }^{\mathrm{TM}}$ Dylight $^{\mathrm{TM}} 650$ (Thermo Fisher Scientific ${ }^{\mathrm{TM}}$ ) and 50 nM Sytox ${ }^{\circledR}$ Green cell death marker (Invitrogen ${ }^{\mathrm{TM}}$ ), or $100 \mu \mathrm{l}$ FACS buffer supplemented with $50 \mathrm{nM}$ Sytox ${ }^{\circledR}$ Green cell death marker (Invitrogen ${ }^{\mathrm{TM}}$ ) only. Cells were incubated in a heating block for 15 minutes at $37^{\circ} \mathrm{C}$ with agitation (400 rpm). Reactions were quenched with $700 \mu \mathrm{l}$ FACS buffer, cells 
harvested, and the pellets washed twice with FACS buffer as before. Washed cells were filtered into $35 \mu \mathrm{m}$ pore cell strainer capped cytometry tubes (Falcon ${ }^{\circledR}, \mathrm{BD}$ Life Sciences), and analysed using an LSR II flow cytometer (BD Life Sciences).

10,000 events were recorded per analysis from viable cells only, characterised by their high ultraviolet autofluorescence (high cellular NADH content) and low Sytox ${ }^{\circledR}$ Green fluorescence (intact membrane, dye exclusion). The Mean Fluorescence Intensity ratio (MFI ratio) was obtained by normalising the average fluorescence intensity from azidosugar labelling against background fluorescence.

\subsubsection{Pancreatic tumour organoid cultures}

Mouse pancreatic tumours were isolated from p48Cre; LSL-KrasG12D (KC) mice (Hingorani et. al. 2003; Westphalen and Olive 2012) by Dr Aarthi Gopinathan (Jodrell Laboratory, CRUK $\mathrm{Cl}$ ) following a protocol from the Tuveson Lab (Available at: http://tuvesonlab.labsites.cshl.edu/protocolsreagents/, 2019). Briefly, this involved dissecting the tumour from the pancreas, disruption of the tumour by mincing, enzymatic digestion, and treatment with DNAase and trypsin. Following centrifugation and resuspension of the cell pellet in Matrigel (BD Biosciences) $25 \mu \mathrm{l}$ Matrigel (BD Biosciences) domes were spotted into a pre-warmed 48-well plate on top of a pre-warmed water bottle (Nunc ${ }^{T M} 175 \mathrm{~cm}^{2}$ flasks (Thermo Fisher Scientific ${ }^{\text {TM}}$ ) filled with Milli-Q $18.2 \Omega$ water), and brought to the Brindle lab for culturing. Organoids were cultured following a protocol from the Tuveson Lab (Available at: http://tuvesonlab.labsites.cshl.edu/protocolsreagents/, 2019), and the "media overlay" method reported in the literature (Baker et. al. 2016) (Figure 3.8a). 
a.

\section{Clevers and Tuveson labs pancreatic ductal organoids}

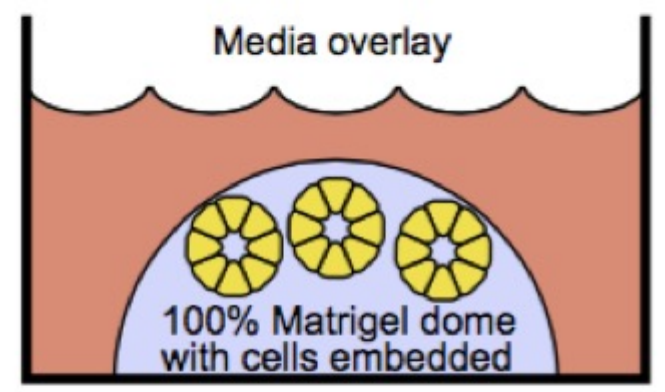

b.

\section{Muthuswamy lab progenitor and PDA organoids}

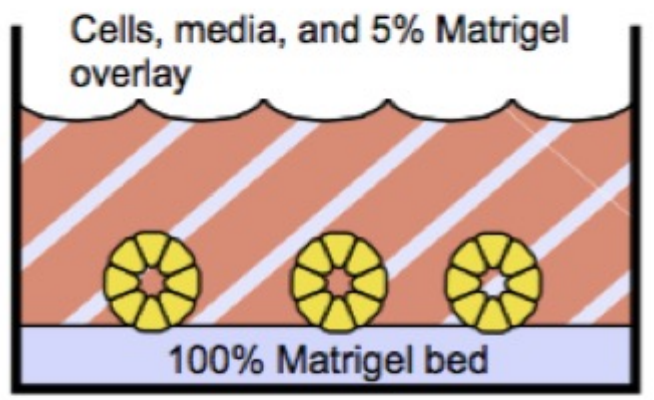

Figure 3.8: Pancreatic organoid cultures. Diagrams show a cross-section of pancreatic organoid culturing methods reported by the Cleavers and Tuveson labs (a) and the Muthuswamy lab (b). Matrigel is depicted in blue, organoids in yellow, and culture medium in brown. (Figure extracted from Baker et. al. 2016).

Generally, $25 \mu$ l domes of Matrigel (BD Biosciences) were melted by resuspension in $500 \mu \mathrm{l}$ ice-cold DMEM with glucose, L-glutamine (Gibco ${ }^{\circledR}$, Invitrogen ${ }^{\mathrm{TM}}$ ) and 5 \% FBS (FBS Gold, PAA Laboratories). The suspension was placed in a $15 \mathrm{ml}$ Falcon tube containing $8 \mathrm{ml}$ ice-cold media. Wells were washed with $500 \mu \mathrm{l}$ ice-cold DMEM to dissolve any residual matrigel and added to the $15 \mathrm{ml}$ Falcon tube. Organoids were pelleted by centrifugation at $700 \times \mathrm{g}$ for 6 minutes and an appropriate amount of the supernatant removed ( $2 \mathrm{ml}$ media remaining). Pellets were resuspended, and media topped up to a total of $10 \mathrm{ml}$. Disrupted organoids were pelleted at $700 \times \mathrm{g}$ for 6 minutes, the supernatant removed and pellets resuspended in fresh Matrigel (25 $\mu$ l per dome) (BD Biosciences). $25 \mu$ l of the matrigel suspension was "spotted" into the centre of each well of a 48 well plate (Corning), and incubated at $37^{\circ} \mathrm{C}$ for 15 minutes on a pre-warmed water bottle to allow the matrigel to set. Wells were topped up with $500 \mu \mathrm{l}$ warm media and incubated at $5 \% \mathrm{CO}_{2}$, in $37^{\circ} \mathrm{C}$ humidified incubators. 


\subsubsection{Bioorthogonal metabolic labelling of pancreatic tumour organoids for confocal microscopy}

Metabolic labelling of pancreatic tumour organoids was conducted based on a modified " $5 \%$ media overlay" culturing method reported in the literature (Baker et. al. 2016) (Figure 3.8b).

Each chamber of a 6-chambered glass coverslip (Ibidi ${ }^{\circledR}$ ) was evenly coated with $100 \mu \mathrm{l}$ fresh Matrigel (BD Biosciences). Coated slides were incubated at $37^{\circ} \mathrm{C}$ for 30 minutes on a prewarmed water bottle to allow the matrigel to set. Organoids were split, as described previously. After removing all the supernatant, the organoid pellet was resuspended in $1 \mathrm{ml}$ pre-warmed TrypLE ${ }^{\mathrm{TM}}$ (Thermo Fisher Scientific ${ }^{\mathrm{TM}}$ ), which is a gentler and more stable alternative to trypsin. Cells were incubated in a $37^{\circ} \mathrm{C}$ incubator for 10 minutes and gently shaken 3 times during the incubation period. TrypLE ${ }^{T M}$ was neutralised by adding $10 \mathrm{ml}$ icecold media, and the solution resuspended to break up the "white spaghetti" characteristically generated after trypsinising organoids. The single-cell suspension was centrifuged at $700 \times \mathrm{g}$ for 6 minutes $\left(4^{\circ} \mathrm{C}\right)$ and the supernatant completely aspirated. The pellet was resuspended in a $3.5 \mathrm{ml}$ solution of warm media containing 5\% Matrigel, and 400 $\mu \mathrm{l}$ of the suspension pipetted over each chamber in a drop-wise manner. The remaining suspension was used to obtain a cell count and measure of viability using the Vi-CELL XR cell viability analyser (Beckman Coulter). After 24 hours, chamber contents were replaced with

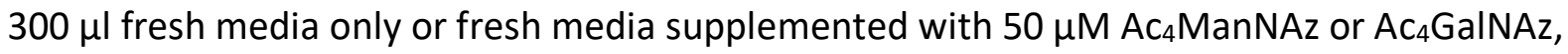
and incubated at $5 \% \mathrm{CO}_{2}$, in $37^{\circ} \mathrm{C}$ humidified incubators for 24 hours.

After 24 hours, media was removed and chambers washed twice in $200 \mu$ l pre-warmed Flow Cytometry Staining (FACS) buffer (PBS with 1\% FBS). A single wash involved pipetting FACS buffer over the wells twice before placing in a $37^{\circ} \mathrm{C}$ incubator for 10 minutes. The wash was removed completely and chambers overlaid with $100 \mu$ I FACS buffer or FACS buffer supplemented with $20 \mu \mathrm{M}$ DIBO-PEG - -biotin, and incubated at $37^{\circ} \mathrm{C}$ for 30 minutes. After 30 minutes, the TMDIBO-biotin was removed completely and the wells washed 4 times with $200 \mu$ FACS buffer. The final wash with FACS buffer was removed completely and chambers overlaid with $100 \mu$ I FACS buffer, or FACS buffer supplemented with $0.835 \mu \mathrm{M}$ NeutAvidin ${ }^{\mathrm{TM}}$ Dylight $^{\mathrm{TM}} 650$ (NA650) (Thermo Fisher Scientific ${ }^{\mathrm{TM}}$ ), and incubated at $37^{\circ} \mathrm{C}$ for 20 minutes. 
After 20 minutes, NA650 was removed, and the wells washed twice with $200 \mu$ l warm FACS buffer. Organoids were fixed in $200 \mu \mathrm{l} 10 \%$ neutral buffered formalin for 10 minutes at room temperature. After 10 minutes, the formalin was removed completely, and the chambers washed 4 times with FACS buffer. Organoids were counterstained with DAPI ( $300 \mathrm{nM})$ and stored in $200 \mu \mathrm{FACS}$ buffer at $4^{\circ} \mathrm{C}$ until confocal imaging using a Leica Tandem confocal microscope.

\subsubsection{Bioorthogonal metabolic labelling of p48Cre; LSL-KrasG12D (KC) mice in vivo and chemoselective ligation of the metabolically labelled membrane proteins ex vivo}

p48Cre; LSL-KrasG12D (KC) mice (Hingorani et. al. 2003; Westphalen and Olive 2012) aged 3, 6 and 12 months, and C57BL/6 control wild-type mice (Serrao et. al. 2015) (age matched to KC mice) were bred in-house at the Cancer Research UK Cambridge Institute, and metabolically labelled with $\mathrm{Ac}_{4} \mathrm{ManNAz}$ in vivo by Dr André Neves (Brindle Laboratory, CRUK $\mathrm{Cl})$.

Mice were dosed daily, either orally by gavage, or intraperitoneally, for up to 3 days, with a solution of $\mathrm{AC}_{4}$ ManNAz (150 mg/kg) in DMSO:PEG400:PBS (5:10:85\%) or with the solvent vehicle alone. 24 hours after the last dose of azido-sugar, mice were sacrificed and the pancreas removed and rapidly frozen in isopentane on dry ice.

The membrane protein fraction was isolated from pancreatic tissue using a modified protocol of the Qproteome ${ }^{\circledR}$ Cell Compartment kit (Qiagen). Briefly, samples of pancreatic tissue were cut into small pieces and transferred to Precellys ${ }^{\circledR} 7 \mathrm{ml}$ bead-containing vials. A weight-adjusted volume of Extraction Buffer CE1 was added ( $5 \mathrm{ml} / \mathrm{g}$ tissue) and the tissue homogenised at $4^{\circ} \mathrm{C}$ using $3 x$ cycles of $6000 \mathrm{rpm}$ for 30 seconds, using a Precellys Evolution homogeniser (Bertin Technologies). Homogenisation was carried out by Ms Sarah McGuire (Brindle Laboratory, CRUK CI). Lysates were transferred to fresh Nostick ${ }^{\circledR}$ hydrophobic microcentrifuge tubes (Scientific Specialties, Inc.), and centrifuged at 1000 x g for 10 minutes at $4^{\circ} \mathrm{C}$. The supernatant, primarily containing cytosolic proteins, was transferred into a fresh Nostick ${ }^{\circledR}$ hydrophobic microcentrifuge tubes (Scientific Specialties, Inc.) and stored at $-20^{\circ} \mathrm{C}$. 
The remaining pellet was resuspended in $1 \mathrm{ml}$ Extraction Buffer CE2 and incubated for 30 minutes at $4^{\circ} \mathrm{C}$ on an end-over-end shaker (30 rpm). Lysates were centrifuged at $6000 \times \mathrm{g}$ for 10 minutes at $4^{\circ} \mathrm{C}$. The supernatant, primarily containing membrane proteins, was transferred into fresh Nostick ${ }^{\circledR}$ hydrophobic microcentrifuge tubes (Scientific Specialties, Inc.). Lysates were stored at $-20^{\circ} \mathrm{C}$.

Four volumes of ice-cold acetone were added to the membrane protein-enriched fractions and incubated for 1 hour on ice. Precipitated proteins were pelleted by centrifugation at 12 , $000 \times \mathrm{g}$ for 10 minutes $\left(4^{\circ} \mathrm{C}\right)$. The supernatant was discarded and the protein pellets air-dried for 15 minutes. Dried protein pellets were resuspended in $100 \mu \mathrm{l}$ Tris-HCL buffer $(50 \mathrm{mM}, \mathrm{pH}$ $8,0.5 \%$ SDS). Residual acetone was removed by the sequential addition of $600 \mu \mathrm{l}$ methanol, $150 \mu \mathrm{l}$ chloroform, and $400 \mu \mathrm{l}$ Milli-Q $18.2 \Omega$ water, with vortexing. After centrifugation at $18,000 \times \mathrm{g}$ for 10 minutes $\left(4^{\circ} \mathrm{C}\right)$, the upper aqueous phase was removed and the precipitated proteins washed twice with $450 \mu \mathrm{l}$ methanol. The pellets were air-dried for a minimum of 15 minutes and resuspended in $100 \mu \mathrm{l}$ Tris-HCL buffer (50 mM, pH 8, 0.5\% SDS). The total protein concentration was determined using the DC ${ }^{\mathrm{TM}}$ Protein Assay (Bio-Rad), using BSA as a standard. Lysates were stored at $-20^{\circ} \mathrm{C}$.

Up to $200 \mu \mathrm{g}$ of metabolically labelled protein was chemoselectively reacted with alkyneAlexa Fluor 488 (Life Technologies), using the Click iT Protein Reaction Kit (Life Technologies), according to the manufacturer's protocol. Residual reactants were removed by methanol/chloroform/Milli-Q $18.2 \Omega$ water precipitation, as described previously. Protein pellets were dried for a minimum of 15 minutes, resuspended in $200 \mu \mathrm{l}$ Tris HCL buffer (50 $\mathrm{mM}, \mathrm{pH} 8,0.5 \% \mathrm{SDS}$ ), and sonicated for 30 seconds ( 9 cycles of 10 seconds ON and 5 seconds OFF) using the Biorupter ${ }^{\circledR}$ Plus (Diagenode). The total protein concentration was determined using the DC ${ }^{\mathrm{TM}}$ Protein Assay (BIO-RAD), using BSA as a standard. Samples were stored at $-20^{\circ} \mathrm{C}$. 


\subsubsection{D gel electrophoresis and in-gel imaging of azidosugar labelled glycoproteins}

Metabolically labelled membrane protein fractions reacted with alkyne-Alexa Fluor 488 in Tris-HCL buffer (50 mM, pH 8, 0.5\% SDS) were loaded on to precast 4-12\% 1mm Bis-Tris gels (Thermo Fisher Scientific ${ }^{\text {TM}}$ ) and resolved in 1x NuPAGE MOPS buffer (Invitrogen) (10 $\mu \mathrm{g} /$ lane; $30 \mathrm{~min}$ at $60 \mathrm{~V}$ and $120 \mathrm{~min}$ for $120 \mathrm{~V}$ ). Alexa Fluor 488 fluorescence (Cy2; $489 \mathrm{~nm}$ maximum excitation wavelength, $506 \mathrm{~nm}$ maximum emission wavelength) was imaged in-gel with a Typhoon scanner (GE Healthcare). Gels were subsequently stained with Instant Blue ${ }^{\mathrm{TM}}$ Coomassie (Expedeon) or silver stain (Pierce Kit) and imaged using an Odyssey CLx Infrared Imaging System (LI-COR Biosciences) or Image Scanner III (GE Healthcare), respectively.

\subsubsection{Statistical analysis}

All p-values were calculated using a one-way ANOVA test. 


\section{Chapter 4: Investigation of differential glycosylation using bioorthogonal chemistry and gel electrophoresis in in vitro and in vivo models of cancer progression}

\section{$\underline{4.1 \text { Introduction }}$}

\subsubsection{Cell surface protein glycosylation in cancer}

Glycans are the complex carbohydrate structures formed when monosaccharides link together via glycosidic bonds. Glycoproteins are defined by the amino acid upon which the glycan structures are installed (Chapter 1: Figure 1.4). The synthesis of the N-linked glycoproteins involves covalent attachment of $\mathrm{N}$-acetylglucosamine to an asparagine (Asn) residue, via its amide side chain, within the target sequence: Asn-X-Serine/Threonine ( $X=$ any amino acid except proline) (Chapter 1: Figure 1.5). The synthesis of the O-linked glycoproteins involves the covalent attachment of carbon 1 in $\mathrm{N}$-acetylgalactosamine to serine or threonine residues via their hydroxyl groups (Pinho and Reis 2015). Within a single polypeptide, multiple asparagine, serine, and threonine residues are available for glycan installation. This can occur at different positions, across different polypeptides, at any given time. Monosaccharides can bond at different carbon positions, between carbons with the same stereochemistry ( $\alpha$-glycosidic bond), between carbons with different stereochemistries ( $\beta$-glycosidic bond), and in linear or branched forms (Seeberger 2017), and can conjugate to acyl (non-sugar) groups within proteins that are embedded in the cell membrane or secreted. Therefore, glycoproteins are highly diverse and their synthesis is dependent on the dynamic interactions between glycoenzymes within a specific cell type. These dynamic interactions are sensitive to physiological and pathological changes (Reily et.al. 2019; Krasnova and Wong 2016; Mariño et. al. 2010) such as in cancer, where abnormal changes in the expression and degradation of glycoproteins have been implicated (Ohtsubo et. al. 2006; Varki et. al. 2017) due to mislocalisation of glycosyltransferases, altered expression of 
glycoenzymes and chaperones, and mutations in genes encoding glycosyltransferases (Stowell et. al. 2015).

Aberrant glycosylation of $\mathrm{N}$-linked (Chapter 1: Figure 1.5) and O-linked glycoproteins can affect growth factor signalling, cell proliferation, adhesion, and migration, and promote tumour progression through enhanced cell-matrix interactions, invasion, angiogenesis, and metastasis (Moremen et. al. 2012) (Chapter 1: Figure 1.8b). For example, the synthesis of complex N-glycan's on E-cadherin by MGAT5 upregulation causes E-cadherin internalisation and disruption of cell-to-cell adhesion sites. O-glycosylated CD44 binds endothelial Eselectin promoting metastasis, and O-glycosylated integrins mediate attachment of tumour cells to the extracellular matrix (Oliveira-Ferrer et. al. 2017). Aberrant O-glycosylation of glycoproteins with an unmodified or sialylated core 10 -glycan called the ThomsenFriedenreich ( $T$ ) antigen (Chapter 1: Figure 1.10), has been suggested as the most common tumour associated glycan alteration on carrier proteins, overexpression of which has been correlated with increases in disease progression, metastasis and poor prognosis in several cancers (Stowell et. al. 2015).

Aberrant O-glycosylation of mucins has been observed in over $90 \%$ of breast cancers and associated with overexpression of $\mathrm{N}$-acetylgalactosaminyltransferases such as GALNACT6, and reduced cell adhesion (Burchell et. al. 2018). In prostate cancer, aberrant glycosylation of O-linked glycoproteins is thought to be mediated by overexpressed glycosyltransferases, such as ST6GALNAC1, which are induced by activated androgen receptors (Munkley 2017) and that synthesise tumour associated antigens such as sialylated Tn antigen and Sialyl Lewis $X$ (Chapter 1: Figure 1.8). Androgen has also been shown to control aberrant mannose trimming of $\mathrm{N}$-glycan's on proteins by inducing the overexpression of mannosidases such as EDEM3 (Munkley et. al. 2016). Elevated levels of Sialy Lewis A antigens (Chapter 1: Figure 1.8) on carrier proteins such as mucin 1 (MUC1) are associated with pancreatic cancer progression and are monitored in the clinical CA 19-9 assay (Pinho and Reis, 2015). Increased fucosylation of mucins, and $\mathrm{N}$-linked integrins and extracellular matrix adhesion proteins have been detected in the tissue or blood of patients with pancreatic intraepithelial neoplasia (PanIN) precursor lesions, pancreatic cancer and metastatic disease, when compared to healthy individuals (Munkley 2019; Pan et. al. 2016). Therefore, cell surface 
glycoproteins and their associated glycan structures facilitate interaction of tumour cells with the tumour microenvironment and are vital for promoting cancer progression (Peixoto et. al. 2019).

\subsubsection{Detection of aberrantly glycosylated cell surface glycoproteins using bioorthogonal chemistry}

Traditional methods for studying aberrant glycosylation include the use of affinity reagents such as lectin arrays and antibodies, which recognise the carbohydrate moieties (Cummings 2009).

Lectins such as wheat germ agglutinin (WGA), which binds preferentially to $\mathrm{N}$ acetylglucosamine residues (Cummings and Etzler 2009), Helix pomatia agglutinin (HPA), which can recognise the $\mathrm{N}$-acetylgalactosamine residues of the Thomsen-nouveau (Tn) antigen (Chapter 1: Figure 1.9), and peanut agglutinin (PNA), which can recognise T antigen (Chapter 1: Figure 1.10), and Core 2 O-glycan's (Oliveira-Ferrer et. al. 2017) (Chapter 1: Figure 1.4, red perforated box), have been used to characterise tumours using histochemistry and lectin blotting. For example, extracts obtained from liver metastases exhibited less WGA binding than lymphosarcoma tissue extracts (Chan et. al. 1984), breast tumour extracts exhibited less WGA binding than extracts from healthy breast tissue ( $\mathrm{Ng}$ et. al. 1987), and increases in HPA binding have been observed in lymph node metastases (Kölbl et. al. 2015), and decreases in WGA binding have been seen during the progression of preinvasive Barrett's oesophagus to adenocarcinoma (Bird-Lieberman et. al. 2012) (Chapter 1: Figure 1.17c). However, steric hinderances can affect interactions of lectins with aberrantly glycosylated glycoproteins (Stowell et. al. 2015).

Anti-carbohydrate antibodies are also in development, which recognise Tn antigen presented on circulating glycoproteins (Kveton et. al. 2017). However, defining the exact glycan sequences recognised by anti-carbohydrate antibodies has proven more difficult than defining the peptide epitopes traditionally targeted by antibodies (Stowell et. al. 2015). Anti- 
Tn antibodies, based on the IgM or IgG immunoglobulin classes, have shown varying affinities and specificities for the Tn antigen (Blixt et. al. 2012).

More novel ways of detecting aberrantly glycosylated proteins involve the use of bioorthogonal monosaccharide analogues containing a functional azide group or "chemical reporter" not normally present in the cell (Laughlin et. al. 2009), which are incorporated metabolically into cellular glycan structures, and subsequently detected by covalent ligation of imaging probes or affinity reagents that rapidly react with the azide group (Saxon and Bertozzi 2000; Saxon et. al. 2002) (Chapter 1: Figure 1.20). We previously demonstrated the use of bioorthogonal chemistry for detecting differential $\mathrm{N}$-azidoacetylmannosamine $\left(\mathrm{Ac}_{4} \mathrm{ManAz}\right)$-specific sialyation and $\mathrm{N}$-azidoacetylgalactosamine ( $\mathrm{Ac}_{4} \mathrm{GalNAz}$-specific Nacetylgalactosamine (Chapter 1: Figure 1.19) glycosylation of plasma membrane glycoproteins and glycolipids in human tumour cell line models of breast (Chapter 3: Figure 3.2) and prostate cancer (Whittaker 2015, Part III dissertation, unpublished data) (Chapter 1: Figure 1.23c) progression, using flow cytometry. In this study, we used gel electrophoresis to visualise how $\mathrm{Ac}_{4} \mathrm{ManNAz}$ and $\mathrm{Ac}_{4} \mathrm{GalNAz}$ (Figure 1.24) differentially label glycoproteins across panels of cell lines, some of which had previously been validated as suitable in vitro models of cancer progression using scratch wound assays, to compare migration, Boyden chambers, to compare invasion, and immunohistochemistry to compare the relative expression of known invasion biomarkers (Pijuan et. al. 2019; Justus et. al. 2014) (Chapter 2). Additionally, we used deglycosylating enzymes to visualise specific azidosugar labelling of $\mathrm{N}$ linked glycoproteins.

The correlation between global increases in glycosylation of cell surface glycans and metastatic potential suggest that detecting changes in glycosylation could be useful for monitoring the propensity of a tumour to metastasise in vivo i.e. for assessing the aggressiveness of the disease. The data also suggest that the glycans differentially detected using flow cytometry (Chapter 1: Figure 1.23) may be part of a larger array of 'metastatic codes' associated with a tumour's glycan profile at any given time point (Fuster and Esko 2005). In other words, differential azidosugar labelling detected by flow cytometry implies the presence of differentially labelled glycoproteins, which may be predictive biomarkers of cancer progression. 


\subsection{Results}

In order to understand the biological basis for the increases in metabolic labelling observed using flow cytometry (Chapter 3: Figure 3.2), the dynamics of azidosugar incorporation into cell surface glycoproteins was assessed using gel electrophoresis.

\subsubsection{Enrichment of the membrane proteome}

Cell extracts were obtained using detergent-based, non-denaturing fractionation. The Qproteome ${ }^{\circledR}$ Cell Compartment kit (Qiagen) was used to remove the cytosolic fraction, followed by solubilisation and isolation of the cell membranes. The membrane-enriched fraction contained plasma membrane proteins and proteins from membrane-bound organelles such as the golgi and mitochondria. Membrane-enriched extracts were desalted using acetone precipitation and methanol, chloroform and water extraction, to generate a purified membrane enriched extract, suitable for further analysis.

Enrichment of the membrane proteome was confirmed by western blot analysis (Figure 4.1). Abundant proteins from the plasma membrane (E-cadherin) and the mitochondria (voltagedependent anion channel, and mitochondrial import inner membrane translocase subunit Timm 23), were detected (Figure 4.1a). As expected, detection of proteins abundant in the cytosol (glyceraldehyde 3-phosphate dehydrogenase) and the nucleus (lamin b) was minimal.

The cellular localisation of proteins within the membrane-enriched extracts (Figure 4.1b) was also confirmed by mass spectrometry. Spectra representing individual peptides generated from in-gel tryptic digestion (Figure 4.1b) were searched against databases of known primary peptide sequences using the Mascot software (http://www.matrixscience.com/search_intro.html, 2019). Proteins identified with Mascot were classified (Table 4.1) by their cellular location using gene ontology analysis (www.pantherdb.org) (Table 4.2). The majority of gene hits within the membrane protein category were designated as plasma or integral membrane proteins (Table 4.3), confirming enrichment of cell surface proteins, the cellular region predominantly labelled using 
bioorthogonal glycan labelling techniques (Chapter 1: Figure 1.20). Plasma membrane proteins consistently accounted for between $37-38 \%$ of designated membrane proteins within the membrane protein category, across different prostate (LNCAP C4-2 and LNCAP C4-2B) and breast (MDA MB 468 and MDA MB 231) cancer cell lines, confirming replication and optimisation of our enrichment and purification protocols in different cancer cell types (Table 4.3).

a.

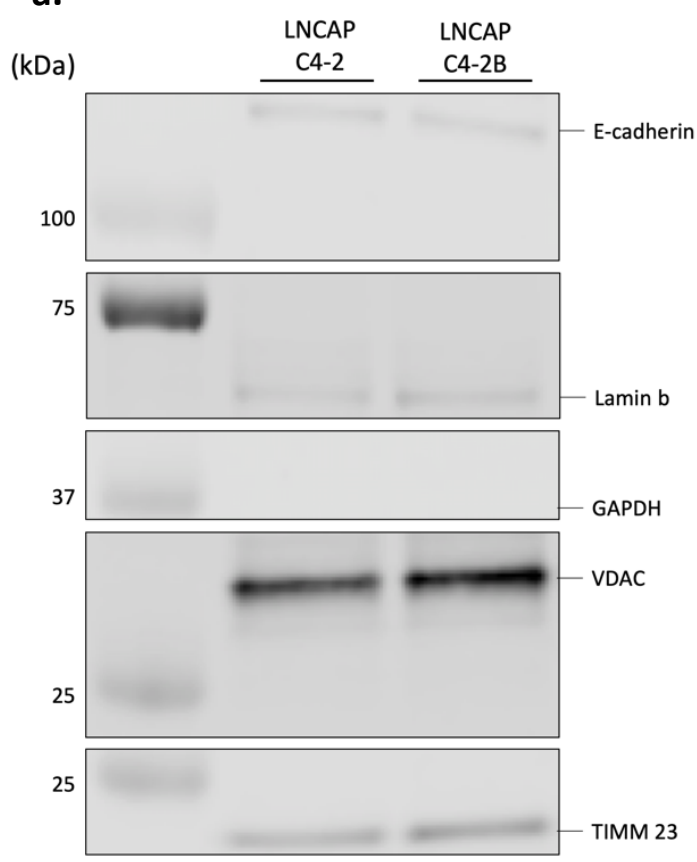

b.

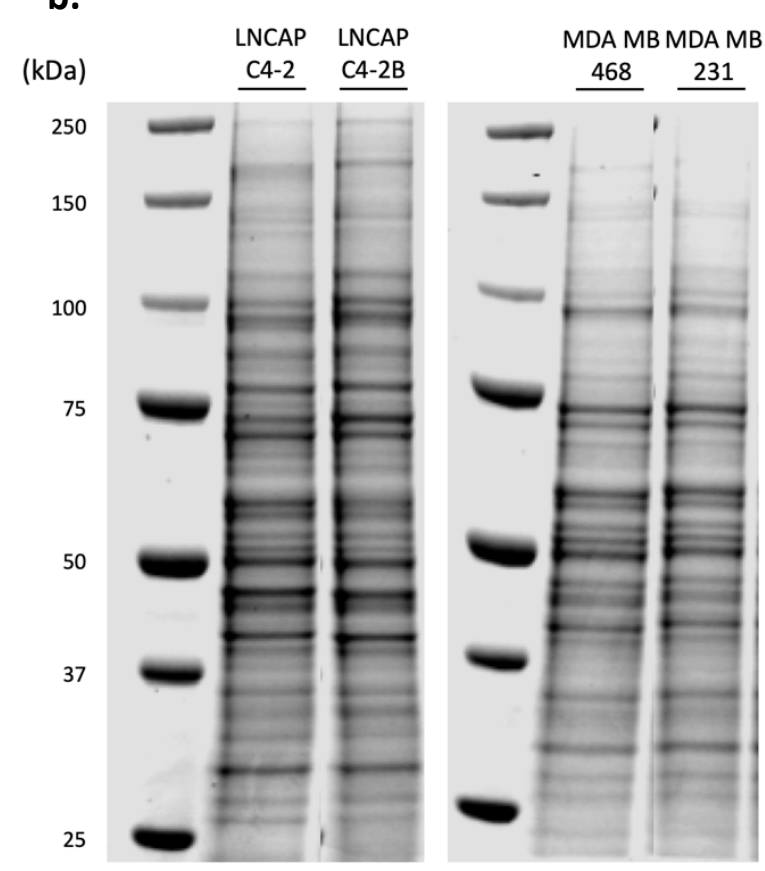

Figure 4.1: Enrichment of the membrane proteome confirmed using western blot.

Membrane-enriched extracts were obtained from two prostate cancer cell lines (LNCAP C4-2 and LNCAP C4-2B) and two breast cancer cell lines (MDA MB 468 and MDA MB 231) using detergent-based fractionation. (a) Representative western blots showing the relative abundance of fraction controls in $10 \mu \mathrm{g}$ of extract from the prostate cancer cell lines: $E$ Cadherin (plasma membrane protein, $97 \mathrm{kDa}$ ), lamin b (nuclear protein, $66 \mathrm{kDa}$ ), glyceraldehyde 3-phosphate dehydrogenase (GAPDH, cytosolic protein, $37 \mathrm{kDa}$ ), voltagedependent anion channel (VDAC, mitochondrial protein, $31 \mathrm{kDa}$ ), mitochondrial import inner membrane translocase subunit Tim23 (TIMM 23, mitochondrial membrane protein, 22 kDa). (b) Coomassie staining of extracts $(10 \mu \mathrm{g})$ resolved by gel electrophoresis, and Coomassie stained. Lanes were segmented into 24 pieces, and subjected to tryptic digestion, followed by protein identification by liquid chromatography-tandem mass spectrometry. 


\begin{tabular}{|c|c|c|c|c|c|c|c|c|c|c|c|c|}
\hline 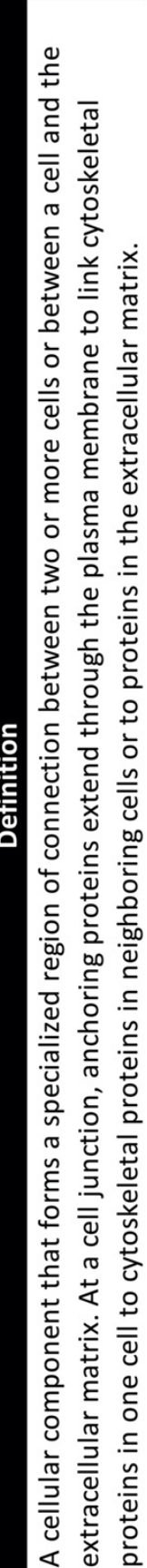 & 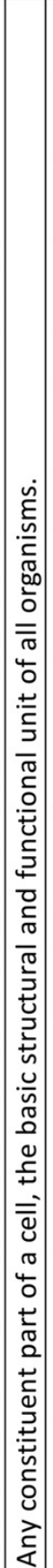 & 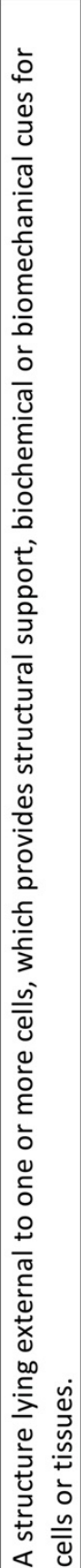 & 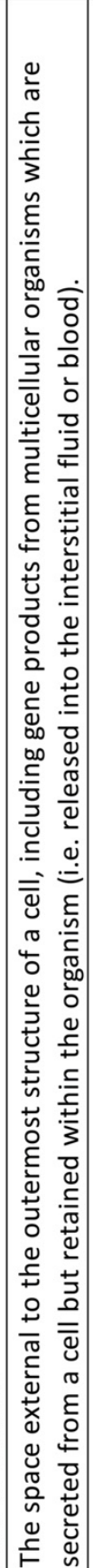 & 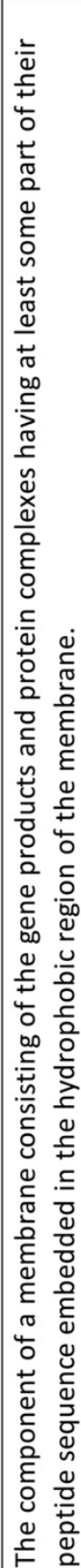 & 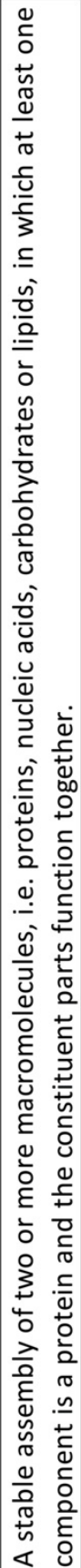 & 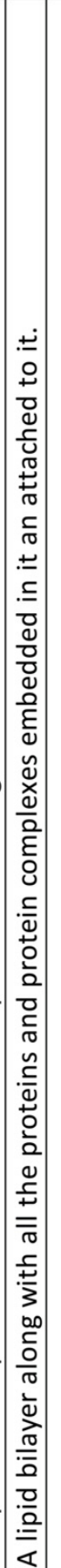 & 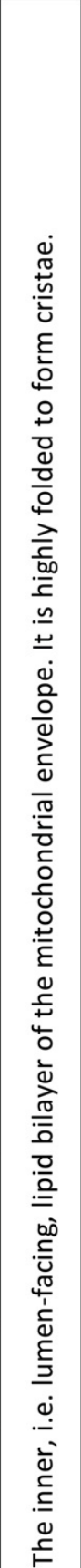 & 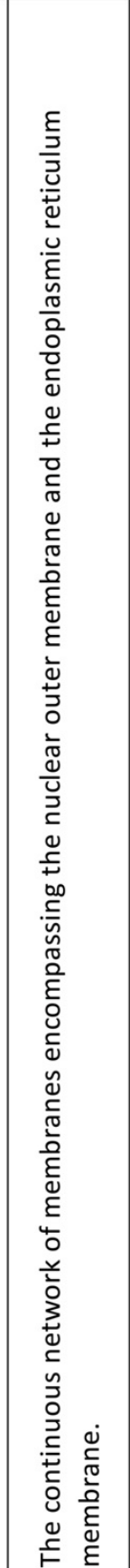 & 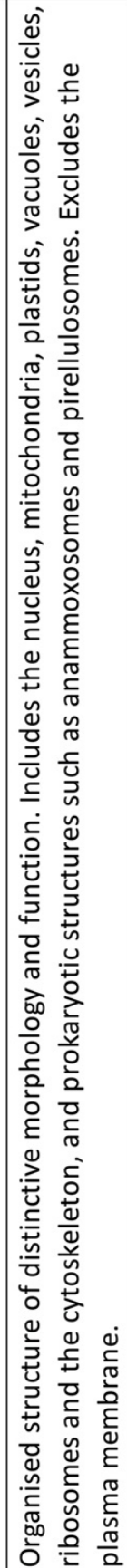 & 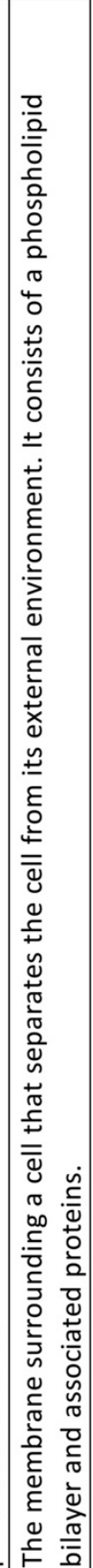 & 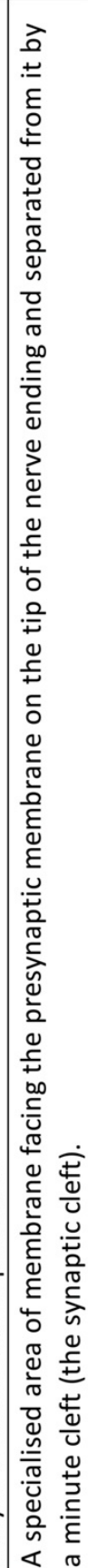 & 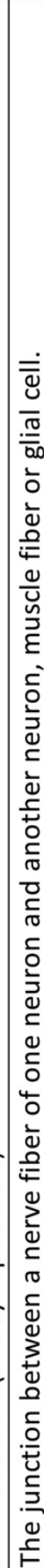 \\
\hline 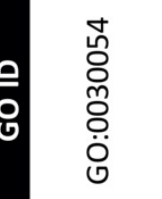 & 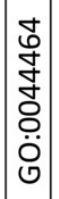 & 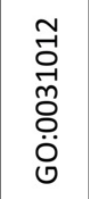 & 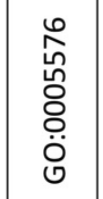 & 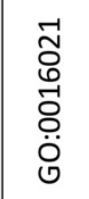 & 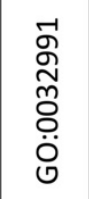 & 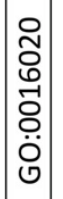 & 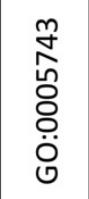 & 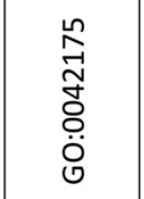 & 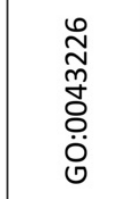 & 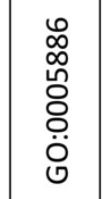 & 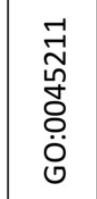 & 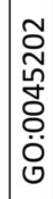 \\
\hline 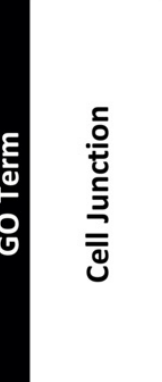 & 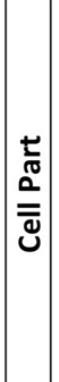 & 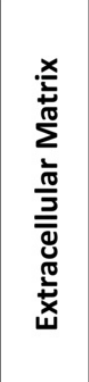 & 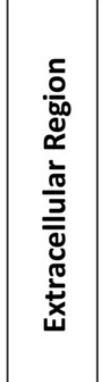 & 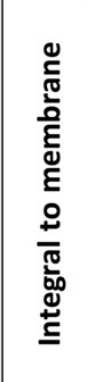 & 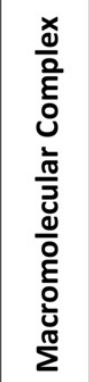 & 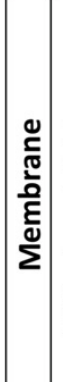 & 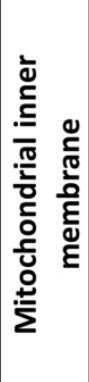 & 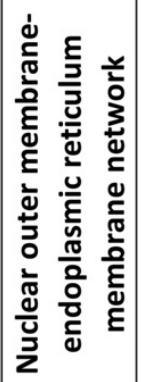 & 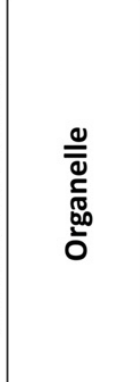 & 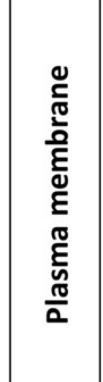 & 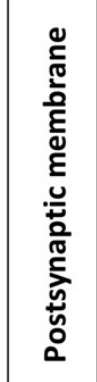 & 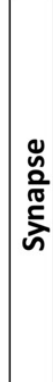 \\
\hline
\end{tabular}


Table 4.1: Protein classifications by GO terms. GO term definitions were collated from The Jackson Laboratory Gene Ontology browser (http://www.informatics.jax.org/vocab/gene_ontology/G0:0005575, 2019) (Author diagram).

\begin{tabular}{|c|c|c|c|c|}
\hline \multirow{2}{*}{ Cellular Component Category } & \multicolumn{4}{|c|}{ Gene Hits } \\
\cline { 2 - 5 } & Prostate cancer cell lines & \multicolumn{2}{c|}{ Breast cancer cell lines } \\
\cline { 2 - 5 } & LNCAP C4-2 & LNCAP C4-2B & MDA MB 468 & MDA MB 231 \\
\hline Cell part & 1437 & 1396 & 1384 & 1257 \\
\hline Organelle & 1065 & 1042 & 992 & 907 \\
\hline Macromolecular complex & 603 & 608 & 569 & 545 \\
\hline Membrane & 202 & 184 & 195 & 177 \\
\hline Extracellular region & 44 & 44 & 44 & 52 \\
\hline Cell junction & 16 & 17 & 15 & 14 \\
\hline Synapse & 9 & 7 & 7 & 8 \\
\hline Extracellular matrix & 6 & 5 & 6 & 11 \\
\hline Membrane category (\% of total) & 5.97 & 5.57 & 6.07 & 5.96 \\
\hline
\end{tabular}

Table 4.2: Proteins identified from the membrane proteome using mass spectrometry classified by cellular component. Proteins identified with Mascot from two prostate cancer cell lines (LNCAP C4-2 and LNCAP C4-2B), and two breast cancer cell lines (MDA MB 468 and MDA MB 231), were classified by their cellular location using gene ontology (GO) analysis (www.pantherdb.org).

\begin{tabular}{|c|c|c|c|c|}
\hline \multirow{2}{*}{ Membrane Component Category } & \multicolumn{4}{|c|}{ Gene Hits } \\
\cline { 2 - 5 } & Prostate cancer cell lines & \multicolumn{2}{c|}{ Breast cancer cell lines } \\
\cline { 2 - 5 } & LNCAP C4-2 & LNCAP C4-2B & MDA MB 468 & MDA MB 231 \\
\hline Plasma membrane & 176 & 168 & 181 & 159 \\
\hline Integral to membrane & 133 & 125 & 135 & 117 \\
\hline $\begin{array}{c}\text { Nuclear outer membrane-endoplasmic } \\
\text { reticulum membrane network }\end{array}$ & 106 & 111 & 111 & 104 \\
\hline Mitochondrial inner membrane & 41 & 40 & 37 & 31 \\
\hline Postsynaptic membrane & 4 & 3 & 3 & 3 \\
\hline Total & 460 & 447 & 467 & 414 \\
\hline Plasma membrane proteins (\% of total) & 38.26 & 37.58 & 38.76 & 38.41 \\
\hline
\end{tabular}


Table 4.3: Proteins identified from the membrane proteome using mass spectrometry classified by membrane component. Proteins identified with Mascot from two prostate cancer cell lines (LNCAP C4-2 and LNCAP C4-2B), and two breast cancer cell lines (MDA MB 468 and MDA MB 231), were classified by membrane location using gene ontology (GO) analysis (www.pantherdb.org).

\subsubsection{Azidosugar labelling of the membrane proteome of prostate cancer cell lines}

Prostate, breast, and pancreatic cancer cell lines, were incubated with precursors of sialic acid and $\mathrm{N}$-acetylgalactosamine that had been labelled with an azide group ( $\mathrm{N}$ azidoacetylmannosamine ( $\mathrm{Ac}_{4} \mathrm{ManNAz}$ ) and $\mathrm{N}$-azidoacetylgalactosamine ( $\mathrm{Ac}_{4} \mathrm{GalNAz}$ ) respectively), and which are incorporated biosynthetically into cell surface glycans. The membrane proteome was isolated and the incorporated azide groups covalently ligated to alkyne-Alexa Fluor 488 using copper-catalysed chemistry. Azidosugar-labelled glycoproteins were resolved by gel electrophoresis and detected directly, in-gel, using Alexa-488 fluorescence (Clark et. al. 2008; Cieniewski-Bernard et. al. 2014).

No background fluorescence was observed in extracts from prostate cancer cells treated with vehicle (0.1\% DMSO) only, or vehicle and the alkyne-Alexa Fluor 488 probe (Figure $4.2 \mathrm{a}$, i). A general increase in the overall Alexa Fluor 488 fluorescence correlated with an increase in the metastatic potential of the prostate cancer cell lines (Figure 4.2a, ii). There was no correlation between increases in $\mathrm{Ac}_{4}$ ManNAz $\left(r^{2}=0.02245\right)$ (Figure 4.2b, i) or Ac ${ }_{4}$ GalNAz $\left(r^{2}=\right.$ 0.4642) (Figure 4.2c, i) labelling of the glycoproteins and doubling time (Figure 4.2b, ii and $4.2 c$, ii). Therefore, the azidosugar labelling of the glycoproteins appeared to be affected by increases in the invasive potential of the prostate cancer cell lines (Chapter 2: Figure 2.3), but not proliferation. 
a.

i.
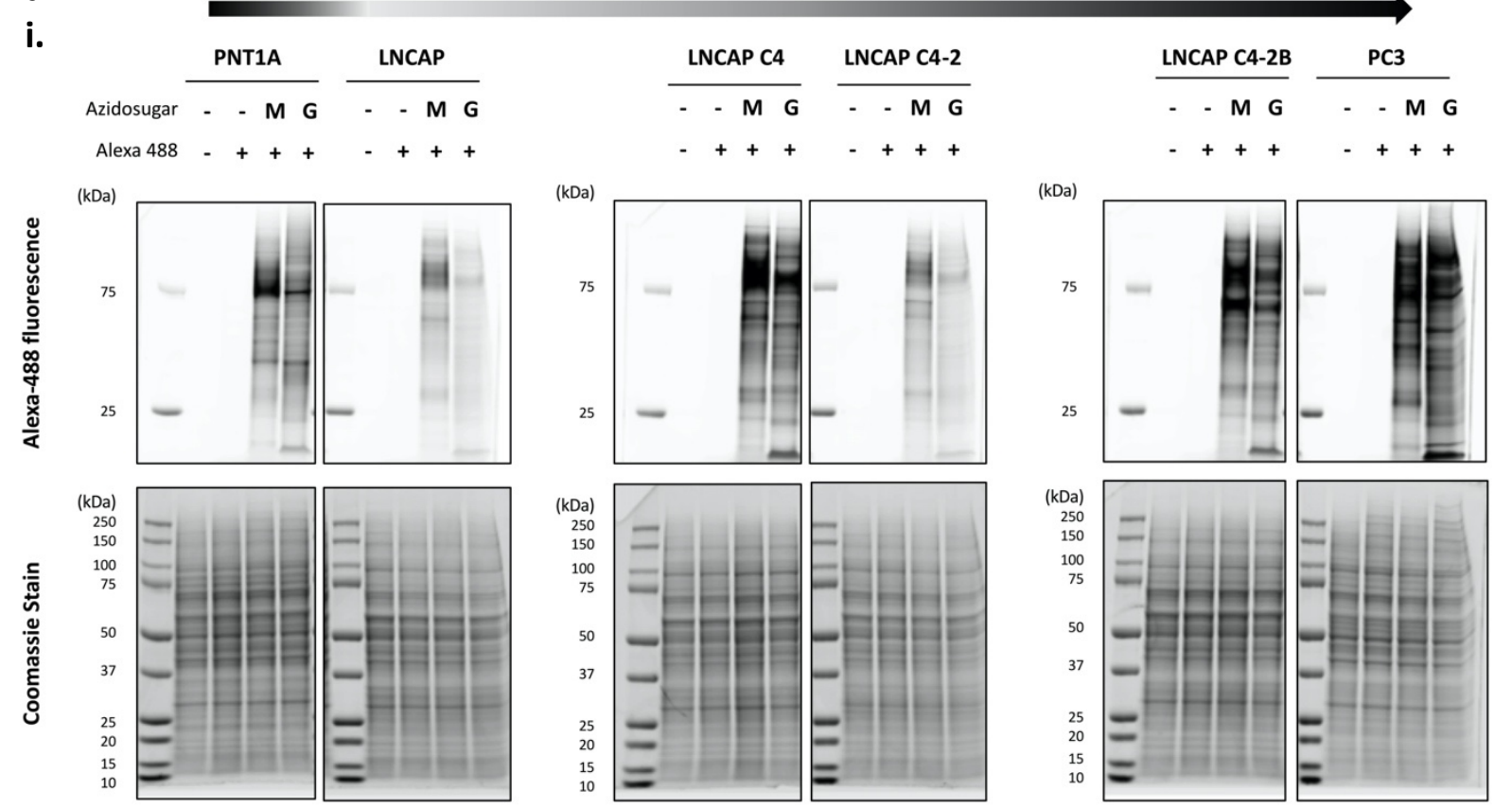

ii.

- $\mathrm{Ac}_{4} \mathrm{ManNAz}$ $\square \mathrm{Ac}_{4} \mathrm{GalNAz}$

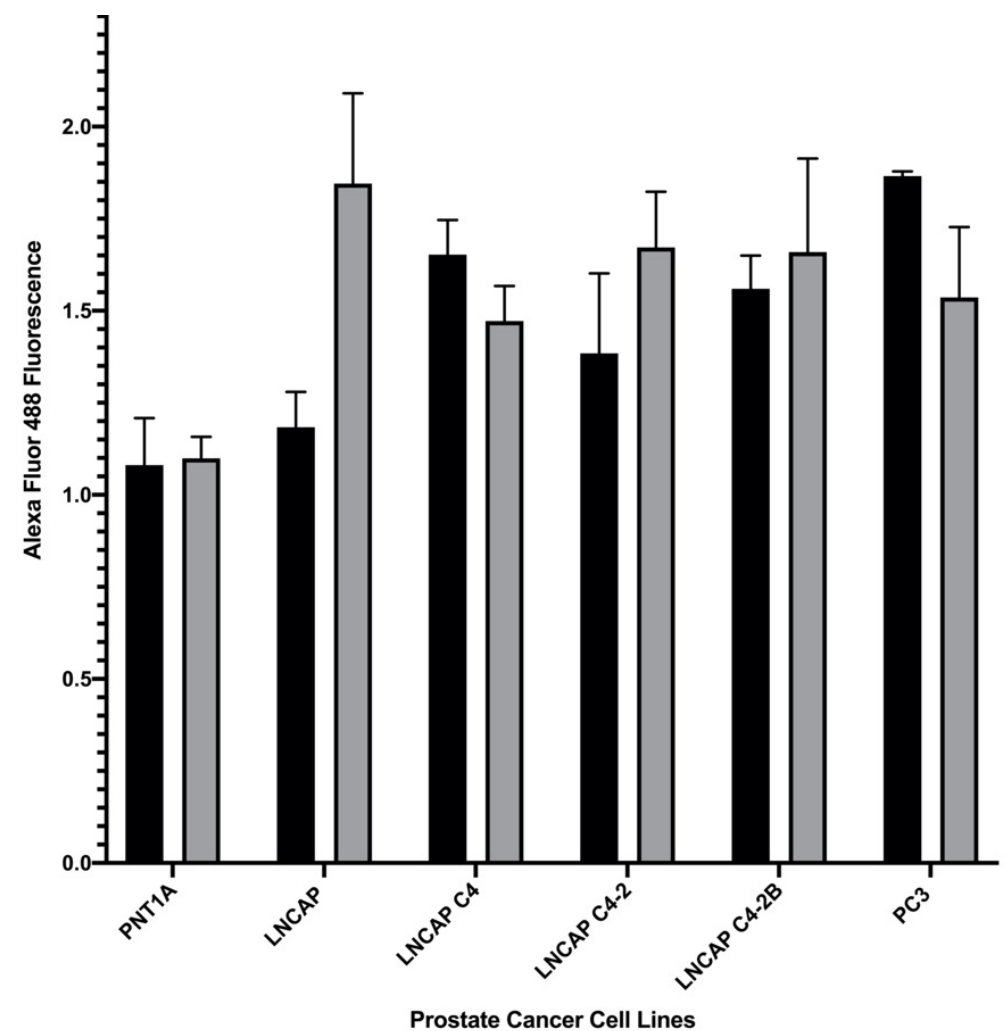




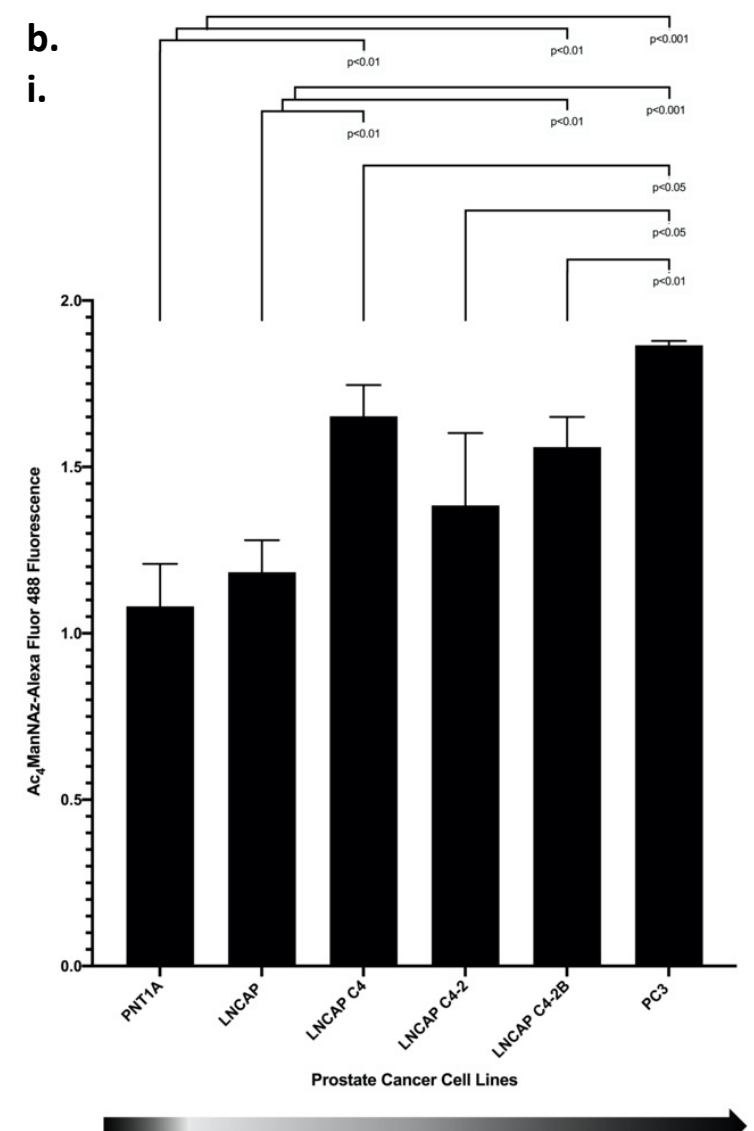

ii.

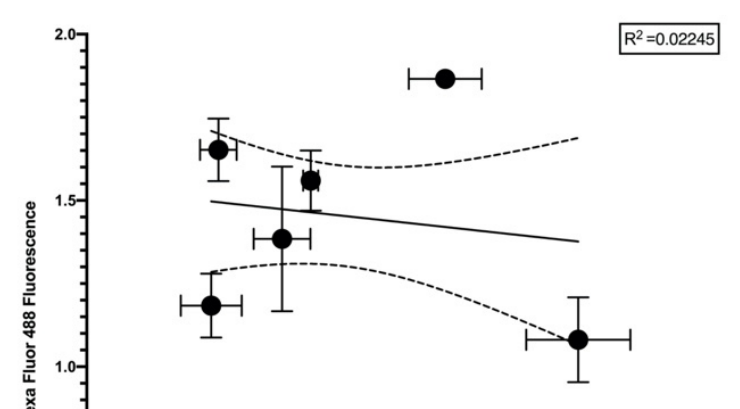

c.

i.

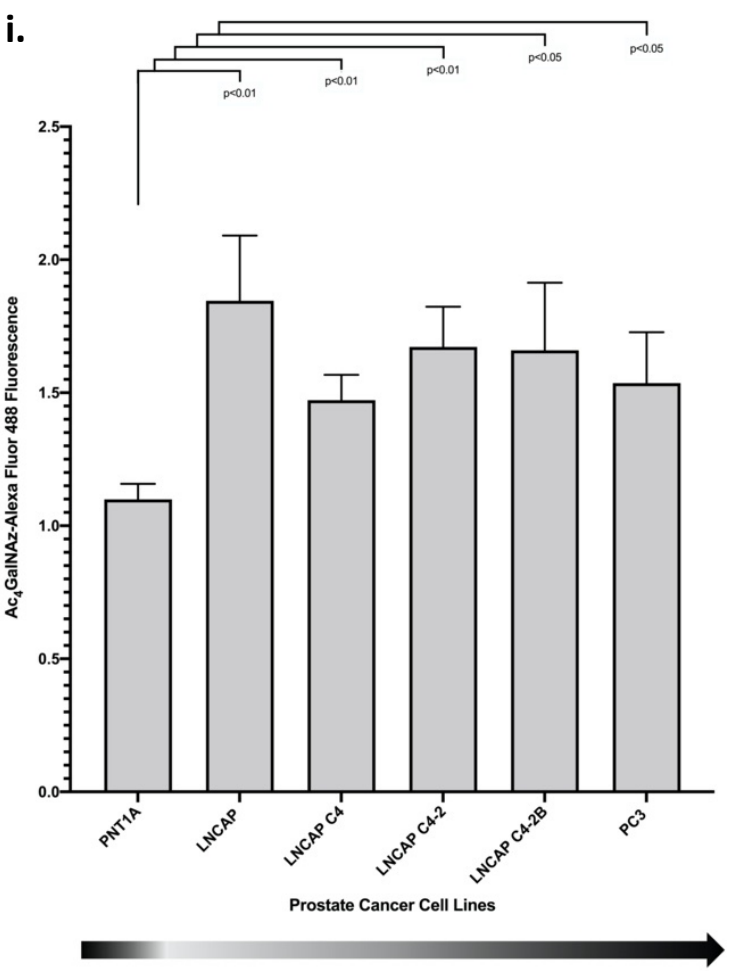

ii.

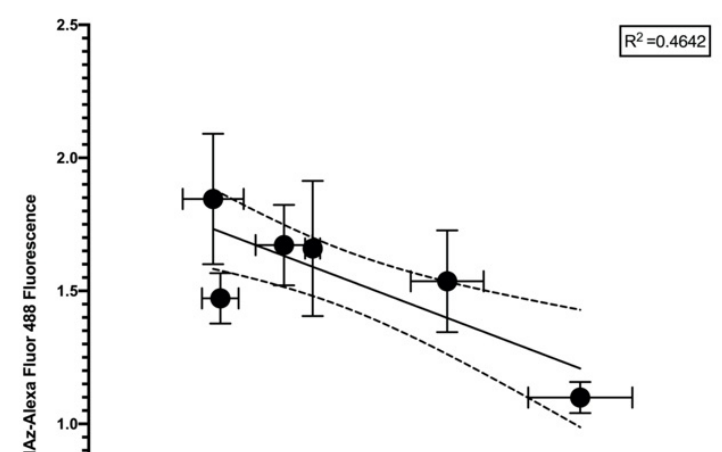


Figure 4.2: In-gel fluorescence detection of azidosugars incorporated into the membrane proteome of prostate cancer cells. (a) Representative in-gel Alexa Fluor 488 fluorescence images from a panel of prostate cell lines with increasing metastatic potential (i). Total protein per lane was visualised using Coomassie staining. Background controls were included to detect non-specific fluorescence. For each lane treated with $\mathrm{N}$-azidoacetylmannosamine (Ac4ManNAz) or N-azidoacetylgalactosamine (Ac4GalNAz), total Alexa Fluor 488 fluorescence was quantified using Fiji Image analysis software, normalised against the Coomassie stain fluorescence within the same lane, and presented as a ratio (ii). Whole cell counts were obtained every 24 hours, over a 72-hour period using the LUNA ${ }^{\text {TM }}$ Automated Cell Counter, and used to plot a growth curve. The gradient of the natural log regression line of each curve was used to calculate the doubling time (hours) for each prostate cancer cell line. (b) Ac ${ }_{4}$ ManNAz-specific fluorescence for each cell line (i) was plotted as a function of doubling time (ii). (c) Ac ${ }_{4}$ GalNAz-specific fluorescence for each cell line (i) was plotted as a function of doubling time (ii). All data represent the average \pm S.D. of three independent experiments, each with 3 replicates. $p$ values represent significance assessed by one-way ANOVA. The darker shades in the arrow's greyscale represent an increase in the metastatic potential of the panel of prostate cancer cell lines. Abbreviations: $M=A c_{4} M a n N A z$ treated, $G$ $=A c_{4}$ GalNAz treated.

\subsubsection{Azidosugar labelling of the membrane proteome of breast cancer cell lines}

No background fluorescence was observed in extracts from breast cancer cells treated with vehicle (0.1\% DMSO) only, or vehicle and the alkyne-Alexa Fluor 488 probe (Figure $4.3 \mathrm{a}, \mathrm{i})$. A general increase in the overall Alexa-488 fluorescence correlated with an increase in the metastatic potential of the breast cancer cell lines (Figure 4.3a, ii). There was no correlation between increases in $A c_{4}$ ManNAz $\left(r^{2}=0.01493\right)$ (Figure 4.3b, i) or Ac ${ }_{4}$ GalNAz $\left(r^{2}=0.04585\right)$ (Figure 4.3c, i) labelling of the glycoproteins and doubling time (Figure 4.3b, ii and 4.3c, ii). Therefore, the azidosugar labelling of the glycoproteins was affected by increases in the invasive potential of the breast cancer cell lines (Chapter 2: Figure 2.6), but not proliferation. 
Previously, we used flow cytometric analysis to quantify global azidosugar labelling of the glycoproteins and glycolipids in the breast cancer cell lines (Chapter 3). Breast cancer cells were incubated with $\mathrm{Ac}_{4} \mathrm{ManNAz}$ or $\mathrm{Ac}_{4} \mathrm{GalNAz}$. The incorporated azide groups were reacted sequentially with TMDIBO-(PEG) ${ }_{3}$-biotin and NeutrAvidin ${ }^{\mathrm{TM}}$ DyLight $^{\mathrm{TM}} 650$ (NA650), followed by fluorescence detection using flow cytometry. The Mean Fluorescence Intensity ratio (MFI ratio) for each cell line was calculated by normalising the average fluorescence intensity from azidosugar-labelled cells against background fluorescence (Chapter 3: Figure 3.1). An increase in $\mathrm{Ac}_{4} \mathrm{ManNAz}$ and $\mathrm{Ac}_{4} \mathrm{GalNAz}$ labelling correlated with an increase in the metastatic potential of the breast cancer cell lines (Chapter 3: Figure 3.2). We observed a strong positive correlation $\left(r^{2}=0.8217\right.$ ) (Figure $4.3 \mathrm{~d}$, i) between increases in Ac4ManNAz-MFI ratios of the breast cancer cell lines, representing global sialylation of the glycoconjugates, and increases in the $\mathrm{Ac}_{4} \mathrm{ManNAz-Alexa}$ Fluor 488 fluorescence (Figure 4.3b, i), representing specific sialylation of the glycoproteins. Therefore, increases in global cell surface sialylation in the breast cancer cell lines detected by flow cytometry (Chapter 3: Figure 3.3) were driven by increased sialyation of the glycoproteins.

There was no correlation $\left(r^{2}=0.2020\right)$ (Figure $4.3 \mathrm{~d}$, ii) between increases in Ac 4 GalNAz-MFI ratios of the breast cancer cell lines, representing global $\mathrm{N}$-acetylgalactosamine glycosylation of the glycoconjugates, and $\mathrm{Ac}_{4}$ GalNAz-Alexa Fluor 488 fluorescence (Figure 4.3c, i), representing specific $\mathrm{N}$-acetylgalactosamine glycosylation of the glycoproteins. Whilst the increases in global $\mathrm{N}$-acetylgalactosamine glycosylation quantified with flow cytometry were statistically significant between the breast cancer cell lines (Chapter 3: Figure 3.3c, i), only a significant difference in specific labelling of the glycoproteins was observed between the least metastatic (MDA MB 453) and most metastatic (MDA MB 231) cell lines in the breast cancer panel. This suggested that the glycoprotein fraction was not principally responsible for the increases in global $\mathrm{N}$-acetylgalactosamine glycosylation observed with flow cytometry. 
a.

i.

\begin{tabular}{|c|c|c|}
\hline & MDA MB 453 & MCF7 \\
\hline Azidosugar & $-\quad-M G$ & $-M G$ \\
\hline & + & ++ \\
\hline
\end{tabular}

MDA MB $468 \quad$ MDA MB 231

- M G - - M G

(kDa)

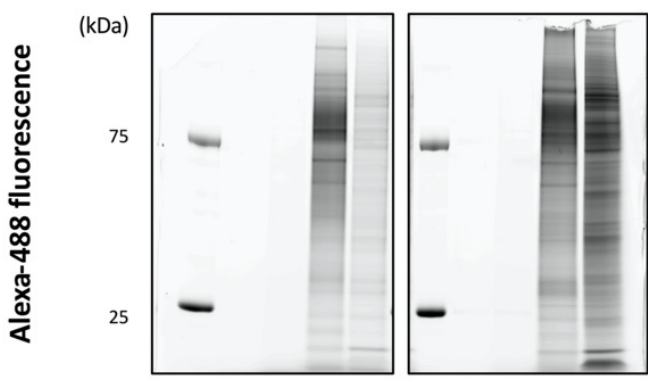

(kDa)
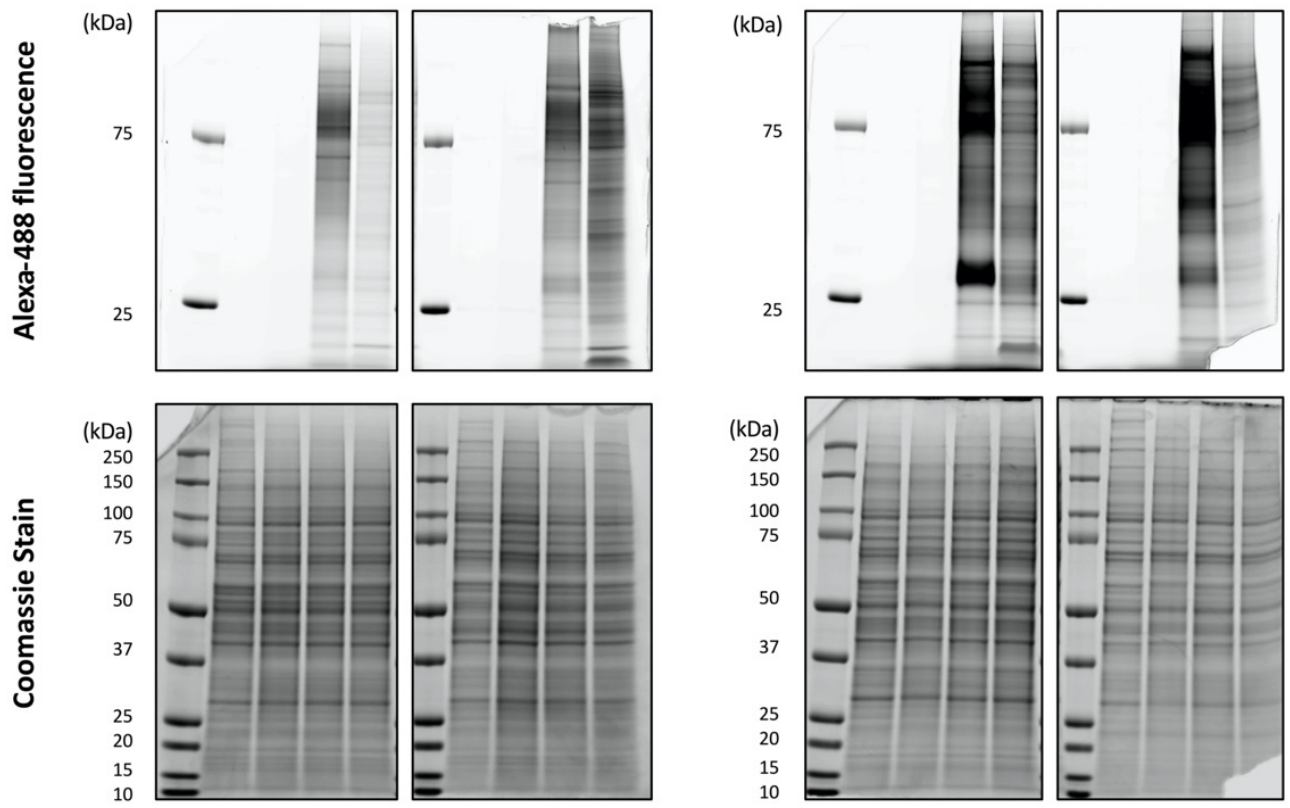

ii.

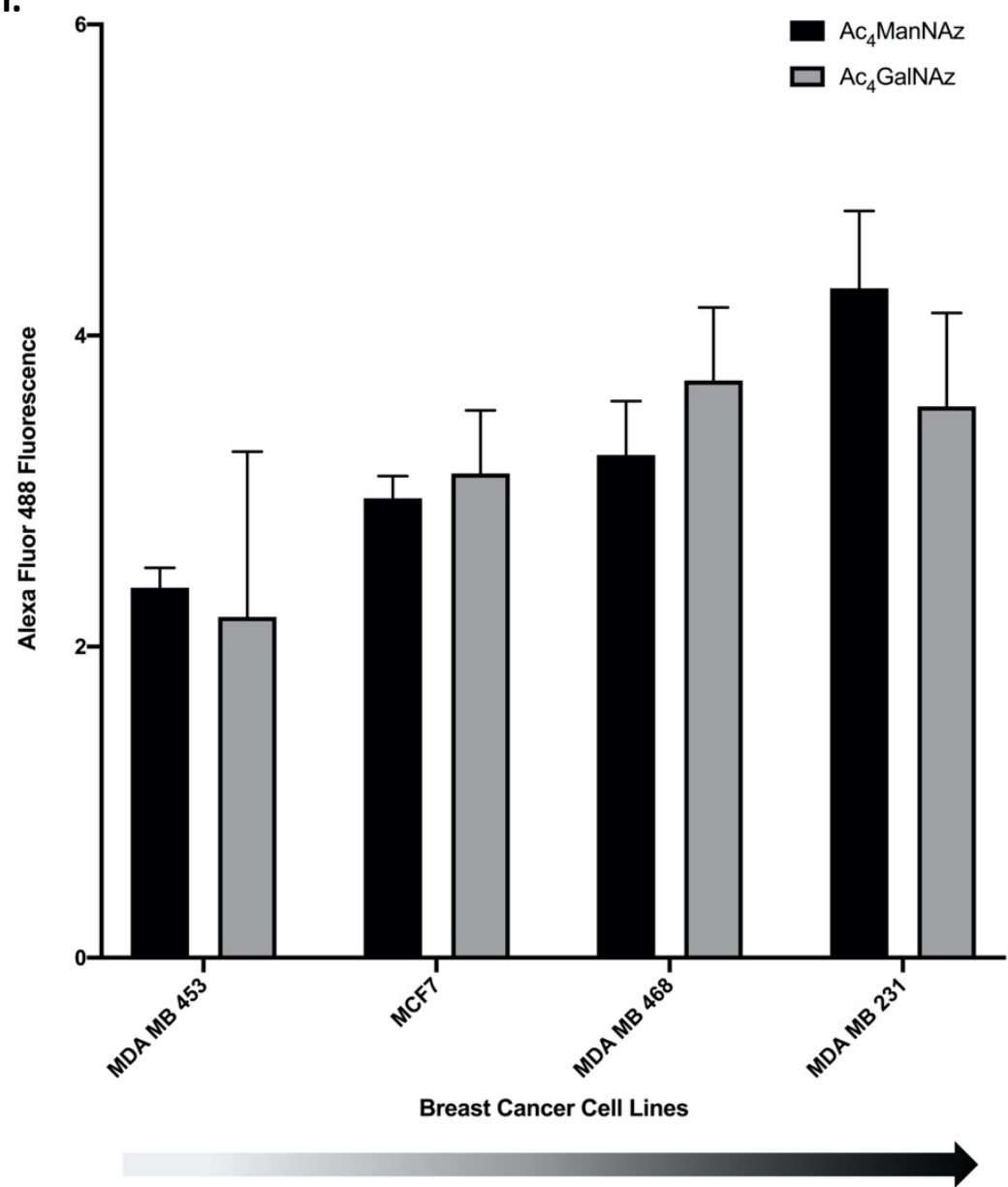




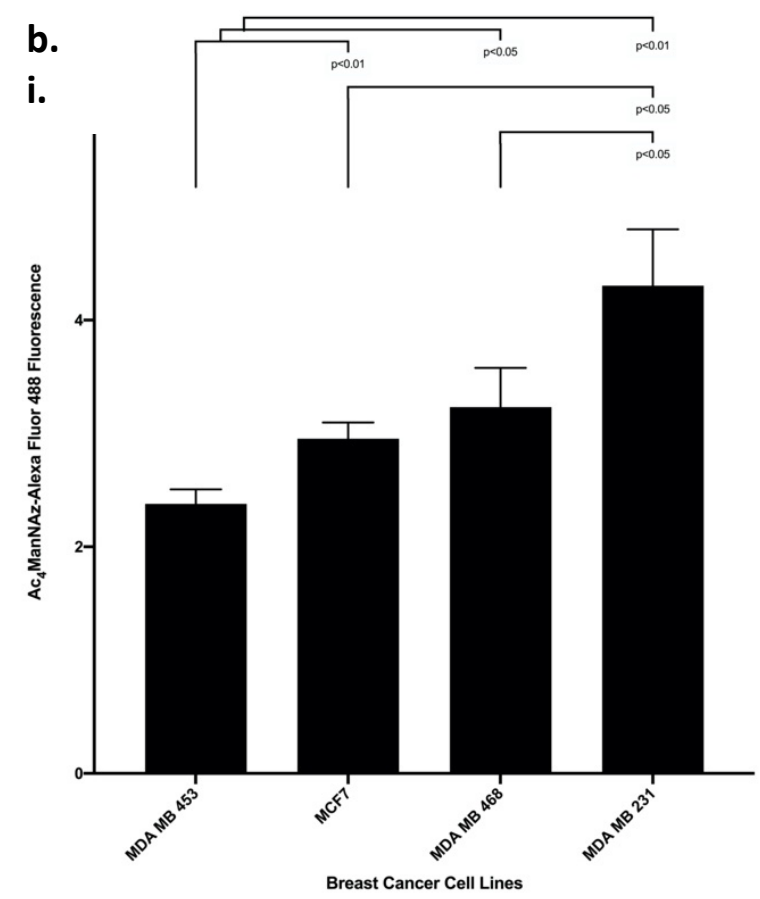

ii.

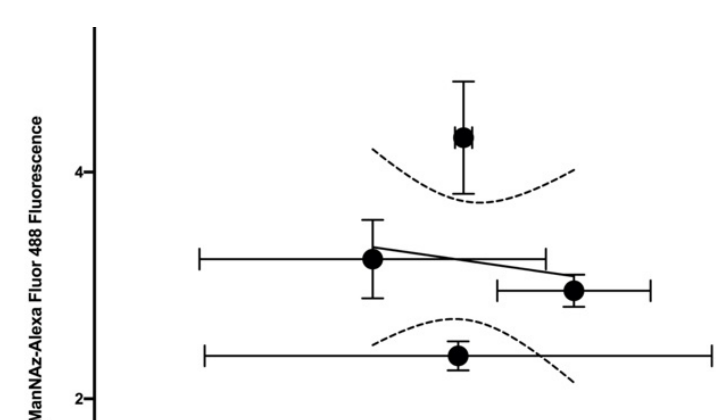

c.

i.

ii.
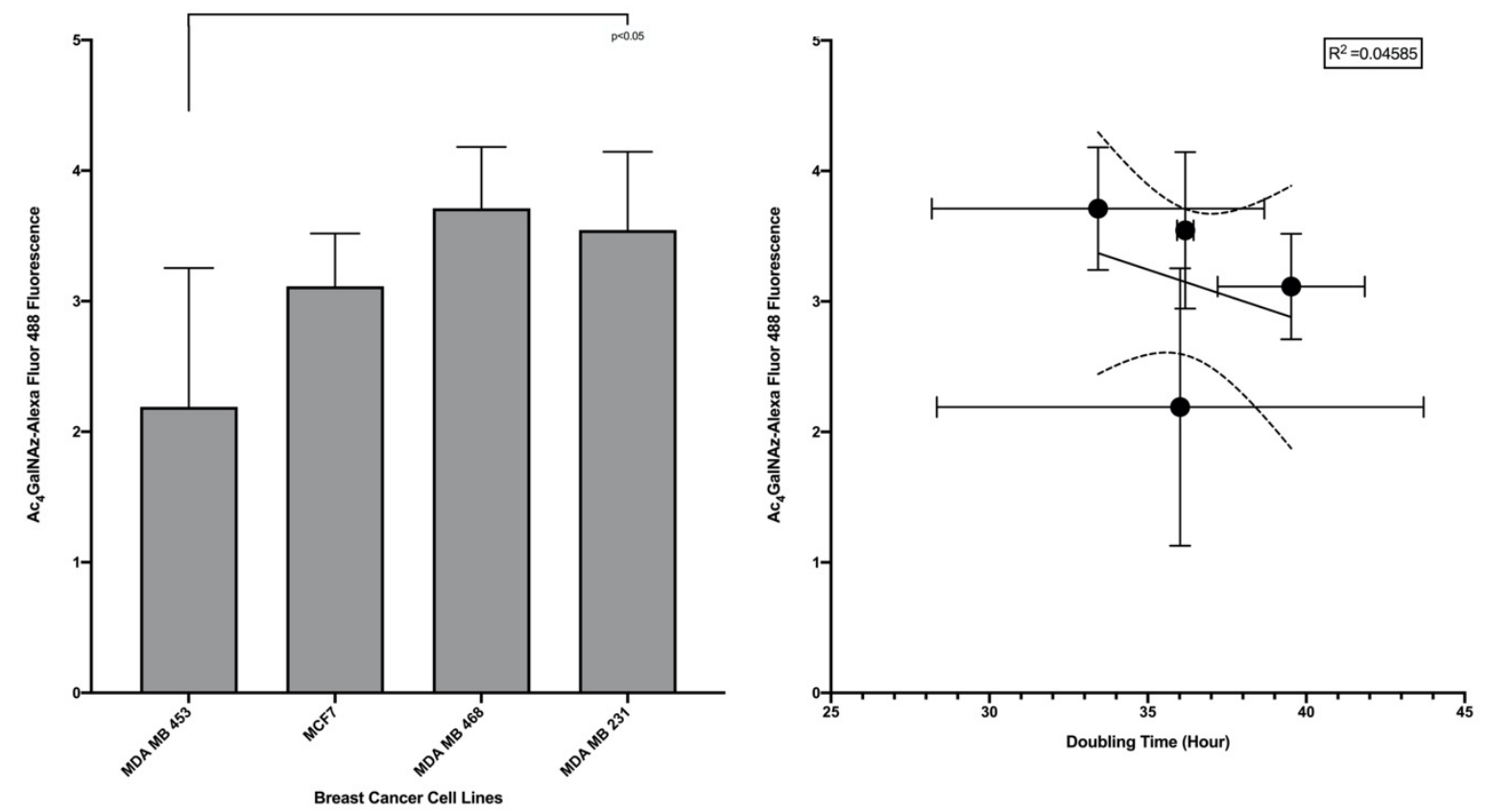
d.

i.

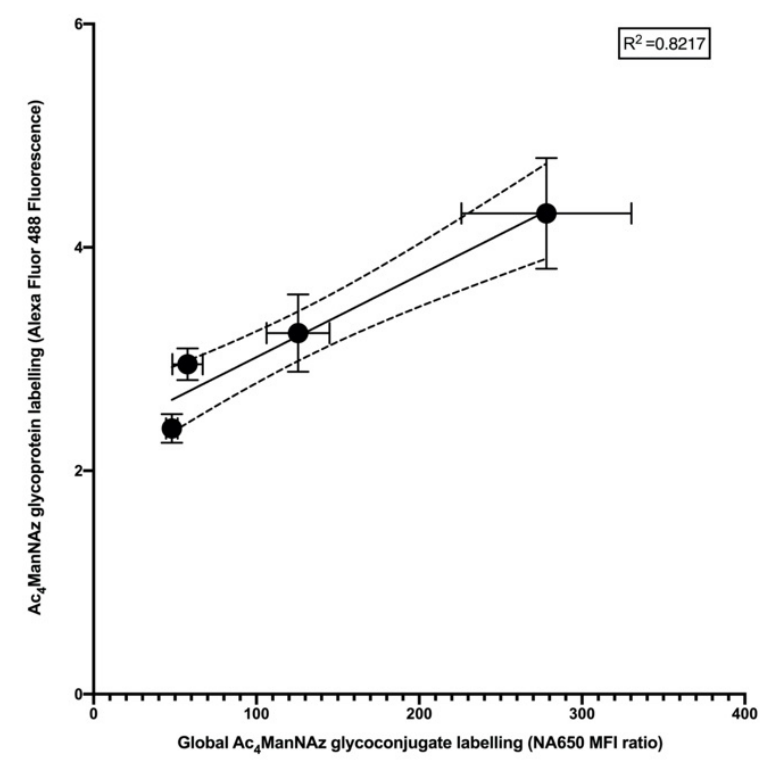

ii.

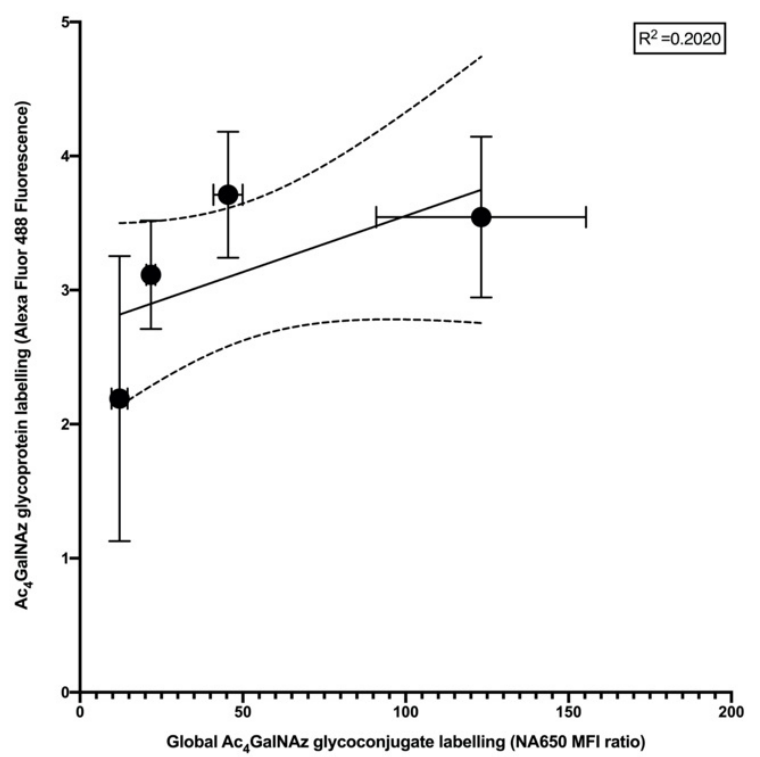

Figure 4.3: In-gel fluorescence detection of azidosugars incorporated into the membrane proteome of breast cancer cells. (a) Representative in-gel Alexa Fluor 488 fluorescence images from a panel of breast cancer cell lines with increasing metastatic potential (i). Total protein per lane was visualised using Coomassie staining. Background controls were included to detect non-specific fluorescence. For each lane treated with $\mathrm{N}$-azidoacetylmannosamine (Ac 4 ManNAz) or N-azidoacetylgalactosamine (Ac 4 GaINAz), total Alexa Fluor 488 fluorescence was quantified using Fiji Image analysis software, normalised against the Coomassie stain fluorescence within the same lane, and presented as a ratio (ii). Whole cell counts were obtained every 24 hours, over a 72-hour period using the LUNA ${ }^{\text {TM }}$ Automated Cell Counter, and used to plot a growth curve. The gradient of the natural log regression line of each curve was used to calculate the doubling time (hours) for each breast cancer cell line. (b) Ac ${ }_{4} M a n N A z-s p e c i f i c$ fluorescence for each cell line (i) were plotted as a function of doubling time (ii). (c) Ac 4 GalNAz-specific fluorescence for each cell line (i) were plotted as a function of doubling time (ii). (d) Breast cancer cells were labelled sequentially with Ac4ManNAz or Ac4GalNAz, TMDIBO-(PEG) 3 -biotin and NeutrAvidin ${ }_{4}^{T M}$ DyLight ${ }^{T M} 650$ (NA650). Fluorescence detection of NA650 using flow cytometric analysis followed. The Mean Fluorescence Intensity ratio (MFI ratio) for each cell line was calculated by normalising the average fluorescence intensity from azidosugar-labelled cells against background fluorescence. MFI ratios for each cell line treated with Ac4ManNAz (i) Ac $c_{4} G a I N A z$ (ii) were plotted as a function of doubling time. All data represent the average \pm S.D. of three 
independent experiments, each with 3 replicates. $p$ values represent significance by one-way ANOVA. The darker shades in the arrow's greyscale represent an increase in the metastatic potential of the panel of prostate cancer cell lines. Abbreviations: $M=A c_{4} M a n N A z$ treated, $G$ $=A c_{4}$ GalNAz treated.

\subsubsection{Azidosugar labelling of the membrane proteome of pancreatic cancer cell lines}

Four human pancreatic cell lines were selected from the literature based on their increasing tumourigenicity (Table 4.4). Azidosugar-labelled glycoproteins were extracted from the pancreatic cell lines, which had been metabolically labelled with $\mathrm{Ac}_{4} \mathrm{ManNAz}$ in vitro, resolved via gel electrophoresis, and detected using Alexa Fluor 488 fluorescence, as described previously.

No background fluorescence was observed in extracts from pancreatic cancer cells treated with vehicle (0.1\% DMSO) only, or vehicle and the alkyne-Alexa Fluor 488 probe (Figure 4.4a). The metastasis derived Capan 1 cell line exhibited higher levels of $A_{4} M a n N A z$ labelling than the tumour derived Capan 2 cell line (Figure 4.4b), consistent with increases in sialylation associated with an increase in metastatic potential (Natoni et. al. 2016). A high degree of $A c_{4} M a n N A z$ metabolic labelling was observed in the human telomerase (hTERT)human pancreatic nestin expressing (HPNE) cell line (H4023) and the hTERT-HPNE derived hTERT-HPNE E6/E7 cell line (H4036), which both represent normal pancreatic epithelium (Table 4.1). This is contrary to reports correlating an increase in sialyation with an increase in cancer aggressiveness (Natoni et. al. 2016). Further independent repeats are needed to confirm if the increases in Alexa Fluor 488 fluorescence are statistically significant. 


\begin{tabular}{|c|c|c|c|c|c|c|}
\hline Cell line & Established & Source & Tumor type & $\begin{array}{l}\text { Tumorigenicity } \\
\text { in mice }\end{array}$ & $\begin{array}{l}\text { Doubling } \\
\text { time (hours) }\end{array}$ & $\begin{array}{l}\text { Source } \\
\text { references }\end{array}$ \\
\hline $\begin{array}{c}\text { hTERT- } \\
\text { HPNE } \\
\text { (H4023) }\end{array}$ & Not specified & $\begin{array}{c}\text { Normal } \\
\text { pancrease of } \\
\text { healthy } 52 \text { year } \\
\text { old caucasian } \\
\text { male }\end{array}$ & $\begin{array}{c}\text { Normal pancreatic } \\
\text { epithelium }\end{array}$ & Non-malignant & 26 & $\begin{array}{c}\text { Lee et. al. } \\
2003\end{array}$ \\
\hline $\begin{array}{l}\text { hTERT- } \\
\text { HPNE } \\
\text { E6/E7 } \\
\text { (H4036) }\end{array}$ & 2000 & $\begin{array}{c}\text { Normal } \\
\text { pancrease of } \\
\text { healthy } 52 \text { year } \\
\text { old caucasian } \\
\text { male }\end{array}$ & $\begin{array}{c}\text { Normal pancreatic } \\
\text { epithelium; derived } \\
\text { from hTERT-HPNE } \\
\text { cell line expressing } \\
\text { E6 and E7 proteins of } \\
\text { HPV16 }\end{array}$ & Non-malignant & 30 & $\begin{array}{l}\text { Lee et. al. } \\
\text { 2005; } \\
\text { Campbell } \\
\text { et. al. } \\
\text { 2007; Lee } \\
\text { et. al. } 2003\end{array}$ \\
\hline Capan 1 & 1974 & $\begin{array}{l}\text { Liver metastasis } \\
\text { from } 40 \text { year old } \\
\text { caucasian male } \\
\text { with pancreatic } \\
\text { adenocarcinoma }\end{array}$ & Adenocarcinoma & Tumourigenic & $49-100$ & $\begin{array}{c}\text { Kyriazis et. } \\
\text { al. 1982; } \\
\text { Deer et. al. } \\
2010\end{array}$ \\
\hline Capan 2 & 1975 & $\begin{array}{c}\text { Pancreatic } \\
\text { adenocarcinoma } \\
\text { from } 56 \text { year old } \\
\text { caucasian male }\end{array}$ & Adenocarcinoma & Tumourigenic & $45-96$ & $\begin{array}{l}\text { Kyriazis et. } \\
\text { al. 1986; } \\
\text { Dahiya et. } \\
\text { al. 1993; } \\
\text { Deer et. al. } \\
2010\end{array}$ \\
\hline
\end{tabular}

Table 4.4: in vitro model of pancreatic cancer progression. Summary of the characteristics of the pancreatic cancer cell lines used in this study (Lee et. al. 2003; Lee et. al. 2005; Campbell et. al. 2007; Kyriazis et. al. 1982; Deer et. al. 2010; Kyriazis et. al. 1986; Dahiya et. al. 1993). Abbreviations: hTERT = human telomerase, $H P N E=$ human pancreatic nestin expressing, and HPV16 = human papilloma virus type 16. (Author diagram). 
a.

\begin{tabular}{|c|c|c|c|c|}
\hline & H4023 & H4036 & Capan 2 & Capan 1 \\
\hline Azidosugar & $-\quad-M$ & $-\quad-M$ & $-M$ & $M$ \\
\hline Alexa 488 & -++ & -++ & $-\quad++$ & + \\
\hline
\end{tabular}
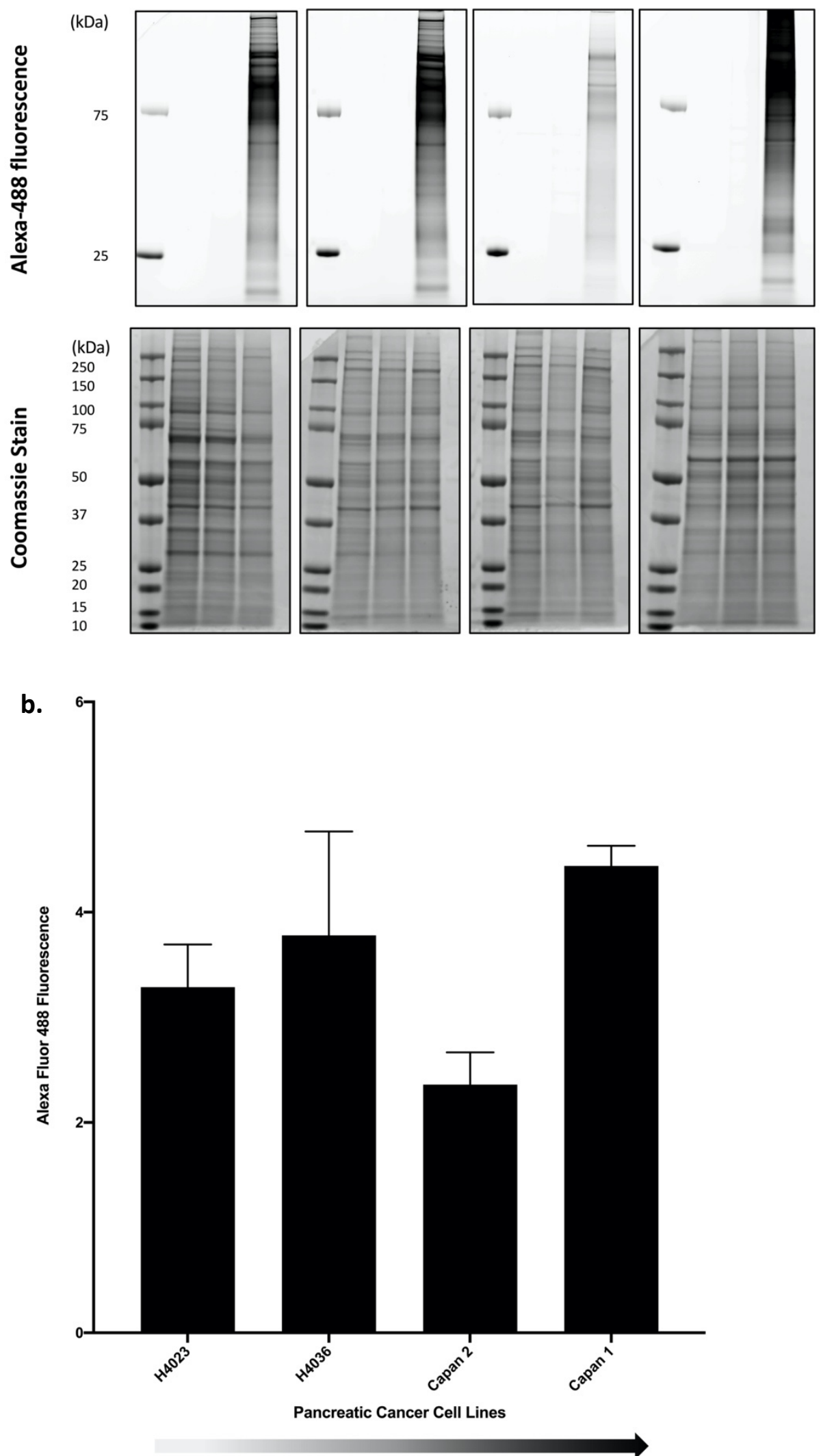
Figure 4.4: In-gel fluorescence detection of azidosugars incorporated into the membrane proteome of pancreatic cancer cells. (a) Representative in-gel Alexa Fluor 488 fluorescence images from a panel of pancreatic cell lines with increasing metastatic potential. Total protein per lane was visualised using Coomassie staining. Background controls were included to detect non-specific fluorescence. (b) For each lane treated with $\mathrm{N}$ azidoacetylmannosamine (Ac4ManNAz), total Alexa Fluor 488 fluorescence was quantified using Fiji Image analysis software, normalised against the Coomassie stain fluorescence within the same lane, and presented as a ratio. All data represent the average \pm S.D. of one experiment, each with 3 replicates. $p$ values represent significance by one-way ANOVA. The darker shades in the arrow's greyscale represent an increase in the metastatic potential of the panel of pancreatic cancer cell lines. Abbreviations: $M=A c_{4} M a n N A z$ treated, $G=$ $\mathrm{Ac}_{4}$ GalNAz treated, $\mathrm{H} 4023$ = human telomerase (hTERT)- Human Pancreatic Nestin Expressing (HPNE) cell line and H4036 = hTERT-HPNE E6/E7 (H4036) cell line.

Overall, an increase in the overall Alexa Flour 488 fluorescence was seen across the prostate (Figure 4.2b, i and c, i), breast (Figure 4.3b, i and c, i) and pancreatic (Figure 4.4b) cancer cell lines. Whether treated with $\mathrm{Ac}_{4} \mathrm{ManNAz}$ or $\mathrm{Ac}_{4} \mathrm{GalNAz}$, the overall intensity of Alexa Fluor 488 fluorescence from the most metastatic cell line in each panel (PC3 prostate cancer cell line, MDA MB 231 breast cancer cell line, and Capan 1 pancreatic cancer cell line) was always significantly higher than the overall intensity of Alexa Fluor 488 fluorescence from the least metastatic cell line in each panel (PNT1A normal prostate cell line, MDA MB 453 breast cancer cell line, and H4023 normal pancreatic cell line). Therefore, a general increase in azidosugar-specific glycosylation was associated with an increase in metastatic potential.

\subsubsection{Specific azidosugar labelling of $\mathbf{N}$-linked glycoproteins probed using deglycosylation enzymes}

Loss of azidosugar-specific fluorescence in metabolically labelled lysates treated with bacterial sialidases (neurominidases), which cleave terminal sialic residues in glycoconjugates (Lewis and Lewis 2012) (Chapter 1: Figure 1.4), has been used previously to 
confirm the presence of bioorthogonally labselled sialylglycoproteins by 1D western blot (Luchansky et. al. 2004; Belardi et. al. 2013; Kang et. al. 2015; Spiciarich et. al. 2017). Therefore, we investigated the azide-specific labelling of N-linked glycoproteins using peptide-N4-( $\mathrm{N}$-acetyl- $\alpha$-glucosaminyl) asparagine amidase (PNGase), an enzyme which specifically cleaves $\mathrm{N}$-glycans from the innermost asparagine residues within the $\mathrm{N}$-linked glycoproteins (Kuhn et. al. 1994).

Cleavage of N-glycans by PNGase F was optimised using the glycoprotein horseradish peroxidase (HRP), and monitored by electrophoretic mobility shifts to a faster migrating product with a lower molecular weight, generated from $\mathrm{N}$-glycan specific changes in mass:charge ratio (Freeze and Kranz 2010; Hofherr et. al. 2014). Deglycosylation of HRP (Figure 4.5a, black arrowhead), was observed as early as 3 hours after treatment with PNGase F (Figure 4.5a, white arrowhead). Next, the LNCAP C4-2B prostate cancer and MDA MB 468 breast cancer cell lines were metabolically labelled with $\mathrm{Ac}_{4} \mathrm{ManNAz}$ or $\mathrm{Ac}_{4} \mathrm{GaINAz}$. Metabolically labelled membrane enriched extracts were labelled with alkyne-Alexa Fluor 488 and treated with PNGase F from Elizabethkingia miricola prior to in-gel fluorescence imaging as described previously. The presence of azidosugar labelled N-glycosylated proteins was confirmed by monitoring electrophoretic mobility shifts in bands of Alexa Fluor 488 fluorescence (Figure 4.5). After 3 hours of treatment with PNGase F, loss of Alexa Fluor 488 fluorescence was observed in $\mathrm{Ac}_{4} \mathrm{ManNAz}$ or $\mathrm{Ac}_{4} \mathrm{GalNAz}$ labelled glycoproteins extracted from the LNCAP C4-2B prostate cancer (Figure 4.5b) and the MDA MB 468 breast cancer (Figure 4.5c) cell lines, particularly between a 50-150 kDa molecular weight range. Remaining bands of $\mathrm{Ac}_{4} \mathrm{ManNAz}$ or $\mathrm{Ac}_{4} \mathrm{GalNAz}$ specific Alexa Fluor 488 fluorescence may represent azidosugar labelled residues inaccessible to PNGase F (Luchansky et. al. 2004).

The core structure of the O-glycosylated proteins contains an innermost serine or threonine residue linked to an $\mathrm{N}$-acetylgalactosamine residue (Chapter 1: Figure 1.4, red perforated boxes). Therefore, we expected the majority of glycoproteins labelled with $\mathrm{Ac}_{4} \mathrm{GalNAz}$, which is a synthetic analogue of $\mathrm{N}$-acetylgalactosamine, to be O-glycans, and consequently not cleavable by PNGase F. This was confirmed by observing negligible shifts in Alexa-488 fluorescence bands in samples treated with $\mathrm{Ac}_{4} \mathrm{GalNAz}$ and PNGase $\mathrm{F}$, in comparison to cell lysates metabolically labelled with $\mathrm{Ac}_{4} \mathrm{ManNAz}$. Overall, our data confirmed azide-specific 
metabolic labelling of $\mathrm{N}$-linked glycoproteins and the potential in using bioorthogonal chemistry to specifically capture aberrantly glycosylated proteins, for identification using mass spectrometry.

a.

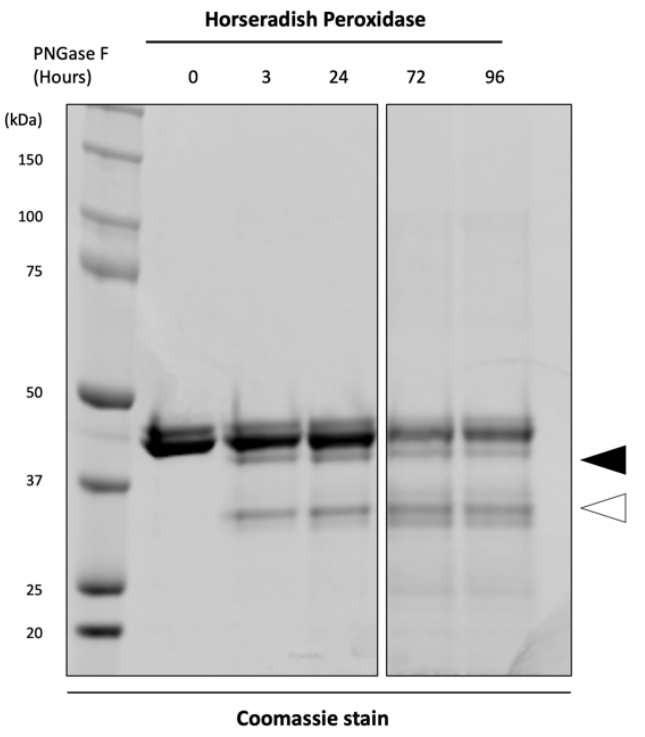

b.
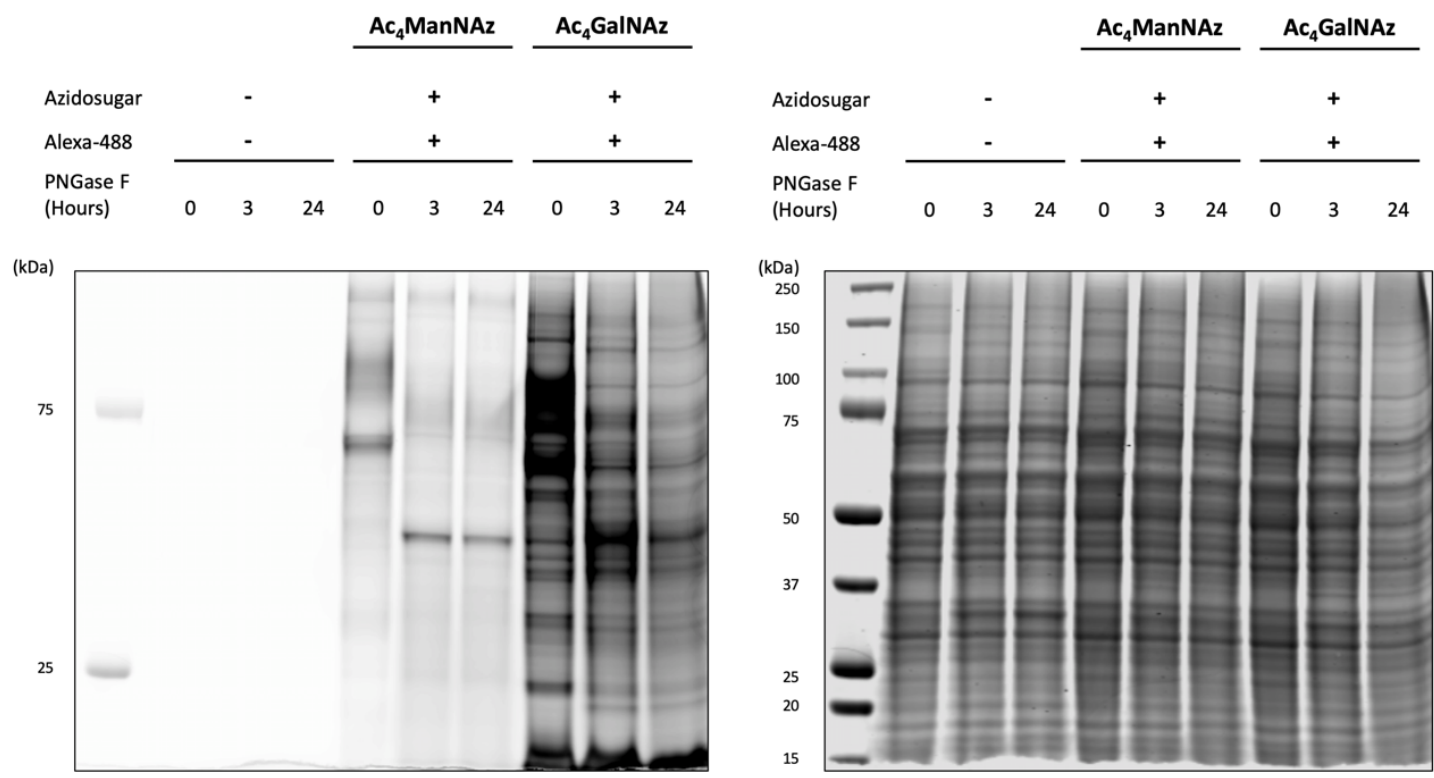

Alexa-488 fluorescence

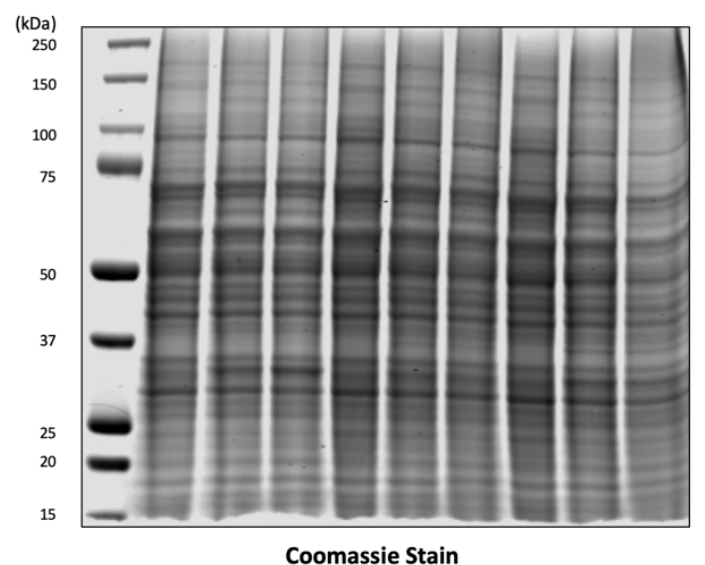


c.

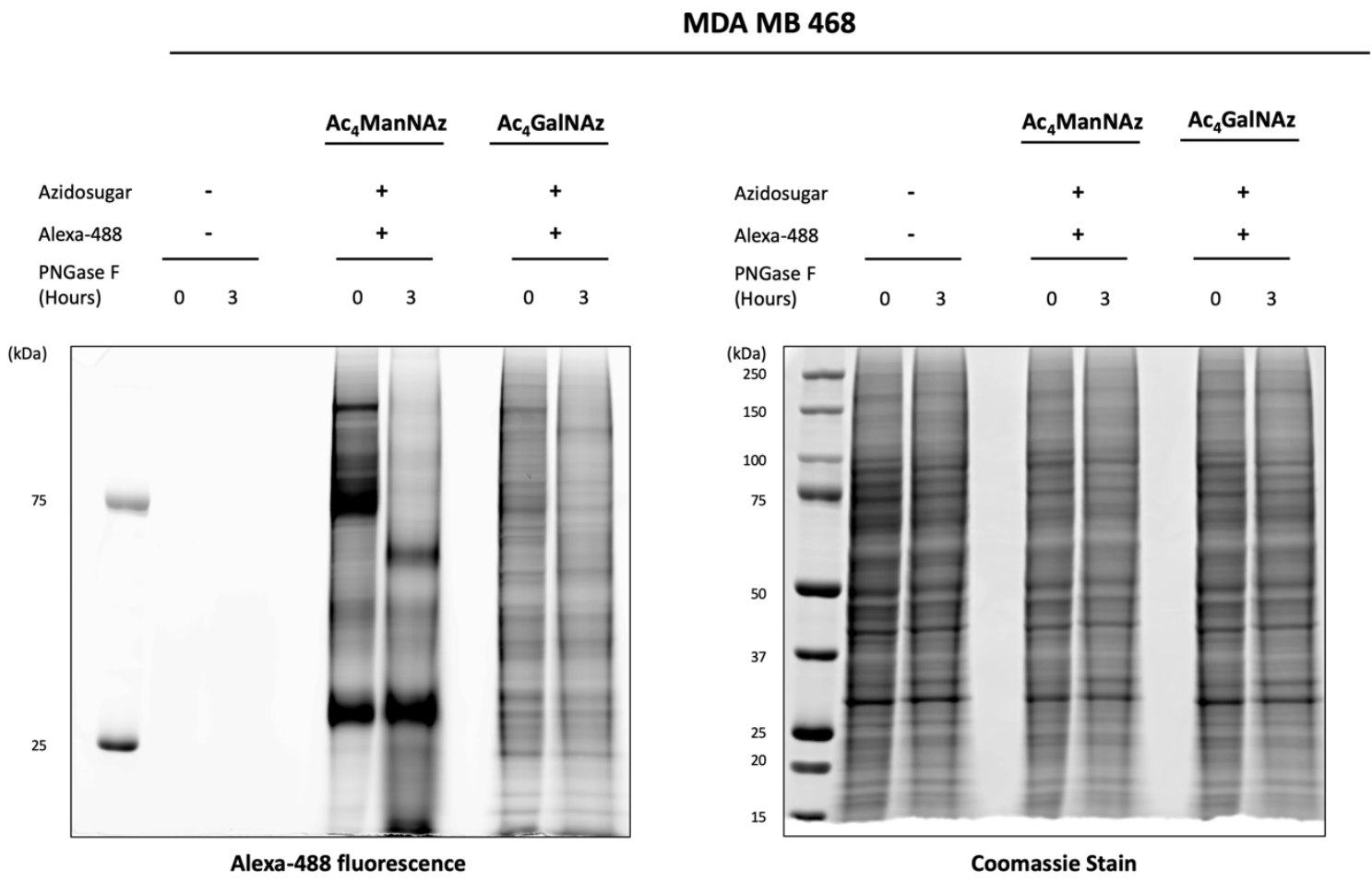

Figure 4.5: In-gel fluorescence detection of azidosugar-labelled N-glycans within the membrane proteome using PNGase F deglycosylation. Horseradish peroxidase and membrane-enriched extracts were treated with PNGase F from Elizabethkingia miricola, for 0, 3, 24, 72 and 96 hours. Total protein (10 $\mu$ g per lane) was visualised using Coomassie staining. (a) Cleavage of $\mathrm{N}$-glycans and deglycosylation of horseradish peroxidase treated with PNGase F (white arrowhead) monitored by mobility shifts (black arrowhead). (b) Representative cleavage of azidosugar-labelled N-glycans monitored by mobility shifts in Alexa-488 fluorescence bands in the LNCAP C4-2B prostate cancer cell line. (c) Representative cleavage of azidosugar-labelled N-glycans monitored by loss of Alexa-488 fluorescence in the MDA MB 468 breast cancer cell line. Background controls were included to detect nonspecific fluorescence. Abbreviations: $M=A c_{4}$ ManNAz treated, $G=A c_{4} G a l N A z$ treated.

\subsubsection{Azidosugar labelling of the membrane proteome analysed using 2D gel electrophoresis}

For a more in-depth assessment of the complex changes in glycoprotein populations and azidosugar incorporation, labelled membrane enriched extracts were resolved based on size 
and charge using 2D-gel electrophoresis. Two prostate cancer cell lines (LNCAP C4-2 and LNCAP (4-2B) with increasing metastatic potential were incubated with $\mathrm{Ac}_{4} \mathrm{ManNAz}$ and $\mathrm{Ac}_{4} \mathrm{GalNAz}$. The membrane proteome was isolated by detergent-based fractionation, the incorporated azide groups covalently ligated to alkyne-biotin, and lysine residues in the labelled proteins tagged with a Cy3 NHS ester. The labelled extracts were resolved by 2D western blot and total protein detected by Cy3 fluorescence (Figure 4.6a). Proteins labelled with $\mathrm{Ac}_{4} \mathrm{ManNAz}$ (Figure 4.6b) or $\mathrm{Ac}_{4} \mathrm{GalNAz}$ (Figure 4.6c) were then detected using Streptavidin-800 fluorescence.

Eight spots exhibiting an increase in both $\mathrm{Ac}_{4} \mathrm{ManNAz}$ and $\mathrm{Ac}_{4} \mathrm{GalNAz}$ labelling were observed (Figure 4.6b and c, white arrowheads). The regions of glycan-specific Streptavidin800 fluorescence and total protein Cy3 fluorescence from each of the eight spots were coregistered and compared between the two prostate cancer cell lines (Figure 4.6d). An increase in azidosugar labelling was observed despite the expression of proteins within the eight spots, appearing similar. This suggested that differential glycosylation may be a more sensitive and specific marker for monitoring cancer progression, than increases in total protein expression alone.

We observed regions exhibiting an increase in $\mathrm{Ac}_{4} \mathrm{GalNAz}$ labelling, that correlated with an increase in the metastatic potential of the prostate cancer cell lines (Figure 4.6c, black arrowheads). Furthermore, more spots of Streptavidin-800 fluorescence were detected when the prostate cancer cell lines were treated with $\mathrm{Ac}_{4} \mathrm{GalNAz}$ (Figure 4.6c), in comparison to $A c_{4} M a n N A z$ (Figure 4.6b). This was expected, as this is due to labelling of the core structure (serine/threonine- $\mathrm{N}$-acetylgalactosamine) present in all O-glycosylated proteins (Chapter 1: Figure 1.4, red perforated boxes). These data demonstrated the use of bioorthogonal chemistry in detecting the differential $\mathrm{Ac}_{4} \mathrm{ManAz-specific}$ sialyation and $\mathrm{Ac}_{4} \mathrm{GalNAz}$-specific $\mathrm{N}$-acetylgalactosamine glycosylation of proteins in an in vitro model of prostate cancer progression. 
a.

LNCAP C4-2
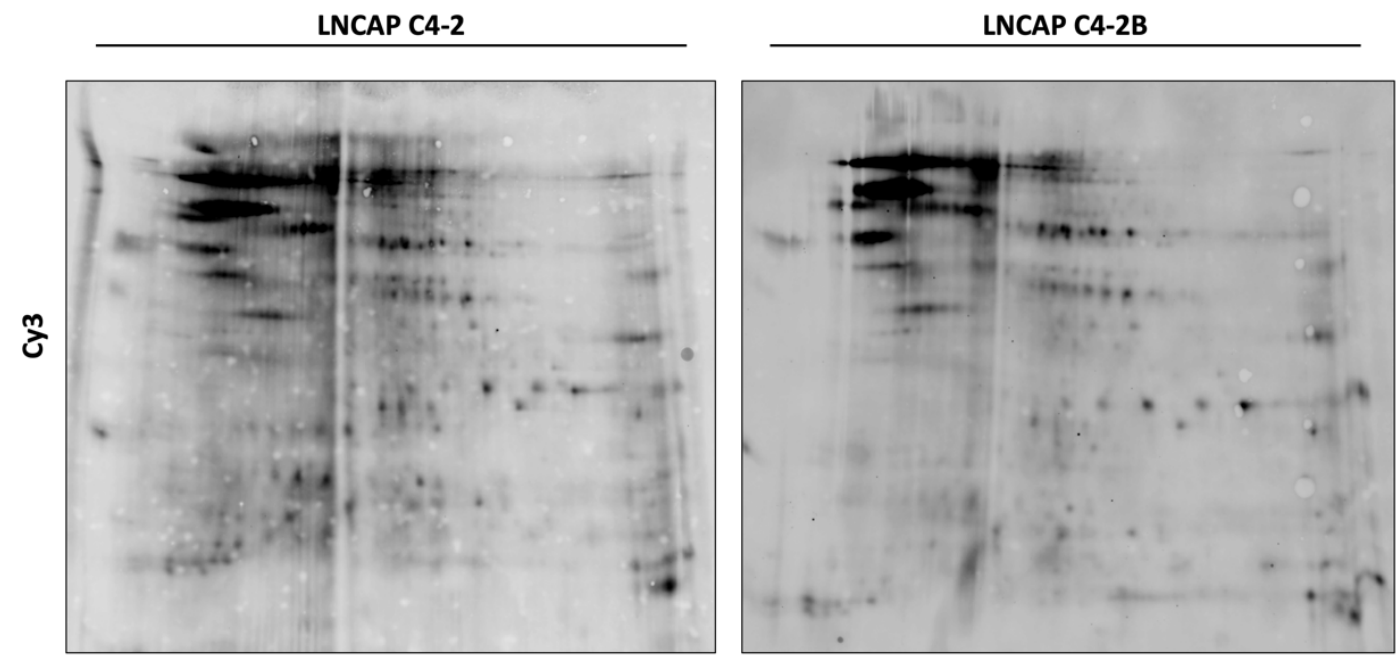

b.

$\mathrm{Ac}_{4}$ ManNAz

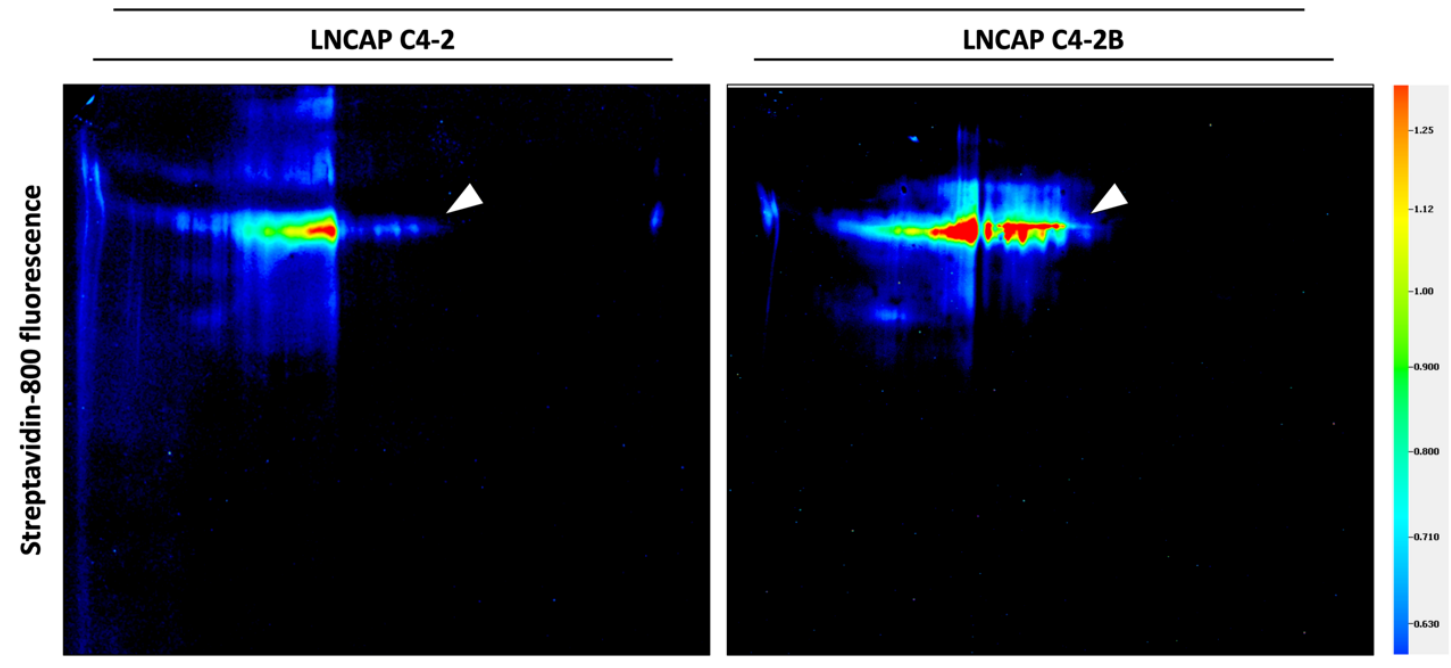


c.

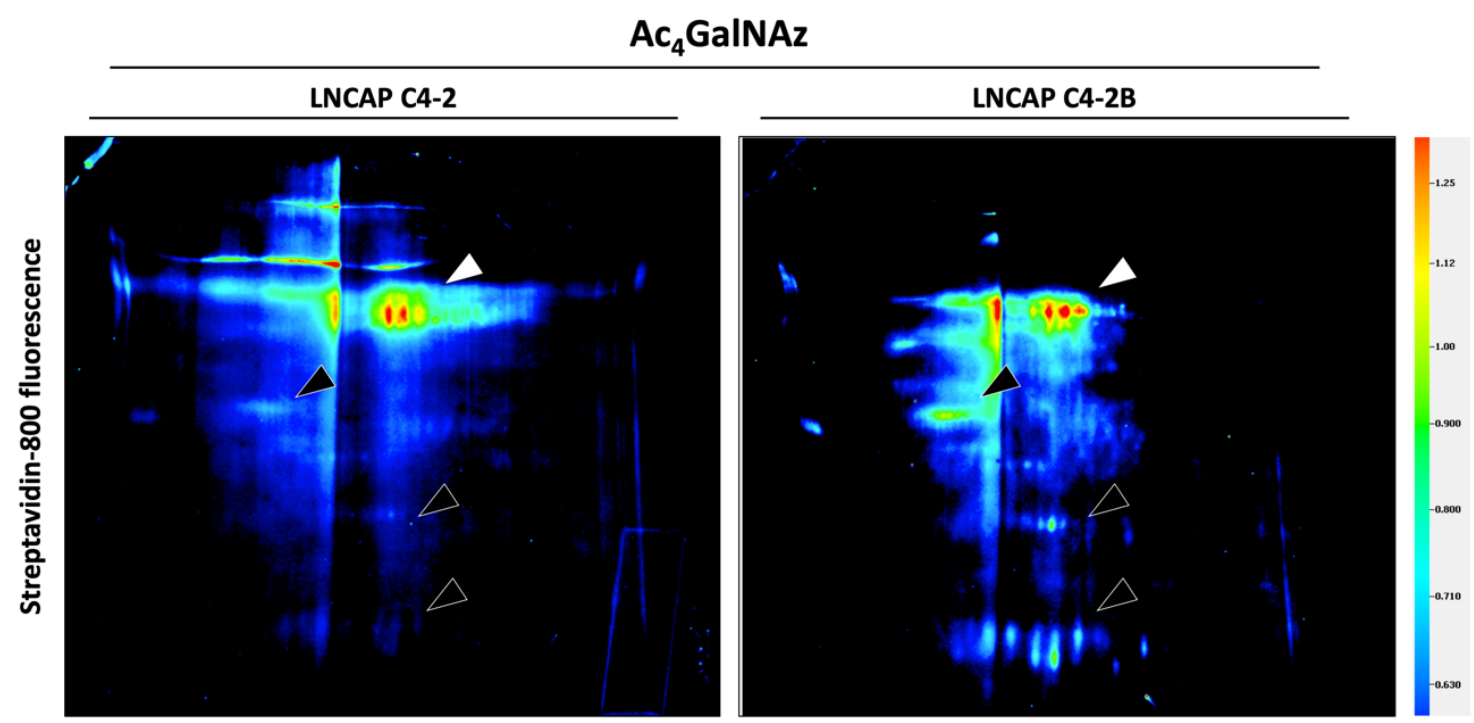

d.

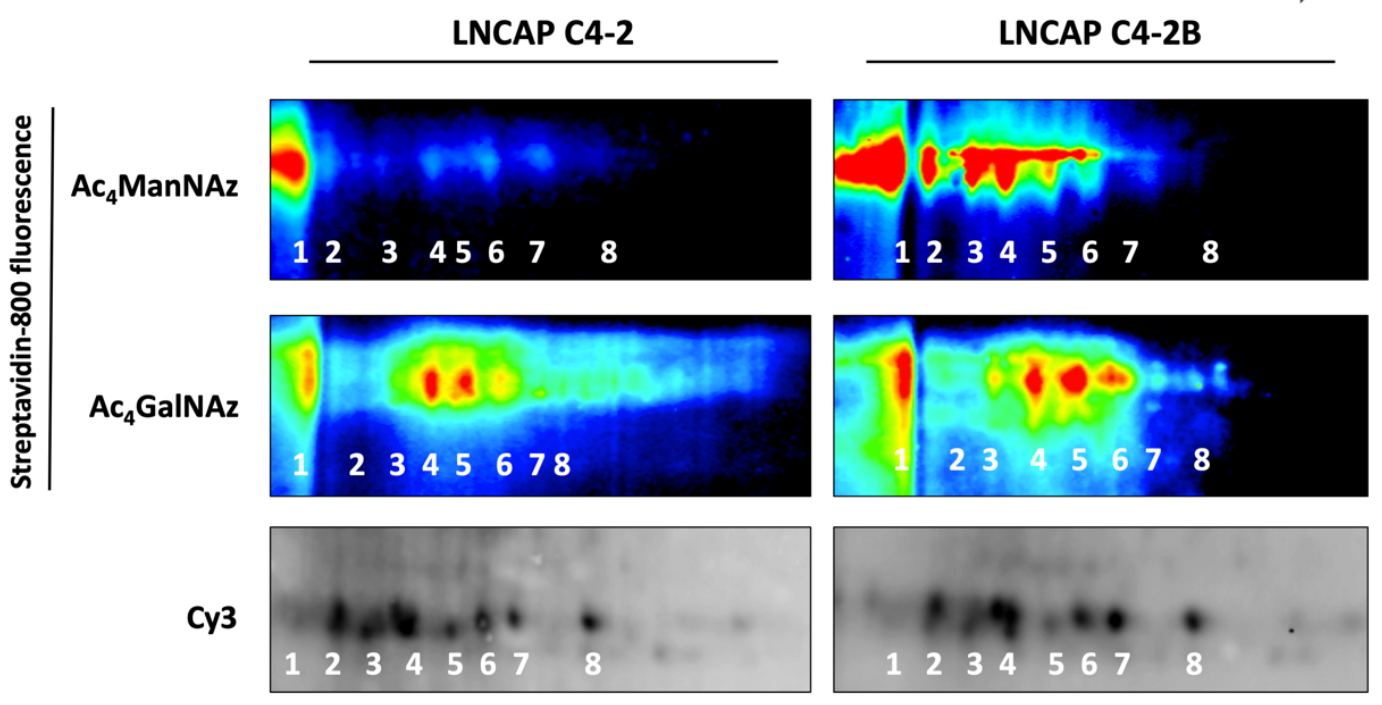

Figure 4.6: Fluorescence detection of azidosugars incorporated into the membrane proteome of prostate cancer cell lines using 2D-gel electrophoresis. The membrane proteome was extracted from two prostate cancer cell lines (LNCAP C4-2 and LNCAP C4-2B) labelled with $\mathrm{N}$-azidoacetylmannosamine (Ac4ManNAz) or $\mathrm{N}$ - azidoacetylgalactosamine (AC4GaINAz), and alkyne-biotin. $35 \mu \mathrm{g}$ of the labelled extracts were reacted Cy3 NHS ester, resolved by $2 D$ western blot and azidosugar-labelled glycoproteins detected using Streptavidin 800 fluorescence. (a) Representative total protein images detected using Cy3 
fluorescence. (b) Detection of Ac4ManNAz-labelled proteins using Streptavidin-800

fluorescence. (c) Detection of Ac4GaINAz-labelled proteins using Streptavidin-800

fluorescence. Regions exhibiting an increase in Ac4GalNAz labelling, that correlated with an increase in the metastatic potential of the prostate cancer cell lines, are highlighted (black arrowheads). (d) Eight spots exhibiting an increase in both $A c_{4} M a n N A z$ and $A c_{4} G a I N A z$ labelling are highlighted (white arrowheads in b and c). Streptavidin-800 fluorescence and Cy3 fluorescence have been co-registered. The darker shades in the arrow's greyscale represent an increase in the metastatic potential of the prostate cancer cell lines.

2D gel electrophoresis analysis of the glycosylated membrane proteome was similarly conducted using two breast cancer cell lines (MDA MB 468 and MDA MB 231) with increasing metastatic potential (Figure 4.7). Azidosugar-labelled extracts were resolved by 2D western blot and total protein detected by Cy3 fluorescence (Figure 4.7a). Proteins labelled with $\mathrm{Ac}_{4} \mathrm{ManNAz}$ (Figure 4.7b) or $\mathrm{Ac}_{4} \mathrm{GalNAz}$ (Figure 4.7c) were then detected using Streptavidin-800 fluorescence.

We observed regions exhibiting an increase in $\mathrm{Ac}_{4}$ ManNAz labelling, that correlated with an increase in the metastatic potential of the breast cancer cell lines (Figure $4.7 \mathrm{~b}$, black arrowheads). Spots exhibiting a decrease in both $A c_{4} M a n N A z$ and $A c_{4} G a l N A z$ labelling were also observed (Figure $4.7 \mathrm{~b}$ and c, white arrowheads). 
a.

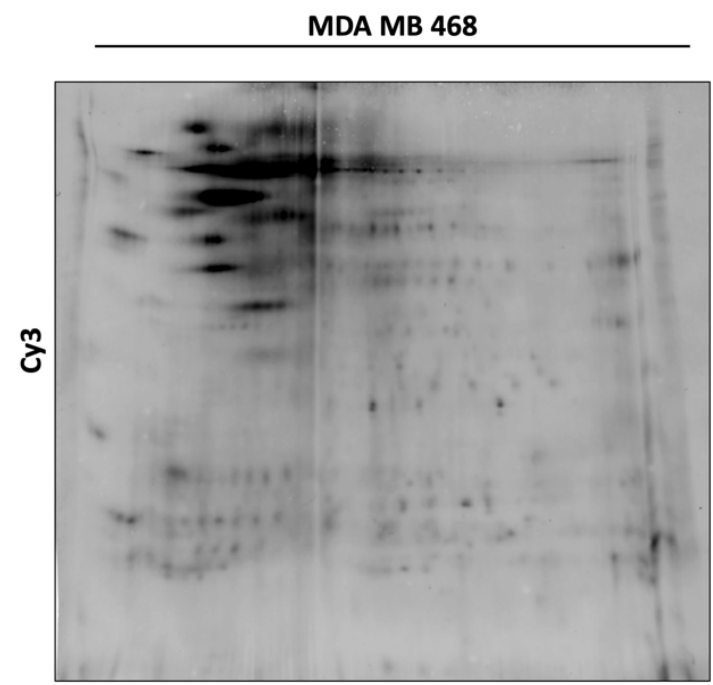

MDA MB 231

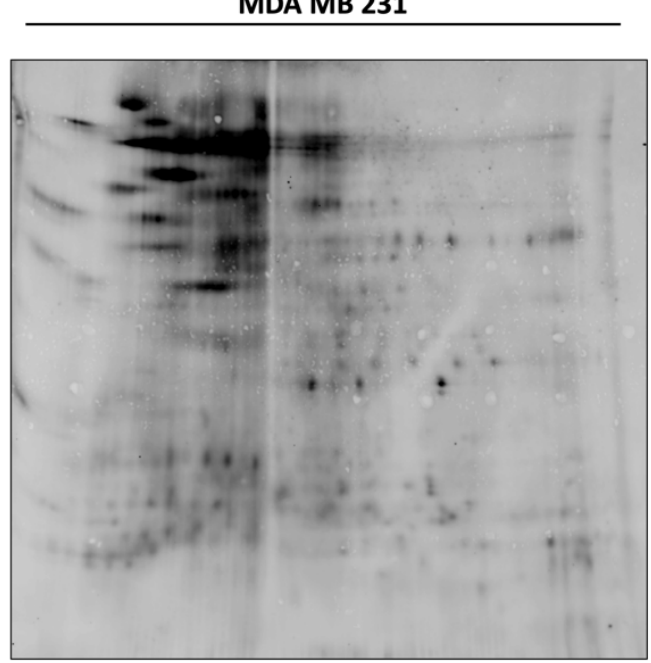

b.

$\mathrm{Ac}_{4}$ ManNAz

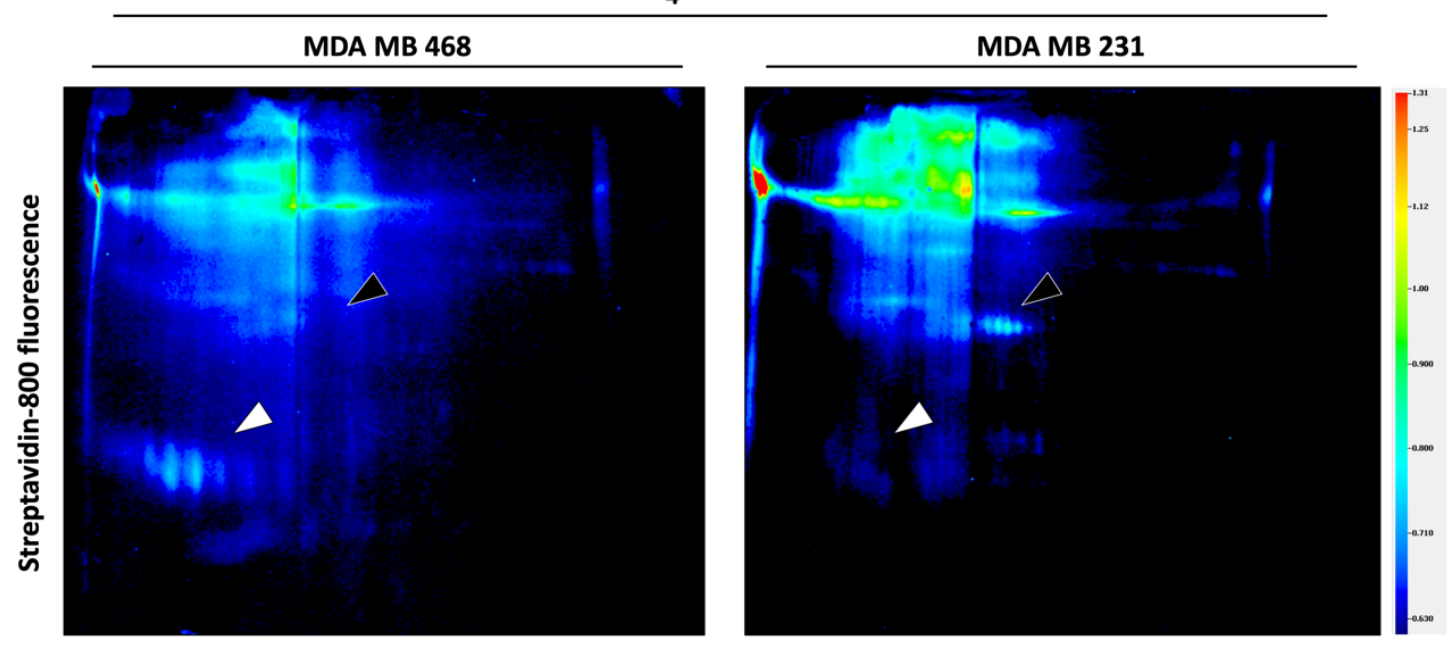


c.

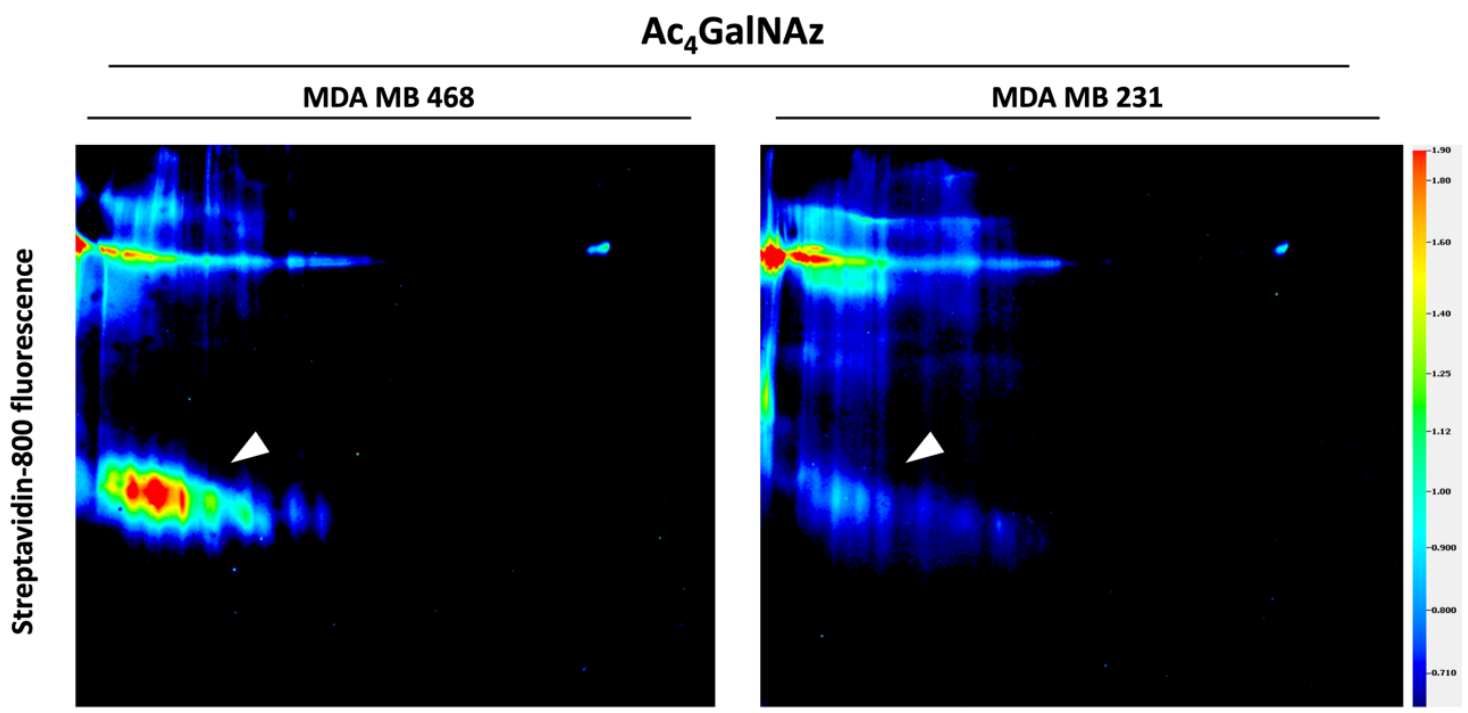

Figure 4.7: Fluorescence detection of azidosugars incorporated into the membrane proteome of breast cancer cell lines using 2D-gel electrophoresis. The membrane proteome was extracted from two breast cancer cell lines (MDA MB 468 and MDA MB 231) labelled

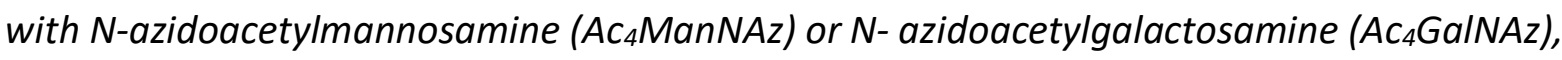
and alkyne-biotin. $35 \mu \mathrm{g}$ of the labelled extracts were minimally reacted with Cy3 NHS ester, resolved via 2D western blot and azidosugar labelled glycoproteins detected using Streptavidin 800 fluorescence. (a) Representative total protein images using Cy3 fluorescence. (b) Detection of Ac4ManNAz-labelled proteins using Streptavidin-800 fluorescence. Regions exhibiting an increase in $A_{4} M a n N A z$ labelling, that correlated with an increase in the metastatic potential of the prostate cancer cell lines, are highlighted (black arrowheads). (c) Detection of Ac 4 GalNAz-labelled proteins using Streptavidin-800 specific fluorescence. Regions exhibiting an increase in both $A c_{4} M a n N A z$ and $A c_{4} G a I N A z$ labelling are highlighted (white arrowheads in b and c). The darker shades in the arrow's greyscale represent an increase in the metastatic potential of the breast cancer cell lines.

Total protein images of membrane-enriched extracts from the prostate (Figure 4.6a) and breast (Figure 4.7a) cancer cell lines were remarkably similar. This suggested that although the population of proteins within the membrane proteome is similar, the proteins that are 
targeted for aberrant glycosylation are influenced by tumour type and the repertoire of glycoenzymes expressed. Collectively, the data continued to demonstrate the use of bioorthogonal chemistry in specifically detecting the differential sialyation and $\mathrm{N}$ acetylgalactosamine glycosylation of proteins from human cell lines representing the progression of prostate, breast, and pancreatic cancer.

\subsection{Discussion}

The link between aberrant protein glycosylation and cancer progression has formed the basis for using glycoprotein detection in the clinical management of cancer. However, the utility of biomarkers has been challenged in recent years due to low cancer specificity and high false-positive rates (Mayeux 2004). Improved aberrantly glycosylated variants of established biomarker have been discovered that improve the clinical utility of these biomarkers, such as AFP-L3, an aberrantly fucosylated version of AFP significantly elevated in hepatocellular carcinoma patients, in comparison to benign liver diseases such as cirrhosis, providing a marker for the onset of hepatocellular carcinoma (Block et. al. 2005; Meany and Chan 2011; Adamczyk et. al. 2012; Pinho and Reis 2015).

Towards the identification of novel aberrantly glycosylated proteins, with the aim of discovering prognostic markers of progression, we utilised bioorthogonal chemistry and gel electrophoresis to visualise differential $\mathrm{N}$-azidoacetylmannosamine ( $\left.\mathrm{cc}_{4} \mathrm{ManAz}\right)$-specific sialyation and $\mathrm{N}$-azidoacetylgalactosamine ( $\left.\mathrm{Ac}_{4} \mathrm{GalNAz}\right)$-specific glycosylation of glycoproteins across panels of cell lines, some of which had been validated previously as suitable in vitro models of cancer progression (Chapter 2). Panels of prostate, breast, and pancreatic cancer cell lines, with increasing metastatic potential, were incubated with $\mathrm{Ac}_{4} \mathrm{ManNAz}$ and $\mathrm{Ac}_{4} \mathrm{GalNAz}$, which were incorporated biosynthetically into cell-surface glycans. The membrane proteome was isolated and the incorporated azide groups covalently ligated to alkyne-Alexa Fluor 488 using copper-catalysed chemistry. Azidosugar-labelled glycoproteins were resolved by gel electrophoresis and detected using Alexa-488 fluorescence. 
An increase in the overall Alexa Fluor 488 fluorescence was seen across the prostate (Figure 4.2b, i and c, i), breast (Figure 4.3b, i and c, i) and pancreatic (Figure 4.4b) cancer cell lines. Whether treated with $\mathrm{Ac}_{4} \mathrm{ManNAz}$ or $\mathrm{Ac}_{4} \mathrm{GalNAz}$, the overall intensity of Alexa Fluor 488 fluorescence from the most metastatic cell line in each panel (PC3 prostate cancer cell line, MDA MB 231 breast cancer cell line, and Capan 1 pancreatic cancer cell line) was always significantly higher than the overall intensity of Alexa Fluor 488 fluorescence from the least metastatic cell line in each panel (PNT1A normal prostate cell line, MDA MB 453 breast cancer cell line, and H4023 normal pancreatic cell line). Therefore, a general increase in azidosugar-specific glycosylation was associated with an increase in metastatic potential. This is consistent with increases in the sialyation (Büll et. al. 2014) of cell surface glycoproteins (Schultz et. al. 2012; Stowell et. al. 2015) observed during disease progression in prostate (Munkley et. al. 2016), breast (Kölbl et. al. 2016), and pancreatic cancers (Munkley 2019), which has been attributed to the generation of tumour-associated antigens on carrier proteins such as the Sialyl Lewis antigens, and unmodified or sialylated Thomsennouveau (Ju et. al. 2011) (Chapter 1: Figure 1.9) and Thomsen-Friedenreich (Springer et. al. 1975) (Chapter 1: Figure 1.10) antigens (Natoni et. al. 2016; Pinho and Reis 2015).

A high degree of $\mathrm{Ac}_{4} \mathrm{ManNAz}$ metabolic labelling was observed in the human telomerase (hTERT)- human pancreatic nestin expressing (HPNE) cell line (H4023) and the hTERT-HPNE derived hTERT-HPNE E6/E7 cell line (H4036), which are both considered to represent normal pancreatic epithelium (Table 4.4). The parent hTERT-HPN cell line has previously been shown with tandem mass spectrometry to have significantly higher levels of $\alpha 2,3$-sialylation and sialyl Lewis $X$ antigen (Chapter 1: Figure 1.8) than the tumourigenic pancreatic adenocarcinoma (PDAC) PaTu-S, PaTu-T, PDAC 1, and PDAC 2 cell lines (Holst et. al. 2017). This is contrary to reports correlating an increase in sialyation with an increase in cancer aggressiveness (Natoni et. al. 2016), but consistent with high levels of Ac 4 ManNAz-specific Alexa Fluor 488 fluorescence observed in metabolically labelled lysates prepared from the hTERT-HPN cell lines (Figure 4.4). Further independent repeats are needed to confirm if the increases in Alexa Fluor 488 fluorescence are statistically significant. Future studies would involve validating the characteristics of each pancreatic cell line using scratch wound assays to compare migration, Boyden chambers to compare invasion and immunohistochemistry to 
compare the relative expression of known invasion biomarkers (Pijuan et. al. 2019; Justus et. al. 2014).

Previously, we used flow cytometric analysis to quantify global azidosugar labelling of the glycoproteins and glycolipids in a panel of breast cancer cell lines (Chapter 3). An increase in global $\mathrm{Ac}_{4} \mathrm{ManNAz}$ and $\mathrm{Ac}_{4} \mathrm{GalNAz}$ labelling correlated with an increase in the metastatic potential of the breast cancer cell lines (Chapter 3: Figure 3.2). We observed a strong positive correlation $\left(r^{2}=0.8217\right.$ ) (Figure $4.3 \mathrm{~d}$, i) between increases in global $\mathrm{Ac}_{4} \mathrm{ManNAz}^{-}$ sialylation of the glycoconjugates observed by flow cytometry, and increases in the $\mathrm{Ac}_{4}$ ManNAz-Alexa Fluor 488 fluorescence (Figure 4.3b, i), representing specific sialylation of the glycoproteins. Therefore, the glycoprotein fraction may be principally responsible for the increased glycan labelling observed with an increase in the metastatic potential of the breast cancer cell lines (Chapter 3: Figure 3.3).

What is yet to be answered is whether increases in bioorthogonal metabolic labelling detects global increases in glycoprotein sialyation or increased turnover (Colley et. al. 2017) of cell surface $\mathrm{N}$ - and $\mathrm{O}$-glycans. It is unknown whether azidosugars are preferentially incorporated into glycan structures via specific glycosidic linkages, and if this occurs at multiple epitopes within a glycoconjugate in a site-specific or tissue-specific manner. This would be influenced by changes in the expression or activity of sialyltransferases in the more metastatic cell lines, which also has not been investigated. We confirmed the azide-specific labelling of $\mathrm{N}$-linked glycoproteins using PNGase $\mathrm{F}$, an enzyme which specifically cleaves $\mathrm{N}$-glycans from the innermost asparagine residues within the $\mathrm{N}$-linked glycoproteins (Kuhn et. al. 1994) (Figure 4.5). Future studies could involve using bacterial sialidases (neuraminidases), which cleave terminal sialic residues in glycoconjugates (Lewis and Lewis 2012) to confirm the presence of bioorthogonally labelled sialoglycoproteins (Luchansky et. al. 2004; Belardi et. al. 2013; Spiciarich et. al. 2017). Furthermore, quantitative mass spectrometry is required to confirm if increases in azidosugar labelling are influenced by the overexpression of glycoproteins across the panels of cell lines used in this study.

For a more in-depth assessment of the complex changes in glycoprotein populations and azidosugar incorporation, labelled membrane enriched extracts were resolved based on size 
and charge using 2D-gel electrophoresis. Populations of azidosugar labelled proteins in prostate (Chapter 4: Figure 4.6, arrowheads) and breast cancer (Chapter 4: 4.7, arrowheads) cell lines, exhibited distinct laddered spot patterns. $\alpha_{1}$-antitrypsin extracted from the serum of patients with congenital disorders of glycosylation type 1 (CGD I), a condition defined by underglycosylation of proteins, and resolved by 2D gel electrophoresis, exhibited a distinct laddered spot pattern (Mills et. al. 2001; Mills et. al. 2003). Subsequent analysis of $\alpha_{1}$ antitrypsin spots using matrix-assisted laser-desorption ionisation-time-of-flight mass spectrometry, revealed 3 subforms: unglycosylated, monoglycosylated or diglycosylated $\alpha_{1-}$ antitrypsin, which differed by one sialic acid residue, observed on 2D gels by an increase in isoelectric point (pl) of $0.1 \mathrm{pH}$ unit (Mills et. al. 2001; Mills et. al. 2003). PSA extracted from the serum of healthy individuals and prostate cancer patients, resolves on 2D gels into 5 spots, representing the 5 isoforms of PSA (F1-F5), with pls of $6.4,6.6,6.8$ and 7.0, respectively (Sarrats et. al. 2010). High-performance liquid chromatography (HPLC) analysis showed that the F1-3 subforms where mono and disialylated, the F4 subform was monosialyated and the F5 subform was unglycosylated (Sarrats et. al. 2010). Therefore, the increase in pls or reduced acidity of the PSA subforms from F1-F5 was attributed to decreases in sialic acid content. Based on these studies, the laddering pattern observed here with the azidosugar labelled proteins may be caused by differential glycan content of protein glycoforms. This is supported by the observation of differences in the Streptavidin-800 fluorescence of protein spots within a single cell type. Additionally, we compared azidosugar labelling of glycoproteins between two prostate cancer cell lines with increasing tumourigenicity. Eight spots exhibited an increase in both $\mathrm{Ac}_{4} \mathrm{ManNAz}$ and $\mathrm{Ac}_{4} \mathrm{GalNAz}$ labelling in the LNCAP C4 and LNCAP C4-2B prostate cancer cell lines (Figure 4.6b and c, white arrowheads), despite the expression of proteins within the eight spots, appearing similar.

Overall, using 1D and 2D gel electrophoresis, we observed differential glycosylation of protein populations within a single line, and between cell lines with increasing metastatic potential. Our data suggest that differential glycosylation may be a more sensitive and specific marker for monitoring cancer progression, than increases in total protein expression alone. 


\subsection{Materials and Methods}

\subsubsection{Cell lines}

The PNT1A, LNCAP, and LNCAP C4-2B human prostate cell lines were obtained from the Cancer Research UK Cambridge Institute Biorepository, based on stocks made available by Joe Kuo. The LNCAP C4, LNCAP C4-2, and PC3 human prostate cancer cell lines were purchased from ATCC. All prostate cell lines were cultured in RPMI with L-glutamine (Gibco ${ }^{\circledR}$, Invitrogen ${ }^{\mathrm{TM}}$ ) and 10\% Foetal bovine serum (FBS Gold, PAA Laboratories).

The MDA MB 453, MCF-7, MDA-MB-468, and MDA MB 231 human breast cancer cell lines were obtained from the Cancer Research UK Cambridge Institute Biorepository, based on stocks made available by Joe Kuo. All breast cell lines were cultured in DMEM with Lglutamine (Gibco ${ }^{\circledR}$, Invitrogen ${ }^{\mathrm{TM}}$ ) and 10\% Foetal bovine serum (FBS Gold, PAA Laboratories).

The human telomerase (hTERT)- human pancreatic nestin expressing (HPNE) (H4023) and hTERT-HPNE E6/E7 (H4036) human pancreatic cell lines were purchased from ATCC and cultured in M3: BaseF media (Incell). The Capan 1 and Capan 2 cell lines were obtained from the Cancer Research UK Cambridge Institute Biorepository, based on stocks made available by the Jodrell group. The Capan 1 and Capan 2 cell lines were cultured initially in IMEM $\left(\right.$ Gibco $^{\circledR}$, Invitrogen ${ }^{\mathrm{TM}}$ ) and Mc Coy's $\left(\mathrm{Gibco}^{\circledR}\right.$, Invitrogen $^{\mathrm{TM}}$ ) media respectively, and $10 \%$ Foetal bovine serum (FBS Gold, PAA Laboratories). All pancreatic cell lines were then adapted to M3: BaseF media (Incell) in a step-wise manner by Ms Laura Brandt (Part III undergraduate student, Brindle Laboratory, $\mathrm{CRUK} \mathrm{Cl}$ ). This involved periodically modifying the old:new media ratio from 50:50, to $75: 25$ and finally 0:100. Culturing of the pancreatic cell lines following adaptation was carried out by Dr. André Neves (Brindle Laboratory, CRUK $\mathrm{Cl})$.

Cell lines were genotyped and authenticated by Short Tandem Repeat (STR) DNA profiling and comparison with available databases from ATCC or DSMZ. All cell lines tested negative for Mycoplasma using the MycoProbe ${ }^{\mathrm{TM}}$ Kit (R\&D Systems). 


\subsubsection{Tissue culture}

Cell lines were maintained in Nunc ${ }^{T M} 175 \mathrm{~cm}^{2}$ flasks (Thermo Fisher Scientific ${ }^{T M}$ ) in $5 \% \mathrm{CO}_{2}$, in $37^{\circ} \mathrm{C}$ humidified incubators, and cultured to $80 \%$ confluence. Briefly, cells were washed once with $20 \mathrm{ml}$ warm Phosphate Buffered Saline (PBS; $8 \mathrm{~g} \mathrm{NaCl}, 0.2 \mathrm{~g} \mathrm{KCl}, 1.44 \mathrm{~g} \mathrm{Na}_{2} \mathrm{HPO}_{4}$, $0.24 \mathrm{~g} \mathrm{KH}_{2} \mathrm{PO}_{4}$ per litre, $\mathrm{pH} 7.4$ ), and trypsinised. Prostate cancer cell lines were trypsinised for 4 minutes at $37^{\circ} \mathrm{C}$, apart from the PC3 cell line, which was trypsinised for 7 minutes. Breast cancer cell lines were trypsinised for 7 minutes at $37^{\circ} \mathrm{C}$. Pancreatic cell lines were trypsinised for $5-10$ minutes at $37^{\circ} \mathrm{C}$. Trypsinised cells were added to equal volumes of media, centrifuged at $700 \times \mathrm{g}$ for 4 minutes $\left(4^{\circ} \mathrm{C}\right)$, and resuspended in fresh media. Viable cell counts were obtained by Trypan blue dye exclusion using a LUNA ${ }^{\mathrm{TM}}$ Automated Cell Counter (Logos Biosystems), and cells seeded at $3 \times 10^{4}$ cells $/ \mathrm{cm}^{2}$.

\subsubsection{Extraction and purification of the membrane protein fraction}

Prostate and breast cell lines were seeded at $3 \times 10^{4}$ cells $/ \mathrm{cm}^{2}$ in Nunc $\mathrm{NM}^{\mathrm{TM}} 175 \mathrm{~cm}^{2}$ flasks (Thermo Fisher Scientific ${ }^{\mathrm{TM}}$ ) and maintained at $5 \% \mathrm{CO}_{2}$, in $37^{\circ} \mathrm{C}$ humidified incubators for 24 hours. After 24 hours, the media was replaced with $20 \mathrm{ml}$ fresh media and cells incubated in $5 \% \mathrm{CO}_{2}$ at $37^{\circ} \mathrm{C}$ for 24 hours. After 24 hours, cells were harvested by trypsinisation and centrifugation and counted using the LUNA ${ }^{\mathrm{TM}}$ Automated Cell Counter (Logos Biosystems), as described previously.

$5 \times 10^{6}$ cells were transferred into Nostick ${ }^{\circledR}$ hydrophobic microcentrifuge tubes (Scientific Specialties, Inc.), washed twice in $2 \mathrm{ml}$ ice-cold PBS, and fractionated using a Qproteome ${ }^{\circledR}$ Cell Compartment kit (Qiagen), according to the manufacturer's protocol. Briefly, washed cell pellets were incubated in $1 \mathrm{ml}$ Extraction Buffer CE1 for 10 minutes at $4^{\circ} \mathrm{C}$ on an endover-end shaker ( $30 \mathrm{rpm})$. Lysates were centrifuged at $1000 \mathrm{x}$ g for 10 minutes at $4^{\circ} \mathrm{C}$. The supernatant, primarily containing cytosolic proteins, was transferred into a fresh Nostick ${ }^{\circledR}$ hydrophobic microcentrifuge tubes (Scientific Specialties, Inc.) and stored at $-20^{\circ} \mathrm{C}$. The remaining pellet was resuspended in $1 \mathrm{ml}$ Extraction Buffer CE2 and incubated for 30 minutes at $4^{\circ} \mathrm{C}$ on an end-over-end shaker (30 rpm). Lysates were centrifuged at $6000 \times \mathrm{g}$ for 
10 minutes at $4^{\circ} \mathrm{C}$. The supernatant, primarily containing membrane proteins, was transferred into fresh Nostick ${ }^{\circledR}$ hydrophobic microcentrifuge tubes (Scientific Specialties, Inc.).

Four volumes of ice-cold acetone were added to the membrane protein-enriched fractions and incubated for 1 hour on ice. Precipitated proteins were pelleted by centrifugation at 12 , $000 \times \mathrm{g}$ for 10 minutes $\left(4^{\circ} \mathrm{C}\right)$. The supernatant was discarded and the protein pellets air-dried for 15 minutes. Dried protein pellets were resuspended in $100 \mu \mathrm{l}$ Tris-HCL buffer $(50 \mathrm{mM}, \mathrm{pH}$ $8,0.5 \%$ SDS). Residual acetone was removed by the sequential addition of $600 \mu$ methanol, $150 \mu \mathrm{l}$ chloroform, and $400 \mu \mathrm{l}$ Milli-Q $18.2 \Omega$ water, with vortexing. After centrifugation at $18,000 \times \mathrm{g}$ for 10 minutes $\left(4^{\circ} \mathrm{C}\right)$, the upper aqueous phase was removed and the precipitated proteins washed twice with $450 \mu$ methanol. The pellets were air-dried for a minimum of 15 minutes, and resuspended in $100 \mu \mathrm{l}$ Tris-HCL buffer ( $50 \mathrm{mM}, \mathrm{pH} 8,0.5 \%$ SDS). The total protein concentration was determined using the DC ${ }^{\mathrm{TM}}$ Protein Assay (Bio-Rad), using BSA as a standard. Lysates were stored at $-20^{\circ} \mathrm{C}$.

\subsubsection{Western blot}

Membrane enriched lysates were resolved on precast 4-12\% 1mm Bis-Tris gels (Thermo Fisher Scientific ${ }^{\mathrm{TM}}$ ) in 1x NuPAGE MOPS buffer (Invitrogen) (10 $\mu \mathrm{g} / \mathrm{lane}, 30 \mathrm{~min} 60 \mathrm{~V}, 120 \mathrm{~min}$ $120 \mathrm{~V})$, and 1D gels transferred onto nitrocellulose membranes using an iBlot 2 (Life). Blots were incubated in $1 x$ Odyssey ${ }^{\circledR}$ PBS block buffer (LI-COR) for 1 hour, followed by an overnight incubation at $4^{\circ} \mathrm{C}$ with a $1: 1000$ dilution of the following primary antibodies in PBS blocking buffer (0.1\% tween, $0.1 \%$ SDS): rabbit monoclonal anti-E-cadherin (Cell Signalling Technologies), rabbit monoclonal anti-lamin b (Cell Signalling Technologies), goat polyclonal anti-GAPDH (Abcam), rabbit monoclonal anti-VDAC (Cell Signalling Technologies), and rabbit polyclonal anti-TIMM 23 (Abcam). After overnight incubation, primary antibodies were removed, and the blots washed 3 times (PBS + Tween (0.1\%), 5 minutes). Blots were incubated for 1 hour at room temperature with a 1:1000 dilution of a goat anti-rabbit IRDye $800 \mathrm{CW}$ secondary antibody (LI-COR) in PBS blocking buffer (0.1\% tween, $0.1 \%$ SDS). Blots 
were washed 3 times in PBS (0.1\% Tween) for 5 minutes, once in PBS for 5 minutes, and imaged on an Odyssey CLx Infrared Imaging System (LI-COR Biosciences).

\subsubsection{LC-MS/MS}

Membrane-enriched lysates were resolved by 1D gel electrophoresis as described above, stained with Instant Blue ${ }^{\mathrm{TM}}$ Coomassie dye (Expedeon), and submitted to the Proteomics Core Facility at the Cancer Research UK Cambridge Institute.

Each gel lane was cut into 24 bands and transferred into a microcentrifuge tube. The bands were cut into pieces, destained, reduced (Dithiothreitol in $100 \mathrm{mM}$ Ammonium bicarbonate $\mathrm{pH}$ 8.5), alkylated (iodoacetamide) and subjected to enzymatic digestion with sequencing grade trypsin overnight at $37^{\circ} \mathrm{C}$. After digestion, the supernatants were suspended in $10 \mu \mathrm{l}$ of $0.1 \%$ formic acid for further LC-MS analysis.

For each band, peptides were loaded on to a reverse-phase trap column ( $2 \mathrm{~cm} \times 100 \mu \mathrm{m}$ i.d.) and separated on an analytical column ( $25 \mathrm{~cm} \times 75 \mu \mathrm{m}$ i.d.) with $5-45 \%$ acetonitrile gradient in $0.1 \%$ formic acid and 5\% DMSO at $300 \mathrm{nl} / \mathrm{min}$ flow rate for 100 minutes. In each data collection cycle, one full MS scan (400 - 1,600 m/z) was acquired in the Orbitrap ion mass analyser (Thermo Fisher Scientific ${ }^{T M}$ ) (60K resolution, automatic gain control (AGC) setting of $3 \times 10^{6}$ and Maximum Injection Time (MIT) of $50 \mathrm{~ms}$ ). The most abundant ions were selected using the data-dependent acquisition algorithm "Top N", for fragmentation by Higher-energy collisional dissociation (HCD). HCD was performed with a collision energy of $30 \%$, an AGC setting of $5 \times 10^{4}$, an isolation window of $2.0 \mathrm{Da}$, a MIT of $200 \mathrm{~ms}$. Previously analysed precursor ions were dynamically excluded for 30 seconds.

\subsubsection{Database searching}

The Proteome Discoverer 1.4. (Thermo Fisher Scientific ${ }^{\mathrm{TM}}$ ) was used for the processing of HCD tandem mass spectra. The SequestHT search engine was used and all the spectra 
searched against the Uniprot Homo sapiens FASTA database (taxon ID 9606 - Version February 2018). All searches were performed using a static modification of Carbamidomethylation at Cysteines (+57.021Da). Methionine oxidation (+15.9949Da) and Deamidation on Asparagine and Glutamine (+0.984) were included as dynamic modifications. Mass spectra were searched using a precursor ion tolerance of $20 \mathrm{ppm}$ and a fragment ion tolerance of $0.02 \mathrm{Da}$. For peptide confidence, 1\% FDR was applied and peptides uniquely matched to a protein were used for further analysis.

\subsubsection{Bioorthogonal metabolic labelling of cell lines in vitro for gel electrophoresis}

$\mathrm{N}$-azidoacetylmannosamine ( $\left.\mathrm{Ac}_{4} \mathrm{ManNAz}\right), \mathrm{N}$-azidoacetylgalactosamine ( $\mathrm{Ac}_{4} \mathrm{GalNAz}$ ) and TMDIBO-PEG 3 -biotin were synthesised by Henning Stöckmann, Department of Chemistry.

Cells were seeded at $1.75 \times 10^{4}$ cells $/ \mathrm{cm}^{2}$ in Nunc ${ }^{\text {TM }} 175 \mathrm{~cm}^{2}$ flasks (Thermo Fisher Scientific ${ }^{\mathrm{TM}}$ ) and maintained in $5 \% \mathrm{CO}_{2}$, in $37^{\circ} \mathrm{C}$ humidified incubators for 3 days before metabolic labelling experiments. Flasks were then washed once with $20 \mathrm{ml}$ warm Phosphate Buffered Saline (PBS; $8 \mathrm{~g} \mathrm{NaCl}, 0.2 \mathrm{~g} \mathrm{KCl}, 1.44 \mathrm{~g} \mathrm{Na}_{2} \mathrm{HPO}_{4}, 0.24 \mathrm{~g} \mathrm{KH_{2 }} \mathrm{PO}_{4}$ per litre, $\mathrm{pH} 7.4$ ), and the cells trypsinised. Prostate cancer cell lines were trypsinised for 4 minutes at $37^{\circ} \mathrm{C}$, apart from the PC3 cell line which was trypsinised for 7 minutes. Breast cancer cell lines were trypsinised for 7 minutes at $37^{\circ} \mathrm{C}$. Pancreatic cell lines were trypsinised for 5-10 minutes at $37^{\circ} \mathrm{C}$. Trypsinised cells were added to equal volumes of media, centrifuged at $700 \times \mathrm{g}$ for 4 minutes $\left(4^{\circ} \mathrm{C}\right)$ and resuspended in fresh media. Viable cell counts were obtained by Trypan blue dye exclusion using a LUNA ${ }^{\mathrm{TM}}$ Automated Cell Counter (Logos Biosystems), and cells seeded at $3 \times 10^{4}$ cells $/ \mathrm{cm}^{2}$ in Nunc ${ }^{\mathrm{TM}} 175 \mathrm{~cm}^{2}$ flasks (Thermo Fisher Scientific ${ }^{\mathrm{TM}}$ ). Plates were incubated in $5 \% \mathrm{CO}_{2}, 37^{\circ} \mathrm{C}$ for 24 hours. After 24 hours, the media was replaced with fresh media only (0.1\% DMSO, vehicle control) or fresh media supplemented with $50 \mu \mathrm{M}$ $\mathrm{Ac}_{4} \mathrm{ManNAz}$ or $\mathrm{Ac}_{4} \mathrm{GalNAz}$, using a surface:area volume ratio equivalent to a 6 well plate (52.5 ml media supplemented with DMSO or azidosugars). Cells were incubated in $5 \% \mathrm{CO}_{2}$ at $37^{\circ} \mathrm{C}$ for 24 hours. After 24 hours, cells were harvested by trypsinisation and counted, as described previously. 
$5 \times 10^{6}$ cells were transferred into Nostick ${ }^{\circledR}$ hydrophobic microcentrifuge tubes (Scientific Specialties, Inc.), washed twice in $2 \mathrm{ml}$ ice-cold PBS, fractionated using a Qproteome ${ }^{\circledR}$ Cell Compartment kit (Qiagen), and purified using acetone precipitation and methanol/chloroform/Milli-Q $18.2 \Omega$ water precipitation, as described previously. The total protein concentration was determined using the DC ${ }^{\mathrm{TM}}$ Protein Assay (BIO-RAD), using BSA as a standard.

Up to $200 \mu \mathrm{g}$ of metabolically labelled protein was chemoselectively reacted with either alkyne-Alexa Fluor 488 (Life Technologies) for 1D gel electrophoresis or alkyne-biotin (Life Technologies) for 2D gel electrophoresis, using the Click iT Protein Reaction Kit (Life Technologies), according to the manufacturer's protocol. Residual reactants were removed by methanol/chloroform/Milli-Q $18.2 \Omega$ water precipitation, as described previously. Protein pellets were dried for a minimum of 15 minutes, and resuspended in $200 \mu$ l Tris HCL buffer ( $50 \mathrm{mM}, \mathrm{pH} 8,0.5 \%$ SDS) and sonicated for 30 seconds ( 3 cycles of 10 seconds ON and 5 seconds OFF) using the Biorupter ${ }^{\circledR}$ Plus (Diagenode). The total protein concentration was determined using the DC ${ }^{\mathrm{TM}}$ Protein Assay (BIO-RAD), using BSA as a standard. Samples were stored at $-20^{\circ} \mathrm{C}$.

\subsubsection{D gel electrophoresis and in-gel imaging of azidosugar labelled glycoproteins}

Metabolically labelled membrane protein fractions reacted with alkyne-Alexa Fluor 488 in Tris-HCL buffer (50 mM, pH 8, 0.5\% SDS) were loaded on precast 4-12\% $1 \mathrm{~mm}$ Bis-Tris gels (Thermo Fisher Scientific ${ }^{\mathrm{TM}}$ ) and resolved in 1x NuPAGE MOPS buffer (Invitrogen) (10 $\mu \mathrm{g} / \mathrm{lane} ; 30 \mathrm{~min}$ at $60 \mathrm{~V}$ and $120 \mathrm{~min}$ for $120 \mathrm{~V})$. Alexa Fluor 488 fluorescence (Cy2; $489 \mathrm{~nm}$ maximum excitation wavelength, $506 \mathrm{~nm}$ maximum emission wavelength) was imaged in-gel with a Typhoon scanner (GE Healthcare). Gels were stained subsequently with Instant Blue ${ }^{\mathrm{TM}}$ Coomassie (Expedeon) or silver stain (Pierce Kit) and imaged using an Odyssey CLx Infrared Imaging System (LI-COR Biosciences) or Image Scanner III (GE Healthcare), respectively.

For each lane treated with $\mathrm{Ac}_{4} \mathrm{ManNAz}$ or $\mathrm{Ac}_{4} \mathrm{GalNAz}$, total Alexa Fluor 488 fluorescence was quantified using the "Gel analysis" pre-installed plug-in on the Fiji image analysis. Generally, 
the Fiji "Rectangular select" tool was used to outline each lane, the relative density within each lane was plotted as a histogram, and the area under each curve plotted. Total Alexa Fluor 488 fluorescence was normalised against the Coomassie stain fluorescence within the same lane, and presented as a ratio.

\subsubsection{Deglycosylation of metabolically labelled glycoproteins using PNGase F}

Metabolically labelled membrane protein fractions reacted with alkyne-Alexa Fluor 488 or horseradish peroxidase (Pierce) were deglycosylated with PNGase F from Elizabethkingia miricola (Sigma). Briefly, lysates ( $5 \mu \mathrm{g}$ ) were adjusted to a final volume of $35 \mu \mathrm{l}$ with Milli-Q $18.2 \Omega$ water and denatured at $100^{\circ} \mathrm{C}$ for 5 minutes in a solution of $250 \mathrm{mM}$ phosphate buffer ( $\mathrm{pH} 7.5$ ), 2\% SDS and 1M 2-mercaptoethanol. Samples were cooled for 5 minutes at $4^{\circ} \mathrm{C}$ and incubated with PNGase F in Triton X-100 (15\% v/v) for 3, 24, 72 or 96 hours.

PNGase F-treated samples were loaded on precast 4-12\% 1mm Bis-Tris gels (Thermo Fisher Scientific $^{\mathrm{TM}}$ ) and resolved in 1x NuPAGE MOPS buffer (Invitrogen) (30 min 60 V, 120 min 120 V). Alexa-488 fluorescence (Cy2; $489 \mathrm{~nm}$ maximum excitation wavelength, $506 \mathrm{~nm}$ maximum emission wavelength) was imaged in-gel with a Typhoon scanner (GE Healthcare). Gels were stained subsequently with Instant Blue ${ }^{\mathrm{TM}}$ Coomassie (Expedeon) and imaged using an Odyssey CLx Infrared Imaging System (LI-COR Biosciences).

\subsubsection{D western blot analysis of azidosugar labelled glycoproteins}

$35 \mu \mathrm{g}$ of metabolically labelled protein reacted with alkyne-biotin were reacted with $0.2 \mathrm{mM}$ Cy3 dye (200 pmoles/ $\mu \mathrm{l})$ at room temperature for 30 minutes. Reactions were quenched with an equal volume of $1 \mathrm{mM}$ lysine. Samples were processed using the 2-D Clean-Up Kit (GE Healthcare), according to the manufacturer's protocol. Briefly, up to $100 \mu \mathrm{l}$ of sample was mixed with $300 \mu$ l Precipitant Solution, incubated on ice for 15 minutes, mixed with 300 $\mu$ Co-precipitate Solution, and incubated on ice for an additional 15 minutes. The precipitated protein was pelleted at maximum speed $(21,000 \times \mathrm{g})$ for 5 minutes, and the 
supernatant removed. Without disturbing the pellet, $40 \mu \mathrm{l}$ Co-precipitate Solution was added to the protein pellet and incubated on ice for 5 minutes. The mixture was centrifuged at maximum speed $(21,000 \times \mathrm{g})$ for 5 minutes, the supernatant removed, and the protein pellet washed in a solution of $25 \mu \mathrm{l}$ Milli-Q $18.2 \Omega$ water, $1 \mathrm{ml}$ ice-cold Wash Buffer and $5 \mu \mathrm{l}$ wash additive for 1 hour (including vortexing of the solution for 30 seconds, every 10 minutes). The mixture was pelleted at maximum speed $(21,000 \times \mathrm{g})$ for 5 minutes, the supernatant removed, and the pellet air dried briefly for 5 minutes.

The dried pellets were resolubilised in $250 \mu \mathrm{l}$ rehydration buffer (7M urea, $2 \mathrm{M}$ thiourea, $2 \%$ ASB-14, 2 mg/ml DTT (fresh), 1\% IPG buffer pH 3-10 non-linear (GE Healthcare (fresh)), heated at $37^{\circ} \mathrm{C}$ for 10 minutes, vortexed for 30 seconds, and centrifuged at maximum speed $(21,000 \times g)$ for 5 minutes to remove insoluble precipitates. Samples were loaded onto precast $13 \mathrm{~cm} \mathrm{pH} \mathrm{3-10} \mathrm{non-linear} \mathrm{IPG} \mathrm{strips} \mathrm{(GE} \mathrm{Healthcare)} \mathrm{and} \mathrm{isoelectric} \mathrm{focusing} \mathrm{carried} \mathrm{out}$ using the Ettan ${ }^{\mathrm{TM}}$ IPGphor ${ }^{\mathrm{TM}} 3$ Isoelectric Focusing System (GE Healthcare) $\left(20^{\circ} \mathrm{C}, 50 \mu \mathrm{A} /\right.$ strip, $3 \mathrm{hr}$ rehydration, $20 \mathrm{~V}$ for $10 \mathrm{hr}, 500 \mathrm{~V}$ for $1 \mathrm{hr}, 1000 \mathrm{~V}$ for $1 \mathrm{hr}, 5000 \mathrm{~V}$ for $5 \mathrm{hr}$ ).

On completion, IPG strips were immediately equilibrated for 20 minutes at room temperature in a reducing equilibration solution ( $75 \mathrm{mM}$ Tris $\mathrm{pH} 8.8,30 \% \mathrm{v} / \mathrm{v}$ glycerol, $6 \mathrm{M}$ Urea, $2 \% \mathrm{w} / \mathrm{v}$ SDS, $5 \mathrm{mg} / \mathrm{ml}$ DTT (fresh)) on an orbital shaker. Equilibrated strips were rinsed in SDS running buffer (25 mM Tris pH 8.3, $192 \mathrm{mM}$ glycine, 0.1\% SDS) and placed on the top

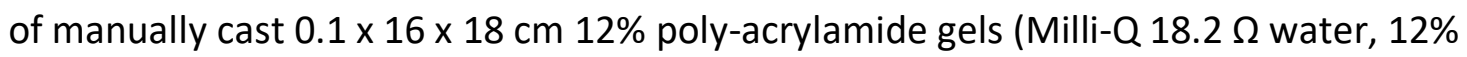
acrylamide (29:1 acrylamide:bis-acrylamide), $0.375 \mathrm{M}$ Tris- $\mathrm{HCl}$ pH 8.8, 0.1\% SDS, 0.1\% ammonium persulphate, 1:250 TEMED). Gels were sealed with an agarose-dye (0.5\% Agarose in SDS running buffer (29 mM Tris pH 8.3, $192 \mathrm{mM}$ Glycine, 0.1\% SDS) + 0.002\% w/v bromophenol blue), placed in the Se600 X-15-1.5 Chroma Vertical Electrophoresis unit (Hoefer) and separated in SDS running buffer (29 mM Tris pH 8.3, $192 \mathrm{mM}$ Glycine, 0.1\% SDS) until the dye front reached the bottom of the gel (20 min at $25 \mathrm{~mA} / \mathrm{gel}, \sim 5$ hours at 40 $\left.\mathrm{mA} / \mathrm{gel}, 15^{\circ} \mathrm{C}\right)$.

After electrophoresis, Cy3 fluorescence ( $548 \mathrm{~nm}$ maximum excitation wavelength, $561 \mathrm{~nm}$ maximum emission wavelength) was imaged in-gel using a Typhoon Scanner (GE Healthcare), and proteins transferred to $0.45 \mu \mathrm{m}$ pore nitrocellulose membranes (Bio-Rad) 
overnight in transfer buffer ( $25 \mathrm{mM}$ Tris pH 8.3, $192 \mathrm{mM}$ glycine, 10\% methanol) using a Hoefer TE62 tank ( $4^{\circ} \mathrm{C}, 100 \mathrm{~mA}, 16-17$ hours). Blots were imaged for Cy3 fluorescence (548 $\mathrm{nm}$ maximum excitation wavelength, $561 \mathrm{~nm}$ maximum emission wavelength) on a Typhoon Scanner (GE Healthcare) post-transfer to assess transfer efficiency. Biotinylated proteins were detected by treating blots with $1 \mathrm{x}$ Odyssey ${ }^{\circledR}$ PBS block buffer (LI-COR) for 1 hour and 1:5000 IRDye 800CW Streptavidin (LI-COR) in PBS block buffer for 15 minutes. Blots were washed 6 times in PBS (0.1\% Tween) for 5 minutes, once in PBS for 5 minutes, and imaged on an Odyssey CLx Infrared Imaging System (LI-COR Biosciences).

\subsubsection{Statistical analysis}

All p-values were calculated using a one-way ANOVA test. 


\section{Chapter 5: Identification of bioorthogonally labelled proteins using liquid chromatography-tandem mass \\ spectrometry.}

\section{$\underline{5.1 \text { Introduction }}$}

\subsubsection{Glycans as biomarkers}

Glycans are the complex carbohydrate structures formed when monosaccharides link together via glycosidic bonds. Glycans act as cellular decorations with roles in intracellular signalling, hormone regulation, cell migration, plasma membrane structuring, immunity, host-pathogen recognition, and drug sensitivity (Pinho and Reis 2015; Varki 2017). In cancer, alterations in the expression of glycoenzymes and their location within the Golgi compartments, alterations to the tertiary conformation of peptide backbones and nascent glycan chains, as well as fluctuations in the abundance of acceptor substrates, sugar nucleotide donors and cofactors (Pinho and Reis 2015; Adamczyk et. al. 2012), cause the generation of aberrant cancer-associated antigens (Chapter 1: Figure 1.7). Aberrant glycosylation can affect growth factor signalling, cell proliferation, adhesion, and migration, and promote tumour progression through enhanced cell-matrix interactions, invasion, angiogenesis, and metastasis (Moremen et. al. 2012).

Based on the role of glycosylation in cancer progression, several studies have gone on to characterise glycome differences between healthy individuals and cancer patients (Abelev et. al. 1963; Springer et. al. 1975; Chan et. al. 1984; Turner et. al. 1985; Ng et. al. 1987), leading to the identification of aberrantly glycosylated glycoproteins or oligosaccharide signatures, known as biomarkers in tissue or serum (Nimse et. al. 2016). Cancer biomarkers are used in clinical practice for cancer diagnosis, therapeutic monitoring, detecting disease recurrence, and examining tumour burden (Peracaula et. al. 2003) (Chapter 1: Figure 1.12). 
However, the utility of biomarkers has been challenged in recent years due to low cancer specificity and high false-positive rates (Mayeux 2004).

\subsubsection{Identification of new glycosylated biomarkers using bioorthogonal chemistry}

Several technologies exist to study the structure, distribution, abundance, and function of glycans in cancer, within cells and tissues, and their variation during cancer (Bertozzi and Sasisekharan 2009; Bennun et. al. 2016), such as glycan-binding lectin and antibody arrays, carbohydrate stains, deglycosylation enzymatic cleavage, genetic mutation models, hydrazide chemistry oxidation of sugars, liquid chromatography-mass spectrometry, hydrophilic interaction chromatography and boronate affinity chromatography (Springer and Gagneux 2016; Pan et. al. 2012; Pan et. al. 2016; Cieniewski-Bernard et. al. 2014).

In recent years, more novel methods of capturing glycoproteins have involved the use of bioorthogonal monosaccharide analogues containing a functional azide group or "chemical reporter" not normally present in the cell (Laughlin et. al. 2009), which are incorporated metabolically into cellular glycan structures, and detected subsequently by covalent ligation of imaging probes or affinity reagents that rapidly react with the azide group (Saxon and Bertozzi 2000; Saxon et. al. 2002) (Chapter 1: Figure 1.20). In the search for new and improved biomarkers, bioorthogonal azide-based chemical reporters, generally called azidosugars, have been combined with mass spectrometry analysis to capture and identify glycosylated proteins, which are overexpressed in small lung cell carcinoma tissue (Pan et. al. 2012), and prostate cancer tissue (Spiciarich et. al. 2017), that were previously missed using less sophisticated lectin- or antibody-based glycoprotein capture methods.

Pan and colleagues incubated A549 lung cell adenocarcinoma, Hela cervical carcinoma, and SW1990 pancreatic adenocarcinoma cell lines, with Ac4ManNAz (Pan et. al. 2012). Incorporated azide groups were covalently ligated to biotin-SS-alkyne, using coppercatalysed chemistry, and the azidosugar labelled proteins enriched using streptavidincoupled beads. Purified azidosugar labelled proteins were identified by mass spectrometry, leading to the identification of neural adhesion molecule, which was uniquely expressed in 
A549 lung cell adenocarcinoma, and subsequently shown to be overexpressed in small lung cell carcinoma tissue using immunohistochemistry (Pan et. al. 2012). Similarly, Spiciarich and colleagues metabolically labelled prostate tissue slices derived from radical prostatectomy specimens with $\mathrm{Ac}_{4} \mathrm{ManNAz}$, captured the azidosugar labelled glycoproteins using biotinalkyne and streptavidin-coupled beads, and then identified the azidosugar labelled proteins using mass spectrometry (Spiciarich et. al. 2017). Legumain was shown to be glycosylated and significantly overexpressed in lysates extracted from normal tissue slice cultures (TSC) in comparison to cancer TSC's (Spiciarich et. al. 2017). These studies highlighted bioorthogonal chemistry as a novel strategy for glycoprotein enrichment and biomarker discovery based on the identification of azidosugar labelled proteins uniquely identified in cancer tissue, or overexpressed in cancer tissues in comparison to normal healthy tissue.

Using bioorthogonal chemistry (Chapter 1: Figure 1.24), flow cytometry (Chapter 3) and gel electrophoresis (Chapter 4), we have previously shown a correlation between increases in $\mathrm{N}$ azidoacetylmannosamine ( $\left.\mathrm{Ac}_{4} \mathrm{ManNAz}\right)$-specific sialyation and $\mathrm{N}$-azidoacetylgalactosamine $\left(\mathrm{Ac}_{4} \mathrm{GalNAz}\right.$-specific glycosylation of plasma membrane glycoconjugates and increases in the metastatic potential of cell lines representing the stages of cancer progression (Chapter 2 ). The data illustrated the utility of bioorthogonal labelling in detecting patterns of aberrant glycosylation associated with progressive disease, and was consistent with a growing body of evidence implicating increased protein glycosylation as a driver in tumourigenesis (Christiansen et. al. 2014). Whilst this trend formed the basis for monitoring tumour glycosylation in vivo (Chapter 3: Figures 3.5-3.8), differential azidosugar labelling may also be useful for identifying biomarkers indicative of cancer progression. The strong labelling of the glycoprotein fraction, observed using 1D (Chapter 4: Figure 4.2-4.4) and 2D gel electrophoresis (Chapter 4: Figure 4.6 and 4.7), suggested that differential glycosylation, specifically within the glycoproteins, may have prognostic value. Therefore, we endeavoured to identify prognostic biomarkers from the prostate cancer membrane proteome using bioorthogonal chemistry and mass spectrometry. 


\subsection{Results}

The metastatic potential of the established prostate cancer cell lines used in the study was confirmed in the scratch wound (Chapter 2: Figure 2.2), Boyden chamber migrations assays (Chapter 2: Figure 2.3a and b) and by demonstrating a correlation with the expression of known invasion biomarkers (Chapter 2: Figures 2.3d). Collectively, the data validated the LNCAP panel of prostate cancer cell lines as a suitable in vitro model of prostate cancer progression. However, given the heterogeneity of human cancers of the same type, we investigated if there were other differences in the prostate cancer cell lines, apart from migration and invasion.

Comparison of the genetic sequences of each cell line revealed vast differences in the consensus profiles of the PNT1A and PC3 cell lines (Figure 5.1a). Furthermore, previous data have shown the PNT1A cell line to be highly motile (Chapter 2: Figure 2.2) and invasive (Chapter 2: Figure 2.3a-c). We deemed the PNT1A cell to be an unsuitable model for normal prostate epithelium and unsuitable for mass spectrometry analysis. As well as being genetically divergent, the PC3 cell line did not express markers commonly associated with prostate cancer in humans, such as prostate-specific antigen (PSA) and prostate-specific membrane antigen (PSMA) (Figure 5.1b). Therefore, only the LNCAP lineage of cell lines (LNCAP, LNCAP C4, LNCAP C4-2, and LNCAP C4-2B), were taken forward for further analysis. They were genetically related (Figure 5.1a) and most representative of the clonal evolution of cancer in humans (Table 2.1a). 
a.

\begin{tabular}{|lllc|}
\hline \multicolumn{1}{|c|}{ Cell Line } & $\begin{array}{c}\text { Consensus } \\
\text { profile }\end{array}$ & $\begin{array}{c}\text { Comparison with } \\
\text { consensus profile }\end{array}$ & $\begin{array}{c}\text { Percentage } \\
\text { match }\end{array}$ \\
\hline LNCAP & LNCAP, ATCC & Exact match & 100 \\
\hline LNCAP C4 & LNCAP, ATCC & Child line of LNCAP & 88 \\
\hline LNCAP C4-2 & LNCAP, ATCC & Child line of LNCAP & 88 \\
\hline LNCAP C4-2B & LNCAP, ATCC & Child line of LNCAP & 86 \\
\hline PC3 & LNCAP, ATCC & Unrelated cell line & 41 \\
\hline PNT1A & LNCAP, ATCC & $\begin{array}{l}\text { Not enough alleles } \\
\text { to compare }\end{array}$ & 30 \\
\hline
\end{tabular}

b.

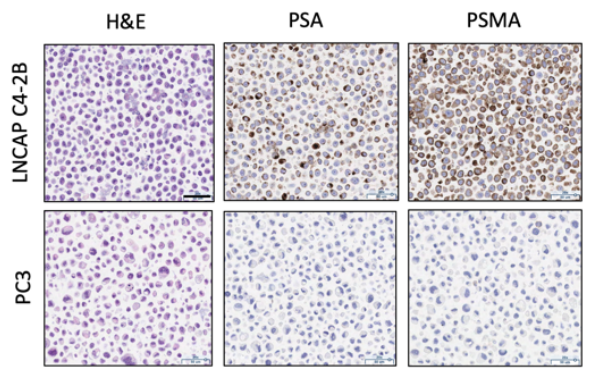

Figure 5.1: Selection of prostate cancer cell lines for mass spectrometric analysis. (a) Gene sequences from the prostate cancer cell lines were compared, against the LNCAP cell line. (b) Immunohistochemistry staining (in brown), was used to measure relative protein expression of known markers of prostate cancer progression: prostate-specific antigen (PSA, column 2), and prostate-specific membrane antigen (PSMA, column 3). Sections were also stained with haematoxylin and eosin (H\&E, column 1). Scale bar in (b) represents $50 \mu m$, (magnification 20x).

\subsubsection{Preparation of azidosugar-labelled proteins for mass spectrometry analysis.}

The LNCAP lineage of prostate cancer cell lines was incubated with precursors of sialic acid and $\mathrm{N}$-acetylgalactosamine that had been labelled with an azide group ( $\mathrm{N}$ azidoacetylmannosamine ( $\mathrm{Ac}_{4} \mathrm{ManNAz}$ ) and $\mathrm{N}$-azidoacetylgalactosamine ( $\left.\mathrm{Ac}_{4} \mathrm{GalNAz}\right)$ ), and which are incorporated biosynthetically into cell surface glycans. The membrane proteome was isolated, and the incorporated azide groups covalently ligated to alkyne-biotin using copper-catalysed chemistry. Proteins were enriched using streptavidin-coupled beads (Figure 5.2a) and resolved by 1D western blot. Specific capture of metabolically labelled proteins was confirmed using Streptavidin-800 fluorescence (Figure 5.2b). 
Biotinylated proteins were detected in samples treated with $A c_{4} M a n N A z$ or $A c_{4} G a l N A z$, confirming specific enrichment of azidosugar labelled proteins within the membrane proteome. The detection of proteins by silver stain, in samples treated with vehicle, indicated some non-specific binding of unlabelled proteins to the streptavidin beads, although this was minimal in comparison to the specific enrichment of proteins treated with the azidosugars (Figure 5.2b). We anticipated detecting more protein from cells treated with $\mathrm{Ac}_{4} \mathrm{GalNAz}$, due to labelling of the core structure (serine/threonine-N-acetylgalactosamine) present in all O-glycosylated proteins. As expected, strong Streptavidin-800 fluorescence and corresponding silver staining were observed in extracts from cells treated with $\mathrm{Ac}_{4} \mathrm{GalNAz}$.

a.

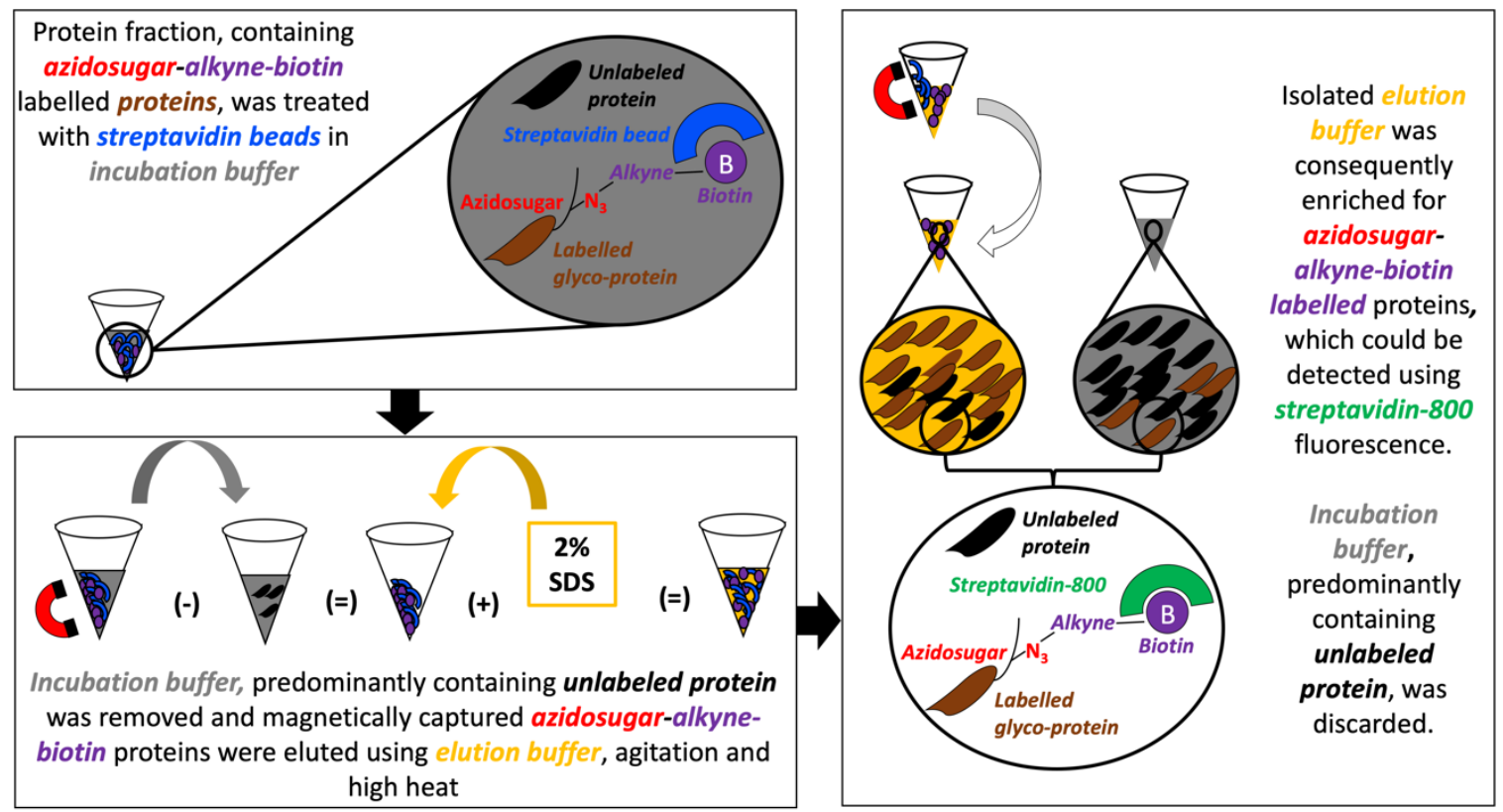


b.

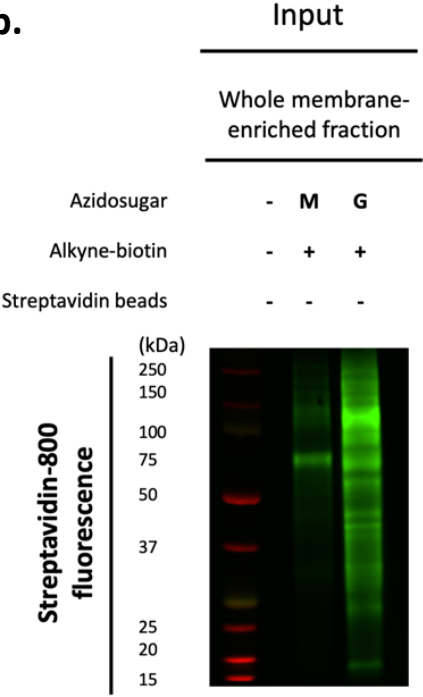

IP: Streptavidin Magsepharose beads
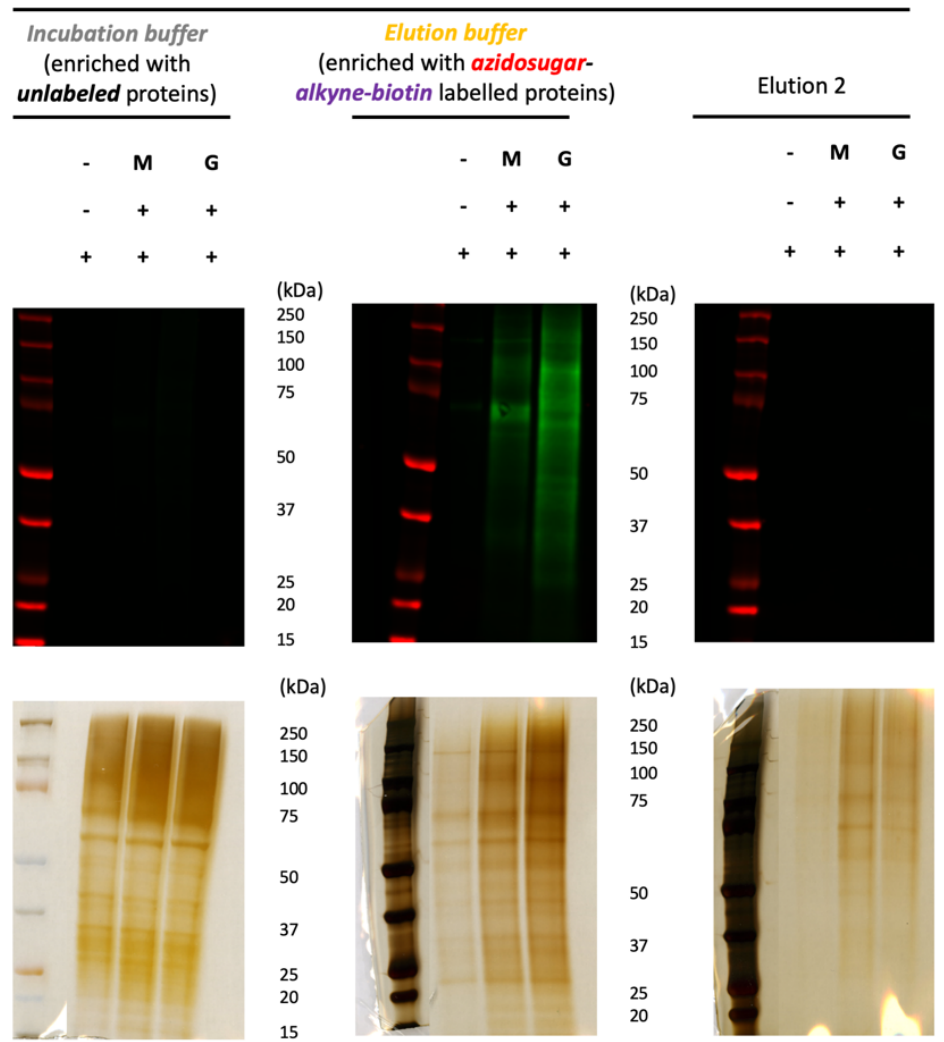

Figure 5.2: Work flow for the enrichment of azidosugar-labelled proteins using streptavidin-coupled magnetic beads. (a) Azidosugar-labelled proteins were enriched from $35 \mu \mathrm{g}$ of alkyne-biotin labelled extract, using streptavidin-coupled beads. Specific capture of azidosugar-labelled proteins was confirmed by western blot and Streptavidin-800 fluorescence. (b) Representative western blots and silver stains are shown from the LNCAP C4-2B prostate cancer cell line. Elutions were conducted twice. Background controls were included to account for non-specific protein enrichment. Abbreviations: $M=A c_{4} M a n N A z$ treated, $G=A c_{4}$ GalNAz treated.

Next, azidosugar-labelled proteins were enriched using streptavidin-coupled beads, resolved by 2D gel electrophoresis in duplicate, and subjected to western blot analysis or silver staining (Figure 5.3a). Metabolically labelled proteins were visualised using Streptavidin-800 fluorescence. Parallel 2D gels were silver stained. Protein spots on the silver stain which coregistered with spots of Streptavidin-800 fluorescence on the western blot were picked, and processed for mass spectrometric analysis (Figure 5.3a, black arrowheads). Streptavidin-800 fluorescence was observed in proteins that were undetectable by silver staining (Figure 5.3a, 
white arrow heads), which was attributed to the sensitivity of bioorthogonal chemistry and its ability to detect low abundance glycoproteins. Traditional mass spectrometry approaches require proteins to be visible by Coomassie or silver staining so that protein spots can be picked manually and analysed (Meleady 2011). Therefore, spots of Streptavidin-800 fluorescence on western blot that could not be co-registered with silver-stained protein spots (Figure 5.3a, white arrowheads) were excluded from the analysis.

To allow all azidosugar-labelled proteins to be identified, including low abundant proteins undetectable by standard protein stains (Figure 5.3a, white arrowheads), an alternative approach was adopted where enriched elutes were analysed directly using mass spectrometry. Leakage of streptavidin monomers from the beads was observed in the eluents (Figure 5.3b) as a result of the high heat and agitation needed to break the streptavidin-biotin binding and maximise elution of the azidosugar-alkyne-biotin labelled glycoproteins from the magnetic beads. Several attempts were made to minimise leakage of streptavidin from the beads such as reducing elution temperatures from $100^{\circ} \mathrm{C}$ to room temperature or using detergent-free buffers (data not shown). Gentler elution conditions resulted in negligible elution of the azidosugar-labelled proteins from the beads. Therefore, we used high heat and agitation to maximise elution of the azidosugar-labelled proteins from the magnetic beads, resolved the elutes by $1 \mathrm{D}$ gel electrophoresis (Figure 5.3c), and then cut the streptavidin bands from the silver-stained gels (Figure 5.3d, red box), excluding them from the analysis. The remaining lanes were segmented and tryptically digested, followed by protein identification using liquid chromatography-tandem mass spectrometry (LCMS) (Savaryn et. al. 2016; Glish and Vachet 2003). This approach was used to identify prognostic biomarkers from the prostate cancer membrane proteome using bioorthogonal chemistry and LCMS (Figure 5.3c) (Küster et. al. 2001). 
a.

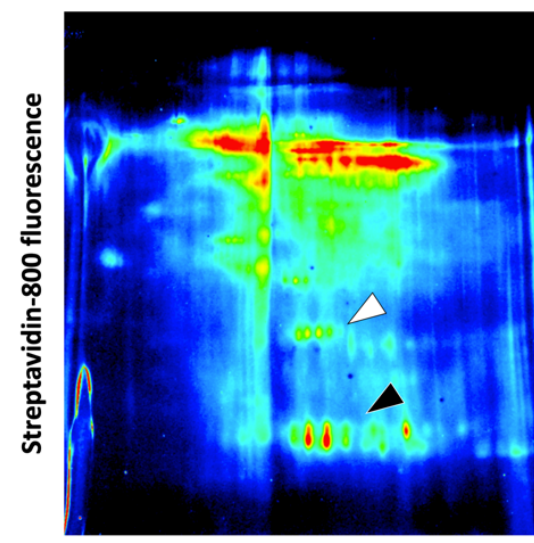

Western blot
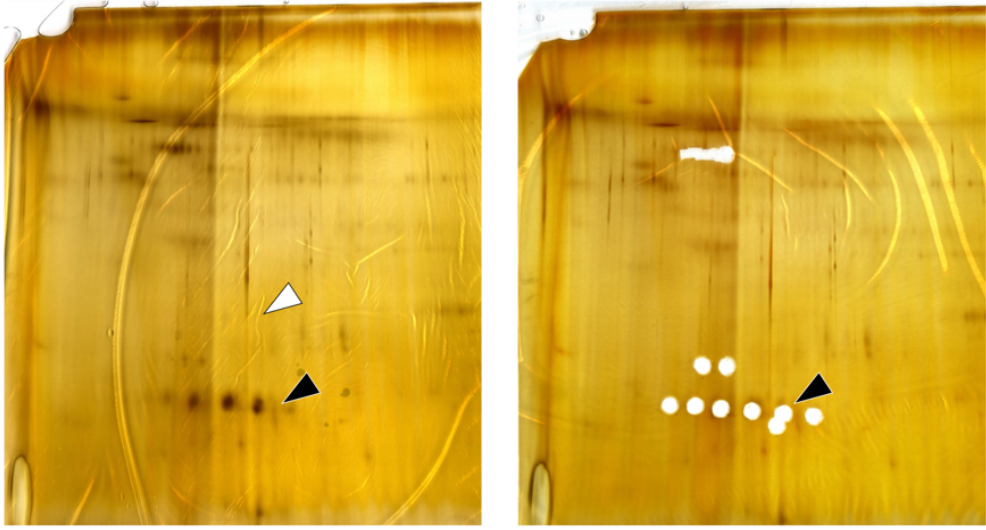

Silver stain

Streptavidin contamination

$\mathrm{m} / \mathrm{z} 402.54$ (3+)

Ion mass: 1204.59 b.

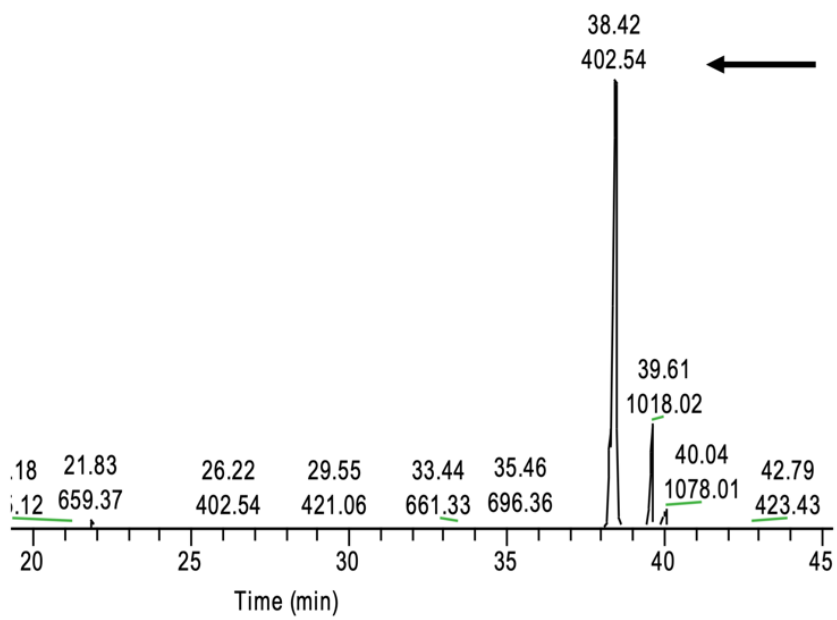

c.

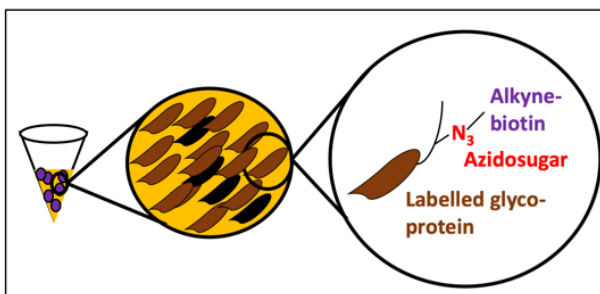

Elution enriched for azidosugar-alkynebiotin labelled proteins

$\downarrow$

1D SDS page and silver stain<smiles>C[AlH]</smiles>

Tryptic digest

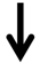

MS-LCMS d.

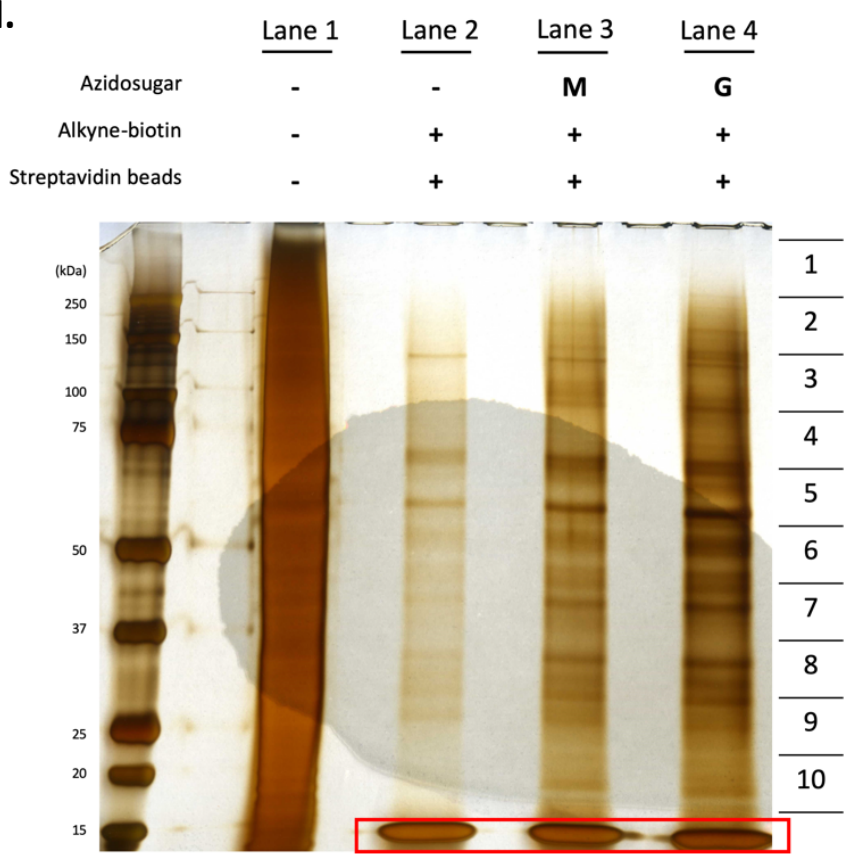


Figure 5.3: Preparation of azidosugar-labelled proteins for mass spectrometry analysis. Azidosugar labelled proteins were enriched from $35 \mu \mathrm{g}$ of azidosugar-alkyne-biotin labelled extract using streptavidin-coupled beads and prepared for mass spectrometry using a variety of approaches. (a) Representative 2D western blot and silver stain of proteins from the LNCAP C4-2B cell line labelled with Ac 4 GaINAz. Regions, where streptavidin 800 fluorescence could be correlated with the silver stain (black arrowheads), were picked and identified by mass spectrometry. Silver-stained protein spots that co-registered with spots of Streptavidin800 fluorescence on the western blot were picked, and processed for mass spectrometry analysis (black arrowheads). Spots of Streptavidin-800 fluorescence on western blot that could not be co-registered with silver-stain protein spots were excluded from the analysis (white arrowheads). (b) Representative chromatograph of the streptavidin bead elute from the LNCAP C4-2 cell line labelled with Ac 4 ManNAz. The abundant peak caused by streptavidin monomers is highlighted (black arrowheads). (c) Elutes were resolved by 1D gel electrophoresis prior to mass spectrometric analysis. (d) Representative silver stain of a gel submitted for mass spectrometric analysis of a membrane protein extract from LNCAP C4-2B cells. Lanes were segmented into 10 pieces, and subjected to tryptic digestion, followed by protein identification by liquid chromatography-tandem mass spectrometry. The strongly stained region due to streptavidin contamination was removed (red box). Abbreviations: $M=$ $A c_{4}$ ManNAz treated, $G=A c_{4} G a l N A z$ treated.

\subsubsection{Identification of azidosugar-labelled proteins using liquid chromatography-tandem mass spectrometry}

Spectra representing individual peptides generated from in-gel tryptic digestion (Figure 5.3c and d) were searched against databases of known primary peptide sequences using the Mascot software (http://www.matrixscience.com/search_intro.html, 2019), leading to the identification of 3555 unique proteins. Identified proteins were subsequently classified by their cellular location using gene ontology (GO) analysis (www.pantherdb.org) (Figure 5.4a). Of the designated membrane proteins, $67 \%$ were classified as plasma membrane proteins (Figure 5.4b), confirming specific azidosugar labelling and glycosylation-dependent enrichment of cell surface proteins. This was consistent with a previous study where $51 \%$ of 
proteins identified from $\mathrm{Ac}_{4} \mathrm{ManNAz}$ treated human prostate cancer tissue slices, were classified as plasma membrane proteins (Spiciarich et. al. 2017).

a.

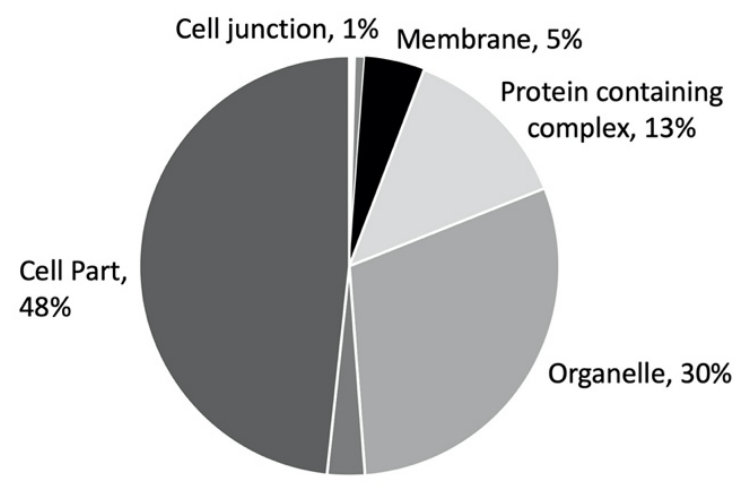

Extracellular region, $3 \%$ b.

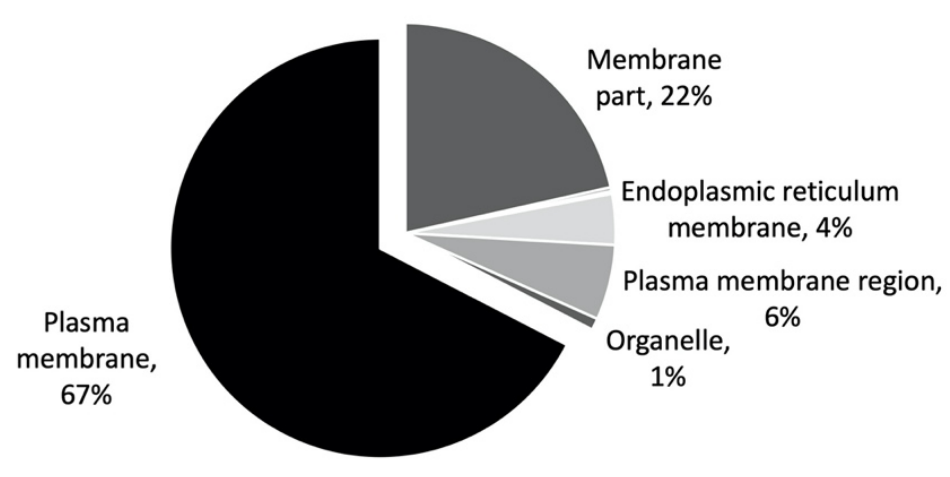

Figure 5.4: Cellular distribution of proteins identified using mass spectrometry. Azidosugar labelled proteins were enriched from the LNCAP lineage of prostate cancer cell lines (LNCAP, LNCAP C4, LNCAP C4-2, and LNCAP C4-2B). Proteins identified with Mascot were classified by their cellular (a) and membrane locations (b) using gene ontology analysis (www.pantherdb.org).

Using the Scaffold proteomic software, proteins were filtered by plasma membrane GO terms. For each of the filtered membrane proteins, the ratio of spectral counts identified from $\mathrm{Ac}_{4}$ ManNAz- (AzM) (Figure 5.3d, lane 3) or $\mathrm{Ac}_{4}$ GalNAz-treated (AzG) (Figure 5.3d, lane 4) samples, and the untreated control samples (U) (Figure 5.3d, lane 2) were calculated. The distribution of $A z / U$ ratios within each cell line was then represented as histograms to distinguish between Ac${ }_{4}$ ManNAz-specific (Figure 5.5a, i), Ac ${ }_{4}$ GalNAz-specific (Figure 5.5a, ii) and non-specific streptavidin bead enrichment. $\mathrm{Ac}_{4}$ ManNAz-labelled proteins (Figure 5.5b, i) or $\mathrm{Ac}_{4}$ GalNAz-labelled proteins (Figure 5.5b, ii) with a relative abundance 4-fold and greater than the untreated control samples (Haun et. al. 2015; Spiciarich et. al. 2017), were selected for further analysis. 
For each cell line, the frequency of proteins identified as having an AzM/U ratio (Figure 5.5c, i) or AzG/U ratio (Figure 5.5c, ii) of 4 and above was summarised. A general increase in the number of azidosugar-labelled proteins meeting the threshold correlated with an increase in the metastatic potential of the cell lines. This was consistent with previous data which has shown a correlation between an increase in metabolic labelling and metastatic potential using flow cytometric analysis (Chapter 3: Figure 3.2) and gel electrophoresis (Chapter 4: Figure 4.2-4.5). Furthermore, more azidosugar labelled proteins were detected in samples treated with $\mathrm{Ac}_{4} \mathrm{GalNAz}$. This is due to the labelling of the core structure (serine/threonine$\mathrm{N}$-acetylgalactosamine) present in all O-glycosylated proteins, as seen with 2D gel electrophoresis (Chapter 4: Figure 4.4).

A subclass of fifteen proteins was created with an $A z M / U$ ratio (Figure $5.5 d$, i) or $A z G / U$ (Figure 5.5d, ii) ratio of 4 and above, in two or more of the prostate cancer cell lines. Of these proteins, five were labelled with both azidosugars (denoted with * in Figure 5.5d). These represent glycoproteins with sialic acid and $\mathrm{N}$-acetylgalactosamine within their glycan structures, which are replaced metabolically with $\mathrm{Ac}_{4} \mathrm{ManNAz}$ and $\mathrm{Ac}_{4} \mathrm{GalNAz}$ respectively. It was reassuring to see that proteins known to be involved in cancer progression met these stringent shortlisting criteria, such as epidermal growth factor (Chang et. al. 2015) and lysosome-associated membrane protein 1 (Okato et al. 2016). Other proteins, currently being investigated as biomarkers for monitoring prostate cancer progression were also identified such as filamin B (Ravipaty et al., 2017). Interestingly, such proteins were enriched based on their overexpression in human prostate cancer tissue, as opposed to their differential glycosylation, which is the method adopted here. Four proteins in our list have not previously been implicated in prostate cancer: $45 \mathrm{kDa}$ calcium-binding protein, serpin B12 (isoform 2), plasma membrane calcium-transporting ATPase 1 (isoform B), and Leucylcystinyl aminopeptidase. Collectively, the data highlights the utility of bioorthogonal chemistry in identifying both known and novel biomarkers. 

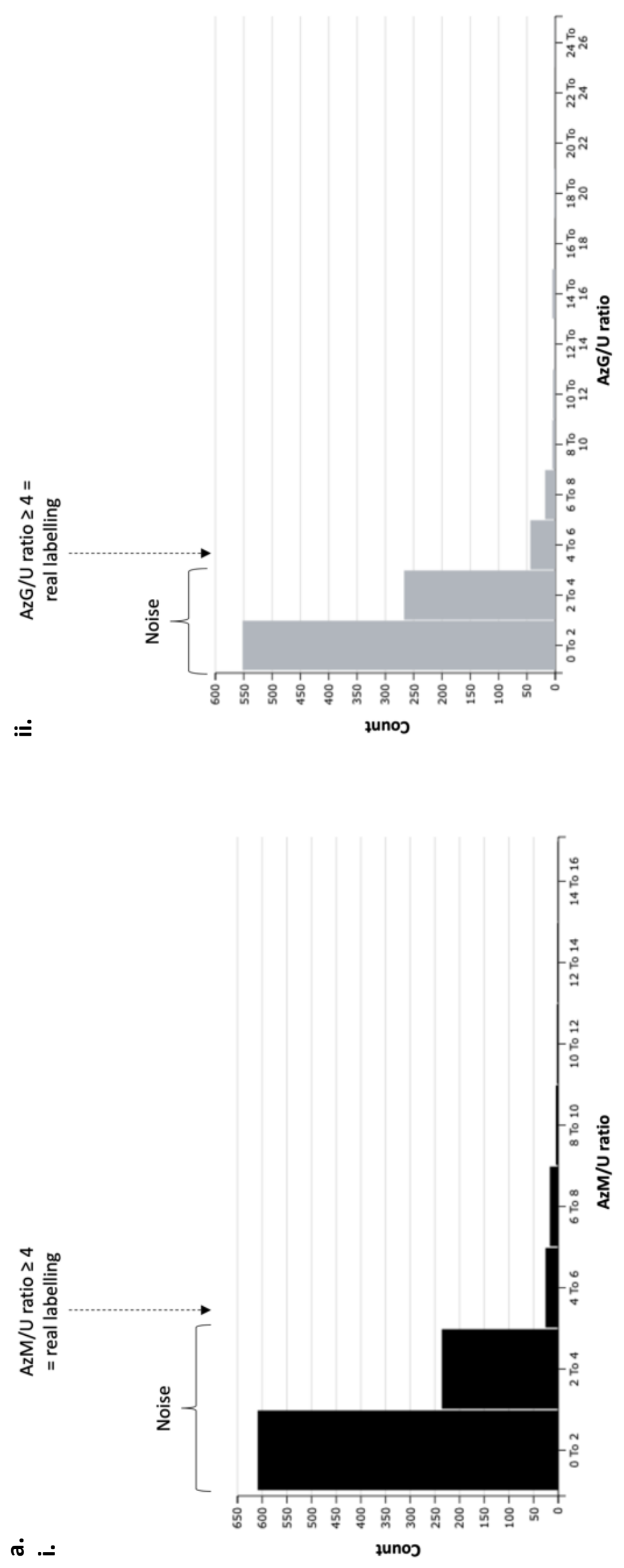


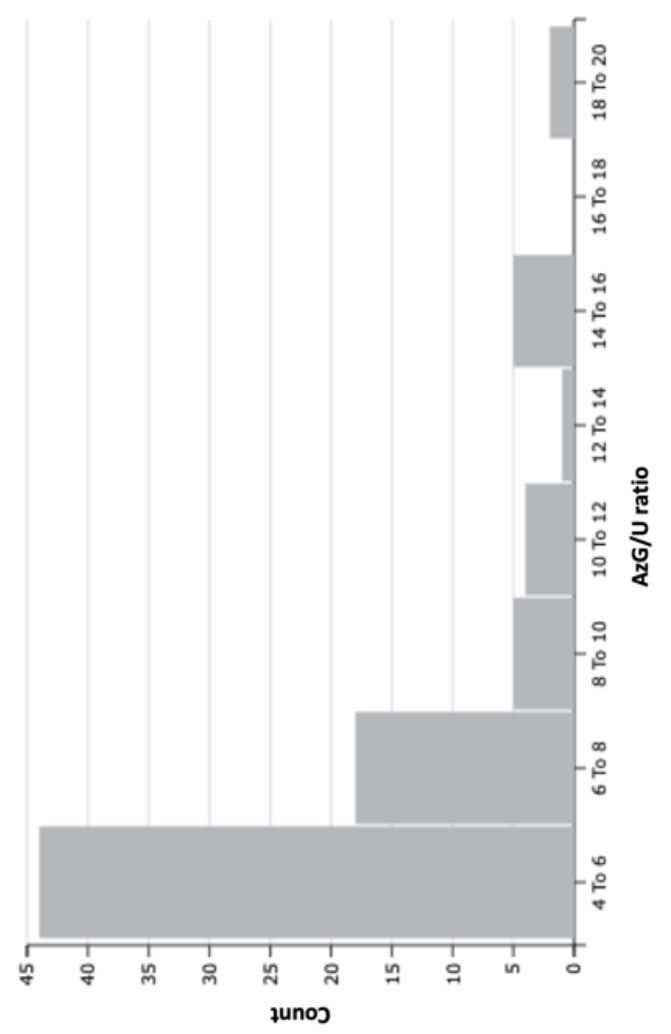

$:=$

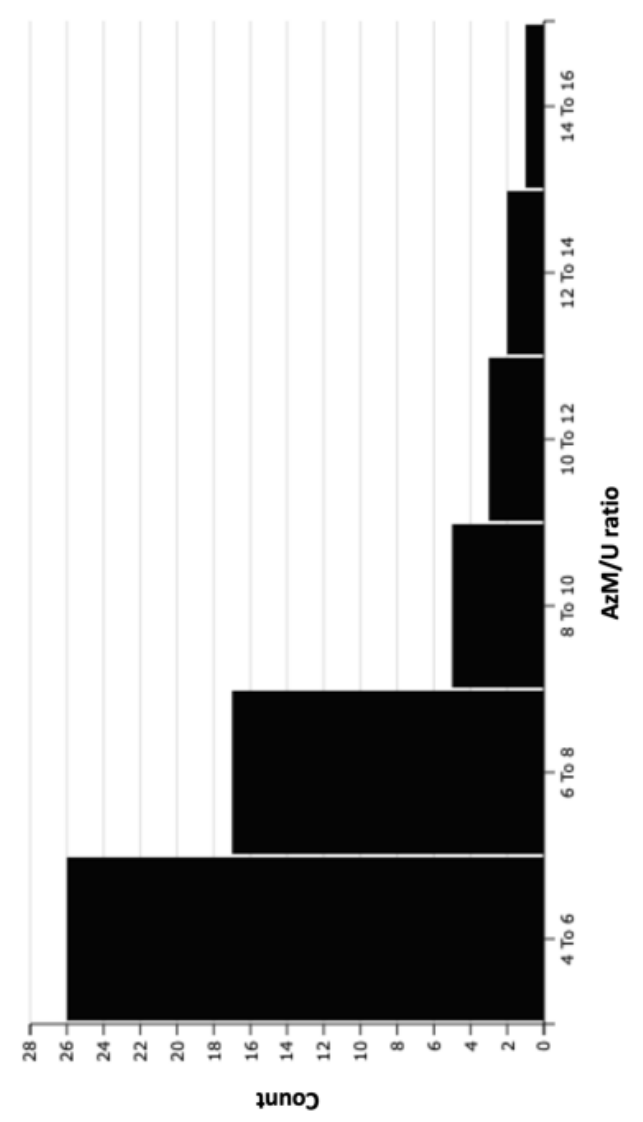




\begin{tabular}{|c|c|c|c|c|c|}
\hline \multirow{2}{*}{$\begin{array}{l}\text { c. } \\
\text { i. }\end{array}$} & & \multicolumn{4}{|c|}{ Frequency of Ac4ManNAz labelled proteins } \\
\hline & & LNCAP & LNCAP C4 & LNCAP C4-2 & LNCAP C4-2B \\
\hline \multirow{28}{*}{ Threshold > } & 0 to 2 & 394 & 183 & 183 & 179 \\
\hline & 2 to 4 & 24 & 31 & 57 & 58 \\
\hline & 4 to 6 & 4 & 11 & 19 & 15 \\
\hline & 6 to 8 & 1 & 1 & 3 & 10 \\
\hline & 8 to 10 & 2 & 2 & 6 & 3 \\
\hline & 10 to 12 & 0 & 0 & 1 & 1 \\
\hline & 12 to 14 & 2 & 1 & 2 & 2 \\
\hline & 14 to 16 & 0 & 0 & 0 & 2 \\
\hline & 16 to 18 & 0 & 1 & 0 & 0 \\
\hline & 18 to 20 & 0 & 1 & 0 & 0 \\
\hline & 20 to 22 & 0 & 0 & 0 & 0 \\
\hline & 22 to 24 & 0 & 0 & 0 & 0 \\
\hline & 24 to 26 & 0 & 0 & 1 & 0 \\
\hline & 26 to 28 & 0 & 0 & 1 & 0 \\
\hline & 28 to 30 & 0 & 0 & 1 & 0 \\
\hline & 30 to 32 & 0 & 0 & 0 & 0 \\
\hline & 32 to 34 & 0 & 0 & 0 & 0 \\
\hline & 34 to 36 & 0 & 0 & 0 & 0 \\
\hline & 36 to 38 & 0 & 0 & 0 & 0 \\
\hline & 38 to 40 & 0 & 0 & 0 & 0 \\
\hline & 40 to 42 & 0 & 0 & 0 & 0 \\
\hline & 42 to 44 & 0 & 1 & 0 & 0 \\
\hline & 44 to 46 & 0 & 0 & 0 & 0 \\
\hline & 46 to 48 & 0 & 0 & 0 & 0 \\
\hline & 48 to 50 & 0 & 0 & 0 & 0 \\
\hline & 50 to 52 & 0 & 0 & 1 & 0 \\
\hline & 52 and above & 0 & 0 & 0 & 0 \\
\hline & $\begin{array}{l}\text { Number of proteins with } \\
\text { AzM/U ratio } 4 \text { fold and above } \\
\text { untreated control }\end{array}$ & 9 & 18 & 35 & 33 \\
\hline
\end{tabular}


ii.

Frequency of Ac4GalNAz labelled proteins

\begin{tabular}{|c|c|c|c|c|c|}
\hline \multirow{31}{*}{ Threshold > } & $\mathrm{AzG} / \mathrm{U}$ ratio & LNCAP & LNCAP C4 & LNCAP C4-2 & LNCAP C4-2B \\
\hline & 0 to 2 & 415 & 201 & 203 & \begin{tabular}{|r|}
167 \\
\end{tabular} \\
\hline & 2 to 4 & 10 & 23 & 44 & 58 \\
\hline & 4 to 6 & 1 & 3 & 11 & 18 \\
\hline & 6 to 8 & 1 & 1 & 4 & 8 \\
\hline & 8 to 10 & 0 & 0 & 4 & 3 \\
\hline & 10 to 12 & 0 & 2 & 4 & 5 \\
\hline & 12 to 14 & 0 & 1 & 0 & 4 \\
\hline & 14 to 16 & 0 & 0 & 2 & 3 \\
\hline & 16 to 18 & 0 & 0 & 0 & 0 \\
\hline & 18 to 20 & 0 & 0 & 0 & 2 \\
\hline & 20 to 22 & 0 & 0 & 0 & 0 \\
\hline & 22 to 24 & 0 & 0 & 0 & 0 \\
\hline & 24 to 26 & 0 & 0 & 0 & 1 \\
\hline & 26 to 28 & 0 & 0 & 0 & 0 \\
\hline & 28 to 30 & 0 & 0 & 0 & 0 \\
\hline & 30 to 32 & 0 & 0 & 1 & 0 \\
\hline & 32 to 34 & 0 & 1 & 0 & 0 \\
\hline & 34 to 36 & 0 & 0 & 0 & 0 \\
\hline & 36 to 38 & 0 & 0 & 0 & 0 \\
\hline & 38 to 40 & 0 & 0 & 0 & 0 \\
\hline & 40 to 42 & 0 & 0 & 0 & 1 \\
\hline & 42 to 44 & 0 & 0 & 0 & 0 \\
\hline & 44 to 46 & 0 & 0 & 0 & 0 \\
\hline & 46 to 48 & 0 & 0 & 0 & 0 \\
\hline & 48 to 50 & 0 & 0 & 1 & 0 \\
\hline & 50 to 55 & 0 & 0 & 0 & 0 \\
\hline & 55 to 60 & 0 & 0 & 0 & 0 \\
\hline & 60 to 65 & 0 & 0 & 1 & 0 \\
\hline & 65 and above & 0 & 0 & 0 & 0 \\
\hline & $\begin{array}{l}\text { Number of proteins with } \\
\text { AzG/U ratio } 4 \text { fold and above } \\
\text { untreated control }\end{array}$ & 2 & 8 & 28 & 45 \\
\hline
\end{tabular}




\begin{tabular}{|c|c|c|c|c|c|c|c|c|c|}
\hline \multirow{3}{*}{ 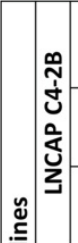 } & $\sum_{x=1}^{2}$ & $\stackrel{\circ}{\circ}$ & $\stackrel{8}{\circ}$ & 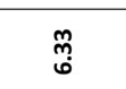 & $\stackrel{8}{0}$ & $\underset{\infty}{\stackrel{\infty}{0}}$ & స్తి & $\underset{j}{\stackrel{g}{g}}$ & $\stackrel{\infty}{\infty}$ \\
\hline & $\sum_{\mathbb{<}}$ & $\begin{array}{l} \\
\text { I } \\
\text { I }\end{array}$ & $\begin{array}{l}8 \\
\text { d } \\
\end{array}$ & $\begin{array}{l}8 \\
\\
9\end{array}$ & $\begin{array}{l}\text { \& } \\
\text { ㄱ }\end{array}$ & $\underset{\infty}{\substack{\infty \\
\infty}}$ & $\begin{array}{l}8 \\
\text { i } \\
\end{array}$ & $\begin{array}{l}8 \\
\text { ¿ }\end{array}$ & $\begin{array}{l}8 \\
\dot{9} \\
\end{array}$ \\
\hline & $\supset$ & 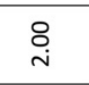 & 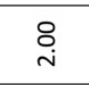 & $\underset{m}{\stackrel{一}{m}}$ & $\underset{i}{\stackrel{i}{i}}$ & $\underset{i}{\stackrel{\leftrightarrow}{+}}$ & i̊ & \&̣̊ & $\begin{array}{l}\stackrel{8}{9} \\
\dot{m}\end{array}$ \\
\hline \multirow{3}{*}{ 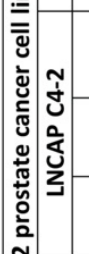 } & 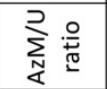 & هั & $\stackrel{\leftrightarrow}{\circ}$ & $\stackrel{8}{\circ}$ & $\stackrel{m}{0}$ & 赵 & న్లి & $\stackrel{8}{+}$ & กุ \\
\hline & $\sum_{<}$ & $\begin{array}{l}\substack{8 \\
\infty \\
\sim \\
\sim} \\
\end{array}$ & $\underset{\infty}{8}$ & $\underset{+}{8}$ & ৪ே & ৪ & $\begin{array}{l}\stackrel{8}{\infty} \\
\text { }\end{array}$ & ষ্ণ & ষ্ণ \\
\hline & $\supset$ & $\underset{\text { i }}{\text { in }}$ & $\underset{\text { i }}{\text { in }}$ & 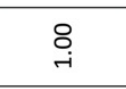 & 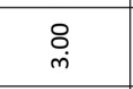 & $\underset{+}{\stackrel{\text { }}{ }}$ & 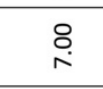 & $\underset{i}{8}$ & $\begin{array}{l}8 \\
\text { Oे } \\
\end{array}$ \\
\hline \multirow{3}{*}{ 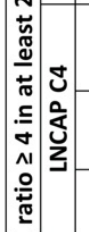 } & 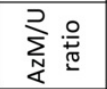 & $\stackrel{n}{i}$ & $\stackrel{0}{0}$ & $\underset{i}{\stackrel{i}{~}}$ & $\stackrel{0}{0}$ & $\stackrel{m}{m}$ & $\underset{f}{J}$ & น̊ำ & 迥 \\
\hline & $\sum_{\mathbb{K}}$ & $\begin{array}{l}\stackrel{8}{\text { d }} \\
\text { in }\end{array}$ & $\begin{array}{l}8 \\
\\
0\end{array}$ & $\underset{\text { i }}{\stackrel{8}{ }}$ & $\begin{array}{l}8 \\
\text { g }\end{array}$ & $\begin{array}{l}8 \\
\infty \\
\sim \\
N\end{array}$ & $\begin{array}{l}\text { ৪ } \\
\text { g̀ }\end{array}$ & চ̊ & 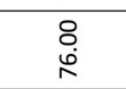 \\
\hline & د & $\begin{array}{l}\underset{\infty}{0} \\
\infty\end{array}$ & 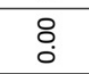 & $\underset{i}{\stackrel{\text { O }}{ }}$ & : & 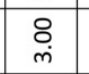 & $\underset{i}{\stackrel{8}{r}}$ & $\underset{\text { i }}{\text { in }}$ & 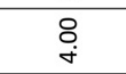 \\
\hline 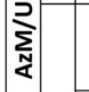 & $\sum_{\substack{\alpha\\
}}^{2} \stackrel{0}{\pi}$ & 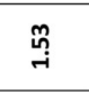 & : & $\stackrel{\infty}{0}$ & $\underset{\text { ని }}{ }$ & $\underset{\sim}{\sim}$ & 芯 & $\stackrel{0}{0}$ & $\underset{0}{\mathscr{0}}$ \\
\hline \multirow[t]{2}{*}{ 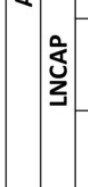 } & $\sum_{\mathbb{x}}^{5}$ & $\underset{\ddot{\sim}}{\stackrel{8}{\sim}}$ & $\underset{i}{\stackrel{\leftrightarrow}{ }}$ & $\stackrel{8}{i}$ & $\begin{array}{l}8 \\
\text { i }\end{array}$ & $\begin{array}{l}\text { வ } \\
\text { ¿ }\end{array}$ & $\begin{array}{l}8 \\
\dot{i}\end{array}$ & i̊ & $\begin{array}{l}\stackrel{8}{\text { p }} \\
\stackrel{\text { g }}{7}\end{array}$ \\
\hline & د & $\begin{array}{l}8 \\
\text { هُ }\end{array}$ & $\stackrel{8}{0}$ & $\underset{0}{8}$ & \&: & $\stackrel{8}{8}$ & $\begin{array}{l}8 \\
\dot{\sim} \\
\text { in }\end{array}$ & : & $\begin{array}{l}8 \\
\\
\end{array}$ \\
\hline & 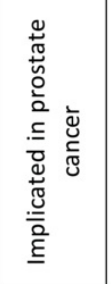 & 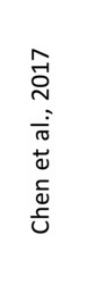 & 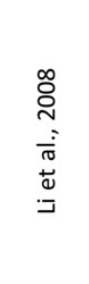 & 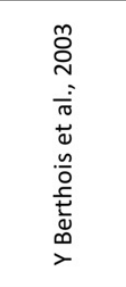 & 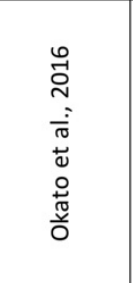 & . & 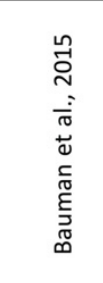 & . & 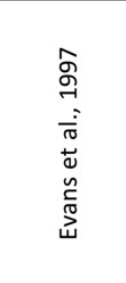 \\
\hline & 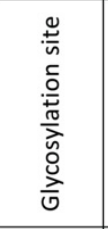 & 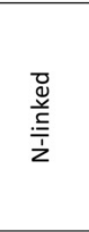 & 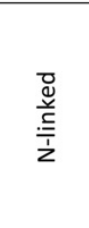 & t & 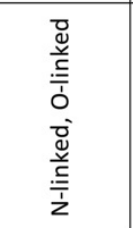 & 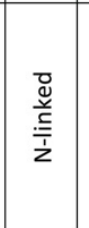 & $\begin{array}{l}\frac{\vec{d}}{\tilde{d}} \\
\stackrel{\underline{\underline{\underline{I}}}}{\bar{z}}\end{array}$ & ' & 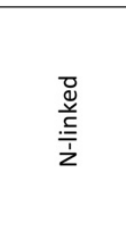 \\
\hline & 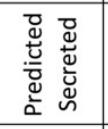 & ' & $>$ & & ' & $>$ & $>$ & . & ' \\
\hline & 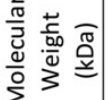 & 占 & $\vec{b}$ & $\stackrel{\sim}{\sim}$ & ஜ & $\mathcal{F}$ & ని & $\stackrel{\infty}{\stackrel{9}{2}}$ & $\stackrel{\mathbb{N}}{\sim}$ \\
\hline & 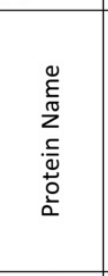 & 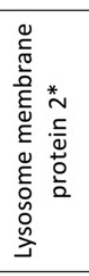 & 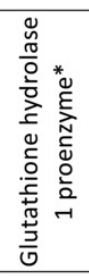 & 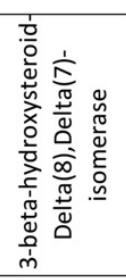 & 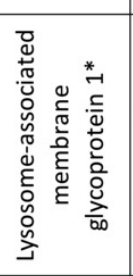 & 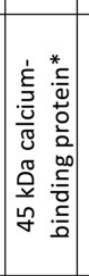 & 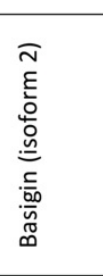 & 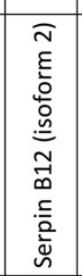 & 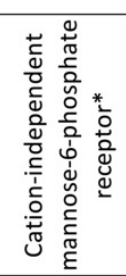 \\
\hline & 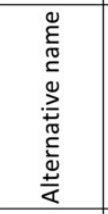 & $\stackrel{\mathscr{0}}{u}$ & $\underset{\widetilde{̃}}{\tilde{U}}$ & 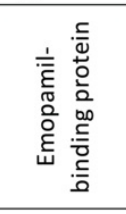 & $\begin{array}{l}\stackrel{0}{0} \\
0 \\
0\end{array}$ & 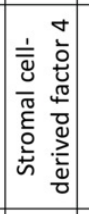 & 守 & . & ָิ̃ \\
\hline & 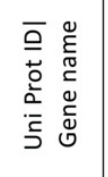 & 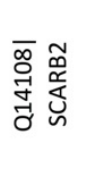 & 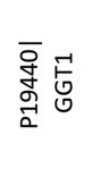 & 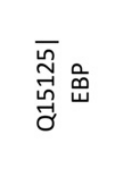 & 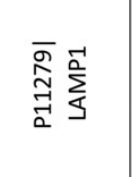 & 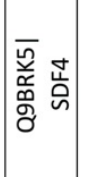 & 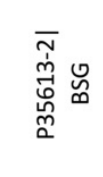 & 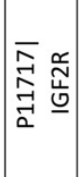 & 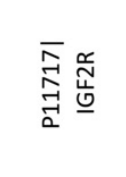 \\
\hline
\end{tabular}




\begin{tabular}{|c|c|c|c|c|c|c|c|c|c|c|c|c|c|}
\hline ๓ & 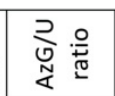 & 总 & $\begin{array}{l}8 \\
\dot{j} \\
\end{array}$ & 今ิ & @̊ & $\underset{0}{\stackrel{0}{0}}$ & ம் & फ़่ & ֻِ̣g & 号 & 导 & ت্ & $\vec{m}$ \\
\hline 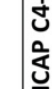 & 导 & $\begin{array}{l}8 \\
\text { in } \\
\text { in }\end{array}$ & $\begin{array}{l}8 \\
\infty \\
\text { i } \\
\end{array}$ & $\begin{array}{l}8 \\
\bar{m} \\
\end{array}$ & $\begin{array}{l}8 \\
\text { İ } \\
\text { ñ }\end{array}$ & 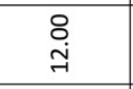 & $\begin{array}{l}8 \\
\\
\end{array}$ & $\begin{array}{l}8 \\
\text { I } \\
\end{array}$ & $\begin{array}{l}8 \\
\substack{\infty \\
\infty \\
n} \\
\end{array}$ & $\begin{array}{l}8 \\
\text { வे } \\
\end{array}$ & $\begin{array}{l}8 \\
\text { in }\end{array}$ & $\begin{array}{l}8 \\
\text { gi } \\
\end{array}$ & $\begin{array}{l} \\
0 \\
0\end{array}$ \\
\hline 岂 & د & $\underset{i}{\stackrel{8}{ }}$ & $\underset{i}{\stackrel{\leftrightarrow}{i}}$ & $\stackrel{8}{0}$ & ọ & $\stackrel{8}{\text { O̊ }}$ & $\underset{m}{\stackrel{8}{m}}$ & $\stackrel{\text { i }}{\text { i }}$ & $\underset{j}{\stackrel{\text { m }}{ }}$ & $\underset{\text { i }}{\text { in }}$ & ị & $\underset{+}{\stackrel{\text { O }}{ }}$ & 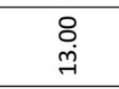 \\
\hline \multirow{3}{*}{ 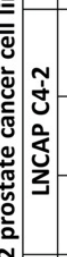 } & 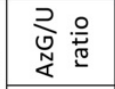 & $\stackrel{\text { ஸે }}{\exists}$ & $\stackrel{\circ}{\circ}$ & $\begin{array}{l}8 \\
\text { ஸे } \\
\end{array}$ & $\stackrel{\mathscr{H}}{+}$ & $\stackrel{\hat{\sigma}}{\circ}$ & $\underset{0}{8}$ & 8 & $\begin{array}{l}8 \\
\dot{+} \\
\end{array}$ & ه̊ & 유. & $\begin{array}{l}8 \\
\\
\end{array}$ & $\underset{g}{q}$ \\
\hline & 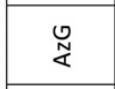 & $\begin{array}{l}8 \\
\dot{y} \\
\dot{y}\end{array}$ & $\begin{array}{l}8 \\
\infty \\
\rightarrow \\
\rightarrow\end{array}$ & $\begin{array}{l}8 \\
\text { in } \\
\text { in }\end{array}$ & $\begin{array}{l}8 \\
\\
\end{array}$ & $\begin{array}{l}8 \\
\\
\end{array}$ & 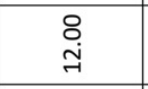 & $\underset{0}{8}$ & $\begin{array}{l}8 \\
\dot{0} \\
\end{array}$ & $\begin{array}{l}8 \\
\text { ì } \\
\end{array}$ & $\begin{array}{l}8 \\
\text { i } \\
\end{array}$ & $\begin{array}{l}8 \\
\\
\end{array}$ & $\begin{array}{l}8 \\
\\
0\end{array}$ \\
\hline & د & $\underset{+}{\circ}$ & $\stackrel{\text { o }}{\text { i }}$ & $\underset{i}{\stackrel{i}{8}}$ & $\underset{+}{\stackrel{8}{+}}$ & $\underset{m}{\stackrel{\leftrightarrow}{m}}$ & 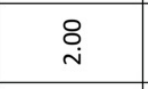 & $\underset{i}{\stackrel{\leftrightarrow}{i}}$ & $\underset{i}{\stackrel{i}{8}}$ & $\underset{i}{\text { \} }} &{\underset{\text { i }}{\text { i }}} &{\text { 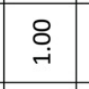 }} &{\begin{array}{l}8 \\
\\
\end{array}} \\
{\hline \multirow{3}{*}{\text { 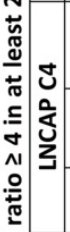 }}\text { 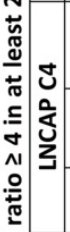 }} &{\text { 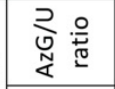 }} &{\stackrel{\text { ¿ }}{\circ}} &{\stackrel{8}{\circ}} &{\text { ষ্ণ }} &{\stackrel{8}{\circ}} &{\stackrel{\circ}{\circ}} &{\stackrel{8}{\circ}} &{\underset{m}{\stackrel{m}{i}}} &{\begin{array}{l}\stackrel{8}{\dot{m}} \\
\text { m. }\end{array}} &{\text { 品 }} &{\text { 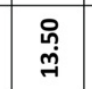 }} &{\stackrel{+}{\circ}} &{\begin{array}{l}\stackrel{n}{\sim} \\
\stackrel{-}{-}\end{array}} \\
{\hline} &{\underset{⿱ 亠 乂}{4}} &{\begin{array}{l}\stackrel{8}{\text { Oे }} \\
\text { ت }\end{array}} &{\text { 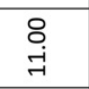 }} &{\text { ị }} &{\stackrel{8}{0}} &{\stackrel{\circ}{\circ}} &{\text { i̊ }} &{\stackrel{8}{+}} &{\underset{m}{\stackrel{\text { }}{m}}} &{\text { 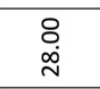 }} &{\text { 足 }} &{\text { 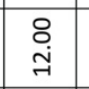 }} &{\begin{array}{l}\stackrel{8}{+} \\
\dot{f}\end{array}} \\
{\hline} &{\supset} &{\begin{array}{l}8 \\
0 \\
0\end{array}} &{\begin{array}{l}8 \\
0 \\
0\end{array}} &{\begin{array}{l}8 \\
0 \\
0\end{array}} &{\begin{array}{l}8 \\
0 \\
0\end{array}} &{\begin{array}{l}8 \\
0 \\
0\end{array}} &{\text { 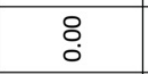 }} &{\text { 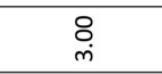 }} &{\underset{i}{\stackrel{8}{0}}} &{\begin{array}{l}8 \\
\infty \\
\infty\end{array}} &{\underset{i}{\text { O্ }}} &{\underset{m}{\stackrel{8}{n}}} &{\underset{+}{8}} \\
{\hline \text { S్ }} &{\text { 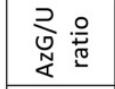 }} &{\stackrel{8}{0}} &{\stackrel{\circ}{\circ}} &{\stackrel{\infty}{0}} &{\text { న్ని }} &{\text { 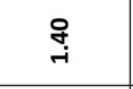 }} &{\stackrel{n}{0}} &{\text { กี }} &{\underset{\mathfrak{T}}{\vec{H}}} &{\stackrel{\Re}{ت}} &{\underset{\Im}{ت}} &{\stackrel{\leftrightarrow}{\circ}} &{\stackrel{\circ}{\circ}} \\
{\hline \multirow[t]{2}{*}{\text { 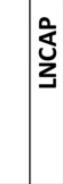 }}\text { 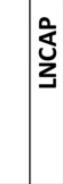 }} &{\text { 足 }} &{\stackrel{8}{0}} &{\underset{i}{\stackrel{\leftrightarrow}{i}}} &{\underset{+}{\stackrel{\leftrightarrow}{+}}} &{\underset{i}{\stackrel{i}{i}}} &{\text { 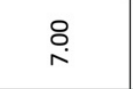 }} &{\text { 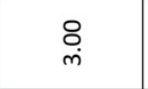 }} &{\text { ì }} &{\begin{array}{l}8 \\
\stackrel{\leftrightarrow}{7}\end{array}} &{\begin{array}{l}8 \\
\end{array}} &{\begin{array}{l}\text { வ } \\
\text { வ }\end{array}} &{\begin{array}{l}8 \\
\text { g }\end{array}} &{\begin{array}{l}8 \\
\dot{0} \\
\infty\end{array}} \\
{\hline} &{\text { د }} &{\stackrel{8}{0}} &{\stackrel{8}{0}} &{\text { ị }} &{\text { 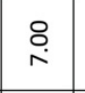 }} &{\text { \&̊. }} &{\begin{array}{l}\stackrel{8}{+} \\
\text { i }\end{array}} &{\text { 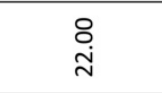 }} &{\begin{array}{l}8 \\
\stackrel{+}{+}\end{array}} &{\begin{array}{l}\text { ه̊ } \\
\text { ف․ }\end{array}} &{\begin{array}{l}\text { \& } \\
\dot{d}\end{array}} &{\begin{array}{l}\stackrel{8}{\circ} \\
\text { ت̇ }\end{array}} &{\text { 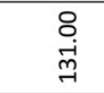 }} \\
{\hline} &{\text { 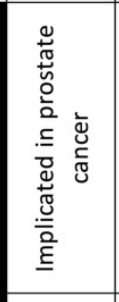 }} &{\text { 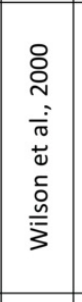 }} &{\text { 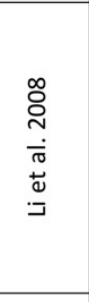 }} &{\text { 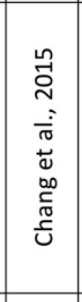 }} &{\text { 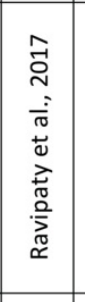 }} &{\text { 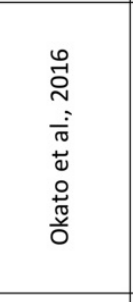 }} &{\text { 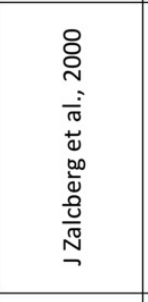 }} &{\text { ' }} &{\text { ' }} &{\text { 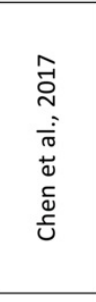 }} &{\text { 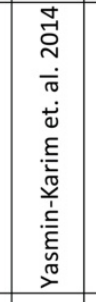 }} &{\text {, }} &{\text { 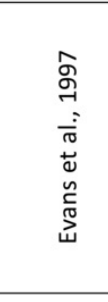 }} \\
{\hline} &{\text { 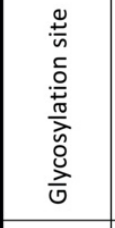 }} &{\text { 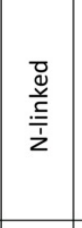 }} &{\text { 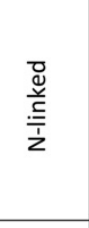 }} &{\text { 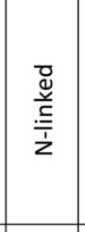 }} &{\text {. }} &{\text { 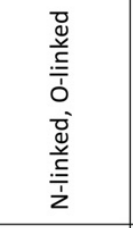 }} &{\text { 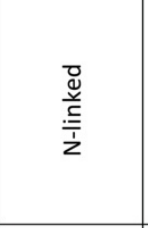 }} &{\text { ' }} &{\text { 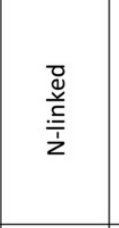 }} &{\text { 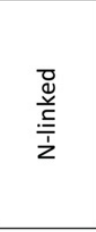 }} &{\text { 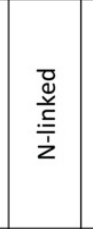 }} &{\text { 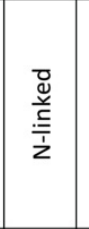 }} &{\begin{array}{l}\frac{\bar{d}}{\tilde{W}} \\
\frac{\underline{\underline{\underline{L}}}}{\dot{z}}\end{array}} \\
{\hline} &{\text { 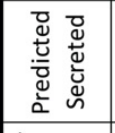 }} &{>} &{>} &{>} &{>} &{\text { ' }} &{\text { ' }} &{\text { ' }} &{\text { ' }} &{\text { ' }} &{>} &{>} &{\text { ' }} \\
{\hline} &{\text { 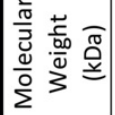 }} &{\infty} &{\vec{b}} &{\underset{\oplus}{\stackrel{\oplus}{*}}} &{\text { 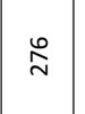 }} &{\text { そ }} &{\underset{\sim}{\stackrel{N}{*}}} &{\stackrel{\stackrel{m}{\rightarrow}}{-1}} &{\exists} &{\text { in }} &{\stackrel{\leftrightarrow}{\rightarrow}} &{\mathcal{F}} &{\stackrel{ \pm}{\sim}} \\
{\hline} &{\text { 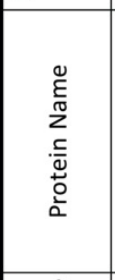 }} &{\text { 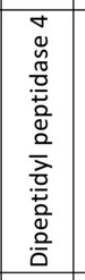 }} &{\text { 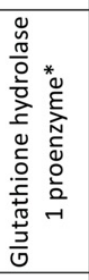 }} &{\text { 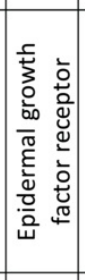 }} &{\text { 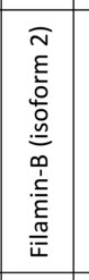 }} &{\text { 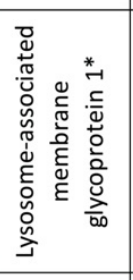 }} &{\text { 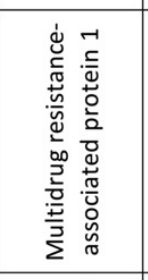 }} &{\text { 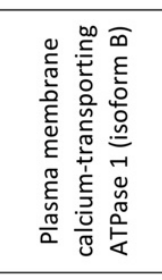 }} &{\text { 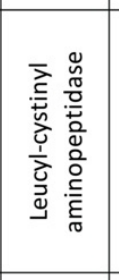 }} &{\text { 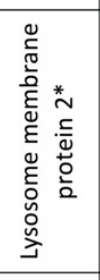 }} &{\text { 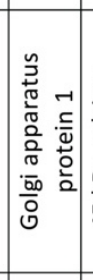 }} &{\text { 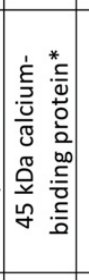 }} &{\text { 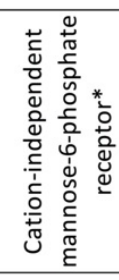 }} \\
{\hline} &{\text { 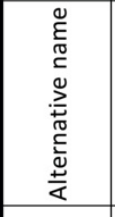 }} &{\text { జ̃ }} &{\text { ָ̃̃ }} &{\text { 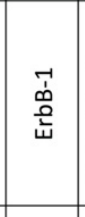 }} &{\text { 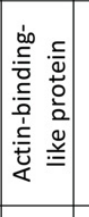 }} &{\text { 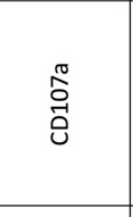 }} &{\text { 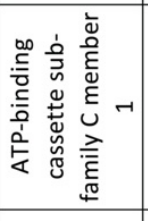 }} &{\text { 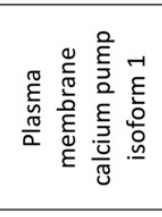 }} &{\text { 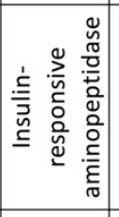 }} &{\text { Ô̊ }} &{\text { 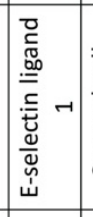 }} &{\text { 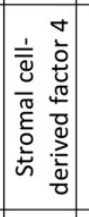 }} &{\text { ญิ }} \\
{\hline} &{\text { 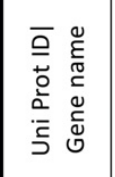 }} &{\text { 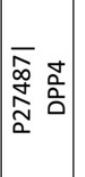 }} &{\text { 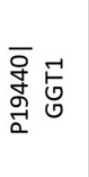 }} &{\text { 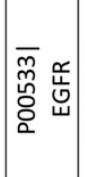 }} &{\text { 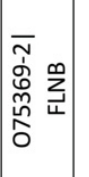 }} &{\text { 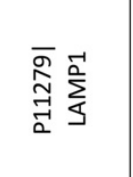 }} &{\text { 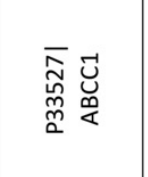 }} &{\text { 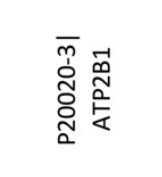 }} &{\text { 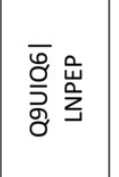 }} &{\text { 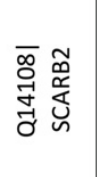 }} &{\text { 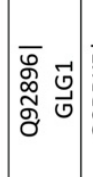 }} &{\mid} &{\text { 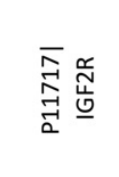 }} \\
$\hline
\end{tabular}


Figure 5.5: Identification of azidosugar-labelled proteins in prostate cancer cell lines.

Azidosugar-labelled proteins were enriched from the LNCAP lineage of prostate cancer cell lines using streptavidin-coupled beads. For each protein identified by liquid chromatographytandem mass spectrometry, the ratio of spectral counts identified from $\mathrm{N}$ -

azidoacetylmannosamine- (Ac4ManNAz or AzM) or N-azidoacetylgalactosamine-labelled (Ac ${ }_{4} G a l N A z$ or $\left.A z G\right)$ samples, and the untreated control samples $(U)$ were calculated. (a) Representative histogram illustrating the distribution of $A z M / U$ (i) and $A z G / U$ ratios (ii) from the highly metastatic LNCAP C4-2B prostate cancer cell line. (b) Ac 4 ManNAz-labelled proteins (i) and Ac $c_{4}$ GalNAz-labelled proteins (ii) with an Az/U ratio of 4 and above were selected for further analysis. (c) For each cell line, the frequency of proteins identified as having an AzM/U ratio (i) or AzG/U ratio (ii) of 4 and above is summarised. (d) The characteristics of proteins identified as having an $A z M / U$ ratio (i) or $A z G / U$ (ii) ratio of 4 and above, in at least two of the prostate cancer cell lines, are summarised. *denotes dual labelled proteins with both AzM/U and AzG/U ratios of 4 and above. (Chen et. al. 2017; Li et. al. 2008; Berthois et. al. 2003); Okato et. al. 2016; Bauman et. al. 2015; Evans et. al. 1997; Wilson et. al. 2000; Chang et. al. 2015; Ravipaty et. al. 2017; Zalcberg et. al. 2000; Yasmin-Karim et. al. 2014).

Next, the Az/U ratios from each of the shortlisted proteins (Figure 5.5) were compared across each of the LNCAP cell lines. We selected azidosugar-labelled proteins exhibiting a general increase in $\mathrm{AzM} / \mathrm{U}$ ratio (Figure 5.6.a, i) or $\mathrm{AzG} / \mathrm{U}$ ratio (Figure 5.6a, ii), which correlated with an increase in the metastatic potential of the cell lines. To confirm if increases in azidosugar labelling were due to increases in the overall expression of the proteins, we used a label-free approach based on spectral counts obtained from the whole membrane enriched proteome (Figure 5.3d, lane 1). Normalised spectral abundance factors (Zybailov et. al. 2006; Byrum et. al. 2013) were calculated to estimate the relative abundance of proteins in cell lines (Figure 5.6b). 


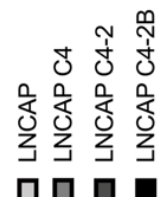

पि०

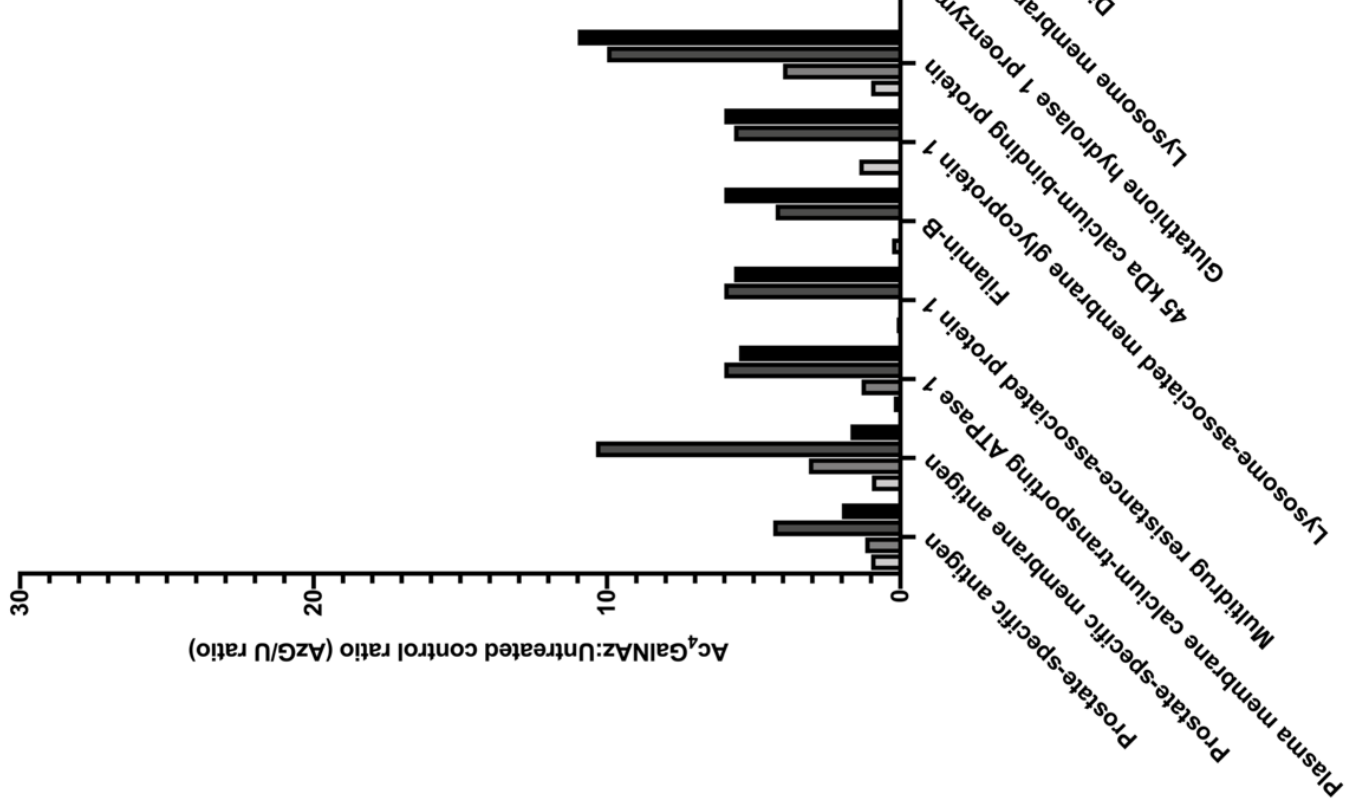

$:=$
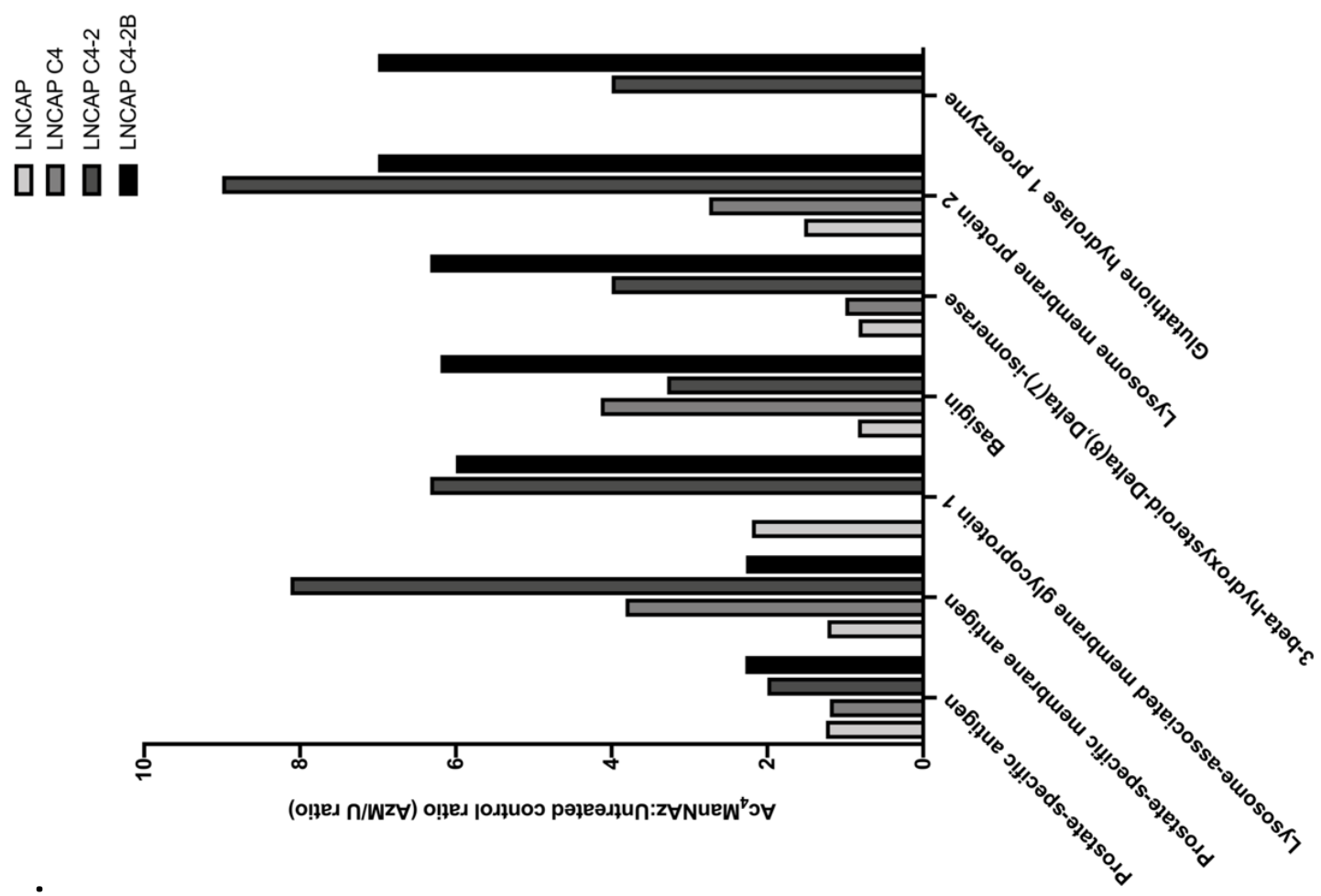

$\dot{\sigma} . \dot{ }$ 
b.

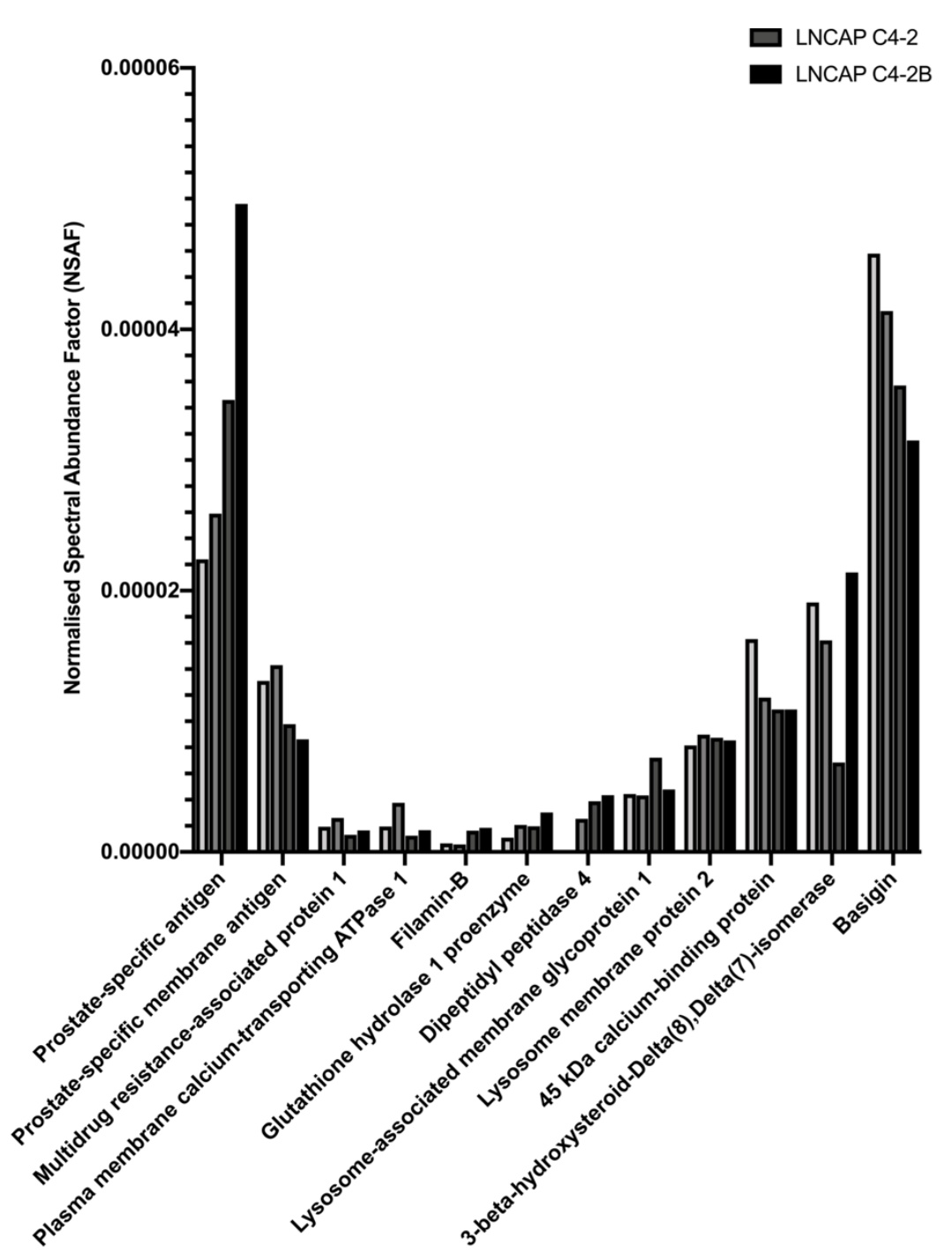

Figure 5.6: Comparison of differential azidosugar-specific glycosylation and relative protein expression. Azidosugar-labelled proteins were identified using mass spectrometry. For each protein, the ratio of spectral counts identified from $N$-azidoacetylmannosamine- (Ac4ManNAz or AzM) or N-azidoacetylgalactosamine-labelled (Ac ${ }_{4} G a I N A z$ or $\left.A z G\right)$ samples, and the untreated control samples $(U)$ were calculated. Proteins with an AzM/U ratio or AzG/U ratio of 4 and above in at least two of the prostate cancer cell lines, were compared across the LNCAP lineage of prostate cancer cell lines. (a) Proteins exhibiting a general increase in AzM/U ratio (i) or $A z G / U$ ratio (ii), which correlated with an increase in the metastatic potential of the cell lines are summarised. (b) Normalised spectral abundance factors (NSAF) for each protein. Ratios and NSAF's for prostate-specific antigen and prostate-specific 
membrane antigen were included for comparison with established markers of prostate cancer progression.

Normalised Spectral Abundance Factors, showed, as expected, a relative increase in the expression of total PSA, but also an increase in $\mathrm{Ac}_{4} \mathrm{ManNAz}$ and $\mathrm{Ac}_{4} \mathrm{GalNAz}$ glycan labelling of PSA, which correlated with an increase in the metastatic potential (Figure 5.6). Glycoprofiling of PSA may reveal prognostic markers of disease aggressiveness, a concept already extensively reviewed in the literature (Tkac et. al. 2019; Llop et. al. 2016). More interesting was the case of basigin (Figure 5.7a) and $45 \mathrm{kDa}$ calcium-binding protein (Figure 5.7b), which showed a general increase in $\mathrm{Ac}_{4} \mathrm{ManNAz}$ and $\mathrm{Ac}_{4} \mathrm{GalNAz}$ labelling, despite their total expression decreasing across the panel of cell lines. Decreased expression of basigin has been reported in prostate cancer in comparison to non-malignant prostate tissue and proposed as a predictor of progression and recurrence after prostatectomy (Bauman et. al. 2015). The molecular weight of basigin ranges between $29-64 \mathrm{kDa}$, based on the extent of $\beta 1,6$ - branched polylactosamine at its three $\mathrm{N}$-glycosylation sites (Tang et. al. 2004). Whilst the low and high molecular weight glycoforms of basigin are well documented in the literature, little is known about the prognostic value of differentially glycosylated basigin in prostate cancer (Figure 5.7a, i). The relative abundance of $45 \mathrm{kDa}$ calcium-binding protein decreased across the panel of LNCAP cell lines, and was low in comparison to prostatespecific antigen (Figure 5.7b, ii). This, once again, highlighted the sensitivity of bioorthogonal chemistry and its utility in detecting glycosylation in low abundance proteins. 
a.

i.

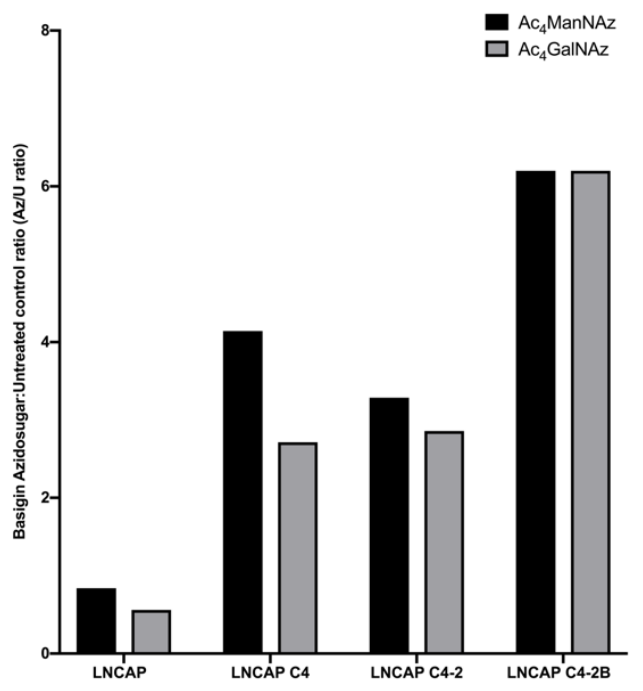

b.

i.

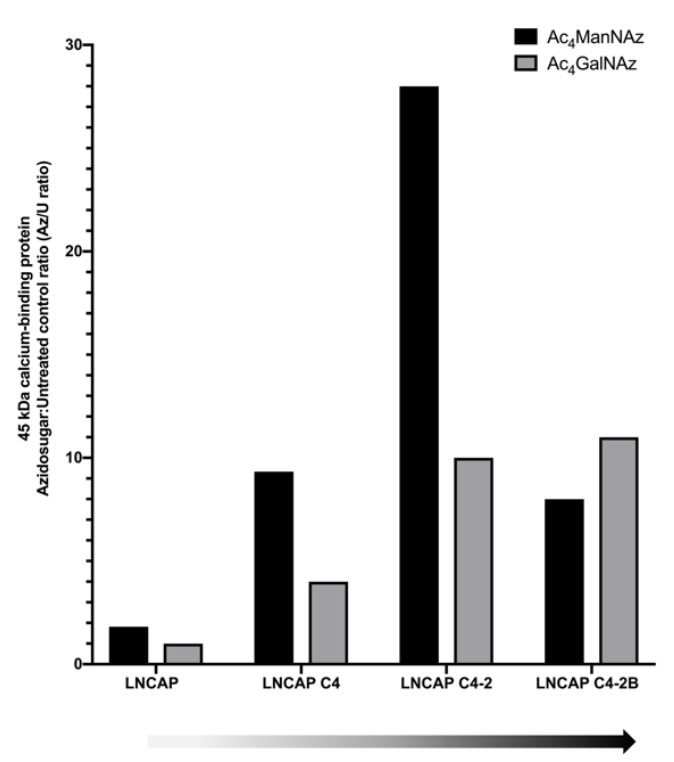

ii.

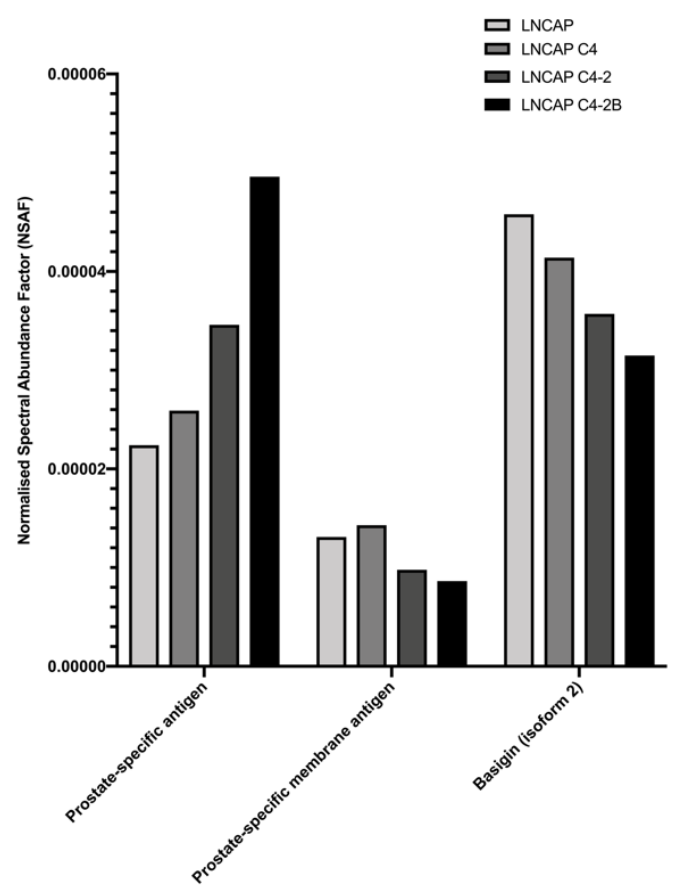

ii.

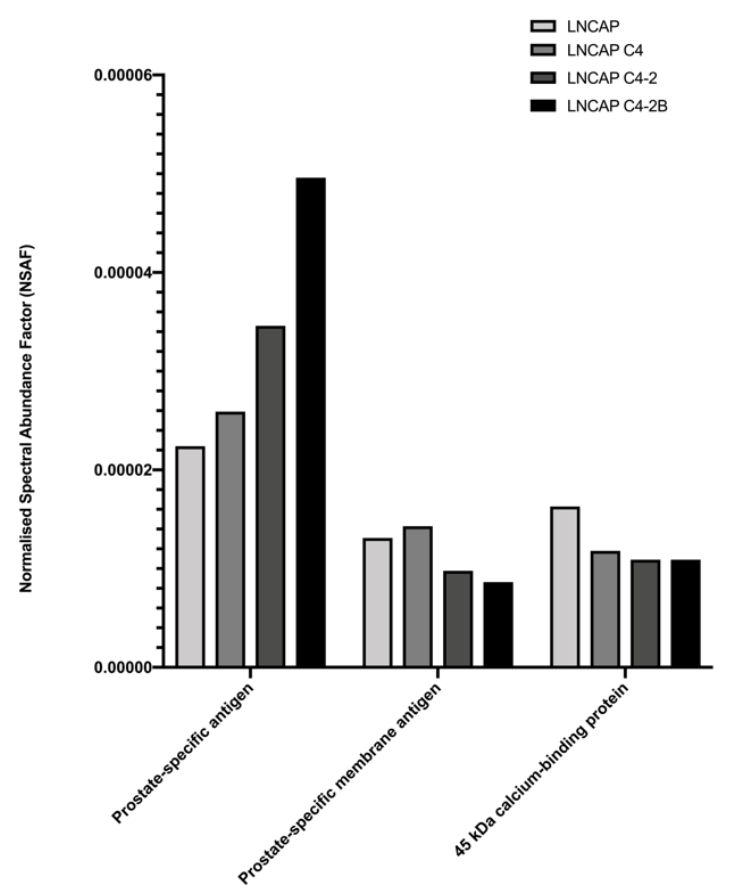

Figure 5.7: Differential glycosylation of basigin and $45 \mathrm{kDa}$ calcium-binding protein. (a) $A$ general increase in N-azidoacetylmannosamine ( $\mathrm{Ac}_{4} \mathrm{ManNAz}$ ) and $\mathrm{N}$ azidoacetylgalactosamine ( $\mathrm{C}_{4}$ GaINAz) labelling of basigin was observed (i) despite the expression of basigin decreasing across the panel of cell lines (ii). (b) A general increase in $A c_{4} M a n N A z$ and $A c_{4} G a l N A z$ labelling of $45 \mathrm{kDa}$ calcium-binding protein was observed (i) despite the expression of $45 \mathrm{kDa}$ calcium-binding protein decreasing across the panel of cell 
lines (ii). Ratios and NSAF's for prostate-specific antigen and prostate-specific membrane antigen are included for comparison with established markers of prostate cancer progression. The darker shades in the arrow's greyscale represent an increase in the metastatic potential of the prostate cancer cell lines.

\subsubsection{Glycoprofiling of membrane bound and secreted basigin using lectin blotting}

Basigin was selected as a candidate of interest as it had a similar abundance to prostatespecific antigen (Figure 5.7b), and was classified as a secreted protein (Figure 5.5d, ii). The expression of membrane-associated basigin in whole-cell lysates and secreted basigin in conditioned media was analysed in the panel of prostate and breast cancer cell lines in order to investigate whether it may have significance as a prognostic biomarker.

Whole-cell lysates were obtained from prostate and breast cancer cell lines grown in complete media. In parallel, conditioned media was collected from cells grown in serumstarved conditions for 24 hours. No significant reduction in cell viability was observed as a result of growing the prostate (Figure 5.8a, i) or breast (Figure 5.8a, ii) cells under serum-free conditions. To show concentrations of proteins in the conditioned media were a result of secretion and not cell death, expression of tubulin was analysed by western blot (Dowling and Clynes 2011) (Figure 5.8b). The low abundance of tubulin in the conditioned media, relative to the whole-cell lysate indicated intact cell membranes (Figure 5.8b, i and ii). 
a.

i.

Complete Medium

$\square$ Serum Free Medium

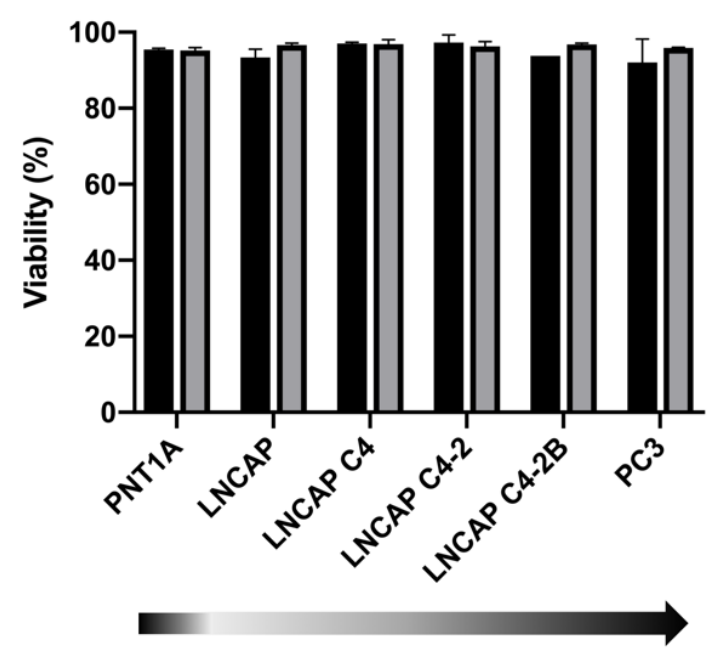

b.

i.
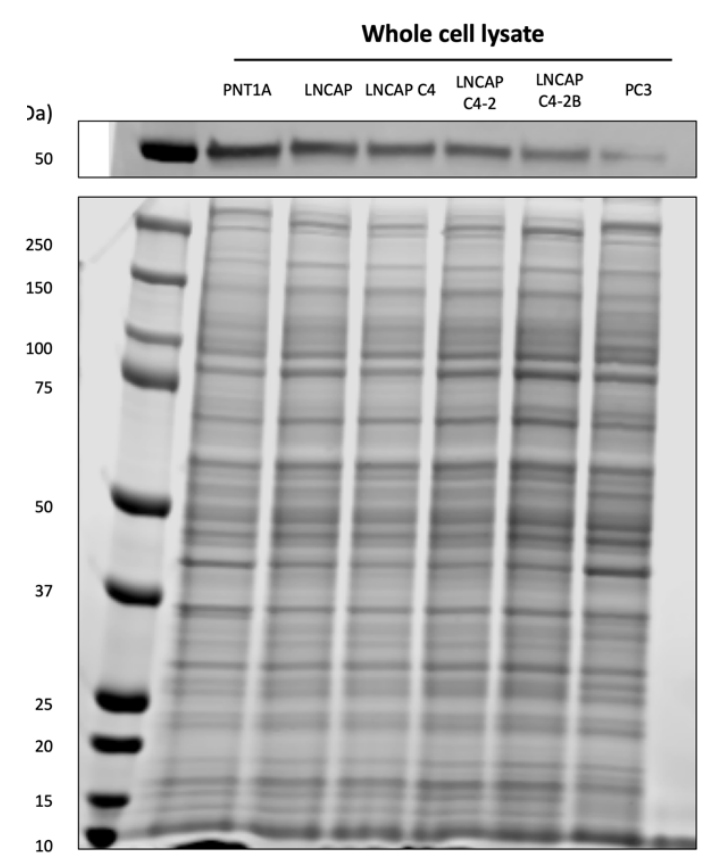

ii.

Complete Medium

$\square$ Serum Free Medium

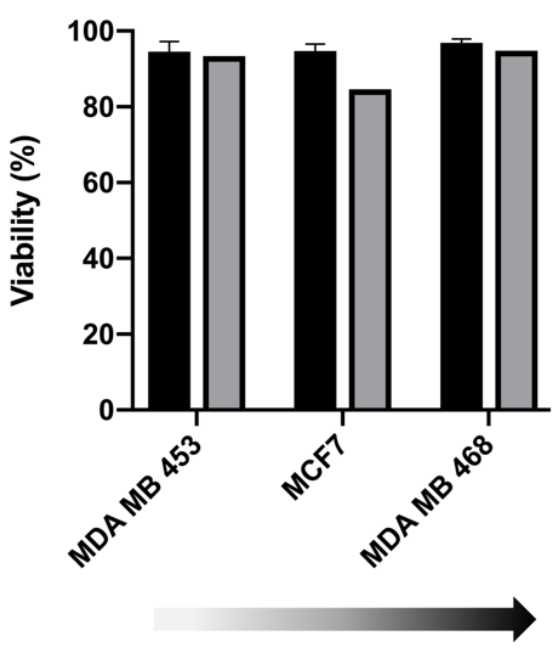

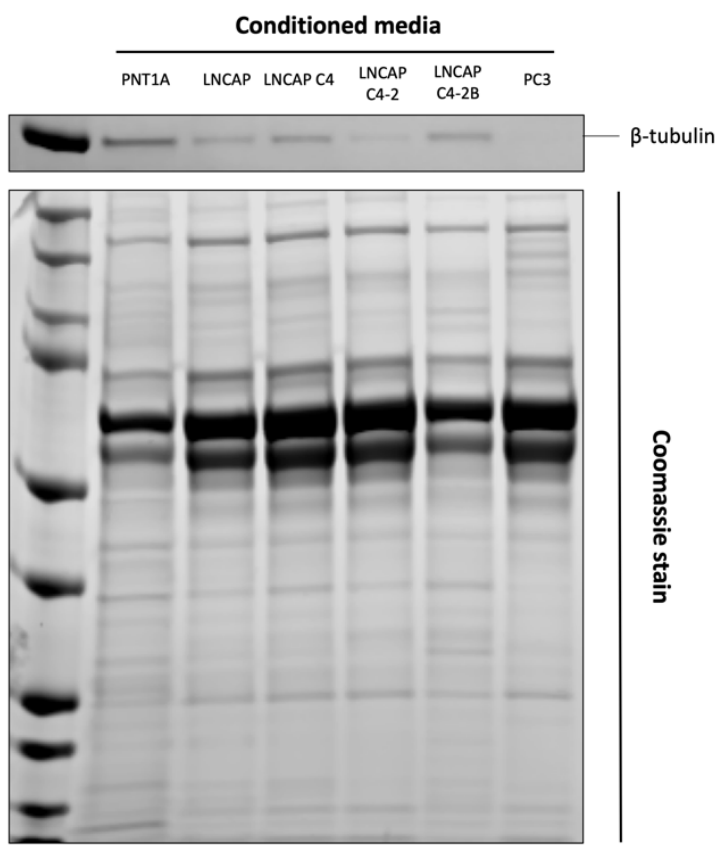


ii.

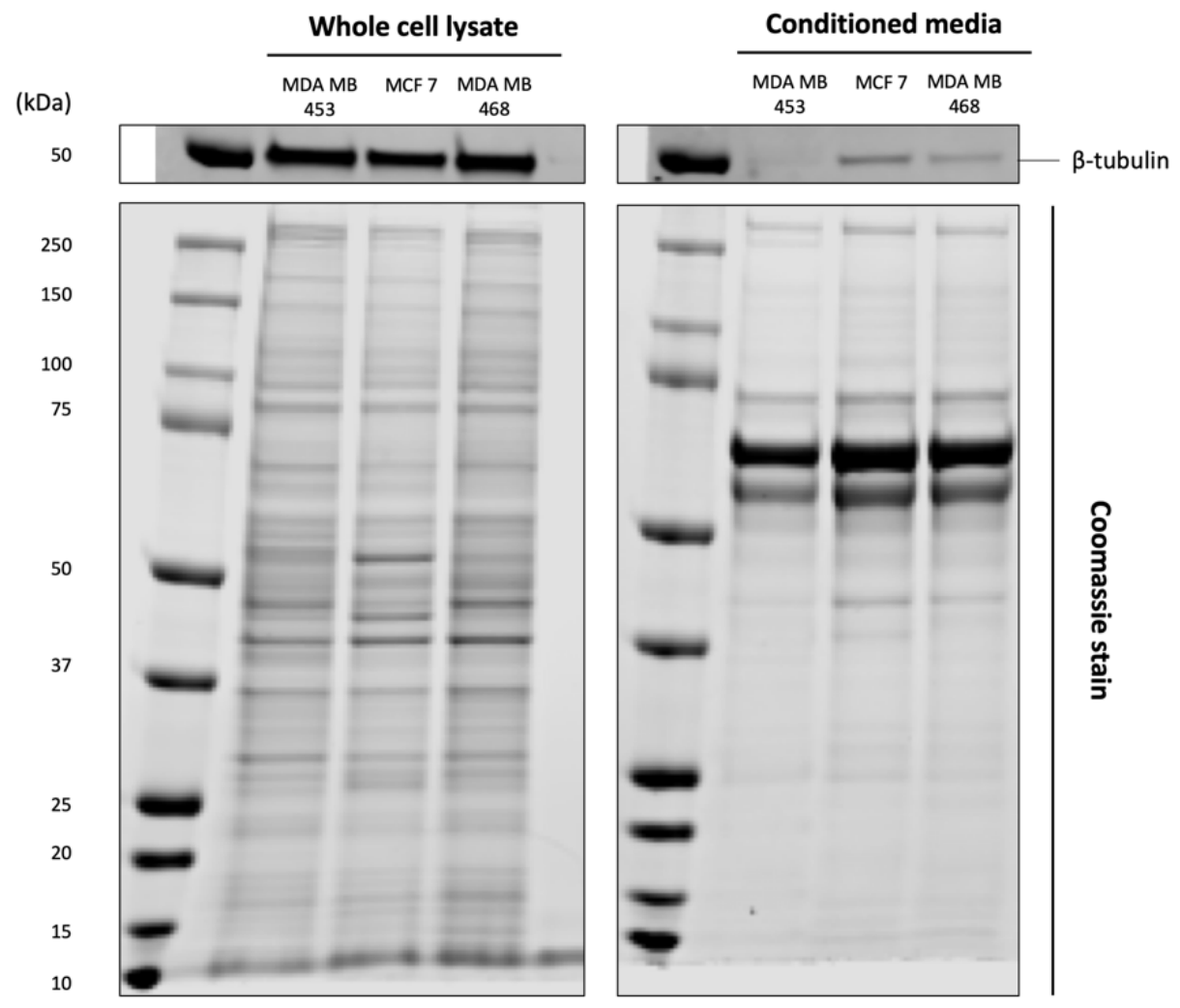

Figure 5.8: Preparation of whole-cell lysates and conditioned media from prostate and breast cell lines. (a) Viability of prostate (i) and breast (ii) cells grown in complete media and serum-free media was compared. (b) The abundance of tubulin in the whole-cell lysate and conditioned media prepared from the prostate (i) and breast (ii) cell lines were compared by western blot (10 $\mu$ g protein loaded per lane). Equal loading was confirmed by total protein Coomassie stain. All data represent the average \pm S.D. of two independent experiments, each with 3 replicates. $p$ values represent significance assessed by one-way ANOVA. The darker shades in the arrow's greyscale represent an increase in the metastatic potential of the panel of prostate and breast cell lines.

Next, the relative expression of high-glycosylated $(\sim 40-60 \mathrm{kDa})$ and low-glycosylated $(\sim 32$ kDa) basigin (Huang et. al. 2013; Tang et. al. 2004) was analysed by western blot (Figure $5.9 a$, i). A greater level of basigin expression was observed in the prostate cancer cell lines compared to the breast cancer cell lines. Increased expression of basigin has been demonstrated in the progression from the non-tumourigenic MDA MB 453 cell line, tumourigenic non-metastatic MCF7 and MDA MB 468 cell lines, and the tumourigenic 
metastatic MDA MB 231 cell line (Kong et. al. 2014), although we could not clearly observe this in $30 \mu \mathrm{g}$ of lysate prepared from the breast cancer cell lines used in this study.

Decreased expression of basigin (Figure 5.9a, ii) across the panel of prostate cancer cell lines was observed in whole-cell lysates, validating the relative expression observed using liquid chromatography-mass spectrometry (Figure 5.7a, ii). Minimal secreted basigin was detected in conditioned media obtained from the prostate cancer cell lines (Figure 5.9b, i).

a.

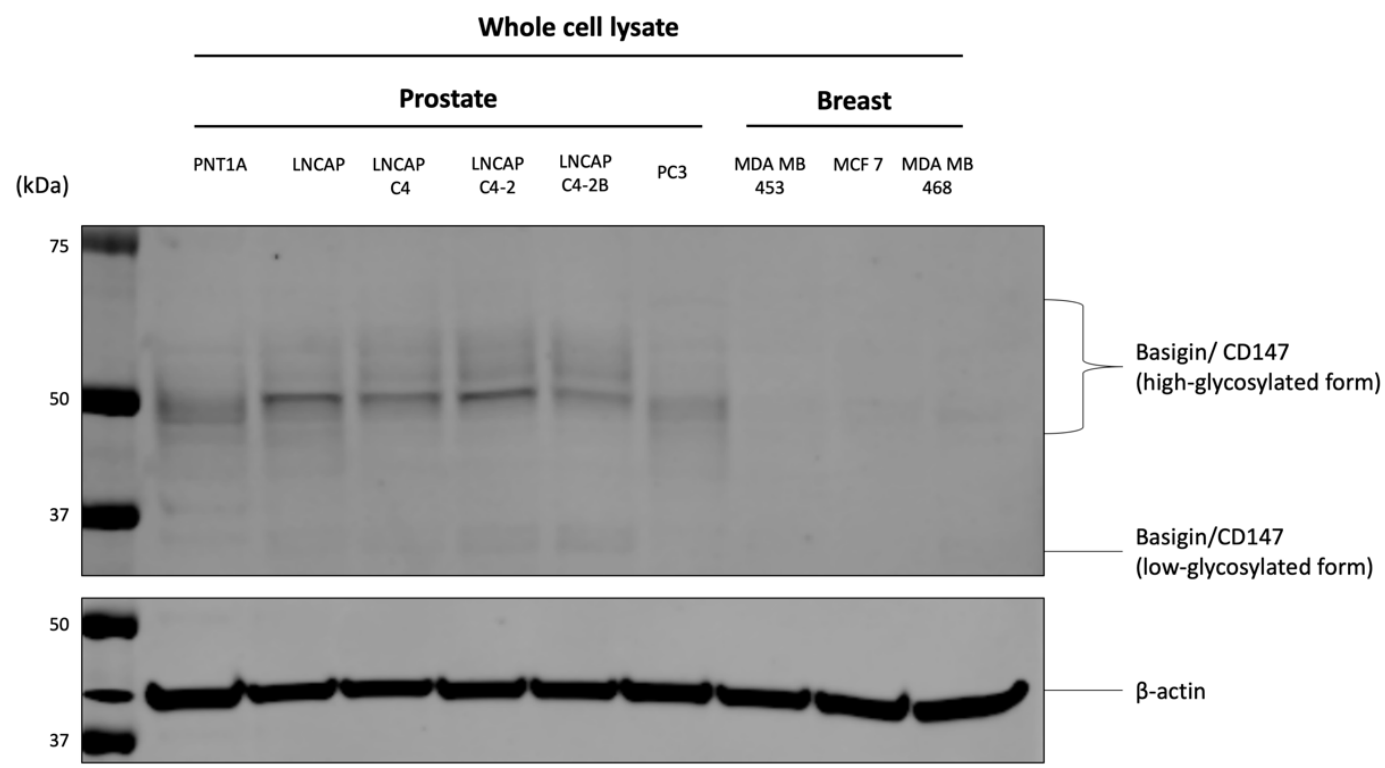

ii.

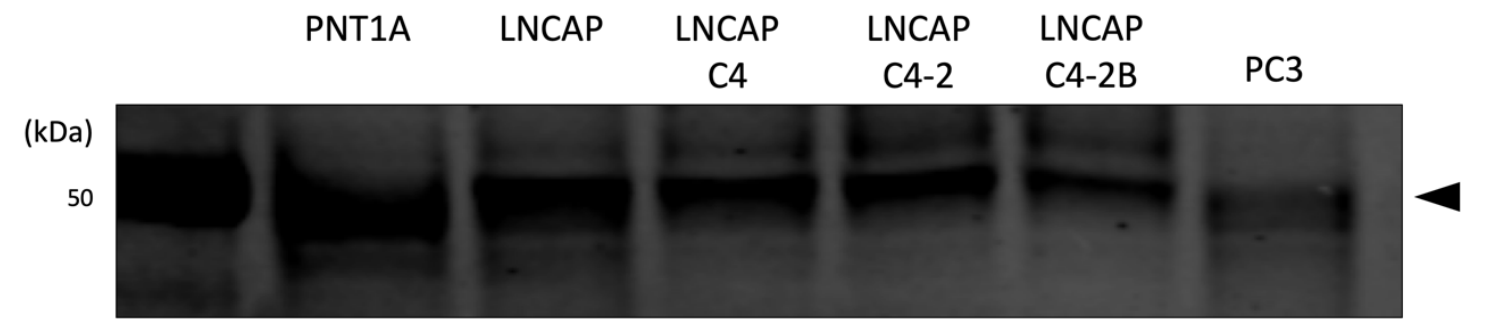

50

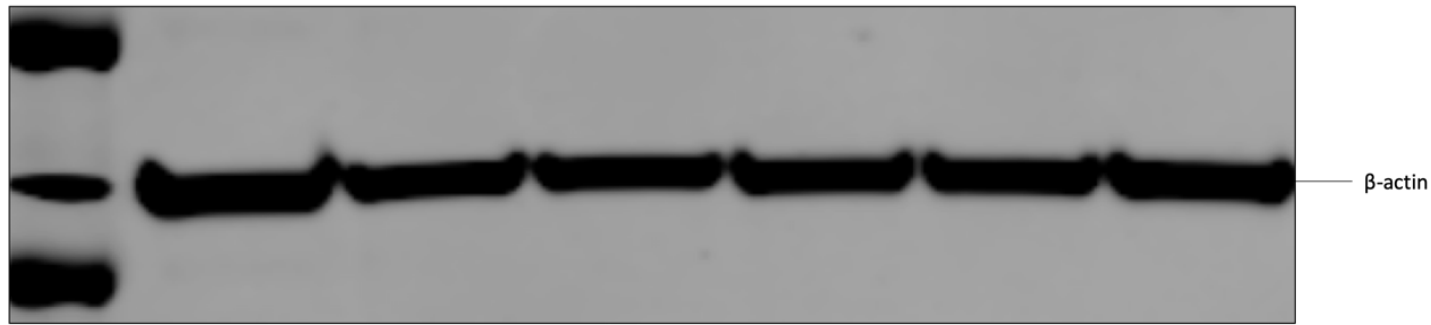


b.

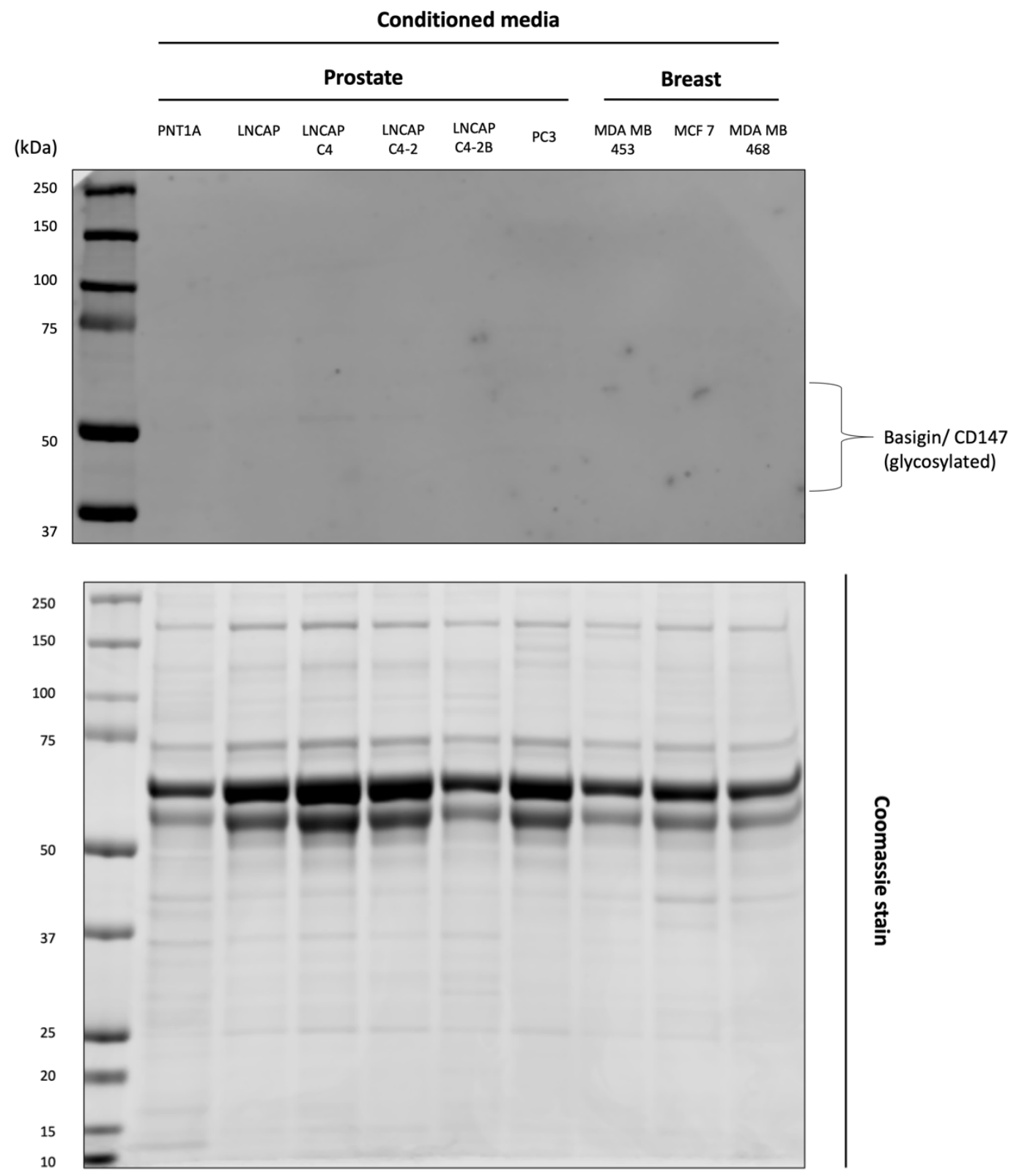


ii.

PNT1A LNCAP LNCAP LNCAP LNCAP

C4 $\quad$ C4-2 $\quad$ C4-2B $\quad$ PC3

(kDa)
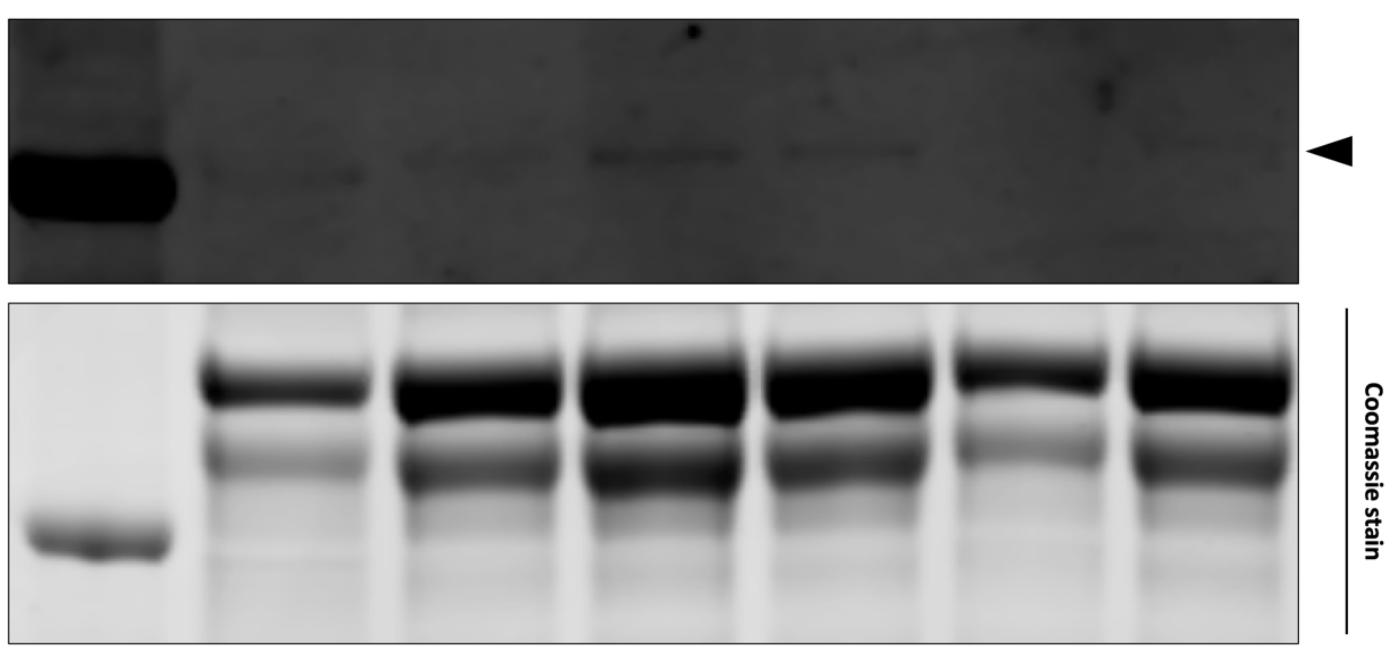

Figure 5.9: Expression of basigin in conditioned media and whole-cell lysates from prostate cancer cell lines. (a) Relative expression of high-glycosylated and low-glycosylated basigin (i) in whole-cell lysates prepared from prostate and breast cancer cell lines analysed by western blot. A decrease in basigin expression (ii, Black arrow head) was observed, validating the decrease in normalised spectral abundance factors (NSAF) observed with mass spectrometry. Actin was used as a loading control. (b) Relative expression of basigin (i) in conditioned media prepared from prostate and breast cancer cell lines. Minimal secreted basigin was detected in the conditioned media (ii, Black arrow head). Equal loading was assessed by total protein Coomassie stain. (30 $\mu \mathrm{g}$ loaded per lane).

Capturing aberrantly glycosylated basigin in patient liquids such as serum or urine, using the techniques adopted in this project (Chapter 1: Figure 1.24), would require biorthogonal labelling of live tissue by administering azidosugars directly to patients in vivo or conducting metabolic labelling of tissue biopsies ex vivo. Tissue explants from subcutaneous xenografts derived from the LNCAP C4-2B prostate cancer cell line, have been previously maintained on dental sponges (Centenera et. al. 2013), and metabolically labelled ex vivo, although cell death and loss of tissue architecture were observed (Kuo 2015, PhD thesis, unpublished). Spiciarich and colleagues successfully metabolically labelled prostate tissue slices derived 
from radical prostatectomy specimens, followed by identification of $\mathrm{Ac}_{4} \mathrm{ManNAz}$ labelled proteins using mass spectrometry (Spiciarich et. al. 2017). However, neither of these ex vivo bioorthogonal metabolic labelling techniques have been translated into clinical practice. Lectins provide an alternative approach to detecting changes in glycosylation. Therefore, we endeavoured to detect the differential glycosylation of basigin, observed with bioorthogonal metabolic labelling (Figure 5.7a), using lectins. This could provide the preliminary data needed to support the development of a clinical assay, which would use sialic acid recognising lectins such as Maackia Amurensis Lectin II and Elderberry bark lectin, or Nacetylgalactosamine recognising lectins such as Vicia Villosa Lectin and Dolichos Biflorus Agglutinin, to capture aberrantly glycosylated basigin, similar to the aberrantly fucosylated alpha fetoprotein (AFP-LC3) capture assay developed by FUJIFILM Wako Diagnostics U.S.A (http://www.wakodiagnostics.com/afpl3test.html, 2019; Block et. al. 2005; Meany and Chan 2011; Adamczyk et. al. 2012; Pinho and Reis 2015) (Chapter 1: Figure 1.16).

Sialylated glycoproteins were detected with fluorescein-conjugated Elderberry bark lectin (EBL; also known as Sambucus nigra lectin) in whole-cell lysates obtained from the panel of prostate and breast cancer cell lines (Figure 5.10a, i). Few glycoproteins were detected with fluorescein-conjugated Maackia amurensis lectin I (MAL I), which recognises galactose, sialic acid at the 3 position of galactose, and lactose (Figure 5.10a, ii). Next, lectin blotting was used to detect a human recombinant glycosylated basigin standard using EBL and MAL I lectins (Figure 5.10b). 
a.

Whole cell lysate
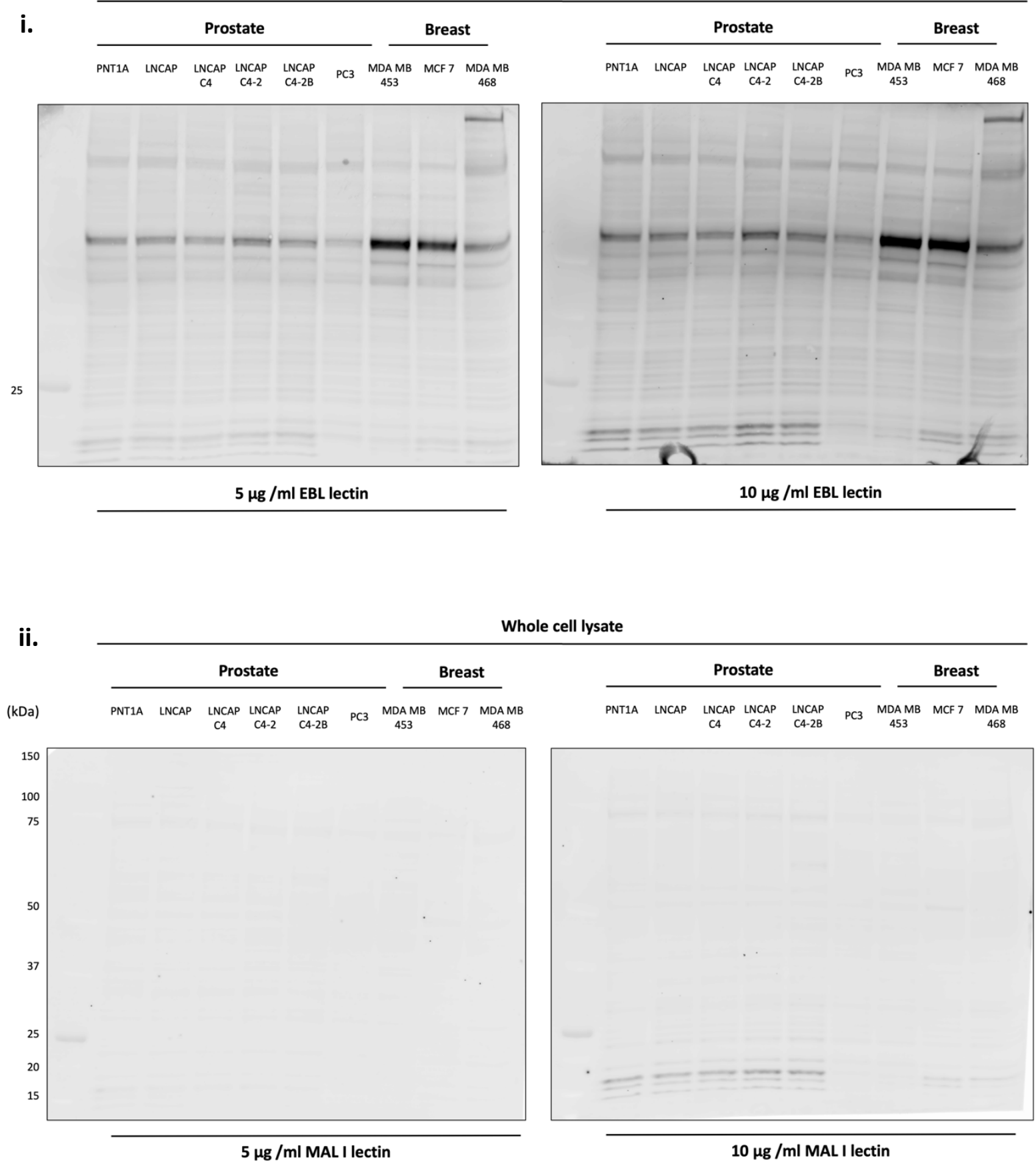


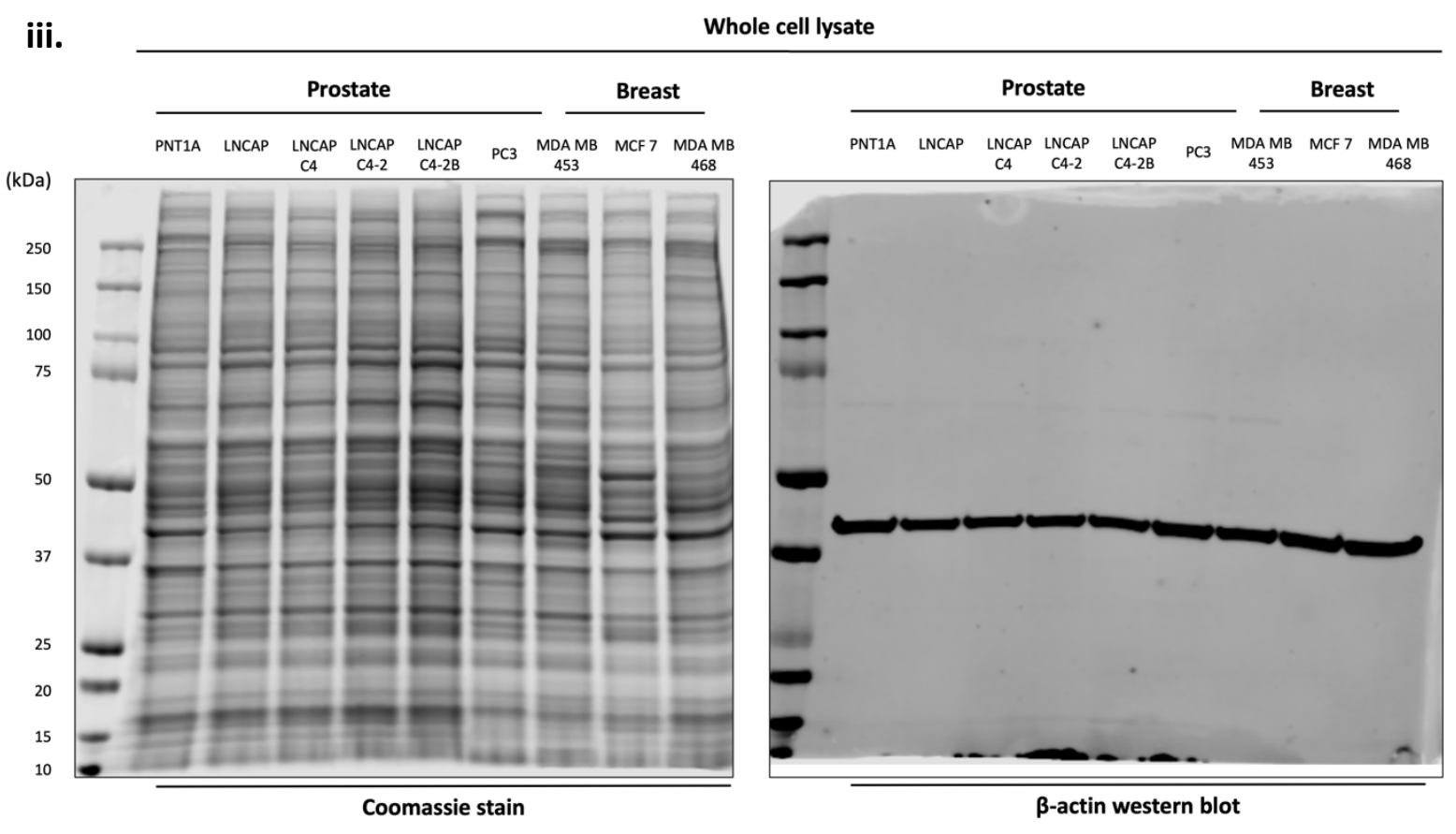

b.

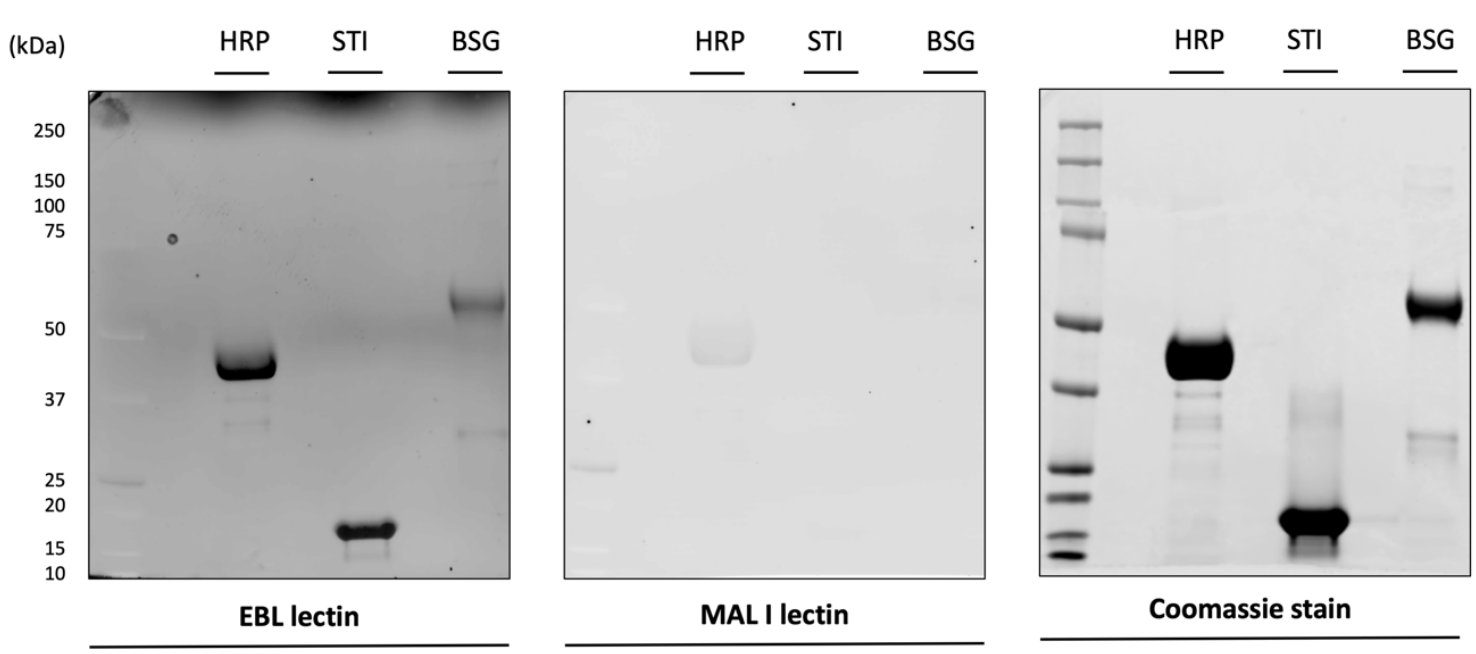

Figure 5.10 Detection of glycoproteins using a lectin blot. (a) Whole-cell lysates (30 $\mathrm{g}$ /lane) prepared from the prostate and breast cancer cell lines were resolved by $1 D$ western blot and probed with $5 \mu \mathrm{g} / \mathrm{ml}$ or $10 \mu \mathrm{g} / \mathrm{ml}$ of Elderberry bark lectin (EBL) to detect sialic acid (i) or Maackia amurensis lectin I (MALI) to detect galactose, sialic acid at the 3 position of galactose, and lactose (ii). Equal loading was confirmed by total protein Coomassie stain and by using actin as a loading control (iii). (b) Glycosylated recombinant proteins (10 $\mu \mathrm{g}$ ) horseradish peroxidase (HRP), soybean trypsin inhibitor (STI) and human basigin (BSG) produced in Sf9, Baculovirus cells were resolved by $1 D$ western blot and probed with $10 \mu \mathrm{g} / \mathrm{ml}$ of EBL or MAL I lectins. Equal loading was confirmed by total protein Coomassie stain. 


\subsubsection{Immunoprecipitation of PSA}

Next, we endeavoured to immunoprecipitate basigin from whole-cell lysates prepared from our panel of cell lines for lectin blot analysis (Figure 5.7a). We selected PSA to optimise immunoprecipitation protocols due to the availability of optimised antibodies and lectin glycoprofiling studies (Tkac et. al. 2019). We could not detect PSA using an anti-PSA antibody (Abcam) (Figure 5.11a) or biotin-conjugated anti-PSA antibody (Abcam) (Figure 5.11b) in whole-cell lysates prepared from the prostate cancer cell lines. Non-specific bands were detected in lysates prepared from the prostate cell lines and a selection of breast cancer cell lines, which do not express PSA (Figure 5.11b, white arrowheads). The expression of PSA in prostate cancer cell lines has previously been confirmed by immunohistochemistry (Chapter 2; Figure 2.3d), and extensively documented in the literature (Chapter 1: Table 2.1a). Therefore, the negative immunoblots were attributed to low immunoreactivity of the antibodies.

We used the direct immunoadsorption approach (Peter et. al. 1999) to pull down PSA from whole-cell lysates obtained from the LNCAP and LNCAP C4-2B prostate cancer cell lines, which express PSA, and the PC3 prostate cancer cell line, which does not express PSA (Figure 5.1b). Generally, streptavidin magnetic beads were blocked with an anti-PSA-biotin antibody (Abcam), followed by addition of the whole-cell lysates for immunocapture of PSA. Captured PSA was eluted by incubation in 2\% SDS and 1x LDS loading buffer (NuPAGE) for 2 hours at room temperature. Elutes were resolved by $1 \mathrm{D}$ western blot followed by detection of PSA using an anti-PSA primary antibody (Abcam). Two faint bands were observed on the anti-PSA blot following immunoprecipitation, which corresponded to the denatured heavy (50 kDa) and light (25 kDa) chains of the biotinylated anti-PSA antibody (Lal et. al. 2005) (Figure 5.11c, black arrowheads). This is a common problem with immunoprecipitation protocols and would mask visualisation of immunoprecipitated basigin in future experiments, as its most abundant form resolves around $50 \mathrm{kDa}$ (Figure 5.9a, i). 
a.

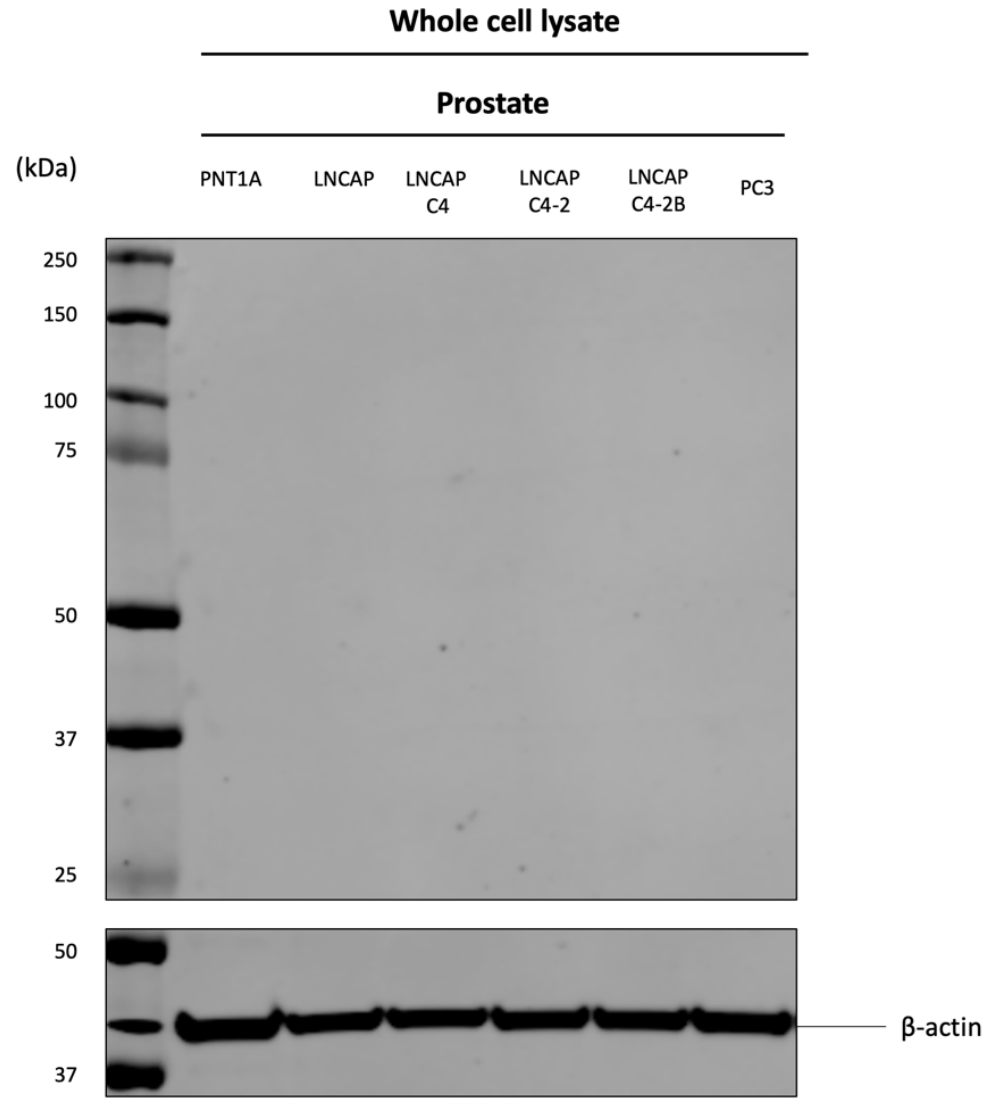

b.

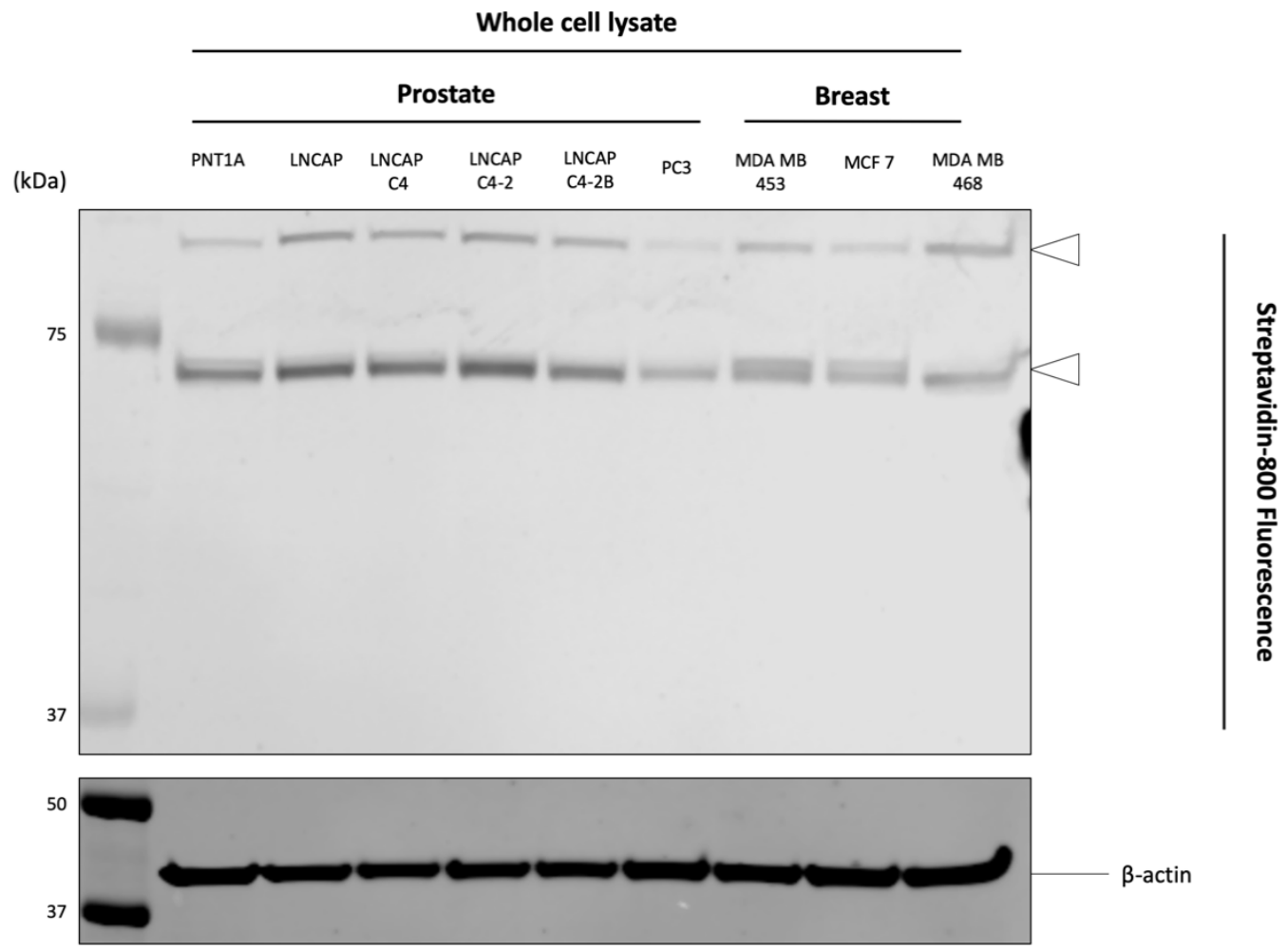


c.

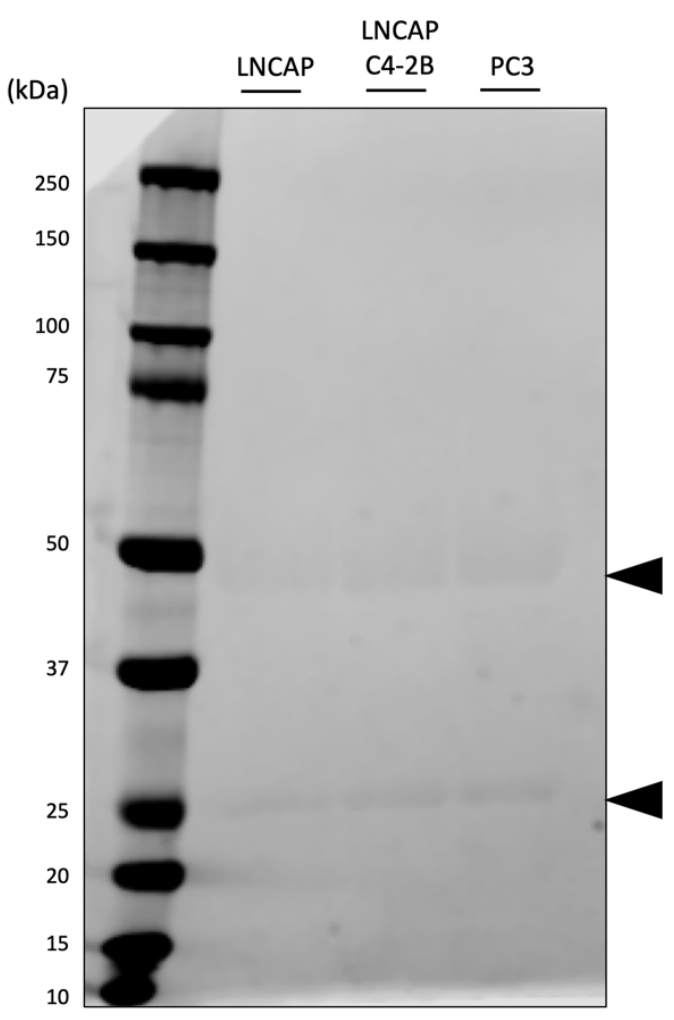

Figure 5.11: Western blot analysis of PSA. (a) Detection of PSA in whole-cell lysates prepared from the prostate and breast cancer cell lines using an Abcam antibody. (b) Detection of PSA in whole-cell lysates prepared from the prostate and breast cancer cell lines using an Abcam biotin-conjugated antibody, which was detected using streptavidin-800 fluorescence. Bands of non-specific streptavidin-800 fluorescence were observed (white arrowheads). (c) Wholecell lysates prepared from the LNCAP, LNCAP C4-2B, and PC3 prostate cancer cell lines were immunoprecipitated using an Abcam biotin-conjugated anti-PSA antibody and streptavidincoupled beads. Precipitates were resolved by gel electrophoresis and analysed by anti-PSA immunoblot. Denatured heavy (50 kDa) and light (25 kDa) chains of the biotinylated anti-PSA antibody were observed (black arrowheads). (30 $\mu \mathrm{g} /$ lane).

We faced challenges obtaining suitable anti-PSA antibodies to optimise the immunoprecipitation protocol. The anti-PSA antibody has now been shown to have variable efficiency in LNCAP cell line lysates (https://www.abcam.com/prostate-specific-antigenantibody-ep1588y-ab76113.html, 2019) and the biotin-conjugated anti-PSA antibody has been discontinued (https://www.abcam.com/prostate-specific-antigen-antibody-5a6-biotin- 
ab182031.html, 2019). Therefore, we were unable to conduct immunoprecipitation of basigin with an optimised protocol to conduct lectin based glycoprofiling within the timeframe of this project.

\subsection{Discussion}

Previously, we assessed the complex changes in glycoprotein populations and azidosugar incorporation in membrane enriched extracts from prostate cancer cell lines using 1D and 2D-gel electrophoresis (Chapter 4). Using 1D gel electrophoresis analysis, an increase in the sialyation $\left(\mathrm{Ac}_{4} \mathrm{ManNAz}\right)$ and $\mathrm{N}$-acetylgalactosamine glycosylation of proteins correlated with an increase in the metastatic potential of panels of prostate (Chapter 4: Figure 4.2b, i and c, i), breast (Chapter 4: Figure 4.3b, i and c, i) and pancreatic (Chapter 4: Figure 4.4b) cancer cell lines. Using 2D gel electrophoresis analysis, eight spots exhibiting an increase in both $\mathrm{Ac}_{4} \mathrm{ManNAz}$ and $\mathrm{Ac}_{4} \mathrm{GalNAz}$ labelling were observed (Figure $4.6 \mathrm{~b}$ and $\mathrm{c}$, white arrowheads), despite the expression of proteins within the eight spots, appearing similar. This suggested that differential glycosylation may be a more sensitive and specific marker for monitoring cancer progression, than increases in total protein expression alone. In this study, we used mass spectrometry to identify differentially azidosugar labelled proteins across the LNCAP in vitro model of prostate cancer progression, which it was anticipated might lead to the discovery of new prognostic biomarkers.

LNCAP cell lines (Chapter 2: Table 2.1a) were treated with $\mathrm{Ac}_{4} \mathrm{ManNAz}$ or $\mathrm{Ac}_{4} \mathrm{GalNAz}$. Metabolically labelled cell-surface glycoproteins were extracted, covalently tagged with an alkyne-biotin bifunctional-biotin probe, and the resulting biotin-tagged azidosugar-labelled proteins enriched using streptavidin-coupled beads. Proteins were then identified using liquid chromatography-tandem mass spectrometry.

We identified a subclass of fifteen differentially glycosylated proteins (Figure $5.5 \mathrm{~d}$ ). Within this subclass, we identified proteins known to be involved in cancer progression, such as epidermal growth factor (Chang et al. 2015) and lysosome-associated membrane protein 1 
(Okato et al. 2016), and proteins currently being investigated as biomarkers for monitoring prostate cancer progression such as filamin B (Ravipaty et al. 2017). Most interesting was the identification of two proteins, basigin (CD147) and $45 \mathrm{kDa}$ calcium-binding protein, which showed a general increase in $\mathrm{Ac}_{4} \mathrm{ManNAz}$ and $\mathrm{Ac}_{4} \mathrm{GalNAz}$ labelling, despite their total expression decreasing across the panel of prostate cancer cell lines (Figure 5.9). Decreased expression of basigin (Figure 5.9a, ii) across the panel of prostate cancer cell lines was observed in whole-cell lysates, validating the relative expression observed using liquid chromatography-mass spectrometry (Figure 5.7a, ii).

Basigin is a transmembrane glycoprotein, which activates monocarboxylate transporters, facilities Plasmodium falciparum erythrocyte invasion during the pathogenesis of malaria, and induces the production of metalloproteases and vascular endothelial growth factor (VEGF) in cancer cells during tumour invasion (Muramatsu 2016). Decreased expression of membrane-bound basigin has been reported using immunohistochemistry (IHC) in prostate cancer tissues in comparison to non-malignant prostate tissue and proposed as a predictor of progression and recurrence after prostatectomy (Bauman et. al. 2015). Basigin has been shown to be highly expressed in normal prostate tissue using IHC, with expression levels decreasing in prostatic intraepithelial neoplasia (PIN) lesions, reducing further in prostate cancer (Pértega-Gomes et. al. 2011). Furthermore, decreased expression of basigin has been strongly associated with ERG (ETS (erythroblast transformation-specific)-related gene) positive prostate cancer (Grupp et. al. 2013), a subclassification characterised by the presence of the TMPRSS2 (transmembrane protease, serine 2) and ERG fusion gene, which are detected in approximately 50\% of prostate cancers (Ayala et. al. 2015). In contrast, elevated basigin expression in prostate cancer tissue, in comparison to benign prostatic hyperplasia, has been observed and correlated with poor prognosis (Han et. al. 2009). Metaanalyses and systematic reviews studying the association of basigin expression with prostate cancer have confirmed the conflicting results in the literature, reporting both increases and decreases in basigin expression (Peng et. al. 2016). However, little is known about the prognostic value of differentially glycosylated basigin in prostate cancer. Similarly, neither the glycosylation or total expression of $45 \mathrm{kDa}$ calcium-binding protein has been implicated in prostate cancer. 
Bioorthogonal azide-based chemical reporters have been combined with mass spectrometry analysis to capture and identify glycosylated proteins, which are overexpressed in small lung cell carcinoma tissue (Pan et. al. 2012), and prostate cancer tissue (Spiciarich et. al. 2017). In this study, we challenge the traditional approach to biomarker discovery, commonly based on identifying proteins that are overexpressed in cancer, instead, giving deliberate attention to proteins exhibiting an increase in glycosylation, despite showing a decrease in total protein expression across an in vitro model of prostate cancer progression. This was based on the observation of eight protein spots on 2D gel western blot, exhibiting an increase in both $\mathrm{Ac}_{4} \mathrm{ManNAz}$ and $\mathrm{Ac}_{4}$ GalNAz labelling, which correlated with an increase in the metastatic potential of two prostate cancer cell lines, despite the total expression of proteins within the eight spots, appearing similar (Chapter 4: Figure 4.6b and c, white arrowheads). Overall, our data suggest that differential glycosylation may be a more sensitive and specific marker for monitoring cancer progression, than increases in total protein expression.

\subsection{Materials and Methods}

\subsubsection{Cell lines}

The PNT1A, LNCAP, and LNCAP C4-2B human prostate cell lines were obtained from the Cancer Research UK Cambridge Institute Biorepository, based on stocks made available by Joe Kuo. The LNCAP C4, LNCAP C4-2, and PC3 human prostate cancer cell lines were purchased from ATCC. All prostate cell lines were cultured in RPMI with L-glutamine $\left(\mathrm{Gibco}^{\circledR}\right.$, Invitrogen ${ }^{\mathrm{TM}}$ ) and 10\% Foetal bovine serum (FBS Gold, PAA Laboratories).

The MDA MB 453, MCF-7, MDA-MB-468, and MDA MB 231 human breast cancer cell lines were obtained from the Cancer Research UK Cambridge Institute Biorepository, based on stocks made available by Joe Kuo. All breast cell lines were cultured in DMEM with Lglutamine (Gibco ${ }^{\circledR}$, Invitrogen ${ }^{\mathrm{TM}}$ ) and 10\% Foetal bovine serum (FBS Gold, PAA Laboratories). 
Cell lines were genotyped and authenticated by Short Tandem Repeat (STR) DNA profiling and comparison with available databases from ATCC or DSMZ. All cell lines tested negative for Mycoplasma using the MycoProbe ${ }^{\mathrm{TM}}$ Kit (R\&D Systems).

\subsubsection{Tissue culture}

Cell lines were maintained in Nunc ${ }^{T M} 175 \mathrm{~cm}^{2}$ flasks (Thermo Fisher Scientific ${ }^{\mathrm{TM}}$ ) in $5 \% \mathrm{CO}_{2}$, in $37^{\circ} \mathrm{C}$ humidified incubators, and cultured to $80 \%$ confluence. Briefly, cells were washed once with warm Phosphate Buffered Saline (PBS; 20 ml/flask; $8 \mathrm{~g} \mathrm{NaCl}, 0.2 \mathrm{~g} \mathrm{KCl}, 1.44 \mathrm{~g}$ $\mathrm{Na}_{2} \mathrm{HPO}_{4}, 0.24 \mathrm{~g} \mathrm{KH}_{2} \mathrm{PO}_{4}$ per litre, $\mathrm{pH} 7.4$ ), and trypsinised. Prostate cancer cell lines were trypsinised for 4 minutes at $37^{\circ} \mathrm{C}$, apart from the PC3 cell line, which was trypsinised for 7 minutes. Breast cancer cell lines were trypsinsed for 7 minutes at $37^{\circ} \mathrm{C}$. Trypsinised cells were added to equal volumes of media, centrifuged at $700 \times \mathrm{g}$ for 4 minutes $\left(4^{\circ} \mathrm{C}\right)$, and resuspended in fresh media. Viable cell counts were obtained by Trypan blue dye exclusion using a LUNA ${ }^{\mathrm{TM}}$ Automated Cell Counter (Logos Biosystems), and cells seeded at $3 \times 10^{4}$ cells $/ \mathrm{cm}^{2}$.

\subsubsection{Bioorthogonal metabolic labelling of cell lines in vitro for mass spectrometry}

$\mathrm{N}$-azidoacetylmannosamine ( $\mathrm{Ac}_{4} \mathrm{ManNAz}$ ), N-azidoacetylgalactosamine ( $\mathrm{Ac}_{4} \mathrm{GalNAz}$ ) and TMDIBO-PEG 3 -biotin were synthesised by Henning Stöckmann, Department of Chemistry.

LNCAP, LNCAP C4, LNCAP C4-2, and LNCAP C4-2B cell lines were seeded at $1.75 \times 10^{4}$ cells $/ \mathrm{cm}^{2}$ in Nunc ${ }^{\mathrm{TM}} 175 \mathrm{~cm}^{2}$ flasks (Thermo Fisher Scientific ${ }^{\mathrm{TM}}$ ) and maintained at $5 \% \mathrm{CO}_{2}$, in $37^{\circ} \mathrm{C}$ humidified incubators for 3 days before metabolic labelling experiments. Flasks were then washed once with $20 \mathrm{ml}$ warm Phosphate Buffered Saline (PBS; $8 \mathrm{~g} \mathrm{NaCl}, 0.2 \mathrm{~g} \mathrm{KCl}, 1.44$ $\mathrm{g} \mathrm{Na}_{2} \mathrm{HPO}_{4}, 0.24 \mathrm{~g} \mathrm{KH}_{2} \mathrm{PO}_{4}$ per litre, $\mathrm{pH} 7.4$ ), and cells trypsinised for 4 minutes at $37^{\circ} \mathrm{C}$. Trypsinised cells were added to equal volumes of media, centrifuged at $700 \times \mathrm{g}$ for 4 minutes $\left(4^{\circ} \mathrm{C}\right)$ and resuspended in fresh media. Viable cell counts were obtained by Trypan blue dye exclusion using a LUNA ${ }^{\mathrm{TM}}$ Automated Cell Counter (Logos Biosystems), and cells seeded at $3 \mathrm{x}$ 
$10^{4}$ cells $/ \mathrm{cm}^{2}$ in Nunc ${ }^{\mathrm{TM}} 175 \mathrm{~cm}^{2}$ flasks (Thermo Fisher Scientific ${ }^{\mathrm{TM}}$ ). Plates were incubated in $5 \% \mathrm{CO}_{2}, 37^{\circ} \mathrm{C}$ for 24 hours. After 24 hours, the media was replaced with fresh media only (0.1\% DMSO, vehicle control) or fresh media supplemented with $50 \mu \mathrm{M} \mathrm{Ac_{4 } M a n N A z \text { or }}$ $\mathrm{Ac}_{4} \mathrm{GalNAz}$, using a surface:area volume ratio equivalent to a 6 well plate $(52.5 \mathrm{ml})$. Cells were incubated in $5 \% \mathrm{CO}_{2}$ at $37^{\circ} \mathrm{C}$ for 24 hours. After 24 hours, cells were harvested by trypsinisation and counted, as described previously.

$5 \times 10^{6}$ cells were transferred into Nostick ${ }^{\circledR}$ hydrophobic microcentrifuge tubes (Scientific Specialties, Inc.), washed twice in $2 \mathrm{ml}$ ice-cold PBS, and fractionated using a Qproteome ${ }^{\circledR}$ Cell Compartment kit (Qiagen), according to the manufacturer's protocol. Briefly, washed cells pellets incubated in $1 \mathrm{ml}$ Extraction Buffer CE1 for 10 minutes at $4^{\circ} \mathrm{C}$ on an end-overend shaker $(30 \mathrm{rpm})$. Lysates were centrifuged at $1000 \mathrm{x}$ for 10 minutes at $4^{\circ} \mathrm{C}$. The supernatant, primarily containing cytosolic proteins, was transferred into a fresh Nostick ${ }^{\circledR}$ hydrophobic microcentrifuge tubes (Scientific Specialties, Inc.) and stored at $-20^{\circ} \mathrm{C}$. The remaining pellet was resuspended in $1 \mathrm{ml}$ Extraction Buffer CE2 and incubated for 30 minutes at $4^{\circ} \mathrm{C}$ on an end-over-end shaker (30 rpm). Lysates were centrifuged at $6000 \mathrm{x}$ for 10 minutes at $4^{\circ} \mathrm{C}$. The supernatant, primarily containing membrane proteins, was transferred into a fresh Nostick ${ }^{\circledR}$ hydrophobic microcentrifuge tubes (Scientific Specialties, Inc.). Samples were stored at $-20^{\circ} \mathrm{C}$.

Four volumes of ice-cold acetone were added to the membrane protein-enriched fractions and incubated for 1 hour on ice. Precipitated proteins were pelleted by centrifugation at 12 , $000 \times \mathrm{g}$ for 10 minutes $\left(4^{\circ} \mathrm{C}\right)$. The supernatant was discarded and the protein pellets air-dried for 15 minutes. Dried proteins pellets were resuspended in $100 \mu$ l Tris-HCL buffer $(50 \mathrm{mM}, \mathrm{pH}$ $8,0.5 \%$ SDS). Residual acetone was removed by the sequential addition of $600 \mu$ methanol, $150 \mu \mathrm{l}$ chloroform, and $400 \mu \mathrm{l}$ Milli-Q $18.2 \Omega$ water, with vortexing. After centrifugation at $18,000 \times \mathrm{g}$ for 10 minutes $\left(4^{\circ} \mathrm{C}\right)$, the upper aqueous phase was removed and the precipitated proteins washed twice with $450 \mu$ methanol. The pellets were air-dried for a minimum of 15 minutes and resuspended in $100 \mu \mathrm{l}$ Tris-HCL buffer (50 mM, pH 8, 0.5\% SDS). The total protein concentration was determined using the DC ${ }^{\text {TM }}$ Protein Assay (Bio-Rad), using BSA as a standard. 
Up to $200 \mu \mathrm{g}$ of metabolically labelled protein was chemoselectively reacted with alkynebiotin (Life Technologies) using the Click iT Protein Reaction Kit (Life Technologies), according to the manufacturer's protocol. Residual reactants were removed by methanol/chloroform/Milli-Q $18.2 \Omega$ water precipitation, as described previously. Protein pellets were dried for a minimum of 15 minutes, and resuspended in $200 \mu$ l Tris HCL buffer ( $50 \mathrm{mM}, \mathrm{pH} 8,0.5 \%$ SDS) and sonicated for 30 seconds ( 3 cycles of 10 seconds ON and 5 seconds OFF) using the Biorupter ${ }^{\circledR}$ Plus (Diagenode). The total protein concentration was determined using the DC ${ }^{\mathrm{TM}}$ Protein Assay (BIO-RAD), using BSA as a standard. Samples were stored at $-20^{\circ} \mathrm{C}$.

\subsubsection{Enrichment of metabolically labelled glycoproteins using Streptavidin Mag Sepharose beads}

$35 \mu \mathrm{g}$ of metabolically labelled protein reacted with alkyne-biotin were incubated with $50 \mu \mathrm{l}$ of Streptavidin Mag Sepharose beads (GE Healthcare), in binding buffer (Tris Buffered Saline (TBS), 50mM Tris-HCL, $150 \mathrm{Mm} \mathrm{NaCl}, \mathrm{pH} 7.5$ ), for 2 hours at room temperature with endover-end mixing (30 rpm). Beads were washed with wash buffer (3x; TBS, 2M Urea, pH 7.5) and transferred to fresh Nostick ${ }^{\circledR}$ hydrophobic microcentrifuge tubes (Scientific Specialties, Inc.). Biotinylated proteins were eluted from the beads with $50 \mu$ l elution buffer ( $2 \%$ SDS) at $100^{\circ} \mathrm{C}$, for 10 minutes with agitation (1000 rpm). Based on Bradford protein quantification analysis (Bio-Rad) of eluted samples, the average amount of eluted proteins was between 11-13 $\mu \mathrm{g}$ of protein.

Elutions $(50 \mu \mathrm{l}$ ) were loaded onto on precast 4-12\% 1mm Bis-Tris gels (Thermo Fisher Scientific $^{\top M}$ ). Gels were resolved in 1x NuPAGE MOPS buffer (Invitrogen) (30 min 60 V, 120 min $120 \mathrm{~V}$ ) and silver stained (Pierce Kit). Silver stained gels were stored in acetic acid (5\%) and submitted to the Cambridge Centre for Proteomics, Department of Biochemistry, for LCMS analysis. 


\subsubsection{Sample preparation for LCMS/MS}

Each gel lane was cut into 10 bands and was transferred into a 96-well PCR plate. Bands were cut into $1 \mathrm{~mm}^{2}$ pieces, destained, reduced (DTT) and alkylated (iodoacetamide) and subjected to enzymatic digestion with sequencing grade trypsin (Promega, Madison, WI, USA) overnight at $37^{\circ} \mathrm{C}$. After digestion, the supernatant was pipetted into a sample vial and loaded onto an autosampler for automated LC-MS/MS analysis.

\subsubsection{LC-MS/MS}

All LC-MS/MS experiments were performed using a Dionex Ultimate 3000 RSLC nanoUPLC (Thermo Fisher Scientific In, Waltham, MA, USA) system and a Q Exactive Orbitrap mass spectrometer (Thermo Fisher Scientific Inc, Waltham, MA, USA). Separation of peptides was performed by reverse-phase chromatography at a flow rate of $300 \mathrm{~nL} / \mathrm{min}$ and a Thermo Scientific reverse-phase nano Easy-spray column (Thermo Scientific PepMap C18, $2 \mu \mathrm{m}$ particle size, 100A pore size, $75 \mu \mathrm{m}$ i.d. $\times 50 \mathrm{~cm}$ length). Peptides were loaded onto a precolumn (Thermo Scientific PepMap 100 C18, $5 \mu \mathrm{m}$ particle size, 100A pore size, $300 \mu \mathrm{m}$ i.d. x $5 \mathrm{~mm}$ length) from the Ultimate 3000 autosampler with $0.1 \%$ formic acid for 3 minutes at a flow rate of $10 \mu \mathrm{L} / \mathrm{min}$. After this period, the column valve was switched to allow elution of peptides from the pre-column onto the analytical column. Solvent $A$ was water $+0.1 \%$ formic acid and solvent B was $80 \%$ acetonitrile, $20 \%$ water $+0.1 \%$ formic acid. The linear gradient employed was $2-40 \%$ B in 30 minutes. Further wash and equilibration steps gave a total run time of 60 minutes.

The LC eluant was sprayed into the mass spectrometer using an Easy-Spray source (Thermo Fisher Scientific Inc.). All $\mathrm{m} / \mathrm{z}$ values of eluting ions were measured in an Orbitrap mass analyzer, set at a resolution of 70000 and was scanned between $\mathrm{m} / \mathrm{z}$ 380-1500. Datadependent scans (Top 20) were employed to automatically isolate and generate fragment ions by higher-energy collisional dissociation (HCD, NCE:25\%) in the HCD collision cell and measurement of the resulting fragment ions was performed in the Orbitrap analyser, set at a resolution of 17500 . Singly charged ions and ions with unassigned charge states were 
excluded from selection for MS/MS and a dynamic exclusion window of 20 seconds was employed.

\subsubsection{Database searching}

Post-run, all MS/MS data were converted to mgf files and the files were then submitted to the Mascot search algorithm (Matrix Science, London UK, version 2.6.0) and searched against the UniProt human database (93609 sequences; 37041084 residues) and common contaminant sequences containing non-specific proteins such as keratins and trypsin (115 sequences, 38274 residues). Variable modifications of oxidation (M) and deamidation (NQ) were applied as well as a fixed modification of carbamidomethyl (C). The peptide and fragment mass tolerances were set to $20 \mathrm{ppm}$ and $0.1 \mathrm{Da}$, respectively. A significance threshold value of $p<0.05$ and a peptide cut-off score of 20 were also applied. All data (DAT files) were then imported into the Scaffold program (Version_4.5.4, Proteome Software Inc, Portland, OR) and spectral counts for each sample exported into an Excel spreadsheet for quantitative analysis.

\subsubsection{Quantitative analysis of protein levels}

A label-free approach based on spectral counts was used to estimate the relative abundance of proteins identified by LCMS (Figure 5.12).

$$
(N A S F)_{k}=\frac{\left(\frac{S p C}{M W}\right) k}{\sum_{i=1}^{N}\left(\frac{S p C}{M W}\right)_{i}}
$$

Figure 5.12: Calculation of Normalised Spectral Abundance Factor (NSAF). Normalised Spectral Abundance Factors (NSAFs) were calculated based on the notion that increases in 
the abundance of a protein are reflected in increases in proteolytically released peptides. Abbreviations: Protein (k), spectral count $(S p C)$, molecular weight $(M W)$, number of proteins in the gel lane (N). (Liu et. al. 2004; Zybailov et. al. 2006; Byrum et. al. 2013). (Figure taken from Byrum et. al. 2013).

The entire spectral count data set was shifted by +0.1 , to enable analysis of proteins with zero spectral counts, and log-transformed. Normalised Spectral Abundance Factors (NSAFs) for each protein within a given lane were then calculated. For a given protein (k), the number of spectral counts $(\mathrm{SpC})$ was divided by its molecular weight (MW) and divided again by the sum of SpC/MW for all proteins (N) within a given lane (Liu et. al. 2004; Zybailov et. al. 2006; Byrum et. al. 2013).

\subsubsection{Preparation of whole-cell lysates and conditioned media}

Prostate and breast cancer cell lines were cultured in phenol red-free media and counted as described previously, seeded at $3 \times 10^{4}$ cells $/ \mathrm{cm}^{2}$ in $100 \mathrm{~mm}$ cell culture dishes $(15 \mathrm{ml}$ media/dish) (Corning), and incubated in $5 \% \mathrm{CO}_{2}$, at $37^{\circ} \mathrm{C}$ for 24 hours. After 24 hours, dishes were washed twice with warmed PBS and replaced with fresh phenol red-free, serum-free media (15 ml media /dish).

After 24 hours, media from each dish was transferred in to $15 \mathrm{ml}$ Falcon tubes and centrifuged at $1000 \mathrm{xg}$ for 10 minutes to pellet cells from the conditioned media. The supernatants were transferred to Amicon Ultra-15 centrifugal filter tubes (Merck Millipore; 3 kDa MWCO) and centrifuged at 3500 rpm for 30 minutes. Centrifugation was repeated, concentrating the supernatants to $200-250 \mu$ volume. Concentrated conditioned media were transferred to fresh Nostick ${ }^{\circledR}$ hydrophobic microcentrifuge tubes (Scientific Specialties, Inc.).

In parallel, the cells from which the conditioned media was obtained were harvested by trypsinisation and centrifugation, as described previously. Cell viability was measured by 
Trypan blue dye exclusion using a LUNA ${ }^{\mathrm{TM}}$ Automated Cell Counter (Logos Biosystems). Cells were incubated with $1 \mathrm{ml}$ RIPA buffer (Thermo Fisher Scientific ${ }^{\top M}$ ) supplemented with $1 \mathrm{x}$ Halt $^{\mathrm{TM}}$ Protease and Phosphatase Inhibitor Cocktail (Thermo Fisher Scientific ${ }^{\mathrm{TM}}$ ) and $1 \times 0.5 \mathrm{M}$ EDTA solution (Thermo Fisher Scientific ${ }^{\mathrm{TM}}$ ), for 30 minutes at $4^{\circ} \mathrm{C}$ on an end-over-end shaker (30 rpm). Lysates were centrifuged at maximum speed $(21,000 \times \mathrm{g})$ for 20 minutes at $4^{\circ} \mathrm{C}$ and the supernatant transferred into a fresh Nostick ${ }^{\circledR}$ hydrophobic microcentrifuge tubes (Scientific Specialties, Inc.).

The total protein concentrations were determined using the $\mathrm{DC}^{\mathrm{TM}}$ Protein Assay (BIO-RAD), using BSA as a standard. Whole-cell lysates and conditioned media fractions were stored at $80^{\circ} \mathrm{C}$.

\subsubsection{Western blot}

Whole-cell lysates or conditioned media were resolved on precast 4-12\% $1 \mathrm{~mm}$ Bis-Tris gels (Thermo Fisher Scientific ${ }^{\mathrm{TM}}$ ) in 1x NuPAGE MOPS buffer (Invitrogen) (10 $\mu \mathrm{g} /$ lane, $30 \mathrm{~min} 60$ V, $120 \mathrm{~min} 120 \mathrm{~V}$ ), and 1D gels transferred onto nitrocellulose membranes using an iBlot 2 (Life). Blots were incubated in $1 x$ Odyssey ${ }^{\circledR}$ PBS block buffer (LI-COR) for 1 hour, followed by an overnight incubation at $4{ }^{\circ} \mathrm{C}$ with the following primary antibodies in PBS blocking buffer (0.1\% tween, $0.1 \%$ SDS): rabbit polyclonal anti-basigin $(1 \mu \mathrm{g} / \mathrm{ml} ;$ Abcam), rabbit monoclonal anti-PSA (1:10,000; Abcam), mouse monoclonal biotin conjugated anti-PSA (1:5000; Abcam), rabbit monoclonal anti-vimentin (1:1000; Cell Signalling Technologies), rabbit monoclonal anti- $\beta$-tubulin (1:1000; Cell Signalling Technologies), and rabbit monoclonal anti- $\beta$-actin (1:1000; Cell Signalling Technologies)

After overnight incubation, primary antibodies were removed, and the blots washed 3 times (PBS + Tween (0.1\%), 5 minutes). Blots were incubated for 1 hour at room temperature with a goat anti-rabbit IRDye 680 RD secondary antibody (1:15, 000; LI-COR) in PBS blocking buffer ( $0.1 \%$ tween, $0.1 \%$ SDS). Blots treated with rabbit antibodies were washed 3 times in PBS (0.1\% Tween) for 5 minutes, once in PBS for 5 minutes, and imaged on an Odyssey CLx Infrared Imaging System (LI-COR Biosciences). Blots treated with mouse monoclonal biotin 
conjugated anti-PSA (1:5000; Abcam) were washed 3 times in PBS (0.1\% Tween) for 5 minutes, incubated in Streptavidin-800 (1:5000; LI-COR) for 15 minutes, washed 6 times in PBS (0.05\% Tween) for 5 minutes, once in PBS for 5 minutes, and imaged on an Odyssey CLx Infrared Imaging System (LI-COR Biosciences).

\subsubsection{Lectin blotting}

Whole-cell lysates, recombinant glycosylated basigin produced in Sf9 Baculovirus cells (Prospec-Tany Technogene), horseradish peroxidase (HRP) (Pierce) and soybean trypsin inhibitor (STI) (Pierce), were resolved on precast 4-12\% 1mm Bis-Tris gels (Thermo Fisher

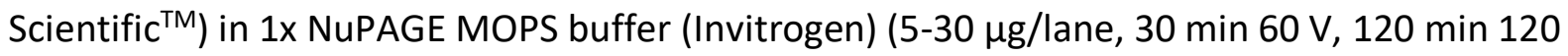
V), and 1D gels transferred onto nitrocellulose membranes using an iBlot 2 (Life). Membranes were blocked in 3\% Bovine Serum Albumin (BSA) (Sigma) in PBST (0.05\% Tween) overnight at $4^{\circ} \mathrm{C}$. After overnight incubation, the BSA block buffer was removed, and the blots washed 3 times (PBS + Tween (0.05\%) for 10 minutes). Blots were incubated for 30 minutes at room temperature with 5 or $10 \mu \mathrm{g} / \mathrm{ml}$ of fluorescein labelled Elderberry Bark Lectin (EBL; Vector Labs) or fluorescein labelled maackia amurensis lectin I (MAL I; Vector Labs). Blots were washed 6 times (PBS + Tween (0.05\%), 5 minutes), and once in PBS for 5 minutes. Cy2 fluorescence (489 nm maximum excitation wavelength, $506 \mathrm{~nm}$ maximum emission wavelength) was imaged using a Typhoon scanner (GE Healthcare)

\subsubsection{Immunoprecipitation of PSA}

$0.4 \mathrm{mg} / \mathrm{ml}$ of mouse monoclonal, biotin conjugated, anti-PSA antibody (Abcam) was incubated with $50 \mu$ l of Streptavidin Mag Sepharose beads (GE Healthcare), in binding buffer (Tris Buffered Saline (TBS), 50mM Tris-HCL, $150 \mathrm{Mm} \mathrm{NaCl}, \mathrm{pH} 7.5$ ), for 30 minutes at room temperature with end-over-end mixing (30 rpm). Beads were washed with wash buffer ( $3 \mathrm{x}$; TBS, $2 \mathrm{M}$ Urea, $\mathrm{pH} 7.5$ ) and incubated with $35 \mu \mathrm{g}$ of unlabelled membrane-enriched protein lysates diluted in binding buffer, for 2 hours at room temperature. Captured proteins were eluted by incubation in 2\% SDS and 1x LDS loading buffer (NuPAGE) for 2 hours at room 
temperature. Elutions were resolved on precast 4-12\% 1mm Bis-Tris gels (Thermo Fisher Scientific $\left.{ }^{\top M}\right)$ ) in 1x NuPAGE MOPS buffer (Invitrogen) (30 min $60 \mathrm{~V}, 120 \mathrm{~min} 120 \mathrm{~V}$ ), and 1D gels transferred onto nitrocellulose membranes using an iBlot 2 (Life). Blots were incubated in 1x Odyssey ${ }^{\circledR}$ PBS block buffer (LI-COR) for 1 hour, followed by an overnight incubation at $4^{\circ} \mathrm{C}$ with a rabbit monoclonal anti-PSA antibody $(1: 10,000 ; A b c a m)$ in PBS blocking buffer (0.1\% tween, $0.1 \%$ SDS).

After overnight incubation, the primary antibody was removed, and the blots washed 3 times (PBS + Tween (0.1\%), 5 minutes). Blots were incubated for 1 hour at room temperature with a goat anti-rabbit IRDye 680 RD secondary antibody $(1: 15,000 ;$ LI-COR $)$ in PBS blocking buffer (0.1\% tween, $0.1 \%$ SDS). Blots were washed 3 times in PBS $(0.1 \%$ Tween) for 5 minutes, once in PBS for 5 minutes, and imaged on an Odyssey CLx Infrared Imaging System (LI-COR Biosciences).

\subsubsection{Statistical analysis}

All p-values were calculated using a one-way ANOVA test. 


\section{Chapter 6: Conclusion}

The link between aberrant glycosylation and cancer progression has formed the basis for the detection of cell surface glycans and glycoproteins in the clinical management of cancer. However, the utility of biomarkers, such as PSA for prostate cancer, and MUC1 for breast cancer, have been challenged in recent years due to low cancer specificity and high falsepositive rates. The aim of this study was to use synthetic analogues of monosaccharides, called azidosugars, to monitor dynamic changes in cell surface glycosylation in models of prostate, breast and pancreatic cancer progression, in order to identify novel biomarkers.

We combined bioorthogonal azide-based chemical reporters with mass spectrometry analysis to capture and identify differentially glycosylated proteins. Our studies led to the identification of basigin (CD147), a transmembrane glycoprotein, which showed a strong increase in sialyation and $\mathrm{N}$-acetylgalactosamine glycosylation, despite its total expression decreasing across a panel of prostate cancer cell lines with increasing metastatic potential. Based on total protein expression alone, basigin, which would become less abundant during cancer progression and consequently more difficult to detect in aggressive disease, appeared to have no clinical value. But in the context of elevated glycosylation, our data suggest that differential glycosylation may be a more sensitive and specific marker for monitoring prostate and breast cancer progression, than increases in total protein expression alone. Differentially glycosylated basigin may be a prognostic marker of prostate cancer progression.

The power of aberrant glycosylation in improving biomarker specificity has already been observed in prostate and liver cancers. Immunoprecipitation of differentially N-glycosylated and sialylated PSA subforms from urine or tissue has been shown to distinguish patients with prostate cancer from those with benign prostate hyperplasia and to improve the cancer specificity of the PSA test (Sarrats et. al. 2010; Pihikova et. al. 2016; Tabarés et. al. 2007; Jia et. al. 2017; Li et. al. 2015; Hsiao et. al. 2016; Meany et. al. 2009; Saldova et. al. 2011). In 2006, the FDA approved the use of alpha fetoprotein (AFP)-L3 for detecting hepatocellular 
carcinoma. AFP-L3 is an aberrantly fucosylated version of AFP, generated by the overexpression of fucosyltransferase FUT 8 (Meany and Chan 2011). AFP-L3 levels are significantly elevated in hepatocellular carcinoma patients in comparison to benign liver diseases such as cirrhosis, providing a marker for the onset of hepatocellular carcinoma (Block et. al. 2005; Meany and Chan 2011; Adamczyk et. al. 2012; Pinho and Reis 2015).

Capturing aberrantly glycosylated basigin in liquid patient samples, such as serum or urine, using the techniques adopted in this project, would require biorthogonal labelling of live tissue by administering azidosugars directly to patients in vivo or conducting metabolic labelling of tissue biopsies ex vivo. We believe that translating bioorthogonal labelling techniques to a clinical setting will pose some challenges, requiring extensive clinical trials, azidosugar dose optimisation, safety studies, and ethical approval. Lectins provide an alternative approach to detecting changes in glycosylation. Their binding specificities are well characterised, they are abundant in nature, inexpensive, stable under a range of experimental conditions, and bind a variety of human tissues (van Kooyk 2008; BirdLieberman et. al. 2012). Lectins have been used to detect differential sialyation of PSA (Meany et. al. 2009) and increased glycosylation of basigin across the stages of hepatocellular carcinoma using Phaseolus vulgaris leucoagglutinin lectin, which recognises galactose and complex $\beta 1$,6-branched N-glycans (Cui et. al. 2018; Huang et. al. 2013). Lectinbased fluorescence imaging has also revealed a link between glycosylation and cancer progression within mucosal surfaces (Bird-Lieberman et. al. 2012; Kuo et. al. 2016) (Chapter 1: Figure 1.17, Figure 1.19 and Figure 1.23).

We were unable to use glycan-binding lectins to confirm differential glycosylation of basigin, within the timeframe of this project. Therefore, future experiments would involve immunoprecipitating basigin from whole-cell lysates prepared from our panel of prostate cancer cell lines to confirm differential glycosylation using lectin blot analysis. This could provide the preliminary data needed to support the development of a clinical assay, similar to the AFP-LC3 capture assay developed by FUJIFILM Wako Diagnostics U.S.A, which uses the Lens culinaris agglutinin (LCA) lectin to capture aberrantly fucosylated AFP-L3 (http://www.wakodiagnostics.com/afpl3test.html, 2019; Block et. al. 2005; Meany and Chan 2011; Adamczyk et. al. 2012; Pinho and Reis 2015) (Chapter 1: Figure 1.16). Glycosylated 
basigin has been observed in prostate, breast (Kong et. al. 2014), liver (Cui et. al. 2018;

Huang et. al. 2013) and pancreatic cancers (Haun et. al. 2015). Therefore, aberrantly glycosylated basigin may be a universal prognostic marker of cancer progression. 


\section{$\underline{\text { References }}$}

Abcam.com. (2019). Anti-Prostate Specific Antigen antibody [5A6] (Biotin) (ab182031) is not available. [online] Available at: https://www.abcam.com/prostate-specific-antigen-antibody5a6-biotin-ab182031.html [Accessed 06 Jun. 2019]

Abcam.com. (2019). Recombinant Anti-Prostate Specific Antigen antibody [EP1588Y] (ab76113) | Abcam. [online] Available at: https://www.abcam.com/prostate-specificantigen-antibody-ep1588y-ab76113.html [Accessed 06 Jun. 2019].

Abelev, G. I., Perova, S. D., Khramkova, N. I., Postnikova, Z. A., Irlin, I. S. (1963), Production of embryonal alpha-globulin by transplantable mouse hepatomas, Transplantation, vol 1, pp. $174-180$

Adamczyk, B., Tharmalingam, T., Rudd, Pauline M. (2012), Glycans as cancer biomarkers, Biochimica et Biophysica Acta, vol. 1820, pp. 1347-1353

Agarwal, P., Beahm, Brendan J., Shieh, P., Bertozzi, C. R., (2015), Systemic Fluorescence Imaging of Zebrafish Glycans with Bioorthogonal Chemistry, Angewandte Chemie, vol. 54, pp. $11504-11510$

Álvarez-Garcia, V., Tawil, Y., Wise, H. M., Leslie, N. R. (2019), Mechanisms of PTEN loss in cancer: It's all about diversity, Seminars in Cancer Biology, pii: S1044-579X(18)30059-2 [ePub ahead of print]

Amur, S., Frueh, F.W., Lesko, L.J. and Huang, S.-M. (2008), Integration and use of biomarkers in drug development, regulation and clinical practice: A US regulatory perspective, Biomarkers in Medicine, vol. 2, pp. 305-311 
Arnold, J. N., Wormald, M. R., Sim, R. B., Rudd, P. M., Dwek, R. A. (2007), The Impact of Glycosylation on the Biological Function and Structure of Human Immunoglobulins, Annual Review of Immunology, vol. 25, pp. 21-50

Arriaga, J., Abate-Shen, C. (2019), Genetically Engineered Mouse Models of Prostate Cancer in the Postgenomic Era, Cold Spring Harbour Prospectives in Medicine, vol. 9, pp. a030528

Arvelo, F., Sojo, F., Cotte, C. (2016), Tumour progression and metastasis, ecancer, vol. 10, pp. 617

Ashwell, G., Harford, J. (1982), Carbohydrate-specific receptors of the liver, Annual Review of Biochemistry, vol. 51, pp. 531-554

Autier, P. (2018), Breast screening and the parallel progression model of cancer, Breast Cancer Research and Treatment, vol. 170, pp. 439-440

Avancès, C., Georget, V., Térouanne, B., Orio, F., Cussenot, O., Mottet, N., Costa, P., Sultan C. (2001), Human prostatic cell line PNT1A, a useful tool for studying androgen receptor transcriptional activity and its differential subnuclear localization in the presence of androgens and antiandrogens, Molecular and Cellular Endocrinology, vol. 184, pp. 13-24

Ayala, G., Frolov, A., Chatterjee, D., He, D., Hilsenbeck, S., Ittmann, M. (2015), Expression of ERG protein in prostate cancer: variability and biological correlates, Endocrine Related Research, vol. 22, pp. 277-287

Baker, L. A., Tiriac, H., Clevers, H., Tuveson, D. A. (2016), Modeling Pancreatic Cancer with Organoids, Trends in Cancer, vol. 2, pp. 176-190

Baker, L., Tiriac, H., Clevers, H., Tuveson, D. (2016), Modeling Pancreatic Cancer with Organoids, Trends in Cancer, vol. 2, pp. 176-190 
Banerjee, D. K., Scher, Malka G., Waechter, C. J. (1981), Amphomycin: Effect of the Lipopeptide Antibiotic on the Glycosylation and Extraction of Dolichyl Monophosphate in Calf Brain Membranes, Biochemistry, vol. 20, pp. 1561-1568

Barbet, J., Bardiès, M., Bourgeois, M., Chérel, M., Davodeau, F., Faivre-chauvet, A., Gestin, J., Kraeber-bodéré, F. (2012), Radiolabeled Antibodies for Cancer Imaging and Therapy, In: Chames P. (eds) Antibody Engineering. Methods in Molecular Biology (Methods and Protocols), vol 907. Humana Press, Totowa, NJ. Available from: https://link.springer.com/protocol/10.1007\%2F978-1-61779-974-7_38

Barkeer, S., Chugh, S., Batra, S. K., Ponnusamy, M. P. (2018), Glycosylation of Cancer Stem Cells: Function in Stemness, Tumorigenesis, and Metastasis, Neoplasia (United States), vol. 20, pp. $813-825$

Bauman, T., Ewald, J., Huang, W., Ricke, W. (2015), CD147 expression predicts biochemical recurrence after prostatectomy independent of histologic and pathologic features, BMC cancer, vol. 15, pp. 549

Beetham, R. (2000), Detection of Bence-Jones protein in practice, Annals of Clinical Biochemistry, vol. 37, pp. 563-570

Belardi, B., De La Zerda, A., Spiciarich, D. R., Maund, S. L., Peehl, D. M., Bertozzi, C. R. (2013), Imaging the glycosylation state of cell surface glycoproteins by two-photon fluorescence lifetime imaging microscopy, Angewandte Chemie, vol. 52, pp. 14045-14049

Bennun, S. V., Hizal, D. B., Heffner, K., Can, O., Zhang, H., Betenbaugh, Michael J. (2016), Systems Glycobiology: Integrating Glycogenomics, Glycoproteomics, Glycomics, and Other 'Omics Data Sets to Characterize Cellular Glycosylation Processes, Journal of Molecular Biology, vol. 428, pp. 3337-3352 
Berg, J. M., Tymoczko, J. L., Stryer, L (2002). Biochemistry. 5th edition. New York: W H Freeman; 2002. Section 11.4, Lectins Are Specific Carbohydrate-Binding Proteins. Available from: https://www.ncbi.nlm.nih.gov/books/NBK22545/

Berger, R. P., Dookwah, M., Steet, R., Dalton, S. (2016), Glycosylation and stem cells: Regulatory roles and application of iPSCs in the study of glycosylation-related disorders, Bioessays, vol. 38, pp. 1255-1265

Berthois, Y., Bourrié, B., Galiègue, S., Vidal, H., Carayon, P., Martin, P. M., Casellas, P. (2003), R31747A is a sigma receptor ligand exhibiting antitumoural activity both in vitro and in vivo, British Journal of Cancer, vol. 88, pp. 438-446

Bertozzi, C. R., Sasisekharan, R. (2009), Glycomics, In: Varki A, Cummings RD, Esko JD, et al., editors. Essentials of Glycobiology. 2nd edition. Cold Spring Harbor (NY): Cold Spring Harbor Laboratory Press; 2009. Chapter 48. Available from:

https://www.ncbi.nlm.nih.gov/books/NBK1965/

Bhide, G. P., Colley, K. J. (2017), Sialylation of N-glycans: mechanism, cellular compartmentalization and function, Histochemistry and Cell Biology, vol. 147, pp. 149-174

Biankin, A. V. (2017), The road to precision oncology, Nature Genetics, vol. 49, pp. 320-321

Bie, H., Yin, J., He, X., Kermode, A. R., Goddard-Borger, Ethan D., Withers, Stephen G., James, M.N.G. (2013), Insights into mucopolysaccharidosis I from the structure and action of $\alpha$-Liduronidase, Nature Chemical Biology, vol. 9, pp. 739-745

Bioscience.co.uk. (2019). Cell chemotaxis assays - CytoSelect ${ }^{\mathrm{TM}}$. [online] Available at: https://www.bioscience.co.uk/cpl/132954 [Accessed 22 May. 2019].

Bird-Lieberman, E. L., Neves, A. A., Lao-Sirieix, P., O’Donovan, M., Novelli, M., Lovat, L., Eng, W., Mahal, L., Brindle, M. K., and Fitzgerald, R. (2012), Molecular imaging using 
fluorescent lectins permits rapid endoscopic identification of dysplasia in Barrett's esophagus, Nature medicine, vol. 18, pp. 315-21

Biskup, K., Braicu, E.I., Sehouli, J., Fotopoulou, C., Tauber, R., Berger, M., Blanchard, V. (2013), Serum glycome profiling-a biomarker for diagnosis of ovarian cancer, Glycoconjugate Journal, vol. 12, pp. 4056-4063

Blixt, O., Lavrova, O. I., Mazurov, D. V., Cló, E., Kračun, S.K., Bovin, N. V., Filatov, A.V. (2012), Analysis of Tn antigenicity with a panel of new IgM and IgG1 monoclonal antibodies raised against leukemic cells, Glycobiology, vol. 22, pp. 529-542

Block, T. M., Comunale, M. A., Lowman, M., Steel, L. F., Romano, P. R., Fimmel, C., Tennant, B. C., London, W. T., Evans, A. A., Blumberg, B. S., Dwek, R. A., Mattu, T. S., Mehta, A. S. (2005), Use of targeted glycoproteomics to identify serum glycoproteins that correlate with liver cancer in woodchucks and humans, Proceedings of the National Academy of Sciences, vol. 102, pp. 779-784

Bogen, S. A., Sompuram, S. R. (2004), Recent trends and advances in immunodiagnostics of solid tumors, BioDrugs, vol. 18, pp. 387-398

Bogenrieder, T., Herlyn, M. (2003), Axis of evil: molecular mechanisms of cancer metastasis, Oncogene, vol. 22, pp. 6524-6536

Boj, S., Hwang, C., Baker, L., Chio, I., Engle, D., Corbo, V., Jager, M., Ponz-Sarvise, M., Tiriac, H., Spector, Gracanin, A., Oni, T., Yu, K., Boxtel, van, Huch, M., Rivera, K., Wilson, J., Feigin, M., Öhlund, D., Handly-Santana, A., Ardito-Abraham, C., Ludwig, M., Elyada, E., Alagesan, B., Biffi, G., Yordanov, G., Delcuze, B., Creighton, B., Wright, K., Park, Y., Morsink, F., Molenaar, I., Rinkes, B., Cuppen, E., Hao, Y., Jin, Y., Nijman, I., Iacobuzio-Donahue, C., Leach, S., Pappin, D., Hammell, M., Klimstra, D., Basturk, O., Hruban, R., Offerhaus, G., Vries, R., Clevers, H., Tuveson, D. (2015), Organoid models of human and mouse ductal pancreatic cancer, Cell, vol. 160, pp. 324-38 
Bojarová, P., Křen, V. (2016), Sugared biomaterial binding lectins: Achievements and perspectives, Biomaterials science, vol. 19, pp. 1142-1160

Bombonati, A., Sgroi, D. (2011), The molecular pathology of breast cancer progression, Journal of Pathology, vol. 223, pp. 307-317

Boogert, M. A.W., Rader, D. J., Holleboom, A. G. (2017), New insights into the role of glycosylation in lipoprotein metabolism, Current Opinion in Lipidology, vol. 28, pp. 502-506 Borrmann, A., van Hest, J. (2014), Bioorthogonal chemistry in living animals, Chemical Science, vol. 5, pp. 2123-2134

Boyle, P., Autier, P., Bartelink, H., Baselga, J., Boffetta, P., Burn, J., Burns, H. J.G., Christensen, L., Denis, L., Dicato, M., Diehl, V., Doll, R., Franceschi, S., Gillis, C. R., Gray, N., Griciute, L., Hackshaw, A., Kasler, M., Kogevinas, M., Kvinnsland, S., La Vecchia, C., Levi, F., McVie, J. G., Maisonneuve, P., Martin-Moreno, J. M., Newton Bishop, J., Oleari, F., Perrin, P., Quinn, M., Richards, M., Ringborg, U., Scully, C., Siracka, E., Storm, H., Tubiana, M., Tursz, T., Veronesi, U., Wald, N., Weber, W., Zaridze, D. G., Zatonski, W., zur Hausen, H. (2003), Annals of Oncology, vol. 13, pp. 973-1005

Briand, P., Petersen, O. W., Van Deurs, B. (1987), A new diploid nontumorigenic human breast epithelial cell line isolated and propagated in chemically defined medium, In Vitro Cellular \& Developmental Biology, vol. 23, pp. 181-188

Brinkley, B. R., Beall, P. T., Wible, L. J., Mace, M. L., Turner, Donna S., Cailleau, R. M. (1980), Variations in Cell Form and Cytoskeleton in Human Breast Carcinoma Cells in Vitro, Cancer Research, vol. 40, pp. 18-31

Brockhausen, I., Stanley, P. (2017), O-GalNAc Glycans. In: Varki A, Cummings RD, Esko JD, et al., editors. Essentials of Glycobiology [Internet]. 3rd edition. Cold Spring Harbor (NY): Cold Spring Harbor Laboratory Press; 2015-2017. Chapter 10. Available from: https://www.ncbi.nlm.nih.gov/books/NBK453030/ doi: 10.1101/glycobiology.3e.010 
Brooks, S. A., Leathem, A.J. (1991), Prediction of lymph node involvement in breast cancer by detection of altered glycosylation in the primary tumour, Lancet, vol. 338, pp. 71-74.

Brooks, S., Hall, D., Buley, I. (2001), GalNAc glycoprotein expression by breast cell lines, primary breast cancer and normal breast epithelial membrane, British Journal of Cancer, vol. 85, pp. 1014-1022

Brown, K. F., Rumgay, H., Dunlop, C., Ryan, M., Quartly, F., Cox, A., Deas, A., Elliss, B., L., Gavin, A., Hounsome, L., Huws, D., Ormiston-Smith, N., Shelton, J., White, C., Parkin, D. M. (2018), The fraction of cancer attributable to modifiable risk factors in England, Wales, Scotland, Northern Ireland, and the United Kingdom in 2015, British Journal of Cancer, vol. 118, pp. $1130-1141$

Bubendorf, L., Schöpfer, A., Wagner, U., Sauter, G., Moch, H., Willi, N., Gasser, T. C., Mihatsch, M. J. (2000), Metastatic patterns of prostate cancer: an autopsy study of 1,589 patients, Human Pathology, vol. 31, pp. 578-583

Büll, C., Stoel, M. A., Den Brok, Martijn, H., Adema, Gosse J. (2014), Sialic acids sweeten a tumor's life, Cancer Research, vol. 74, pp. 3199-3204

Burchell, Joy M., Beatson, R., Graham, R., Taylor-Papadimitriou, J., Tajadura-Ortega, V. (2018), O-linked mucin-type glycosylation in breast cancer, Biochemical Society Transactions, vol. 46 , pp. $779-788$

Burdall, S.E., Hanby, A.M., Lansdown, M.R., Speirs, V. (2003), Breast cancer cell lines: Friend or foe?, Breast Cancer Research, vol. 5, pp. 89-95

Burton, L. J., Hawsawi, O., Loyd, Q., Henderson, V., Howard, S., Harlemon, M., Ragin, C., Roberts, R., Bowen, N., Gacii, A., Odero-Marah, V. (2018), Association of Epithelial Mesenchymal Transition with prostate and breast health disparities, PLOS ONE, vol. 13, pp. e0203855 
Buscaglia, Carlos A., Bossi, M., Yu, H, Chen, X., Bertozzi, Carolyn R., Mucci, J., Campetella, O. (2016), Sialic Acid Glycobiology Unveils Trypanosoma cruzi Trypomastigote Membrane Physiology, PLoS Pathogens, vol. 12, pp. 1-29

Byler, S., Goldgar, S., Heerboth, S., Leary, M., Housman, G., Moulton, K., Sarkar, S. (2014), Genetic and epigenetic aspects of breast cancer progression and therapy, Anticancer Research, vol. 34, pp. 1071-1077

Byrum, S., Larson, S., Avaritt, N., Moreland, L., Mackintosh, S., Cheung, W., Tackett, A. (2013), Quantitative Proteomics Identifies Activation of Hallmark Pathways of Cancer in Patient Melanoma, Journal of Proteomics and Bioinformatics, vol. 6, pp 43-50

Cailleau, R., Young, R., Olivé, M., Reeves, W. J. Jr. (1974), Breast tumor cell lines from pleural effusions, Journal of the National Cancer Institute, vol. 53, pp 661-674

Camp, L. A., Chauhan, P., Farrar, J. D., Lehrman, M. A. (1993), Defective mannosylation of glycosylphosphatidylinositol in Lec35 Chinese hamster ovary cells, Journal of Biological Chemistry, vol. 268, pp. 6721-6728

Campbell, P. M., Groehler, A. L., Lee, K. M., Ouellette, M. M., Khazak, V. D. C. J. (2007), K-Ras promotes growth transformation and invasion of immortalized human pancreatic cells by raf and phosphatidylinositol 3-kinase signalling, Cancer Research, vol. 67, pp 2098-2106

Carioli, G., Malvezzi, M., Rodriguez, T., Bertuccio, P., Negri, E., La Vecchia, C. (2018), Trends and predictions to 2020 in breast cancer mortality: Americas and Australasia, Breast, vol. 37, pp. $163-169$

Carioli, G., Malvezzi, M., Rodriguez, T., Bertuccio, P., Negri, E., La Vecchia, C. (2017), Trends and predictions to 2020 in breast cancer mortality in Europe, Breast, vol. 36, pp. 89-95 
Centenera, M. M., Raj, Ganesh V., Knudsen, K. E., Tilley, W. D., Butler, L. M. (2013), Ex vivo culture of human prostate tissue and drug development, Nature Reviews: Urology, vol. 10, pp. $483-487$

Chan, W. S., Jackson, A., Turner, G. A. (1984), Differences in surface expression of WGAbinding proteins of cells from a lymphosarcoma and its liver metastases, British Journal of Cancer, vol. 49, pp. 181-191

Chang, P.V., Prescher, J. A., Hangauer, M. J., Bertozzi, C. R. (2007), Imaging cell surface glycans with bioorthogonal chemical reporters, Journal of the American Chemical Society, vol. 129 , pp. $8400-8401$

Chang, Y. S., Chen, W. Y., Yin, J. J., Sheppard-Tillman, H., Huang, J., Liu, Y. N. (2015), EGF Receptor Promotes Prostate Cancer Bone Metastasis by Downregulating miR-1 and Activating TWIST1, Cancer Research, vol. 75, pp. 3077-3086

Chapoval, A. I., Legutki, J. B., Stafford, P., Trebukhov, Andrey V., Johnston, S.A., Shoikhet, Yakov N., Lazarev, A.F. (2015), Immunosignature: Serum antibody profiling for cancer diagnostics, Asian Pacific Journal of Cancer Prevention, vol. 16, pp. 4833-4837

Chavez, K., Garimella, S. and Lipkowitz, S. (2010), Triple negative breast cancer cell lines: One tool in the search for better treatment of triple negative breast cancer, Breast disease., vol. 32 , pp. $35-48$

Chen, Jung T., Chen, Chein H., Ku, Ko L., Hsiao, M., Chiang, Chun P., Hsu, Tsui L., Chen, Min H., Wong, Chi H. (2015), Glycoprotein B7-H3 overexpression and aberrant glycosylation in oral cancer and immune response, Proceedings of the National Academy of Sciences of the United States of America, vol. 112, pp. 13057-13062

Chen, T., You, Y., Jiang, H., Wang, Z. Z. (2017), Epithelial-mesenchymal transition (EMT): A biological process in the development, stem cell differentiation, and tumorigenesis, Journal of Cellular Physiology, vol. 232, pp. 3261-3272. 
Chen, T., You, Y., Jiang, H., Wang, Zack Z. (2017), Epithelial-mesenchymal transition (EMT): A biological process in the development, stem cell differentiation, and tumorigenesis, Journal of Cellular Physiology, vol. 232, pp. 3261-3272

Chen. C., Tang, C., Lin, L., Tsai, C., Chu, C., Lin, T., Huang, Y. (2017), Thrombospondin-2 promotes prostate cancer bone metastasis by the up-regulation of matrix metalloproteinase-2 through down-regulating miR-376c expression, Journal of Hematology and Oncology, vol. 10, pp. 33

Cheng, B., Xie, R., Dong, L. and Chen, X. (2016), Metabolic remodeling of cell- surface Sialic acids: Principles, applications, and recent advances, ChemBioChem, vol. 17, pp. 11-27

Cheng, B., Xie, R., Dong, L., Chen, X. (2016), Metabolic Remodeling of Cell-Surface Sialic Acids: Principles, Applications, and Recent Advances, ChemBioChem, vol. 17, pp. 11-27

Christiansen, M. N., Chik, J., Lee, L., Anugraham, M., Abrahams, J. L., Packer, N. H. (2014), Cell surface protein glycosylation in cancer, Proteomics, vol. 14, pp. 525-546

Cieniewski-Bernard, C., Dupont, E., Deracinois, B., Lambert, M., Bastide, B. (2014), Multiplexed detection of O-GIcNAcome, phosphoproteome, and whole proteome within the same gel, Frontiers in Endocrinology, vol. 5, pp. 1-10

Clark, P.M., Dweck, J. F., Mason, D.E., Hart, C. R., Buck, S. B., Peters, E.C., Agnew, B. J., HsiehWilson, L. C. (2008), Direct in-gel fluorescence detection and cellular imaging of O-GIcNACmodified proteins, Journal of the American Chemical Society, vol. 130, pp. 11576-11577

Colley, K. J, Varki, A., Kinoshita, T. (2017), Cellular Organization of Glycosylation. In: Varki A, Cummings RD, Esko JD, et al., editors. Essentials of Glycobiology [Internet]. 3rd edition. Cold Spring Harbor (NY): Cold Spring Harbor Laboratory Press; 2015-2017. Chapter 4. Available from: https://www.ncbi.nlm.nih.gov/books/NBK453052/ doi: 10.1101/glycobiology.3e.004 
Cook, P. J., Doll, R., Fellingham, S. A. (1969), A mathematical model for the age distribution of cancer in man, International Journal of Cancer, vol. 4, pp. 93-112

Corfield, A. (2017), Eukaryotic protein glycosylation: a primer for histochemists and cell biologists, Histochemistry and Cell Biology, vol. 147, pp. 119-147

Cowell, C. F., Weigelt, B., Sakr, R. A., Ng, C. K., Hicks, J., King, T. A., Reis-Filho, J. S. (2013), Progression from ductal carcinoma in situ to invasive breast cancer: Revisited, Molecular Oncology, vol. 7, pp. 859-869

Cui, J., Huang, W., Wu, B., Jin, J., Jing, L., Shi, Wen P., Liu, Zhen Y., Yuan, L., Luo, D., Li, L., Chen, Zhi N., Jiang, Jian L. (2018), N-glycosylation by N-acetylglucosaminyltransferase V enhances the interaction of CD147/basigin with integrin $\$ \beta \$ 1$ and promotes HCC metastasis, Journal of Pathology, vol. 245, pp. 41-52

Cummings RD, Etzler ME. (2009), Antibodies and Lectins in Glycan Analysis. In: Varki A, Cummings RD, Esko JD, et al., editors. Essentials of Glycobiology. 2nd edition. Cold Spring Harbor (NY): Cold Spring Harbor Laboratory Press; 2009. Chapter 45. Available from: https://www.ncbi.nlm.nih.gov/books/NBK1919/

Cummings, R. D, Liu, F. T, Vasta, G. R. (2017), Galectins, In: Varki A, Cummings RD, Esko JD, et al., editors. Essentials of Glycobiology [Internet]. 3rd edition. Cold Spring Harbor (NY): Cold Spring Harbor Laboratory Press; 2015-2017. Chapter 36. Available from: https://www.ncbi.nlm.nih.gov/books/NBK453091/doi: 10.1101/glycobiology.3e.036

Cummings, R. D. (2009), The repertoire of glycan determinants in the human glycome, Molecular BioSystems, vol. 5, pp. 1087-1104

Cunningham, D., You, Z. (2015), In vitro and in vivo model systems used in prostate cancer research, Journal of Biological Methods, vol. 2, pp. e17 
Dahiya, R., Kwak, K. S., Byrd, J. C., Ho, S., Yoon, W. H., Kim, Y. S. (1993), Mucin synthesis and secretion in various human epithelial cancer cell lines that express the MUC-1 mucin gene, Cancer Research, vol. 15, pp. 1437-1443

Dai, X., Li, T., Bai, Z., Yang, Y., Liu, X., Zhan, J., Shi, B. (2015), Breast cancer intrinsic subtype classification, clinical use and future trends, American Journal of Cancer Research, vol. 5, pp. 2929-2943.

Daniotti, Jose L., Vilcaes, Aldo A., Demichelis, Vanina T., Ruggiero, Fernando M., RodriguezWalker, M. (2013), Glycosylation of glycolipids in cancer: Basis for development of novel therapeutic approaches, Frontiers in Oncology, vol. 3, pp. 1-12

Davidson, J.M., Gorringe, K.L., Chin, S.-F., Orsetti, B., Besret, C., Courtay-Cahen, C., Roberts, I., Theillet, C., Caldas, C., Edwards, P.A.W. (2000), Molecular cytogenetic analysis of breast cancer cell lines, British Journal of Cancer, vol. 83, pp. 1309-1317

Debes, J., Tindall, D. (2002), The role of androgens and the androgen receptor in prostate cancer, Cancer Letters, vol. 187, pp. 1-7

Deer, E. L., Gonzalez-Hernandez, J., Coursen, J. D., Shea, J.E., Ngatia, J., Scaife, C. L., Firpo, M. A., Mulvihill, S. J. (2010), Phenotype and Genotype of Pancreatic Cancer Cell Lines, Pancreas, vol. 39 , pp. $425-435$

Denmeade, S., Sokoll, L., Dalrymple, S., Rosen, M., Gady, A., Bruzek, D., Ricklis, R., Isaacs, J. (2003), Dissociation Between Androgen Responsiveness for Malignant Growth vs. Expression of Prostate Specific Differentiation Markers PSA, hK2, and PSMA in Human Prostate Cancer Models, The Prostate, vol. 54, pp.249-257

Dennis, J. W., Granovsky, M., Warren, C.E. (1999), Glycoprotein glycosylation and cancer progression, Biochimica et Biophysica Acta, vol. 1473, pp. 21-34

Dhillon, S. (2015), Dinutuximab: First global approval, Drugs, vol. 75, pp. 923-927 
Dijkers, E. C., Oude Munnink, T. H., Kosterink, J. G., Brouwers, A. H., Jager, P. L., De Jong, J. R., Van Dongen, G. A., Schröder, C. P., Lub-De Hooge, M. N., De Vries, E. G. (2010), Biodistribution of $89 \mathrm{Zr}$-trastuzumab and PET Imaging of HER2-Positive Lesions in Patients with Metastatic Breast Cancer, Clinical Pharmacology and Therapeutics, vol. 87, pp. 586-592

Dong, J. (2006), Prevalent Mutations in Prostate Cancer, Journal of Cellular Biochemistry, vol. 97, pp. 433-447

Dowling, P., Clynes, M. (2011), Conditioned media from cell lines: A complementary model to clinical specimens for the discovery of disease-specific biomarkers, Proteomics, vol. 11, pp. $794-804$

Drost, J., Karthaus, W., Gao, D., Driehuis, E., Sawyers, C., Chen., Y, Clevers, H. (2016), Organoid culture systems for prostate epithelial and cancer tissue, Nature Protocols, vol. 2, pp. 347-358

Drudge-Coates, L., Oh, W. K., Tombal, B., Delacruz, A., Tomlinson, B., Ripley, Aimee V., Mastris, K., O'Sullivan, J. M., Shore, N. D., (2018), Recognizing Symptom Burden in Advanced Prostate Cancer: A Global Patient and Caregiver Survey, Clinical Genitourinary Cancer, vol. 16, pp. e411-e419

Dube, D. H., Bertozzi, C. R. (2003), Metabolic oligosaccharide engineering as a tool for glycobiology, Current Opinion in Chemical Biology, vol. 7, pp. 616-625

Duffy, M. J. (2012), Tumor markers in clinical practice: A review focusing on common solid cancers, Medical Principles and Practice, vol. 22, pp. 4-11

Dutt, S., Gao, A. (2009), Molecular mechanisms of castration-resistant prostate cancer progression, Future Oncology, vol. 5, pp. 1403-1413 
Eeles, R., Goh, C., Castro, E., Bancroft, E., Guy, M., Olama, A., Easton, D., Kote-Jarai, Z. (2014), The genetic epidemiology of prostate cancer and its clinical implications, Nature Reviews: Urology, vol. 11, pp. 18-31

Epstein, Ri. J., Lin, F. P. (2017), Cancer and the omics revolution, Australian Family Physician, vol. 46, pp. 189-193

Evans, C. P., Elfman, F., Cunha, G., Shuman, M. A. (1997), Decreased prostate cancer cell migration by inhibition of the insulin-like growth factor II/Mannose-6-Phosphate receptor, Urological Oncology, vol. 3, pp. 166-170

Fantozzi, A., Christofori, G. (2006), Mouse models of breast cancer metastasis, Breast Cancer Research, vol. 8, pp. 212

Fitzmaurice, C., Dicker, D., Pain, A., Hamavid, H., Moradi-Lakeh, M., Maclntyre, M., Allen, C., Hansen, G., Woodbrook, R., Wolfe, C., Hamadeh, R., Moore, A., Werdecker, A., Gessner, B., Te Ao, B., McMahon, B., Karimkhani, C., Yu, C., Cooke, G., Schwebel, D., Carpenter, D., Pereira, D., Nash, D., Kazi, D., De Leo, D., Plass, D., Ukwaja, K., Thurston, G., Yun Jin, K., Simard, E., Mills, E., Park, E., Catalá-López, F., deVeber, G., Gotay, C., Khan, G., Hosgood, H., Santos, I., Leasher, J., Singh, J., Leigh, J., Jonas, J., Sanabria, J., Beardsley, J., Jacobsen, K., Takahashi, K., Franklin, R., Ronfani, L., Montico, M., Naldi, L., Tonelli, M., Geleijnse, J., Petzold, M., Shrime, M., Younis, M., Yonemoto, N., Breitborde, N., Yip, P., Pourmalek, F., Lotufo, P., Esteghamati, A., Hankey, G., Ali, R., Lunevicius, R., Malekzadeh, R., Dellavalle, R., Weintraub, R., Lucas, R., Hay, R., Rojas-Rueda, D., Westerman, R., Sepanlou, S., Nolte, S., Patten, S., Weichenthal, S., Abera, S., Fereshtehnejad, S., Shiue, I., Driscoll, T., Vasankari, T., Alsharif, U., Rahimi-Movaghar, V., Vlassov, V., Marcenes, W., Mekonnen, W., Melaku, Y., Yano, Y., Artaman, A., Campos, I., MacLachlan, J., Mueller, U., Kim, D., Trillini, M., Eshrati, B., Williams, H., Shibuya, K., Dandona, R., Murthy, K., Cowie, B., Amare, A., Antonio, C., Castañeda-Orjuela, C., van Gool, C., Violante, F., Oh, I., Deribe, K., Soreide, K., Knibbs, L., Kereselidze, M., Green, M., Cardenas, R., Roy, N., Tillmann, T., Li, Y., Krueger, H., Monasta, L., Dey, S., Sheikhbahaei, S., Hafezi-Nejad, N., Kumar, G., Sreeramareddy, C., Dandona, L., Wang, H., Vollset, S., Mokdad, A., Salomon, J., Lozano, R., Vos, T., Forouzanfar, M., Lopez, A., 
Murray, C. and Naghavi, M. (2015). The Global Burden of Cancer 2013, JAMA Oncol, vol. 1, no. 4 , pp. 505-27.

Ford, T. F., Butcher, D. N., Masters, J. R., Parkinson, M. C. (1985), Immunocytochemical localisation of prostate-specific antigen: specificity and application to clinical practice, British Journal of Urology, vol. 57, pp. 50-55

Foreman, K., Marquez, N., Dolgert, A., Fukutaki, K., Fullman, N., McGaughey, M., Pletcher, M., Smith, A., Tang, K., Yuan, C., Brown, J., Friedman, J., He, J., Heuton, K., Holmberg, M., Patel, D., Reidy, P., Carter, A., Cercy, K., Chapin, A., Douwes-Schultz, D., Frank, T., Goettsch, F., Liu, P., Nandakumar, V., Reitsma, M., Reuter, V., Sadat, N., Sorensen, R., Srinivasan, V., Updike, R., York, H., Lopez, A., Lozano, R., Lim, S., Mokdad, A., Vollset, S., Murray, C. (2018). Forecasting life expectancy, years of life lost, and all-cause and cause-specific mortality for 250 causes of death: reference and alternative scenarios for 2016-40 for 195 countries and territories, The Lancet, vol. 392, pp. 2052-2090.

Freeze, H., Eklund, E., Ng, B., Patterson, M. (2015), Neurological aspects of human glycosylation disorders, Annual Review of Neuroscience, vol. 38, pp. 105-125

Freeze, H., Kranz, C. (2010), Endoglycosidase and Glycoamidase Release of N-Linked Glycan's, Current Protocols in Immunology, vol. 83, pp. 8.15.1-8.15.26

FUJIFILM Wako Diagnostics-AFP-L3 Test. [online] Wakodiagnostics.com. Available at: http://www.wakodiagnostics.com/afpl3test.html [Accessed 25 May. 2019].

Fuster, M. and Esko, J. (2005), The sweet and sour of cancer: Glycans as novel therapeutic targets', Nature reviews: Cancer, vol. 5, pp. 526-542

Gene Ontology Resource. (2019). GO enrichment analysis. [online] Available at: http://geneontology.org/docs/go-enrichment-analysis/ [Accessed 22 Apr. 2019]. 
Ghazarian, H., Idoni, B., Oppenheimer, S. (2011), A glycobiology review: Carbohydrates, lectins and implications in cancer therapeutics, Acta histochemica, vol. 113, pp. 236-47

Ghosh, A., Wang, X., Klein, E., Heston, W. D. (2005), Novel role of prostate-specific membrane antigen in suppressing prostate cancer invasiveness, Cancer Research, vol. 65, pp. $727-731$

Glish, G. L., Vachet, R. W. (2003), The basics of mass spectrometry in the twenty-first century, Nature Reviews: Drug Discovery, vol. 2, pp. 140-150

Goon, S. S. B., Tullius, M. V., Gibson, B.W., Bertozzi, C. R. (2003), Metabolic incorporation of unnatural sialic acids into Haemophilus ducreyi lipooligosaccharides, Proceedings of the National Academy of Sciences of the United States of America, vol. 100, pp. 3089-3094

Gottlieb, C., Marie Skinner, A, Kornfeld, S. (1974), Isolation of a Clone of Chinese Hamster Ovary Cells Deficient in Plant Lectin-Binding Sites, Proceedings of the National Academy of Sciences of the United States of America, vol. 71, pp. 1078-1082

Granovsky, M., Fata, J., Pawling, J., Muller, W. J., Khokha, R., Dennis, J. W. (2000), Suppression of tumor growth and metastasis in Mgat5-deficient mice, Nature Medicine, vol. 6, pp. 306-312

Greaves, M., Maley, C.C. (2012), Clonal Evolution in Cancer, Nature, vol. 481, pp. 306-313

Grigoriadis, A., Mackay, A., Noel, E., Wu, Pei J., Natrajan, R., Frankum, J. Reis-Filho, Jorge S. Grönberg, H., Adolfsson, J., Aly, M., Nordström, T., Wiklund, P., Brandberg, Y., Thompson, J., Wiklund, F., Lindberg, J., Clements, M., Egevad, L., Eklund, M. (2015), Prostate cancer screening in men aged 50-69 years (STHLM3): A prospective population-based diagnostic study, The Lancet Oncology, vol. 16, pp. 1667-1676 
Grosso, G., Bella, F., Godos, J., Sciacca, S., Del Rio, D., Ray, S., Galvano, F., Giovannucci, E. L. (2017), Possible role of diet in cancer: Systematic review and multiple meta-analyses of dietary patterns, lifestyle factors, and cancer risk, Nutrition Reviews, vol. 75, pp. 405-419

Grupp, K., Höhne, Thorsten S., Prien, K., Hube-Magg, C., Tsourlakis, Maria C., Sirma, H., Pham, T., Heinzer, H., Graefen, M., Michl, U., Simon, R., Wilczak, W., Izbicki, J., Sauter, G., Minner, S., Schlomm, T., Steurer, S. (2013), Reduced CD147 expression is linked to ERG fusion-positive prostate cancers but lacks substantial impact on PSA recurrence in patients treated by radical prostatectomy, Experimental and Molecular Pathology, vol. 95, pp. 227234

Haji-Ghassemi, O., Blackler, Ryan J., Young, N. M., Evans, Stephen V. (2015), Antibody recognition of carbohydrate epitopes, Glycobiology, vol. 25, pp. 920-952

Hakomori, S. (1985), Aberrant Glycosylation in Cancer Cell Membranes as Focused on Glycolipids: Overview and Perspectives, Cancer Research, vol. 45, pp. 2405-2414

Haltiwanger, R. S., Lowe, J. B. (2004), Role of Glycosylation in Development, Annual Review of Biochemistry, vol. 73, pp. 491-537

Hama, Y., Urano, Y., Koyama, Y., Choyke, P., Kobayashi, H. (2006), Targeted optical imaging of cancer cells using lectin-binding BODIPY conjugated avidin, Biochemical and biophysical research communications, vol. 348, pp. 807-13

Hama, Y., Urano, Y., Koyama, Y., Kamiya, M., Bernardo, M., Paik, R., Krishna, M., Choyke, P., Kobayashi, H. (2006), In vivo spectral fluorescence imaging of submillimeter peritoneal cancer implants using a lectin-targeted optical agent, Neoplasia (New York, N.Y.), vol. 8, pp. $607-12$

Hamdy, F. C., Donovan, J. L., Lane, J. A., Mason, M., Metcalfe, C., Holding, P., Davis, M., Peters, T. J., Turner, E. L., Martin, R. M., Oxley, J., Robinson, M., Staffurth, J., Walsh, E., Bollina, P., Catto, J., Doble, A., Doherty, A., Gillatt, D., Kockelbergh, R., Kynaston, H., Paul, A., 
Powell, P, Prescott, S., Rosario, Derek J., Rowe, E., Neal, D. E. (2016), 10-Year Outcomes After Monitoring, Surgery, or Radiotherapy for Localized Prostate Cancer, New England Journal of Medicine, vol. 375, pp. 1415-1424

Han, Z. D., Bi, X. C., Qin, W. J., He, H.C., Dai, Q. S., Zou, J., Ye, Y. K, Liang, Y. X, Zeng, G. H, Chen, Z. N., Zhong, W. D. (2009), CD147 Expression Indicates Unfavourable Prognosis in Prostate Cancer, Pathology \& Oncology Research, vol. 15, pp. 69-374

Hanahan, D., Weinberg, R. A. (2011), Hallmarks of cancer: The next generation, Cell, vol. 144, pp. $646-674$

Hart, G. W., Copeland, R. J. (2010), Glycomics hits the big time, Cell, vol. 143, pp. 672-676

Haun, R. S., Quick, C. M., Siegel, E.R., Raju, I., Mackintosh, S.G., Tackett, A. J. (2015), Bioorthogonal labeling cell-surface proteins expressed in pancreatic cancer cells to identify potential diagnostic/therapeutic biomarkers, Cancer Biology and Therapy, vol. 16, pp. 15571565

Helenius, A., Sitia, R., Swoboda, B. E.P. (2001), Quality control in the secretory assembly line, Philosophical Transactions of the Royal Society B: Biological Sciences, vol. 356, pp. 147-150

Higai, K., Miyazaki, N., Azuma, Y., Matsumoto, K. (2006), Interleukin-1ß induces sialyl Lewis X on hepatocellular carcinoma HuH-7 cells via enhanced expression of ST3Gal IV and FUT VI gene, FEBS Letters, vol. 580, pp. 6069-6075

Higuero, A. M., Díez-Revuelta, N., Abad-Rodríguez, J. (2017), The sugar code in neuronal physiology, Histochemistry and Cell Biology, vol. 147, pp. 257-267

Hingorani, S. R., Petricoin, E. F., Maitra, A., R., V., King, C., Jacobetz, M. A., Ross, S., Conrads, T. P., Veenstra, Timothy D., Hitt, B. A., Kawaguchi, Y., Johann, D., Liotta, Lance A., Crawford, H.C., Putt, Mary E., Jacks, T., Wright, Christopher V E., Hruban, R. H., Lowy, Andrew M., 
Tuveson, D. A. (2003), Preinvasive and invasive ductal pancreatic cancer and its early detection in the mouse, Cancer Cell, vol. 4, pp. 437-450

Hizal, D. B., Wolozny, D., Colao, J., Jacobson, E., Tian, Y., Krag, S. S., Betenbaugh, M. J., Zhang, H. (2014), Glycoproteomic and glycomic databases, Clinical Proteomics, vol. 11, pp. 1-10

Hofherr, A., Wagner, C., Fedeles, S., Somlo, S., Köttgen, M. (2014), N-glycosylation determines the abundance of the transient receptor potential channel TRPP2, Journal of Biological Chemistry, vol. 289, pp. 14854-14867

Holen, I., Speirs, V., Morrissey, B., Blyth, K. (2017), In vivo models in breast cancer research: progress, challenges and future directions, Disease Models \& Mechanisms, vol. 10, pp. 359371

Holliday, D., Speirs, V. (2011), Choosing the right cell line for breast cancer research, Breast Cancer Research, vol. 13, pp. 215

Horne, H. N., Oh, H., Sherman, M. E., Palakal, M., Hewitt, S. M., Schmidt, M. K., Milne, R. L., Hardisson, D., Benitez, J, Blomqvist, C., Bolla, M. K., Brenner, H., Chang-Claude, J., Cora, R., Couch, F. J., Cuk, K., Devilee, P., Easton, D. F., Eccles, D. M., Eilber, U., Hartikainen, J. M., Heikkilä, P., Holleczek, B., Hooning, M. J., Jones, M., Keeman, R., Mannermaa, A., Martens, J. W. M., Muranen, T. A., Nevanlinna, H., Olson, J. E., Orr, N., Perez, J. I. A., Pharoah, P. D. P., Ruddy, K. J., Saum, K. U., Schoemaker, M. J., Seynaeve, C., Sironen, R., Smit, V. T. H. B. M, Swerdlow, A. J., Tengström, M., Thomas, A. S., Timmermans, A. M., Tollenaar, R. A. E. M., Troester, M. A., van Asperen, C. J., van Deurzen, C. H. M., Van Leeuwen, F. F., Van't Veer, L. J., García-Closas, M., Figueroa, J. D. (2018), E-cadherin breast tumor expression, risk factors and survival: Pooled analysis of 5,933 cases from 12 studies in the Breast Cancer Association Consortium, Nature Scientific Reports, vol. 8, pp. 6574

Hounsell, E. F., Young, M., Davies, M. J. (1997), Glycoprotein changes in tumours: A renaissance in clinical applications, Clinical Science, vol. 93, pp. 287-293 
Hsiao, Chun J., Tzai, Tzong S., Chen, Chein H., Yang, Wen H., Chen, Chung H. (2016), Analysis of Urinary Prostate-Specific Antigen Glycoforms in Samples of Prostate Cancer and Benign Prostate Hyperplasia, Disease Markers, ePub: 8915809.

Hsu, T.-L., Hanson, S. R., Kishikawa, K., Wang, S.-K., Sawa, M., Wong, C.-H. (2007), Alkynyl sugar analogs for the labeling and visualization of glycoconjugates in cells, Proceedings of the National Academy of Sciences, vol. 104, pp. 2614-2619

Huang , Y., Cheng, C., Zhang, C., Zhang, Y., Chen, M., Strand, D., Jiang, M. (2016), Advances in prostate cancer research models: From transgenic mice to tumor xenografting models, Asian Journal of Urology, vol. 3, pp. 64-74

Huang, K., Wu, Li D. (2008), Aggrecanase and Aggrecan degradation in osteoarthritis: A review, Journal of International Medical Research, vol. 36, pp. 1149-1160

Huang, W., Luo, Wen J., Zhu, P., Tang, J., Yu, Xiao L., Cui, Hong Y., Wang, B., Zhang, Y., Jiang, Jian L., Chen, Zhi N. (2013), Modulation of CD147-induced matrix metalloproteinase activity: Role of CD147 N-glycosylation, Biochemical Journal, vol. 449, pp. 437-448

Hudak, J. E., Bertozzi, C. R. (2014), Glycotherapy: new advances inspire a reemergence of glycans in medicine, Chemistry and Biology, vol. 21, pp. 16-37

Hugo, H. J., Gunasinghe, N. P.A.D., Hollier, B. G., Tanaka, T., Blick, T., Toh, A., Hill, P., Gilles, C., Waltham, M., Thompson, E. W. (2017), Epithelial requirement for in vitro proliferation and xenograft growth and metastasis of MDA-MB-468 human breast cancer cells: Oncogenic rather than tumor-suppressive role of E-cadherin, Breast Cancer Research, vol. 19, pp. 1-25

International Agency for Research on Cancer (IARC), European Commission, the World Health Organisation (WHO), European Code Against Cancer: 12 ways to reduce your cancer risk [online] Available at: https://cancer-code-europe.iarc.fr/index.php/en/ [Accessed 21 Jun. 2019]. 
Ishikawa, T., Shimizu, D., Kito, A., Ota, I., Sasaki, T., Tanabe, M., Yamada, A., Arioka, H., Shimizu, S., Wakasugi, J., Mori, R., Chishima, T., Ichikawa, Y., Endo, I. (2012), Breast cancer manifested by hematologic disorders, Journal of Thoracic Disease, vol. 4, pp. 650-654

Ittmann, M., Huang, J., Radaelli, E., Martin, P., Signoretti, S., Sullivan, R., Simons, B. W., Ward, J. M., Robinson, B. D., Chu, G. C., Loda, M., Thomas, G., Borowsky, A., Cardiff, R. D. (2013), Animal models of human prostate cancer: The Consensus Report of the New York Meeting of the Mouse Models of Human Cancers Consortium Prostate Pathology Committee, Cancer Research, vol. 73, pp. 2718-2736

Jia, G., Dong, Z., Sun, C., Wen, F., Wang, H., Guo, H., Gao, X., Xu, C., Yang, C., Sun, Y. (2017), Alterations in expressed prostate secretion-urine PSA N-glycosylation discriminate prostate cancer from benign prostate hyperplasia, Oncotarget, vol. 8, pp. 76987-76999

Jin, J., Dayyani, F., Gallick, G. (2011), Steps in Prostate Cancer Progression that lead to Bone Metastasis, International Journal of Cancer, vol. 128, pp. 2545-2561

Ju, T., Otto, V., Cummings, R. (2011), The Tn antigena-structural simplicity and biological complexity, Angewandte Chemie, vol. 50, pp. 1770-1791

Justus, C. R., Leffler, N., Ruiz-Echevarria, M., Yang, Li V. (2014), Migration and Invasion Assays, Journal of Visualized Experiments, vol. 88, pp. e51046

Kang, K., Joo, S., Choi, Ji Y., Geum, S., Hong, Seok P., Lee, Seung Y., Kim, Yong H., Kim, Seong M., Yoon, Myung H., Nam, Y., Lee, Kyung B., Lee, Hee Y., Choi, Insung S. (2015), Tissue-based metabolic labeling of polysialic acids in living primary hippocampal neurons, Proceedings of the National Academy of Sciences of the United States of America, vol. 112, pp. e241-e248

Kang, M. S., Spencer, J. P., Elbein, A. D. (1978), Amphomycin inhibition of mannose and GIcNAc incorporation into lipid-linked saccharides, Journal of Biological Chemistry, vol. 253, pp. $8860-8866$ 
Kelly, S. P., Anderson, W. F., Rosenberg, P. S., Cook, M. B. (2018), European Urology Focus, vol. 4, pp. $121-127$

Kimbunga, S., Lomana, N., Hedenfalk, I., (2015), Clinical and molecular complexity of breast cancer metastases, Seminars in Cancer Biology, vol. 35, pp. 85-95

Klein, C. (2009), Parallel progression of primary tumours and metastases, Nature Reviews: Cancer, vol. 9, pp. 302-312

Kojima, S., Jay, M. (1986), Application of lectins to tumor imaging radiopharmaceuticals, European Journal of Nuclear Medicine, vol. 12, pp. 385-389

Kölbl, A. C., Andergassen, U., Jeschke, U. (2015), The role of glycosylation in breast cancer metastasis and cancer control, Frontiers in Oncology, vol. 5, pp. a219

Kong, Ling M., Liao, Cheng G., Zhang, Y., Xu, J., Li, Y., Huang, W., Zhang, Y., Bian, H., Chen, Zhi N. (2014), A regulatory loop involving miR-22, Sp1, and c-Myc modulates CD147 expression in breast cancer invasion and metastasis, Cancer Research, vol. 74, pp. 3764-3778

Kopitz, J. (2017), Lipid glycosylation: a primer for histochemists and cell biologists, Histochemistry and Cell Biology, vol. 147, pp. 175-198

Krasnova, L., Wong, C. (2016), Understanding the chemistry and biology of Glycosylation with Glycan synthesis, Annual review of biochemistry, vol. 85, pp. 599-630.

Kuhn, P., Tarentino, A. L., Plummer, T. H., Van Roey, P. (1994), Crystallization and preliminary crystallographic analysis of peptide- $\mathrm{N} 4-(\mathrm{N}$-acetyl- $\beta$-D-glucosaminyl)asparagine amidase PNGase F, Journal of Molecular Biology, vol. 241, pp. 622-623

Kuo, J. (2015), Imaging glycosylation changes in cancer progression and its potential clinical applications, PhD Thesis (Unpublished) 
Kuo, J., Ibrahim, A., Dawson, S., Parashar, D., Howat, W., Guttula, K., Miller, R., Fearnhead, N., Winton, D., Neves, A., Brindle, K. (2016), Detection of colorectal dysplasia using fluorescently labelled lectins, Nature Scientific reports, vol. 6: 24231

Küster, B., Krogh, T. N., Mørtz, E., Harvey, D. J. (2001), Glycosylation analysis of gel-separated proteins, Proteomics, vol. 1, pp. 350-361

Kveton, F., Blšáková, A., Hushegyi, A., Damborsky, P., Blixt, O., Jansson, B., Tkac, J. (2017), Optimization of the Small Glycan Presentation for Binding a Tumor-Associated Antibody: Application to the Construction of an Ultrasensitive Glycan Biosensor, Langmuir, vol. 33, pp. $2709-2716$

Kyriazis, A. P., Kyriazis, A. A., Scarpelli, D. G., Fogh, J., Rao, M. S., Lepera, R. (1982), Human pancreatic adenocarcinoma line Capan-1 in tissue culture and the nude mouse: morphologic, biologic, and biochemical characteristics. American Association of Pathologists, vol. 106, pp. $250-260$

Kyriazis, A. P., Kyriazis, Aikaterini A., Sternberg, C. N., Sloane, N. H., Loveless, J. D. (1986), Morphological, biological, biochemical, and karyotypic characteristics of human pancreatic ductal adenocarcinoma capan-2 in tissue culture and the nude mouse, Cancer Research, vol. 46, pp. $5810-5815$

Laidler, P., Dulińska, J., Lekka, M., Lekki, J. (2005), Expression of prostate specific membrane antigen in androgen-independent prostate cancer cell line PC-3, Archives of Biochemistry and Biophysics, vol. 435, pp. 1-14

Lamouille, S., Xu, J., Derynck, R. (2014), Molecular mechanisms of epithelial-mesenchymal transition, Nature Reviews: Molecular Cell Biology, vol. 15, pp. 178- 196

Lancaster, M., Knoblich, J. (2014), Organogenesis in a dish: Modeling development and disease using organoid technologies, Science, vol. 345, pp. e1247125 
Lantos, A. B., Carlevaro, G., Araoz, B., Ruiz Diaz, P., Camara, María de los M Lardizabal, J., Ding, J., Delwar, Z., Rennie, P. S., Jia. W. (2018), A TRAMP-derived orthotopic prostate syngeneic (TOPS) cancer model for investigating anti-tumor treatments, The Prostate, vol. 78, pp. 457-468

Laughlin, S., Agard, N., Baskin, J., Carrico, I., Chang, P., Ganguli, A., Hangauer, M., Lo, A., Prescher, J., Bertozzi, C. (2006), Metabolic labeling of glycans with azido sugars for visualization and glycoproteomics, Methods in enzymology, vol. 415, pp. 230-50

Laughlin, S., Baskin, J., Amacher, S., Bertozzi, C. (2008), In vivo imaging of membraneassociated glycans in developing zebrafish, Science (New York, N.Y.), vol. 320, pp. 664-7

Laughlin, S., Bertozzi, C. (2009), Imaging the glycome, Proceedings of the National Academy of Sciences of the United States of America, vol. 106, pp. 12-7.

Laughlin, S.T., Bertozzi, C.R. (2009), In vivo imaging of Caenorhabditis elegans Glycans, ACS Chemical Biology, vol. 4, pp. 1068-1072

Lee, K. M., Nguyen, C., Ulrich, A. B., Pour, P. M., Ouellette, M. M. (2003), Immortalization with telomerase of the Nestin-positive cells of the human pancreas, Biochemical and Biophysical Research Communications, vol. 301, pp. 1038-1044

Lee, K. M., Yasuda, H., Hollingsworth, M. A., Ouellette, M. M. (2005), Notch2-positive progenitors with the intrinsic ability to give rise to pancreatic ductal cells, Laboratory Investigation, vol. 85, pp. 1003-1012

Lee, Y. T., Tan, Y. J., Oon, C. E. (2018), Molecular targeted therapy: Treating cancer with specificity, European Journal of Pharmacology, vol. 834, pp. 188-196

Lewis, A. L., Lewis, W. G. (2012), Host sialoglycans and bacterial sialidases: A mucosal perspective, Cellular Microbiology, vol. 14, pp. 1174-1182 
Li, Qing K., Chen, L., Ao, Ming H., Chiu, Joyce H., Zhang, Z., Zhang, H., Chan, Daniel W. (2015), Serum fucosylated prostate-specific antigen (PSA) improves the differentiation of aggressive from non-aggressive prostate cancers, Theranostics, vol. 5, pp. 267-276

Li, R., Guo, Y., Han, B. M., Yan, X., Utleg, A. G., Li, W., Tu, L. C., Wang, J., Hood, L., Xia, S., Lin, B. (2008), Proteomics cataloging analysis of human expressed prostatic secretions reveals rich source of biomarker candidates, Proteomics - Clinical Applications, vol. 2, pp. 543-555

Li, S., Shen, Y., Wang, M., Yang, J., Lv, M., Li, P., Chen, Z., Yang, J. (2017), Loss of PTEN expression in breast cancer: Association with clinicopathological characteristics and prognosis, Oncotarget, vol. 8, pp. 32043-32054

Liu, H., Sadygov, R. G., Yates, J. R. 3rd. (2004), A Model for Random Sampling and Estimation of Relative Protein Abundance in Shotgun Proteomics, Analytical Chemistry, vol. 76, pp. 4193-4201

Liu, X., Ory, V., Chapman, S., Yuan, H., Albanese, C., Kallakury, B., Timofeeva, O. A., Nealon, C., Dakic, A., Simic, V., Haddad, B. R., Rhim, J. S., Dritschilo, A., Riegel, A., McBride, A., Schlegel, R. (2012), ROCK inhibitor and feeder cells induce the conditional reprogramming of epithelial cells, American Journal of Pathology, vol. 108, pp. 599-607

Llop, E., Ferrer-Batallé, M., Barrabés, S., Guerrero, P. E., Ramírez, M., Saldova, R., Rudd, P. M., Aleixandre, R. N., Comet, J., de Llorens, R., Peracaula, R. (2016), Improvement of Prostate Cancer Diagnosis by Detecting PSA Glycosylation-Specific Changes, Theranostics, vol. 6, pp. $1190-1204$

Logothetis, C. J., Gallick, G. E., Maity, S. N., Kim, J., Aparicio, A., Efstathiou, E., Lin, S. H. (2013), Cancer Discovery, vol. 3, pp. 849-861

Longueville, F., Lacroix, M., Barbuto, A. M., Bertholet, V., Gallo, D., Larsimont, D., Marcq, L., Zammatteo, N., Boffe, S., Leclercq, G., Remacle, J. (2005), Molecular characterization of 
breast cancer cell lines by a low-density microarray, International Journal of Oncology, vol. 27 , pp. $881-892$

Lovat, L. B., Eng, W. S., Mahal, L. K., Brindle, K. M., Fitzgerald, R. C. (2012), Molecular imaging using fluorescent lectins permits rapid endoscopic identification of dysplasia in Barrett's esophagus, Nature Medicine, vol. 18, pp. 315-321

Lowe, J. B., Marth, J. D. (2003), A Genetic Approach to Mammalian Glycan Function, Annual Review of Biochemistry, vol. 72, pp. 643-691

Luchansky, S. J., Argade, S., Hayes, B. K., Bertozzi, C. R. (2004), Metabolic functionalization of recombinant glycoproteins, Biochemistry, vol. 43, pp. 12358-12366

Madden, D. L. (2018), From a Patient Advocate's Perspective: Does Cancer Immunotherapy Represent a Paradigm Shift?, Current Oncology Reports, vol. 20, pp. 1-7

Magnani, J. L. (2004), The discovery, biology, and drug development of sialyl Lea and sialyl Lex, Archives of Biochemistry and Biophysics, vol. 426, pp. 122-131

Mankoff, D. A. (2007), A definition of molecular imaging, Journal of nuclear medicine, vol. 48, pp. $18 \mathrm{~N}, 21 \mathrm{~N}$

Mankoff, D.A., Farwell, M. D., Clark, A.S., Pryma, D. A. (2017), Making molecular imaging a clinical tool for precision oncology: A review, JAMA Oncology, vol. 3, pp. 695-701

Mariño, K., Bones, J., Kattla, J. J., Rudd, P. M. (2010), A systematic approach to protein glycosylation analysis: A path through the maze, Nature Chemical Biology, vol. 6, pp. 713-723

Marquardt, T., Lühn, K., Srikrishna, G., Freeze, H. H., Harms, E., Vestweber, D. (1999), Correction of leukocyte adhesion deficiency type II with oral fucose, Blood, vol. 94, pp. 39763985 
Marques-da-Silva, D., dos Reis Ferreira, V., Monticelli, M., Janeiro, P., Videira, P. A., Witters, P., Jaeken, J., Cassiman, D. (2017), Journal of Inherited Metabolic Disease, vol. 40, pp. 195207

Matrixscience.com. (2019). Mascot overview | Protein identification software for mass spec data. [online] Available at: http://www.matrixscience.com/search_intro.html [Accessed 05 Aug. 2019].

Mayeux, R. (2004), Biomarkers: Potential uses and limitations, The Journal of the American Society for Experimental NeuroTherapeutics, vol. 1, pp. 182-188.

Maylié-Pfenninger, M., Jamieson, J. (1980), Development of cell surface saccharides on embryonic pancreatic cells, Journal of Cell Biology, vol. 86, pp. 96-103

McCarthy, C., Saldova, R., Wormald, M. R., Rudd, P.M., McElvaney, N. G., Reeves, E. P. (2014), The role and importance of glycosylation of acute phase proteins with focus on alpha-1 antitrypsin in acute and chronic inflammatory conditions, Journal of Proteome Research, vol. 13, pp. 3131-3143

Meany, D. L., Zhang, Z., Sokoll L. J., Zhang, H., Chan, D. W. (2009), Glycoproteomics for Prostate Cancer Detection: Changes in Serum PSA Glycosylation Patterns, Journal of Proteome Research, vol. 8, pp. 613-619

Meeusen, E., Lim, E., Mathivanan, S. (2017), Secreted Tumor Antigens - Immune Biomarkers for Diagnosis and Therapy, Proteomics, vol. 17, pp. 1-7

Meleady, P. (2011), 2D gel electrophoresis and mass spectrometry identification and analysis of proteins, Methods in Molecular Biology, vol. 784, pp. 123-137

Milella, M., Falcone, I., Conciatori, F., Incani, Ursula C., Del Curatolo, A., Inzerilli, N., Nuzzo, Carmen M.A., Vaccaro, V., Vari, S., Cognetti, F., Ciuffreda, L. PTEN: Multiple functions in human malignant tumors, Frontiers in Oncology, vol. 5, pp. 1-14 
Mills, K., Mills, P. B., Clayton, P. T., Johnson, A. W., Whitehouse, D. B., Winchester, B. G. (2001), Identification of $\alpha 1$-antitrypsin variants in plasma with the use of proteomic technology, Clinical Chemistry, vol. 47, pp. 2012-2022

Mills, K., Mills, P. B., Clayton, P.T., Mian, N., Johnson, A. W., Winchester, B.G. (2003), The underglycosylation of plasma $\alpha 1$-antitrypsin in congenital disorders of glycosylation type I is not random, Glycobiology, vol. 13, pp. 73-85

Mitchell, S., Abel, P., Ware, M., Stamp, G., Lalani, E. (2000), Phenotypic and genotypic characterization of commonly used human prostatic cell lines, BJU International, vol. 85, pp. 932-944

Mitchell, S., Abel, P., Ware, M., Stamp, G., Lalani, El N. (2000), Phenotypic and genotypic characterization of commonly used human prostatic cell lines, BJU International, vol. 85, pp. 932-944

Morell A. G, Gregoriadis G, Scheinberg I. H, Hickman J, Ashwell G. (1971), The role of sialic acid in determining the survival of circulating interferon, The Journal of Biological Chemistry, vol. 246, pp. 1461-1467

Moremen, K., Tiemeyer, M., Nairn, A. (2012), Vertebrate protein glycosylation: Diversity, synthesis and function, Nature reviews: Molecular cell biology, vol. 13, pp. 448-62.

Munkley, J. (2019), The glycosylation landscape of pancreatic cancer (Review), Oncology Letters, vol. 17, pp. 2569-2575

Munkley, J., Elliott, David J. (2016), Hallmarks of glycosylation in cancer, Oncotarget, vol. 7, pp. $35478-35489$

Munkley, J., Mills, Ian G, Elliott, D. J. (2016), The role of glycans in the development and progression of prostate cancer, Nature Reviews: Urology, vol. 13, pp. 324-333 
Munkley, J., Vodak, D., Livermore, K. E., James, K., Wilson, B. T., Knight, B., Mccullagh, P., Mcgrath, J., Crundwell, M., Harries, L. W., Leung, H. Y., Robson, C. N., Mills, I. G., Rajan, P., Elliott, D. J. (2016), Glycosylation is an Androgen-Regulated Process Essential for Prostate Cancer Cell Viability, EBioMedicine, vol. 8, pp. 103-116

Muramatsu, T. (2016), Basigin (CD147), a multifunctional transmembrane glycoprotein with various binding partners, Journal of Biochemistry, vol. 159, pp. 481-490

Murray, C. J. L., Lopez, A. D. (1997), Mortality by cause for eight regions of the world: Global Burden of Disease Study, Lancet, vo. 349, no. 9061, pp. 1269-1276.

Murugaesu, N., Iravani, M., Weverwijk, van, Ivetic, A., Johnson, D., Antonopoulos, A., Fearns, A., Jamal-Hanjani, M., Sims, D., Fenwick, K., Mitsopoulos, C., Gao, Q., Orr, N., Zvelebil, M., Haslam, S., Dell, A., Yarwood, H., Lord, C., Ashworth, A., Isacke, C. (2014), An in vivo functional screen identifies ST6GaINAc2 sialyltransferase as a breast cancer metastasis suppressor, Cancer discovery, vol. 4, pp. 304-17.

Nagae, M., Yamaguchi, Y. (2012), Function and 3D structure of the N-glycans on glycoproteins, International Journal of Molecular Sciences, vol. 13, pp. 8398-8429

Naghavi, M., Wang, H., Lozano, R., Davis, A., Liang, X., Zhou, M., Vollset, S. E.; Ozgoren, A. A.; Abdalla, S., Abd-Allah, F., et al. (2015), The Lancet, vol. 385, pp 117-71.

Naito, S., Takahashi, T., Onoda, J., Uemura, S., Ohyabu, N., Takemoto, H., Yamane, S., Fujii, I., Nishimura, Shin I., Numata, Y. (2017), Generation of Novel Anti-MUC1 Monoclonal Antibodies with Designed Carbohydrate Specificities Using MUC1 Glycopeptide Library, ACS Omega, vol. 2, pp. 7493-7505

Nangia-Makker, P., Conklin, J., Hogan, V., Raz, A. (2002), Carbohydrate-binding proteins in cancer, and their ligands as therapeutic agents, Trends in Molecular Medicine, vol. 8, pp. $187-192$ 
National Cancer Institute (NIH), Dictionary of Cancer Terms, [online] Available at: https://www.cancer.gov/publications/dictionaries/cancer-terms/def/molecular-medicine [Accessed 22 Jun. 2019]

Natoni, A., Macauley, M.S., O'Dwyer, M. E. (2016), Targeting selectins and their ligands in cancer, Frontiers in Oncology, vol. 6, pp. 1-12

Negm, R., Verma, M., Srivastava, S. (2002), The promise of biomarkers in colorectal cancer detection, TRENDS in Molecular Medicine, vol. 8, 288-293

Neve, R. M., Chin, K., Fridlyand, J., Yeh, J., Baehner, F. L., Fevr, T., Clark, L., Bayani, N., Coppe, J. P., Tong, F., Speed, T., Spellman, P. T., DeVries, S., Lapuk, A., Wang, N. J., Kuo, W. L., Stilwell, J. L., Pinkel, D., Albertson, D. G., Waldman, F. M., McCormick, F., Dickson, R. B., Johnson, M. D., Lippman, M., Ethier, S., Gazdar, A., Gray, J. W. (2006), A collection of breast cancer cell lines for the study of functionally distinct cancer subtypes, Cancer Cell, vol. 10, pp. $515-527$

Neves, A., Brindle, K. (2014), Imaging cell death, Journal of nuclear medicine: official publication, Society of Nuclear Medicine, vol. 55, pp. 1-4

Neves, A., Stöckmann, H., Harmston, R., Pryor, H., Alam, I., Ireland-Zecchini, H., Lewis, D., Lyons, S., Leeper, F., Brindle, K. (2011), Imaging sialylated tumor cell glycans in vivo, FASEB journal: official publication of the Federation of American Societies for Experimental Biology, vol. 25 , pp. 2528-37

Neves, A., Stöckmann, H., Wainman, Y., Kuo, J., Fawcett, S., Leeper, F. and Brindle, K. (2013), Imaging cell surface glycosylation in vivo using "double click" chemistry, Bioconjugate chemistry, vol. 24, pp. 934-41

Neves, A., Wainman, Y., Wright, A., Kettunen, M., Rodrigues, T., McGuire, S., Hu, D., Bulat, F., Crich, G., Stöckmann, H., Leeper, F., Brindle, K. (2015), Imaging Glycosylation in vivo by 
metabolic labeling and magnetic resonance imaging, Angewandte Chemie (International ed. in English), vol. 55, pp. 1286-90

Ng, R. C.Y., Roberts, A. N., Wilson, R. G., Latner, A. L., Turner, G. A. (1987), Analyses of protein extracts of human breast cancers: Changes in glycoprotein content linked to the malignant phenotype, British Journal of Cancer, vol. 55, pp. 249-254

Nimse, S. B., Sonawane, M. D., Song, K. S, Kim, T. (2016), Biomarker detection technologies and future directions, Analyst, vol. 141, pp. 740-755

Northcott, J. M., Dean, I. S., Mouw, J. K., Weaver, V. M. (2018), Feeling Stress: The Mechanics of Cancer Progression and Aggression, Frontiers in Cell and Developmental Biology, vol. 6, pp. a17

Ntziachristos, V. (2006), Fluorescence molecular imaging, Annual review of biomedical engineering, vol. 8, pp. 1-33

Ohtsubo, K., Marth, Jamey D. (2006), Glycosylation in Cellular Mechanisms of Health and Disease, Cell, vol. 126, pp. 855-867

Okato, A., Goto, Y., Kurozumi, A., Kato, M., Kojima, S., Matsushita, R., Yonemori, M., Miyamoto, K., Ichikawa, T., Seki, N. (2016), Direct regulation of LAMP1 by tumor-suppressive microRNA-320a in prostate cancer, International Journal of Oncology, vol. 49, pp. 111-122

Oliveira-Ferrer, L., Legler, K., Milde-Langosch, K. (2017), Role of protein glycosylation in cancer metastasis, Seminars in Cancer Biology, vol. 44, pp. 141-152

Pan, P. W., Zhang, Q. Hou, J., Liu, Z., Bai, F., Cao, Mei R., Sun, T., Bai, G. (2012), Cell surface glycoprotein profiling of cancer cells based on bioorthogonal chemistry, Analytical and Bioanalytical Chemistry, vol. 403, pp. 1661-1670 
Pan, S., Brentnall, T. A., Chen, R. (2016), Glycoproteins and glycoproteomics in pancreatic cancer, World Journal of Gastroenterology, vol. 22, pp. 9288-9299

Pandeya, N., McLeod, D. S., Balasubramaniam, K., Baade, P. D., Youl, P. H., Bain, C. J., Allison, R., Jordan, S. J. (2016), Increasing thyroid cancer incidence in Queensland, Australia 19822008 - True increase or overdiagnosis, Clinical Endocrinology, vol. 84, pp. 257-264

Parisotto, M., Metzger, D. (2013), Genetically engineered mouse models of prostate cancer, Molecular Oncology, vol. 7, pp. 190-205

Park, M., Lee, C., Lee, H. (2018), Mouse models of breast cancer in preclinical research, Laboratory Animal Research, vol. 34, pp. 160-165

Peixoto, A., Relvas-Santos, M., Azevedo, R., Lara Santos, L., Ferreira, José A. (2019), Protein glycosylation and tumor microenvironment alterations driving cancer hallmarks, Frontiers in Oncology, vol. 9, pp. 1-24

Peng, F., Li, H., Ning, Z., Yang, Z., Li, H., Wang, Y., Chen, F., Wu, Y. (2016), CD147 and Prostate Cancer: A Systematic Review and Meta-Analysis, PLoS ONE, vol. 11, pp. e0163678

Penny, L. K., Wallace, H. M. (2015), The challenges for cancer chemoprevention, Chemical Society Reviews, vol. 44, pp. 8836-8847

Peracaula, R., Barrabés, S., Sarrats, A., Rudd, P. M., De Llorens, R. (2008), Altered glycosylation in tumours focused to cancer diagnosis, Disease Markers, vol. 25, pp. 207-218

Peracaula, R., Tabarés, G., Royle, L., Harvey, David J., Dwek, R. A., Rudd, P. M., de Llorens, R. (2003), Altered glycosylation pattern allows the distinction between prostate-specific antigen (PSA) from normal and tumor origins, Glycobiology, vol. 13, pp. 457-470

Perik, P. J., Lub-De Hooge, M. N., Gietema, J. A., Van Der Graaf, Winette T.A., De Korte, M. A., Jonkman, S., Kosterink, Jos G.W., Van Veldhuisen, Dirk J., Sleijfer, Dirk T., Jager, P. L., De 
Vries, E. G.E. (2006), Indium-111-labeled trastuzumab scintigraphy in patients with human epidermal growth factor receptor 2-positive metastatic breast cancer, Journal of Clinical Oncology, vol. 24, pp. 2276-2282

Pértega-Gomes, N., Vizcaíno, J. R., Miranda-Gonçalves, V., Pinheiro, C., Silva, J., Pereira, H., Monteiro, P., Henrique, R. M., Reis, R. M., Lopes, C., Baltazar, F. (2011), Monocarboxylate transporter 4 (MCT4) and CD147 overexpression is associated with poor prognosis in prostate cancer, BMC Cancer, vol. 11, pp. 312

Peter, J., Unverzagt, C., Lenz, H., Hoesel, W. (1999), Purification of prostate-specific antigen from human serum by indirect immunosorption and elution with a hapten, Analytical Biochemistry, vol. 273, pp. 98-104

Petrova, Y. I., Schecterson, L., Gumbiner, Barry M. (2016), Roles for E-cadherin cell surface regulation in cancer, Molecular Biology of the Cell, vol. 27, pp. 3233-3244

Pienta, K. J., Abate-shen, C., Agus, D. B., Attar, R. M., Leland, W. K., Greenberg, N. M., Hahn, W. C., Isaacs, J. T., Navone, N. M., Peehl, D. M., Simons, J. W., Solit, D. B., Soule, H. R., Terry, A. (2008), Prostate, vol. 68, pp. 629-639

Pihikova, D., Kasak, P., Kubanikova, P., Sokol, R., Tkac, J. (2016), Aberrant sialylation of a prostate-specific antigen: Electrochemical label-free glycoprofiling in prostate cancer serum samples, Analytica Chimica Acta, vol. 934, pp. 72-79

Pijuan, J., Barceló, C., Moreno, David F., Maiques, O., Sisó, P., Marti, Rosa M., Macià, A., Panosa, A. (2019), In vitro Cell Migration, Invasion, and Adhesion Assays: From Cell Imaging to Data Analysis, Frontiers in Cell and Developmental Biology, vol. 7, pp. 1-16

Pinho, S., Reis, C. (2015), Glycosylation in cancer: Mechanisms and clinical implications, Nature reviews: Cancer, vol. 15, pp. 540-55. 
Potosky A. L., Kessler, L., Gridley, G., Brown, C. C., Horm, J. W. (1990), Rise in prostatic cancer incidence associated with increased use of transurethral resection, Journal of the National Cancer Institute, vol. 82, pp. 1624-1628

Prestegard, J. H., Liu, J., Widmalm, G. (2017), Oligosaccharides and Polysaccharides. In: Varki A, Cummings RD, Esko JD, et al., editors. Essentials of Glycobiology [Internet]. 3rd edition. Cold Spring Harbor (NY): Cold Spring Harbor Laboratory Press; 2015-2017. Chapter 3. Available from: https://www.ncbi.nlm.nih.gov/books/NBK453048/ doi: 10.1101/glycobiology.3e.003

Qu, Y., Han, B., Yu, Y., Yao, W., Bose, S., Karlan, Beth Y., Giuliano, Armando E., Cui, X. (2015), Evaluation of MCF10A as a reliable model for normal human mammary epithelial cells, PLoS ONE, vol. 10, pp. 1-16

Quigley, D. A., Dang, H. X., Zhao, S. G., Lloyd, P., Aggarwal, R., Alumkal, J. J., Foye, A., Kothari, V., Perry, M. D., Bailey, A. M., Playdle, D., Barnard, T. J., Zhang, L., Zhang, J., Youngren, J. F., Cieslik, M. P., Parolia, A., Beer, T. M., Thomas, G., Chi, K. N., Gleave, M., Lack, N. A., Zoubeidi, A., Reiter, R. E., Rettig, M. B., Witte, O., Ryan, C. J., Fong, L., Kim, W., Friedlander, T., Chou, J., Li, H., Das, R., Li, H., Moussavi-Baygi, R., Goodarzi, H., Gilbert, L. A., Lara, P. N. Jr, Evans, C. P., Goldstein, T. C., Stuart, J. M., Tomlins, S. A., Spratt, D. E., Cheetham, R. K., Cheng, D. T., Farh, K., Gehring, J. S., Hakenberg, J., Liao, A., Febbo, P. G., Shon, J., Sickler, B., Batzoglou, S., Knudsen, K. E., He, H. H, Huang, J., Wyatt, A. W., Dehm, S.M., Ashworth, A., Chinnaiyan, A. M., Maher, C. A., Small, E. J., Feng, F. Y. (2018), Cell, vol. 174, pp. 758-769

Quinn, M. J., D'Onofrio, A., Møller, B., Black, R., Martinez-Garcia, C., Møller, H., Rahu, M., Robertson, C., Schouten, L. J., La Vecchia, C., Boyle, P. (2003), Cancer mortality trends in the EU and acceding countries up to 2015, Annals of Oncology, vol. 14, pp. 1148-1152

Ravipaty, S., Wu, W., Dalvi, A., Tanna, N., Andreazi, J., Friss, T., Klotz, A., Liao, C., Garren, J., Schofield, S., Diamandis, E. P., Klein, E. A., Dobi, A., Srivastava, S., Tekumalla, P., Kiebish, M. A., Vishnudas, V., Sarangarajan, R. P., Narain, N. R., Akmaev, V. R. (2017), Clinical Validation 
of a Serum Protein Panel (FLNA, FLNB and KRT19) for Diagnosis of Prostate Cancer, Journal of Molecular Biomarker Design, vol. 8, pp. 323 (ePub)

Reily, C., Stewart, Tyler J., Renfrow, M. B., Novak, J. (2019), Glycosylation in health and disease, Nature Reviews: Nephrology, vol. 15, pp. 346-366

Renkonen, J., Tynninen, O., Häyry, P., Paavonen, T., Renkonen, R. (2002), Glycosylation might provide endothelial zip codes for organ-specific leukocyte traffic into inflammatory sites, American Journal of Pathology, vol. 2, pp. 543-550

Reticker-Flynn, N., Bhatia, S. (2015), Aberrant glycosylation promotes lung cancer metastasis through adhesion to galectins in the metastatic niche, Cancer discovery, vol. 5, pp. 168-81.

Reusch, D., Tejada, M. L. (2015), Fc glycans of therapeutic antibodies as critical quality attributes, Glycobiology, vol. 25, pp. 1325-1334

Rhodes, A., Jasani, B., Couturier, J., McKinley, M. J., Morgan, J. M., Dodson, A. R., Navabi, H., Miller, K. D., Balaton, A. J. (2002), A Formalin-Fixed, Paraffin-Processed Cell Line Standard for Quality Control of Immunohistochemical Assay of HER-2/neu Expression in Breast Cancer, American Journal of Clinical Pathology, vol. 117, pp. 81-89

Roehrborn, C. G., Black, L. K. (2011), The economic burden of prostate cancer, BJU International, vol. 108, pp. 806-813

Rush, J. S., Panneerselvam, K., Waechter, C. J., Freeze, Hudson H. (2000), Mannose supplementation corrects GDP-mannose deficiency in cultured fibroblasts from some patients with congenital disorders of glycosylation (CDG), Glycobiology, vol. 10, pp. 829-835

Russell P.J., Kingsley E.A. (2003) Human Prostate Cancer Cell Lines. In: Russell P.J., Jackson P., Kingsley E.A. (eds) Prostate Cancer Methods and Protocols. Methods in Molecular Medicine $^{\mathrm{TM}}$, vol 81. Springer, Totowa, NJ. Available at: https://link.springer.com/book/10.1385/1592593720 
Russell, P., Kingsley, E. (2003). Human Prostate Cancer Cell Lines. Methods in molecular medicine. 81. 21-39. 10.1385/1-59259-372-0:21

Sakamoto, K., Schmidt, J. W., Wagner, K. U. (2015), Mouse models of breast cancer, Methods in Molecular Biology, vol. 1267, pp. 47-71

Saldova, R., Fan, Y., Fitzpatrick, J. M., Watson, R. William G, Rudd, P. M. (2011), Core fucosylation and $\alpha 2-3$ sialylation in serum $\mathrm{N}$-glycome is significantly increased in prostate cancer comparing to benign prostate hyperplasia, Glycobiology, vol. 21, pp. 195-205

Saldova, R., Reuben, J., Hamid, A., Rudd, P. and Cristofanilli, M. (2011), Levels of specific serum $\mathrm{N}$-glycans identify breast cancer patients with higher circulating tumor cell counts, Annuals of oncology: official journal of the European Society for Medical Oncology, vol. 22, pp. 1113-9

Sarrats, A., Comet, J., Tabarés, G., Ramírez, M., Aleixandre, R. N., De Llorens, R., Peracaula, R. (2010), Differential percentage of serum prostate-specific antigen subforms suggests a new way to improve prostate cancer diagnosis, Prostate, vol. 70, pp. 1-9

Sarrats, A., Saldova, R., Comet, J., O'Donoghue, N., De Llorens, R., Rudd, Pauline M., Peracaula, R. (2010), Glycan characterization of PSA 2-DE subforms from serum and seminal plasma, OMICS A Journal of Integrative Biology, vol. 14, pp. 465-474

Satelli, A., Li, S. (2011), Vimentin as a potential molecular target in cancer therapy Or Vimentin, an overview and its potential as a molecular target for cancer therapy, Cellular and Molecular Life Sciences, vol. 68, pp. 3033-3046

Savaryn, J., Toby, T., Kelleher, N. (2016), A Researcher's Guide to Mass Spectrometry-Based Proteomics, Proteomics, vol. 16, pp. 2435-2443 
Sawa, M., Hsu, Tsui L., Itoh, T., Sugiyama, M, Hanson, S. R., Vogt, Peter K., Wong, Chi H. (2006), Glycoproteomic probes for fluorescent imaging of fucosylated glycans in vivo, Proceedings of the National Academy of Sciences of the United States of America, vol. 103, pp. $12371-12376$

Saxon, E., Bertozzi, C. R. (2000), Cell Surface Engineering by a Modi ed Staudinger Reaction, Science, vol. 287, pp. 2007-2010

Saxon, E., Luchansky, S. J., Hang, Howard, C., Yu, C., Lee, S. C., Bertozzi, C. R. (2002), Investigating cellular metabolism of synthetic azidosugars with the Staudinger ligation, vol. 124, pp. 14893-14902

Schedin-Weiss, S., Winblad, B., Tjernberg, L. O. (2014), The role of protein glycosylation in Alzheimer disease, FEBS Journal, vol. 281, pp. 46-62

Schmidt, K. T., Chau, C. H., Price, D. K., Figg, W. D. (2016), Precision Oncology Medicine: The Clinical Relevance of Patient-Specific Biomarkers Used to Optimize Cancer Treatment, Journal of Clinical Pharmacology, vol. 56, pp. 1484-1499

Schnaar, R. L. (2016), Concise Introduction to Glycobiology for the Allergist, Journal of Allergy and Clinical Immunology, vol. 135, pp. 609-615

Schröder, F. H., Hugosson, J., Roobol, M. J., Tammela, T. L. J., Ciatto, S., Nelen, V., Kwiatkowski, M., Lujan, M., Lilja, H., Zappa, M., Denis, L. J., Recker, F., Määpttänen, A. B., Bangma, Chris H., Aus, G., Villers, A., Rebillard, X., Van Kwast, T., Blijenberg, B. G., Moss, Sue M., De Koning, H. J., Auvinen, A. (2009), Screening and prostate-cancer mortality in a randomized European study, New England Journal of Medicine, vol. 260, pp. 1320-1328

Schröder, F. H., Hugosson, J., Roobol, M. J., Tammela, T. L.J., Ciatto, S., Nelen, V., Kwiatkowski, M., Lujan, M., Lilja, H., Zappa, M., Denis, L. J., Recker, F., Määpttänen, A. B., Bangma, C. H., Aus, G., Villers, A., Rebillard, X., Van Kwast, T., Blijenberg, B. G., Moss, S. M., 
De Koning, H. J., Auvinen, A. (2009), Screening and prostate-cancer mortality in a randomized european study, New England Journal of Medicine, vol. 360, pp. 1320-1328

Schultz, Matthew J., Swindall, Amanda F., Bellis, Susan L. (2012), Regulation of the metastatic cell phenotype by sialylated glycan's, Cancer and Metastasis Reviews, vol. 31, pp. 501-518

Seeberger PH. (2017), Monosaccharide Diversity. In: Varki A, Cummings RD, Esko JD, et al., editors. Essentials of Glycobiology [Internet]. 3rd edition. Cold Spring Harbor (NY): Cold Spring Harbor Laboratory Press; 2015-2017. Chapter 2. Available from: https://www.ncbi.nlm.nih.gov/books/NBK453086/ doi: 10.1101/glycobiology.3e.002

Seeling, M., Brückner, C., Nimmerjahn, F. (2017), Differential antibody glycosylation in autoimmunity: Sweet biomarker or modulator of disease activity?, Nature Reviews: Rheumatology, vol. 13 , pp. $621-630$

Serrao, E. M., Kettunen, M. I., Rodrigues, T. B., Dzien, P., Wright, A. J., Gopinathan, A., Gallagher, F. A., et al. (2015), MRI with hyperpolarised [1-13C]pyruvate detects advanced pancreatic preneoplasia prior to invasive disease in a mouse model, Gut, vol. 65, pp. 465-475

Sharma, S. (2009), Tumor markers in clinical practice: General principles and guidelines, Indian Journal of Medical and Paediatric Oncology, vol. 30, pp. 1-8

Sharon, N., Lis, H. (1989), Lectins as cell recognition molecules, Science, vol. 246, pp. 227-234

Shieh, Y., Eklund, M., Sawaya, G. F., Black, W. C., Kramer, Barnett S., Esserman, L. J. (2016), Population-based screening for cancer: Hope and hype, Nature Reviews Clinical Oncology, vol. 13 , pp. $550-565$

Shinde, S., El-Schich, Z., Malakpour, A., Wan, W., Dizeyi, N., Mohammadi, R., Rurack, K., Gjörloff Wingren, A., Sellergren, B. (2015), Sialic Acid-Imprinted Fluorescent Core-Shell Particles for Selective Labeling of Cell Surface Glycans, Journal of the American Chemical Society, vol. 137, pp. 13908-13912 
Singhai, R., Patil, V. W., Jaiswal, S. R., Patil, Shital D., Tayade, M. B., Patil, A. V. (2011), ECadherin as a diagnostic biomarker in breast cancer, North American Journal of Medical Sciences, vol. 3, pp. 227-233

Smith-Palmer, J., Takizawa, C., Valentine, W. (2019), Literature review of the burden of prostate cancer in Germany, France, the United Kingdom and Canada, BMC Urology, vol. 19, pp. 1-16

Smittenaar, C. R., Petersen, K. A., Stewart, K., Moitt, N. (2016), Cancer incidence and mortality projections in the UK until 2035, British Journal of Cancer, vol. 115, pp. 1147-1155

Soliman, C., Yuriev, E., Ramsland, Paul A. (2017), Antibody recognition of aberrant glycosylation on the surface of cancer cells, Current Opinion in Structural Biology, vol. 44, pp. 1-8

Soule, H. D., Vazguez, J., Long, A., Albert, S., Brennan, M., (1973), A human cell line from a pleural effusion derived from a breast carcinoma, Journal of the National Cancer Institute, vol. 51, pp. 1409-1416

Soysala, S., Tzankovb, A., Muenst, S. (2015), Role of the Tumor Microenvironment in Breast Cancer, Pathobiology, vol. 82, pp. 142-152

Spencer, J. P., Elbein, A. D. (1980), Transfer of mannose from GDP-mannose to lipid-linked oligosaccharide by soluble mannosyl transferase, Proceedings of the National Academy of Sciences of the United States of America, vol. 77, pp. 2524-2527

Spiciarich, D. R., Nolley, R., Maund, S. L., Purcell, S. C., Herschel, J., lavarone, A. T., Peehl, D. M., Bertozzi, C. R. (2017), Bioorthogonal Labeling of Human Prostate Cancer Tissue Slice Cultures for Glycoproteomics, Angewandte Chemie, vol. 56, pp. 8992-8997 
Springer, G. F., Desai, P. R., Banatwala, I. (1975), Blood group MN antigens and precursors in normal and malignant human breast glandular tissue, Journal of the National Cancer Institute, vol. 54, pp. 335-359

Springer, S. A., Gagneux, P. (2016), Glycomics: Revealing the Dynamic Ecology and Evolution of Sugar Molecules, Journal of Proteomics, vol. 135, pp. 90-100

St. Amand, M. M., Tran, K., Radhakrishnan, D., Robinson, A. S., Ogunnaike, B. A. (2014), Controllability analysis of protein Glycosylation in Cho cells, PLoS ONE, vol. 9, pp. e87973

Stamps, A. C., Davies, S.C., Burman, J., O'Hare, M. J. (1994), Analysis of proviral integration in human mammary epithelial cell lines immortalized by retroviral infection with a temperature-sensitive SV40 T-antigen construct, International Journal of Cancer, vol. 57, pp. 865-874

Stanley, P., Narasimhan, S., Siminovitch, L., Schachter, H. (1975), Chinese hamster ovary cells selected for resistance to the cytotoxicity of phytohemagglutinin are deficient in a UDP $N$ acetylglucosamine glycoprotein $\mathrm{N}$ acetylglucosaminyltransferase activity, Proceedings of the National Academy of Sciences of the United States of America, vol. 72, pp. 3323-3327

Stanley, P., Taniguchi, N., Aebi, M. (2017), N-Glycans. In: Varki A, Cummings RD, Esko JD, et al., editors. Essentials of Glycobiology [Internet]. 3rd edition. Cold Spring Harbor (NY): Cold Spring Harbor Laboratory Press; 2015-2017. Chapter 9. Available from:

https://www.ncbi.nIm.nih.gov/books/NBK453020/ doi: 10.1101/glycobiology.3e.009

Stöckmann, H., Neves, A.A., Stairs, S., Ireland-Zecchini, H., Brindle, K.M., Leeper, F.J. (2011), Development and evaluation of new cyclooctynes for cell surface glycan imaging in cancer cells, Chemical Science, vol. 2, pp. 932-936

Stowell, S. R., Ju, T., Cummings, R. D. (2015), Protein Glycosylation in Cancer, Annual Review of Pathology: Mechanisms of Disease, vol. 10. pp. 473-510 
Ström, P., Nordström, T., Aly, M., Egevad, L., Grönberg, H., Eklund, M. (2018), The Stockholm-3 Model for Prostate Cancer Detection: Algorithm Update, Biomarker Contribution, and Reflex Test Potential, European Urology, vol. 74, pp. 204-210

Tabarés, G., Jung, K., Reiche, J., Stephan, C., Lein, M., Peracaula, R., de Llorens, R., Hoesel, W. (2007), Free PSA forms in prostatic tissue and sera of prostate cancer patients: Analysis by 2DE and western blotting of immunopurified samples, Clinical Biochemistry, vol. 40, pp. 343350

Tai, S., Sun, Y., Squires, J. M., Zhang, H., Oh, W. K., Liang, C. Z., Huang, J. (2011), PC3 is a cell line characteristic of prostatic small cell carcinoma, Prostate, vol. 71, pp. 1668-1679

Taichman, R., Loberg, R., Mehra, R., Pienta, K. (2007), The evolving biology and treatment of prostate cancer, Journal of Clinical Investigation, vol. 117, pp. 2351- 2361

Tang, W., Chang, S., Hemler, M. (2004), Links between CD147 Function, Glycosylation, and Caveolin-1, Molecular Cell Biology, vol. 15, pp. 4043-4050

Tao, S. C., Li, Y., Zhou, J., Qian, J., Schnaar, R. L., Zhang, Y., Goldstein, I. J., Zhu, H., Schneck, J. P. (2008), Lectin microarrays identify cell-specific and functionally significant cell surface glycan markers, Glycobiology, vol. 18, pp. 761-769

Thalmann, G. N., Anezinis, P. E., Chang, S. M., Zhau, H. E., Kim, E. E., Hopwood, V. L., Pathak, S., von Eschenbach, A. C., Chung, L. W. (1994), Androgen-independent Cancer Progression and Bone Metastasis in the LNCaP Model of Human Prostate Cancer, Cancer Research, vol. 54, pp. $2577-2581$

Tkac, J., Gajdosova, V., Hroncekova, S., Bertok, T., Hires, M., Jane, E., Lorencova, L., Kasak, P. (2019), Prostate-specific antigen glycoprofiling as diagnostic and prognostic biomarker of prostate cancer, Interface Focus, vol. 9, pp. a 20180077 
Todeschini, R., Hakomori, S. (2008), Functional role of glycosphingolipids and gangliosides in control of cell adhesion, motility, and growth, through glycosynaptic microdomains, Biochimica et Biophysica Acta, vol. 1780, pp. 421-433

Turner, G. A., Skillen, A. W., Buamah, P., Guthrie, D., Welsh, J., Harrison, J., Kowalski, A. (1985), Relation between raised concentrations of fucose, sialic acid, and acute phase proteins in serum from patients with cancer: Choosing suitable serum glycoprotein markers, Journal of Clinical Pathology, vol. 38, pp. 588-592

Tutt, A. (2012), Molecular characterisation of cell line models for triple-negative breast cancers, BMC Genomics, vol. 13, pp. 1-14

Tuvesonlab.labsites.cshl.edu. (2019). Tuveson Lab | Protocols and Requests for Reagents. [online] Available at: http://tuvesonlab.labsites.cshl.edu/protocolsreagents/ [Accessed 15 Aug. 2019].

Uemura, T., Shiozaki, K., Yamaguchi, K., Miyazaki, S., Satomi, S., Kato, K., Sakuraba, H., Miyagi, T. (2009), Contribution of sialidase NEU1 to suppression of metastasis of human colon cancer cells through desialylation of integrin $\beta 4$, Oncogene, vol. 28, pp. 1218-1229

Vajaria, B. N., Patel, P. S. (2017), Glycosylation: a hallmark of cancer?, Glycoconjugate Journal, vol. 34, pp. 147-156

van Kooyk, Y. and Rabinovich, G.A. (2008), Protein-glycan interactions in the control of innate and adaptive immune responses, Nature Immunology, vol. 9, pp. 593-601

van Steenbrugge, G. J., Groen, M., van Dongen, J. W., Bolt, J., van der Korput, H., Trapman, J., Hasenson, M., Horoszewicz, J. (1989), The human prostatic carcinoma cell line LNCaP and its derivatives. An overview, Urology Research, vol. 17, pp. 71-77

Varki, A. (2017), Biological roles of glycans, Glycobiology, vol. 27, pp. 3-49 
Virji, M. A., Mercer, D. W., Herberman, R. B. (1988), Tumor Markers in Cancer Diagnosis and Prognosis, CA: A Cancer Journal for Clinicians, vol. 38, pp. 104-126

Wagenblast, E., Soto, M., Gutiérrez-Ángel, S., Hartl, C., Gable, A., Maceli, A., Erard, N., Williams, A., Kim, S., Dickopf, S., Harrell, J., Smith, A., Perou, C., Wilkinson, J., Hannon, G., Knott, S. (2015), A model of breast cancer heterogeneity reveals vascular mimicry as a driver of metastasis, Nature, vol. 520, pp. 358-62

Wallis, M. G. (2018), How do we manage overdiagnosis/overtreatment in breast screening?, Clinical Radiology, vol. 73, pp. 372-380

Wang, S., Yin, D., Wang, W., Shen, X., Zhu, J.-J., Chen, H.-Y., Liu, Z. (2016), Targeting and imaging of cancer cells via Monosaccharide-Imprinted fluorescent Nanoparticles, Nature Scientific Reports, vol. 6:22757

Warram, J. M., De Boer, E., Sorace, A. G., Chung, T. K, Kim, H., Pleijhuis, R.G., van Dam, Gooitzen M., Rosenthal, Eben LEben L R. (2014), Antibody Based Imaging Strategies of Cancer, Cancer and Metastasis Reviews, vol. 33, pp. 809-822

Wei, J., Xu, G., Wu, M., Zhang, Y., Li, Q., Liu, P., Zhu, T., Song, A., Zhao, L., Han, Z., Chen, G., Wang, S., Meng, L., Zhou, J., Lu, Y., Ma, D. (2008), Overexpression of vimentin contributes to prostate cancer invasion and metastasis via Src regulation, Anticancer Research, vol. 28, pp. $327-334$

Weigelt, B., Peterse, J., van 't Veer, L. (2005), Breast Cancer Metastasis: Markers and Models, Nature Reviews: Cancer, vol. 5, pp. 591-602

Weiner, L., Murray, J., Shuptrine, C. (2012), Antibody-based immunotherapy of cancer: New insights, new targets, Cell, vol. 148, pp. 1081-1084 
Westphalen, C. B., Olive, K. (2012), Genetically Engineered Mouse Models of Pancreatic Cancer: The KPC Model, its Variants and Their Application in Immuno-oncology Drug Discovery, Cancer J, vol. 18, pp. 502-510

Whittaker, T. (2015), Imaging Malignancy using Metabolic Glycan Imaging, Part III Dissertation (Unpublished)

Whittle, J., Lewis, M., Lindeman, G., Visvader, J. (2015), Patient-derived xenograft models of breast cancer and their predictive power, Breast Cancer Research, vol. 17, pp. 17

Wilson, M. J., Ruhland, A. R., Quast, B. J., Reddy, P. K., Ewing, S. L., Sinha, A. A. (2000), Dipeptidylpeptidase IV activities are elevated in prostate cancers and adjacent benign hyperplastic glands, Journal of Andrology, vol. 21, pp. 220-226

Wu, X., Gong, S., Roy-Burman, P., Lee, P., Culig, Z. (2013), Current mouse and cell models in prostate cancer research, Endocrine Related Research, vol. 20, pp. R155-R170

Xie, R., Dong, L., Huang, R., Hong, S., Lei, R. and Chen, X. (2014), Targeted imaging and proteomic analysis of tumor-associated glycans in living animals, Angewandte Chemie (International ed. in English), vol. 53, pp. 14082-6

Xie, R., Hong, S., Feng, L., Rong, J. and Chen, X. (2012), Cell-selective metabolic glycan labeling based on ligand-targeted liposomes, Journal of the American Chemical Society, vol. 134, pp. 9914-7

Yasmin-Karim, S., King, M. R., Messing, E. M., Lee, Y. F. (2014), E-selectin ligand-1 controls circulating prostate cancer cell rolling/adhesion and metastasis, Oncotarget, vol. 5, pp. $12097-12110$

Yokata, J. (2000), Tumor progression and metastasis Carcinogenesis, vol. 21, pp.497-503 Yu, X., Marshall, M. J.E., Cragg, Mark S., Crispin, M. (2017), Improving Antibody-Based Cancer Therapeutics Through Glycan Engineering, BioDrugs, vol. 31, pp. 151-166 
Zalcberg, J, Hu, X. F., Slater, A., Parisot, J., El-Osta, S., Kantharidis, P., Chou, S. T., Parkin, J. D. (2000), MRP1 not MDR1 gene expression is the predominant mechanism of acquired multidrug resistance in two prostate carcinoma cell lines, Prostate Cancer and Prostatic Disease, vol. 2, pp. 543-555

Zardavas, D., Irrthum, A., Swanton, C., Piccart, M. (2015), Clinical management of breast cancer heterogeneity, Nature Reviews: Clinical Oncology, vol. 12, pp. 381-394

Zhang, Z., Wuhrer, M., Holst, S. (2018), Serum sialylation changes in cancer, Glycoconjugate Journal, vol. 35, pp. 139-160

Zhou, C. K., Check, D. P., Lortet-Tieulent, J., Laversanne, M., Jemal, A., Ferlay, J., Bray, F., Cook, M. B., Devesa, S. S. (2016), Prostate cancer incidence in 43 populations worldwide: An analysis of time trends overall and by age group, International Journal of Cancer, vol. 138, pp. $1388-1400$

Zhou, Shu M., Cheng, L., Guo, Shu J., Wang, Y., Czajkowsky, Daniel M., Gao, H., Hu, Xiao F., Tao, Sheng C. (2015), Lectin RCA-I specifically binds to metastasis-associated cell surface glycans in triple-negative breast cancer, Breast Cancer Research, vol. 17, pp. 1-14

Zybailov, B., Mosley, A. L., Sardiu, M. E., Coleman, M. K., Florens, L., Washburn, M. P. (2006), Statistical Analysis of Membrane Proteome Expression Changes in Saccharomyces cerevisiae, Journal of Proteomic Research, vol. 5, pp. 2339-2347 